\title{
Dynamics of Langmuir Circulation in Oceanic Surface Layers
}

by

Anand Gnanadesikan

A.B. Princeton University, 1988

Submitted in Partial Fulfillment of the Requirements for the Degree of

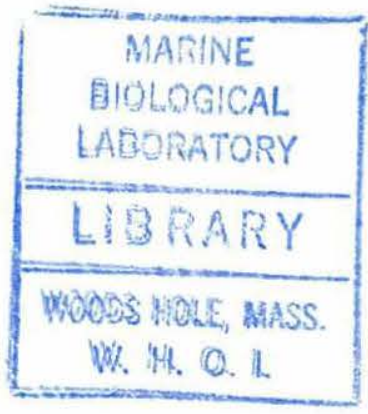

\author{
DOCTOR OF PHILOSOPHY
}

at the

\section{MASSACHUSETTS INSTITUTE OF TECHNOLOGY \\ and the \\ WOODS HOLE OCEANOGRAPHIC INSTITUTION}

September, 1994

(C)Anand Gnanadesikan

The author hereby grants to MIT and WHOI permission to reproduce and distribute copies of this thesis document in whole or in part

Signature of Author Joint Program in Physical Oceanography Massachusetts Institute of Technology/ Woods Hole Oceanographic Institution

Certified by

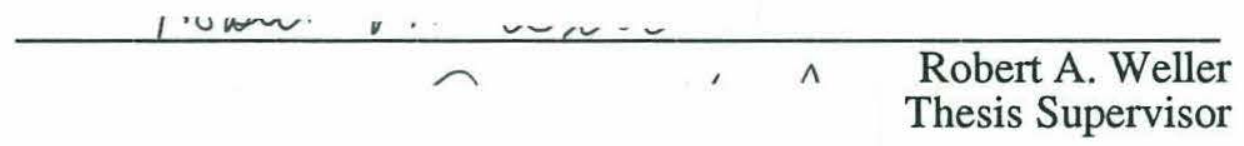

Accepted by

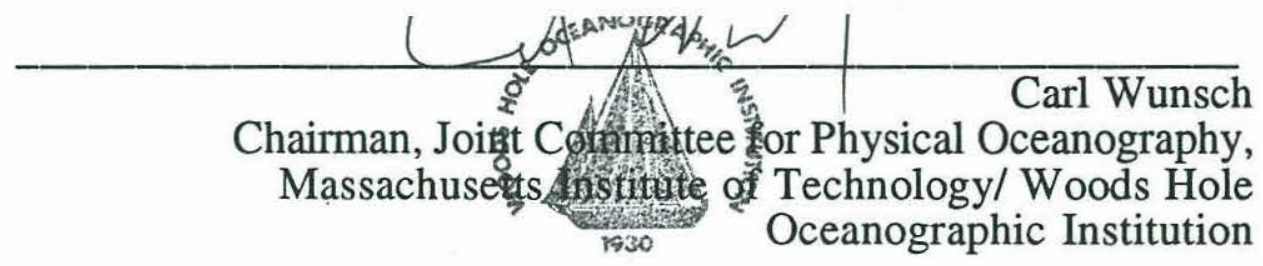




\title{
DYNAMICS OF LANGMUIR CIRCULATION IN OCEANIC SURFACE LAYERS
}

by

\author{
ANAND GNANADESIKAN \\ Submitted to the Joint Program in Physical Oceanography \\ in partial fulfillment of the requirements for the Degree of \\ Doctor of Philosophy in Oceanography
}

\begin{abstract}
This work investigates whether large-scale coherent vortex structures driven by wave-current interaction (Langmuir circulation) are responsible for maintaining the oceanic mixed layer. Langmuir circulations dominate the near-surface vertical transport of momentum and density when the characteristic scale for forcing (defined as the Craik-Leibovich instability parameter $\gamma_{\text {CLS }}$ ) is stronger than the characteristic scale for diffusive decay $\gamma_{\text {diff. }}$. Since the wave-current forcing is concentrated near the surface both terms depend on the cell geometry. Cells with long wavelengths penetrate more deeply into the water column. These cells grow more slowly than the fastest growing mode for most cases, but always dominate the solution in the absence of Coriolis forces. In the presence of Coriolis forces, the horizontal wavelength and thus the depth of penetration are limited. When a cell geometry is found such that $\gamma_{\text {CLS }}$ » $\gamma_{\text {diff, }}$, the current profile produced by smallscale diffusion is unstable to Langmuir cells and the cells replace small-scale diffusion as the dominant vertical transport mechanism for momentum and density. The perturbation crosscell shear is predicted to scale as $\gamma_{\text {CLS }}$. Such a scaling is observed during two field experiments. The observed velocity profile during these experiments is more sheared than predicted by a model which implicitly assumes instantaneous mixing by large eddies, but less sheared than predicted by a model which assumes small-scale mixing by near-isotropic turbulence. The latter profile is unstable to Langmuir cells when waves are present. The inclusion of cells driven by wave-current interaction explains the failure of the mixed layer to restratify on two days with high waves and low wind. Wave-current interaction introduces a small but efficient source of energy for transporting density which goes as the surface stress times the Stokes drift.
\end{abstract}

Thesis Supervisor: Robert A. Weller

Title: Senior Scientist, Department of Physical Oceanography, Woods Hole Oceanographic Institution 


\section{Acknowledgments}

While the errors and shortcomings of this work are my own, such strengths as may be found within it owe much to others.

My advisor Bob Weller played a huge role in this thesis. Much of our current knowledge of Langmuir cells is due to his persistence over the last two decades in pushing for accurate instrumentation with which to measure them, making the case time and again to funding agencies and the scientific community. Bob offered me the freedom to approach this rich and challenging problem in my own way, while providing tremendous resources and opportunities. He taught me the importance of grounding theoretical work in data, not simply assuming that the ocean behaves in a mathematically convenient way. He also was instrumental in teaching me to think scientifically.

Each member of my committee contributed to this document. Joe Pedlosky provided numerous ideas, comments, and suggestions for improving the theoretical chapters. His insistence on consistency and rigor has greatly improved this work. Paola Rizzoli's detailed comments on early drafts were instrumental in helping me to define critical ideas, and her encouragement was also important at critical times. Paola has the gift of being able to give criticism without making the recipient feel stupid. Dave Chapman was instrumental in getting me started on the numerical modelling portions of the thesis. Gene Terray was always a good sounding board for wacky ideas, and helped me formulate ideas about wavecurrent interaction and numerical modelling at a number of points. $\mathrm{Al}$ Plueddemann was consistently encouraging, and helped greatly in defining the issues for the data chapters.

A number of other scientists deserve special mention. Jim Price was a wonderful chairman of my defense, conscientous and thorough. Chris Garrett helped me in the early stages of my work to clarify the important questions.

The two datasets which I used were the result of a great deal of hard work by a large number of people. Jerry Dean, Erika Francis, Melora Park-Samelson, Rick Trask, and Bryan Way played especially critical roles in generating these data sets, as did the crew of the Research Platform FLIP. I would also like to thank those who shared data with me, in particular Jerry Smith and Rolf Lueck.

The Office of Naval Research supported me throughout graduate school, first as an ONR Graduate Fellow. and later as a research assistant under the Surface Waves Processes Program (ONR Grant N00014-90-J-1495). 
My deep thanks to friends, both from school and church, and family whose love and support helped me through graduate school. Special thanks are due to my wife Amalia. Her love, confidence, and support have kept me going. She also put long hours in editing this thesis, making innumerable suggestions to improve clarity and style, as well as laying out figure, proofreading, and just asking me questions and making me explain just what I was trying to say.

They that go down to the sea in ships, that do business in great waters. These see the works of the Lord, and his wonders in the deep.

Oh that men would praise the Lord for his goodness, and for his wonderful works to the children of men!

Psalms 107:23-24,31 


\section{Table of Contents}

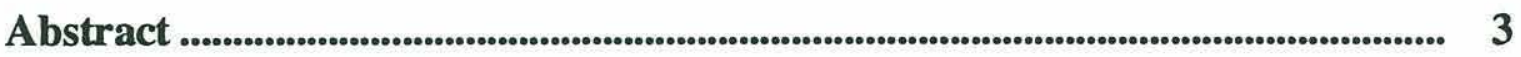

Acknowledgments ...................................................................................................... 5

Table of Contents ........................................................................................................... 7

Chapter 1: Langmuir Circulation in the Oceanic Surface Layer ................. 9

Chapter 2: The Instability of Langmuir Cells in Fluid Layers with

No Coriolis Forces ................................................................................................................ 22

Chapter 3: Structure and Instability of an Ekman Spiral in the Presence

of Surface Gravity Waves ...................................................................................................... 50

Chapter 4: The Spatial Scale of Equilibrium Langmuir Circulations ......... 92

Chapter 5: The Velocity and Density Structure of Fluid Layers with

Finite-Amplitude Langmuir Circulations ............................................................ 129

Chapter 6: Langmuir Circulation during the Mixed Layer Dynamics

Experiment ....................................................................................................................................170

Chapter 7: Langmuir Circulation during the Surface Waves Processes

Program .................................................................................................................................................225

Chapter 8: Finite-Amplitude Langmuir Circulation during MILDEX and

SWAPP ............................................................................................................................. 274

Chapter 9: Conclusions and Discussion ............................................................................... 299

Appendix A: Derivation of Huang's Equations ..............................................................310

Appendix B: The Spectral Instability Code ..................................................................... 325

Appendix C: The Finite-Difference Code ......................................................................... 331

Appendix D: Measurement Errors for Current Meters on FLIP.................... 332

Appendix E: Thruster Contamination during MILDEX ....................................... 339

References ............................................................................................................................................. 344

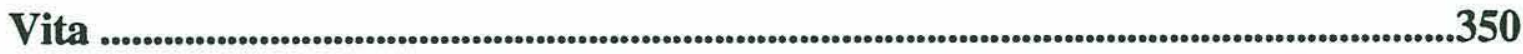




\section{Chapter 1: Langmuir Circulation in the Oceanic Surface Layer}

\subsection{Introduction: The Oceanic Surface Layer}

Oceanographers have long been aware that the uppermost layer of oceans and lakes is relatively well mixed in contrast with the strongly stratified main thermocline lying directly below it. In the ocean this surface layer is home to the majority of primary production and the site of almost all oceanic photochemistry. It connects the atmosphere and the deep ocean and is involved in a vast number of biological, physical, and chemical processes of interest to oceanographers.

At many times, the upper portion of the surface layer is well-mixed with respect to conservative scalar quantities like temperature and salinity. This mixed layer is not, however, well-mixed with respect to velocity. Figure 1.1a shows typical one-hour average profiles of velocity and temperature within the surface layer. The data shown were taken off the Research Platform FLIP in 1983. While the temperature varies less than 0.01 degrees down to 40 meters, it is hard to determine the mixed layer base from the velocity measurements. The mixed layer also contains relatively large time-varying shears. Figure $1.1 \mathrm{~b}$ shows a one-hour time series of the velocity difference between $4.5 \mathrm{~m}$ and $6.75 \mathrm{~m}$ in an unstratified mixed layer. The data were also taken off FLIP. The data were band-passed for periods between 100 and 10000 seconds to eliminate the effect of surface gravity waves and inertial oscillations and subsampled to one sample per minute. There are velocity differences of several $\mathrm{cm} / \mathrm{s}$ within the layer which display noticeable variability over time.

There are at present two views of how the mixed layer is maintained. One view (exemplified by the work of Mellor and Yamada, 1974; Klein and Coste, 1984) holds that the processes responsible have small spatial scales in comparison with the layer depth. The trajectory of a particle within the mixed layer is a random walk as it is passed from one small eddy to another. Models based on this view parameterize mixing in terms of a small-scale eddy viscosity and produce horizontally averaged velocity profiles with a great deal of shear within a relatively isothermal mixed layer. The other view (exemplified by the work of Davis et al., 1981; Price et al., 1986) holds that motions with vertical and horizontal scales comparable to the mixed layer are responsible for its maintainence. Available models which adhere to this view, however, postulate that 

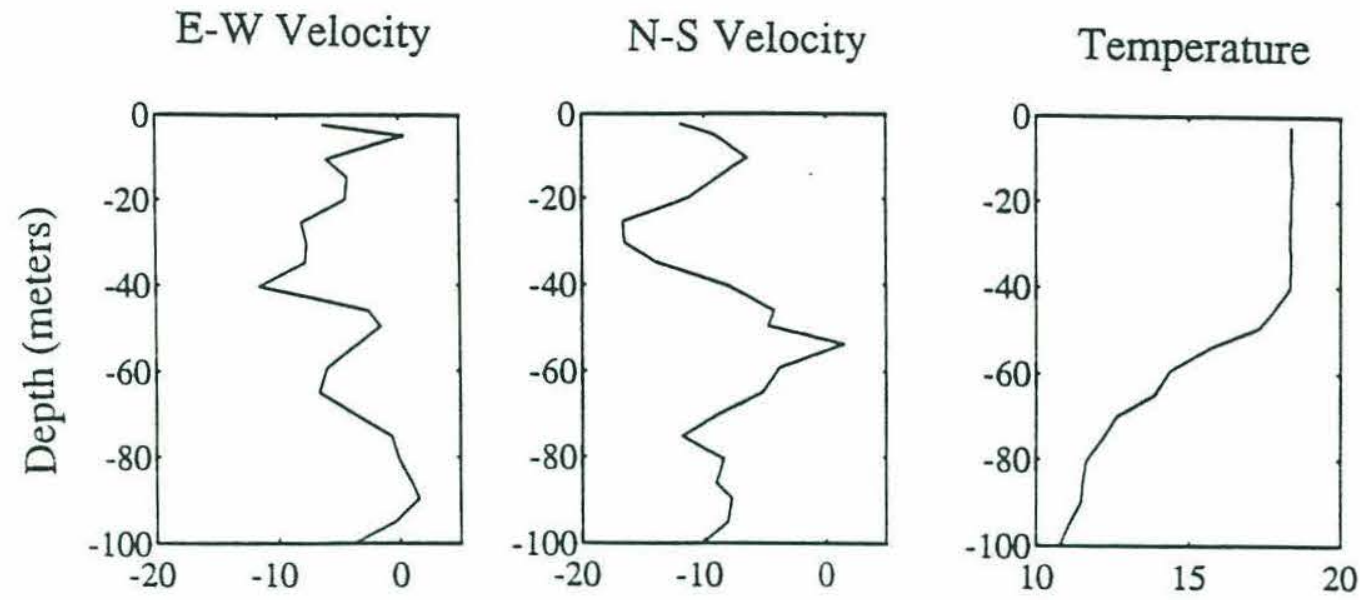

Velocity in $\mathrm{cm} / \mathrm{s}$

Velocity in $\mathrm{cm} / \mathrm{s}$

(a)

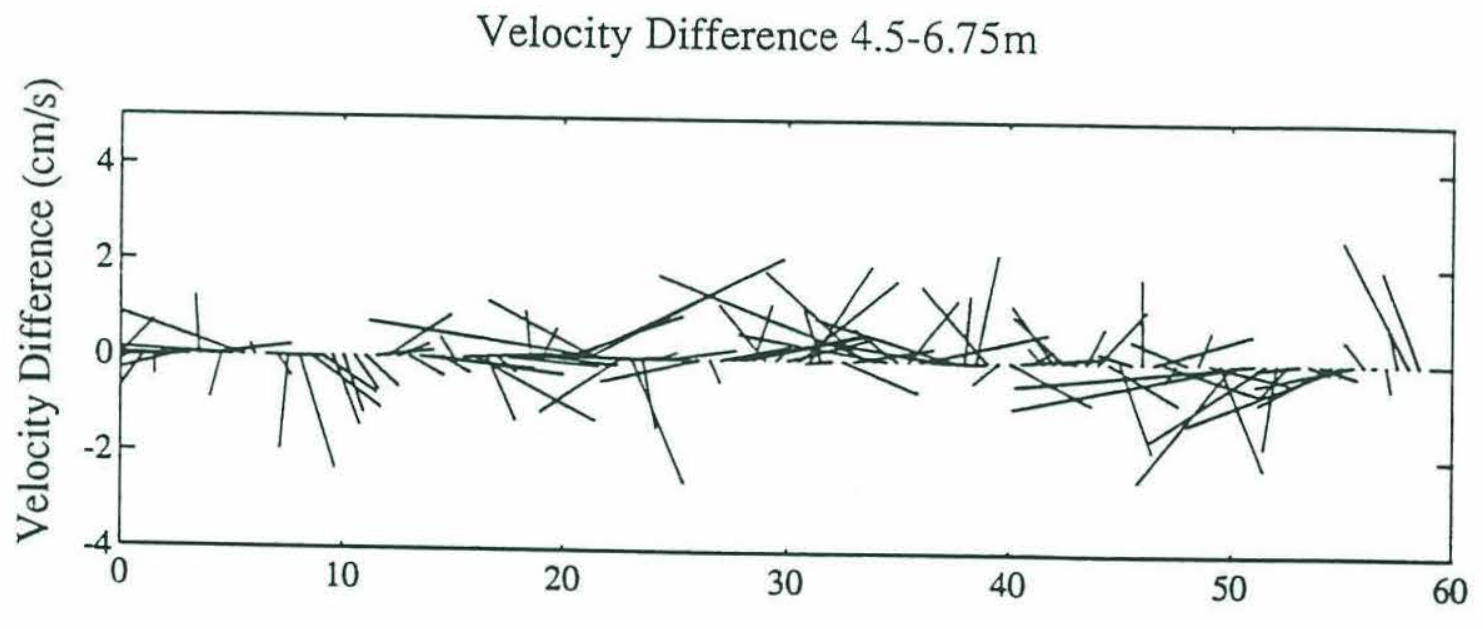

Time in Minutes

(b)

Figure 1.1: Velocity Structure within oceanic mixed layers. (a) Hourly-averaged profiles of velocity in the east (left panel), and north (central panel) direction and temperature (right panel). Profiles are averaged from 1100-1200 local time on November 9th, 1983 off of R/P FLIP. (b) Stick plot of the velocity difference between 4.5 and $6.75 \mathrm{~m}$. Velocity data was collected at $0.5 \mathrm{~Hz}$, band-passed for frequencies in the $0.01-0.0001 \mathrm{~Hz}$ band and subsampled to once per minute. Start time is $0000 \mathrm{Z}$ on March 5th, 1990. 
the mixed layer is a slab within which the horizontally-averaged velocity is completely homogenized. In the real world, however, there are persistent velocity differences within the mixed layer (as seen in Figure 1.1).

This thesis focuses on a mixing process involving eddies with spatial scales comparable to the mixed layer depth, the two-dimensional roll vortices known as Langmuir circulation or Langmuir cells. The following questions are asked: -Under what conditions do Langmuir cells replace small-scale mixing as the principal mechanism by which the mixed layer is stirred?

-When do Langmuir cells produce large spatially and temporally-varying velocity shears within the mixed layer?

-How do the cells affect the energy balance of the mixed layer?

In order to answer these questions certain subsidiary issues must be addressed:

-When are Langmuir cells present in the mixed layer?

-What is the spatial structure of the cells?

-What is the equilibrium population of cells?

Before plunging into the strategies which are used to answer these questions, some observational studies of Langmuir circulation are considered. These studies demonstrate that the cells are of the appropriate scale to affect the dynamics of the mixed layer.

\subsection{Observations of Langmuir Cells}

Langmuir (1938) was the first to make quantitative observations of the circulations which bear his name. Motivated by personal observations from an ocean liner of rows of seaweed and debris lined up with the wind, he established many of the major features of the cells in an ingenious series of experiments on Lake George. Figure 1.2 shows a schematic of Langmuir circulation, illustrating some of the major features established by Langmuir and subsequent investigators. The circulation involves roll vortices whose axes are horizontal and oriented at an angle $\alpha$ relative to the wind. The vortices have width $\mathrm{L}_{\text {cell }} / 2$. The typical velocities associated with these rolls are denoted by $\mathrm{U}$ in the crosscell ( $\mathrm{x}$ ) direction and $\mathrm{W}_{\mathrm{up}}$ and $\mathrm{W}_{\text {down }}$ in the vertical (z).The vortices are in general asymmetric, with downwelling velocities exceeding upwelling velocities. The downwelling zones are associated with jets of water of width $\mathrm{L}_{\text {jet }}$ and characteristic perturbation velocity $\mathrm{V}_{\text {jet }}$ moving in the alongcell (+y) direction. The depth of the cells is $\mathrm{D}$.

Often, the cells are associated with a surface layer in which there is a large velocity shear. The characteristic velocity in this layer is denoted in Figure 1.2 by 
$\mathrm{V}_{\text {surf }}$ and the depth of this layer by $\mathrm{D}_{\text {surf }}$. Although the figure shows $\mathrm{D}_{\text {surf }}$ as being much smaller than the cell depth $\mathrm{D}$, there are some published cases (e.g. Van Straaten,1950) where the cells are embedded within the shear layer. Velocities within the shear layer are often of order $10 \mathrm{~cm} / \mathrm{s}$.

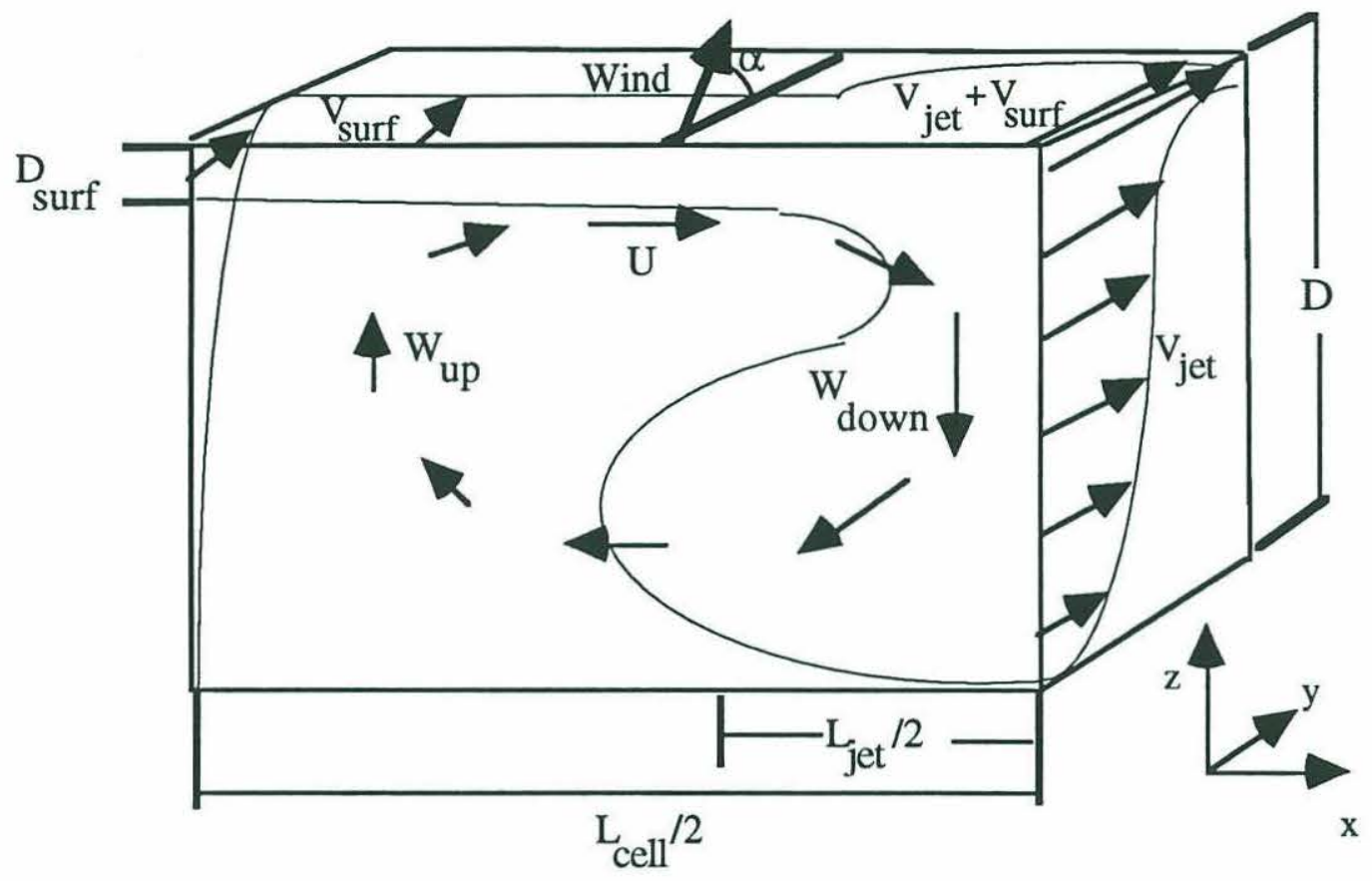

Figure 1.2: A schematic of Langmuir circulation illustrating the concepts found in the text

Properties of the cells have been described in the literature as follows:

- Geometry: The cells vary much more slowly in the alongcell than in the crosscell direction. Estimates of the ratio of alongcell length to crosscell spacing $\mathrm{L}_{\text {cell }}$ range from 3-4 (Thorpe, 1993) to of order 100 (Kenney, 1977). Cell spacing scales as the depth of the fluid (Van Straaten,1950, for cells seen on a tidal flat) or as the mixed layer depth (Smith et al., 1987). $\alpha$ ranges from 0-20 degrees (Faller, 1964).

- Vertical Velocities: Early observations of vertical velocities were made in lakes or in relatively calm conditions fairly close to surface, and the velocities seen were roughly $1-4 \mathrm{~cm} / \mathrm{s}$ in the downwelling regions, and about $1-2 \mathrm{~cm} / \mathrm{s}$ in the upwelling regions (Langmuir, 1938; Gordon, 1970). Recent work in more strongly forced layers (Weller et al.,1985, Weller and Price, 1988, Zedel and Farmer, 1991) has demonstrated the existence of stronger vertical velocities of order $5-25 \mathrm{~cm} / \mathrm{s}$. - Horizontal Velocities: Velocities associated with the downwind jets have been estimated to be quite large, generally falling in the $5-10 \mathrm{~cm} / \mathrm{s}$ range (Langmuir, 
1938; Harris and Lott, 1973; Kenney, 1977; Ryanzhin, 1983; Smith et al., 1987). Crosscell velocities have been less frequently measured, but estimates of their magnitude fall into the same general range.

- Occurrence: Cells are often seen in stormy conditions where the waves and wind stress are large and where the surface is being cooled (Weller and Price,1988) but they have also been observed at wind speeds of 2-4 m/s (Owen, 1966; Scott, 1970; Kenney, 1977) when the stratification was stable (Faller and Woodcock,1964) and when the mixed layer was being heated (Kenney,1977).

- Associated Phenomena: A number of investigators (Langmuir, 1938; Woodcock, 1944; Sutcliffe et al., 1963) report that seaweed or other biological debris is swept into surface convergence zones. Thorpe (1984), Smith et al (1987), and Zedel and Farmer (1991) show that bubbles generated by breaking waves are swept into cell convergence zones, producing curtains of bubbles which can be detected with sonars.

The cells as presented above are of the right order of magnitude to cause strong vertical transports of momentum and large horizontal variability in the mixed layer transport. This can be demonstrated as follows. Consider the vertical transport of momentum. The vertical and alongwind velocities associated with the cells are correlated. Let the momentum flux carried by the cells be defined as ${\rho u_{*}^{2}}_{2}^{2}$, The downward transport of momentum in the downwelling zone may be estimated as $-\rho \mathrm{W}_{\text {down }} \mathrm{V}_{\text {jet }}$ The transport of momentum in the upwelling zone $-\rho W_{\text {up }} \mathrm{V}_{\text {up }}$ may be estimated by noting that $\mathrm{W}_{\text {down }} \mathrm{L}_{\text {jet }}=\mathrm{W}_{\text {up }}\left(\mathrm{L}_{\text {cell }}-\mathrm{L}_{\text {jet }}\right)$ and $\mathrm{V}_{\text {up }}=$ $\mathrm{V}_{\text {jet }} \mathrm{L}_{\text {jet }} /\left(\mathrm{L}_{\text {cell }}-\mathrm{L}_{\text {jet }}\right)$ since $\mathrm{V}_{\text {up }}, \mathrm{V}_{\text {jet }}, \mathrm{W}_{\text {down }}$, and $\mathrm{W}_{\text {jet }}$ are all perturbations from the mean mixed layer velocity. Averaging over the width of the cell:

$$
\mathrm{u}_{* \mathrm{La}}^{2}=\frac{1}{\mathrm{~L}_{\text {cell }}} \int_{0}^{\mathrm{L}_{\text {cell }}} \mathrm{v}^{\prime} \mathrm{w}^{\prime} \mathrm{dx} \sim 2 \mathrm{~V}_{\text {jet }} \mathrm{W}_{\text {down }} \mathrm{L}_{\text {jet }} / \mathrm{L}_{\text {cell }}
$$

If $\mathrm{V}_{\text {jet }}$ and $\mathrm{W}_{\text {down }}$ are approximately $2 \mathrm{~cm} / \mathrm{s}$ and $\mathrm{W}_{\text {up }}$ is of order $1 \mathrm{~cm} / \mathrm{s}$, then since $\mathrm{L}_{\mathrm{jet}} / \mathrm{L}_{\text {cell }}=1 / 3, \mathrm{u}_{* \mathrm{La}}^{2}$ is $1.3 \mathrm{~cm}^{2} / \mathrm{s}^{2}$. This corresponds to a the stress caused by a wind of about $7.5 \mathrm{~m} / \mathrm{s}$ - a fairly stiff breeze. Larger estimates for cell velocities yield larger estimates for momentum fluxes. Langmuir cells are of the right order of magnitude to transport momentum within the mixed layer.

The variability in horizontal transport associated with Langmuir cells can also be large compared to the mean Ekman transport. We can estimate the amplitude of this variability by estimating the size of the transport relative to the base of the mixed layer carried in the jets. This "jet transport" $\mathrm{M}_{\text {jet }}$ is defined as 


$$
\mathrm{M}_{\mathrm{jet}}=\mathrm{V}_{\mathrm{jet}} * \mathrm{D}_{\mathrm{jet}} * \frac{\mathrm{L}_{\mathrm{jet}}}{\mathrm{L}_{\mathrm{cell}}}
$$

with these quantities are defined as in Figure 1.2. The Ekman transport (the total volume transport when the surface stress is wholly balanced by Coriolis force). is

$$
\mathrm{M}_{\text {ekman }}=\frac{\tau}{\rho f}
$$

where $\tau$ is the surface stress, $\rho$ the density and $f$ the Coriolis force. Table 1.1 shows a comparison of these two quantities for several published observations of Langmuir cells. $\mathrm{M}_{\mathrm{jet}}$ is often large compared with $\mathrm{M}_{\mathrm{ekman}}$. This does not mean that the cells alter the value of the Ekman transport, but that the structure of this transport is strongly influenced by the presence of cells. Once again Langmuir cells can determine the velocity structure within the mixed layer.

\begin{tabular}{|l|l|l|l|}
\hline Source & $\begin{array}{l}\text { Jet Transport } \\
\mathrm{M}_{\text {iet }}\left(\mathrm{m}^{2} \mathrm{~s}^{-1}\right)\end{array}$ & $\begin{array}{l}\text { Ekman Transport } \\
\mathrm{M}_{\text {ekman }}\left(\mathrm{m}^{2} \mathrm{~s}^{-1}\right)\end{array}$ & $\begin{array}{l}\text { Ratio } \\
\mathrm{M}_{\text {iet }} / \mathrm{M}_{\text {ekman }}\end{array}$ \\
\hline Langmuir (1938) & $0.06-0.33$ & $0.37-1.5$ & $0.04-0.8$ \\
\hline Gordon (1970) & $0.16-0.42$ & 0.4 & $0.4-1.0$ \\
\hline Kenney (1977) & $0.1-0.2$ & $0.2-1.0$ & $0.1-1.0$ \\
\hline Ryanzhin (1983) & 0.3 & 0.46 & 0.65 \\
\hline Weller and Price (1988) & $1.0-4.0$ & $1.8-3.5$ & $0.3-2.0$ \\
\hline
\end{tabular}

Table 1.1: Relative size of the Ekman transport and the variability in that transport due to Langmuir cells.

\subsection{Equations for mixed layer evolution and Langmuir circulation}

While Langmuir cells were long thought to play a critical role in upper ocean mixing, the dynamics of the instability process giving rise to the cells remained obscure for almost forty years. The situation was rectified in the 1970s by a series of papers (Craik, 1970; Craik and Leibovich, 1976; Leibovich,1977a,b; Huang,1979) which developed a set of equations for the evolution of a layer of fluid in the presence of surface gravity waves, stratification, Coriolis forces, and Langmuir cells. The equations are presented below as developed by Huang (1979).

$$
\begin{gathered}
\frac{\partial \Omega}{\partial \mathrm{t}}+\left(\mathrm{u}+\mathrm{u}_{\mathrm{s}}\right) \frac{\partial \Omega}{\partial \mathrm{x}}+\mathrm{w} \frac{\partial \Omega}{\partial \mathrm{z}}=\mathrm{F}\left(\frac{\partial \mathrm{v}}{\partial \mathrm{z}}+\frac{\partial \mathrm{v}_{\mathrm{S}}}{\partial \mathrm{z}}\right)+\frac{\partial \mathrm{v}_{\mathrm{s}} \partial \mathrm{v}}{\partial \mathrm{z}}+\mathrm{Ri} \frac{\partial \rho}{\partial \mathrm{x}}+\mathrm{La} \nabla^{2} \Omega \\
\frac{\partial \mathrm{v}}{\partial \mathrm{t}}+\left(\mathrm{u}+\mathrm{u}_{\mathrm{s}}\right) \frac{\partial \mathrm{v}}{\partial \mathrm{x}}+\mathrm{w} \frac{\partial \mathrm{v}}{\partial \mathrm{z}}=-\frac{1 \partial \mathrm{p}}{\rho \partial \mathrm{y}}-\mathrm{F}\left(\mathrm{u}+\mathrm{u}_{\mathrm{S}}\right)+\mathrm{La} \nabla^{2} \mathrm{v}
\end{gathered}
$$




$$
\begin{gathered}
\frac{\partial \rho}{\partial \mathrm{t}}+\left(\mathrm{u}+\mathrm{u}_{\mathrm{s}}\right) \frac{\partial \rho}{\partial \mathrm{x}}+\mathrm{w} \frac{\partial \rho}{\partial \mathrm{z}}=\mathrm{La} \nabla^{2} \rho \\
\Omega=\nabla^{2} \psi \\
\frac{\partial \psi}{\partial \mathrm{x}}=-\mathrm{w} \quad \frac{\partial \psi}{\partial \mathrm{z}}=\mathrm{u} \\
\mathrm{La}=\frac{\mathrm{ve}_{\mathrm{e}}}{\mathrm{a}^{2} \sigma} \quad \mathrm{F}=\frac{\mathrm{f}}{\mathrm{k}_{\mathrm{w}}^{2} \mathrm{a}^{2} \sigma} \quad \mathrm{Ri}=\frac{\mathrm{N}^{2}}{\mathrm{k}_{\mathrm{w}}^{4} \mathrm{a}^{4} \sigma^{2}} \\
\mathrm{k}_{\mathrm{w}}^{-1}(\mathrm{x}, \mathrm{y}, \mathrm{z})=(x, y, z) \\
\left(\mathrm{k}_{\mathrm{w}} \mathrm{a}\right)^{2} \frac{\sigma}{\mathrm{k}_{\mathrm{w}}}\left(\mathrm{u}, \mathrm{u}_{\mathrm{s}}, \mathrm{v}, \mathrm{v}_{\mathrm{S}}, \mathrm{w}\right)=\left(u, u_{s}, v, v_{s}, w\right) \\
\frac{1}{\mathrm{k}_{\mathrm{w}}^{2} \mathrm{a}^{2} \sigma} \mathrm{t}=t
\end{gathered}
$$

In the above equations, $\mathrm{k}_{\mathrm{w}}, \mathrm{a}$, and $\sigma$ are the wavenumber, amplitude and frequency of the driving surface gravity waves. $v_{\mathrm{e}}$ is the eddy viscosity, $\mathrm{f}$ the Coriolis parameter, $\mathrm{N}$ the buoyancy frequency, and $\mathrm{u}_{\mathrm{S}}$ and $\mathrm{v}_{\mathrm{S}}$ the Stokes Drift in the crosscell ( $+\mathrm{x})$ and alongcell $(+\mathrm{y})$ directions respectively. $\mathrm{u}$ and $\mathrm{w}$ are the horizontal and vertical velocities respectively in the crosscell (xz) plane. $\Omega$ and $\mathrm{v}$ are the vorticity and velocity respectively in the alongcell direction. Equations 1-4(a-e) are for dimensionless quantities, with equations 1-4(g-i) giving the conversion from dimensionless distance, velocity and time to dimensional (italicized) form. Equation (1-4f) defines three important dimensionless numbers. La is the Langmuir number, which is a scaled eddy viscosity or inverse Reynolds number. F is a scaled Coriolis parameter. $\mathrm{Ri}$ is the Richardson number, the square of the ratio between a characteristic buoyancy frequency and a characteristic Stokes drift shear.

These equations are derived from a perturbation expansion (presented in full in Appendix A) in which the following scaling assumptions are made.

1. Cell velocities are small (of order $\varepsilon=\mathrm{k}_{\mathrm{w}} \mathrm{a}$ ) in comparison with wave orbital velocities.

2. The cells evolve on time scales which are slow (order $\varepsilon^{2}$ ) in comparison with the wave frequency.

3. The cells are capable of carrying vertical fluxes of horizontal momentum of the same order as the surface stress $\left.\mathrm{La} \frac{\partial \mathrm{V}}{\partial \mathrm{z}}\right|_{\mathrm{z}=0}$. 
4. The Coriolis force is of the right order to balance the surface stress.

5. The cells are capable of carrying density fluxes of the same order as the surface density flux $\left.L \frac{\partial \rho}{\partial z}\right|_{z=0}$.

6. The turbulent eddy viscosity and eddy diffusivity are the same.

In combination, assumptions 3 and 4 mean that nonlinear terms $\frac{\partial v}{\partial z}$ must be of the same order as the Coriolis force terms and the diffusive terms.

In addition to the scaling assumptions, Huang's equations contain one other major assumption, that of a constant mixing coefficient. This is in many ways the weakest part of the equations. In the presence of wave breaking, for example, the mixing would be stronger near the surface, while in the presence of KelvinHelmholtz instability at the mixed layer base it would be stronger there. When interpreting results derived from these equations, the Langmuir number should be thought of as setting the order of magnitude of the diffusive decay.

The approximation of constant mixing coefficient has a major effect on the density profile. In the absence of cells, the only possible steady-state solutions are those for which

$$
\nabla^{2} \rho=\frac{\partial^{2} \rho}{\partial z^{2}}=0
$$

Thus the only possible solutions are those for which the density profile is constant or linear. In the absence of cells, the equations cannot support a solution with a thermocline and a mixed layer. Whether cells are present or not, at steady-state the density flux must be constant with depth. This is an unrealistic representation of mixed layer density evolution given that the time scales for mixing density through the thermocline (which are of order weeks or months) are different from the time scales for adding heat to the mixed layer (of order hours or days).

The appearance of the Stokes drift in equations (1-4a,b) demands some extra explanation. The Stokes drift arises because irrotational surface gravity waves have larger alongwave velocities at the crest of the wave than at the trough. Averaging over a wave period, the mean velocity following a particle is nonzero, even though the mean Eulerian velocity at depths below the wave zone is zero (see Phillips, 1960 for a discussion). Given a monochromatic deep-water wave with wavenumber $\mathrm{k}_{\mathrm{w}}$, frequency $\sigma$, and amplitude a, the Stokes drift is

$$
\mathrm{v}_{\mathrm{S}}(\mathrm{z})=\mathrm{k}_{\mathrm{w}} \mathrm{a}^{2} \sigma \exp \left(2 \mathrm{k}_{\mathrm{w}} \mathrm{z}\right)
$$

This Lagrangian drift acts to tilt both planetary and relative vortex lines. In an Eulerian framework, this vortex interaction arises through nonlinear interactions 
between the Eulerian-mean vorticity and the wave orbital velocity. Figure 1.3 illustrates these interactions for a vortex tube oriented in the $+\mathrm{z}$ direction for surface gravity waves propagating in the $+y$ direction. In the presence of surface gravity waves, this tube is stretched at the wave crests, compressed at the wave trough and tilted in between. This means that vorticity perturbations in the $y$ direction $\Omega^{\prime}$ are created which are in phase with the vertical wave velocity $w^{\prime}$, and with the divergence of the horizontal wave velocity $\partial v^{\prime} / \partial y$. Likewise vorticity perturbations in the vertical direction $z^{\prime}$ are created in phase with the vertical shear associated the waves $\partial v^{\prime} / \partial z$. As a result there are mean sources of vorticity $\frac{\partial}{\partial z} \overline{\Omega^{\prime} w^{\prime}}$, $\overline{\Omega^{\prime} \partial v^{\prime} / \partial \mathrm{x}}$, and $\overline{\zeta^{\prime} \partial \mathrm{v}^{\prime} / \partial \mathrm{z}}$ where the overbar denotes averaging over a wave period. These terms go as the square of the wave velocity shear, which goes as the Stokes drift shear.

The research presented here differs from previous work in several ways. -It focuses on cases where the bottom boundary is a no-stress boundary. This is based on the observations (Weller ,1981; Price, Weller, and Schudlich, 1987) showing that the momentum balance can generally be closed by integrating to the top of the main thermocline. (This is not true near the Coriolis frequency, but the propagation of inertial energy into the thermocline is beyond the scope of this thesis). In the majority of previous results, either the bottom boundary is a no-slip bottom (Lele, 1985), the stress on the bottom boundary is the same as at the top boundary, (Lele, 1985; Leibovich et al., 1989; Cox and Leibovich, 1993) or the water column is infinitely deep (Leibovich, 1977a).

-The mixing coefficients for density and velocity are the same. Previous authors (Leibovich and Paolucci, 1981; Lele,1985; Leibovich et al., 1989; Cox and Leibovich, 1993) considered cases where the turbulent Prandtl number (given by the ratio between the eddy viscosity and diffusivity) is equal to its molecular value of 7. This is not a good approximation for strongly turbulent mixed layers. Many mixed layer models assume a turbulent Prandtl number of 1 (Denman, 1973; Price et al. 1986) while others have it close to 1 (Mellor and Yamada, 1974). Setting the Prandtl number to be greater than one can lead to time-dependent solutions which are not necessarily realistic for oceanic cases.

-The effect of cells on the equilibrium profile when the surface forcing is balanced by small-scale mixing rather at the transient problem of how cells and small-scale mixing combine to establish a mixed layer is considered. The focus is on which process maintains the mixed layer rather than on how that layer is created. 

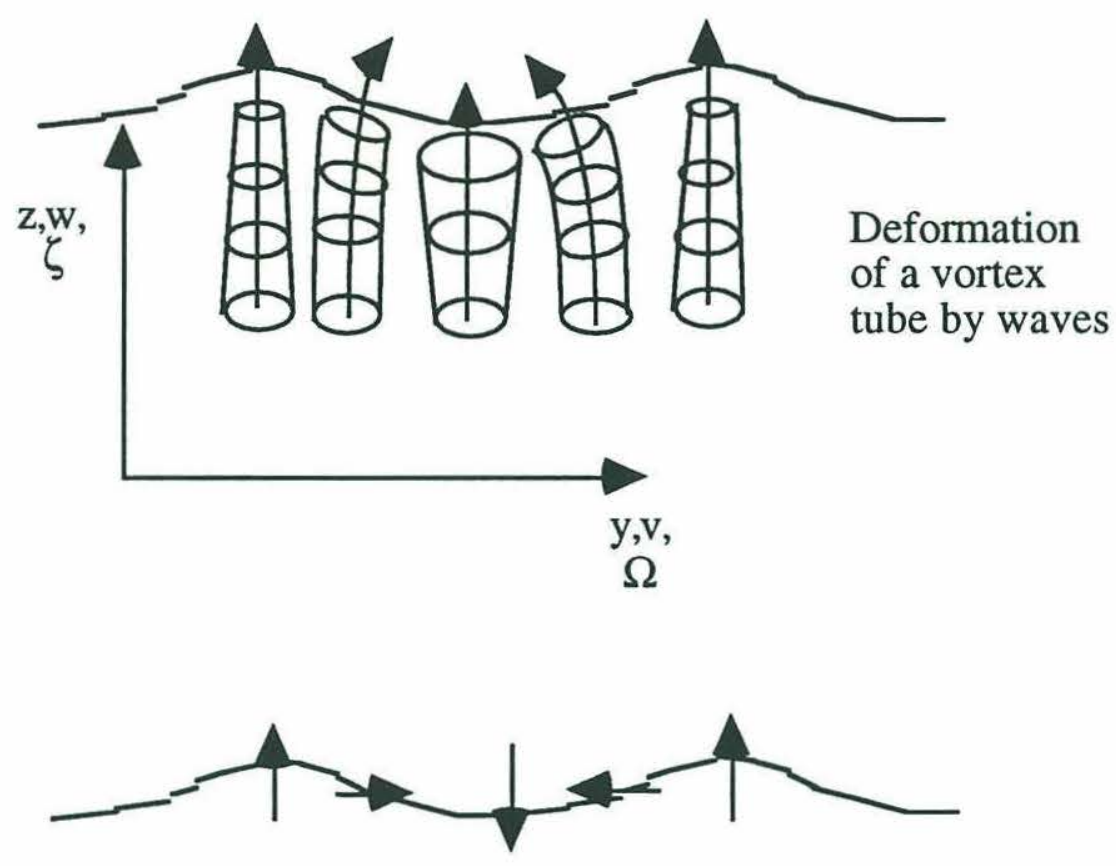

Perturbation vorticity associated with tilting (y-direction) and stretching (z-direction).

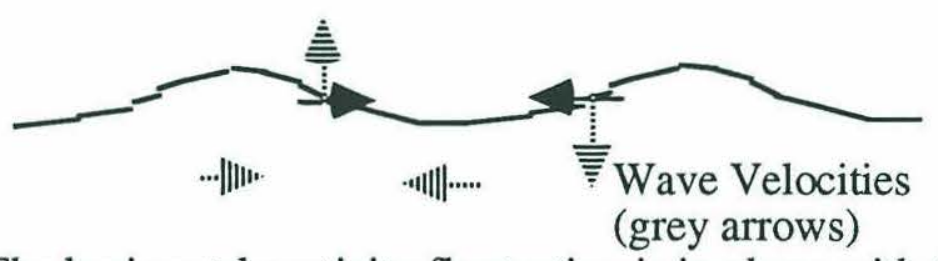

The horizontal vorticity fluctuation is in phase with the vertical wave velocity, resulting in a flux, and with the convergence of the horizontal wave velocity, resulting in a mean stretching.

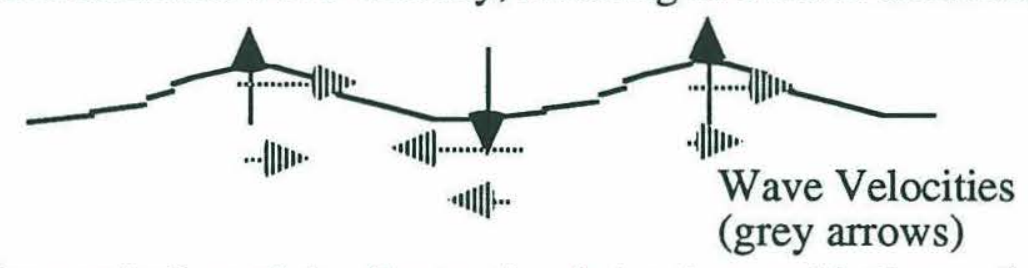

The vertical vorticity fluctuation is in phase with the vertical shear in the horizontal wave velocity. This also acts as a source of vorticity through tilting.

Figure 1.3: Schematic of the production of horizontal vorticity by waves interacting with a vertical vortex tube. 
-The Stokes drift shear decays with depth rather than being a constant. Although some investigators (Leibovich, 1977a; Lele, 1985) have considered how the decay of the Stokes drift shear affects the instability problem, the effect on the structure of the cells at equilibrium has not been considered. When the Stokes drift and Eulerian shears within the mixed layer are nonuniform the dominant mode at equilibrium generally has a longer wavelength than the most unstable mode.

-The Coriolis force is nonzero. An implication of including Coriolis forces is that the Eulerian and Stokes drift shears will not necessarily be parallel and that the depth over which the cells penetrate is limited.

\subsection{Langmuir Cells vs. Small Scale Mixing: The Plan of Attack}

The equations introduced in the previous section are used over the course of this thesis to study when mixed layer profiles produced by small-scale mixing are unstable to cells, and to characterize the modification of these equilibrium profiles produced by finite-amplitude cells.

The fact that the forcing is concentrated near the surface of the layer makes for some difficulty. This may be seen more clearly by contrasting the problem at hand with the well-studied Rayleigh-Benard problem. For buoyant convection between two flat plates the strength of the the forcing is given by the buoyancy frequency $\mathrm{N}$

$$
N=\sqrt{-\frac{g \partial \rho}{\rho \partial z}}
$$

where $\mathrm{g}$ is gravity, $\rho$ is density and $\mathrm{z}$ is the vertical coordinate. The characteristic diffusive decay scale is given by

$$
\gamma_{\text {diff }}=\sqrt{v \kappa}\left(k^{2}+\pi^{2} / \mathrm{D}^{2}\right)
$$

where $v$ is the viscosity, $K$ the diffusivity, $k$ the horizontal wavenumber, and $D$ the depth. If $N » \gamma_{\text {diff }}$ one expects instability to occur and that the finite-amplitude cells will erase much of the initial stratification. For Langmuir cells, however, the Stokes drift decreases exponentially with depth scale $\mathrm{k}_{\mathrm{w}}^{-1} / 2$ (of order 10-20 meters). As a result it is unclear what the analogue of equation (1-7) should be. It is also unclear that the Craik-Leibovich instability mechanism will be able to force cells which can homogenize mixed layers with depths greater than $\mathrm{k}_{\mathrm{w}}^{-1}$.

Chapters 2 and 3 attack the problem of defining analogues to the stratification and Rayleigh number for Langmuir circulation. It is shown that for infinitesimal cells one can define the Craik-Leibovich instability parameter $\gamma_{C L S}$, which characterizes the strength of the forcing on the vortices (page 39). 


$$
\gamma_{\text {CLS }}^{2}(\mathrm{D})=\frac{4}{\mathrm{D}}\left(\int_{-\mathrm{D}}^{0} \mathrm{~F}(\mathrm{z}) \frac{\partial \mathrm{v}_{\mathrm{s}}}{\partial \mathrm{z}} \mathrm{dz} \int_{-\mathrm{D}}^{0} \mathrm{~F}(\mathrm{z}) \frac{\partial \mathrm{V}}{\partial \mathrm{z}} \mathrm{dz}-\int_{-\mathrm{D}}^{0} \mathrm{G}(\mathrm{z}) \frac{\mathrm{g})}{\rho \partial \mathrm{z} \rho} \mathrm{dz}\right)
$$

where $\mathrm{F}(\mathrm{z})$ and $\mathrm{G}(\mathrm{z})$ are weighting functions which are proportional to the nonlinear momentum and density fluxes carried by the infinitesimal cells. $\mathrm{V}$ and $\mathrm{v}_{\mathrm{s}}$ are the Eulerian velocity and Stokes drift, respectively, which are parallel to the cell axis. If $\gamma_{\text {diff }}$ is a characteristic diffusive decay scale for the infinitesimal cells, the stratified Craik-Leibovich Rayleigh number RaCLS is defined as:

$$
\mathrm{RaCLS}=\gamma_{\mathrm{CLS}}^{2} / \gamma_{\text {diff }}^{2}
$$

When $\operatorname{Ra}_{C L S}>1$ (and additionally $\gamma_{C L S}$ is greater than the frequency with which the cells are tilted by the crosscell shear), cells with a particular geometry are unstable.

An important implication of this result is that $\gamma_{C L S}$ and RaCLS depend on the vertical structure of the cells. Chapters 2 and 3 discuss how this vertical structure depends on the cell spacing, Langmuir number, Stokes drift profile, and boundary conditions. The cell spacing is particularly important,with long-wavelength cells penetrating deeper into the water column.

Since RaCLS depends on the spatial structure of the cells the question of which horizontal scales dominate the solution at equilibrium is important. Chapter 4 shows that in the absence of Coriolis forces, energy flows to the gravest modes. This evolution is very slow once the cells reach some quasi-equilibrium mixed layer depth. Mathematically, stratification does not limit the depth of penetration of the cells, but the growth may be slow enough so that penetration is limited for geophysically interesting time scales. The presence of Coriolis forces acts to limit the horizontal length scale and thus the depth of penetration of the cells.

Suppose there is a cell geometry for which RaCLS 1 for infinitesimal cells. In Chapter 5 it is shown that at finite-amplitude, these Langmuir cells replace small scale eddy diffusion as the dominant means by which momentum and density are transported within the mixed layer. In such cases, the characteristic scale for shear within the mixed layer will go roughly as $\gamma_{\text {CLS. }}$ The shear is a natural index of cell strength which can be used to isolate the forcing mechanism.

Chapters 6 and 7 use the framework developed in Chapters 2-5 to look at data from two experiments off the coast of southern California, the Mixed Layer Dynamics Experiment (MILDEX, Chapter 6) and the Surface Waves Processes Program (SWAPP, Chapter 7). The time-varying shear in a band from $1-36 \mathrm{cph}$ is used as an index of cell strength. The level of this shear correlates extremely well 
with an estimate of $\gamma_{C L S}$ assuming cells of roughly $10 \mathrm{~m}$ depth. These results represent the first prediction of cell strength in the field and support the idea that the cells are driven by wave-current interaction. RacLS is shown to be large for extended periods of time, indicating that Langmuir cells rather than small-scale mixing should be the dominant mechanism by which the mixed layer is stirred.

Comparisons between the observations and two one-dimensional mixing models further support this picture. When the cells are strong, the observed lowfrequency $(0.01-0.05 \mathrm{cph})$ shear profile has less shear within the mixed layer than predicted by a model which assumes small scale mixing, but more shear than predicted by a model which treats the mixed layer as a slab. The velocity profile which results from small-scale mixing unstable to Langmuir cells when waves are present. Additionally, both one-dimensional models predict restratification on two days during SWAPP when estimates of the energy balance of Langmuir cells indicate that such restratification should not occur. In Chapter 8, finite-difference code runs demonstrate that the cells should indeed replace small-scale diffusion as the dominant transport mechanism within the mixed layer, homogenizing the velocity profile predicted by small-scale mixing. The result strongly supports the theses that cells are important in stirring the mixed layer and that wave-current interaction is important in driving the cells.

There are differences between theory and observations. Chapter 8 lists some of these shortcomings with respect to the predicted equilibrium cell population, mean shear and velocity structure, and total transport. Some possible remedies are suggested. Chapter 9 concludes the thesis and suggests some avenues for future work.

\subsection{Conclusions}

This thesis argues that Langmuir circulations driven by wave-current interaction are the dominant mechanism for stirring strongly mixed oceanic surface layers. When the surface forcing is strong, Langmuir cells are more important than small-scale diffusion driven by buoyant overturning and shear instability. In some cases, the cells are the reason why a mixed layer is seen at all. Although small-scale turbulent processes are potentially still important in the initiation of mixing (Chapter 9), as the mixed layer develops they become less important than large-scale Langmuir circulations. 


\section{Chapter 2: The Instability of Langmuir Cells in Fluid Layers with No Coriolis Forces}

\subsection{Introduction}

This thesis argues that Langmuir circulation driven by wave-current interaction, rather than small-scale diffusion driven by shear instability and local buoyant overturning, is primarily responsible for maintaining the mixed layer. In Chapter 1, a set of equations were introduced for the evolution of a layer of fluid in the presence of waves, Coriolis force, and Langmuir circulation. This chapter uses these equations to answer the following questions.

- Under what circumstances is the equilibrium solution set up by small-scale turbulent diffusion unstable to Langmuir cells?

- How do diffusion, stratification, Stokes drift profile, layer depth, and cell spacing affect the growth rate and vertical structure of the unstable modes?

-What is the effect of the boundary conditions for density on the growth rate and structure of the unstable modes?

The cases examined assume that the Coriolis force is zero, the cell axis, waves, and Eulerian shear are parallel $\left(\mathrm{u}_{\mathrm{s}}=0\right)$, and that the wind stress is balanced by a pressure gradient. The goal is to reduce the many different parameters to a few important numbers. These turn out to be:

- The stratified Craik-Leibovich instability parameter $\gamma_{C L S}$ (a measure of the strength of the vortex forcing due to wave-current interaction and buoyancy).

- The stratified Craik-Leibovich Rayleigh number RaCLS (a measure of the strength of the forcing relative to the diffusive decay).

- The aspect ratio of the cells $D_{\max } / \mathrm{L}$ where $\mathrm{D}_{\max }$ is the depth at which the maximum vertical velocity occurs.

The results can be extended to provide a basis for understanding the dynamics of infinitesimal and finite-amplitude cells in the presence of Coriolis forces.

Before embarking on this study we briefly note related work. Leibovich (1977a) studied the instability of an undisturbed column of infinitely deep water. $\mathrm{He}$ found that the growth rate of Langmuir cells was a strong function both of the Langmuir number and the horizontal wavenumber. Leibovich (1977b) showed that the maximum inviscid growth rate for cells in the presence of stratification was:

$$
\gamma=\max \left(\sqrt{\frac{\partial \mathrm{V} \partial \mathrm{v}_{\mathrm{s}}}{\partial \mathrm{z} \partial \mathrm{z}}-\mathrm{N}^{2}}\right)
$$


so that high stratification suppressed the instability. Lele (1985) showed that the marginal instability for Langmuir cells occurred at infinite cell spacing $(\mathrm{k}=0)$ when the bottom boundary was a no-stress bottom. Cox and Leibovich (1993) considered the effect of changing this boundary condition on the instability.

This study uses a different initial condition from Leibovich (1977a), namely the equilibrium flow set up by small-scale diffusion in the absence of Langmuir cells. This initial condition was chosen since the goal of the thesis is to determine whether Langmuir cells or small-scale diffusion is the dominant transport mechanism in an equilibrium mixed layer. This initial condition is not wholly unrealistic, since in real oceans and lakes there is almost always some pre-existing shear as a result of pressure gradients, internal waves, or inertial oscillations. As noted in Chapter 1, an additional difference between this work and that of previous investigators is that the turbulent Prandtl number is set equal to 1 instead of its molecular value of 7 .

\subsection{Equations of Motion and Methods of Solution}

The equations of motion are (Leibovich,1977a)

$$
\begin{gathered}
\frac{\partial \Omega}{\partial \mathrm{t}}+\mathrm{u} \frac{\partial \Omega}{\partial \mathrm{x}}+\mathrm{w} \frac{\partial \Omega}{\partial \mathrm{z}}=\frac{\partial \mathrm{v}_{\mathrm{s}} \partial \mathrm{v}}{\partial \mathrm{z}}+\mathrm{Ri} \frac{\partial \rho}{\partial \mathrm{x}}+\mathrm{La} \nabla^{2} \Omega \\
\frac{\partial \mathrm{v}}{\partial \mathrm{t}}+\mathrm{uv} \frac{\partial \mathrm{v}}{\partial \mathrm{x}}+\mathrm{w} \frac{\partial \mathrm{v}}{\partial \mathrm{z}}=-\frac{1 \partial \mathrm{p}}{\rho \partial \mathrm{y}}+\mathrm{La} \nabla^{2} \mathrm{v} \\
\frac{\partial \rho}{\partial \mathrm{t}}+\mathrm{u} \frac{\partial \rho}{\partial \mathrm{x}}+\mathrm{w} \frac{\partial \rho}{\partial \mathrm{z}}=\mathrm{La} \nabla^{2} \rho \\
\Omega=\nabla^{2} \psi \\
\frac{\partial \psi}{\partial \mathrm{x}^{2}}=-\mathrm{w} \quad \frac{\partial \psi}{\partial \mathrm{z}}=\mathrm{u} \\
\mathrm{La}=\frac{\mathrm{v}_{\mathrm{e}}}{\mathrm{a}^{2} \sigma} \quad \mathrm{Ri}=\mathrm{N}^{2} \\
\mathrm{k}_{\mathrm{w}}^{4} \mathrm{a}^{4} \sigma^{2} \\
\mathrm{k}_{\mathrm{w}}^{-1}(\mathrm{x}, \mathrm{y}, \mathrm{z})=(x, y, z) \\
\left(\mathrm{k}_{\mathrm{w}} \mathrm{a}\right)^{2} \frac{\sigma}{\mathrm{k}_{\mathrm{w}}}\left(\mathrm{u}, \mathrm{v}, \mathrm{v}_{\mathrm{s}}, \mathrm{w}\right)=\left(u, v, v_{s}, w\right) \\
\frac{1}{\mathrm{k}_{\mathrm{w}}^{2} \mathrm{a}^{2} \sigma} \mathrm{t}=t
\end{gathered}
$$


In these equations $\mathrm{k}_{\mathrm{w}}, \mathrm{a}$, and $\sigma$ are the wavenumber, amplitude, and frequency of the driving surface gravity waves, $v_{\mathrm{e}}$ isthe eddy viscosity, $\mathrm{N}$ is the buoyancy frequency, and $\mathrm{v}_{\mathrm{s}}$ is the Stokes Drift. The italicized quantities are dimensional, with equations $(2-2 \mathrm{~g}-\mathrm{i})$ giving the conversion to nondimensional units. The key nondimensional numbers are the Langmuir number La and the Richardson number $\mathrm{Ri}$. The boundary conditions on the velocity are

$$
\begin{gathered}
\left.\mathrm{La} \frac{\partial \mathrm{v}}{\partial \mathrm{z}}\right|_{\mathrm{z}=-\mathrm{D}}=\left.\Omega\right|_{\mathrm{z}=0}=\left.\Omega\right|_{\mathrm{z}=-\mathrm{D}}=0 \\
=\frac{\tau}{\rho}
\end{gathered}
$$

In the absence of Langmuir cells after a diffusive equilibrium is set up the mean Eulerian velocity in such a layer is given by

$$
\mathrm{V}(\mathrm{z})=\frac{\tau(\mathrm{z}+\mathrm{D})^{2}}{\rho \operatorname{La} 2 \mathrm{D}}+\left(\frac{\tau}{\rho \mathrm{D}}-\frac{1 \partial \mathrm{p}}{\rho \partial \mathrm{y}}\right) \mathrm{t}+\mathrm{C}
$$

Where $\mathrm{C}$ is an undetermined constant which can be set equal to zero without altering the fundamental dynamics. In order to obtain a constant solution, the pressure gradient which is required to balance the wind stress is $\partial p / \partial y=\tau / D$.

This scenario is not strictly realistic for the majority of oceanic cases, in which the primary balance is between wind stress and Coriolis force. However, it may be applicable to the flow in the interior of lakes where the spacing between convergence zones is small compared with the distance across the lake.

Additionally, as shown in Chapter 5, some results for mixed layers without Coriolis force can be applied to mixed layers with Coriolis force when the Ekman depth $\sqrt{\frac{2 v_{e}}{f}}$ (f the Coriolis frequency) is large compared with the layer depth.

The density equation deserves some special consideration. As noted in the previous chapter, the mixing parameterization adopted for this study requires either that $\rho$ be constant throughout the layer, or that it vary linearly from top to bottom. In both cases the density flux is constant throughout the layer. Since the deep ocean may be thought of as a reservoir of cold, dense water, the density is held fixed on the lower boundary.

$$
\rho l_{z=-D}=D
$$

The upper boundary condition on density is less clear. The effect of two possible conditions are considered, one for which the density is fixed on the upper boundary, and another for which the density flux is fixed on the upper boundary.

$$
\begin{gathered}
\rho l_{z=0}=0 \\
\partial \rho /\left.\partial z\right|_{z=0}=-1
\end{gathered}
$$


If the density is fixed, the density flux carried by the cells is set by the internal dynamics of the system, a somewhat more interesting case. However, it is unclear that it is a physical case, since the atmosphere is more likely to set the flux than it is to fix the density at the upper boundary.

In addition to the boundary conditions, there are a large number of parameters which have a potential effect on the instability; namely the cell spacing $\mathrm{L}$, layer depth $\mathrm{D}$, Langmuir number La, Richardson number Ri, surface shear $\frac{\partial \mathrm{V}}{\partial \mathrm{z}} \mathrm{I}_{\mathrm{z}=0}$, and Stokes drift shear profile $\frac{\partial \mathrm{v}_{\mathrm{s}}}{\partial \mathrm{z}}$. In order to reduce the parameter space which must be considered, it is useful to choose parameter ranges which are reasonable for oceanic environments. For oceanic cases, the frequency of the driving waves is of order $0.5-1 \mathrm{rad} / \mathrm{s}$, corresponding to periods of order 6-12 seconds. The e-folding depth for wave velocity decay $1 / \mathrm{k}_{\mathrm{w}}$ for such waves is roughly 8-32 meters. This thesis concentrates on layers with nondimensional depths ranging from 2 to 6 , corresponding to layer depths of about 15-200 meters. Reasonable values for oceanic eddy viscosities range from $10-1000 \mathrm{~cm}^{2} / \mathrm{s}$ (Huang,1979; Weller,1981). Depending on how one calculates the quantity $a^{2} \sigma$ (either by integrating over a spectrum or simply choosing values from the spectral peak) one may obtain a range of Langmuir numbers from $\mathrm{O}\left(10^{-4}\right)$ to $\mathrm{O}(1)$.

Two reasonable approximations for the nondimensional Stokes drift are used in this work, one for a monochromatic wave train and another for that given by the Pierson-Moskowitz spectrum (Pierson and Moskowitz, 1964):

$$
\begin{array}{cl}
\mathrm{v}_{\mathrm{s}}(\mathrm{z})=\int_{f=0}^{\infty} \frac{5}{\mathrm{v}_{\mathrm{s}}}(\mathrm{z})=\exp (2 \mathrm{z}) & \text { Monochromatic } \\
\ln \left(-1.25 \frac{1}{f^{4}}\right) \exp \left(2 f^{2} \mathrm{z}\right) \mathrm{d} f & \text { P-M. Spectrum }
\end{array}
$$

where $f$ is a dummy variable representing the nondimensional frequency. The Pierson-Moskowitz spectrum is chosen to have the same amplitude and peak frequency as the monochromatic wave train. Relative to the monochromatic wave train, the Pierson-Moskowitz spectrum yields a larger Stokes drift (by a factor of 4) and much larger Stokes drift shear (infinite at $\mathrm{z}=0$ ) near the surface. ${ }^{*}$

The instability problem is cast as follows. The streamfunction, alongcell velocity, and Stokes drift are represented as

\footnotetext{
* Note that an infinite surface Stokes drift shear means that the inviscid limit on the growth rate (2-1) is always infinite and that a finite value of stratification will never act to limit the growth rate. This presents a major obstacle to applying the theory to the real ocean, and serves to motivate the development of an instability theory which does not depend solely on a local parameter.
} 


$$
\begin{gathered}
\psi=\delta \psi^{\prime}(\mathrm{x}, \mathrm{z}, \mathrm{t})+\Psi(\mathrm{z})=\delta \mathrm{e}^{\mathrm{ikx}} \sum_{\mathrm{m}=1}^{\mathrm{M}} \Psi_{\mathrm{m}}(\mathrm{t}) \sin \left(\frac{\mathrm{m} \pi \mathrm{z}}{\mathrm{D}}\right)+\sum_{\mathrm{m}=1}^{\mathrm{M}} \Psi_{\mathrm{m}} \sin \left(\frac{\mathrm{m} \pi \mathrm{z}}{\mathrm{D}}\right) \\
\mathrm{v}=\delta \mathrm{v}^{\prime}(\mathrm{x}, \mathrm{z}, \mathrm{t})+\mathrm{V}(\mathrm{z})=\delta \mathrm{e}^{\mathrm{ikx}} \sum_{\mathrm{m}=0}^{\mathrm{M}} \mathrm{v}_{\mathrm{m}}(\mathrm{t}) \cos \left(\frac{\mathrm{m} \pi \mathrm{z}}{\mathrm{D}}\right)+\sum_{\mathrm{m}=0}^{\mathrm{M}} \mathrm{V}_{\mathrm{m}} \cos \left(\frac{\mathrm{m} \pi \mathrm{z}}{\mathrm{D}}\right) \\
\mathrm{v}_{\mathrm{s}}=\sum_{\mathrm{m}=0}^{\mathrm{M}} \mathrm{V}_{\mathrm{m}} \cos \left(\frac{\mathrm{m} \pi \mathrm{z}}{\mathrm{D}}\right)
\end{gathered}
$$

where $\delta$ is a small number. Let the density field be

$$
\rho=\delta \rho^{\prime}(x, z, t)+P(z)=\delta e^{i k x} \sum_{m=1}^{M} \rho_{m}(t) \sin \left(\frac{n \pi z}{D}\right)+\sum_{m=1}^{M} P_{m} \sin \left(\frac{m \pi z}{D}\right)
$$

for density fixed on top and bottom boundaries or

$$
\rho=\delta e^{i k x} \sum_{m=1}^{M} \rho_{n}(t) \cos \left(\frac{(2 m-1) \pi z}{2 D}\right)+\sum_{m=1}^{M} P_{m} \cos \left(\frac{(2 m-1) \pi z}{2 D}\right)
$$

for density fixed on the bottom boundary and density flux fixed on the top boundary. These expansions may be substituted into equations (2-2) and expanded in terms of $\delta$. To zeroth order in $\delta$ this procedure yields a Fourier-series representation of the steady state solution in the absence of cells. At first order in $\delta$, the growth rate of the linearly most unstable mode and the structure of that mode can be cast as a linear eigenvalue problem in terms of the the coefficients $\psi_{\mathrm{m}}, \mathrm{v}_{\mathrm{m}}, \rho_{\mathrm{m}}$. The value of the largest positive eigenvalue is a function of the number of modes in the truncation, but it converges as $\mathrm{M}$ becomes large. The results in this chapter are for $M=40$, a value for which all results presented here converged.

The vertical velocity for such an unstable mode is given by

$$
\mathrm{w}=\mathrm{ik} \sum_{\mathrm{m}=1}^{\mathrm{M}} \psi_{\mathrm{m}} \sin (\mathrm{m} \pi \mathrm{z} / \mathrm{D}) \mathrm{e}^{\mathrm{ikx}}=\mathrm{ik} \psi^{\prime}
$$

The depth at which the maximum vertical velocity occurs $\left(D_{\max }\right)$ is the depth at which $|\psi|$ ' is a maximum.

In section 2.3 a spectral instability code of the type outlined above is used to characterize the dependence of the growth rate and $\mathrm{D}_{\max }$ on layer depth, Stokes 
drift shear profile, stratification, and boundary conditions. The growth rate and $\mathrm{D}_{\max }$ are closely linked.

In section 2.4 a simple understanding of these complicated dependencies is sought. Linearized energy balance equations for the instability are derived which give a sense of how quickly cells with a given shape grow. By making some simplifying assumptions, such as using two simple truncations to approximate the shape of the unstable modes, closed-form analytical solutions for the growth rate are obtained. These solutions are used to infer the important physical parameters which determine the growth rate and cell structure of the linearly unstable modes.

\subsection{Craik-Leibovich Instability in Nonrotating Mixed Layers: Results from an Instability Code}

\subsubsection{Results for Idealized Unstratified Surface Layers}

This section focuses on two primary questions

1. How does the growth rate $\gamma$ of the linearly most unstable mode depend on the horizontal spacing of the cells L, the layer depth D, the Stokes drift profile, and the Langmuir number La?

2. How does the $D_{\max }$ depend on these same parameters?

It is important to note the limitations of $\mathrm{D}_{\max }$ as an index of cell penetration. Since the cell structure is not invariant it cannot be assumed that the
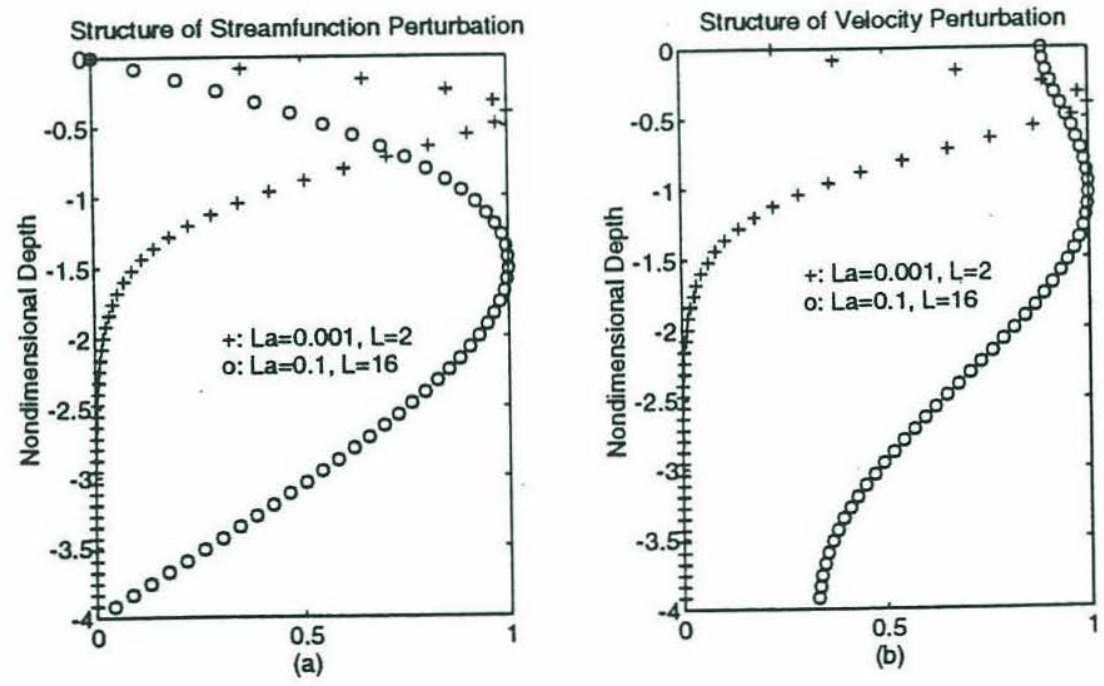

Figure 2.1: Vertical structure of the most unstable mode assuming monochromatic waves and a surface Eulerian shear of 1. (+) Langmuir number La=0.001, L=2. (o) $\mathrm{La}=0.1, \mathrm{~L}=16$. (a) Streamfunction perturbation. (b) Velocity perturbation. 
cells have no effect at depths more than twice $\mathrm{D}_{\max }$. Figure 2.1a shows the streamfunction perturbation for the most unstable mode for $\mathrm{La}=0.001, \mathrm{k}=2 \pi / 2(+)$ and $\mathrm{La}=0.1, \mathrm{k}=2 \pi / 16(\mathrm{o})$. Figure $2.1 \mathrm{~b}$ shows the velocity perturbation. When $\mathrm{La}$ and $\mathrm{L}$ are small, the streamfunction perturbation is concentrated near the surface and resembles the velocity perturbation. When they are large, the streamfunction perturbation penetrates over the depth of the mixed layer and is very different from the velocity perturbation. Looking at $\mathrm{D}_{\max }$ alone neglects these changes in structure. Nonetheless $\mathrm{D}_{\max }$ is a useful diagnostic for cell penetration.

The linkage between growth rate and depth of penetration can be seen by considering a simple case. Suppose a monochromatic wave train is propagating in a direction parallel to the wind, so that the Stokes drift is given by (2-7a) and that the surface Eulerian shear $\left.\frac{\partial \mathrm{V}}{\partial \mathrm{z}}\right|_{\mathrm{z}=0}=1$. The maximum inviscid growth rate for this scenario is $\gamma_{\mathrm{CL}}^{\max }=\sqrt{2}$. Figure 2.2a shows the growth rate of the most unstable mode $\gamma=\gamma(\mathrm{L}, \mathrm{N}=0, \mathrm{La})$ and $2.2 \mathrm{~b}$ the depth of the maximum vertical velocity $D_{\max }=D_{\max }(\mathrm{L}, \mathrm{N}=0, \mathrm{La})$ for a layer depth of 2 . The horizontal axis is $\log _{10} \mathrm{La}(1 / \mathrm{La}$ is analogous to Reynolds number), while the vertical axis is the horizontal wavenumber $k=2 \pi / \mathrm{L}$. $\mathrm{D}_{\max }$ and $\gamma$ are linked as follows:

- Given a constant value of $\mathrm{k}$, as La decreases $\gamma$ increases and $\mathrm{D}_{\max }$ decreases.

- As La becomes very small, both $\gamma$ and $\mathrm{D}_{\max }$ asymptote to a constant value.

- At very low values of $\mathrm{La}$, large values of $\gamma$ occur when $\mathrm{D}_{\max }$ is small.

This linkage is relatively insensitive to layer depth. Figure $2.2 \mathrm{c}$ and $2.2 \mathrm{~d}$ show $\gamma$ and $D_{\max }$ for a layer depth of 4 , and 2.2e and 2.2f show $\gamma$ and $D_{\max }$ for a layer depth of 6 . As La becomes small and $\mathrm{k}$ approaches $2 \pi$ the growth rate of the unstable mode approaches 0.8 , slightly more than half of $\gamma_{\mathrm{CL}}^{\max }$ and $\mathrm{D}_{\max }$ is about 0.3 for all three values of depth. For larger wavenumbers, larger values of $\gamma$ coupled to smaller values of $\mathrm{D}_{\max }$ are seen. For $\mathrm{La}=10^{-5}$ the largest growth rate of 1.19 occurs for cell spacing $\mathrm{L}=0.1(\mathrm{k}=20 \pi)$. $\mathrm{D}_{\max }$ for this unstable mode is 0.06 .

However, there are some parts of parameter space where the layer depth matters. In particular, at low wavenumbers and high La:

- $\mathrm{D}_{\max }$ is approximately half the layer depth.

- The stability boundary depends on the layer depth.

The importance of the layer depth for such cases is explained in Section 2.4.

The effect of changing the Stokes drift profile from a monochromatic wave train to one corresponding to a Pierson-Moskowitz spectrum is shown in Figure 


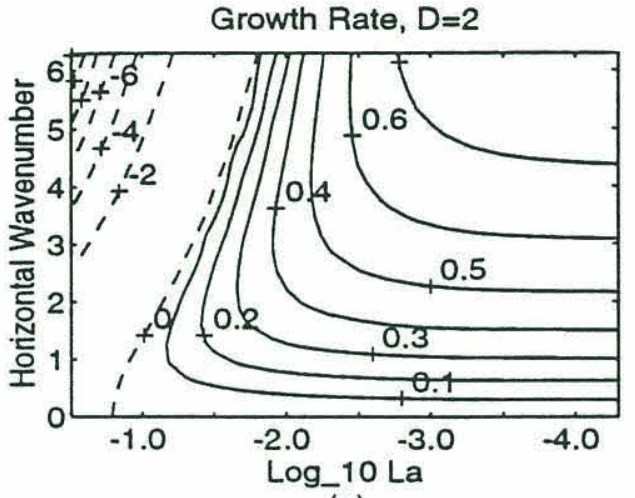

(a)

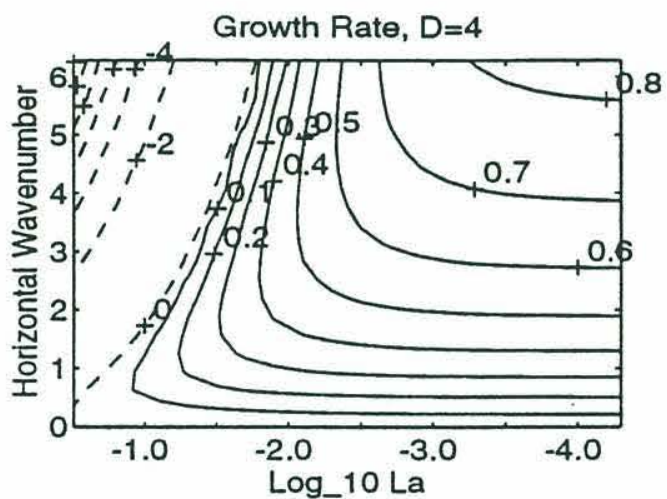

(c)

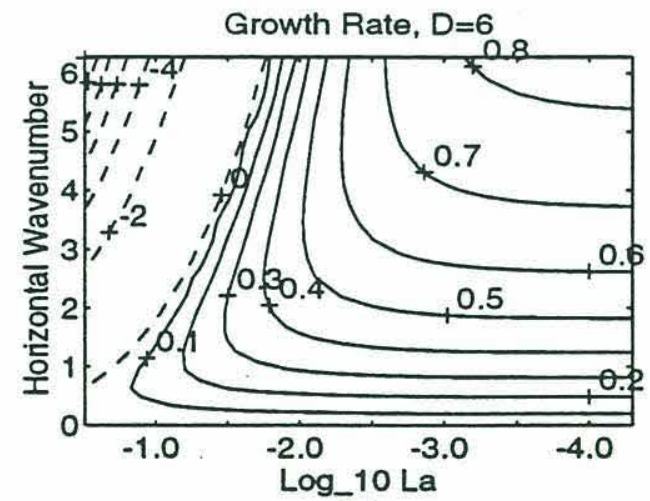

(e)

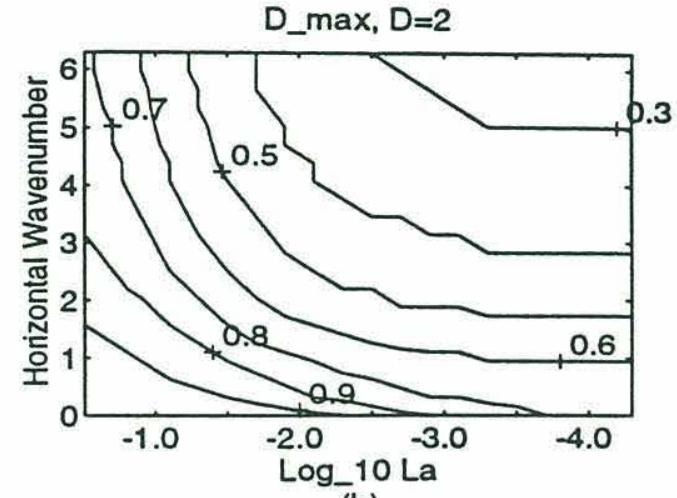

(b)

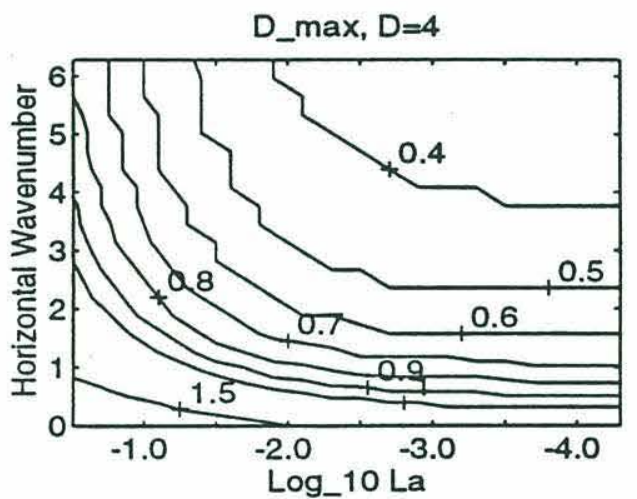

(d)

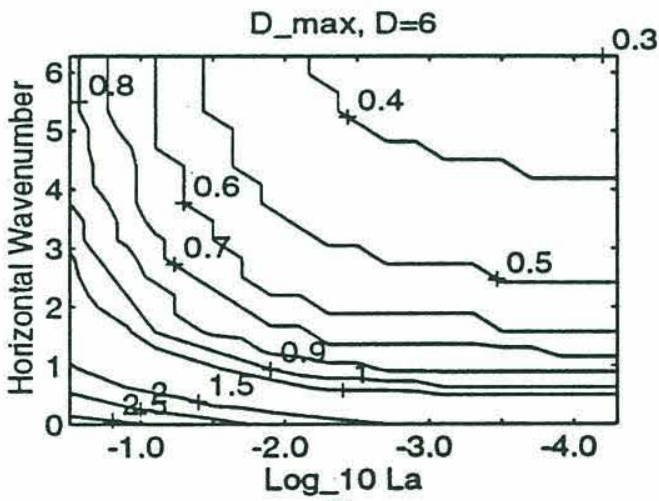

(f)

Figure 2.2: Growth rate and depth of maximum vertical velocity $D_{\max }$ as a function of horizontal wavenumber and Langmuir number given monochromatic waves, no Coriolis force, and a surface Eulerian shear $=1$. Waves, wind and cell axis are assumed collinear. (a) Growth rate, Layer depth $D=2$. (b) $D_{\max }, D=2$. (c) Growth rate, $D=4$. (d) $D_{\max }, D=4$. (e) Growth rate, $D=6$. (f) $D_{\max }, D=6$. 
2.3. Figure 2.3a shows the Stokes drift for a Pierson-Moskowitz spectrum and $2.3 \mathrm{~b}$ the Stokes drift shear. Using a wave spectrum rather than a monochromatic wave train increases the Stokes drift and Stokes drift shear for $\mathrm{z}>-1$, decreases them for $-1>\mathrm{z}>-3$ increases them for $\mathrm{z}<-3$. Figure $2.3 \mathrm{c}$ shows $\gamma$ and $2.3 d \mathrm{D}_{\max }$ for a layer depth of 4 and a surface shear of 1 (corresponding to Figure $2.2 \mathrm{c}$ and d). The effect of changing the profile is to increase the growth rate for all values of horizontal wavenumber and Langmuir number, with the largest changes being at high wavenumber and low Langmuir number. $\mathrm{D}_{\max }$ decreases fairly uniformly, with the mean decrease being close to 0.2 . Increases in growth rate are correlated to decreases in $\mathrm{D}_{\max }$.

Lastly, the behavior of the instability at low wavenumbers is considered. Figure 2.4a shows the behavior of the growth rate of the most unstable mode for $\mathrm{La}=0.001$ as $\mathrm{k}$ goes to zero for $\mathrm{D}=2,4$, and 6 given a monochromatic wave train and a surface Eulerian shear of 1.0. The growth rates decrease approximately quadratically, with marginal instability occurring at $\mathrm{k}=0$ (infinite wavenumber). The growth rates are clearly strongly affected by the depth of the layer, with larger depths corresponding to larger growth rates. Figure $2.4 \mathrm{~b}$ shows the depth of penetration, which asymptotes to somewhat less than half the layer depth in all cases as the wavenumber $\mathrm{k}$ goes to zero.

In summary, the main results of the unstratified runs are:

- At high wavenumbers and low Langmuir numbers, the growth rate and depth of penetration are largely independent of layer depth and Langmuir number but strongly dependent on cell spacing.

- At low wavenumbers, the growth rate depends on the value of $\mathrm{La}$, the cell spacing and the layer depth.

- Changing the Stokes drift profile from one given by monochromatic waves to one given by a Pierson-Moskowitz spectrum increases the growth rates sharply.

\subsubsection{Instability in Idealized Stratified Mixed Layers}

Turning now to stratified Craik-Leibovich instability, this section considers cases where $\mathrm{D}=4$, the waves are monochromatic, and the surface shear=1. From Leibovich (1977b) the maximum inviscid growth rate in the presence of stratification is given by the maximum of $\sqrt{\frac{\partial V \partial v_{s}}{\partial z \partial z}-R i}$. For the shear profiles in this chapter given a monochromatic wave train, the first product has a maximum 


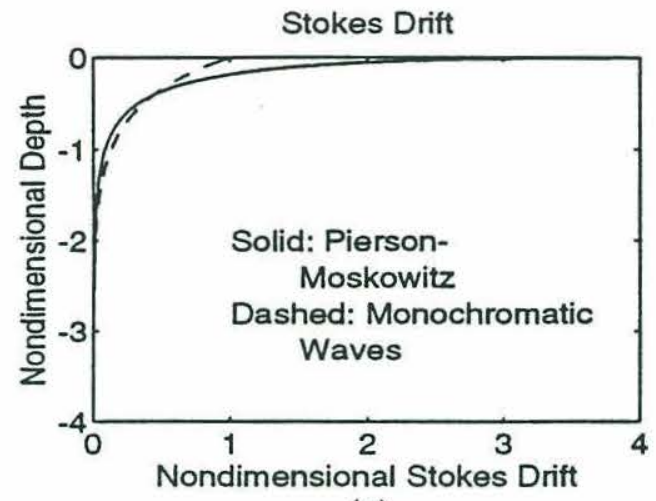

(a)

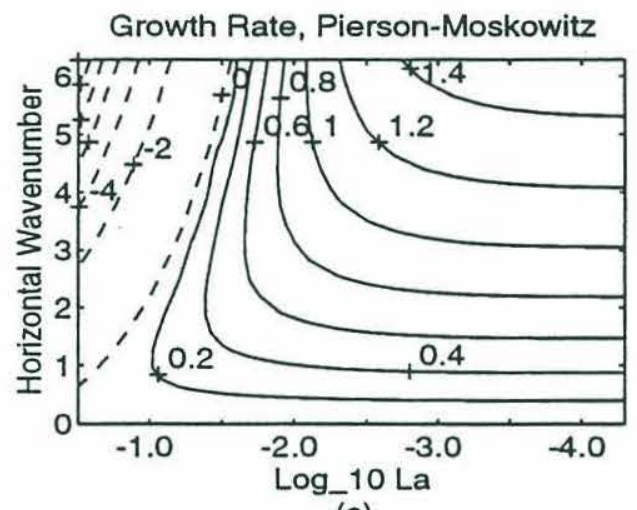

(c)

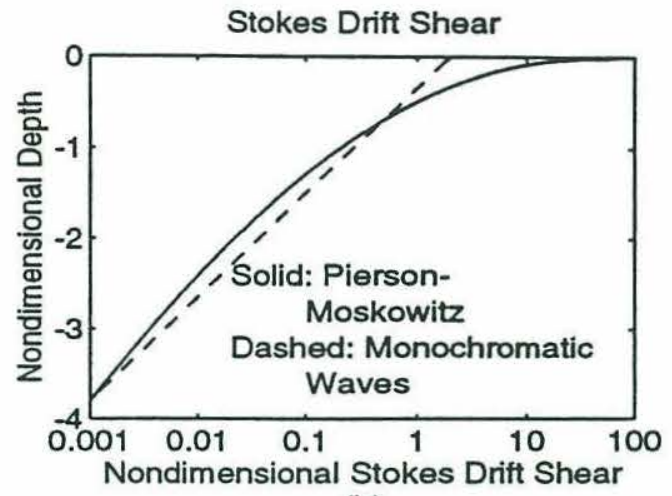

(b)

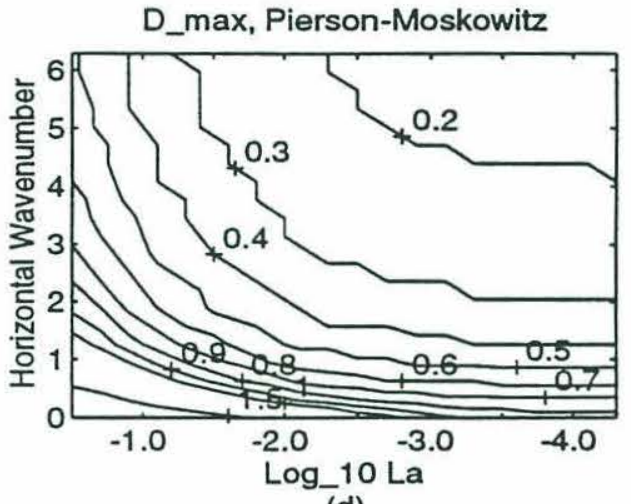

(d)

Figure 2.3: Effect on Craik-Leibovich instability of changing the waves from monochromatic to a Pierson-Moskowitz spectrum. (a) Stokes drift for PiersonMoskowitz spectrum (solid)and monochromatic waves (dashed) with same peak frequency and amplitude. (b) Same as (a) but for Stokes Drift shear. (c) Growth rate from 40 -mode instability code assuming $\mathrm{V}_{0}(\mathrm{z})=(\mathrm{z}+\mathrm{D})^{2} / 2 \mathrm{D}, \mathrm{D}=4$ and waves given by a Pierson-Moskowitz spectrum. (d) Same as (c) but for depth of maximum vertical velocity $D_{\max }$.

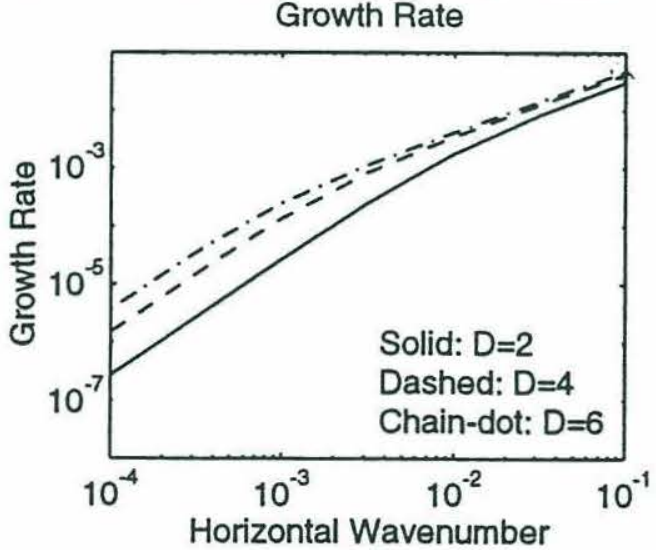

(a)

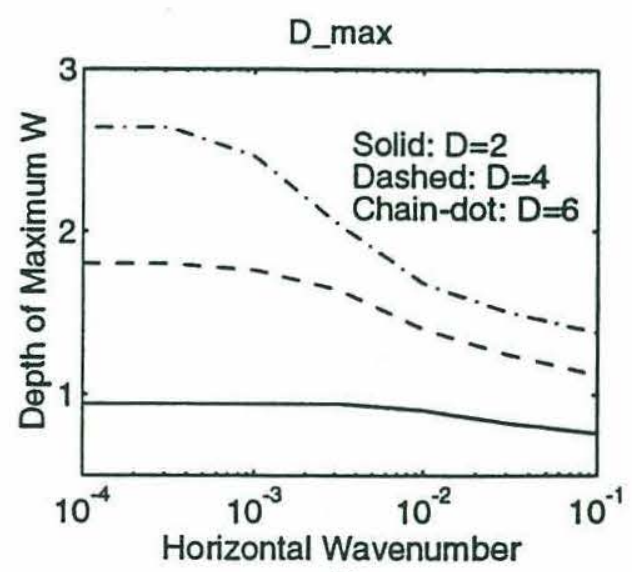

(b)

Figure 2.4: Growth rate and depth of maximum vertical velocity $D_{\max }$ at low wavenumber for three values of layer depth assuming monochromatic waves, $V_{0}(z)=(z+D)^{2} / 2 D$, and $L a=0.001$. Solid lines are for $D=2$, dashed for $D=4$ and chain-dotted for $\mathrm{D}=6$. (a) Growth rate. (b) $\mathrm{D}_{\max }$. 
of 2 . The investigation is divided into cases where $\mathrm{Ri} \ll 2.0$ (weak stratification), $\mathrm{Ri}$ somewhat smaller than 2.0 (moderate stratification) and Ri 2.0 (strong stratification).

This section has two main purposes. The first is to investigate when the boundary conditions for density are important for determining the growth rate and structure of the instability. This will guide the choice of a boundary condition for the finite-difference code runs in Chapters 4 and 5. The second purpose is to extend results from 2.3.1 to stratified cases to determine how the stratification affects the cell structure and growth rate.

Figure 2.5 shows growth rates and depth of cell penetration for cases of weak to moderate stratification. Figure $2.5 \mathrm{a}$ shows the growth rate and $2.5 \mathrm{~b} \mathrm{D}_{\max }$ for $\mathrm{Ri}=0.05$ (weak stratification) with the density fixed on top and bottom boundaries. Comparison with Figure $2.2 \mathrm{c}$ and $\mathrm{d}$ shows very little change in either the growth rate or the depth of maximum vertical velocity. Weak stratification does not affect the instability at high wavenumbers to any great degree.

Figure $2.5 \mathrm{c}$ and $\mathrm{d}$ repeat $2.5 \mathrm{a}$ and $\mathrm{b}$ for $\mathrm{Ri}=0.5$ (moderate stratification). The growth rates decrease in the presence of moderate stratification, and $D_{\max }$ decreases as well. Given a fixed wavenumber, stratification can play a role in limiting the depth of penetration of the cells. Similar results were found by Lele (1985) and Li and Garrett (1993b).

Figure $2.5 \mathrm{e}$ and $\mathrm{f}$ repeat $2.5 \mathrm{c}$ and $\mathrm{d}$, but for the density flux, rather than the density, fixed on the upper boundary. Changing the boundary condition produces very little difference in the growth rate or depth of maximum vertical velocity. Even for moderate values of stratification, the physics of the instability are relatively insensitive to the upper boundary condition.

This lack of sensitivity to boundary conditions does not hold when the stratification is strong. Figure 2.6a shows $\gamma$ and $2.6 b \mathrm{D}_{\max }$ as a function of wavenumber and $\mathrm{La}$ for $\mathrm{Ri}=2.0$ and for density fixed on upper and lower boundaries. In the absence of viscosity, there is no instability for $\mathrm{Ri}=2.0$. This is not the case in the presence of viscosity. The growth rates have a very interesting pattern, showing a maximum in Langmuir number.

This pattern is strongly dependent on the upper boundary condition. Figure $2.6 \mathrm{c}$ and $2.6 \mathrm{~d}$ show the growth rate and $\mathrm{D}_{\max }$ for $\mathrm{Ri}=2.0$ but with the density flux fixed on the upper boundary instead of the density. The instability is damped except at very low wavenumber and Langmuir number. 


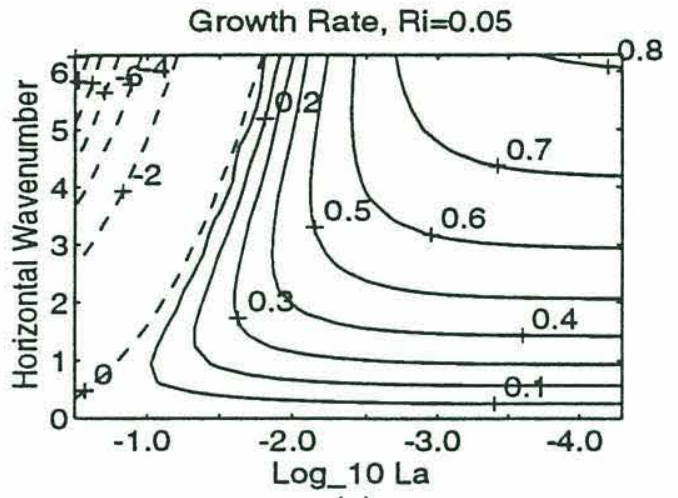

(a)

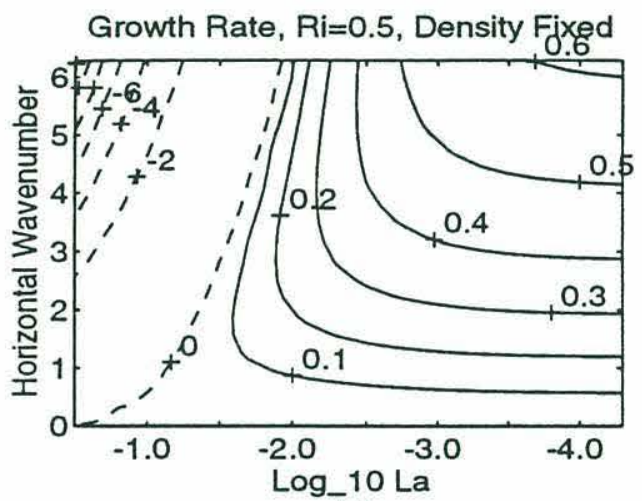

(c)

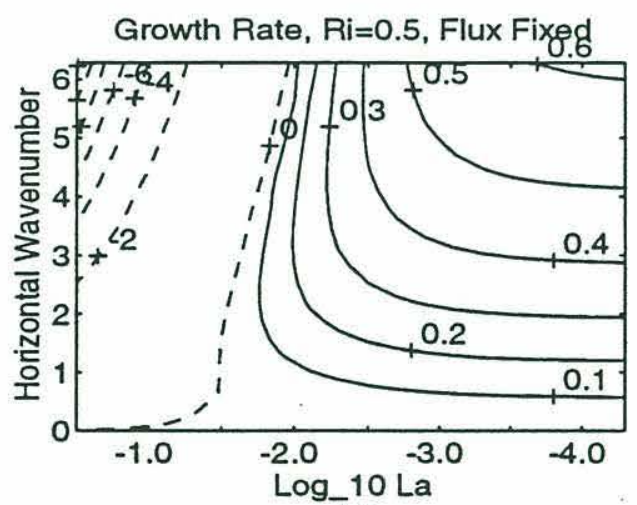

(e)

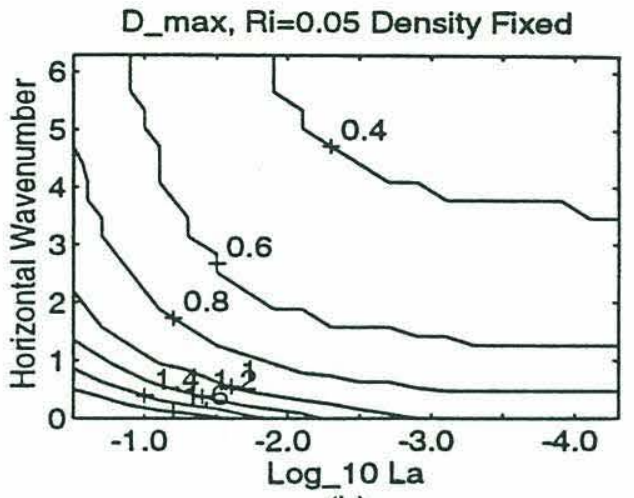

(b)

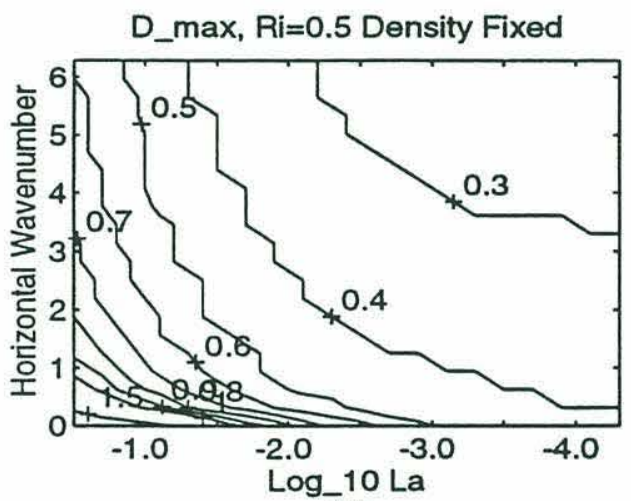

(d)

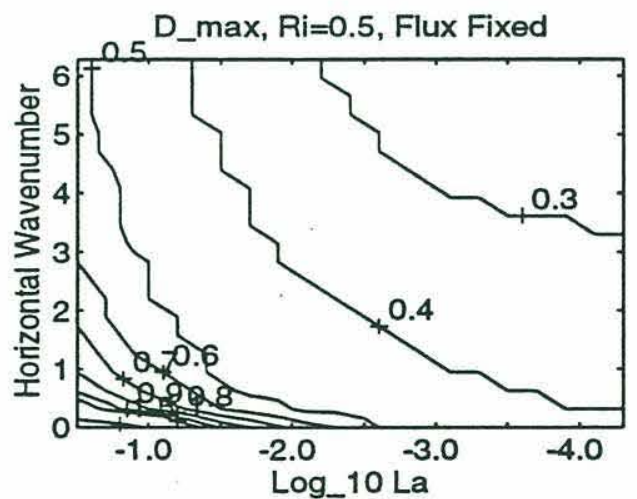

(f)

Figure 2.5: Instability of Langmuir cells in the presence of low to moderate stratification. All cases assume layer depth $\mathrm{D}=4$, monochromatic waves, no Coriolis forces and a surface Eulerian shear of 1 . (a) Growth Rate, $\mathrm{Ri}=0.05$ density fixed on both boundaries. (b) $\mathrm{D}_{\max }, \mathrm{Ri}=0.05$, density fixed on both boundaries. (c) Same as (a), but for $\mathrm{Ri}=0.5$. (d) Same as (b) but for $\mathrm{Ri}=0.5$. (e) Same as (c) but for density flux fixed on upper boundary. (f) Same as (d) but for density flux fixed on upper boundary. 
Growth Rate, Ri=2.0 Density Fixed

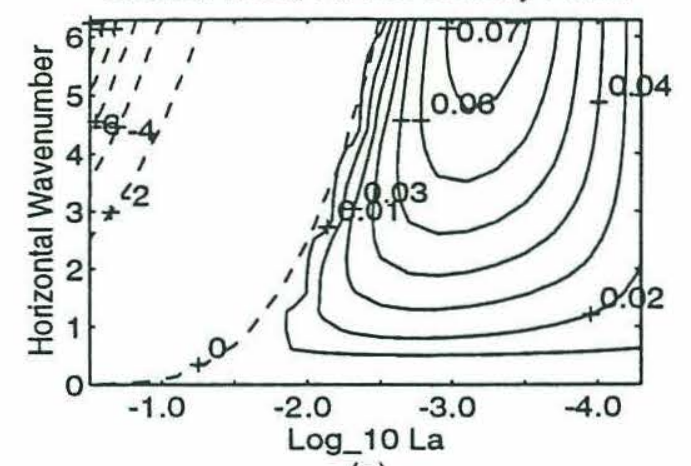

(a)

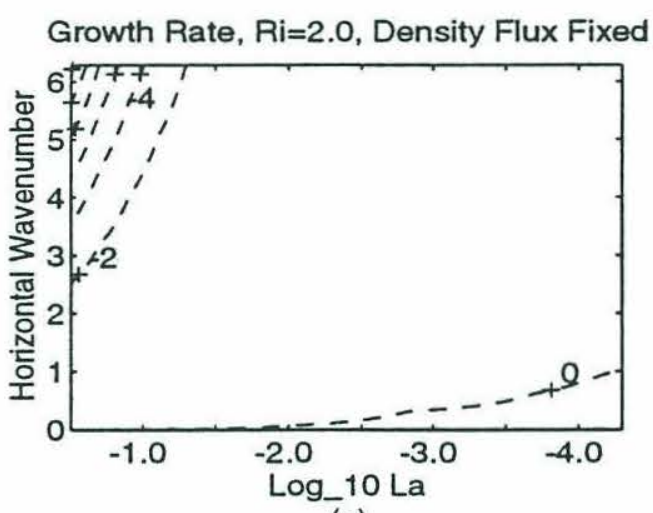

(c)

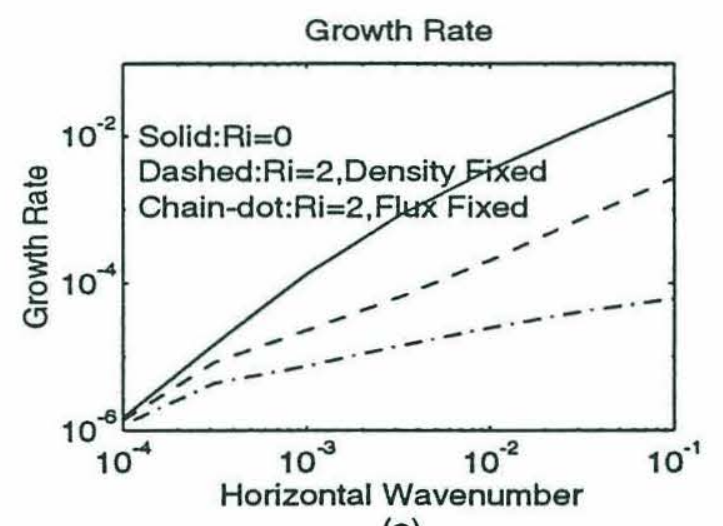

(e)

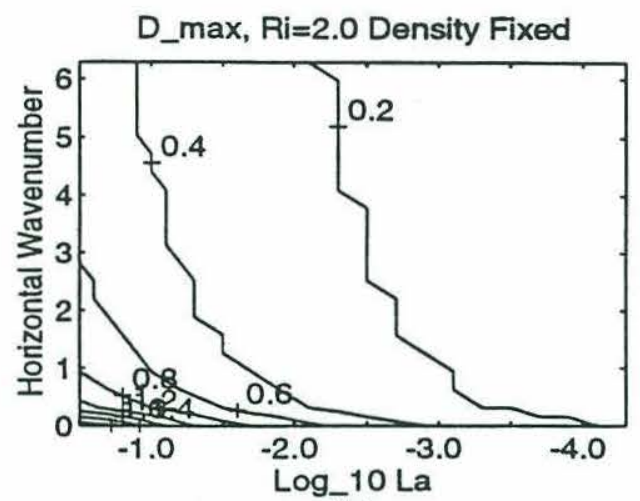

(b)

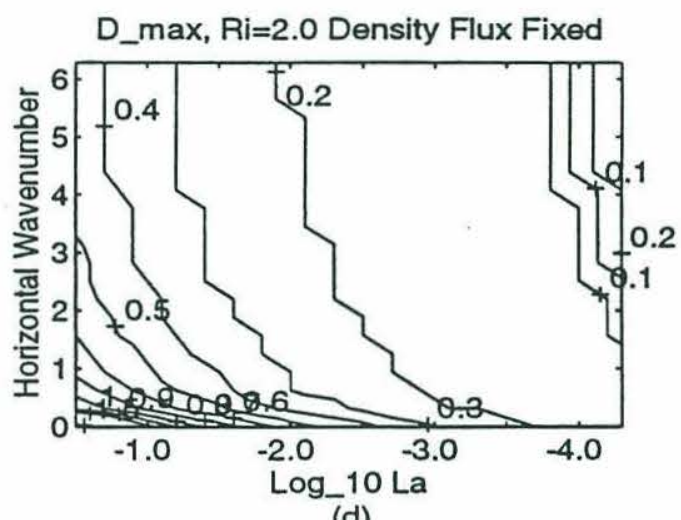

(d)

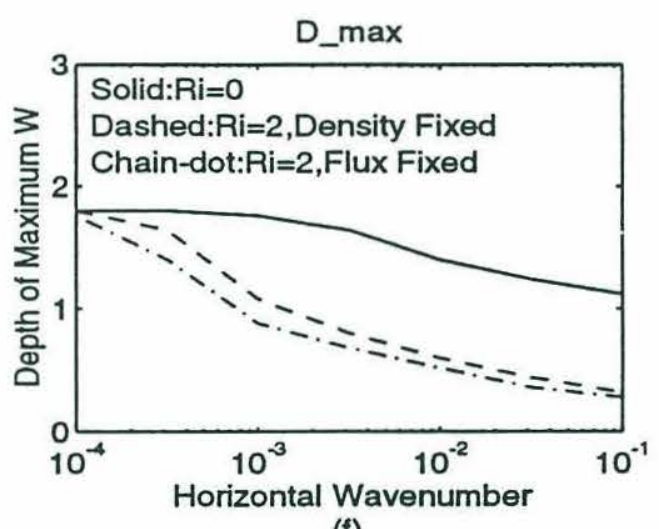

(f)

Figure 2.6: Instability of Langmuir cells at high values of stratification. All runs shown assume monochromatic waves, layer depth $\mathrm{D}=4$, no Coriolis forces and a surface Eulerian shear of 1.0. (a) Growth rate, $\mathrm{Ri}=2.0$, density fixed on top and bottom boundaries. (b) $\mathrm{D}_{\max }, \mathrm{Ri}=2.0$, density fixed on top and bottom boundaries. (c) Same as (a) but for density flux fixed on upper boundary. (d) Same as (b) but for density flux fixed on upper boundary. (e) Growth rate at very low wavenumber given $\mathrm{La}=0.001$. Solid line is for $\mathrm{Ri}=0.0$, dashed for $\mathrm{Ri}=2.0$ with density fixed on both boundaries. chain-dotted for $\mathrm{Ri}=2.0$ with density flux fixed on upper boundary. (f) Same as (e) but for $D_{\max }$. 
At low wavenumber and Langmuir number, there is some instability even when the stratification is strong. Figure 2.6e shows $\gamma$ and Figure $2.6 f \mathrm{D}_{\max }$ for $\mathrm{La}=0.001, \mathrm{D}=4$, given a monochromatic wave train and a surface shear of 1.0. The solid line is for $\mathrm{Ri}=0.0$. The dashed line is for $\mathrm{Ri}=2.0$ with density fixed on the top and bottom boundaries. The chain-dotted line is for $\mathrm{Ri}=2.0$ with the density fixed on the lower boundary and the density flux fixed on the top boundary. The growth rates are smaller for the two stratified cases and $D_{\max }$ is smaller as well, indicating that the cells are trapped closer to the surface. The upper boundary condition is also important for the growth rates at low wavenumbers, with a flux boundary condition on the upper boundary giving lower growth rates. As the wavenumber becomes very small, both the growth rate and depth of maximum vertical velocity asymptote to the unstratified value.

The four major results for stratified Langmuir cells are thus -At weak to moderate values of stratification, the growth rates and depth of maximum vertical velocity for the linearly unstable modes is not greatly affected by the upper boundary condition and the overall pattern resembles that in the absence of stratification.

-Stratification reduces both the growth rate and depth at which the maximum vertical velocity occurs.

- For strong values of stratification, the value of $\mathrm{La}$ as well as the upper boundary condition is critical in determining the growth rate and depth of maximum vertical velocity for the linearly unstable modes.

- At low wavenumbers, however, the stratified results asymptote to the unstratified results even for high values of stratification.

\subsection{The Physics of Craik-Leibovich Instability}

\subsubsection{Energetics of the Instability}

In order to understand the results of Section 2.3, we will now derive equations for the energy balance which demonstrate how the cell structure determines the growth rate, and how the Langmuir number, Stokes drift profile, stratification, and boundary conditions determine the cell structure. Take the linearized equations of motion. As in Section 2.2 let $\delta \psi^{\prime}, \delta v^{\prime}, \delta \rho^{\prime}$ represent the perturbation streamfunction, velocity, and density fields, while $\Psi_{0}, \mathrm{~V}_{0}$ and $\mathrm{P}_{0}$ represent the equilibrium fields in the absence of cells. Substituting into (2-2), the equations to zeroth order in $\delta$ are:

$$
\Psi_{0}(\mathrm{z})=0
$$




$$
\begin{gathered}
\mathrm{V}_{0}(\mathrm{z})=\frac{(\mathrm{z}+\mathrm{D})^{2}}{2 \mathrm{D}} \\
\mathrm{P}_{0}(\mathrm{z})=-\mathrm{z}
\end{gathered}
$$

while to first order in d:

$$
\begin{gathered}
\frac{\partial}{\partial \mathrm{t}} \nabla^{2} \psi^{\prime}=\frac{\partial \mathrm{v}_{\mathrm{s}} \partial \mathrm{v}^{\prime}}{\partial \mathrm{z} \partial \mathrm{x}}+\mathrm{Ri} \frac{\partial \rho^{\prime}}{\partial \mathrm{x}}+\mathrm{La}^{4} \psi^{\prime} \\
\frac{\partial \mathrm{v}^{\prime}}{\partial \mathrm{t}}=\frac{\partial \mathrm{v}_{0} \partial \psi^{\prime}}{\partial \mathrm{z} \partial \mathrm{x}}+\mathrm{La} \nabla^{2} \mathrm{v}^{\prime} \\
\frac{\partial \rho^{\prime}}{\partial \mathrm{t}}=\frac{\partial \mathrm{P}_{0} \partial \psi^{\prime}}{\partial \mathrm{z} \partial \mathrm{x}}+\mathrm{La} \nabla^{2} \rho^{\prime}
\end{gathered}
$$

Multiplying equation (2-13a) by $\psi^{\prime},(2-13 b)$ by v', (2-13c) by $\rho$ ', and designating horizontal averaging by an overbar gives the perturbation variance equations:

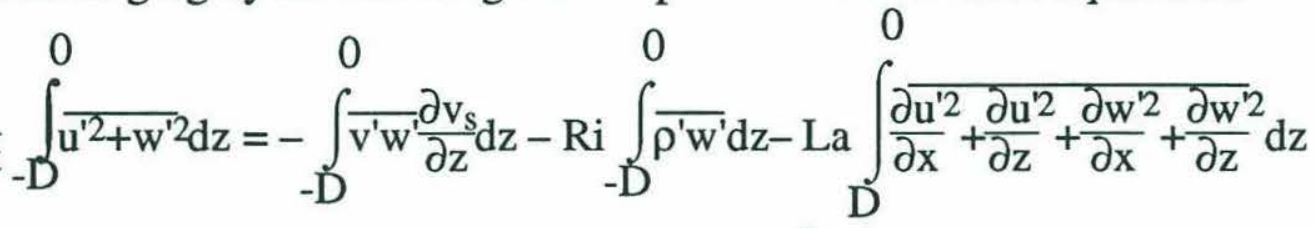

$$
\frac{\partial}{\partial t} \int_{-D}^{0} \overline{v^{\prime 2}} d z=-\int_{-D}^{0} \overline{v^{\prime} w} \frac{\partial V_{0}}{d z} d z-L a \int_{-D}^{0} \frac{\partial v^{\prime 2}}{\partial x}+\frac{\partial v^{2}}{\partial z} d z
$$

$$
\frac{\partial}{\partial t} \int_{-D}^{0} \overline{\rho^{\prime 2}} d z=\int_{-D}^{0} \frac{\rho^{\prime} w^{\prime}}{\frac{\partial P_{0}}{d z}} d z-L a \int_{-D}^{0} \frac{\partial \rho^{2}}{\partial x}+\frac{\partial \rho^{2}}{\partial z} d z
$$

Then the energy balance is

$$
\begin{gathered}
\frac{\partial}{\partial t} E_{c c}=P_{\text {stokes }}-B_{\text {trans }}-\varepsilon_{c c} \\
\frac{\partial}{\partial t} E_{a c}=P_{a c}-\varepsilon_{a c} \\
\frac{\partial}{\partial t} E_{\rho}=P_{\rho}-\varepsilon_{\rho}
\end{gathered}
$$

where $\mathrm{E}_{\mathrm{cc}, \mathrm{ac}}$ are the energies associated with flow in the crosscell and alongcell directions respectively. $\mathrm{E}_{\rho}$ is the density variance. $\mathrm{P}_{\text {stokes }}$ is the Stokes production (the work done by the waves on the cell vortices). $\mathrm{P}_{\mathrm{ac}}$ is the shear production. $\mathrm{P}_{\rho}$ is the density variance production. $\mathrm{B}_{\text {trans }}$ is the buoyancy transport. $\varepsilon_{\mathrm{cc}, \mathrm{ac}, \rho}$ are the dissipation terms associated with the crosscell velocities, alongcell velocity, and density respectively.

\section{Define}

$$
\begin{aligned}
& \mathrm{v}^{\prime}=\mathrm{v}_{1} \mathrm{e} \gamma \mathrm{t} V(\mathrm{z}) \cos (\mathrm{kx}) \\
& \rho^{\prime}=\rho_{1} \mathrm{e} \gamma \mathrm{t} \rho(\mathrm{z}) \cos (\mathrm{kx})
\end{aligned}
$$




$$
\psi^{\prime}=\psi_{1} \mathrm{e} \gamma \mathrm{t} \psi(\mathrm{z}) \sin (\mathrm{kx})
$$

so that the perturbation structure of each field is given by a shape function multiplied by an amplitude. The structure functions $V, \psi, \rho$ are normalized so that:

$$
\frac{2}{\mathrm{D}} \int_{-\mathrm{D}}^{0}|(V, \psi, \rho)|^{2} \mathrm{~d} z=1
$$

Substituting into the crosscell energy balance and letting $\gamma$ be the growth rate then yields the following relation between the three amplitudes

$$
\text { 8) } \frac{\gamma \psi_{1}^{2}}{2} \int_{-D}^{0}\left(\frac{\partial \psi}{\partial z}\right)^{2}+k^{2} \psi^{2} d z=\psi_{1} v_{1} \frac{k}{2} \int_{-D}^{0} \psi(z) V(z) \frac{\partial v_{s}}{\partial z} d z+
$$

$$
\operatorname{Ri} \psi_{1} \rho \frac{\mathrm{k}}{2} \int_{-\mathrm{D}}^{0} \psi(\mathrm{z}) \rho(\mathrm{z}) \mathrm{dz}-\frac{\psi_{1}^{2} \mathrm{La}}{2} \int_{-\mathrm{D}}^{0}\left(\frac{\partial^{2} \psi}{\partial \mathrm{z}^{2}}\right)^{2}+2 \mathrm{k}^{2}\left(\frac{\partial \psi}{\partial \mathrm{z}}\right)+\mathrm{k}^{4} \psi^{2} \mathrm{dz}
$$

Defining

$$
\begin{gathered}
\hat{V_{s}} s_{z}=\frac{2}{D} \int_{-D}^{0} \psi(z) V(z) \frac{\partial v_{s}}{\partial z} d z \\
\beta=\frac{2}{D} \int_{-D}^{0} \psi(z) \rho(z) d z \\
k_{\psi}^{2}=\frac{2}{D} \int_{-D}^{0}\left(\frac{\partial \psi}{\partial z}\right)^{2}+k^{2} \psi^{2} d z \\
K_{\psi}^{4}=\frac{2}{D} \int_{-D}^{0}\left(\frac{\partial^{2} \psi}{\partial z^{2}}\right)^{2}+2 k^{2}\left(\frac{\partial \psi}{\partial z}\right)^{2}+k^{4} \psi^{2} d z
\end{gathered}
$$

and dividing out common terms, yields

$$
\left(\gamma+\mathrm{La} \mathrm{K}_{\psi}^{4} / \mathrm{k}_{\psi}^{2}\right) \psi_{1}=\mathrm{kV} \mathrm{V}_{\mathrm{z}} / \mathrm{k}_{\psi}^{2} \mathrm{v}_{1}+\mathrm{Ri} \beta \mathrm{k} / \mathrm{k}_{\psi}^{2} \rho_{1}
$$

Similarly, by defining

$$
\hat{\mathrm{V}}_{\mathrm{z}} \stackrel{2}{=} \int_{-\mathrm{D}}^{0} \psi(\mathrm{z}) V(\mathrm{z}) \frac{\partial \mathrm{V}_{0}}{\partial \mathrm{z}} \mathrm{dz}
$$

$$
\hat{\mathrm{P}}_{0 \mathrm{z}}=\frac{2}{\mathrm{D}} \int_{-\mathrm{D}}^{0} \psi(\mathrm{z}) \rho(\mathrm{z}) \frac{\partial \mathrm{P}_{0}}{\partial \mathrm{z}} \mathrm{dz}
$$




$$
\mathrm{k}_{\mathrm{v}}^{2}=\frac{2}{\mathrm{D}} \int_{-\mathrm{D}}^{0}\left(\frac{\partial V}{\partial \mathrm{z}}\right)^{2}+\mathrm{k}^{2} \mathrm{~V}^{2} \mathrm{dz} \quad \mathrm{k}_{\rho}^{2}=\frac{2}{\mathrm{D}} \int_{-\mathrm{D}}^{0}\left(\frac{\partial \rho}{\partial \mathrm{z}}\right)^{2}+\mathrm{k}^{2} \rho^{2} \mathrm{dz}
$$

the equations for alongcell velocity and density may also be obtained

$$
\begin{aligned}
& \left(\gamma+\operatorname{Lak}_{\mathrm{v}}^{2}\right) \mathrm{v}_{1}=\mathrm{k} \hat{\mathrm{V}}_{\mathrm{z}} \psi_{1} \\
& \left(\gamma+\mathrm{Lak}_{\rho}^{2}\right) \rho_{1}=\mathrm{k} \hat{\mathrm{P}}_{0 \mathrm{z}} \psi_{1}
\end{aligned}
$$

Substituting into (2-20) and letting

$$
\begin{gathered}
\gamma_{\mathrm{CL}}^{2}=\hat{\mathrm{V}}_{\mathrm{z}} \hat{\mathrm{V}}_{\mathrm{s}_{\mathrm{z}}} \\
\mathrm{N}^{2}=\operatorname{Ri} \beta \hat{\mathrm{P}}_{\mathrm{z}}
\end{gathered}
$$

yields the following, cubic equation for $\gamma$

$$
\begin{aligned}
& \left(\gamma+\mathrm{LaK}_{\psi}^{4} / \mathrm{k}_{\psi}^{2}\right)\left(\gamma+\mathrm{Lak}_{\mathrm{v}}^{2}\right)\left(\gamma+\mathrm{Lak}_{\rho}^{2}\right)= \\
& \mathrm{k}^{2} \gamma_{\mathrm{CL}}^{2} / \mathrm{k}_{\psi}^{2}\left(\gamma+\mathrm{Lak}_{\rho}^{2}\right)+\mathrm{k}^{2} \mathrm{~N}^{2} / \mathrm{k}_{\psi}^{2}\left(\gamma+\mathrm{La} \mathrm{k}_{\mathrm{v}}^{2}\right)
\end{aligned}
$$

By considering some simple solutions of equation (2-24) it is possible to understand the physics behind the results of section 2.3. This is done during the remainder of this section.

\subsubsection{Linking Cell Structure and Growth Rate at High Wavenumber}

Suppose that the density and velocity perturbations have identical structure functions, so that $V(\mathrm{z})=\rho(\mathrm{z})$, and $\mathrm{k}_{\rho}^{2}=\mathrm{k}^{2}$. Then the solution to $(2-24)$ is

$$
\sqrt{\left.\frac{\mathrm{La}^{2}\left(\mathrm{k}_{\mathrm{v}}^{2}+\mathrm{K}_{\psi}^{4} / \mathrm{k}_{\psi}^{2}\right)^{2}}{4}+\frac{\mathrm{La}\left(\mathrm{k}_{\mathrm{v}}^{2}+\mathrm{K}_{\psi}^{4} / \mathrm{k}_{\psi}^{2}\right)}{2}+\mathrm{k}_{\psi}^{2} \gamma_{\mathrm{CL}}^{2}-\mathrm{N}^{2}\right)-\mathrm{La} \mathrm{k}_{\mathrm{v}}^{2} \mathrm{~K}_{\psi}^{4}}
$$

The stratified Craik-Leibovich instability parameter $\gamma_{C L S}$ and the characteristic diffusive decay scale $\gamma_{\text {diff }}$ may then be defined as follows:

$$
\begin{gathered}
\gamma_{C L S}=\sqrt{\gamma_{C L}^{2}-N^{2}} \\
\gamma_{\text {diff }}=\text { La k }{ }_{v}^{2} K_{\psi}^{4} k_{\psi}^{2} / k^{2}
\end{gathered}
$$

Then the necessary condition for instability is that

$$
\operatorname{RaCLS}=\frac{\gamma_{\text {CLS }}^{2}}{\gamma_{\text {diff }}^{2}}>1
$$

As La becomes very small, the growth rate becomes 


$$
\gamma=\frac{\mathrm{k}}{\mathrm{k}_{\psi}} \sqrt{\gamma_{\mathrm{CL}}^{2}-\mathrm{N}^{2}}=\frac{\mathrm{k}}{\mathrm{k}_{\psi}} \gamma_{\mathrm{CLS}}
$$

Equation (2-26a) may be squared and rewritten as follows

$$
\begin{array}{r}
\gamma_{\mathrm{CLS}}^{2}(\mathrm{D})=\left(\frac{2}{\mathrm{D}}\right)^{2} \int_{-\mathrm{D}}^{0} \psi(\mathrm{z}) V(\mathrm{z}) \frac{\partial \mathrm{V}_{0}}{\partial \mathrm{z}} \mathrm{dz} \int_{-\mathrm{D}}^{0} \psi(\mathrm{z}) V(\mathrm{z}) \frac{\partial \mathrm{v}_{\mathrm{s}}}{\partial \mathrm{z}} \mathrm{dz}- \\
\quad \int_{-\mathrm{D}}^{0} \psi(\mathrm{z}) \rho(\mathrm{z}) \frac{\partial \mathrm{P}_{0}}{\partial \mathrm{z}} \mathrm{dz} \int_{-\mathrm{D}}^{0} \psi(\mathrm{z}) \rho(\mathrm{z}) \mathrm{dz}
\end{array}
$$

Which may be rewritten in dimensional form as:

$$
\gamma_{C L S}^{2}(D)=\frac{4}{D^{2}}\left(\int_{-D}^{0} F(z) \frac{\partial v_{S}}{\partial z} d z \int_{-D}^{0} F(z) \frac{\partial V}{\partial z} d z-\int_{-D}^{0} G(z) \frac{g \partial \rho}{\rho \partial z} d z\right)
$$

so that $\gamma_{\text {CLS }}$ corresponds to the local instability parameter defined by Leibovich (1977b) and shown in (2-1), but with the various components defined by depthaverages rather than by local values. The weighting functions $F(z)$ and $G(z)$ used to define the depth-average depend on the shape of the momentum and density transport carried by the cells.

In order to get a better feel for the what the various terms mean, the streamfunction, density, and alongcell velocity structure functions may be approximated as follows

$$
\begin{array}{cc}
\psi=\sin \left(\pi \mathrm{z} / \mathrm{D}^{\prime}\right) & \mathrm{z}>-\mathrm{D}^{\prime} \\
V, \rho=\cos \left(\pi \mathrm{z} / 2 \mathrm{D}^{\prime}\right) & \mathrm{z}>-\mathrm{D}^{\prime} \\
\psi, V, \rho=0 & \mathrm{z}<-\mathrm{D}^{\prime}
\end{array}
$$

so that $D^{\prime}=2 D_{\max }$. In this truncated representation of cell structure, the cells penetrate over D', but have no effect below $\mathrm{D}^{\prime}$. This truncation will be denoted T1. Because the cell penetration depth is limited, Truncation T1 models cells which are not affected by the different bottom boundary conditions on velocity and density.

Defining $\gamma_{C L S 1}$ as $\gamma_{C L S}$ when the structure functions are given by Truncation $\mathrm{T} 1$, and noting that $\mathrm{k}_{\psi}=\mathrm{K}_{\psi}=\sqrt{\mathrm{k}^{2}+\pi^{2} / \mathrm{D}^{\prime 2}}$ then

$$
\gamma_{\text {diff }}=\mathrm{La}\left(\mathrm{k}^{2}+\pi^{2} / \mathrm{D}^{\prime 2}\right) \sqrt{\mathrm{k}^{2}+\pi^{2} / 4 \mathrm{D}^{2} / \mathrm{k}}
$$

$$
\gamma^{\mathrm{T} 1}=-\mathrm{La}\left(\mathrm{k}^{2}+\left(5 \pi / 8 \mathrm{D}^{\prime}\right)^{2}\right)+\sqrt{\mathrm{La}^{2}\left(\mathrm{k}^{2}+\left(5 \pi / 8 \mathrm{D}^{\prime}\right)^{2}\right)+\frac{\mathrm{k}^{2}}{\mathrm{k}^{2}+\pi^{2} / \mathrm{D}^{\prime 2}}\left(\gamma_{\mathrm{CLS} 1}^{2}-\gamma_{\text {diff }}^{2}\right)}
$$




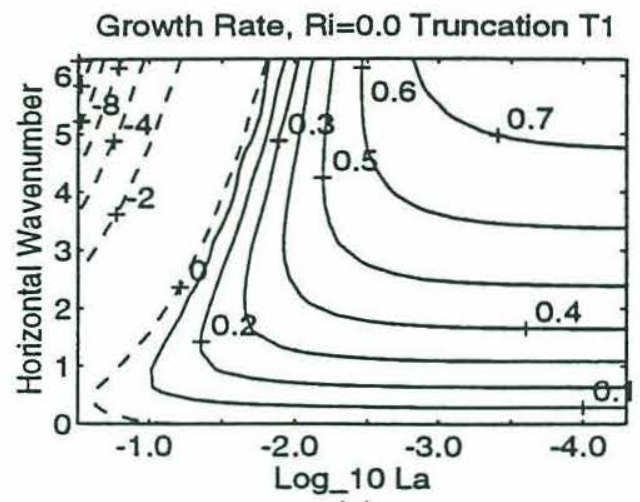

(a)

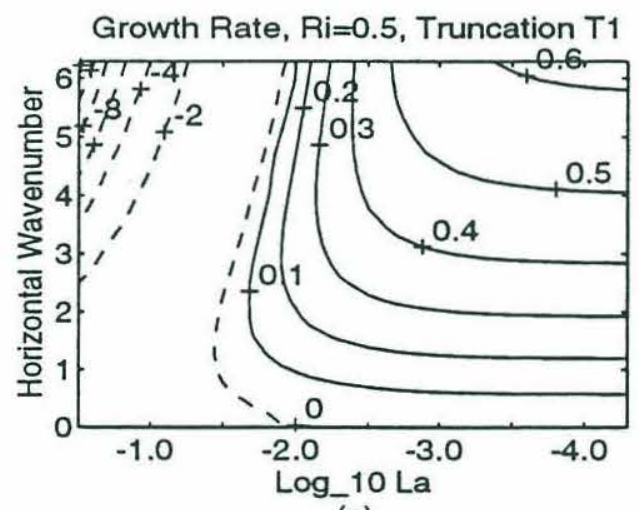

(c)

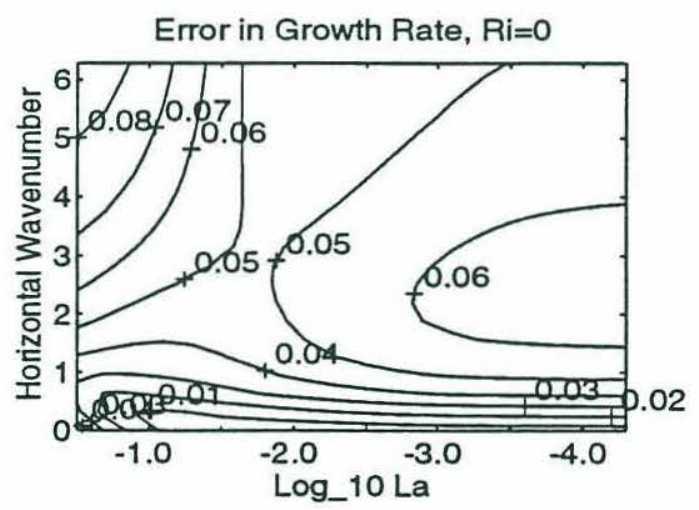

(e)

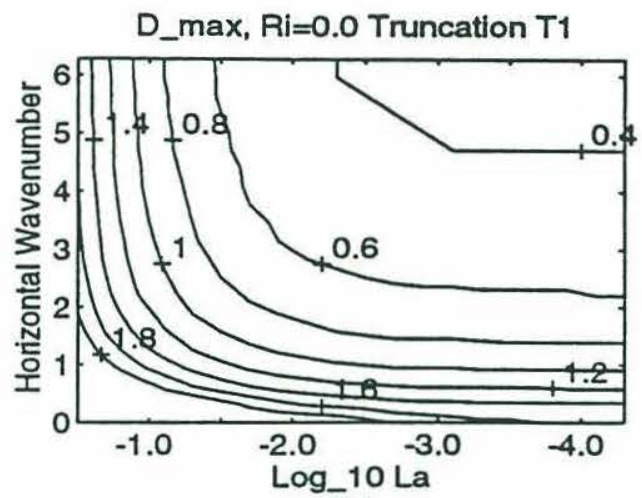

(b)

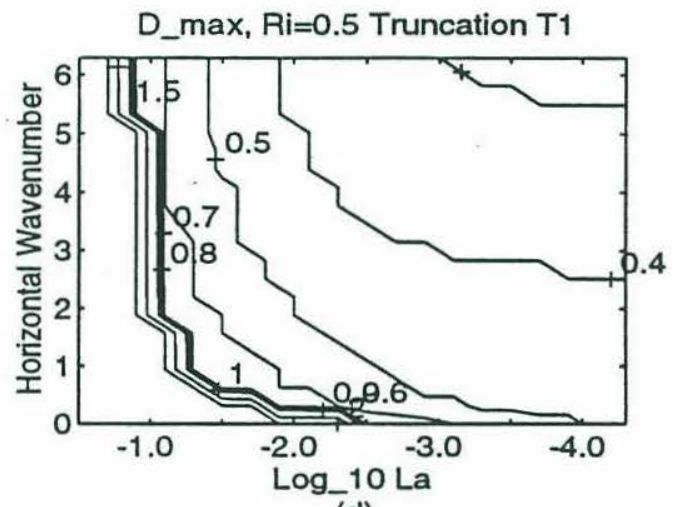

(d)

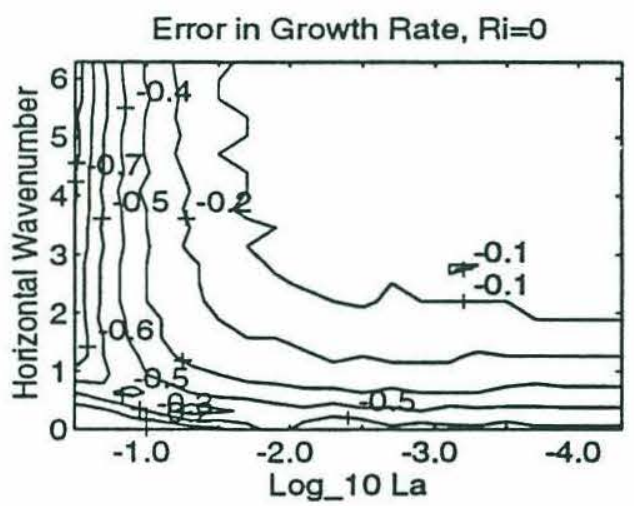

(f)

Figure 2.7: Ability of truncated models to capture the physics of Langmuir cell instability. All runs assume $\mathrm{D}=4$, monochromatic waves parallel with the wind and cell axis, no Coriolis force and a surface Eulerian shear of 1. (a) Growth rate, Truncation T1. Ri=0.5. (b) Depth of maximum vertical velocity, Truncation T1 $\mathrm{D}_{\max }, \mathrm{Ri}=0.0$. (c) Growth rate, Truncation $\mathrm{T} 1$. $\mathrm{Ri}=0.0$. (d) Depth of maximum vertical velocity, Truncation $T 1 D_{\max }, R i=0.5$. (e) Error in growth rate caused by using Truncation $T 1$ for $R i=0.0$. (f) Error in $D_{\max }$ caused by using Truncation $T 1$ for $\mathrm{Ri}=0.0$. 


$$
\operatorname{Ra}_{C L S 1}=\gamma_{\text {CLS1 }}^{2} / \gamma_{\text {diff }}^{2}
$$

For large values of RaCLS1

$$
\gamma \sim \frac{\mathrm{k}}{\sqrt{\mathrm{k}^{2}+\pi^{2} / \mathrm{D}^{\prime 2}}} \gamma_{\mathrm{CLS}}
$$

How well does this approximate truncation predict growth rates and cell structure? Suppose that the growth rate of the most unstable mode $\gamma(\mathrm{k}, \mathrm{N}=0, \mathrm{La})$ is given by maximizing $\gamma^{\mathrm{T} 1}\left(\mathrm{D}^{\prime}\right)$ with respect to $\mathrm{D}^{\prime}$ and that the depth of maximum vertical velocity is given by half the value of $\mathrm{D}^{\prime}$ for which $\gamma^{\mathrm{T} 1}$ is maximized. Figures $2.7 \mathrm{a}$ and $\mathrm{b}$ show the resulting predictions for growth rate and $\mathrm{D}_{\max }$ as a function of horizontal wavenumber and Langmuir number for $\mathrm{D}=4$, surface shear $=1, \mathrm{Ri}=0.0$, and a monochromatic wave train (corresponding to Figures $2.2 \mathrm{c}$ and d). Figure $2.7 \mathrm{c}$ and $\mathrm{d}$ show the growth rate and $\mathrm{D}_{\max }$ for $\mathrm{Ri}=0.5$, corresponding to Figure $2.5 \mathrm{e}$ and $\mathrm{f}$. Figures $2.7 \mathrm{e}$ and $\mathrm{f}$ show the difference between the growth rate and $D_{\max }$ predicted by Truncation $T 1$ and that predicted by the full instability code for $\mathrm{D}=4$ and $\mathrm{Ri}=0.0$.

The truncated model does very well at capturing the dependence of the growth rate on horizontal wavenumber and Langmuir number, not only qualitatively but quantitatively as well. Making the approximations that the density and velocity structures are identical and that they are essentially zero below $2 \mathrm{D}_{\max }$ does not change the solutions substantially. The mean error introduced by using the truncation is 0.05 , a small error given growth rates ranging from -12 to 0.7 . The truncation does not predict the depth of maximum vertical velocity as accurately, overestimating it for large $\mathrm{La}$ and low wavenumber. Nonetheless the truncation does still capture the reduction in $\mathrm{D}_{\max }$ associated with decreasing $\mathrm{La}$ and cell spacing. The agreement between the trucnation and the full instability code means that the closed-form solution in equation (2-32b) can be used to isolate the important physics governing the relationship between cell structure and growth rate (except when the Langmuir number or the wavelength is very large).

Suppose that $\mathrm{La}=0$. Then the growth rate is given by

$$
\gamma=\frac{\mathrm{k}}{\sqrt{\mathrm{k}^{2}+\pi^{2} / \mathrm{D}^{\prime 2}}} \gamma_{\mathrm{CLS} 1}=\mathrm{G}\left(\mathrm{k}, \mathrm{D}^{\prime}\right) \gamma_{\mathrm{CLS} 1}\left(\mathrm{D}^{\prime}\right)
$$

where $G\left(k, D^{\prime}\right)=k / \sqrt{k^{2}+\pi^{2} / D^{\prime 2}}$, is a geometric factor. The geometric factor is a monotonically increasing function of $\mathrm{D}^{\prime}$, going as $\mathrm{D}^{\prime}$ as for $\mathrm{D}^{\prime} \ll 2 / \mathrm{k}$ and asymptoting to a value of 1 if $D^{\prime} » 2 / k$. The stratified Craik-Leibovich instability parameter is a monotonically decreasing function of D', since the Eulerian and Stokes' Drift shears are maximal at $\mathrm{z}=0$ and decrease with depth. The growth rate then is 
determined by a tradeoff between maximizing $\gamma_{C L S}$ (favoring cells trapped near the surface) and maximizing $\mathrm{kD}^{\prime}$ (favoring cells penetrating to great depth).

The details of this tradeoff are strongly dependent on the vertical structure of the $\gamma_{C L S}\left(D^{\prime}\right)$. If only the amplitude of the forcing is changed (say by doubling the surface shear), $\gamma^{\mathrm{T} 1}$ will increase by a constant factor but the depth $\mathrm{D}_{\max }$ at which the maximum in $\gamma^{\mathrm{T} 1}$ (D') occurs will not change. On the other hand, if the structure of the forcing is changed, (say by changing from a monochromatic wave train to a Pierson-Moskowitz spectrum) $\mathrm{D}_{\max }$ will change.

The physics behind the tradeoff between the aspect ratio and the forcing can be understood for the unstratified case as follows (the stratified case is more complicated mathematically but the basic idea is identical). Let, as before, $\mathrm{E}_{\mathrm{cc}}$ be the crosscell perturbation energy and $\mathrm{E}_{\mathrm{ac}}$ be the alongcell perturbation energy, and let $\mathrm{u}^{\prime}, \mathrm{v}^{\prime}$, and $\mathrm{w}^{\prime}$ be characteristic perturbation velocities in the crosscell horizontal, alongcell horizontal and vertical directions respectively. Then

$$
\begin{gathered}
\frac{\partial}{\partial t} E_{c c} \sim \frac{\partial}{\partial t}\left(u^{\prime 2}+w^{\prime 2}\right) \sim \text { Stress*Stokes Drift Shear } \\
\frac{\partial}{\partial t} E_{a c} \sim \frac{\partial}{\partial t} v^{\prime 2} \sim \text { Stress*Eulerian Shear }
\end{gathered}
$$

Solving for the change in stress in terms of the change in energies gives

$\frac{\partial}{\partial t}$ Stress $\sim \frac{\partial}{\partial t} v^{\prime} w^{\prime} \sim$ Stress $* \sqrt{\text { Eulerian Shear*Stokes Drift Shear* } \frac{w^{\prime 2}}{u^{\prime 2}+w^{\prime 2}}}$

$$
\sim \sqrt{\text { Stokes Production*Shear Production } *} \frac{\mathrm{k}}{\sqrt{\mathrm{k}^{2}+\pi^{2} / \mathrm{D}^{\prime 2}}}
$$

So maximizing $\gamma_{\mathrm{CL}}$ optimizes the energy release resulting from a given stress, while maximizing $\mathrm{kD}$ ' optimizes the efficiency of the released energy at increasing the stress.

If $\mathrm{La} \neq 0$ an additional factor is introduced. As $\mathrm{D}^{\prime}$ gets very small, the diffusive decay scale, which for small $\mathrm{D}^{\prime}$ goes as $\mathrm{La} / \mathrm{D}^{\prime 2}$, gets very large and can overwhelm the Craik-Leibovich instability parameter within equation (2-32b). Minimizing the diffusive decay scale favors cells with a deeper penetration. This means that the growth rate decreases as $\mathrm{La}$ increases for two reasons, greater diffusive damping and a decreased ability to take advantage of the higher shears near the surface.

In the absence of density stratification $(\mathrm{Ri}=0) \gamma_{\mathrm{CLS}}$ is always positive. In the presence of such stratification, however, there is a depth below which $\gamma_{C L}\left(2 D_{\max }\right)$ $=\mathrm{N}$. This serves as a lower limit for cell penetration so long as the cells do not feel the lower boundary. For $\mathrm{Ri}=0.5$, monochromatic waves, a layer depth of 4 and a 
surface shear of 1 , the depth $D_{\max }$ for which $\gamma_{C L 1}=\mathrm{N}$ is 0.8 . In Figure $2.5 \mathrm{f}$ the unstable modes have $D_{\max }<0.8$ except at very low wavenumber.

An implication of this result is that Langmuir cells do not require that the water column be unstable over their entire depth of penetration. Mathematically this would mean that $\sqrt{\frac{\partial V \partial v_{s}}{\partial z \partial z}-N^{2}}>0$ for all $z>-2 D_{\max }$. If this were the case, given $\mathrm{Ri}=0.5, \mathrm{D}_{\max }$ would be no more than 0.3 , much smaller than is seen in most of the cases presented in Figure 2.5. The upper limit on the depth of penetration is determined by the requirement that the average wave-current forcing must be stronger than the average stabilizing stratification, rather than the wave-current forcing needing to be stronger over the entire cell depth of penetration.

This upper limit on cell penetration only holds when the velocity and density perturbation have similar structures. The boundary conditions on density and velocity are different in the present formulation of the problem. This means that the velocity and density structures may be quite different. As shown in the following section, when the cells "feel the boundaries" the stratification does not necessarily limit the depth of penetration.

To summarize then, given cells which do not feel the effect of the bottom boundary, so that density and velocity perturbations have similar structures:

-The growth rate and structure of nonrotating Langmuir cells are determined by a complex tradeoff between maximizing the strength of the forcing (given by $\gamma_{C L S}$ ), maximizing efficiency of the forcing (given by the aspect ratio $\mathrm{kD}$ ), and minimizing the strength of diffusion (given by $\gamma_{\text {diff }}$ )

-The necessary condition for instability of cells of a given geometry is that RaCLS $=\gamma_{\text {CLS }}^{2} / \gamma_{\text {diff }}^{2}>1$.

-Cell depth of penetration decreases for decreasing cell spacing and diffusion and increasing stratification.

\subsubsection{Cell Structure and Growth Rate at Low Wavenumber and High Langmuir Number}

In Section 2.3 it was demonstrated that at long wavelengths the growth rate $\gamma$ becomes very small. It can also be shown that the alongcell velocity structure function becomes constant at long wavelengths

$$
V(z) \rightarrow 1 / \sqrt{2}
$$


so that the diffusive decay scale for the velocity perturbation $\mathrm{Lak}_{\mathrm{v}}^{2} \rightarrow \mathrm{La} \mathrm{k}^{2}$ which is also very small. Qualitative evidence of this trend towards smoothing the velocity perturbation at long wavelength can be seen in Figure 2.1. The structure functions for the streamfunction and density perturbations, however, must have some vertical structure so as to satisfy the boundary conditions, so that the diffusive decay scales for these fields asymptote to a nonzero value. At long wavelengths then

$$
\gamma, \operatorname{Lak}_{\mathrm{v}}^{2} \ll \operatorname{LaK}_{\psi}^{4} / \mathrm{k}_{\psi}^{2}, \mathrm{Lak}_{\mathrm{p}}^{2}
$$

so that equation (2-24) simplifies to

$$
\operatorname{LaK}_{\psi}^{4} / \mathrm{k}_{\psi}^{2}\left(\gamma+\mathrm{Lak}_{\mathrm{v}}^{2}\right) \mathrm{La} \mathrm{k}_{\rho}^{2}=\mathrm{k}^{2} \gamma_{\mathrm{CL}}^{2} / \mathrm{k}_{\psi}^{2} \mathrm{La} \mathrm{k}_{\rho}^{2}
$$

yielding the following solution for the growth rate

$$
\gamma=\frac{\mathrm{k}^{2}}{\mathrm{La} \mathrm{K}_{\psi}^{4}}\left(\gamma_{\mathrm{CL}}^{2}-\mathrm{La} \mathrm{K}_{\psi}^{4} \mathrm{k}_{\mathrm{V}}^{2} / \mathrm{k}^{2}\right)
$$

The density vanishes from the problem altogether, a result seen in the instability code. The mathematical development above demonstrates that this lack of dependence on stratification results from the boundary conditions. In order to satisfy the boundary conditions, the density perturbation must have some vertical structure. As a result, it is much more strongly damped than the velocity perturbation, and so ceases to play an important role in the dynamics.

A truncation which captures these dynamics is one for which the cells penetrate over the entire layer of fluid

$$
\begin{gathered}
\psi=\psi_{0} \mathrm{e}^{\mathrm{ikx}} \sin (\pi \mathrm{z} / \mathrm{D}) \\
\mathrm{v}=\mathrm{v}_{1} \mathrm{e}^{\mathrm{ikx}}+\mathrm{V}_{0}(\mathrm{z})
\end{gathered}
$$

We will refer to this truncation as T2. The most salient feature of this truncation is that the velocity perturbation is strongly affected by both the upper and the lower boundaries, and thus has a uniform structure with depth, while the density perturbation is ignored altogether. Defining

$$
\begin{gathered}
\hat{\mathrm{V}}_{\mathrm{z} 2}=\frac{\sqrt{2}}{\mathrm{D}} \int_{-\mathrm{D}^{\prime}}^{0} \frac{\partial \mathrm{V}}{\partial \mathrm{z}} \sin \left(\frac{\pi \mathrm{z}}{\mathrm{D}}\right) \mathrm{dz} \\
\hat{\mathrm{V}}_{\mathrm{z} \mathrm{z} 2}=\frac{\sqrt{2}}{\mathrm{D}} \int_{-\mathrm{D}^{\prime}}^{0} \frac{\partial \mathrm{v}_{\mathrm{s}}}{\partial \mathrm{z}} \sin \left(\frac{\pi \mathrm{z}}{\mathrm{D}}\right) \mathrm{dz} \\
\gamma_{\mathrm{CL} 2}=\sqrt{\hat{\mathrm{V}}_{\mathrm{z} 2} \hat{\mathrm{V}}_{\mathrm{z} 2}}
\end{gathered}
$$




$$
\gamma_{\text {diff } 2}=\mathrm{La}\left(\mathrm{k}^{2}+\frac{\pi^{2}}{\mathrm{D}^{2}}\right)
$$

the growth rate of the most unstable mode given fixed $\mathrm{La}, \mathrm{k}$ and $\mathrm{D}$ is

$$
\gamma^{\mathrm{T} 2}=-\mathrm{La}\left(\mathrm{k}^{2}+\frac{\pi^{2}}{2 \mathrm{D}^{2}}\right)+\sqrt{\mathrm{La}^{2}\left(\mathrm{k}^{2}+\frac{\pi^{2}}{2 \mathrm{D}^{2}}\right)^{2}+\frac{\mathrm{k}^{2}}{\mathrm{k}^{2}+\pi^{2} / \mathrm{D}^{2}}\left(\gamma_{\mathrm{CL} 2}^{2}-\gamma_{\text {diff }}^{2}\right)}
$$

As k goes to zero, (2-43) asymptotes to

$$
\gamma^{\mathrm{T} 2}=\left(\frac{\mathrm{kD}}{\pi}\right)^{2} \gamma_{\text {diff } 2}\left(\frac{\gamma_{\mathrm{CL2}}{ }^{2}}{\gamma_{\text {diff } 2^{2}}}-1\right)
$$

So that the necessary condition for instability is

$$
\mathrm{Ra}_{\mathrm{CL} 2}=\gamma_{\mathrm{CL} 2}^{2} / \gamma_{\text {diff }}^{2}>1
$$

This truncation gives good predictions of the growth rate at very low wavenumber.

If there is a wavenumber $\mathrm{k}_{\mathrm{c}}$ such that the cells penetrate over the entire domain $\mathrm{D}$, then for all $\mathrm{k}<\mathrm{k}_{\mathrm{c}}$ the diffusive decay scale $\gamma_{\text {diff2 }}(\mathrm{k}, \mathrm{D})<\gamma_{\text {diff2 }}\left(\mathrm{k}_{\mathrm{c}}, \mathrm{D}\right)$, while $\gamma_{\mathrm{CL} 2}$ does not change. From (2-45) then, there is a similar unstable mode filling the domain for all $\mathrm{k}<\mathrm{k}_{\mathrm{c}}$. It should be noted, however, that the growth rates at small values of $\mathrm{k}$ are very small, going as $\mathrm{k}^{2}$.

One of the questions with which this thesis began was whether or not the strongly surface-trapped forcing associated with wave-current interaction could force cells which penetrate over a deep mixed layer. Insight into this question may be gained by considering the dependence of $\gamma_{\mathrm{CL} 2}$ on $\mathrm{D}$. Assuming monochromatic waves, no Coriolis force, and a surface shear of 1 ,

$$
\gamma_{\mathrm{CL} 2}^{2}=\frac{4}{2 \mathrm{D}^{2}+\pi^{2}} \sim \frac{2}{\mathrm{D}^{2}}
$$

for large D. By contrast $\gamma_{\text {diff }}^{2}$ goes as $\mathrm{D}^{-4}$, so the forcing falls off less quickly with depth than does the diffusion. The implication is that deep mixed layers in the absence of Coriolis force are unstable to very long-wavelength cells. Given that the boundary conditions chosen for this study are realistic, cells which are long enough will penetrate over the entire depth of the mixed layer.

The main results of this section can be summarized as follows:

-At low wavenumbers, the fixing density on the lower boundary causes the density perturbation to be damped preferentially. As a result density does not limit the growth rate or depth of penetration of the cells at long enough wavenumber. -Given density fixed on the lower boundary, the condition for instability to occur at some wavenumber is that $\operatorname{RaCL}_{2}>1$, regardless of the size of the stratification. 


\subsubsection{How are the cell depth and growth rate determined at high wavenumbers for high stratification?}

We now turn to the question of how $\mathrm{D}_{\max }$ and $\gamma$ are determined at high values of stratification and high wavenumbers. A particular question is why the upper boundary condition determines the presence of instability and why the growth rate exhibits a maximum with respect to Langmuir number. In Section 2.4.3 it was shown that at low wavenumbers the effect of density was removed when the density perturbation was preferentially damped. A similar effect occurs at high wavenumbers and high stratification.

In order for instability to occur, equation (2-24) must have a positive root. A sufficient condition for this is that the constant terms in the polynomial be negative.

$$
\frac{1}{\mathrm{k}_{\psi}^{2}}\left(\mathrm{La}^{3} \mathrm{~K}_{\psi}^{4} \mathrm{k}_{\mathrm{p}}^{2} \mathrm{k}_{\mathrm{v}}^{2}-\mathrm{La} \mathrm{k}{ }^{2} \gamma_{\mathrm{CL}}^{2} \mathrm{k}_{\mathrm{p}}^{2}+\mathrm{La} \mathrm{k} \mathrm{N}^{2} \mathrm{k}_{\mathrm{v}}^{2}\right)<0
$$

This is true if and only if

$$
\begin{gathered}
\gamma_{\mathrm{CL}}^{2}-\mathrm{La}^{2} \mathrm{~K}_{\psi}^{4} \mathrm{k}_{\mathrm{v}}^{2} / \mathrm{k}^{2}>0 \\
\frac{\gamma_{\mathrm{CL}}^{2}-\mathrm{La}^{2} \mathrm{~K}_{\psi}^{4} \mathrm{k}_{\mathrm{v}}^{2} / \mathrm{k}^{2}}{\mathrm{~N}^{2}}>\frac{\mathrm{k}_{\mathrm{v}}^{2}}{\mathrm{k}_{\mathrm{p}}^{2}}
\end{gathered}
$$

The first of these conditions corresponds to the unstratified instability condition. Given a situation which is stable in the absence of stratification, adding stratification will not make it unstable. The second condition (2-48b) means that if $\mathrm{N}^{2}$ is larger than $\gamma_{\mathrm{CL}}^{2}$ then the density perturbation must be more heavily damped than the velocity perturbation. When the density is fixed on the upper boundary, given a depth of penetration $\mathrm{D}^{\prime}$

$$
\begin{gathered}
\rho \sim \sin \left(\pi \mathrm{z} / \mathrm{D}^{\prime}\right) \\
V \sim \cos \left(\pi \mathrm{z} / 2 \mathrm{D}^{\prime}\right)
\end{gathered}
$$$$
\mathrm{z}>-\mathrm{D}^{\prime}
$$$$
\mathrm{z}>-\mathrm{D}^{\prime}
$$

equation (2-48) becomes

$$
\frac{\gamma_{C L}^{2}-L^{2} K_{\psi}^{4} k_{v}^{2} / k^{2}}{N^{2}}>\frac{k^{2}+\pi^{2} / 4 D^{\prime 2}}{k^{2}+\pi^{2} / D^{\prime 2}}
$$

When density is fixed on the upper boundary, instability is possible for $\mathrm{N}^{2}<4 \gamma_{\mathrm{CL}}$. This constrasts with the inviscid case (2-1) or that for which density flux is fixed on the upper boundary, where instability is only possible when $\mathrm{N}^{2}<\gamma_{\mathrm{CL}}$. When the boundary conditions are such so that the density (which is stabilizing) is more 
strongly damped than the alongcell velocity (which is destabilizing), the effect is to reduce the stabilizing effect of density.

\subsection{Conclusions}

This thesis has set out to determine whether small-scale diffusion or Langmuir circulation is the dominant mixing mechanism within the surface layer. This chapter moves towards that goal by determining when an equilibrium velocity profile in a layer with no Coriolis forces becomes unstable to cells. The necessary condition is that a cell geometry be found such that the forcing of the roll vortices by wave current interaction and buoyancy (which goes as the Craik-Leibovich instability parameter $\gamma_{C L S}$ ) is stronger than the characteristic diffusive decay (which goes as $\gamma_{\text {diff }}$ ).

Given a fixed cell spacing, the growth rate and cell structure are determined by a tradeoff between maximizing the forcing, minimizing the diffusion, and maximizing the efficiency of the cells at reinforcing themselves (determined by the aspect ratio $\mathrm{kD}$ ). The upshot of this tradeoff is that cells with long wavelengths penetrate deeper into the mixed layer while cells with small wavelengths are trapped nearer to the surface. Langmuir cells differ in this sense from classical Rayleigh-Benard convection, where all the unstable modes penetrate over the fluid depth. In order to understand the effect of the cells on the vertical transport of momentum and density, it is necessary to capture the horizontal structure of the cells. This is shown to be important when the structure of an idealized surface layer with equilibrium Langmuir cells predicted by a finite-difference code is compared to that observed in two field experiments in Chapter 8.

Given a fixed cell spacing, stratification reduces the growth rate and depth of penetration of cells. The degree to which the stratification limits the depth of penetration in a global sense, however, is dependent on the boundary conditions. When the boundary conditions on density result in perturbations in the density being more heavily damped than perturbations in the velocity, the effect of density is limited. Given the boundary conditions chosen for this study density does not limit the depth of penetration of the cells in a mathematical sense. In a geophysical sense however, density may limit the penetration depth, since the cells which do penetrate over the depth of a stratified fluid layer have very long wavelengths and correspondingly small growth rates. 
Because the wave-current interaction mechanism is most intense near the surface, $\gamma_{C L S}$ and $\gamma_{\text {diff }}$ are closely linked to the vertical structure of the cells. Equation (2-29) may be rewritten when $\mathrm{Ri}=0$ as:

$$
\gamma_{C L}^{2} \sim \frac{\int_{-D}^{0} v^{\prime} w^{\prime} \frac{\partial v s}{\partial z} d z \int_{-D}^{0} v^{\prime} w^{\prime} \frac{\partial v}{\partial z} d z}{0} \frac{0}{\int_{-D}^{\prime} v^{\prime 2} d z \int_{-D}^{\prime} w^{\prime 2} d z}
$$

This means that the "effective Stokes drift shear" felt by cells of a given depth $\gamma_{C L S}$ is not that right at the surface (where v'w' is zero) but rather that where v'w' is strong. Figure 2.8 shows the depth at which $\frac{\partial \mathrm{v}_{\mathrm{s}}}{\partial \mathrm{z}}=\hat{\mathrm{V}}_{\mathrm{s}_{\mathrm{z} 1}}\left(\mathrm{D}^{\prime}\right)$ for monochromatic waves and for waves given by a Pierson-Moskowitz spectrum. For monochromatic waves, the depth at which the "effective" Stokes drift shear occurs varies from 30$70 \%$ of the depth of penetration, while for the Pierson-Moskowitz spectrum, the depth at which the effective shear occurs is approximately $30 \%$ of the depth of penetration for a wide range of penetration depths. Thus the Stokes drift shears

Depth of Effective Shear as a Function of Depth of Penetration

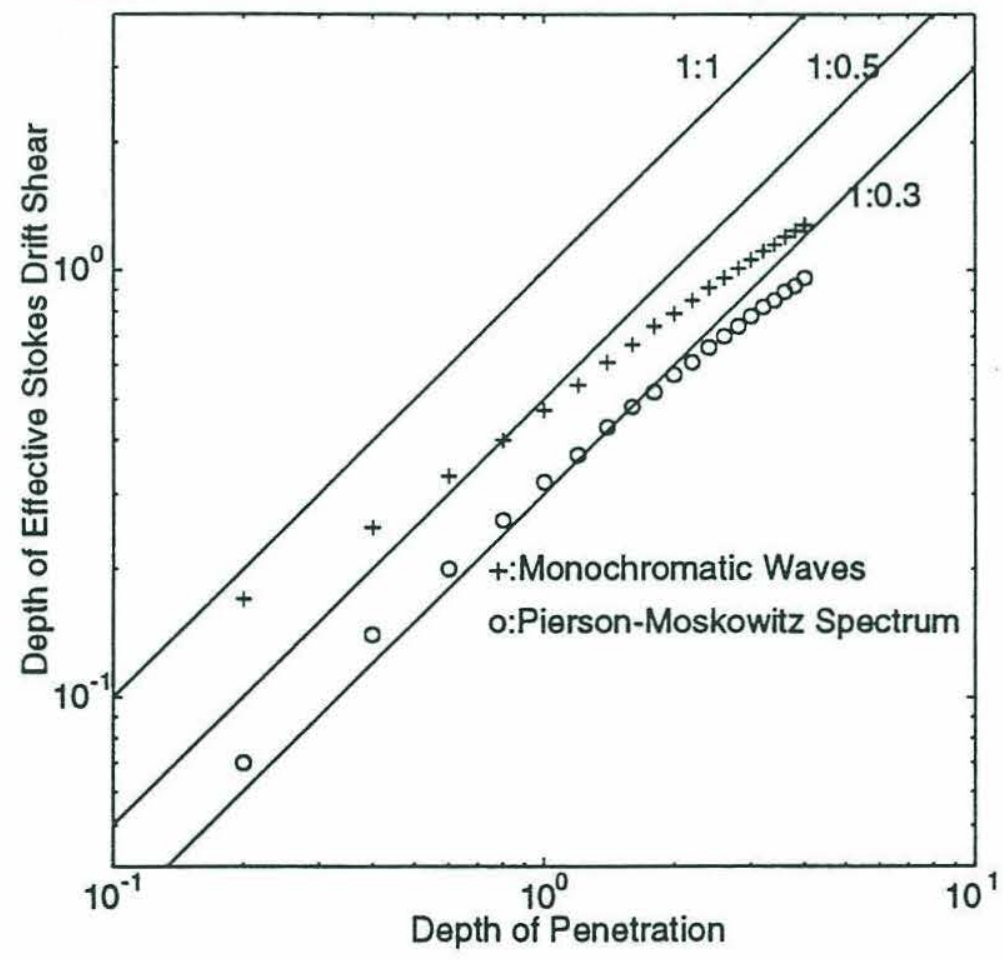

Figure 2.8: Depth of "effective" Stokes drift shear vs. depth of penetration of cells, given cell shapes given by Truncation T1. 
caused by short, high-frequency waves are only important for cells which do not penetrate very deeply into the mixed layer. Such cells may be important for transient problems of mixed layer creation, but are less important at equilibrium This is important for observationalists, since it is difficult to measure such highfrequency waves in the field.

The results outlined here have implications for numerical modelling of the cells. Given a box of width $\mathrm{L}$ and depth $\mathrm{D}$ the depth over which the cells penetrate will be very sensitive to $L$ if $L$ «D. If the results are to be applied to realistic situations modelling must proceed in boxes which are wide enough for modes which penetrate over the entire depth to be at least theoretically accessible. If this is not computationally possible (say in cases where the stratification is strong) the failure to include such modes should at least be noted.

A number of issues raised by this chapter are covered in the remainder of the thesis. One such issue is the effect of the assumption that the cell axis, wave and wind direction all parallel and the Coriolis force equal to zero. Chapter 3 considers what happens when these assumptions are relaxed. The angle of cell orientation is then determined by a tradeoff between maximizing the strength of the forcing and minimizing the crosscell shear, similar to the tradeoff which determines the depth of penetration in the nonrotating case.

Another question of interest is how the horizontal scale of the cells is selected at equilibrium. As noted already, the answer to this question has major implications for the depth of penetration of cells and thus for the horizontally averaged velocity and density structure. The fact that marginal instability occurs for $\mathrm{k}=0$ suggests that energy will cascade to large scales without limit (Foster, 1969; Chapman and Proctor, 1980; Cox and Leibovich, 1993). In Chapter 4 it is shown that this is in fact the case in the absence of Coriolis forces. Since cells with longer wavelengths penetrate deeper into the water column, this means that stratification cannot limit the depth of penetration of the cells in a mathematical sense. Geophysically, however, this may not be true, since the energy takes a long time to cascade to the longest scales, and these scales have very small growth rates.

A final question is whether having RaCLS » 1 for infinitesimal cells with a given geometry means that finite-amplitude cells with roughly the same geometry replace small-scale diffusion as the primary mixing mechanism within the surface layer. In Chapter 5 it will be shown that the answer is yes. In Chapters 6 and 7 it is shown that RacLS is often large in oceanic mixed layers. 


\section{Chapter 3: Structure and Instability of an Ekman Spiral in the Presence of Surface Gravity Waves}

\section{1: Introduction}

A major objective of this thesis is to isolate the conditions for which Langmuir cells replace small-scale diffusion as the principal process stirring the mixed layer. A first step is to compute the instability of the equilibrium profile set up by small-scale diffusion to Langmuir cells. The next step is to identify the important processes affecting to the instability and the characteristic scales associated with them.

Chapter 2 considered the physics behind the instability of Langmuir cells in an idealized layer in which the wind stress is balanced by a pressure gradient, and the waves are parallel with the cells and wind. The three important processes which determine the strength of the instability are the production of vorticity through wave-current interaction, the production of vorticity by horizontal fluctuations in density, and diffusion. The strength of the two sources of vorticity is given by the stratified Craik-Leibovich instability parameter $\gamma_{C L S}$ (page 39):

$$
\gamma_{C L S}^{2}=\frac{4}{D^{2}}\left(\int_{-D^{\prime}}^{0} F(z) \frac{\partial v_{s}}{\partial z} d z \int_{-D}^{0} F(z) \frac{\partial V}{\partial z} d z-\int_{-D}^{0} G(z) \frac{g \partial \rho}{\rho \partial z} d z\right)
$$

where $\mathrm{v}_{\mathrm{s}}$ and $\mathrm{V}$ are the Stokes drift and Eulerian velocity, $\mathrm{z}$ is the vertical axis and $\rho$ is the density. The functions $F(z)$ and $G(z)$ are weighting functions which depend on the boundary conditions and are proportional to the shape of momentum and buoyancy transport carried by the most unstable mode at a given horizontal wavenumber. In Section 2.5 the Craik-Leibovich instability parameter was shown to play a role analogous to the buoyancy frequency in Rayleigh-Benard convection and the ratio

$$
\text { RaCLS }=\gamma_{\text {CLS }}^{2} / \gamma_{\text {diff }}^{2}
$$

where $\gamma_{\text {diff }}$ is a characteristic diffusive scale was shown to be analogous to the Rayleigh number for Rayleigh-Benard convection. When RaCLS is large for cells with a particular geometry, those cells are unstable. An important difference between Langmuir cells and classical Rayleigh-Benard convection is that the depth of penetration of the cells is important in determining $\gamma_{C L S}$, and that the horizontal spacing of the cells is important in determining this depth. 
This chapter considers the growth rate and structure of Langmuir cells when the wind stress is balanced by the Coriolis force. This is a physically more realistic condition for oceanic mixed layers (Weller, 1981) but introduces a number of complications. In particular, the waves, Eulerian shear, and cell axis are no longer necessarily parallel. The equations of motion are introduced in Section 3.2. In Section 3.3 the equilibrium state set up by small-scale diffusion alone in the presence of waves (but absence of cells) is derived. The instability of this state to Langmuir cells is computed in Section 3.4. In order to interpret the results, simple truncated models of the instability (similar to those used in Chapter 2) are used in Section 3.5 to obtain closed-form solutions from which the important physical processes may be extracted. Additionally, modified instability codes are used to evaluate the effects of crosscell shear, Coriolis forces, and wave-current interaction on Langmuir cells within an Ekman layer.

The major results of the chapter are as follows:

- The presence of waves is predicted to produce an Eulerian flow whose transport is equal and opposite to that associated with the Stokes drift and whose structure is determined by the Ekman number La/F and the layer depth $\mathrm{D}$.

- The Ekman spiral in the presence of waves is strongly unstable to roll vortices with growth rates much larger than the Coriolis frequency for a wide range of $\mathrm{La}$ and $\mathrm{F}$ which are reasonable for oceanographic cases.

-Wave-current interaction is the dominant forcing mechanism, except at very long wavelengths, when Coriolis forces are also important.

- In order to maximize the strength of wave-current forcing, the cells are oriented along an axis lying between the wave propagation axis and the Eulerian shear. - The presence of shear in the crosscell direction which tilts the cells acts to limit the growth, in some cases suppressing cells altogether at low wavelengths.

\subsection{Equations of Motion and Methods of Solution}

Consider a layer of fluid of depth D, as shown in Figure 3.1. The alongcell (+y) axis is taken to lie at some angle $\alpha$ to the right of the wind. If $\alpha<0$, the axis of cell orientation is to the left of the wind. Letting the velocity in the alongcell direction be denoted by $\mathrm{v}$, the vorticity in the alongcell direction by $\Omega$, the horizontal velocity in the crosscell direction by $u$, and the vertical velocity by $w$, the equations of motion become:

$$
\frac{\partial \Omega}{\partial \mathrm{t}}+\left(\mathrm{u}+\mathrm{u}_{\mathrm{s}}\right) \frac{\partial \Omega}{\partial \mathrm{x}}+\mathrm{w} \frac{\partial \Omega}{\partial \mathrm{z}}=\mathrm{F} \frac{\partial}{\partial \mathrm{z}}\left(\mathrm{v}+\mathrm{v}_{\mathrm{s}}\right)+\frac{\partial \mathrm{v}_{\mathrm{s}} \partial \mathrm{v}}{\partial \mathrm{z}}+\mathrm{R} \mathrm{x} \frac{\partial \rho}{\partial \mathrm{x}}+\mathrm{La} \nabla^{2} \Omega
$$




$$
\begin{gathered}
\frac{\partial \mathrm{v}}{\partial \mathrm{t}}+\left(\mathrm{u}+\mathrm{u}_{\mathrm{s}}\right) \frac{\partial \mathrm{v}}{\partial \mathrm{x}}+\mathrm{w} \frac{\partial \mathrm{v}}{\partial \mathrm{z}}=-\mathrm{F}\left(\mathrm{u}+\mathrm{u}_{\mathrm{s}}\right)+\mathrm{La} \nabla^{2} \mathrm{v} \\
\frac{\partial \rho}{\partial \mathrm{t}}+\left(\mathrm{u}+\mathrm{u}_{\mathrm{s}}\right) \frac{\partial \rho}{\partial \mathrm{x}}+\mathrm{w} \frac{\partial \rho}{\partial \mathrm{z}}=\mathrm{La} \nabla^{2} \rho \\
\Omega=\nabla^{2} \psi \\
\frac{\partial \psi}{\partial \mathrm{x}}=-\mathrm{w} \quad \frac{\partial \psi}{\partial \mathrm{z}}=\mathrm{u} \\
\mathrm{La}=\frac{\mathrm{ve}}{\mathrm{a}^{2} \sigma} \quad \mathrm{F}=\frac{\mathrm{f}}{\mathrm{k}_{\mathrm{w}}^{2} \mathrm{a}^{2} \sigma} \quad \mathrm{Ri}=\frac{\mathrm{N}^{2}}{\mathrm{k}_{\mathrm{w}}^{4} \mathrm{a}^{4} \sigma^{2}}
\end{gathered}
$$

In these equations, $\mathrm{k}_{\mathrm{w}}, \mathrm{a}$ and $\sigma$ are the wavenumber, amplitude, and frequency of the driving waves, $v_{e}$ the eddy viscosity, $f$ the Coriolis parameter, and $\mathrm{N}$ the buoyancy frequency. A brief discussion of these equations is given in Chapter 1, with a full derivation in Appendix A.

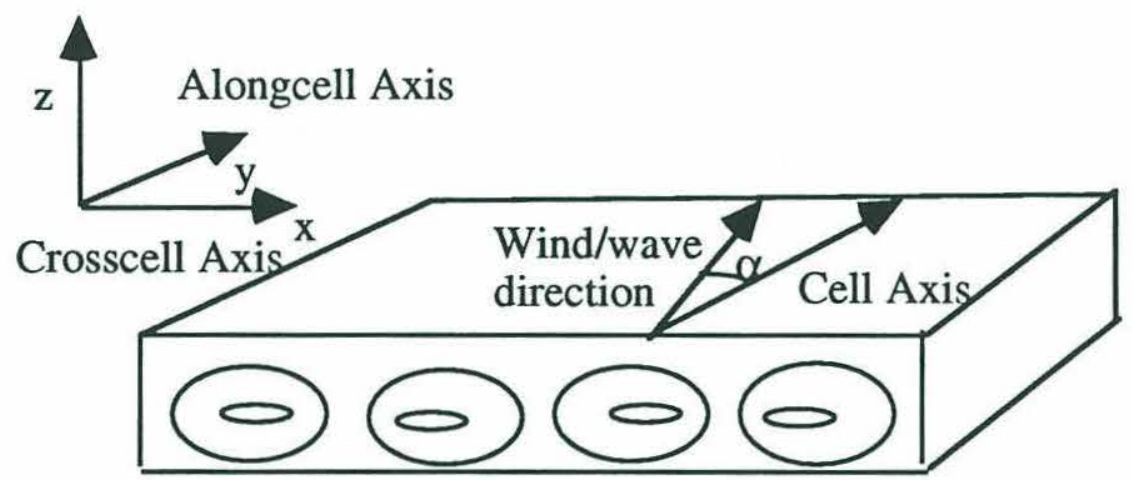

Figure 3.1: Schematic of the problem as solved in this chapter.

The cells are aligned at an angle $\alpha$ to the right of the wind, so that the boundary conditions on velocity and density are :

$$
\begin{gathered}
\mathrm{La} \frac{\partial \mathrm{v}}{\partial \mathrm{z}=0}=\frac{\tau}{\rho} \cos (\alpha),\left.\quad \Omega\right|_{\mathrm{z}=0}=-\frac{\tau}{\mathrm{La} \rho} \sin (\alpha) \\
\left.\frac{\partial \mathrm{v}}{\partial \mathrm{z}}\right|_{\mathrm{z}=-\mathrm{D}}=\left.\Omega\right|_{\mathrm{z}=-\mathrm{D}}=0 \\
\left.\frac{\partial \rho}{\partial \mathrm{z}}\right|_{\mathrm{z}=0}=1
\end{gathered}
$$




$$
\left.\rho\right|_{\mathrm{z}=-\mathrm{D}}=\mathrm{D}
$$

The fluxes of density and velocity are set on the upper boundary and the velocity flux and value of density are set on the bottom boundary. The effect of fixing density on the bottom boundary was shown to be important at high values of Langmuir number and/or stratification and for very large values of cell spacing in Chapter 2. For such cases, the fact that perturbations in the density are damped more strongly than perturbations in alongcell velocity can reduce or remove the effect of stratification.

There are four important differences between these equations and those studied in Chapter 2.

-The momentum equations contain a term proportional to the Coriolis frequency times the total Lagrangian velocity.

-The pressure gradient term has been dropped.

-The cell axis is no longer necessarily parallel with the axis of wave propagation, so that the Stokes drift may contribute to crosscell advection.

-The cell axis is no longer necessarily parallel with the wind stress, so that the vorticity is no longer zero on the upper boundary.

The instability problem is cast as follows. Let the streamfunction, alongcell velocity, density, and Stokes drift be approximated by the following expansions:
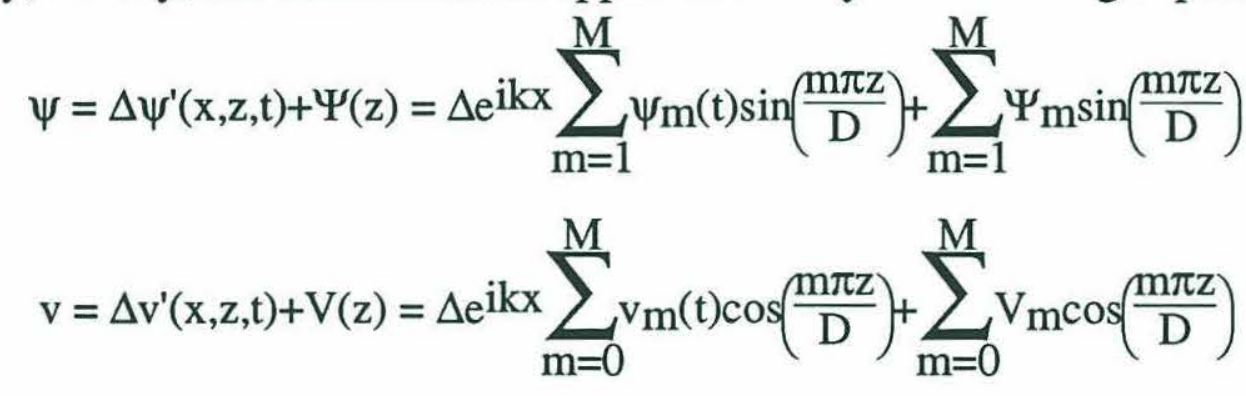

$$
\mathrm{u}_{\mathrm{s}}=\sum_{\mathrm{m}=0}^{\mathrm{M}} \mathrm{U} \mathrm{s}_{\mathrm{m}} \cos \left(\frac{\mathrm{m} \pi \mathrm{z}}{\mathrm{D}}\right) \quad \mathrm{v}_{\mathrm{s}}=\sum_{\mathrm{m}=0}^{\mathrm{M}} \mathrm{V} \mathrm{s}_{\mathrm{m}} \cos \left(\frac{\mathrm{m} \pi \mathrm{z}}{\mathrm{D}}\right)
$$

$(3-5 d) \rho=\Delta \rho^{\prime}(x, z, t)+P_{0}(z)=\Delta e^{i k x} \sum_{m=1}^{M} \rho_{m}(t) \cos \left(\frac{(2 m-1) \pi z}{2 D}\right)+\sum_{m=1}^{M} P_{m} \cos \left(\frac{(2 m-1) \pi z}{2 D}\right)$

where $\Delta$ is a small number and the capital letters refer to the time-mean flow and stratification in the absence of Langmuir cells. The wavelength of the unstable mode $\mathrm{k}$ is $2 \pi / \mathrm{L}$ where $\mathrm{L}$ is the cell spacing. Substituting into (3-3) yields to zeroth order in $\Delta$ the following equations for the horizontally-averaged fields. 


$$
\frac{\partial}{\partial t} \frac{\partial U}{\partial z}-F \frac{\partial}{\partial z}\left(v_{s}+V\right)=L a \frac{\partial^{2} U}{\partial z^{2}}
$$

$$
\frac{\partial \mathrm{V}}{\partial \mathrm{t}}+\mathrm{F}\left(\mathrm{u}_{\mathrm{s}}+\mathrm{U}\right)=\mathrm{La} \frac{\partial^{2} \mathrm{~V}}{\partial \mathrm{z}^{2}}
$$

Given the boundary conditions in (3-4), the steady-state solution of equation (3-6a) is just $P_{0}(z)=-z$. The velocity structure is not so easily derived. Integrating (3-6b) with respect to $\mathrm{z}$ and defining the complex velocity and Stokes drift profiles:

$$
\begin{aligned}
& \mathrm{W}(\mathrm{z})=\mathrm{U}(\mathrm{z})+\mathrm{i} \mathrm{V}(\mathrm{z}) \\
& \mathrm{W}_{\mathrm{s}}(\mathrm{z})=\mathrm{u}_{\mathrm{s}}(\mathrm{z})+\mathrm{i} \mathrm{v}_{\mathrm{s}}(\mathrm{z})
\end{aligned}
$$

yields the following equation for complex velocity:

$$
\frac{\partial \mathrm{W}}{\partial \mathrm{t}}+\mathrm{iF}\left(\mathrm{W}_{\mathrm{s}}+\mathrm{W}\right)=\mathrm{La} \frac{\partial^{2} \mathrm{~W}}{\partial \mathrm{z}^{2}}
$$

At present there is no solution of these equations given the boundary condition of no stress at $\mathrm{z}=-\mathrm{D}$. Such a solution is derived in Section 3.3, thereby enabling the calculation to be carried forward to higher order in $\Delta$.

At first order in $\Delta$ the problem of finding the growth rate and structure of the unstable modes becomes a linear eigenvalue problem in the coefficients $\psi_{\mathrm{m}}$, $v_{m}$ and $\rho_{m}$. For each set of $k, L a, R i$ and $\alpha$ there are a number of eigenfunctions, some of which may have positive eigenvalues and correspond to unstably growing modes. Section 3.4 considers the growth rates of the most unstable mode given a fixed set of $\mathrm{k}, \mathrm{La}, \mathrm{Ri}$, and $\alpha$. The depth $\mathrm{D}_{\max }$ at which the maximum downwelling velocity occurs is used as a proxy for cell structure. As in Chapter 2, $D_{\max }$ is the depth at which the maximum perturbation streamfunction amplitude $\left|\psi^{\prime}(\mathrm{z})\right|$ occurs.

Section 3.5 moves from models of Langmuir cell instability which are formally correct to some models of instability which approximate the equations of motion so as to extract important physical parameters and include or exclude various physical processes. These models are used to interpret the results of Section 3.4. The first of these simple models is a truncated model which will be referred to as truncation T3. The equations of this truncation are

$$
\begin{array}{cc}
\psi(\mathrm{x}, \mathrm{z}, \mathrm{t})=\psi_{0} \mathrm{e}^{\gamma} \sin (\mathrm{kx}) \sin \left(\pi \mathrm{z} / \mathrm{D}^{\prime}\right)+\psi_{1} \mathrm{e}^{\gamma \mathrm{t}} \cos (\mathrm{kx}) \sin \left(2 \pi \mathrm{z} / \mathrm{D}^{\prime}\right)+\Psi_{0}(\mathrm{z}) & \mathrm{z}>-\mathrm{D}^{\prime} \\
\mathrm{v}(\mathrm{x}, \mathrm{z}, \mathrm{t})=\mathrm{v}_{0} \mathrm{e} \mathrm{e}^{\prime} \cos (\mathrm{kx}) \sin \left(\pi \mathrm{z} / \mathrm{D}^{\prime}\right)+\mathrm{v}_{1} \mathrm{e}^{\gamma} \sin (\mathrm{kx}) \sin \left(2 \pi \mathrm{z} / \mathrm{D}^{\prime}\right)+\mathrm{V}_{0}(\mathrm{z}) & \mathrm{z}>-\mathrm{D}^{\prime} \\
\psi=\Psi_{0}(\mathrm{z}) \mathrm{V}=\mathrm{V}_{0}(\mathrm{z}) & \mathrm{z}<-\mathrm{D}^{\prime}
\end{array}
$$

This truncation differs from those developed in Chapter 2 in that it does not exactly satisfy the boundary conditions on velocity. The basic idea, however, is 
still to approximate the shape of the most unstable mode as closely as possible while keeping the problem simple enough to obtain a closed-form solution for the growth rate. The truncation is accurate for small values of $\mathrm{La}$. As noted in Chapter 2 , truncated models of this type are inaccurate when they fail to reproduce critical features of the unstably growing cells.

In addition to the truncated model, two modified spectral instability codes are also used to look at the importance of various processes for causing instability. The first code computes the instability of the equilibrium velocity and density profile as though there were no Coriolis force. Since the Coriolis force is involved in maintaining the equilibrium current profile upon which the instabilities grow this is not, strictly speaking, a well-posed problem. ${ }^{1}$ The purpose of using the modified code for such scenarios is to diagnose the importance of Ekman instability (Gammelsrød, 1975; Lele, 1985). If the growth rate of cells in the absence of Coriolis force is smaller than the growth rate in the presence of Coriolis force, Ekman instability probably contributes to cell growth. If the growth rate does not change significantly, Ekman instability most likely does not contribute. The growth rates calculated from this code are referred to as being computed "in the absence of Ekman instability".

A second modified instability code examines the importance of shear instability by expanding the streamfunction and crosscell Stokes drift alone

$$
\begin{gathered}
\psi=\Delta \mathrm{e}^{\mathrm{ikx}} \sum_{\mathrm{m}=1}^{\mathrm{M}} \psi_{\mathrm{m}} \sin \left(\frac{\mathrm{m} \pi \mathrm{z}}{\mathrm{D}}\right)+\sum_{\mathrm{m}=1}^{\mathrm{M}} \Psi_{\mathrm{m}} \sin \left(\frac{\mathrm{m} \pi \mathrm{z}}{\mathrm{D}}\right) \\
\mathrm{u}_{\mathrm{s}}=\sum_{\mathrm{m}=1}^{\mathrm{M}} \mathrm{us}_{\mathrm{m}} \cos \left(\frac{\mathrm{m} \pi \mathrm{z}}{\mathrm{D}}\right)
\end{gathered}
$$

Linearizing the equations (3-3) with respect to $\Delta$, the instability problem for shear instability is cast as a linear eigenvalue problem in the coefficients $\psi_{\mathrm{m}}$. The growth rates from this code will be referred to as due to "shear instability alone".

The investigation is limited to cases where the layer depth $\mathrm{D}=4$ and the surface Eulerian shear is 1 . The choice of $\mathrm{D}=4$ is made so as to look at a mixed layer where the Stokes drift falls off within the mixed layer, but slowly enough so that the region where it falls off can still be resolved by a relatively small number

\footnotetext{
${ }^{1}$ In the limit when the growth rate of the most unstable mode $\gamma$ is large in comparison with the scaled Coriolis frequency one can rescale the equations in terms of $\mathrm{F} / \gamma$ to obtain a formally valid expression for the growth rate. However, in many of the cases for which this procedure is used, the growth rates are of the same order as the Coriolis frequency, so that even this procedure is not formally valid.
} 
of modes. Keeping the surface shear equal to one facilitates comparison with other published work. It also means that the Craik-Leibovich instability parameter changes only as the result of changes in the mean structure and not the amplitude of shear, simplifying the interpretation of the results. It should be emphasized that the fact that surface stress and Langmuir number vary together in this work is not to be taken as an assertion that they are linearly related in the real world.

\subsection{The Ekman Spiral in the Presence of Surface Gravity Waves}

\subsubsection{The Solution of the Equations}

In order to compute the instability of a given equilibrium state to Langmuir cells, one must first calculate the structure of that equilibrium state. This is done by finding a general solution to equations (3-8) for time-varying monochromatic waves and taking the steady-state solution as a special case. Assume a complex

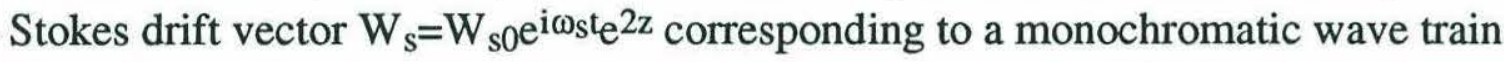
whose direction of propagation rotates with frequency $\omega_{\mathrm{s}}$. Defining $\delta\left(\omega_{\mathrm{S}}\right)=\sqrt{2 \mathrm{La} /\left(\mathrm{F}+\omega_{\mathrm{S}}\right)}$, the solution to equation (3-8) takes the form

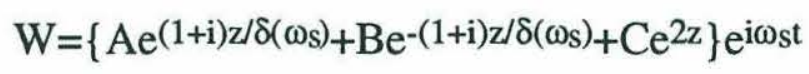

where

$$
\begin{gathered}
\mathrm{A}=-\frac{\delta\left(\omega_{\mathrm{s}}\right)}{\delta(0)^{2}}(1+\mathrm{i}) \frac{1}{2-\mathrm{i} / \delta\left(\omega_{\mathrm{s}}\right)^{2}} \frac{1-2 \mathrm{e}^{-2 \mathrm{D}_{\mathrm{e}}-(1+\mathrm{i}) \mathrm{D} / \delta\left(\omega_{\mathrm{s}}\right)}}{1-\mathrm{e}^{-2(1+\mathrm{i}) \mathrm{D} / \delta\left(\omega_{\mathrm{s}}\right)}} \mathrm{W}_{\mathrm{S} 0} \\
\mathrm{~B}=\frac{\delta\left(\omega_{\mathrm{S}}\right)}{\delta(0)^{2}}(1+\mathrm{i}) \frac{1}{2-\mathrm{i} / \delta\left(\omega_{\mathrm{s}}\right)^{2}} \frac{1-2 \mathrm{e}^{-2 \mathrm{D}^{(}(1+\mathrm{i}) \mathrm{D} / \delta\left(\omega_{\mathrm{S}}\right)}}{1-\mathrm{e}^{2(1+\mathrm{i}) \mathrm{D} / \delta\left(\omega_{\mathrm{s}}\right)}} \mathrm{W}_{\mathrm{S} 0}
\end{gathered}
$$

Given that the equations are linear, the response to any monochromatic wave train whose direction and amplitude vary with time can now be solved by Fourier transforming the complex Stokes drift vector $\mathrm{W}_{\mathrm{s}}(\mathrm{t})=\mathrm{u}_{\mathrm{s}}(\mathrm{t})+\mathrm{iv}_{\mathrm{s}}(\mathrm{t})$ and superimposing solutions for each frequency $\omega$ from equations $(3-11,12)$.

Integrating the solution given by equations $(3-11,12)$ to obtain the Eulerian mass transport yields

$$
\int_{-D}^{0} W d z=-\frac{F}{2\left(\omega_{s}+F\right)} W_{s 0}\left(1-e^{-2 D}\right)=-\frac{F}{\omega_{S}+F} \int_{-D}^{0} W_{s 0} e^{2 z} d z
$$


So that if $\omega_{\mathrm{s}}=0$, the mean Eulerian transport exactly balances the Stokes drift. This satisfies the condition, first stated by Ursell (1950), that the total Lagrangian mass flux associated with a train of surface gravity waves on a rotating earth be zero.

The Ekman number Ek may be defined as follows:

$$
\mathrm{Ek}=\frac{\mathrm{La}}{\mathrm{F}}=\frac{\mathrm{V}_{\mathrm{e}} \mathrm{k}_{\mathrm{w}}{ }^{2}}{\mathrm{f}}
$$

where $v_{\mathrm{e}}$ is the eddy viscosity, $f$ the dimensional Coriolis frequency, and $\mathrm{k}_{\mathrm{w}}$ is the wavenumber of the gravity waves at the peak of the spectrum. When Ek is large, the wave velocities fall off on spatial scales smaller than the Ekman depth $\delta(0)$. When Ek is small, the wave velocities fall off on spatial scales which are large compared with an Ekman depth.

Figure 3.2 illustrates a number of time-mean $\left(\omega_{\mathrm{s}}=0\right)$ Eulerian current spirals over a no-stress bottom for various values of $E k$ with $D=4$ assuming no shear (and hence no stress) at the upper surface. The waves are propagating in the $+y$ direction. Five values of $\mathrm{Ek}$ are shown, ranging from 0.01 to 100 (corresponding to a range of $\delta(0)$ from 7.07 to 0.07 ). For the largest value of Ek (largest value of $\delta$ ), the flow parallel to the wave axis is essentially uniform with depth, with an integrated flow equal and opposite to that of the Stokes Drift. Some shear is seen to the right of the wave axis. For Ek=1, there is a clear spiral current pattern with more shear to the right of the waves. For Ek $<1$, the Eulerian current is equal and opposite to the Stokes drift over most of the depth of the fluid.

Suppose now that instead of a monochromatic wave train we have an arbitrary Stokes drift profile. Then equation (3-8) may be solved using the method of Green's functions.

$$
W(z)=\int_{-D}^{0} G\left(z, z_{0}\right) W_{S}\left(z_{0}\right) d z_{0}
$$

where $\mathrm{G}$ is given by

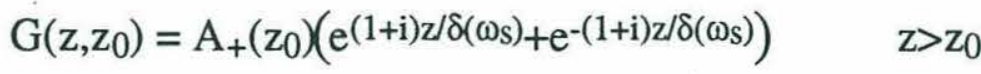

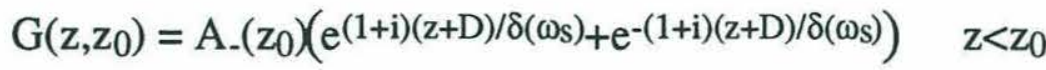

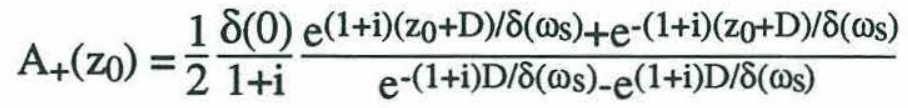

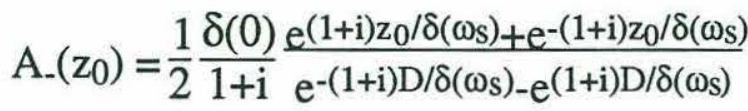




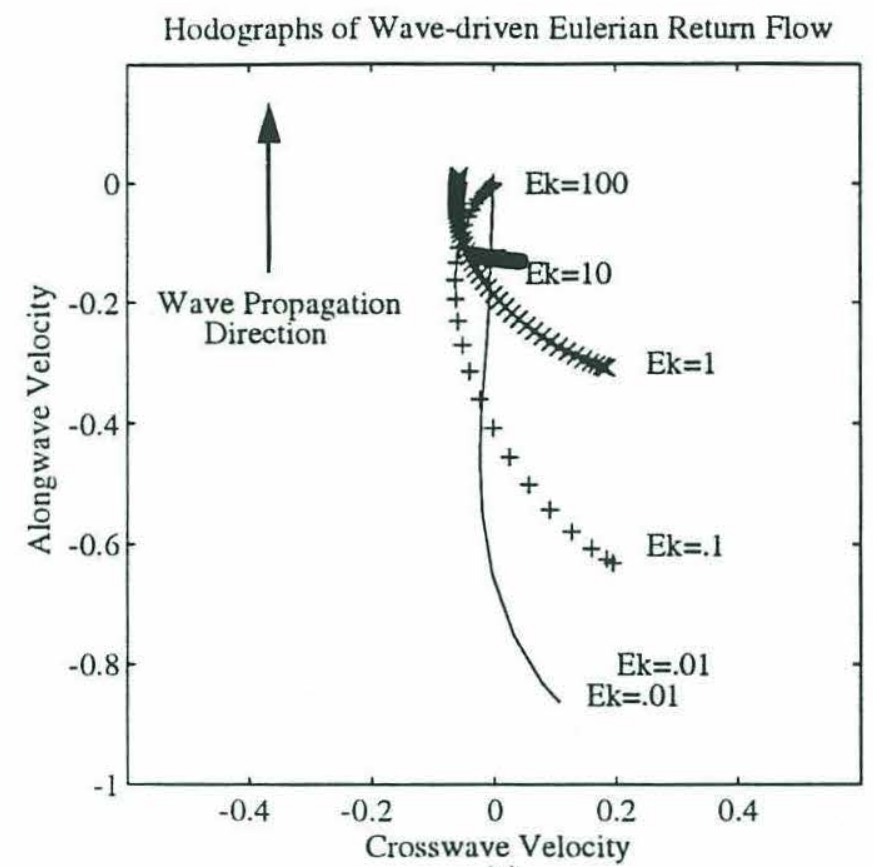

(a)

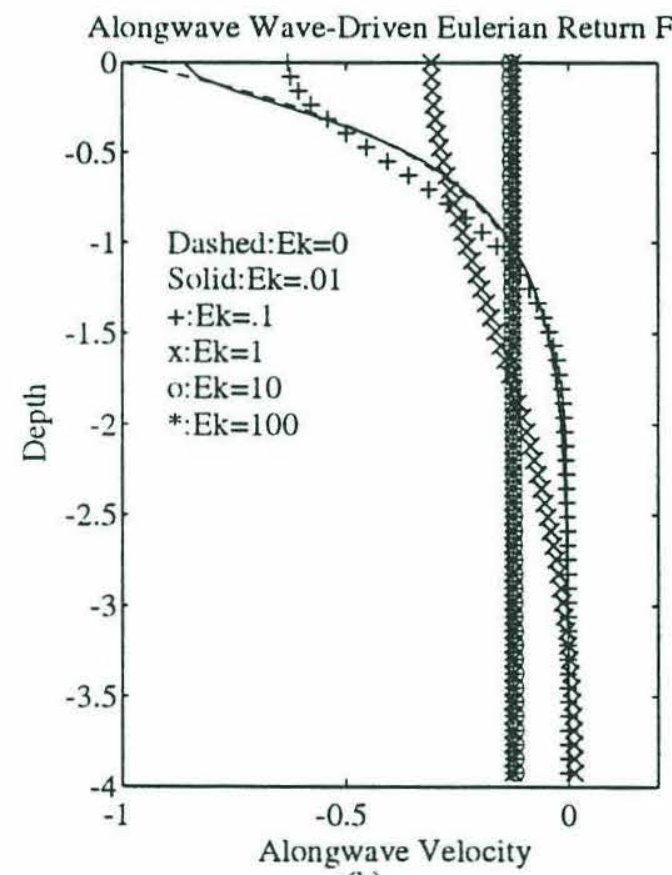

(b)

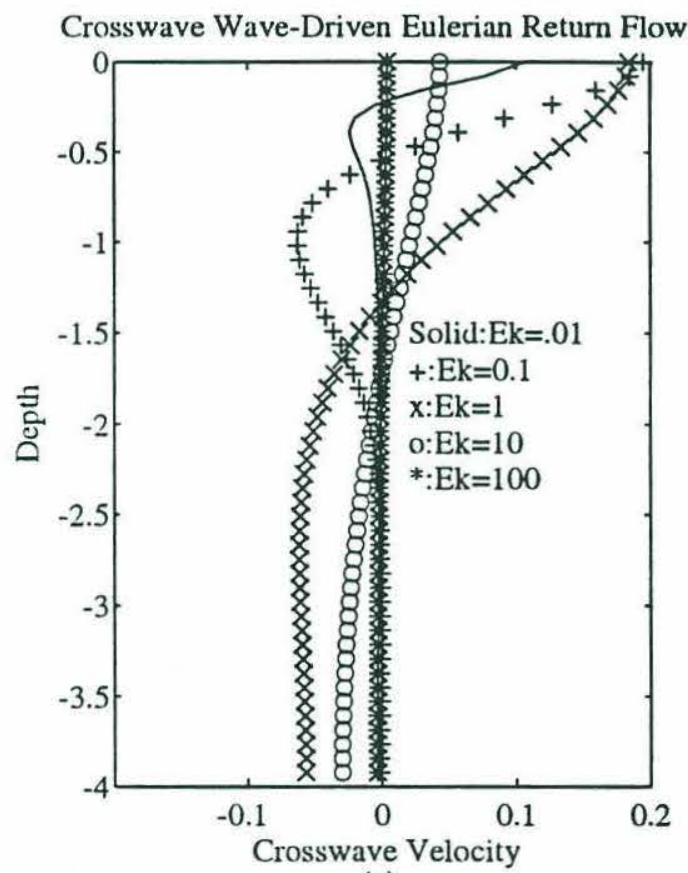

(c)

Figure 3.2: Ekman spiral in the presence of waves for different values of Ekman number (solution given by (3-11) and (3-12))). In all cases wave train is monochromatic so that the Stokes drift $\mathrm{v}_{S}=\mathrm{e}^{2 \mathrm{z}}$, the mixed layer depth $\mathrm{D}$ is 4 and the surface stress is zero. Symbols are for different values of Ek: 100 (solid line), $10(+), 1$ (x), 0.1, (o), $0.01\left(^{*}\right)$. (a) Velocity hodograph, crosswave vs. downwave current. (b) Downwave Eulerian current vs. depth. (c) Crosswave Eulerian current vs. depth. 


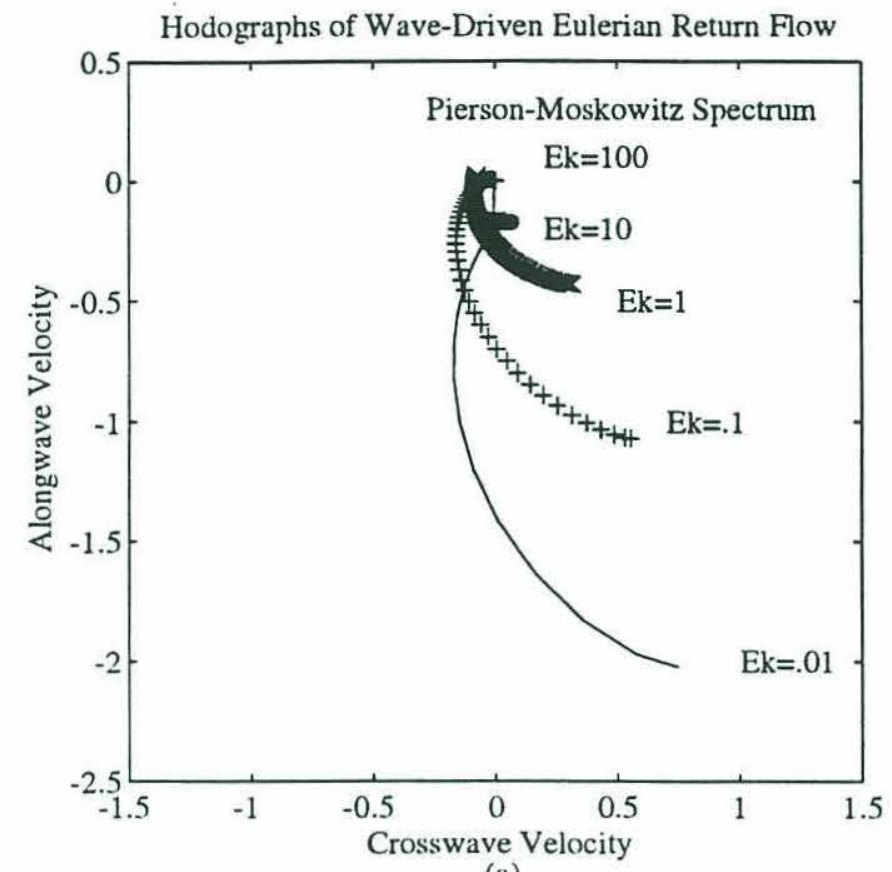

(a)

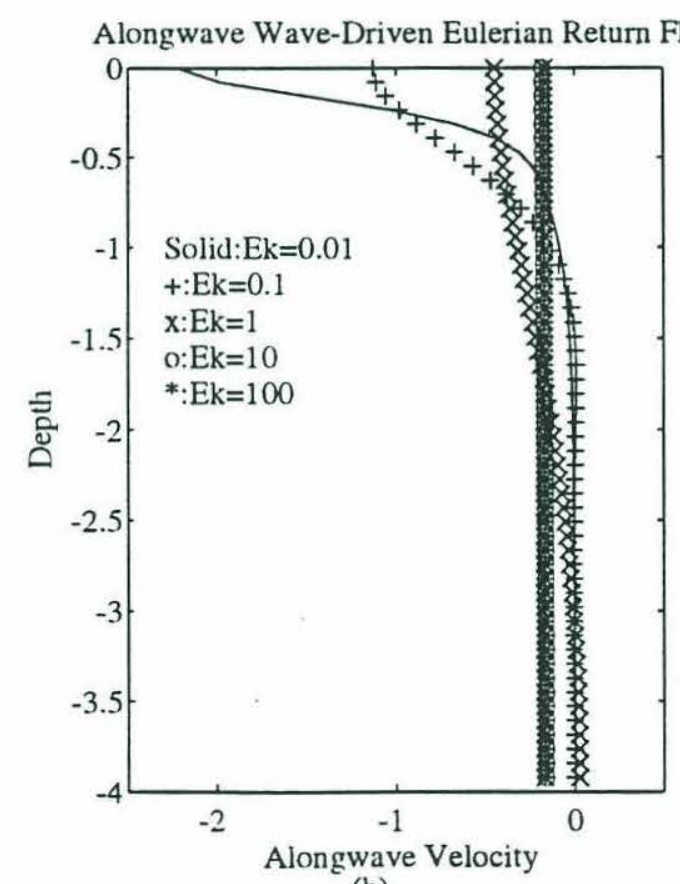

(b)

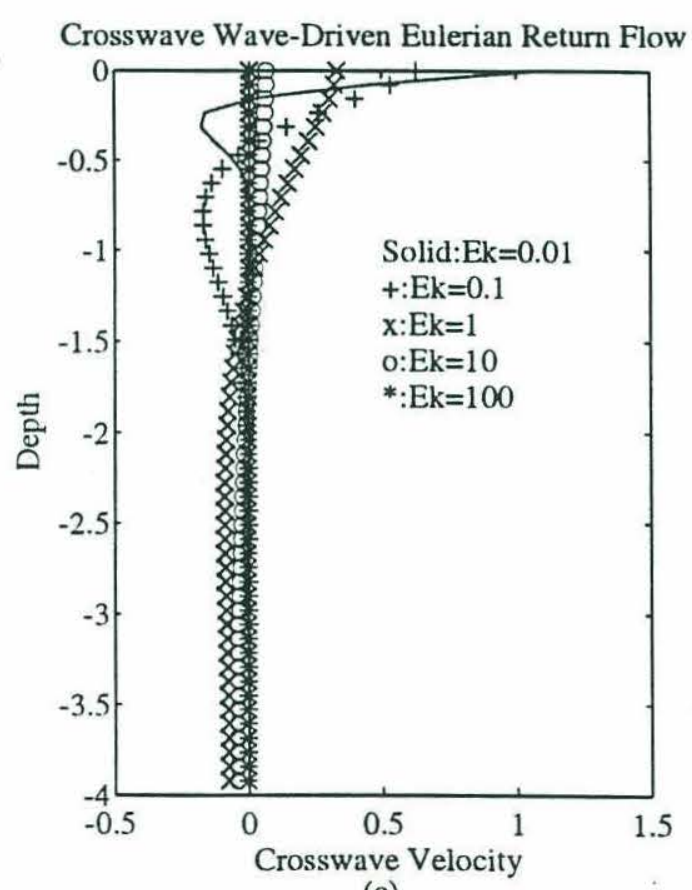

(c)

Figure 3.3: Same as 3.2 but for waves given by a Pierson-Moskowitz spectrum (Equations 3-15,16,17). 
Figure 3.3 repeats Figure 3.2 for a Stokes drift profile corresponding to a Pierson-Moskowitz spectrum identical to that of Chapter 2.

$$
\mathrm{v}_{\mathrm{s}}(\mathrm{z})=\int_{f=0}^{\infty} \frac{5}{f^{2}} \exp \left(-1.25 \frac{1}{f^{4}}\right) \exp \left(2 f^{2} \mathrm{z}\right) \mathrm{d} f
$$

The peak frequency and total energy in this spectrum is the same as the monochromatic wave train for which the solution in $(3-11,12)$ was derived. Note that the scale of the axes in Figure 3.3 is much larger than that in Figure 3.2. The total transport is 1.34 as large as that for a monochromatic wave train. For large values of Ek, the profile again asymptotes to one in which the Coriolis-driven return flow is distributed over the entire mixed layer. As for a monochromatic wave train, the addition of a wave spectrum adds only a small crosswind shear to the Eulerian current profile. For small values of Ek, on the other hand, there is more shear for the Pierson-Moskowitz spectrum than for the monochromatic wave train, and the surface Eulerian currents are significantly more upwind.

The main results of this section are summarized as follows:

-The presence of surface gravity waves in a rotating mixed layer changes the mean Eulerian transport, introducing an Eulerian return flow which balances the Lagrangian Stokes drift.

-If the Ekman number $\mathrm{La} / \mathrm{F}$ is large, the return flow is distributed over scales of an Ekman depth, and the shears involved are much smaller than the Stokes drift shears.

-If the Ekman number is small, the return flow is essentially equal and opposite to the Stokes drift at depths below an Ekman depth.

\subsubsection{Discussion}

The presence of a wave-Coriolis force interaction term is important in setting the horizontally averaged initial condition and the total Eulerian transport within the surface layer. An explanation of this term in terms of vorticity was given in Chapter 1. While demonstrating how the force arises mathematically, such a derivation does not lead to much insight into why there is an Eulerian-mean transport balancing the Stokes drift. This shortcoming may be overcome by considering the vertically integrated momentum balance in the presence of waves. Suppose a train of surface gravity waves with surface amplitude a propagates in the $+y$ direction, so that the free surface and alongwave velocity are given by 


$$
\begin{aligned}
& \eta=\mathrm{a} \cos \left(\mathrm{k}_{\mathrm{w}} \mathrm{y}-\sigma \mathrm{t}\right) \\
& \mathrm{v}=\mathrm{a} \sigma \mathrm{e}^{\mathrm{kwz}} \cos \left(\mathrm{k}_{\mathrm{w}} \mathrm{y}-\sigma \mathrm{t}\right)
\end{aligned}
$$

and further suppose that $\varepsilon=\mathrm{ka}$ is a small parameter. Then to first order in $\mathrm{ka}$, any surface $\zeta$ defined by

$$
\zeta=z_{0}+a e^{k w z 0} \cos (k y-\sigma t)
$$

is a material surface whose temporally averaged depth is $\mathrm{z}_{0}$. Integrating the Coriolis force on the waves $\mathrm{F}_{\mathrm{cor}}(\mathrm{z})=\mathrm{f} * \mathrm{v}_{\mathrm{w}}$ from $\mathrm{z}=-\infty$ to the material surface $\zeta$ over a wave period and defining [ ] as temporal averaging over a wave period:

$$
(3-20)\left[\int_{-\infty}^{\zeta} F_{c o r}(z) d z\right]=\frac{\sigma}{2 \pi} \int_{0}^{2 \pi / \sigma} \int_{-\infty}^{\zeta} f v_{w} d z d t=\frac{1}{2 \pi} \int_{0}^{2 \pi / \sigma} \frac{f a \sigma}{k_{w}} e^{k w} \zeta \cos \left(k_{w} y-\sigma t\right) d t
$$

which to first order in $\mathrm{k}_{\mathrm{w}}$ a gives

$$
\begin{aligned}
& 2 \pi / \sigma
\end{aligned}
$$

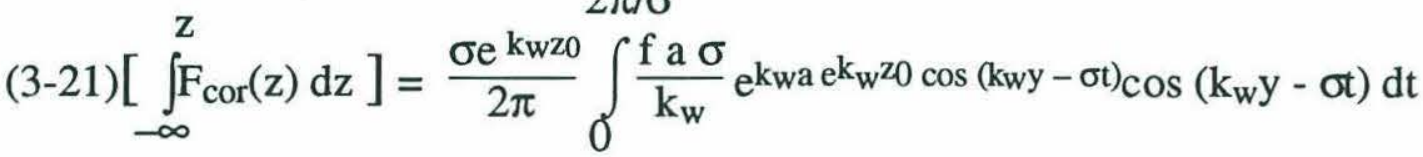

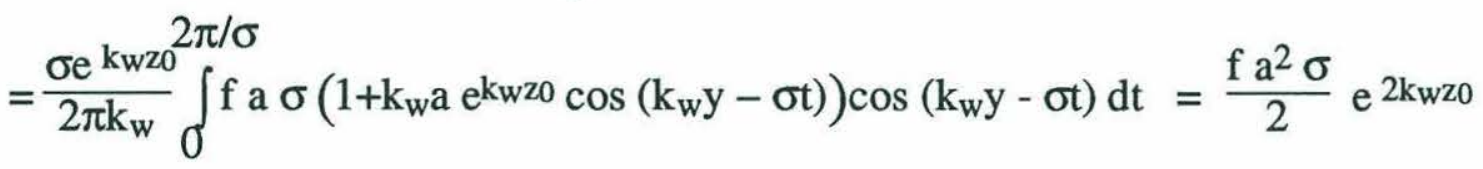

Then the time-averaged Coriolis force at an average depth $\mathrm{z}_{0}$ is given by

$$
\mathrm{F}\left(\mathrm{z}_{0}\right)=\mathrm{f}_{\mathrm{w}} \mathrm{a}^{2} \sigma \mathrm{e}^{2 \mathrm{kwz}_{0}}=\mathrm{f} * \mathrm{v}_{\mathrm{s}}
$$

This force arises because a time-varying force at a time-varying depth leads to a time-averaged force at a time-averaged depth. This Coriolis force drives a mean Eulerian flow which balances the Lagrangian transport associated with the Stokes drift, a result predicted by a number of authors (Ursell, 1950; Hasselmann, 1970; Pollard, 1970; Weber, 1983). The first three of these authors considered cases where $\mathrm{La}=0$ so that the Eulerian flow was found to exactly cancel the Stokes drift at each depth —a result reproduced here as Ek goes to zero. Weber (1983) considered monochromatic waves in an infinitely deep fluid within a purely Lagrangian framework. The Lagrangian transport predicted by adding the Stokes drift to the solution in $(3-11,12)$ asymptotes to his result as the layer depth goes to infinity. In the absence of waves, the solution asymptotes to that for an Ekman spiral over a finite-depth, no-stress bottom boundary.

The range of values of $\mathrm{Ek}(0.01-100)$ chosen in Section 3.3.1 are reasonable for many oceanic conditions. In the field, eddy viscosities are quite 
large, of order $0.001-0.1 \mathrm{~m}^{2} / \mathrm{s}$ ( compared with $10^{-5}-10^{-4} \mathrm{~m}^{2} / \mathrm{s}$ in the thermocline). This implies that the Ekman depth is approximately $3-30 \mathrm{~m}$, while the e-folding depth $1 / \mathrm{k}_{\mathrm{w}}$ for the velocities associated with surface gravity waves is of order 10 $25 \mathrm{~m}$ for oceanic surface gravity waves with periods of 6-10 seconds. This gives a rough range of Ek of $0.01-10$. The extreme cases where Ek is very small or very large are rare. Very large values of Ek might occur when the wind is strong but the waves are duration or fetch-limited (so that the waves would be small, even though the turbulence would be quite strong). Small values of Ek (corresponding to high waves with little turbulence) might occur after a large storm or as the result of strong swell propagation. In the cases considered for SWAPP and MILDEX in Chapters $6-8$, Ek is of order 1 . This is exactly in the middle of the range chosen for the current investigation.

\subsection{Instability of the Mean Current Spiral over a No-stress Bottom}

Having calculated the structure of the horizontally averaged Ekman response in the absence of Langmuir circulation in section 3.3 we now proceed to calculate the instability of the time-mean Ekman profile. As noted above, the investigation is limited to cases where the nondimensional layer depth $\mathrm{D}=4$ and the surface shear $=1$.

The case of a Stokes drift oriented at some angle to the Eulerian shear has not been studied in the published literature. The fact that Stokes drift and Eulerian shear are no longer aligned means that the cells cannot be assumed to be aligned with the wind and waves. ${ }^{*}$ In order to fully explore the instability of the current profiles derived in the last section, the growth rate of the most unstable mode is calculated as a function of angle of orientation as well as horizontal wavenumber.

Figure 3.4 shows hodographs of the Ekman spiral for $\mathrm{F}=0.001,0.01$, and 0.1 and $\mathrm{La}=0.001,0.01$, and 0 . In all cases the waves are monochromatic and propagate parallel to the wind. For these values of $\mathrm{La}$ and $\mathrm{F}, \mathrm{Ek}$ varies from 0.01 $(\mathrm{La}=0.001, \mathrm{~F}=0.1)$ to $100(\mathrm{La}=0.1, \mathrm{~F}=0.001)$. Lines of constant $\mathrm{Ek}$ run from the upper left to the lower right. As in the previous chapter, changing $\mathrm{La}$ implies that the surface stress also changes. The dashed lines show spirals without waves while the solid lines show spirals with waves. The open triangles mark the value of the surface current. Since the surface shear is constant, the structure of the spirals is a

\footnotetext{
${ }^{*}$ Mourad (pers. comm.) has studied the effect of shear instability driven by inflection points in the crosscell velocity in reinforcing the cells, but to my knowledge he does not consider the effect of such shears on cell orientation.
} 


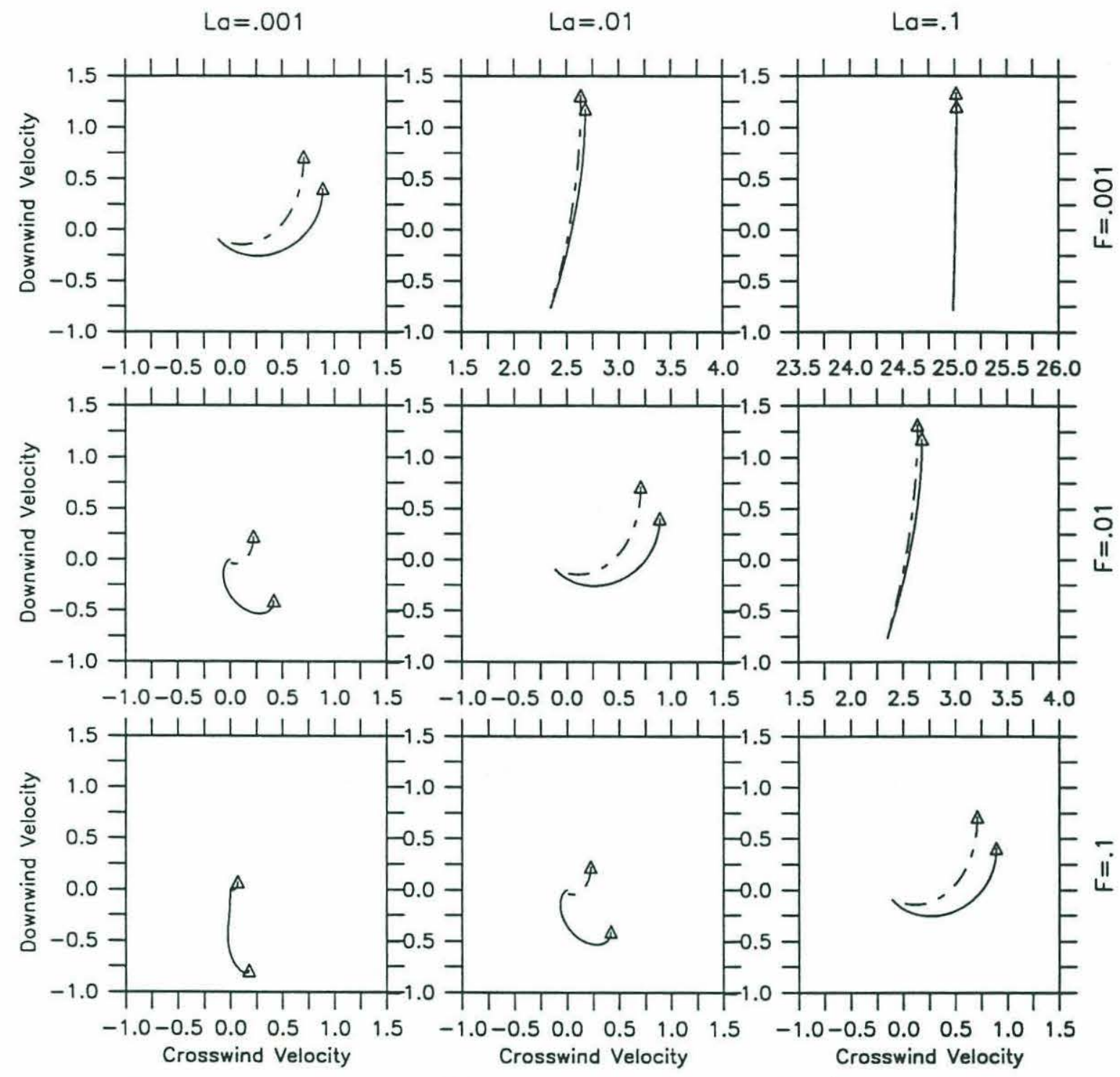

Ekman Spirals with (Solid) and without (Dashed) Monochomatic Waves

Figure 3.4: Hodographs of Ekman spirals with (solid) and without (dashed) waves. The vertical axis is the alongwind velocity, the horizontal axis the crosswind velocity.The surface velocity is shown by open triangles. All cases are for mixed layer depth $\mathrm{D}=4$ and $\left.\frac{\partial \mathrm{V}}{\partial \mathrm{z}}\right|_{\mathrm{z}=0}=1$, waves parallel with wind. Top left: $\mathrm{F}=0.001, \mathrm{La}=0.001$. Top center: $\mathrm{F}=0.001, \mathrm{La}=0.01$. Top right: $\mathrm{F}=0.001, \mathrm{La}=0.1$. Middle left: $\mathrm{F}=0.01, \mathrm{La}=0.001$. Middle center: $\mathrm{F}=0.01, \mathrm{La}=0.01$. Middle right: $\mathrm{F}=0.01, \mathrm{La}=0.1$. Bottom left: $\mathrm{F}=0.1, \mathrm{La}=0.001$. Bottom center: $\mathrm{F}=0.1, \mathrm{La}=0.01$. Bottom right: $\mathrm{F}=0.1, \mathrm{La}=0.1$ 
function of Ek alone. As Ek becomes very large (as in the upper right-hand corner) the current profile is essentially the same as that for the flow treated in Chapter 2 with an offset due to the Ekman transport and Coriolis-driven wave return flow. The Ekman transport for this case is much larger than the wave return flow. As Ek becomes very small, as in the lower left-hand corner, the orientation of the current is essentially upwind over much of the depth, with a spiral near the top of the water column. The Ekman transport when Ek is small is much smaller than the wave return flow (not often a realistic condition, but one which is possible if the waves are large and the wind very weak).

Figure 3.5 shows contours of the growth rate of the most unstable mode for the spirals without waves in Figure 3.4. For these current spirals the only possible instability mechanisms are Ekman instability and shear instability. In general there is only growth for $\mathrm{Ek} \leq 1$. Growth rates for these cases are still very small compared with the Eulerian and Stokes drift shears. Given $\mathrm{La}=0.001$, the maximum growth rates are approximately 0.005 for $\mathrm{F}=0.001$ ( $\mathrm{Ek}=1), 0.01$ for $\mathrm{F}=0.01$ ( $\mathrm{Ek}=0.1)$, and 0.005 for $\mathrm{F}=0.1$ ( $\mathrm{Ek}=0.01)$. The unstable rolls are generally oriented to the right of the wind, as much as 70 degrees to the right of the wind for $\mathrm{La}=0.001, \mathrm{~F}=0.1$.

The picture presented above changes drastically in the presence of surface gravity waves. Figure 3.6 has the same layout as Figure 3.5, but with the difference that the maximum growth rate is now calculated in the presence of surface gravity waves. For $E k=1-100$ growth rates are of order $0.1-0.5$, while in the absence of surface gravity waves the surface layer was predicted to be essentially stable to two-dimensional disturbances. For Ek $<1$ the growth rates are smaller than for large values of Ek, but they are generally still at least an order of magnitude larger than the growth rates in the absence of waves. This supports one of the principal hypotheses of the thesis, namely that wave-current interaction is the main driving mechanism for Langmuir cells. The forcing required to drive these cells is relatively weak by oceanic standards. Assuming an eddy viscosity of $0.01 \mathrm{~m}^{2} / \mathrm{s}$, a scenario for which $\mathrm{La}=\mathrm{F}=0.01$ with a surface shear of 1 corresponds to $2 \mathrm{~m}$ high waves with a period of 8 seconds and a wind stress of $0.1 \mathrm{~Pa}$. Neither the wave height not the wind stress is particularly large.

A number of other conclusions about the cell structure can be drawn from Figure 3.6. These are summarized below:

-For $\mathrm{Ek} » \mathrm{O}(1)$ the shear is essentially downwind and the axis of maximum instability points essentially downwind too. As Ek decreases, the shear 

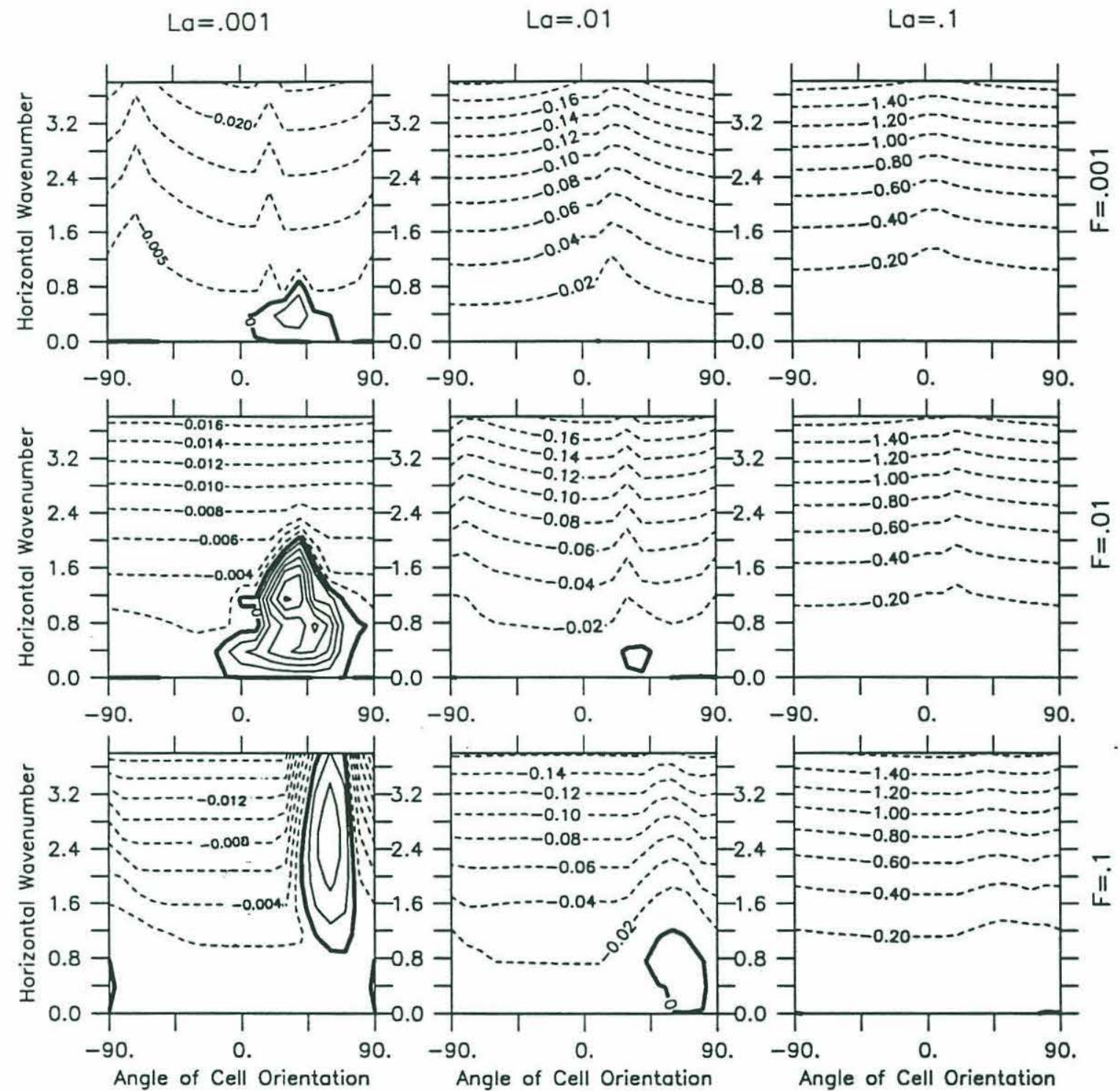

Growth Rates in the Absence of Surface Gravity Waves

Figure 3.5: Growth rate of the most unstable mode as a function of horizontal wavenumber (vertical axis), and angle of orientation $\alpha$ of the cell axis relative to the wind (horizontal axis 0 is parallel with the wind and waves, \pm 90 is perpendicular to the wind). Calculations are for the dashed spirals in Figure 3.4 from a spectral instability code excluding the effects of waves on the current and on the instability. Growth rates for $\alpha \pm 180$ are the same as for $\alpha$. Dashed lines are negative contours. Top left: $\mathrm{F}=0.001, \mathrm{La}=0.001$. Top center: $\mathrm{F}=0.001, \mathrm{La}=0.01$. Top right: $\mathrm{F}=0.001, \mathrm{La}=0.1$. Middle left: $\mathrm{F}=0.01, \mathrm{La}=0.001$. Middle center: $\mathrm{F}=0.01, \mathrm{La}=0.01$. Middle right: $\mathrm{F}=0.01, \mathrm{La}=0.1$. Bottom left: $\mathrm{F}=0.1, \mathrm{La}=0.001$. Bottom center: $\mathrm{F}=0.1, \mathrm{La}=0.01$. Bottom right: $\mathrm{F}=0.1, \mathrm{La}=0.1$. 

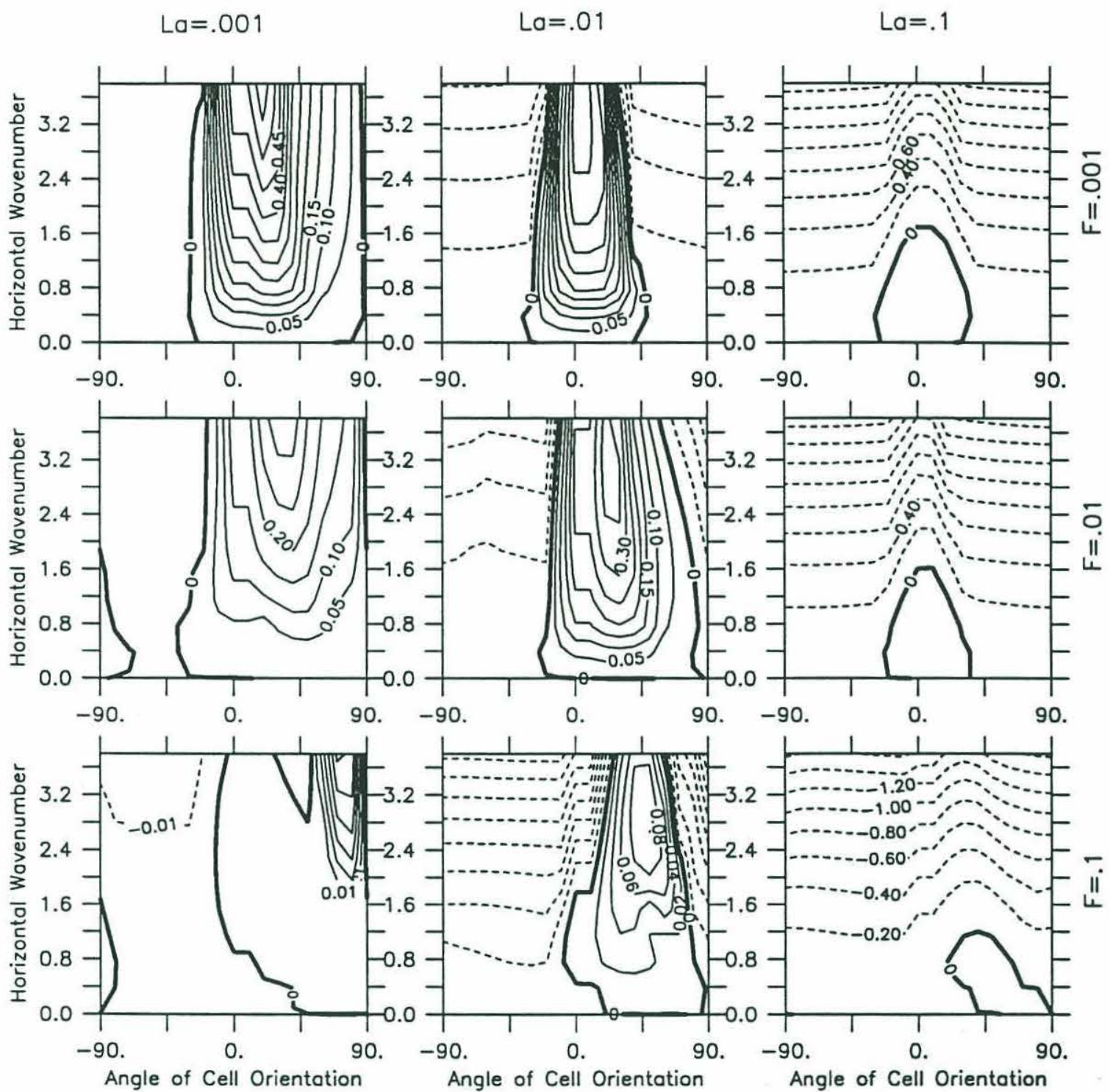

Growth Rates in the Presence of Monochromatic Surface Gravity Waves

Figure 3.6: Growth rate of the most unstable mode as a function of horizontal wavenumber (vertical axis), and angle of orientation $\alpha$ of the cell axis relative to the wind (horizontal axis 0 is parallel with the wind and waves, \pm 90 is perpendicular to the wind). Calculations are for the solid spirals in Figure 3.4 from a spectral instability code. Growth rates for $\alpha \pm 180$ are the same as for $\alpha$. Dashed lines are negative contours. Top left: $\mathrm{F}=0.001, \mathrm{La}=0.001$. Top center: $\mathrm{F}=0.001$, $\mathrm{La}=0.01$. Top right: $\mathrm{F}=0.001, \mathrm{La}=0.1$. Middle left: $\mathrm{F}=0.01, \mathrm{La}=0.001$. Middle center: $\mathrm{F}=0.01, \mathrm{La}=0.01$. Middle right: $\mathrm{F}=0.01, \mathrm{La}=0.1$. Bottom left: $\mathrm{F}=0.1$, $\mathrm{La}=0.001$. Bottom center: $\mathrm{F}=0.1, \mathrm{La}=0.01$. Bottom right: $\mathrm{F}=0.1, \mathrm{La}=0.1$. 

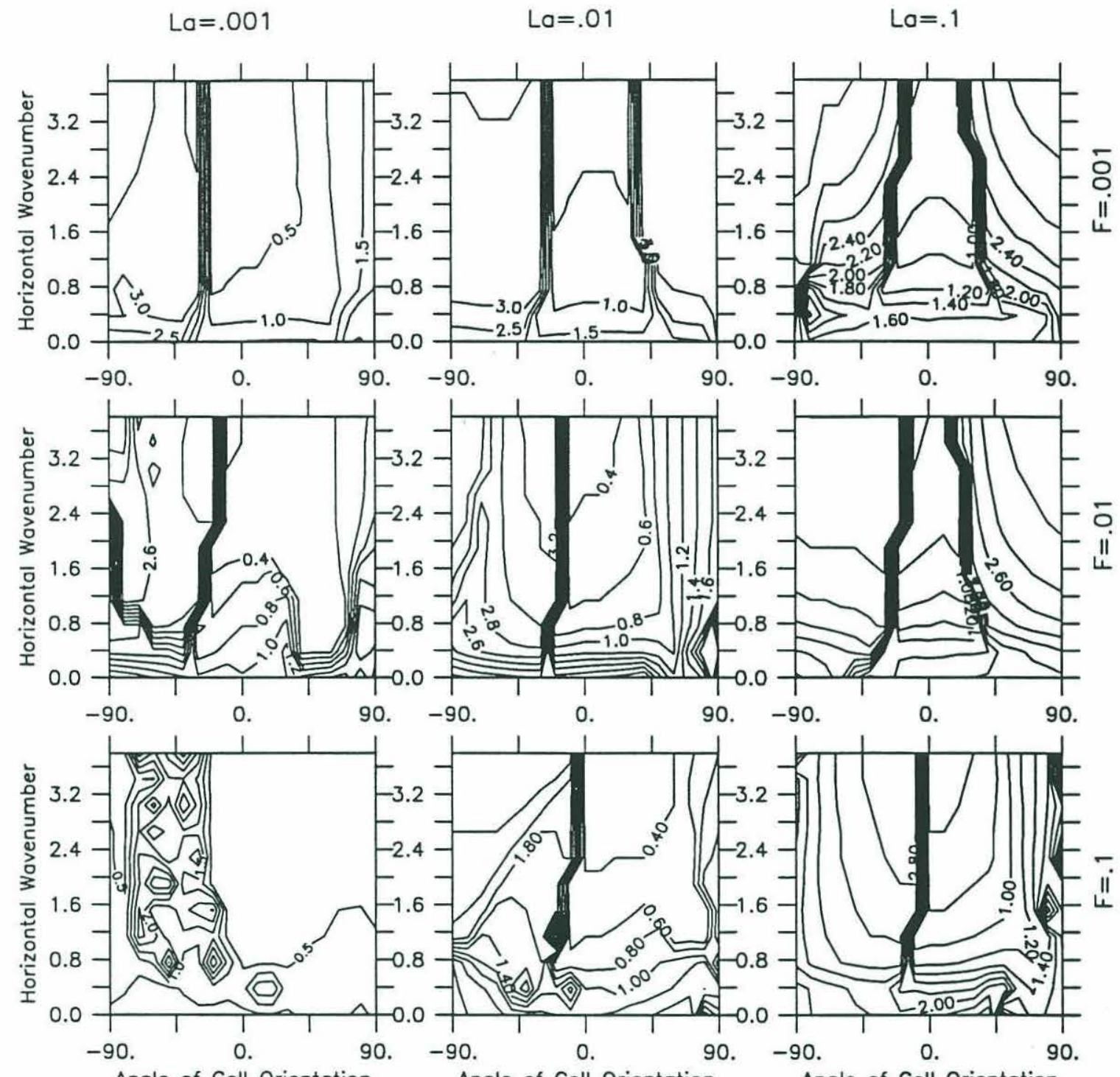

Angle of Cell Orientation Angle of Cell Orientation Angle of Cell Orientation

$D_{\max }$ in the Presence of Monochromatic Surface Gravity Waves

Figure 3.7: Depth of maximum vertical velocity for the most unstable mode as a function of horizontal wavenumber (vertical axis), and angle of orientation $\alpha$ of the cell axis relative to the wind (horizontal axis 0 is parallel with the wind and waves, \pm 90 is perpendicular to the wind). Calculations are for the solid spirals in Figure 3.4 from a spectral instability code. Growth rates for $\alpha \pm 180$ are the same as for $\alpha$. Dashed lines are negative contours. Top left: $\mathrm{F}=0.001, \mathrm{La}=0.001$. Top center: $\mathrm{F}=0.001, \mathrm{La}=0.01$. Top right: $\mathrm{F}=0.001, \mathrm{La}=0.1$. Middle left: $\mathrm{F}=0.01$, $\mathrm{La}=0.001$. Middle center: $\mathrm{F}=0.01, \mathrm{La}=0.01$. Middle right: $\mathrm{F}=0.01, \mathrm{La}=0.1$. Bottom left: $\mathrm{F}=0.1, \mathrm{La}=0.001$. Bottom center: $\mathrm{F}=0.1, \mathrm{La}=0.01$. Bottom right: $\mathrm{F}=0.1$, $\mathrm{La}=0.1$. 
turns more and more to the right of the wind. As this happens the growth rates decrease and the angle at which the unstable growth rates are largest also shifts to be more to the right of the wind.

- For small values of Ek a subsidiary maximum in growth rate sometimes occurs to the left of the axis of the main instability.

- For a given value of Ek (fixed current profile), the growth rates, range of unstable angles and wavenumber of maximum instability are a function of $\mathrm{La}$. As $\mathrm{La}$ increases, the maximum growth rates, range of unstable angles, and wavenumber of maximum instability all decrease.

In Chapter 2, it was shown that the cell structure, as revealed by the depth of maximum vertical velocity $D_{\max }$, was linked to the growth rate. Figure 3.7 shows $D_{\max }$ for the nine current spirals in 3.4. The patterns are again rather complicated, but a number of points can be made.

- For unstable modes, $D_{\max }$ occurs near the surface. As the wavenumber increases (smaller and smaller cell spacings) $D_{\max }$ decreases. For a given value of wavenumber, $D_{\max }$ is a function of the angle of cell orientation. Minima in $\mathrm{D}_{\max }$ with respect to angle of cell orientation reflect maxima in the growth rate of the most unstable mode.

- For damped modes, $\mathrm{D}_{\max }$ is found near the bottom of the fluid layer. As the horizontal wavenumber increases, so does $D_{\max }$, so that the least damped modes are compressed more and more near the base of the layer.

-For a given value of Ek (fixed current profile), as La increases, $\mathrm{D}_{\max }$ moves closer to the middle of the layer.

It is also notable that for $\mathrm{La}=0.001, \mathrm{~F}=0.1, \mathrm{D}_{\max }$ behaves erratically, jumping between large and small values. Examination of the modal structure reveals that the modes involved have two maxima. Most of the jumpiness is the result of $D_{\max }$ jumping between the two maxima. Luckily, the modes for which this is the case are stable and so are not of interest for the present analysis.

In the real ocean, wave energy is spread out over a spectrum rather than being concentrated in a monochromatic wave train. In Chapter 2 spreading energy over a spectrum was found to increase the growth rates. This is also the case in the presence of the Coriolis force. Figure 3.8 shows the hodographs of Ekman spirals corresponding to those in Figure 3.4, for waves given by a Pierson-Moskowitz spectrum rather than as a monochromatic wave train. Figure 3.9 shows contours of growth rate. The strength of the instability as a function of the angle of orientation can be partially understood by looking at the shear. Consider the changes produced 


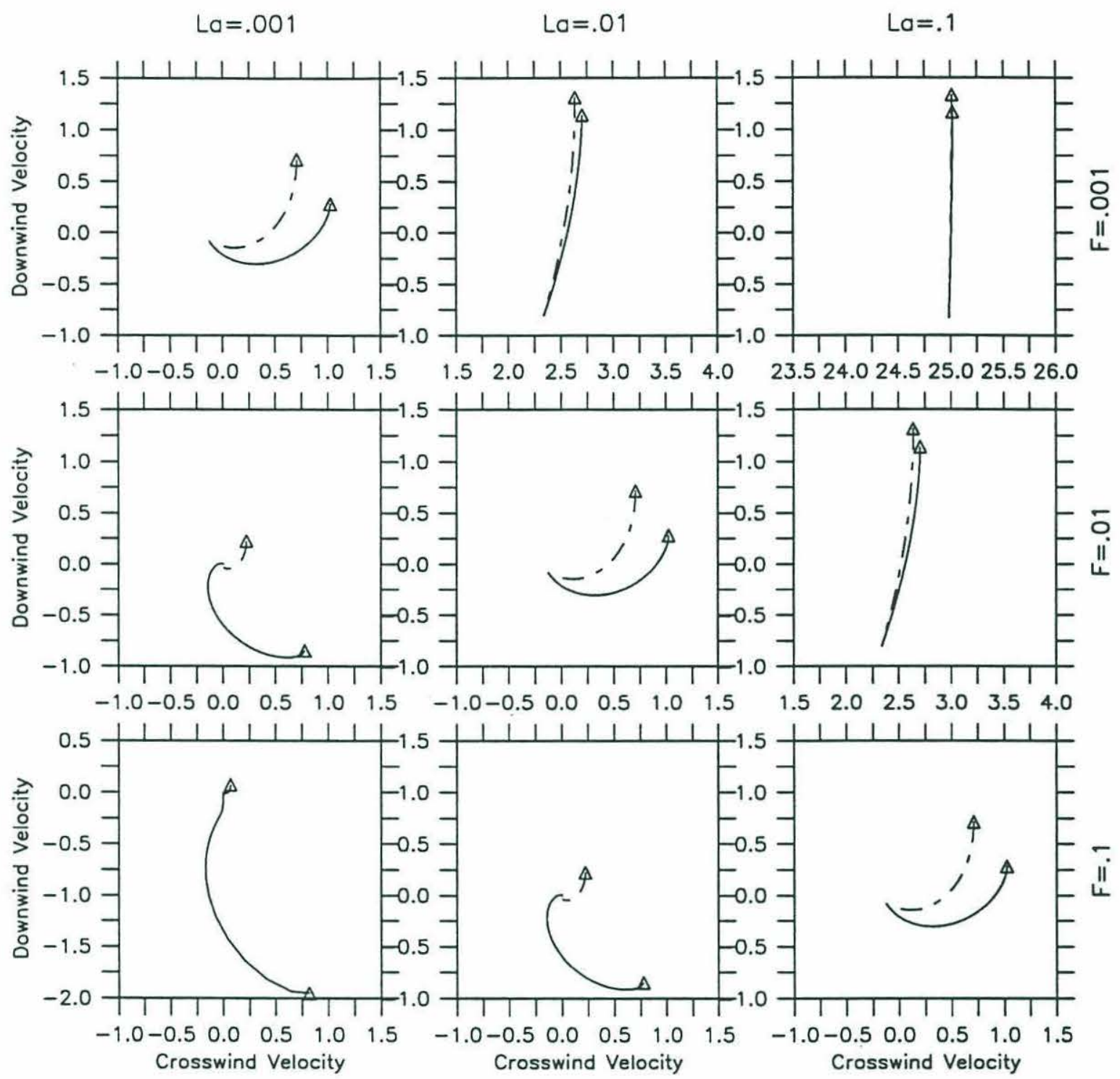

Ekman Spirals with (Solid) and without (Dashed) Wave Spectrum

Figure 3.8: Hodographs of Ekman spirals with (solid) and without (dashed) waves. Waves given by Pierson -Moskowitz spectrum. The vertical axis is the alongwind velocity, the horizontal axis the crosswind velocity.The surface velocity is shown by open triangles. All cases are for mixed layer depth $\mathrm{D}=4$ and $\left.\frac{\partial \mathrm{V}}{\partial \mathrm{z}}\right|_{\mathrm{z}=0}=1$, waves parallel with wind. Top left: $\mathrm{F}=0.001, \mathrm{La}=0.001$. Top center: $\mathrm{F}=0.001$, $\mathrm{La}=0.01$. Top right: $\mathrm{F}=0.001, \mathrm{La}=0.1$. Middle left: $\mathrm{F}=0.01, \mathrm{La}=0.001$. Middle center: $\mathrm{F}=0.01, \mathrm{La}=0.01$. Middle right: $\mathrm{F}=0.01, \mathrm{La}=0.1$. Bottom left: $\mathrm{F}=0.1$, $\mathrm{La}=0.001$. Bottom center: $\mathrm{F}=0.1, \mathrm{La}=0.01$. Bottom right: $\mathrm{F}=0.1, \mathrm{La}=0.1$ 

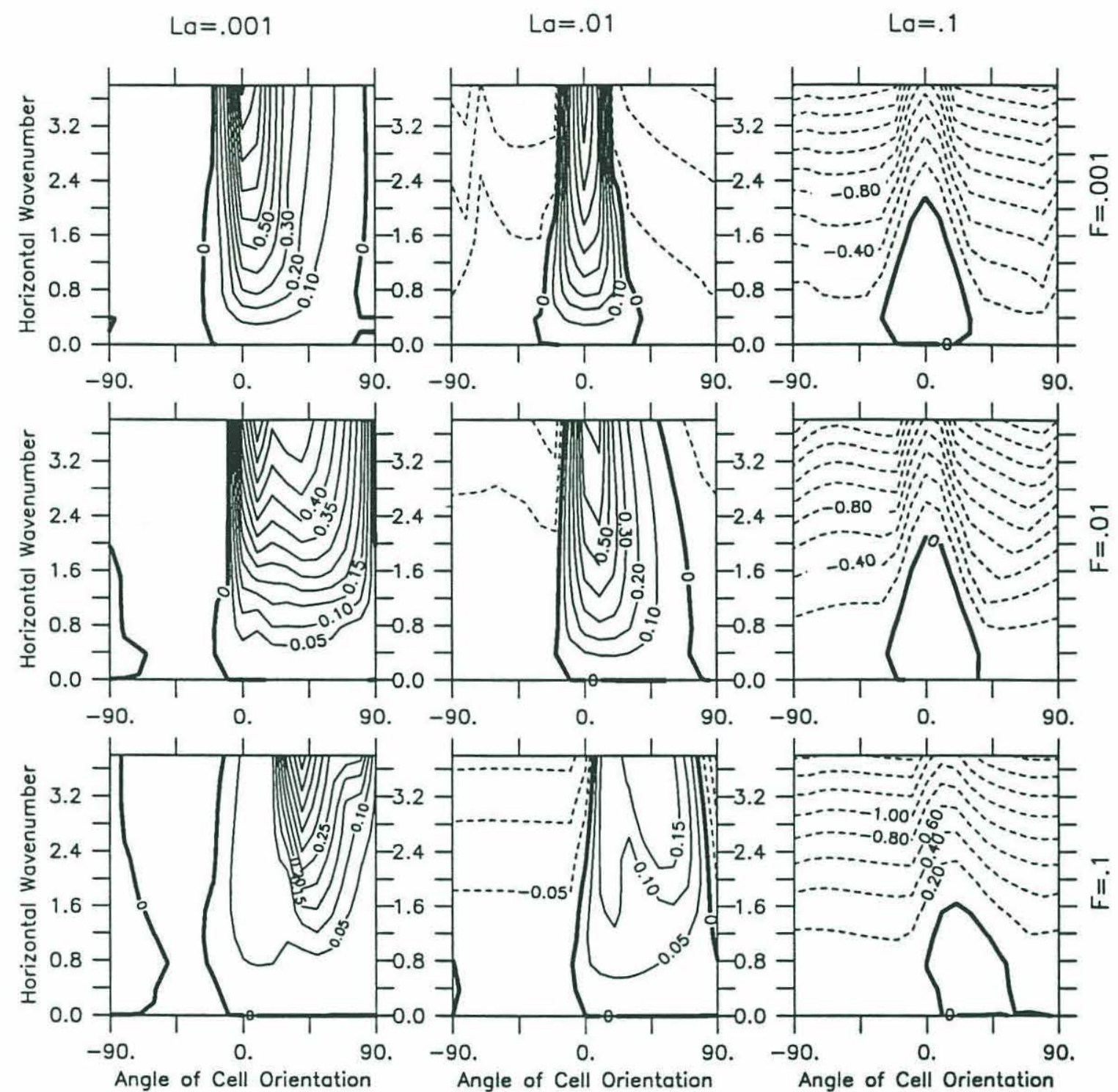

Growth Rates in the Presence of Wave Spectrum (Pierson-Moskowitz)

Figure 3.9: Growth rate of the most unstable mode as a function of horizontal wavenumber (vertical axis), and angle of orientation $\alpha$ of the cell axis relative to the wind (horizontal axis 0 is parallel with the wind and waves, \pm 90 is perpendicular to the wind). Calculations are for the solid spirals in Figure 3.8 from a spectral instability code. Waves are given by a Pierson-Moskowitz spectrum. Growth rates for $\alpha \pm 180$ are the same as for $\alpha$. Dashed lines are negative contours. Top left: $\mathrm{F}=0.001, \mathrm{La}=0.001$. Top center: $\mathrm{F}=0.001, \mathrm{La}=0.01$. Top right: $\mathrm{F}=0.001$, $\mathrm{La}=0.1$. Middle left: $\mathrm{F}=0.01, \mathrm{La}=0.001$. Middle center: $\mathrm{F}=0.01, \mathrm{La}=0.01$. Middle right: $\mathrm{F}=0.01, \mathrm{La}=0.1$. Bottom left: $\mathrm{F}=0.1, \mathrm{La}=0.001$. Bottom center: $\mathrm{F}=0.1$, $\mathrm{La}=0.01$. Bottom right: $\mathrm{F}=0.1, \mathrm{La}=0.1$. 
in the growth rate by looking at cases when $\mathrm{Ek}>1$ (top right), Ek=1 (central diagonal), or $\mathrm{Ek}<1$ (lower left).

-When Ek »1 changing from a monochromatic wave train to a Pierson-

Moskowitz spectrum does not produce a significant change in the Eulerian shear profile, whereas the Stokes drift shear increases sharply. Growth rates essentially double.

-When Ek=1, changing to a Pierson-Moskowitz spectrum shifts the Eulerian shear slightly more to the right of the wind, while increasing its magnitude slightly as well, while the Stokes drift shear increases sharply. The Stokes drift shear "wins", and the axis of maximum instability moves from being at about 15 degrees to roughly 5 degrees to the right of the wind. The growth rates increase by a factor of slightly more than 2 . -When Ek<1 changing from a monochromatic wave train to a PiersonMoskowitz spectrum results in giving much larger Stokes' drift and Eulerian shears near the surface. Once again, the change in the Stokes' drift shifts the angle of maximum instability upwind. For $E \mathrm{k}=0.01$, corresponding to $\mathrm{La}=0.001, \mathrm{~F}=0.1$, the axis of maximum instability shifts from approximately 75 degrees to the right of the wind to roughly 50 degrees to the right of the wind. Interestingly, there are some unstably growing modes at more than 90 degrees to the right of the wind. Growth rates increase by a factor of 4 or more.

The effect of stratification on the strength of the instability is shown in Figure 3.10. Contours of growth rate vs. angle of orientation and horizontal wavenumber are shown for $\mathrm{La}=0.01, \mathrm{~F}=0.001,0.01$, and 0.1 (corresponding to the middle column of Figure 3.6), for $\mathrm{Ri}=0.05,0.2$, and 1.0. The growth rates are

\begin{tabular}{|l|l|l|l|l|l|l|}
\hline \multicolumn{1}{|c|}{$\alpha$} & 0 & 15 & 30 & 45 & 60 & 75 \\
\hline 0.01 & 171 & 242 & 252 & 217 & $>10^{6}$ & $>10^{6}$ \\
\hline 0.025 & 68 & 96 & 100 & 86 & $>10^{6}$ & $>10^{6}$ \\
\hline 0.05 & 33 & 46 & 49 & 42 & $>10^{6}$ & $>10^{6}$ \\
\hline 0.075 & 21 & 29 & 31 & 26 & $>10^{6}$ & $>10^{6}$ \\
\hline 0.1 & Stable & 21 & 22 & 19 & $>10^{6}$ & $>10^{6}$ \\
\hline
\end{tabular}

Table 3.1: Maximum integer wavelength which was unstable given $\mathrm{La}=\mathrm{F}$, monochromatic waves and a surface shear of 1 for differing values of $\mathrm{La}$, angle of cell orientation $\alpha$. 

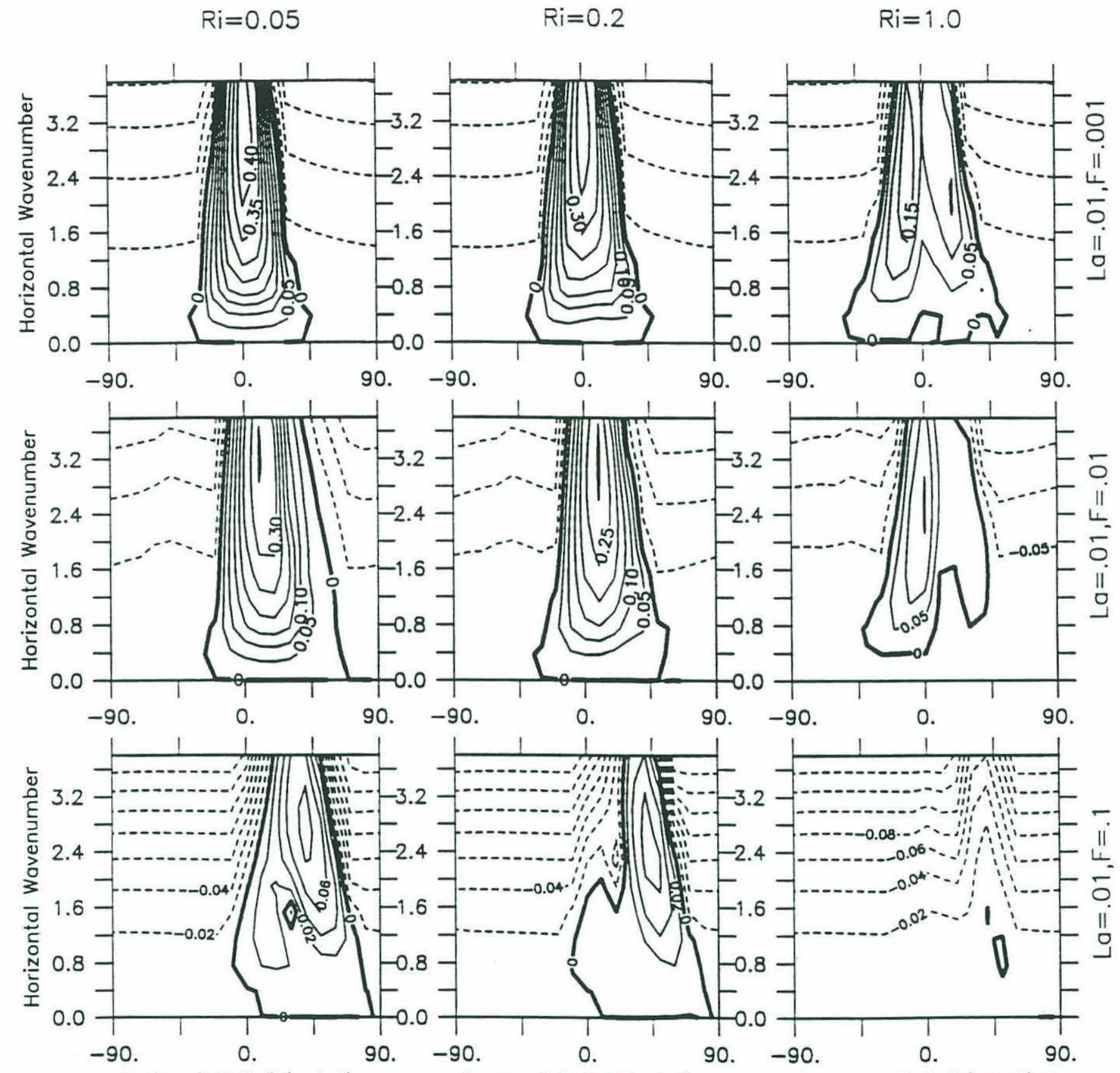

Angle of Cell Orientation

Angle of Cell Orientation

Angle of Cell Orientation

Growth Rates in the Presence of Monochromatic Surface Gravity Waves

Figure 3.10: Growth Rate as a function of horizontal wavenumber (vertical axis) and angle of orientation of cell axis relative to the wind (horizontal axis; 0 is parallel, \pm 90 is perpendicular to wind and waves) for the spirals in the central column of Figure $3.6(\mathrm{La}=0.01, \mathrm{D}=4$, surface shear $=1)$ given different values of stratification. Top left: $\mathrm{F}=0.001$. $\mathrm{Ri}=0.05$. Top center: $\mathrm{F}=0.001, \mathrm{Ri}=0.2$. Top right: $\mathrm{F}=0.001, \mathrm{Ri}=1.0$. Middle left: $\mathrm{F}=0.01$. $\mathrm{Ri}=0.05$. Middle center: $\mathrm{F}=0.01, \mathrm{Ri}=0.2$. Middle right: $F=0.01, R i=1.0$. Bottom left: $F=0.1$. $R i=0.05$. Bottom center: $F=0.1$, $\mathrm{Ri}=0.2$. Bottom right: $\mathrm{F}=0.1, \mathrm{Ri}=1.0$. 
Growth Rate: $L a=0.01, F=0.01, L=32$

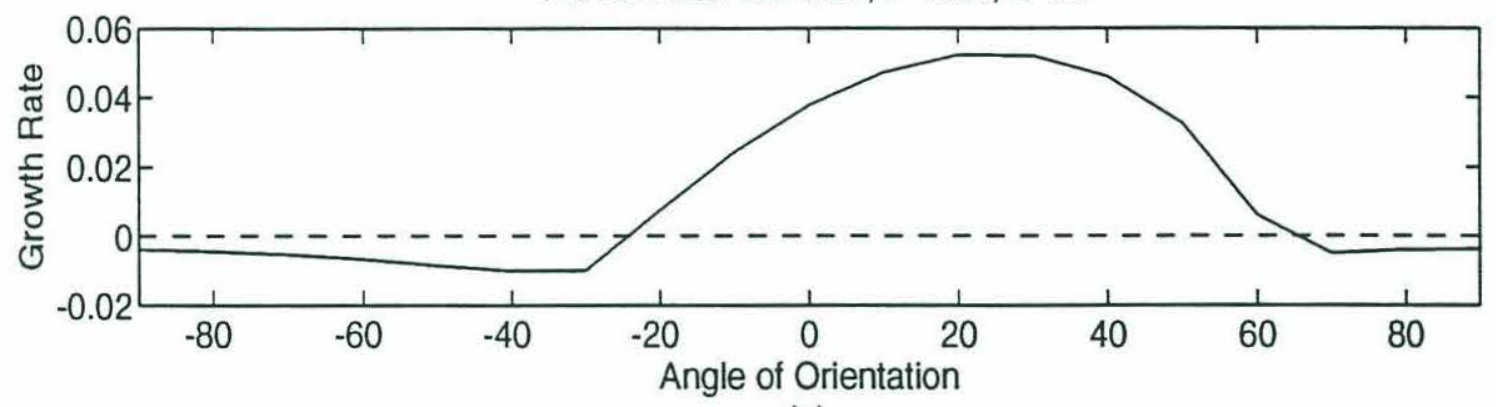

(a)

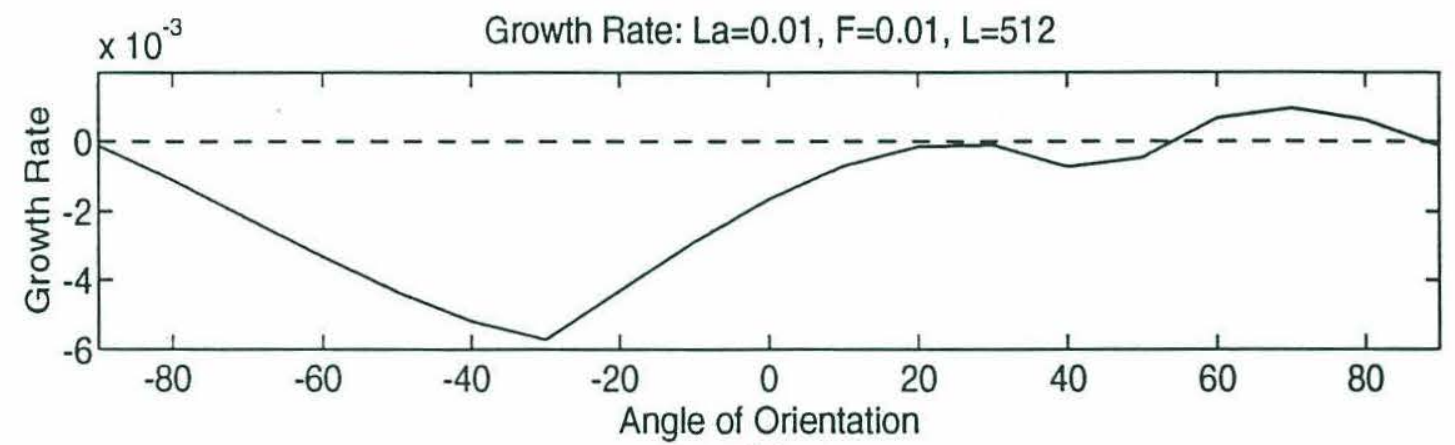

(b)

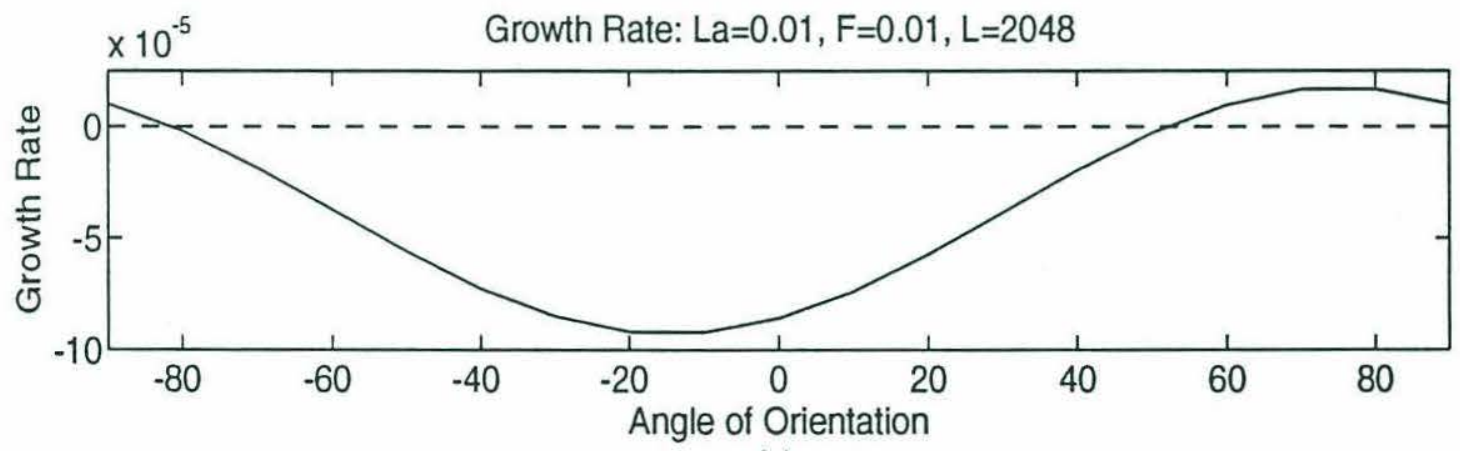

(c)

Figure 3.11: Langmuir cell instability at very low wavenumber. Growth rate as function of angle of cell orientation given $\mathrm{La}=\mathrm{F}=0.01, \mathrm{D}=4$, monochromatic waves and a surface shear of 1. (a) Growth rate vs. angle of orientation, cell spacing $\mathrm{L}=32$.(b) Growth rate vs. angle of orientation. $\mathrm{L}=512$. (c) Growth rate vs. angle of orientation, $\mathrm{L}=2048$. 
reduced as the stratification increases. For high enough values of Ri there is a low wavenumber cutoff for wavelengths which are only a few times as large as the mixed layer depth (see the lower right-hand corner of 3.10). In addition the axis of maximum instability is a strong function of wavenumber. This is especially true for the smallest value of Ek shown ( $E k=0.1$, the bottom row).

The presence of a cutoff in the instability at low wavenumber is particularly interesting. It may occur when the corresponding wavelengths are not extremely large compared with the depth of the fluid layer. The pattern of the cutoff, however, is extremely complicated. Table 3.1 shows the wavelength of the marginally stable cell to within 1.0 for spirals where $\mathrm{Ek}=1(\mathrm{La}=\mathrm{F})$, given different values of $\mathrm{La}$ and angle of orientation. For cells oriented between 0 and 45 degrees to the right of the wind, there is a low wavenumber cutoff which depends strongly on the Langmuir number (varying approximately as $1 / \mathrm{La}$ ). For $\mathrm{La}=60$ and $\mathrm{La}=75$ however, there does not appear to be a low wavenumber cutoff.

Figure 3.11 shows plots of the growth rate vs the angle of orientation for $\mathrm{La}=\mathrm{F}=0.01$ for three values of cell spacing $\mathrm{L}(32,512$, and 2048). Two different physical regimes appear. For cells oriented between 20 degrees to the left of the wind and about 50 degrees to the right of the wind, there is a cutoff at low wavenumber. For cells oriented between about 50 degrees to the right of the wind and 90 degrees to the right of the wind roll vortices are only unstable at low wavenumber. This is an indication that the fundamental physics driving the cells could be different in the two regimes. Section 3.5 explores this question further.

To summarize, the major results of this section are that:

- The presence of surface gravity waves can destabilize the mean current profile in a fluid layer with a no-stress bottom boundary.

- The instability is a strong function of the angle at which the cells are oriented relative to the wind and waves, with the axis of maximum instability lying in between the axis along which the Eulerian shear is oriented and that along which the waves are propagating.

-There is sometimes a low-wavenumber cutoff.

-The cell structure is a strong function of the angle of orientation of the cells.

The dependence of the growth rate and $\mathrm{D}_{\max }$ on horizontal wavenumber and Langmuir number is understandable given the results of Chapter 2. The Stokes drift and Eulerian shears are largest near the surface, so that in order to maximize the forcing $\mathrm{D}_{\max }$ would be much less than $\mathrm{L}$, the cell spacing. On the other hand, in order to maximize the efficiency of this forcing in producing cells $\mathrm{D}_{\max }$ would be 
much larger than L. Additionally, cells for which $\mathrm{D}_{\max }$ is much smaller than $\mathrm{L}$ will have larger diffusive decay associated with them. The resulting tradeoff leads to the depth of penetration of the cells scaling with the cell spacing. Additionally, it means that an increase in La causes an increase in depth of penetration.

The results of Chapter 2 do not, however, provide immediate answers to three questions which arise from the results of this section:

1. How is the angle of orientation of the cells determined, and how is the angle of orientation linked to the growth rate and depth of penetration?

2. How important are Craik-Leibovich instability, Ekman instability, and shear instability in driving unstable roll vortices in an Ekman layer with waves?

3. What processes account for the cutoff of the instability at low wavenumbers? These questions are addressed in Section 3.5.

\subsection{Understanding the physics behind instability of an Ekman layer with surface gravity waves}

\subsubsection{Models of the instability}

This examines the physics of the instability of the Ekman spiral to Langmuir cells using the approach developed in Chapter 2. Simple truncated representations of the velocity and streamfunction fields are used to approximate the instability code results and to provide a simple understanding of the processes involved. Additionally, modified instability codes which omit certain physical processes are also used to look at the importance of Ekman instability and shear instability in causing Langmuir cells.

The truncation which will be used in this section (T3) was introduced in equations (3-9). The linear instability problem which one derives by substituting these equations into the equations of motion (3-3) is one in which the Coriolis force does not play a role. The truncation can thus be used to estimate the importance of the Coriolis force in directly causing instability. If it fails to reproduce the observed characteristics of the instability, Coriolis forces are important.

Consider the growth rate when the viscosity is zero. Following Chapter 2 the following definitions can be made

$$
\hat{\mathrm{V}}_{\mathrm{z} 3}^{(1,2)}\left(\mathrm{D}^{\prime}\right)=\frac{2}{\mathrm{D}^{\prime}} \int_{-\mathrm{D}^{\prime}}^{0} \frac{\partial \mathrm{V}_{0}}{\partial \mathrm{z}} \sin ^{2}\left(\frac{(1,2) \pi \mathrm{z}}{\mathrm{D}^{\prime}}\right) \mathrm{dz}
$$




$$
\widehat{V}_{\mathrm{sz3}}^{(1,2)}\left(\mathrm{D}^{\prime}\right)=\frac{2}{\mathrm{D}^{\prime}} \int_{-\mathrm{D}^{\prime}}^{0} \frac{\partial \mathrm{v}}{\partial \mathrm{z}} \sin 2\left(\frac{(1,2) \pi \mathrm{z}}{\mathrm{D}^{\prime}}\right) \mathrm{dz}
$$

$$
\sigma=\frac{2 \mathrm{k}}{\mathrm{D}^{\prime}} \int_{-\mathrm{D}^{\prime}}^{0}\left(\frac{\partial \Psi_{0}}{\partial \mathrm{z}}+\mathrm{u}_{\mathrm{s}}\right) \sin \left(\frac{\pi \mathrm{z}}{\mathrm{D}^{\prime}}\right) \sin \left(\frac{2 \pi \mathrm{z}}{\mathrm{D}^{\prime}}\right) \mathrm{dz}
$$
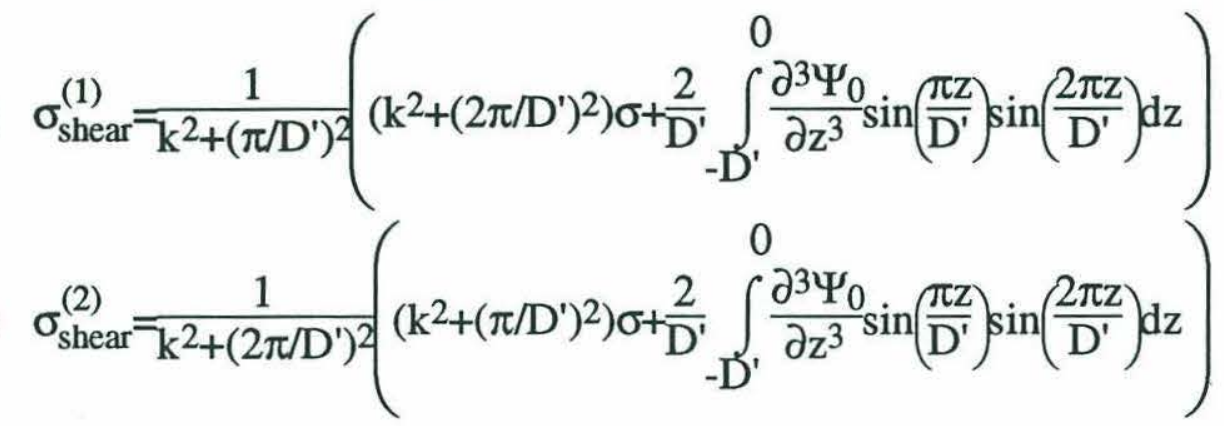

where the superscripts refer to the vertical wavenumber of the perturbations. $\vartheta_{\mathrm{sz3}}^{(1,2)}$ and $\widehat{\vartheta}_{\mathrm{z} 3}^{(1,2)}$ represent the depth-weighted averages of the alongcell Stokes drift and Eulerian shears respectively, where the weighting function is proportional to the momentum transport carried by Langmuir cell with either one or two maxima in vertical velocity. These terms correspond closely to the terms $\hat{V}_{\mathrm{z}}$ and $\hat{V}_{\mathrm{z}}$ derived in Chapter 2. The frequencies $\sigma_{\text {shear }}^{(1)}, \sigma_{\text {shear, }}^{(2)}$, and $\sigma$ are measures of the effectiveness of the crosscell shear at coupling cells with different vertical wavenumbers. These terms scale as the aspect ratio $\mathrm{kD}$ times the crosscell shear. $\sigma$ will be referred to as the crosscell tilting frequency. Substituting into equations (3-3) yields the following linear eigenvalue problem.

$$
\left(\begin{array}{cccc}
-\gamma & -\sigma_{\text {shear }}^{(1)} & \frac{\mathrm{ik}}{\mathrm{k}^{2}+\left(\pi / \mathrm{D}^{\prime}\right)^{2}} \widehat{\mathrm{v}}_{\mathrm{sz} 3}^{(1)} & 0 \\
\sigma_{\text {shear }}^{(2)} & -\gamma & 0 & -\frac{\mathrm{ik}}{\mathrm{k}^{2}+4\left(\pi / \mathrm{D}^{\prime}\right)^{2}} \widehat{\mathrm{V}}_{\mathrm{sz} 3}^{(2)} \\
-\mathrm{ik} \hat{\mathrm{V}}_{\mathrm{z} 3}^{(1)} & 0 & -\gamma & -\sigma \\
0 & \mathrm{ik} \hat{\mathrm{V}}_{\mathrm{z} 3}^{(2)} & \sigma & -\gamma
\end{array}\right)\left(\begin{array}{c}
\psi_{0} \\
\psi_{1} \\
\mathrm{v}_{0} \\
\mathrm{v}_{1}
\end{array}\right)=0
$$

The growth rate of the most unstable mode may be solved for analytically.

Defining

$$
\gamma_{3}^{(1)}=\sqrt{\frac{k^{2}}{k^{2}+\left(\pi / D^{\prime}\right)^{2}} \widehat{V}_{\mathrm{z} 3}^{(1)} \widehat{\bigvee}_{\mathrm{sz} 3}^{(1)}}
$$




$$
\gamma_{3}^{(2)}=\sqrt{\frac{\mathrm{k}^{2}}{\mathrm{k}^{2}+\left(2 \pi / \mathrm{D}^{\prime}\right)^{2}} \widehat{V}_{\mathrm{z} 3}^{(2)} \widehat{ }_{\mathrm{sz} 3}^{(2)}}
$$

The growth rate is given by

$$
\begin{aligned}
(3-27) \gamma^{2} & =\frac{1}{2}\left(\gamma_{3}^{(1)^{2}}+\gamma_{3}^{(2)^{2}}-\sigma_{\text {shear }}^{(1)} \sigma_{\text {shear }}^{(2)}-\sigma^{2}\right)+ \\
& \sqrt{\frac{1}{4}\left(\gamma_{3}^{(1)^{2}}+\gamma_{3}^{(2)}-\sigma_{\text {shear }}^{(1)} \sigma_{\text {shear }}^{(2)}-\sigma^{2}\right)^{2}+\sigma_{\text {shear }}^{(1)} \sigma_{\text {shear }}^{(2)} \sigma^{2}-\gamma_{3}^{(1)^{2}} \gamma_{3}^{(2)^{2}}}
\end{aligned}
$$

This complicated expression contains some interesting physics which is useful for understanding the behavior of the cells observed thus far.

In the presence of small amounts of viscosity truncation T3 may still appoximately capture the relevant structure of the cells. Equation (3-25) is then replaced by

$$
\left(\begin{array}{ccc}
-\gamma-\gamma_{\text {diff }}^{(1)} & -\sigma_{\text {shear }}^{(1)} & \frac{\mathrm{ik}}{\mathrm{k}^{2}+\left(\pi / \mathrm{D}^{\prime}\right)^{2}} \widehat{\mathrm{V}}_{\mathrm{sz} 3}^{(1)} \\
\sigma_{\text {shear }}^{(2)} & -\gamma-\gamma_{\text {diff }}^{(2)} & 0 \\
-\mathrm{ik} \hat{\mathrm{V}}_{\mathrm{z3}}^{(1)} & 0 & -\gamma-\gamma_{\text {diff }}^{(1)} \\
0 & \mathrm{ik} \widehat{V}_{\mathrm{z} 3}^{(2)} & \sigma
\end{array}\right.
$$

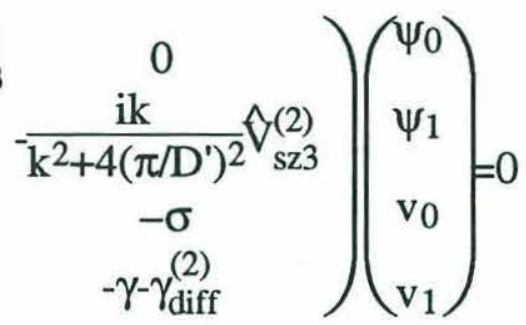

where $\gamma_{\text {diff }}^{(1)}=\mathrm{La}\left(\mathrm{k}^{2}+\left(\pi / \mathrm{D}^{\prime}\right)^{2}\right)$ and $\gamma_{\text {diff }}^{(2)}=\mathrm{La}\left(\mathrm{k}^{2}+\left(2 \pi / \mathrm{D}^{\prime}\right)^{2}\right)$ The damping terms on the diagonal render it impossible to derive a closed-form solution. The most unstable growth rate $\gamma$ from (3-28) in the presence of viscosity is compared to that from (327 ) to evaluate the importance of diffusion in determining the cell structure and growth rate.

\subsubsection{Verification of the truncated model}

The proof of the usefulness of truncated models such as those derived in the last section is that they reproduce the relevant results. Figure 3.12 shows the growth rate and depth of maximum vertical velocity for an initial current profile which is the equilibrium solution of equation (3-7) given $\mathrm{La}=0.001, \mathrm{~F}=0.01$, monochromatic waves and $\left.\frac{\partial \mathrm{V}}{\partial \mathrm{z}}\right|_{\mathrm{z}=0}=1$. This current profile corresponds to the solid line in the middle row, left-hand column of Figure 3.4. The top row shows the predicted growth rate and depth of maximum vertical velocity from the full instability code, the middle row the predictions from (3-27) and the bottom row the predictions from solving equation (3-28). Over the range of unstable cases, 


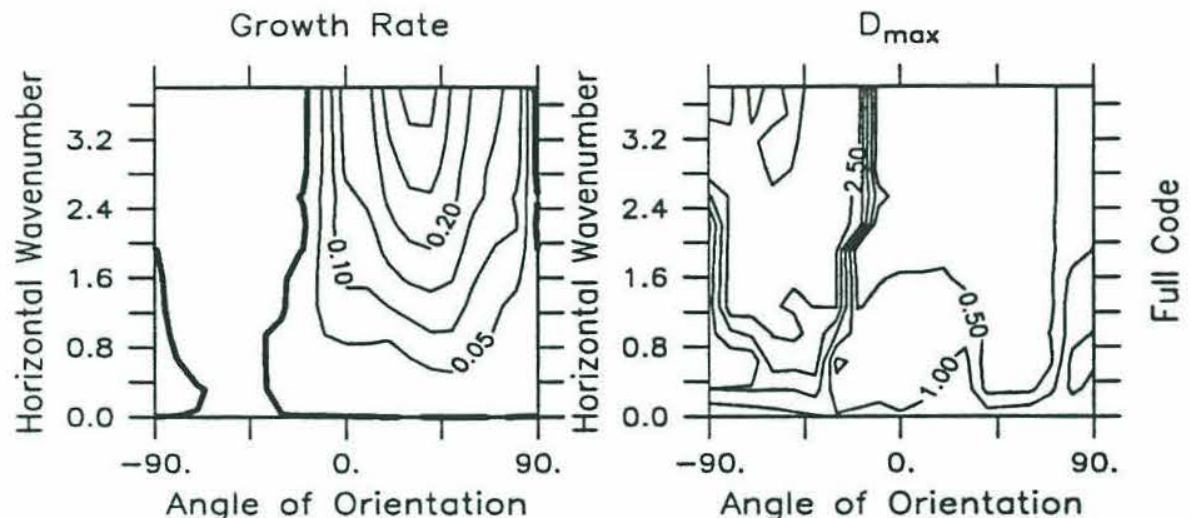

(a)

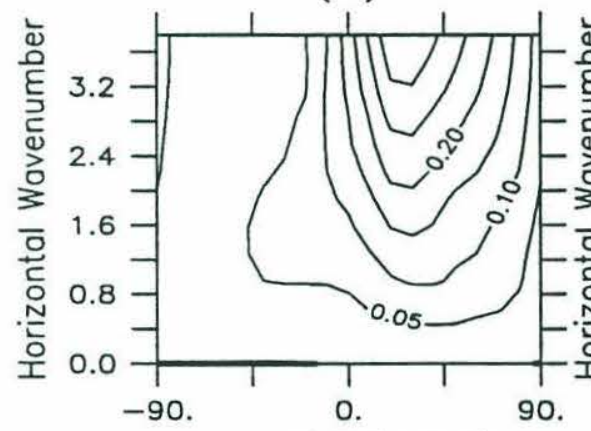

Angle of Orientation

(c)

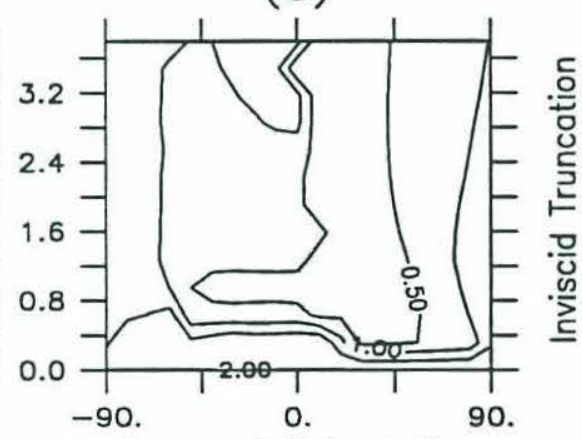

Angle of Orientation

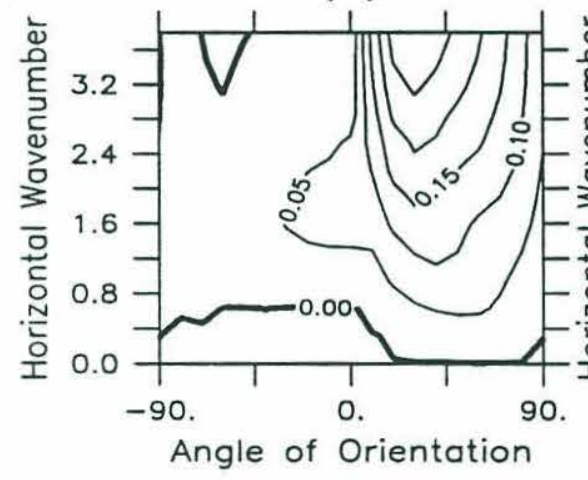

(e) (d)

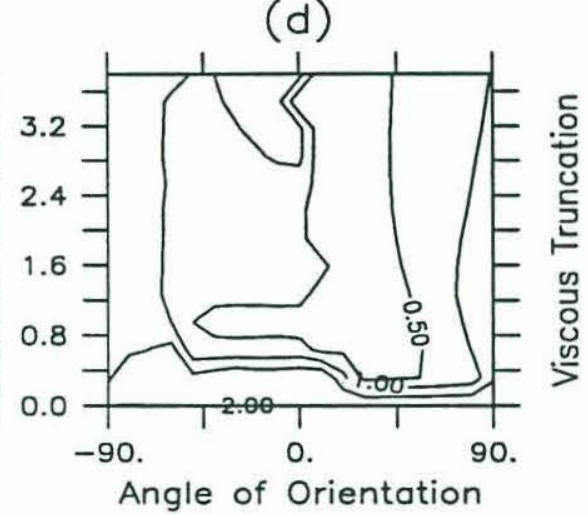

(f)

Figure 3.12: A comparison between the truncated models and the spectral instability code $\mathrm{La}=0.001, \mathrm{~F}=0.01, \mathrm{D}=4$, monochromatic waves oriented parallel to the wind, and a surface Eulerian shear of 1 . Growth rate and depth at which vertical velocity is maximum $D_{\max }$ are plotted against horizontal wavenumber (vertical axis) and angle of cell orientation (horizontal axis). (a) Growth rate, full instability code. (b) $\mathrm{D}_{\max }$, full instability code. (c) Growth rate, Truncation T3 (largest growth rate from equation 3-27). (d) $\mathrm{D}_{\max }$, producing the largest growth rate in equation (3-27).(e) Largest growth rate from solving equation (3-28).

(f) $\mathrm{D}_{\max }$ which produced largest growth rate in equation (3-28). 
both the inviscid and viscous truncations do very well at predicting the growth rates. The root-mean-square deviation over the entire plot is 0.026 for the inviscid case and 0.035 for the viscous case. Adding viscosity narrows the range of unstable angles somewhat and reduces the growth rates at high wavenumber.

The qualitative structure of the depth of maximum vertical velocity is well captured by both of the truncations. There is a low in $\mathrm{D}_{\max }$ in the region where is cells are unstable and a high region where they are stable. For cases when the growth rate is positive, using equation (3-27) results in a mean error in predicting $D_{\max }$ of 0.13 while using the viscous truncation results in a mean error of 0.26 . Both of these errors are quite small, given a water column depth of 4 . The truncations do not do as well at predicting $\mathrm{D}_{\max }$ when the growth rate is stable (note those cases when $\alpha<0$ ), generally underestimating it. However, this is of less importance, since the primary concern of this chapter is unstable Langmuir cells.

Since the truncations predict certain characteristics of the unstable modes, it makes sense to use them to analyze the physical process which go into determining the dependence of growth rate and depth of maximum vertical velocity on the angle of orientation. This is done in section 3.5.3.

The fact that truncation $\mathrm{T} 3$ gives reasonable predictions for $\mathrm{La}=0.001$, $\mathrm{F}=0.01$ implies that the relevant physical processes for this scenario are shear instability and Craik-Leibovich instability. It also implies that Ekman instability is not important for these parameter settings. Section 3.5.4 explores this question in more detail using modified instability codes and shows that Ekman instability can play a role in generating vortices with low horizontal wavenumber. Section 3.5.5 looks at the low wavenumber regime in more detail and presents a simple model for low-wavenumber cutoffs.

\subsubsection{How is the dependence of growth rate and depth of maximum downwelling on angle of orientation determined?}

In Chapter 2, the growth rate of the most unstable Langmuir cell mode in the nonrotating case was shown to be determined by the following tradeoff: -Maximizing the forcing on the vortices (given by the stratified Craik-Leibovich instability parameter $\gamma_{C L S}$ ).

-Maximizing efficiency of the cells at reinforcing themselves (given by the aspect ratio $\mathrm{kD}^{\prime}=4 \pi \mathrm{D}_{\max } / \mathrm{L}$ ).

-Minimizing the diffusion (given by $\gamma_{\text {diff }} \sim \mathrm{La} / \mathrm{D}_{\max }{ }^{2}$ ). 
The next few sections show that for rotating mixed layers this tradeoff still holds, but that with the additional constraint that the crosscell tilting frequency is minimized. This is done by considering some scenarios where equation (3-26) becomes simpler.

The first scenario is illustrated in Figure 3.13. Assume that the Stokes drift and Eulerian shears are constant with depth and that the Stokes drift shear is oriented along an axis $\theta$ degrees to the right of the Eulerian shear. This means that the terms which go as the second derivative of the Eulerian velocity vanish so that $\sigma_{\text {shear }}^{(1)}=\frac{k^{2}+\left(4 \pi / D^{\prime}\right)^{2}}{k^{2}+\left(\pi / D^{\prime}\right)^{2}} \sigma$ and $\sigma_{\text {shear }}^{(2)} \frac{k^{2}+\left(\pi / D^{\prime}\right)^{2}}{k^{2}+\left(4 \pi / D^{\prime}\right)^{2}} \sigma$. Then the growth rate in (3-27) becomes

$$
\gamma^{2}=\frac{1}{2}\left(\gamma_{3}^{(1)^{2}}+\gamma_{3}^{(2)^{2}}-2 \sigma^{2}\right)+\sqrt{\left(\gamma_{3}^{(1)^{2}}+\gamma_{3}^{(2)^{2}}-2 \sigma^{2}\right)^{2}+4 \sigma^{2}-4 \gamma_{3}^{(1)^{2}} \gamma_{3}^{(2)^{2}}}
$$

where

$$
\gamma_{3}^{(1,2)}=\sqrt{\frac{\mathrm{k}^{2}}{\mathrm{k}^{2}+(1,4) \mathrm{l}^{2 \partial \mathrm{z} \partial \mathrm{V}} \mathrm{z}} \cos (\alpha) \cos (\theta-\alpha)}
$$

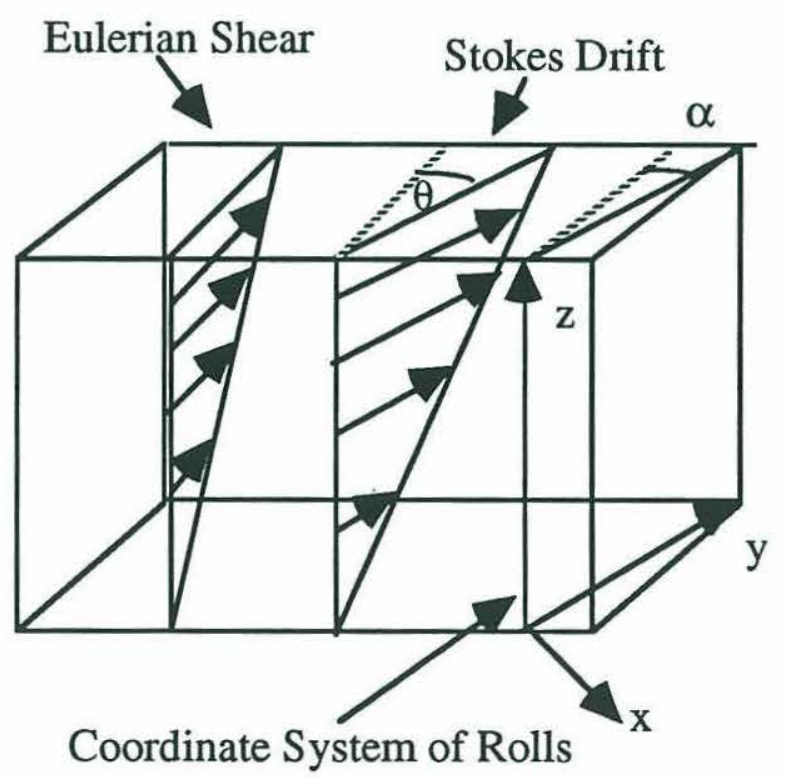

Figure 3.13: Schematic of simplified case used in Section 3.5.3 to explore effect of changing the direction of the Stokes Drift relative to the shear. 
Maximizing the growth rate for this scenario requires maximizing $\gamma_{3}^{(1,2)}$, and minimizing $\sigma$. Since for this simple scenario, the shears are constant over depth maximizing $\gamma_{1}$ and $\gamma_{2}$ for a given angle of orientation also means maximizing the aspect ratio $2 \pi \mathrm{D}^{\prime} / \mathrm{L}=\mathrm{kD}$ '. On the other hand minimizing $\sigma$, which goes as $\mathrm{kD}$ ' times the crosscell shear, means minimizing $\mathrm{kD}$ '.

Assume that the cells penetrate over the depth of the layer (an assumption which is not correct when the crosscell shear is large). The angle of maximum instability is determined by a tradeoff between minimizing $\sigma$ and maximizing the product $\cos (\alpha) \cos (\theta-\alpha)$. The former occurs for the present case when

$$
\frac{\partial u}{\partial z}+\frac{\partial u_{S}}{d z}=-\frac{\partial V}{\partial z} \sin (\alpha)+\frac{\partial V_{S}}{\partial z} \sin (\theta-\alpha)=0
$$

while the latter occurs when $\alpha=\theta / 2$. In the special case where the Stokes drift shear and Eulerian shear are equal then, $\alpha=\theta / 2$ maximizes both the product of the projections of the Stokes drift shear and Eulerian shear on the axis of cell orientation and minimizes the crosscell shear. The growth rate in this case is just

$$
\gamma_{\max }=\sqrt{\frac{\mathrm{k}^{2} \partial \mathrm{V} \partial \mathrm{V}_{\mathrm{S}}}{\mathrm{k}^{2}+\mathrm{1}^{2} \partial \mathrm{z} \partial \mathrm{z}} \cos ^{2}(\theta / 2)}
$$

Increasing the angle between Eulerian shear and Stokes drift shear decreases the growth rate and increases the angle of orientation of the cells relative to the wind. This is consistent with the picture developed in Section 3.4.

In Chapter 2, the growth rate when diffusion was weak was shown to go as

$$
\gamma=\sqrt{\frac{\mathrm{k}^{2}}{\mathrm{k}^{2}+\mathrm{l}^{2}} \hat{\vartheta}_{\mathrm{z}} \hat{\nabla}_{\mathrm{z}}}=\frac{\mathrm{k}}{\sqrt{\mathrm{k}^{2}+\mathrm{1}^{2}}} \gamma_{\mathrm{CL}}
$$

Where $\gamma_{\mathrm{CL}}$, the Craik-Leibovich instability parameter, defined the strength of the wave-current forcing mechanism. Examining (3-32) it can be seen that

$$
\gamma_{C L}=\sqrt{\frac{\partial V \partial V_{S}}{\partial z \partial z} \cos ^{2}(\theta / 2)}
$$

provides the equivalent definition for $\gamma_{C L}$ in the system at hand. This demonstrates that the relevant shears for wave-current interaction are the Eulerian and Stokes drift shears parallel to the axis of cell orientation.

Further insight into the physics of Langmuir cell instability can be gained by considering the behavior of the solution of the solution to (3-26) when $\mathrm{L} a \neq 0$, but the crosscell shear terms are equal to zero. In this case the fastest growing mode is given by the maximum of 


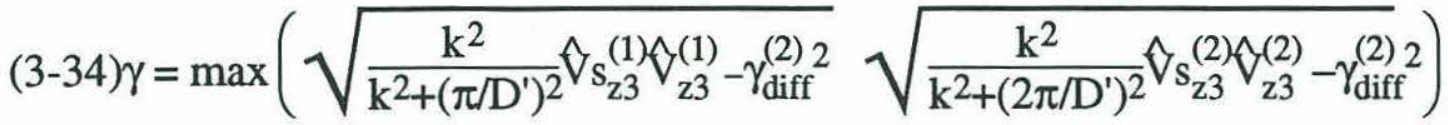
If $\gamma_{\mathrm{CL} 3}^{(1,2)}=\sqrt{\hat{V s}_{\mathrm{z} 3}^{(1,2)} \hat{V}_{\mathrm{z} 3}^{(1,2)}}$ then the presence of instability then, hinges on finding a cell geometry such that

$$
\operatorname{RaCL} 3=\left(\left(\mathrm{k}^{2}+\left((1,2) \pi / \mathrm{D}^{\prime}\right)^{2}\right) \gamma_{\mathrm{CL} 3}^{(1,2)} / \gamma_{\text {diff }}^{(1,2)} \mathrm{k}^{2}\right)^{2}>1
$$

The physics behind what determines $\gamma$ in the absence of crosscell shears are very similar to the physics in the absence of Coriolis forces in Chapter 2.

The structure and growth rate of linearly unstable Langmuir cells, given Ek of order 0.1 or larger, are largely governed by the following tradeoff:

-Maximizing the forcing (given by $\gamma_{\mathrm{CL}}$ ). Tends to favor cells concentrated near the surface and oriented along an axis midway between the Eulerian and Stokes drift shears.

-Maximizing efficiency of the cells at reinforcing themselves (given by the aspect ratio $D_{\max } / L$ ). Tends to favor cells which have deeper penetration.

-Minimizing the crosscell tilting frequency $\sigma$. Tends to favor orientation along an axis where the difference between the Eulerian and Stokes drift shears is minimized and concentration at depths at which the crosscell shear is small. Also tends to favor cells much wider than they are deep.

-Minimizing diffusion, given by the diffusive decay $\gamma_{\text {diff. }}$ Favors deeper penetration of the cells.

This tradeoff provides answers to the following questions about the results in Section 3.4 which are important for understanding the larger problem:

1. Given a constant surface stress and Langmuir number, and assuming waves and wind collinear, what is the dependence of the growth rate, cell structure, and orientation of the cells on Ek?

When Ek is large (F small) the Eulerian shear and Stokes drift shear are also collinear. The tendency to maximize $\gamma_{C L S}$ and minimize $\sigma$ will result in the axis of maximum instability lying parallel to the wind and waves. As the wavenumber becomes large, the aspect ratio $\mathrm{kD}^{\prime}$ of the cells becomes of order unity for shallower and shallower depths. As is the case with the nonrotating instability cases which were studied in the last chapter, the result is that $D_{\max }$ becomes smaller and smaller.

As Ek decreases, the shear shifts to the right of the wind as the depth increases. Near the surface, the two shears are still collinear, but the conditions on 
maximizing the aspect ratio and minimizing diffusion mean that the cells cannot take advantage of this fact. The axis of maximum instability will thus tend to move to the right of the wind. As a result, the projection of the Eulerian and Stokes drift shears on the cell axis decreases and the growth rates drop.

\section{What changes in instability structure occur when the waves are represented} with a Pierson-Moskowitz spectrum?

When Ek is large, changing from monochromatic waves to a PiersonMoskowitz spectrum increases only the Stokes drift. The condition on minimizing crosscell shear draws the axis of maximum instability closer to the direction of wave propagation. Since the Eulerian shear does not change very much, the growth rates essentially double, as they did for the nonrotating case.

When Ek is small, changing the representation of the waves from a monochromatic wave train to a spectrum increases the Eulerian shear as well as the Stokes drift shear. The increase in the growth rate is thus larger than for low $\mathrm{La}$, and the change in angle is smaller.

\subsubsection{What processes are involved in causing the instability?}

In the last section, the Craik-Leibovich wave-current interaction mechanism was shown to be very important in creating Langmuir cells. A natural question which arises is the role of other instability processes, in particular shear instability and Ekman instability (Gammelsrød, 1975). A number of spectral codes (noted in Section 3.2) were developed to estimate the importance of these various processes.

Figure $3.14 \mathrm{a}$ and $\mathrm{b}$ show growth rates for $\mathrm{La}=0.001, \mathrm{~F}=0.01$. This case has already been shown to be well described by the truncation T3. The solid lines are for the full spectral instability code, the dashed lines for a modified code where the effect of the Coriolis parameter on the cells was set to zero, the chain-dotted lines are for shear instability alone. The $\mathrm{x}$ marks are for the growth rate derived from (327). Figure 3.14a shows growth rates for $\mathrm{L}=4(\mathrm{k}=\pi / 2)$ vs angle of orientation. The absence of Coriolis forces makes little difference to the growth rate, with the growth rates in the presence of Coriolis force being almost identical to those in the absence of Coriolis force. There is some evidence of shear instability, but the magnitude of the growth rates associated with it are small compared with the growth rates calculated by the full code. Figure $3.14 \mathrm{~b}$ shows the growth rate vs $\log _{10}$ horizontal wavenumber using the same conventions as Figure 3.14a for $\alpha=40$. Once again the presence or absence of Coriolis forces makes little 


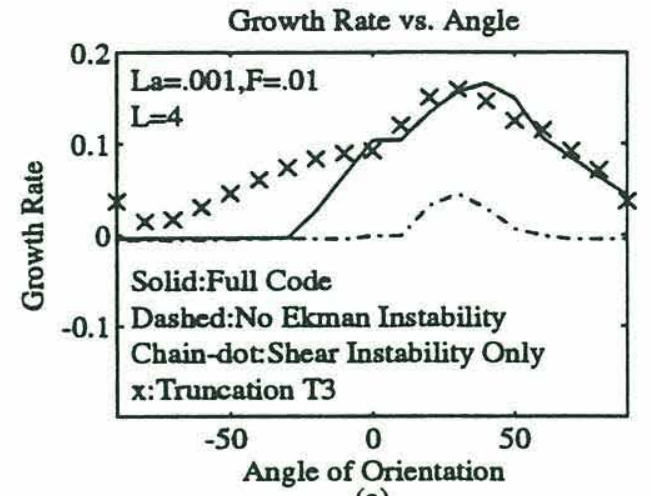

(a)

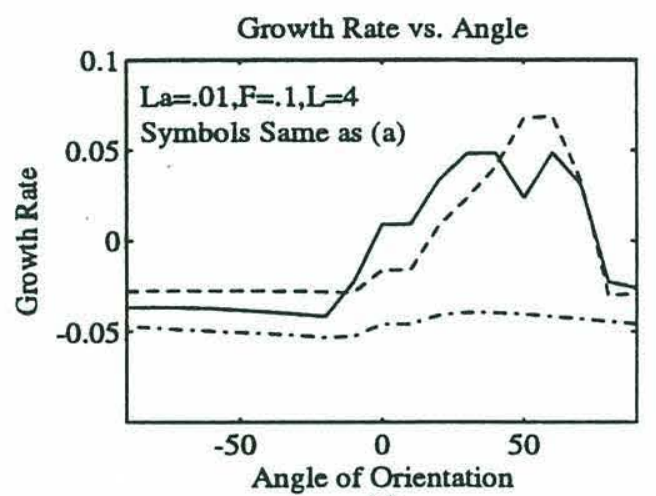

(c)

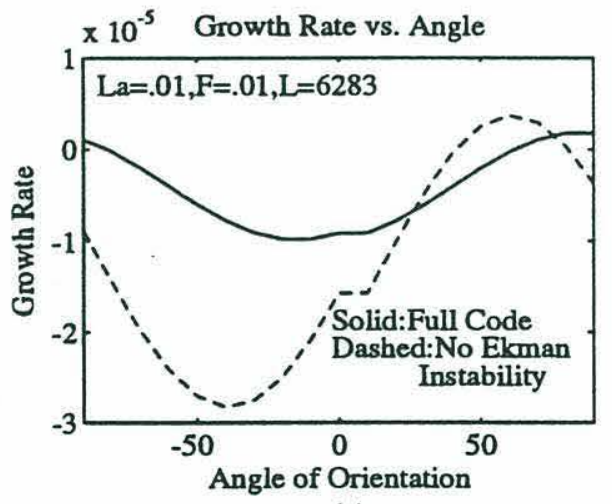

(e)

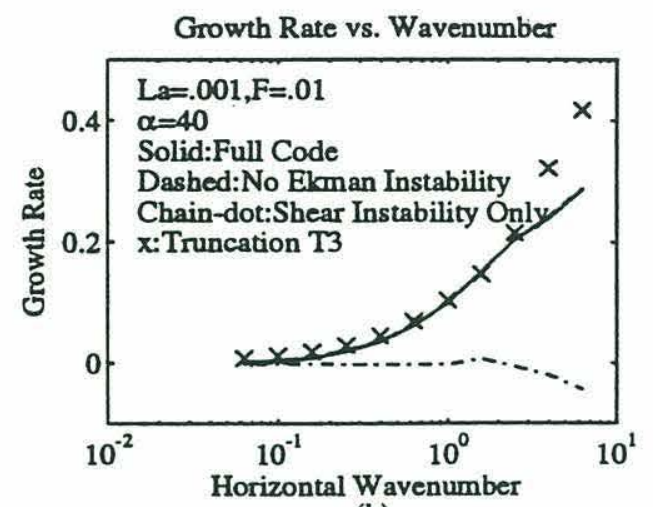

(b)

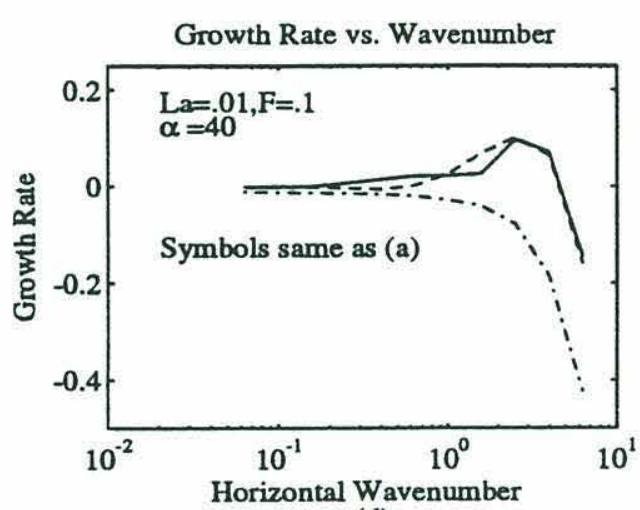

(d)

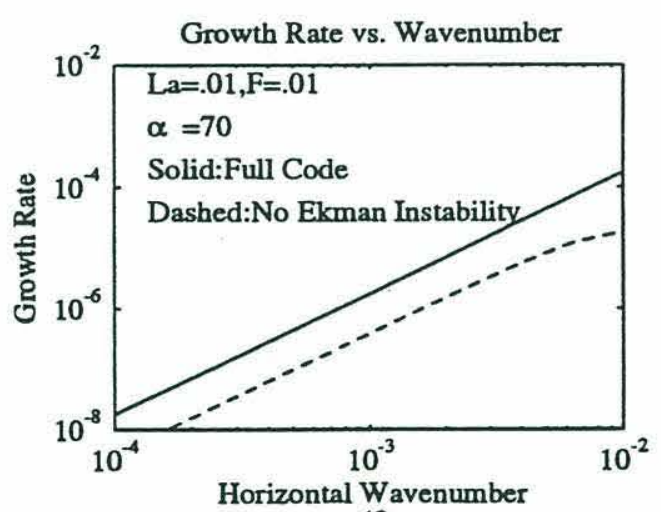

(f)

Figure 3.14: Physics of instability at low Ekman number Ek=La/F. Growth rates are shown with various physical processes excluded. Solid lines are for the full spectral instability code. Dashed lines have Ekman instability excluded. Chaindotted lines are for a code which only allows shear instability. $x$-marks show results from Truncation T3 (equation 3-27). (a) $\mathrm{La}=0.001, \mathrm{~F}=0.01$. Growth rate vs. angle of orientation $\alpha$ for crosscell spacing $L=4$. (b) $L a=0.001, F=0.01$. Growth rate vs $\log _{10}$ horizontal wavenumber. $\alpha=40$ (near maximum angle of instability). (c) Same as (a) but for $\mathrm{La}=0.01, \mathrm{~F}=0.1$. (c) Same as (b), but for $\mathrm{La}=0.01, \mathrm{~F}=0.1$. (e) Growth rate vs angle of orientation, $\mathrm{La}=\mathrm{F}=0.01, \mathrm{~L}=6283$ ( $\mathrm{k}=0.001)$. (f) $\mathrm{Log}_{10}$ growth rate vs $\log _{10}$ wavenumber $\mathrm{La}=0.01, \mathrm{~F}=0.01, \alpha=70$. 
difference, while the presence or absence of Craik-Leibovich instability makes a big difference. The presence of diffusion affects the cells at high wave numbers. While Craik-Leibovich instability and diffusion are critical in setting the growth rate of the cells for this particular case, Ekman instability and shear instability are not that important for small enough $\mathrm{La}$ and $\mathrm{F}$.

The situation is different for a scenario where $\mathrm{La}=0.01$ and $\mathrm{F}=0.1$. This corresponds to increasing the effect of diffusion and Coriolis force, but retaining the same velocity profile. Figures $3.14 \mathrm{c}$ and $\mathrm{d}$ show plots corresponding to $3.14 \mathrm{a}$ and $\mathrm{b}$ with all parameters other than $\mathrm{La}$ and $\mathrm{F}$ the same. The presence or absence of Coriolis forces can make a substantial difference in the growth rate. The Coriolis force destabilizes some cases for low wavenumbers when the cell axis points approximately downwind, while reducing the growth rate for $\mathrm{k}$ of order $\pi / 4$. Shear instability is not sufficient to account for the observed growth rates.

The Coriolis force is also a key player in accounting for the presence of instability at low wavenumber. Figure $3.14 \mathrm{e}$ shows the pattern of growth rate vs. angle in when Ekman instability was allowed to act on the cells (solid) and when it was not (dashed) for cells with a horizontal wavenumber $\mathrm{k}$ of 0.001 given an initial velocity profile corresponding to that for monochromatic waves with $\mathrm{La}=\mathrm{F}=.01$ and a surface shear of 1 . Again, the size of the growth rate changes sharply in the presence of Ekman instability, with vortices whose axes are oriented approximately crosswind being destabilized, and those oriented about 50 degrees to the right of the wind being stabilized. For the cases shown in $3.14 \mathrm{e}$ and $\mathrm{f}$ shear instability was found not to play a role (the growth rates associated with shear instability alone were approximately -0.0062 ). Figure $3.14 \mathrm{f}$ shows a plot of $\log _{10}$ growth rate vs $\log _{10}$ horizontal wavenumber for $\mathrm{La}=\mathrm{F}=0.01$, given an angle of cell orientation $\alpha=70$. The solid lines show the results of the full code, the dashed lines the growth rate when the Coriolis force does not act on the cells so that Ekman instability is disallowed. In Table 3.1 the growth rate predicted by the full code did not cut off at low wavenumbers for this angle of orientation. In the absence of Ekman instability, the growth rate is sharply reduced, dropping by a factor of 10 over most of the range.

Although Craik-Leibovich instability is responsible for the growth of highwavenumber cells which quickly destabilize the Ekman layer, Ekman instability can play a role as well. The Ekman instability process (Gammelsrød, 1975) produces very slowly growing, long wavelength cells when the scaled Coriolis parameter $\mathrm{F}$ is large. 


\subsubsection{How are Langmuir cells stabilized at low wavenumber in the presence of}

\section{Coriolis forces?}

The presence of Coriolis forces can stabilize long-wavelength Langmuir cells (given the appropriate angle of orientation). The presence of a lowwavenumber cutoff has implications for the final state of the mixed layer, since it can serve to limit the depth to which Langmuir cells can penetrate. It is therefore worthwhile to try to understand the physics which cause such a phenomenon. Two hypotheses which might explain a low-wavenumber cutoff in the presence of Coriolis force are the following:

1. The presence of Coriolis force stabilizes cells with long wavelengths.

2. The presence of Coriolis force produces crosscell shears which stabilize the cells.

The presence of a low-wavenumber cutoff is not attributable to the effects of the Coriolis force on the instability. The best piece of evidence for this result is that the modified instability code used in 3.5.4, which removed the effects due to Coriolis forces on the cells. For some cases where Ek was small, removing the Coriolis force reduced the growth rate at low wavenumbers.

Crosscell shears can act to stabilize long-wavelength cells. An extremely simplified instability model can be used to demonstrate this fact. Consider a case with unstable mode $\mathrm{A}$ and stable mode $\mathrm{B}$ which are coupled as follows

$$
\begin{aligned}
& \frac{\partial A}{\partial t}=\Gamma_{1} A-\Phi_{1} B \\
& \frac{\partial B}{\partial t}=-\Gamma_{2} A+\Phi_{2} B
\end{aligned}
$$

where $\Gamma_{1}, \Gamma_{2}, \Phi_{1}$, and $\Phi_{2}$ are positive numbers. $\Gamma_{1}$ represents the growth rate of the unstable mode. $\Gamma_{2}$ represents the decay rate of the stable mode. $\Phi_{1}$, and $\Phi_{2}$ represent coupling coefficients between the two modes. In terms of the phenomenon at hand, $\Phi_{2}$ represents the rate at which crosscell shears tilt a linearly unstable mode with a particular vertical structure into a vertical structure associated with a stable mode. $\Phi_{1}$ represents the rate at which the shear tilts the stable mode (whose growth rate would be $-\Gamma_{2}$ ) back into the unstable mode (whose growth rate would be $\Gamma_{1}$ ). The growth rate of the coupled instability is given by

$$
\gamma=\frac{\Gamma_{1}-\Gamma_{2}}{2}\left(-1+\sqrt{1-\Phi_{1} \Phi_{2} /\left(\Gamma_{1}-\Gamma_{2}\right)^{2}}\right)
$$

If $\left|\Gamma_{1}\right|<\left|\Gamma_{2}\right|$ any coupling at all between the two modes will damp out the instability. 


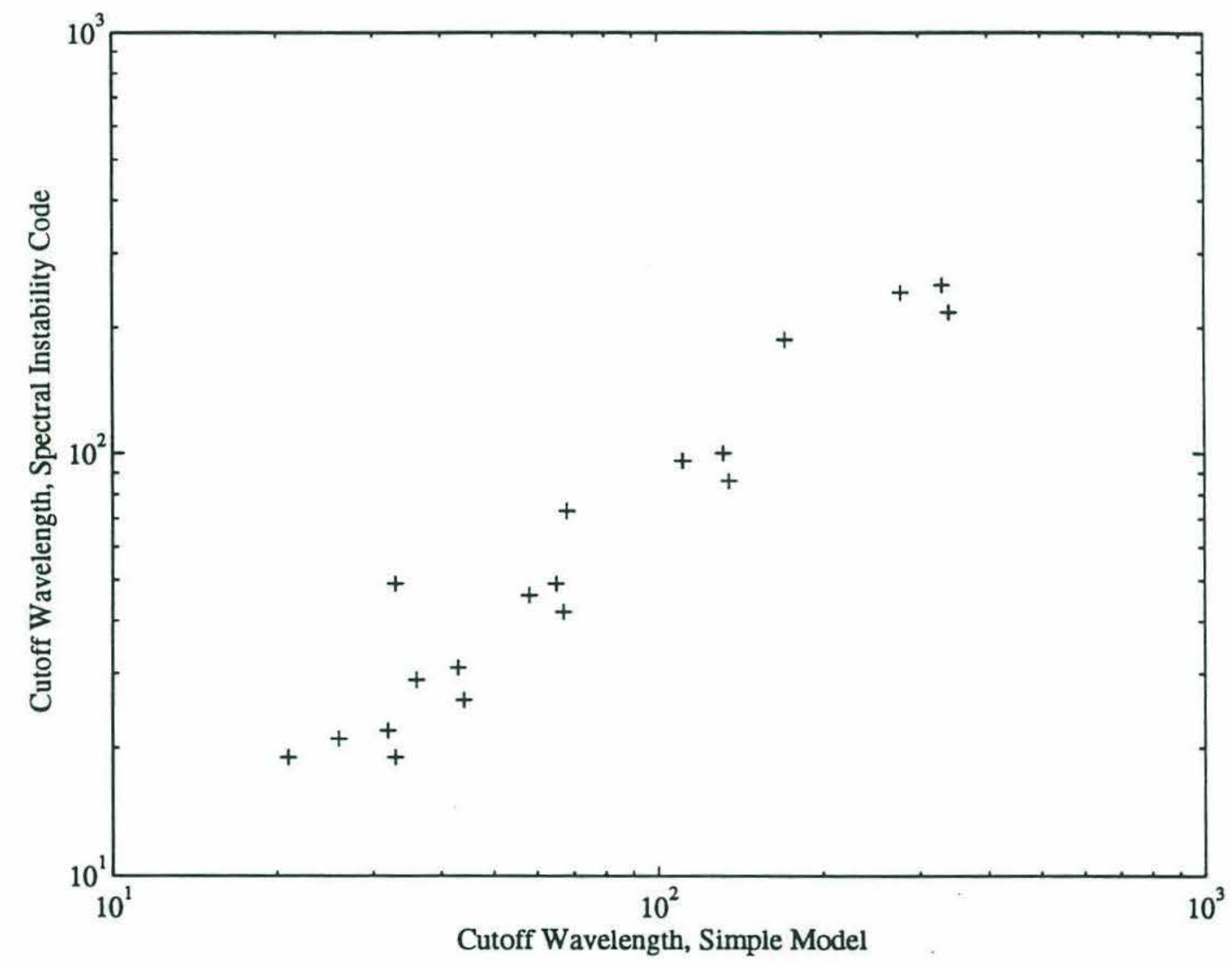

Figure 3.15: Predicting the low-wavenumber cutoff. Horizontal axis is $\log _{10}$ predicted cutoff wavelength from equation (3-37), vertical axis is $\log 10$ cutoff wavelength from the spectral instability code. 
This simple model may be applied to the case at hand as follows. As $\mathrm{k} \rightarrow 0$, the most unstable mode when the effects of crosscell shears are excluded goes to zero as $\Gamma_{1}=\frac{\mathrm{k}^{2} \gamma_{\mathrm{CL}}{ }^{2}}{\left(\mathrm{k}^{2}+\pi^{2} / \mathrm{D}^{2}\right) \gamma_{\text {diff }}}$ where $\gamma_{\mathrm{CL}}$ is the Craik-Leibovich instability parameter and $\gamma_{\text {diff }}=\mathrm{La}\left(\mathrm{k}^{2}+\pi^{2} / \mathrm{D}^{2}\right)$ (Section 2.4.5). The next most stable mode has vertical structure in the velocity perturbation. It will have a decay rate of order $\gamma_{\text {diff }}$ which is the diffusive time scale for damping out this velocity perturbation when $\mathrm{kD}$ is very small. One may estimate a cutoff wavelength for the cells using the hypothesis that it occurs when $\Gamma_{1}=\Gamma_{2}$. Then if $\mathrm{k}_{\min } \mathrm{D} / \pi_{\ll 1} \mathrm{k}_{\min }$ is given by

$$
\mathrm{k}_{\min }=\frac{\pi \gamma_{\text {diff }}}{\mathrm{D} \gamma_{\mathrm{CL}}}
$$

Figure 3.15 shows the predicted and actual cutoff wavelengths for $\mathrm{La}=\mathrm{F}$ (Ek $=1$ ) as a function of $\mathrm{La}$ for different values of angle of orientatation. When the cells have a low wavenumber cutoff, the simple theory from equation (3-37) does a reasonable job at predicting at least the order of magnitude of the cutoff wavelength. Thissupports the hypothesis that crosscell shear is responsible for causing the cutoff of instability at long wavelengths.

\subsection{Conclusions and Discussion}

This chapter demonstrates that the Ekman layer in the presence of surface gravity waves can be unstable to Langmuir cells with growth rates much larger than the Coriolis frequency. With the exception of some cases at low $\mathrm{La}$ and wavenumber, wave-current interaction is the dominant forcing mechanism. In order for cells to grow, the forcing of the vortices due to wave-current interaction and density (which scales as the stratified Craik-Leibovich instability parameter $\gamma_{\text {CLS }}$ ) must be stronger than diffusion (which scales as $\gamma_{\text {diff }}$ ) and frequency with which the cells are tilted in the crosscell direction $\sigma$. The presence of Coriolis force does not directly affect the instability for Ek of order 1 or larger, but it can affect the instability indirectly by changing the angle between the Stokes drift and Eulerian shears. At low values of $\mathrm{La}$, Ek, and horizontal wavenumber Ekman instability can also play an important role in creating unstable roll vortices.

This chapter also demonstrates that within the framework of Huang's equations irrotational surface gravity waves are predicted to drive an Eulerian return flow whose transport is equal and opposite that associated with the Lagrangian Stokes drift. The vertical structure of this transport depends on the ratio between the inverse wavenumber of the surface gravity waves and the Ekman 
depth. When the wave velocities fall off on spatial scales longer than an Ekman depth, the return flow is equal and opposite to the Stokes drift over most of the water column. When the wave velocities fall off on spatial scales much smaller than an Ekman depth the return flow is smoothed out over an Ekman depth.

These results support the main premise of this thesis, namely that Langmuir cells driven by wave-current interaction play an important role in stirring the mixed layer. By defining the characteristic scales associated with the important physical processes, this chapter provides a framework within which the premise can be tested with field data.

In addition to supporting the overall premise, this chapter provides insight into the dynamics governing the orientation and structure of the cells in the presence of Coriolis force. In Chapter 2, the depth of penetration and growth rate of the cells in the absence of Coriolis forces were shown to be determined by a tradeoff between maximizing the strength of the vortex forcing (maximizing $\gamma_{C L S}$ ), maximizing the efficiency of this forcing (maximizing $\mathrm{D}_{\max } / \mathrm{L}$ ) and minimizing the strength of diffusion $\left(\gamma_{\text {diff }}\right)$. This chapter demonstrates that a similar tradeoff (with one additional constraint) determines the growth rate, structure and orientation of the cells in the presence of Coriolis forces. This constraint is that the crosscell shear (which tilts the cells) be minimized. This tradeoff has the following results:

- As in the absence of Coriolis forces, short-wavelength cells do not penetrate as deeply into the mixed layer as long-wavelength cells.

- For longer-wavelength cells with depths of penetration of the same order as the Ekman depth, the Eulerian shear over the depth of penetration is not oriented in the same direction as the wind and waves. In order to maximize the wave-current forcing and minimize the crosscell shear, the cell axis is oriented in between the waves and shear (to the right of the wind in the northern hemisphere).

- Increasing $\mathrm{F}$ for constant $\mathrm{La}$ and surface stress results in concentrating the shear closer and closer to the surface. If the cell geometry is held constant as F increases, assuming truncation $\mathrm{T} 3$ to be approximately valid

$(3-38) \gamma_{C L 3} \sim \frac{2}{D} \sqrt{\int_{-D}^{0} \sin ^{2}(\pi z / D) e^{2 k w z} d z \int_{-D}^{0} \sin ^{2}(\pi z / D) e^{z / \delta} d z \sim \frac{2 \sqrt{k_{w}^{-1} \delta}}{D}}$ where $\mathrm{k}_{\mathrm{w}}$ is the wavelength of the surface gravity waves and $\delta$ is the Ekman depth. 
Increasing $F$ decreases $\gamma_{C L S}$, both by increasing the angle between Stokes drift shear and Eulerian shear and by trapping the Eulerian shear closer to the surface.

These facts have some interesting implications for cell dynamics. One of the most important concerns the question of whether the surface-intensified forcing associated with wave-current interaction can drive cells which penetrate over the depth of the mixed layer. The scaling of $\gamma_{\mathrm{CL} 3}$ in equation (3-38) shows that it goes as $1 / \mathrm{D}$. The diffusive decay rate, on the other hand, goes as $1 / \mathrm{D}^{2}$. Thus, given a deep enough unstratified mixed layer, the surface forcing will in fact be able to drive cells which penetrate over the depth of the layer.

Crosscell shear, however, may combine with diffusion to limit the depth of cell penetration. This is because the depth to which the cells penetrate is limited by their horizontal spacing, and the presence of crosscell shear may limit this spacing. Thus rotation may effectively limit the depth of penetration of the cells by producing crosscell shears. The degree to which this is true depends on a number of factors, including the effectiveness of Coriolis force in producing Ekman instability, a question which remains to be sorted out in detail for particular cases. The fact that crosscell shear can limit cell growth also has some implications for the effect of initial conditions on cell growth.

The presence of a cutoff in the instability at low wavenumber has implications for the effect of stratification on the depth of cell penetration. In the absence of rotation and associated crosscell shears, the boundary conditions on density result in long-wavelength cells not feeling the effect of density and growing in spite of stratification (Chapter 2). In the presence of rotation, cells have a maximum cell spacing, which allows stratification to set the depth to which cells can penetrate.

Another interesting implication of this work is the light it sheds on what happens to the growth rate and angle of maximum instability when the waves are propagating at an angle $\theta$ to the wind. Such a situation may occur when swell propagation is important, though it is most likely not important during the experiments studied later in this thesis. When Ek is very large, the presence of waves does not change the Eulerian shear profile much and the Eulerian shear is largely downwind over depths at which there is strong Stokes drift shear. As a result increasing $|\theta|$ will cause the growth rate to decrease, with the maximum growth rate occurring at angles in between $\theta$ and 0 but lying closer to the larger of the two shears. 
For Ek 1, however, having waves propagate to the right of the wind produces a decrease in the magnitude of the Eulerian shear, while having the waves propagate to the left of the wind increases the magnitude of the Eulerian shear. For such cases there will be a strong asymmetry in the dependence of the instability on $\theta$, with the water column being more unstable when waves are to the left of the wind than when they are to the right of the wind.

When $\theta=180$, it will be very difficult to find a situation in which there is instability. When Ek is large, the Eulerian shear will largely be set by the wind stress and will be opposite to the Stokes drift shear. When Ek is small the Eulerian velocity below an Ekman depth or so will be essentially equal and opposite to the Stokes drift.

A number of issues raised by this chapter are covered in more detail in the remainder of this thesis. The question of which horizontal scales are chosen at equilibrium is covered in Chapter 4. The question of how the resulting cells grow to finite amplitude, come to equilibrium, and transport momentum and density is considered in Chapter 5. Actual instability calculations for real mixed layers are presented in Chapters 6 and 7 for the SWAPP and MILDEX experiments. The existence of an Eulerian return flow is also considered in these chapters as well as in Chapter 8. 


\section{Chapter 4: The Spatial Scale of Equilibrium Langmuir Circulations}

\subsection{Introduction}

The strength of the forcing of Langmuir circulations due to wave-current interaction and buoyancy, damping due to diffusion, and the ratio between the two depends on the depth to which the cells penetrate. This in turn depends on the horizontal wavelength of the cells. Long-wavelength cells have smaller growth rates than short-wavelength cells but penetrate more deeply into the water column (Chapters 2 and 3). This chapter uses a finite-difference code to examine which horizontal length scales are chosen when the cells grow to equilibrium.

Relatively few papers have considered what determines the horizontal scale of Langmuir cells at equilibrium for realistic oceanic conditions. Leibovich (1977a) showed that the horizontal scale of growing cells in an infinitely deep fluid evolved to larger scales over time, but could not integrate the equations out to a equilibrium. Lele (1985) and $\mathrm{Li}$ and Garrett (1993a) reported cells growing to fill the model domain. None of these papers, however, consider the effects of Coriolis forces or use model domains which are much wider than they are deep.

The finite-difference code looks at the evolution of the cells over time, rather than looking for a fixed-point solution of the equations of motion. This approach has a number of advantages when it comes to making comparisons with field data. First, there are a number of possible equilibrium states, corresponding to differing cell spacing. It is difficult to decide which one will dominate the equilibrium state a priori. Second, any given fixed-point equilibrium state may itself be unstable, so that the final state may be unsteady. Finally, as will be seen, it may take an unrealistically long time to approach the equilibrium state-so that it may never actually be seen in the field.

The approach taken is as follows. Section 4.2 reviews the equations of motion, and introduces the mathematical methods used to solve them. Section 4.3 considers Langmuir cells in the absence of Coriolis forces, and analyzes possible mechanisms for energy transfer at long-wavelengths. Section 4.4 examines scale selection in the presence of Coriolis forces. Section 4.5 concludes this chapter.

The principal results are as follows:

- Finite-amplitude Langmuir cells generally have horizontal spacings larger than that associated with the fastest growing mode for all horizontal wavelengths. 
- In the absence of Coriolis forces, energy is passed to larger and larger horizontal scales (implying deeper and deeper vertical penetration).

-In the presence of Coriolis forces, this evolution is arrested. As a result, the depth of penetration of Langmuir cells is limited.

\subsection{Equations of Motion and Methods of Solution}

Figure 4.1 shows the physical setup of the problem for this chapter. The waves propagate parallel to the wind, and the alongcell ( $+y)$ axis is oriented at some angle $\alpha$ to the right of the wind and waves. Then if the $+x$ axis is across the cells and the $+\mathrm{z}$ axis is in the vertical, the velocities $\mathrm{u}, \mathrm{v}$, and $\mathrm{w}$ are defined as being the velocities in the $\mathrm{x}, \mathrm{y}$, and $\mathrm{z}$ directions and the vorticity $\Omega$ is defined as being the vorticity in the $+\mathrm{y}$ direction the equations of motion are:

$$
\frac{\partial \Omega}{\partial t}+\left(u+u_{s}\right) \frac{\partial \Omega}{\partial x}+w \frac{\partial \Omega}{\partial z}=-\frac{\partial p}{\partial y}+F \frac{\partial}{\partial z}\left(v+v_{s}\right)+\frac{\partial v_{s} \partial v}{\partial z} \partial x+R i \frac{\partial \rho}{\partial x}+L a \nabla^{2} \Omega
$$

$$
\frac{\partial v}{\partial t}+\left(u+u_{s}\right) \frac{\partial v}{\partial x}+w \frac{\partial v}{\partial z}=-F\left(u+u_{s}\right)+L a \nabla^{2} v
$$

$$
\frac{\partial \rho}{\partial t}+\left(u+u_{s}\right) \frac{\partial \rho}{\partial x}+w \frac{\partial \rho}{\partial z}=L a \nabla^{2} \rho
$$

$$
\Omega=\nabla^{2} \psi
$$

$$
\frac{\partial \psi}{\partial \mathrm{x}}=-\mathrm{w} \quad \frac{\partial \psi}{\partial \mathrm{z}}=\mathrm{u}
$$

$$
\mathrm{La}=\frac{\mathrm{ve}_{\mathrm{e}}}{\mathrm{a}^{2} \sigma} \quad \mathrm{F}=\frac{\mathrm{f}}{\mathrm{k}_{\mathrm{w}}^{2} \mathrm{a}^{2} \sigma} \quad \mathrm{Ri}=\frac{\mathrm{N}^{2}}{\mathrm{k}_{\mathrm{w}}^{4} \mathrm{a}^{4} \sigma^{2}}
$$

$$
\mathrm{k}_{\mathrm{w}}^{-1}(\mathrm{x}, \mathrm{y}, \mathrm{z})=(x, y, z)
$$

$$
\begin{gathered}
\left(\mathrm{k}_{\mathrm{w}} \mathrm{a}\right)^{2} \frac{\sigma}{\mathrm{k}_{\mathrm{w}}}\left(\mathrm{u}, \mathrm{u}_{\mathrm{s}}, \mathrm{v}, \mathrm{v}_{\mathrm{s}}, \mathrm{w}\right)=\left(u, u_{s}, v, v_{s}, w\right) \\
\frac{1}{\mathrm{k}_{\mathrm{w}}^{2} \mathrm{a}^{2} \sigma} \mathrm{t}=t
\end{gathered}
$$

In these equations, $\mathrm{k}_{\mathrm{w}}, \mathrm{a}$, and $\sigma$ are the wavenumber, amplitude and frequency of the driving waves, $v_{\mathrm{e}}$ the eddy viscosity, $\mathrm{N}$ the buoyancy frequency, $\mathrm{f}$ the Coriolis parameter, and $\mathrm{u}_{\mathrm{S}}$ and $\mathrm{v}_{\mathrm{S}}$ the Stokes Drift. La represents the Langmuir number, which is a scaled eddy viscosity or inverse Reynolds number. $\mathrm{Ri}$ is the square of 
the scaled buoyancy frequency and $\mathrm{F}$ is the scaled Coriolis parameter. Equations (4-1a-e) are for dimensionless variables, with equations (4-1g-i) showing how these variables are converted to dimensional (italicized) form. The equations differ from those in Chapter 3 in that the pressure gradient is not necessarily zero.

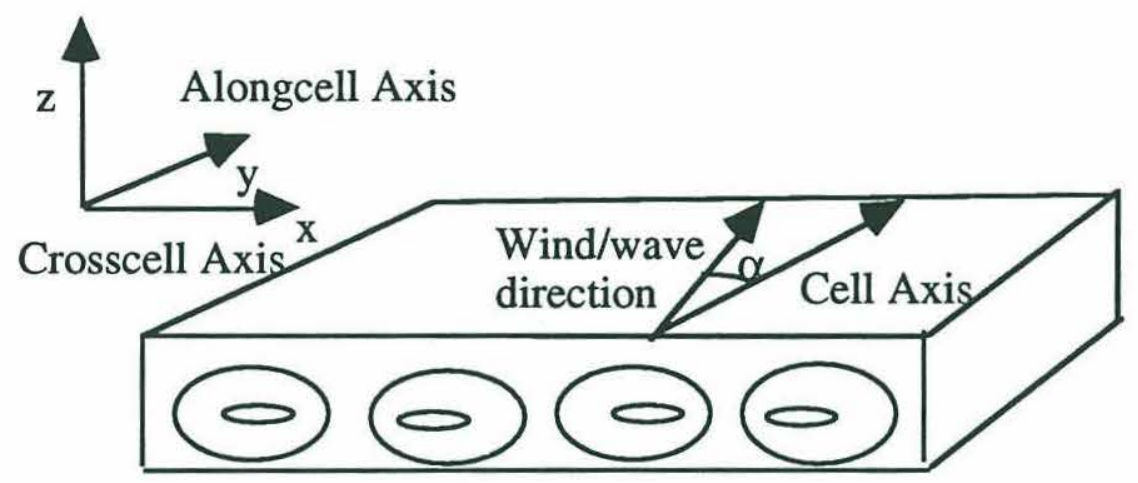

Figure 4.1: Schematic of the problem as solved in this chapter. The waves and wind are collinear and propagate along an axis $\alpha$ degrees to the left of the cell axis.

The boundary conditions on the velocity and density are

$$
\begin{gathered}
\left.\mathrm{La} \frac{\partial \mathrm{v}}{\mathrm{z}}\right|_{\mathrm{z}=0}=\frac{\tau}{\rho} \cos \alpha \\
\left.\mathrm{La} \Omega\right|_{\mathrm{z}=0}=-\frac{\tau}{\rho} \sin \alpha \\
\left.\mathrm{La} \frac{\partial \mathrm{v}}{\partial \mathrm{z}}\right|_{\mathrm{z}=-\mathrm{D}}=\left.\Omega\right|_{\mathrm{z}=-\mathrm{D}}=0 \\
\left.\mathrm{La} \frac{\partial \rho}{\partial \mathrm{z}}\right|_{\mathrm{z}=0}=1 \\
\left.\rho\right|_{\mathrm{z}=-\mathrm{D}}=-\mathrm{D}
\end{gathered}
$$

where $\alpha$ is the angle of orientation of the cells relative to the wind and $D$ is the depth of the fluid layer. The boundary conditions on velocity are just those which have been used throughout the thesis thus far. The boundary conditions on density were discussed in some detail in Chapters 1 and 2. The effect of fixing density on the bottom boundary is to eliminate its effect on the instability at very long wavelengths (of order tens to hundreds of times the layer depth).

Two basic scenarios are considered in this chapter. In the first (identical to that studied in Chapter 2), $F=0, u_{s}=0, \frac{\partial p}{\partial y}=\frac{\tau}{D}$ and $\alpha=0$, so that the waves, wind and cell axis are all parallel, and a pressure gradient balances the wind stress. In the second scenario, $F \neq 0$ and $\frac{\partial p}{\partial y}=0$, so that the wind stress is balanced by the Coriolis force. For the second set of cases, $\alpha$ is chosen by taking the angle of maximum 
instability from the results of the spectral instability code discussed in Chapter 3. When $\alpha \neq 0$ there are crosscell Stokes drifts $\left(u_{s} \neq 0\right)$.

The equations are approached using a finite-difference code. The code is a relatively simple one, based on the methods described by Roache (1977). The time-stepping is simple forward-difference. The Jacobian terms are treated using the method of Arakawa (1966) which conserves finite difference analogues of the first and second moments of momentum, vorticity, and density. Diffusion was handled using simple centered differencing. Some details of the code, plus some runs which were made to verify the physics, are shown in Appendix C.

There are clearly a large number of parameters which may be varied. The investigation in this chapter is limited to scenarios with Langmuir numbers between 0.01 and 0.1 , scaled Coriolis parameters between 0.001 and 0.1 and layer depths of 2-6. $\mathrm{Ri}$ (scaled $\mathrm{N}^{2}$ ) ranges from 0 to 0.5 . All runs presented in this chapter are for Stokes drift profiles corresponding to monochromatic waves. The surface Eulerian shear is set to 1 (other values of surface Eulerian shear were tried but the results did not differ in any substantial way). For purposes of the analysis presented here, this means that the surface stress and Langmuir number are linearly related. As in Chapters 2 and 3 this should not be taken as an assertion about the real ocean.

The initial condition for all the runs was to take an equilibrium current profile in the absence of waves and impose a pattern of jets and a small crosscell flow upon it. Defining $L$ as the domain width, the initial velocity perturbation has the structure

$$
\begin{aligned}
\mathrm{v}= & \mathrm{V}_{0}(\mathrm{z})+\delta\left(\sin (2 \pi \mathrm{x} / \mathrm{L})+\frac{1}{2} \sin (4 \pi \mathrm{x} / \mathrm{L})+\frac{1}{3} \sin (6 \pi \mathrm{x} / \mathrm{L})\right) \\
& +\delta\left(\sin (8 \pi \mathrm{x} / \mathrm{L})+\frac{1}{2} \sin (16 \pi \mathrm{x} / \mathrm{L})+\frac{1}{3} \sin (24 \pi \mathrm{x} / \mathrm{L})\right)
\end{aligned}
$$

where $\delta$ is a small number. When the domain size changes but the predicted structure remains the same, the internal dynamics of the system rather than the initial conditions set the wavenumber of the equilibrium disturbance.

In order to interpret the results of the finite-difference code two simple truncated models of equilibrated Langmuir cells are also considered. The first truncation is a nonlinear version of the depth-limited truncation $\mathrm{T} 1$ considered in Chapter 2. This truncation includes the effects of density stratification, and assumes that Langmuir cells do not alter the momentum balance or the density structure below a certain depth $\mathrm{z}=-\mathrm{D}^{\prime}$. It is denoted as truncation $\mathrm{T} 1 \mathrm{~N}$, and is 
appropriate to use when the cells do not feel the effect of the bottom boundary because of stratification or because diffusion is small. The truncation is presented below:

$$
\begin{aligned}
& \psi=\psi_{11} \sin \left(\pi \mathrm{z} / \mathrm{D}^{\prime}\right) \mathrm{e}^{\mathrm{ik} 1 \mathrm{x}}+\psi_{21} \sin \left(\pi \mathrm{z} / \mathrm{D}^{\prime}\right) \mathrm{e}^{\mathrm{ik} 2 \mathrm{x}} \quad \mathrm{z}>-\mathrm{D}^{\prime} \\
& \mathrm{v}=\mathrm{v}_{01} \cos \left(\pi \mathrm{z} / 2 \mathrm{D}^{\prime}\right)+\mathrm{v}_{02} \cos \left(3 \pi \mathrm{z} / 2 \mathrm{D}^{\prime}\right)+\mathrm{v}_{11} \cos \left(\pi \mathrm{z} / 2 \mathrm{D}^{\prime}\right) \mathrm{e}^{\mathrm{ik} 1 \mathrm{x}}
\end{aligned}
$$

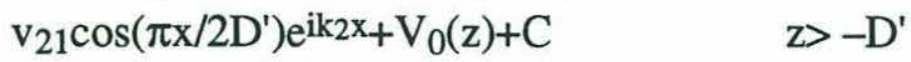

$$
\begin{aligned}
& \rho=\rho_{01} \cos \left(\pi z / 2 D^{\prime}\right)+\rho_{02} \cos \left(3 \pi z / 2 D^{\prime}\right)+\rho_{11} \cos \left(\pi x / 2 D^{\prime}\right) e^{i k_{1} x} \\
& +\rho_{21} \cos \left(\pi x / 2 D^{\prime}\right) e^{i k 2 x}+P_{0}(z) \\
& \mathrm{z}>-\mathrm{D}^{\prime} \\
& \psi=0, v=V_{0}(z)+C, \rho=P_{0}(z) \\
& \mathrm{Z}<-\mathrm{D}^{\prime}
\end{aligned}
$$

where the constant $C$ is determined by the initial momentum of the system. The subscripts refer to the horizontal and vertical wavenumbers of the Fourier modes respectively. Substituting this truncation into the energy balance equations in Chapter 2 yields a set of nonlinear evolution equations for the amplitudes $\psi_{11,21}, \mathrm{v}_{01,02,11,21} 1$ and $\rho_{01,02,11,21}$. If $\mathrm{v}_{21}=\psi_{21}=\rho_{21}=0$, the equilibrium state of this system of equations has a simple analytic solution. Letting $\gamma_{C L 1}\left(D^{\prime}\right)=\sqrt{\hat{V}_{\mathrm{z}_{\mathrm{z} 1}} \hat{\mathrm{V}}_{\mathrm{z} 1}}$, $\gamma_{\text {diff }}=\mathrm{La}\left(\mathrm{k}^{2}+\left(\pi / \mathrm{D}^{\prime}\right)^{2}\right), \mathrm{N}_{1}=(8 / 3 \pi) \sqrt{\mathrm{Ri}}$, where

$$
\hat{\mathrm{V}}_{\mathrm{z} 1} \frac{2}{\mathrm{D}^{\prime}} \int_{-\mathrm{D}^{\prime}}^{0} \frac{\partial \mathrm{V}}{\partial \mathrm{z}} \sin \left(\pi \mathrm{z} / \mathrm{D}^{\prime}\right) \cos \left(\pi \mathrm{z} / 2 \mathrm{D}^{\prime}\right) \mathrm{dz}
$$

\section{0}

$$
\hat{V} s_{\mathrm{z} 1}=\frac{2}{\mathrm{D}^{\prime}} \int_{-\mathrm{D}^{\prime}}^{0} \frac{\partial \mathrm{v}_{\mathrm{s}}}{\partial \mathrm{z}} \sin \left(\pi \mathrm{z} / \mathrm{D}^{\prime}\right) \cos \left(\pi \mathrm{z} / 2 \mathrm{D}^{\prime}\right) \mathrm{dz}
$$

yields the equilibrium solutions

$$
\begin{aligned}
& \psi_{11}=\frac{1}{\mathrm{k}_{1}^{2}+\pi^{2} / \mathrm{D}^{\prime 2}} \sqrt{\gamma_{\mathrm{CL} 1^{2}-\mathrm{N}_{1}^{2}-\gamma_{\mathrm{diff}}{ }^{2}\left(\mathrm{k}_{1}^{2}+\left(\pi / 2 \mathrm{D}^{\prime}\right)^{2}\right) / \mathrm{k}_{1}^{2}}}
\end{aligned}
$$

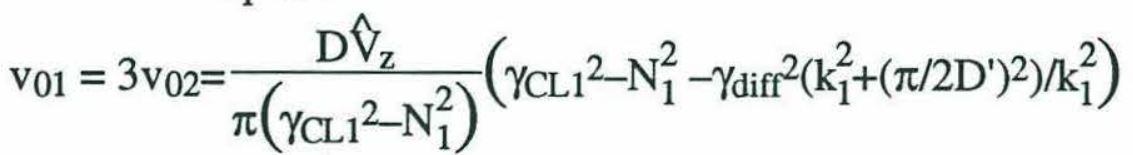

$$
\begin{aligned}
& \mathrm{v}_{11}=\frac{\gamma_{\mathrm{diff}} \hat{\mathrm{V}}_{\mathrm{z}}}{\mathrm{k}_{1}\left(\gamma_{\mathrm{CL} 1^{2}}-\mathrm{N}_{1}^{2}\right)} \sqrt{\gamma_{\mathrm{CL1} 1^{2}-\mathrm{N}_{1}^{2}-\gamma_{\mathrm{diff}}{ }^{2}\left(\mathrm{k}_{1}^{2}+\left(\pi / 2 \mathrm{D}^{\prime}\right)^{2}\right) / \mathrm{k}_{1}^{2}}} \\
& \rho_{01}=3 \rho_{02}=\frac{8 \mathrm{DP}_{\mathrm{Z}}}{3 \pi^{2}\left(\gamma_{\mathrm{CL} 1^{2}}-\mathrm{N}_{1}^{2}\right)}\left(\gamma_{\mathrm{CL} 1^{2}}-\mathrm{N}_{1}^{2}-\gamma_{\mathrm{diff}}{ }^{2}\left(\mathrm{k}_{1}^{2}+\left(\pi / 2 \mathrm{D}^{\prime}\right)^{2}\right) / \mathrm{k}_{1}^{2}\right) \\
& \rho_{11}=\frac{8 \gamma_{\text {diff }} \mathrm{P}_{\mathrm{z}}}{3 \pi \mathrm{k}_{1}\left(\gamma_{\left.\mathrm{CL} 1^{2}-\mathrm{N}_{1}^{2}\right)}\right.} \sqrt{\gamma_{\mathrm{CL} 1^{2}-\mathrm{N}_{1}^{2}-\gamma_{\mathrm{diff}}{ }^{2}\left(\mathrm{k}_{1}^{2}+\left(\pi / 2 \mathrm{D}^{\prime}\right)^{2}\right) / \mathrm{k}_{1}^{2}}}
\end{aligned}
$$


In section 4.3 .2 the stability of this equilibrium state is examined to perturbations with respect to $\psi_{21}$ and $v_{21}$.

The second truncation is one where the cells fill the entire depth of the fluid layer, and is denoted as T2N. It can be used when $\mathrm{La}$ and $\mathrm{L}$ are large. If $\mathrm{D}$ is the depth of the layer.

$$
\begin{gathered}
\psi=\psi_{1} \sin \left(\pi z / D^{\prime}\right) e^{i k_{1} x}+\psi_{2} \sin (\pi z / D) e^{i k 2 x} \\
v=v_{0} \cos (\pi z / D)+v_{1} e^{i k_{1} x_{2}}+v_{2} e^{i k 2 x}+v_{0}(z)
\end{gathered}
$$

where the subscripts now refer only to the horizontal wavenumber of each term (all terms correspond to the gravest vertical wavenumber). Substituting this truncation into the equations of motion yields a set of nonlinear evolution equations for the amplitudes $\psi_{1,2}, \mathrm{v}_{0,1,2}$ When $\mathrm{v}_{2}=\psi_{2}=0$, the equilibrium state of this system of equations has a simple analytic solution. Defining $\gamma_{\mathrm{CL} 2}(\mathrm{D})=\sqrt{\hat{\mathrm{V}}_{\mathrm{z} 2} \hat{\mathrm{V}}_{\mathrm{z} 2}}, \gamma_{\text {diff } 2}=\mathrm{La}\left(\mathrm{k}_{1}^{2}+(\pi / \mathrm{D})^{2}\right)$, where

$$
\hat{\mathrm{V}}_{\mathrm{z} 2}=\frac{2}{\mathrm{D}} \int_{-\mathrm{D}}^{0} \frac{\partial \mathrm{V}}{\partial \mathrm{z}} \sin (\pi \mathrm{z} / \mathrm{D}) \mathrm{dz}
$$

$$
\left.\hat{\mathrm{V}}_{\mathrm{z} 2}=\frac{2}{\mathrm{D}} \int_{-\mathrm{D}} \frac{\partial \mathrm{v}_{\mathrm{s}}}{\partial \mathrm{z}} \sin (\pi \mathrm{z} / \mathrm{D}) \mathrm{dz}\right)
$$

yields equilibrium solutions

$$
\begin{gathered}
\psi_{1}=\frac{2}{\mathrm{k}_{1}^{2}+\pi^{2} / \mathrm{D}^{\prime 2}} \sqrt{\frac{1}{2} \gamma_{\mathrm{CL} 2^{2}-\gamma_{\mathrm{diff} 2^{2}}}} \\
\mathrm{v}_{0}=\frac{\mathrm{D} \hat{\mathrm{V}}_{z}}{\pi}\left(1-\frac{2 \gamma_{\text {diff } 2}^{2}}{\gamma_{\mathrm{CL} 2}^{2}}\right) \\
\mathrm{v}_{1}=\frac{\gamma_{\text {diff } 2}}{\mathrm{k}_{1} \hat{\mathrm{V}_{\mathrm{z} 2}}} \sqrt{\frac{1}{2} \gamma_{\mathrm{CL} 2}{ }^{2}-\gamma_{\text {diff } 2}{ }^{2}}
\end{gathered}
$$

The strength of truncated models is that they can be used to derive closedform solutions from which important physical processes can be isolated. A concommitant weakness is that if the truncated models do not accurately approximate the spatial structure of the cells, they cannot capture the relevant physics which determine cell evolution. One such weakness of these truncations is that they assume that cells with different spacings have the same vertical structure. As seen in Chapters 2 and 3 this is only true when the wavelength is relatively long. A second weakness of these simple truncations is their inability to model 
triad interactions, which are responsible for the flow of energy to large scales in two-dimensional turbulence. As a result, the truncations are only really useful at modelling interactions between long-wavelength cells where (as shown later in the chapter) triad interactions are not as important and the cell structure is relatively constant as a function of cell spacing.

\subsection{Scale Selection in the Absence of Coriolis Force}

\subsubsection{Results from the Finite-Difference Code}

A certain understanding of the evolution of the cell structure can be gained by considering the development of the eddy kinetic energy. Figure 4.2 shows a schematic of the energetic evolution of the cells when the model domain is much wider than the depth. There is an initial phase during which the cells grow strongly, overshoot an equilibrium level, and return to this level. This phase will be referred to as the initial growth phase. The initial growth phase generally lasts approximately 20-100 nondimensional time units. Given that oceanic surface gravity waves have periods ranging from 6-10 seconds and steepnesses of about 0.06 , one nondimensional time period corresponds to a dimensional time of roughly 4 minutes. Dimensionally, then, the initial growth phase corresponds to time periods of 1-5 hours. In lakes, where the waves have shorter periods, the initial growth phase would most likely itself be shorter. The structure of the streamfunction, velocity, and density fields during this phase are expected to be a strong function of the initial conditions. Numerical results during the initial growth phase cannot be interpreted in terms of the mean forcing functions alone. For this reason, the evolution of cell structure is briefly examined in one case below, but is not studied in detail during this chapter.

The equilibrium which is set up after the initial growth phase is not a steady state for all times. It does, however, last for periods of up to 1200 nondimensional time units, corresponding to dimensional times of about 2-4 days. This stage of development is termed the "first quasiequilibrium phase". During this phase, there is an identifiable and relatively constant cell spacing. This stage of cell development is examined in more detail, since it appears to be an appropriate one for comparison with much of the data. Given that forcing functions in the field often change over periods of a few days, an equilibrium state which requires 4-5 days to develop will rarely be seen. Chapter 5 considers the necessary condition 
for the cells to replace small-scale diffusion as the dominant transport mechanism during a given quasiequilibrium stage.

The first quasiequilibrium stage is followed by a time period during which some or all of the cells within the domain cells merge, referred to below as the "first merging phase". There is generally a rise in the level of eddy kinetic energy associated with this merging process. The merging is surprisingly slow. For the runs which have been analysed for this work, the merging took a few days in general, and sometimes even longer. The first merging phase is followed by a second quasi-equilibrium phase, which in turn is followed by more cycles of merging and equilibrium.

An illustration of these concepts can be seen for a scenario with $L a=0.01$, $R i=0$, and $D=2$. The domain width $L$ is 32 . The most unstable mode for this case has a wavelength of 1.85 and a growth rate of 0.437 . Figure 4.3 illustrates the development of this scenario during the initial growth phase. The evolution of the eddy kinetic energy is shown in Figure 4.3a and the development of the perturbation streamfunction during the initial growth phase for times $T=8,15$, and 40 is shown in Figures $4.3 \mathrm{~b}, \mathrm{c}$ and $\mathrm{d}$ respectively. The streamfunction field initially contains several scales of cells. Over time these tilt and merge into larger cells. By $\mathrm{T}=40$ a spacing of $\mathrm{L}=8$ has developed. This spacing persists for quite a long time. Figure 4.4 shows the streamfunction at $\mathrm{T}=320,640$, and 2880 . The last of these corresponds to a dimensional time of 4-9 days. For $\mathrm{T}=320(4.4 \mathrm{~b})$ there are still four pairs of cells in the model domain, although there are hints that some of them are becoming smaller. At $\mathrm{T}=640$ the pair of cells in the rightmost part of the domain has merged. At $\mathrm{T}=2880$ the cells in the middle of the domain have been squeezed out (a day later there is only one pair of cells left in the domain). As a pair of cells is "gobbled up" by the cells around it the location at which the downwelling occurs shifts to a region where there was previously upwelling.

The merging process shown here was observed in a large number of runs. In all cases the merging involved two downwelling zones, with their associated velocity plumes moving closer and closer together. As this happened, the pair of cells in between the downwelling plumes was squeezed into a smaller and smaller space, eventually collapsing as the two plumes coalesced into a single plume.

The first equilibrium stage in unstratified mixed layers is often characterized by a ratio between cell spacing and layer depth of approximately 4:1. Figure 4.5 shows a number of unstratified runs with different parameter settings. 


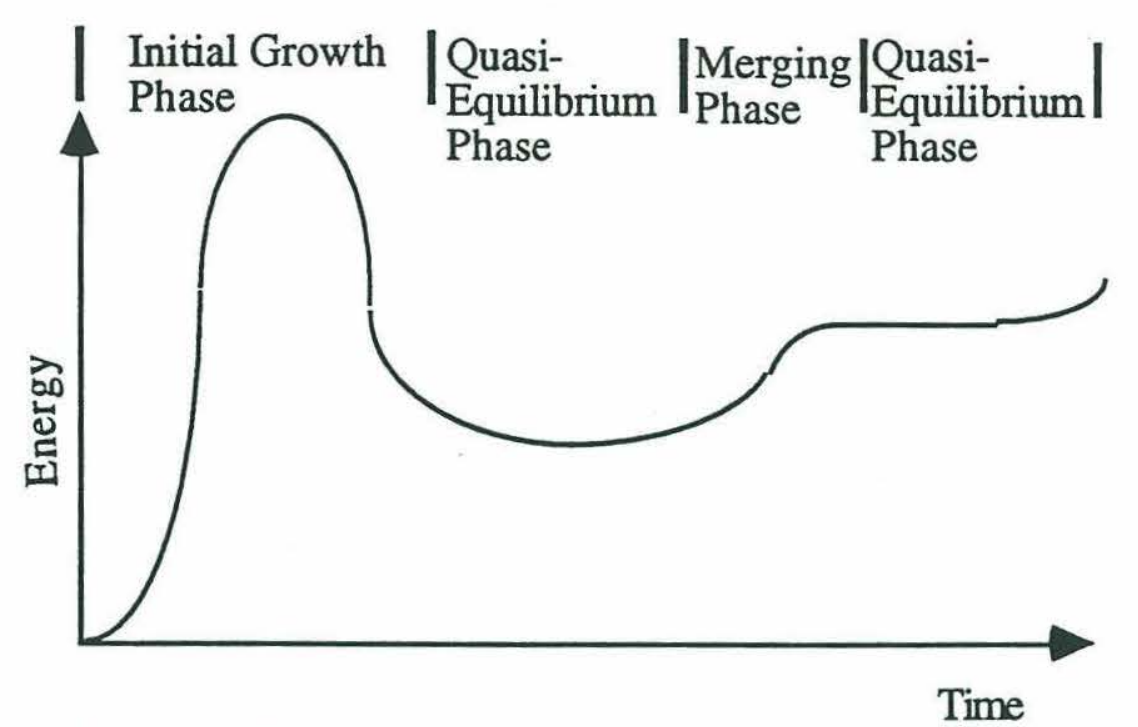

Figure 4.2: Schematic of the evolution of eddy kinetic energy during the development of Langmuir cells, illustrating the various stages of cell evolution.
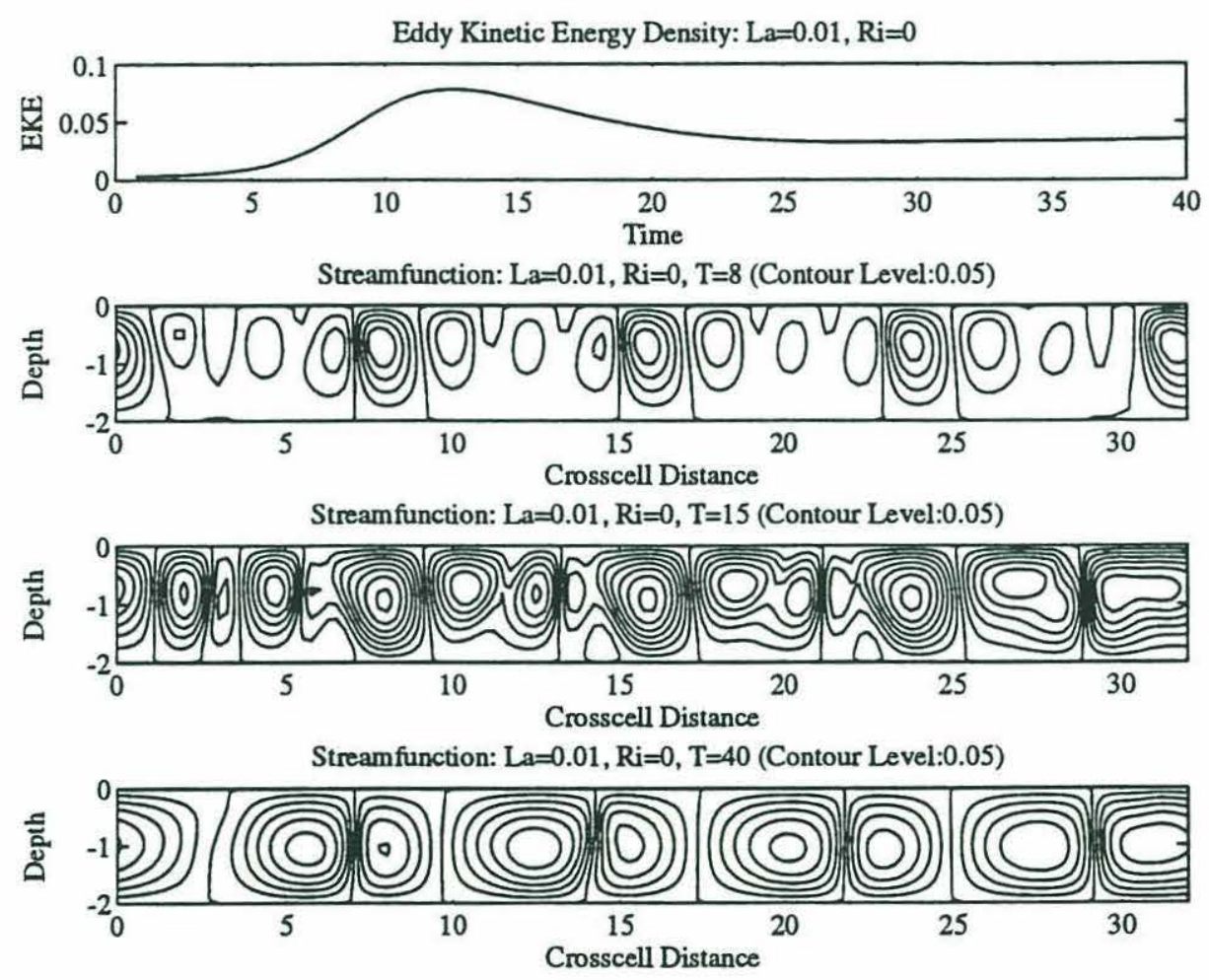

Figure 4.3: Short-time evolution of Langmuir circulations. $\mathrm{La}=0.01, \mathrm{Ri}=0.0$, $\mathrm{D}=2$. (a) Evolution of the eddy kinetic energy. (b) Streamfunction field $\mathrm{T}=8$. Contour level is 0.05 (c) Streamfunction field, $T=15$ (d) Streamfunction field, $\mathrm{T}=40$. 

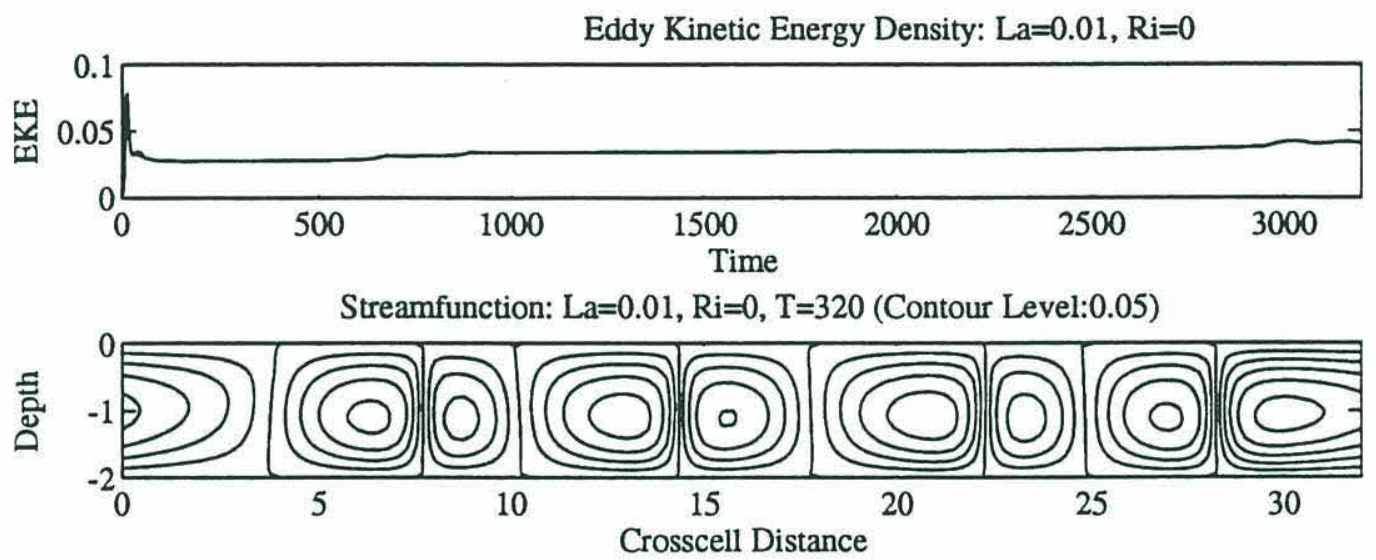

Streamfunction: $\mathrm{La}=0.01, \mathrm{Ri}=0, \mathrm{~T}=640$ (Contour Level:0.05)

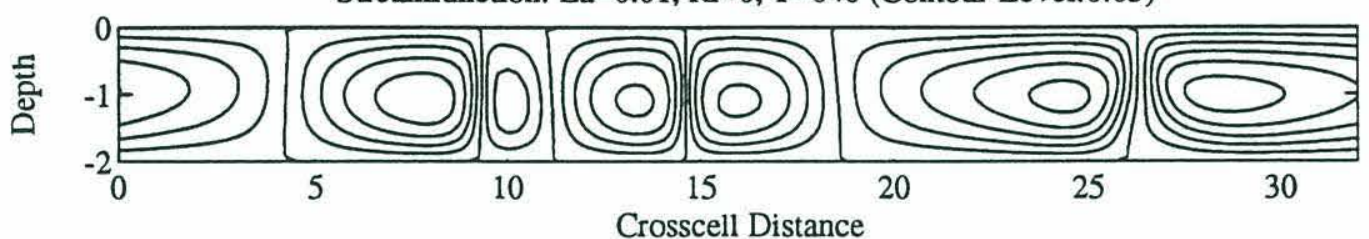

Streamfunction: $\mathrm{La}=0.01, \mathrm{Ri}=0, \mathrm{~T}=2880$ (Contour Level:0.05)

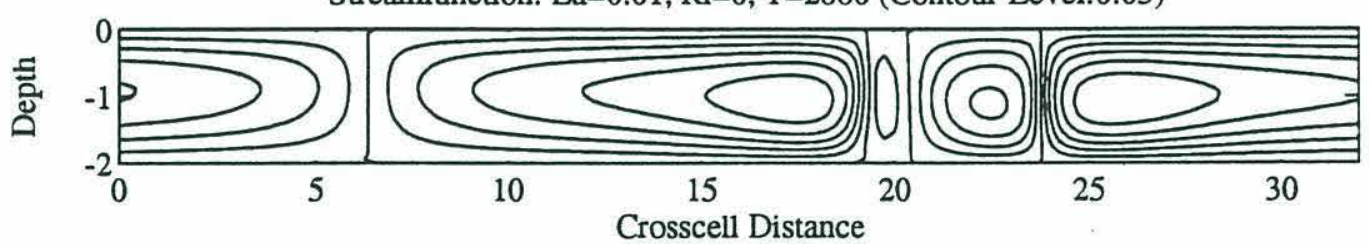

Figure 4.4: Long-time evolution of Langmuir circulations. $\mathrm{La}=.01, \mathrm{Ri}=0.0, \mathrm{D}=2$. (a) Evolution of the eddy kinetic energy. (b) Streamfunction field $\mathrm{T}=320$. Contour level is .05. (c) Streamfunction field, $T=640$. (d) Streamfunction field, $T=2880$. 
Figure 4.5a shows contours of streamfunction for a run where $\mathrm{La}=0.01, \mathrm{Ri}=0$, $\mathrm{D}=2$, and $\mathrm{L}=64$. There are now 8 pairs of cells in the domain. Doubling the domain size does not make a difference in the solution at this time. Figure $4.5 \mathrm{~b}$ shows contours of streamfunction for $\mathrm{La}=0.1, \mathrm{Ri}=0, \mathrm{D}=2$, and $\mathrm{L}=32$ at $\mathrm{T}=400$. Changing the value of $\mathrm{La}$ by a factor of 10 also does not change the crosscell spacing for the quasi-equilbrium state. Figure $4.5 \mathrm{c}$ shows contours of streamfunction for $\mathrm{La}=0.1$, $\mathrm{Ri}=0, \mathrm{D}=4$, and $\mathrm{L}=32$ at $\mathrm{T}=400$. There are now only two pairs of cells in the model domain (so that the ratio between cell spacing and depth is still 4:1). This turns out to be the first quasiequilibrium state for $\mathrm{D}=4$ for $\mathrm{La}=0.01,0.025$, and 0.05 as well. Interestingly, Langmuir cells in the field appear to have a similar ratio between cell spacing and mixed layer depth of 3-4 (Smith et al.,1987).

Stratification does not greatly inhibit the transfer of energy to large scales. Figure 4.6a shows contours of streamfunction for $\mathrm{La}=0.005, \mathrm{Ri}=0.1$ at $\mathrm{T}=400$. Figure $4.6 \mathrm{~b}$ shows the same field at $\mathrm{T}=800$. For $\mathrm{T}=400$, the depth of cell penetration is limited to about half the depth. As time progresses and cells merge the penetration increases. This process takes quite a bit of time, however (again the $\mathrm{T}=400$ is roughly 1 day). When the temporal evolution of this particular run is examined in more detail, it becomes clear that the merging process is still continuing at $\mathrm{T}=800$. The implication is that the long wavelength-cells eventually dominate the flow field, but that this takes a long time to occur. Figure $4.6 \mathrm{c}$ shows the streamfunction for $\mathrm{La}=0.01, \mathrm{Ri}=0.2$ at $\mathrm{T}=800$. Once again, the horizontal spacing is limited over time periods of days, thus limiting the vertical penetration of the cells.

Knowing the cell spacing provides a certain amount of information about the vertical structure of the cells. When there is only one linearly unstable mode at a given wavenumber, the normalized streamfunction perturbation strongly resembles this mode. Figures 4.7 and 4.8 show the standard deviation of the streamfunction as a function of depth, normalized so that the peak value is 1 for a number of scenarios. The + marks denote the model runs, the solid lines show the most unstable mode from the spectral instability code derived in Appendix B. The dashed lines show the second most unstable mode (when there is one) at the cell spacing observed in the finite-difference code. The cases shown in Figure 4.7 correspond to those in Figure 4.5. Figure 4.7a shows a case (corresponding to that in Figure 4.5a) where $\mathrm{La}=0.01, \mathrm{Ri}=0$, at a time $\mathrm{T}=400$. There are two unstable modes, and the streamfunction splits the difference between them. Figure $4.7 \mathrm{~b}$ shows a case (corresponding to Figure $4.5 b$ ) where $\mathrm{La}=0.1, \mathrm{Ri}=0.0, \mathrm{D}=4$. For this 

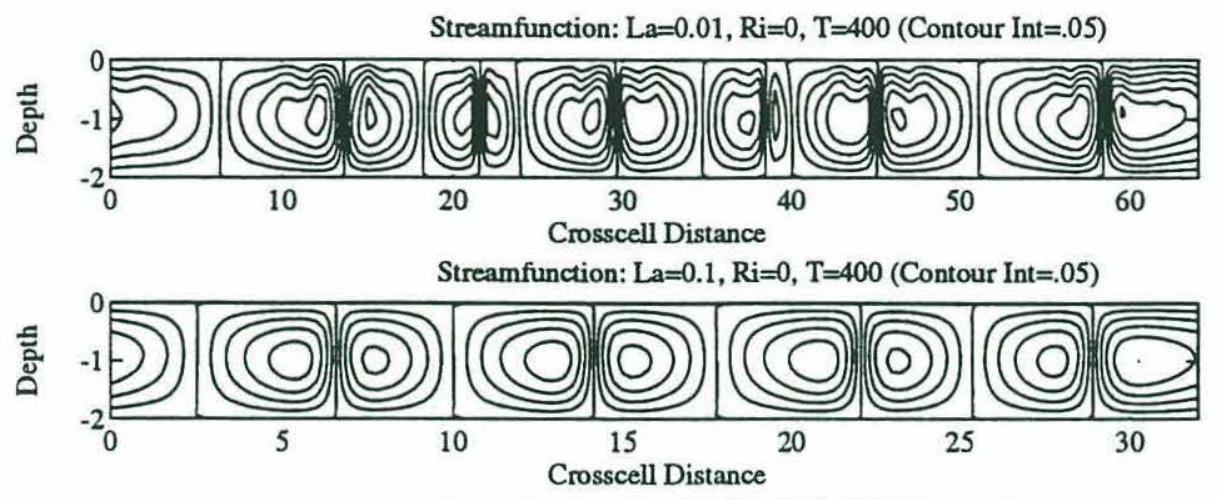

Streamfunction: $\mathrm{La}=0.1, \mathrm{Ri}=0, \mathrm{~T}=400$ (Contour $\mathrm{Int}=.05$ )

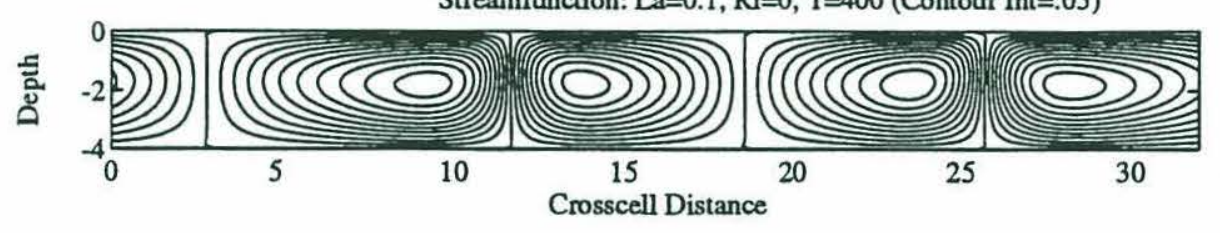

Figure 4.5: Quasiequilibrium states of Langmuir circulations. All plots are contours of streamfunction at time $\mathrm{T}=400$. Contour interval is 0.05 . (a) $\mathrm{La}=0.01$, $\mathrm{Ri}=0.0, \mathrm{D}=2$. Domain size $\mathrm{L}=64$. (b) $\mathrm{La}=0.1, \mathrm{Ri}=0, \mathrm{D}=2, \mathrm{~L}=32$. (c) $\mathrm{La}=0.1, \mathrm{Ri}=0$, $\mathrm{D}=4, \mathrm{~L}=32$.
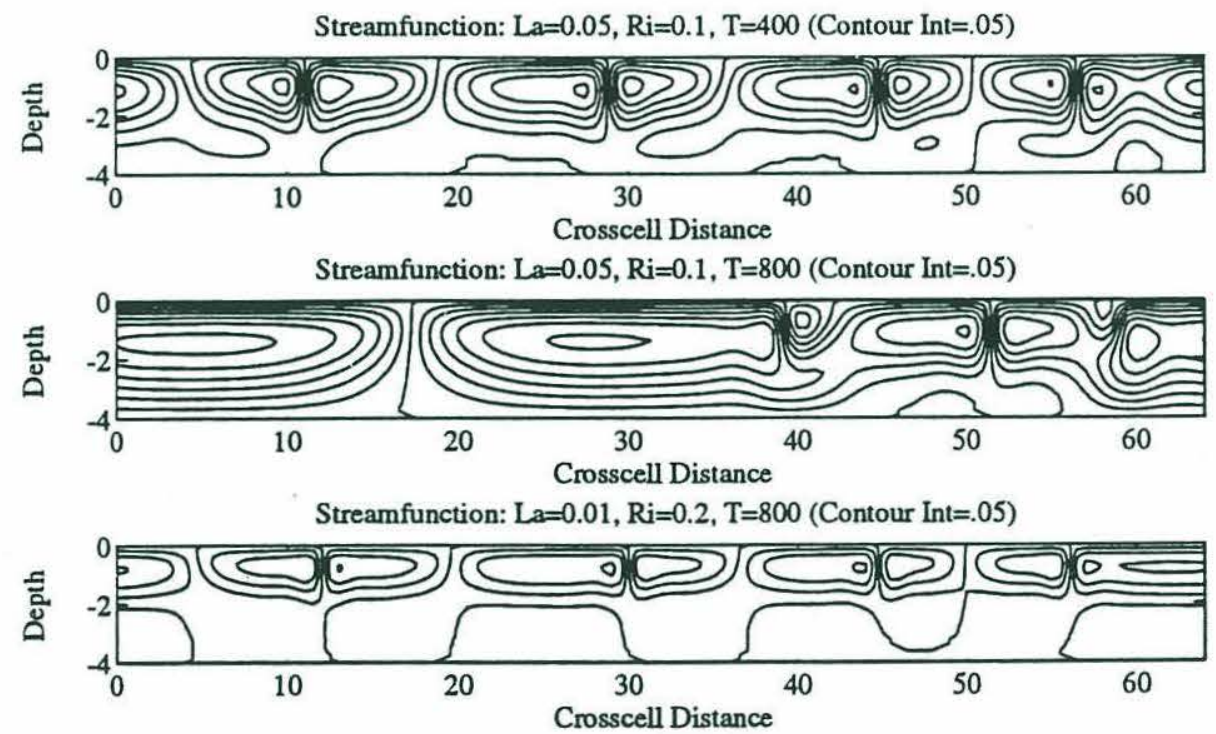

Figure 4.6: Quasiequilibrium states of Langmuir circulations. All plots are contours of streamfunction. Contour interval is $0.05 . \mathrm{D}=4, \mathrm{~L}=64$. (a) $\mathrm{La}=0.05$, $\mathrm{Ri}=0.1, \mathrm{~T}=400$ (b) $\mathrm{La}=0.1, \mathrm{Ri}=0.1, \mathrm{~T}=800$. (c) $\mathrm{La}=0.01, \mathrm{Ri}=0.2, \mathrm{~T}=800$. 


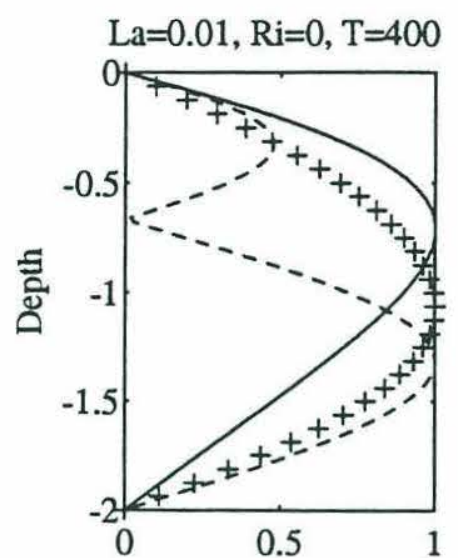

(a)

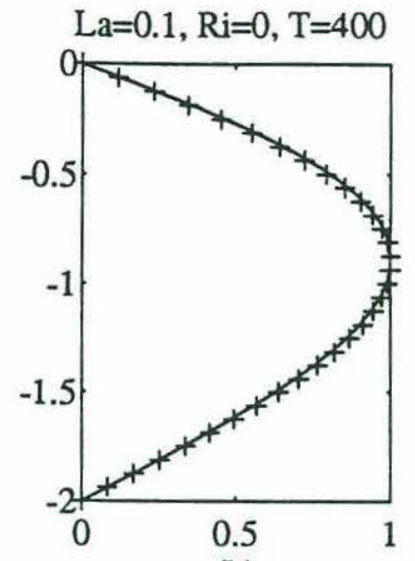

(b)

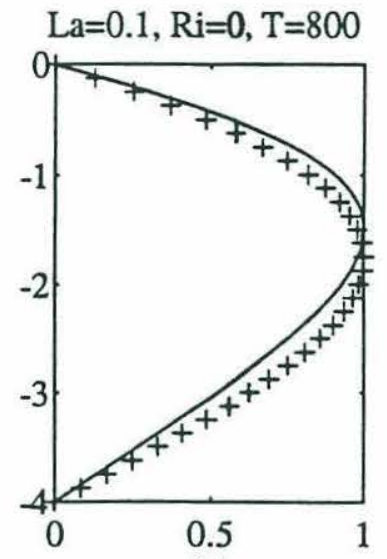

(c)

Figure 4.7: Structure of the streamfunction perturbation compared with the spectral instability code. In all figures, the solid line represents the most linearly unstable mode from code in Appendix B,dashed lines, if present, the 2nd most unstable mode from the code, and + marks the output of the finite-difference code. All fields are normalized to a maximum value of 1 , since the instability code only predicts structure, not amplitude. Cases shown correspond to those in Figure 4.5. (a) $\mathrm{La}=0.01, \mathrm{Ri}=0, \mathrm{D}=2, \mathrm{~T}=400$. (b) $\mathrm{La}=0.1, \mathrm{Ri}=0, \mathrm{D}=2, \mathrm{~T}=400$. (c) $\mathrm{La}=0.1, \mathrm{Ri}=0$, $\mathrm{D}=2, \mathrm{~T}=800$.

case, given the modelled cell spacing of 16 there is only one linearly unstable mode, and the vertical structure of the perturbation streamfunction corresponds closely to this mode. Figure $4.7 \mathrm{c}$ shows the perturbation streamfunction for $\mathrm{T}=800$. The cell spacing is now 32 . Once again, the streamfunction perturbation looks very similar to that associated with the linearly unstable mode.

In the presence of stratification, the instability theory can be even more useful at predicting the cell structure. Figure 4.8 shows the streamfunction perturbation for the three cases in Figure 4.6, $\mathrm{La}=0.05, \mathrm{Ri}=0.1, \mathrm{D}=4$ and $\mathrm{T}=400$ (Figures 4.6a and 4.8a). $\mathrm{La}=0.05, \mathrm{Ri}=0.1, \mathrm{D}=4$, and $\mathrm{T}=800$ (Figures $4.6 \mathrm{~b}$ and $4.8 b$ ), and $\mathrm{La}=0.01, \mathrm{Ri}=0.2, \mathrm{D}=4$ and $\mathrm{T}=800$ (Figures $4.6 \mathrm{c}$ and $4.8 \mathrm{c}$ ). In all three cases the instability code captures the vertical structure of the streamfunction perturbation. The difference between the case shown in Figure 4.8a and that in Figure $4.8 \mathrm{~b}$ is that the cell spacing $\mathrm{L}$ is 8 in Figure $4.8 \mathrm{a}$ and 16 in Figure $4.8 \mathrm{~b}$. The instability code captures the change in the depth of penetration associated with this increase in the cell spacing. The reason for the deeper penetration of cells with larger wavelengths was discussed in Chapter 2. 


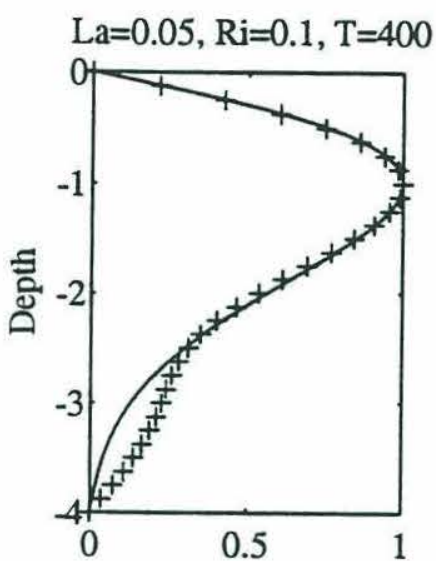

(a)

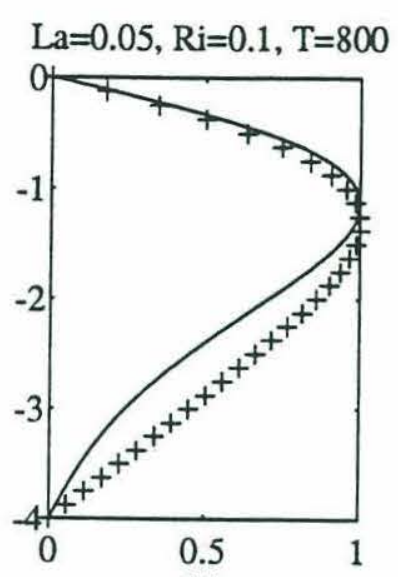

(b)

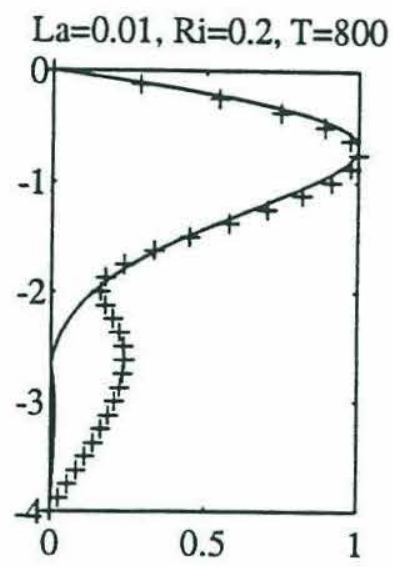

(c)

Figure 4.8: Same as Figure 4.7, but for three cases corresponding to Figure 4.6. All cases have $\mathrm{D}=4$. (a) $\mathrm{La}=0.05, \mathrm{Ri}=0.1, \mathrm{~T}=400$. (b) $\mathrm{La}=0.05, \mathrm{R}=0.1, \mathrm{~T}=800$. (c) $\mathrm{La}=0.01, \mathrm{Ri}=0.2, \mathrm{~T}=800$.

The instability code qualitatively reproduces the vertical structure of the alongcell velocity perturbations as well. Figure 4.9a shows the standard deviation of the alongcell velocity as a function of depth for $\mathrm{La}=0.01, \mathrm{Ri}=0, \mathrm{D}=2$ (shown in Figures 4.2-4.4) at $\mathrm{T}=1500$. The velocity perturbation for this case is basically constant with depth, although there is some variability. The instability code predicts a structure which is also relatively constant with depth, but the details are different. At larger values of $\mathrm{La}$, the structure predicted by finite-difference code is much more similar to that predicted by the instability code. Figure $4.9 \mathrm{~b}$ shows the standard deviation of the alongcell velocity for $\mathrm{La}=0.1, \mathrm{Ri}=0, \mathrm{~T}=400$ (considered in Figure 4.5a and 4.7a). The agreement between linear theory and the finite difference code is much closer. The constancy of the velocity perturbation with depth implies that Truncation $\mathrm{T} 2$ is an appropriate approximation with which to extract the relevant dynamics.

In the presence of stratification, the velocity structure also becomes surfacetrapped. Figure $4.9 \mathrm{c}$ shows the standard deviation of the alongcell velocity as a function of depth for $\mathrm{La}=0.01, \mathrm{Ri}=0.5, \mathrm{~T}=400$. For this case, there are different scales of cells in the model, but the most energetic wavelength is $\mathrm{L}=16$. The velocity perturbation now falls off quite sharply with depth in both the finitedifference code and the linear instability code. It is arguable that the appropriate truncation to use for this case is Truncation T1. 


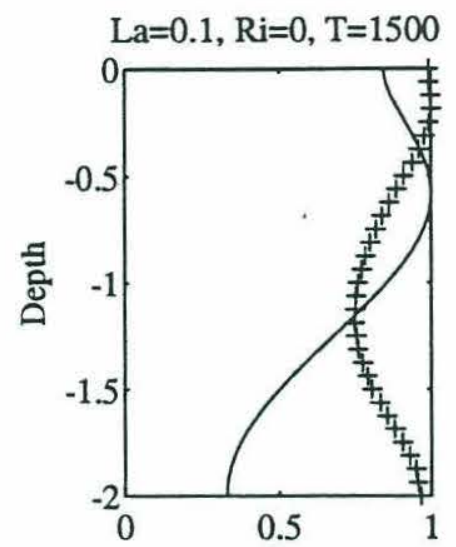

(a)

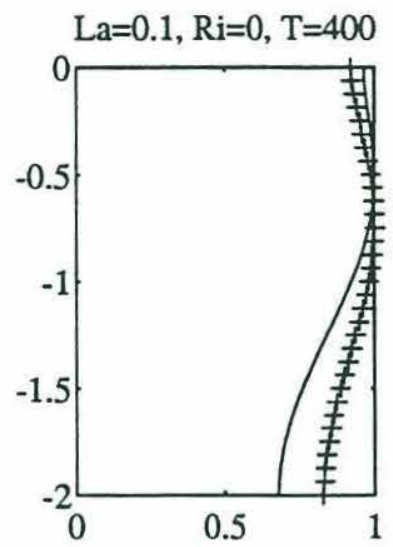

(b)

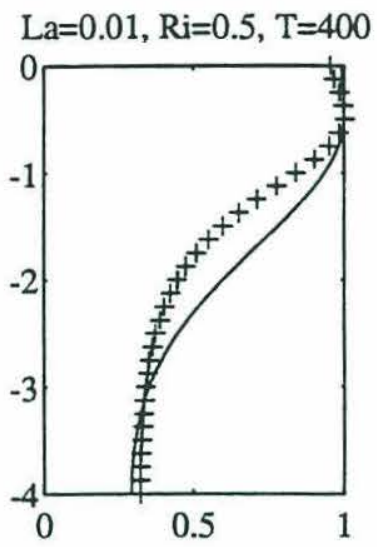

(c)

Figure 4.9: Vertical structure of the alongcell velocity perturbation. Solid lines are from instability code, + marks from finite-difference code. (a) $\mathrm{La}=0.01, \mathrm{Ri}=0, \mathrm{D}=2$, $\mathrm{T}=1500$. (Case shown in Figures 4.3-4.4).(b) $\mathrm{La}=0.1, \mathrm{Ri}=0, \mathrm{D}=2, \mathrm{~T}=400$. (cf.

Figure 4.5b). (c) $\mathrm{La}=0.01, \mathrm{Ri}=0.5, \mathrm{~T}=400$.

This section concludes by examining the merging process in detail for a single run. The current profiles and total eddy kinetic energy for this run do not change substantially after a nondimensional time of about 30 , so that the momentum transport carried by the cells and the energy balance are relatively constant. Figure 4.10 shows the evolution of the amplitude of the Fourier modes of the crosscell and alongcell velocity as a function of horizontal and vertical wavenumber over time for the case studied in the Figures 4.3 and 4.4 where $\mathrm{La}=0.01, \mathrm{Ri}=0$, and $\mathrm{D}=2$. The domain width $\mathrm{L}$ is 32 . Figure 4.10 shows the evolution of streamfunction modes with the form $\psi_{\mathrm{nm}} \exp (2 \mathrm{i} \pi \mathrm{nx} / \mathrm{L}) \sin (\mathrm{m} \pi \mathrm{z} / \mathrm{D})$ and velocity modes with the form $v_{n m} \exp (2 \mathrm{i} \pi \mathrm{nx} / \mathrm{L}) \cos (\mathrm{m} \pi \mathrm{z} / \mathrm{D})$. The four gravest modes in the horizontal ( $n=1,2,3,4$ corresponding to lengths of $32,16,10.67$, and 8 ) are considered, as well as the $n=8$ mode (corresponding to a length of 4 ). In the vertical direction, the two gravest modes (for velocity, $m=0,1$, and for streamfunction $\mathrm{m}=1,2$ ) are considered.

Figure 4.10a shows the energy for the streamfunction modes with one cycle in the vertical and 1,2,3,4 and 8 cycles in the horizontal during the time period shown in Figure 4.4. During the time period shown, energy moves to larger and larger scales in the horizontal. The pattern is one in which the smaller-wavelength mode grows to large amplitude, then decays as the larger wavelength mode grows. The time required for a mode with $\mathrm{L}=8$ ( $\mathrm{n}=4$, marked by + marks) to replace 

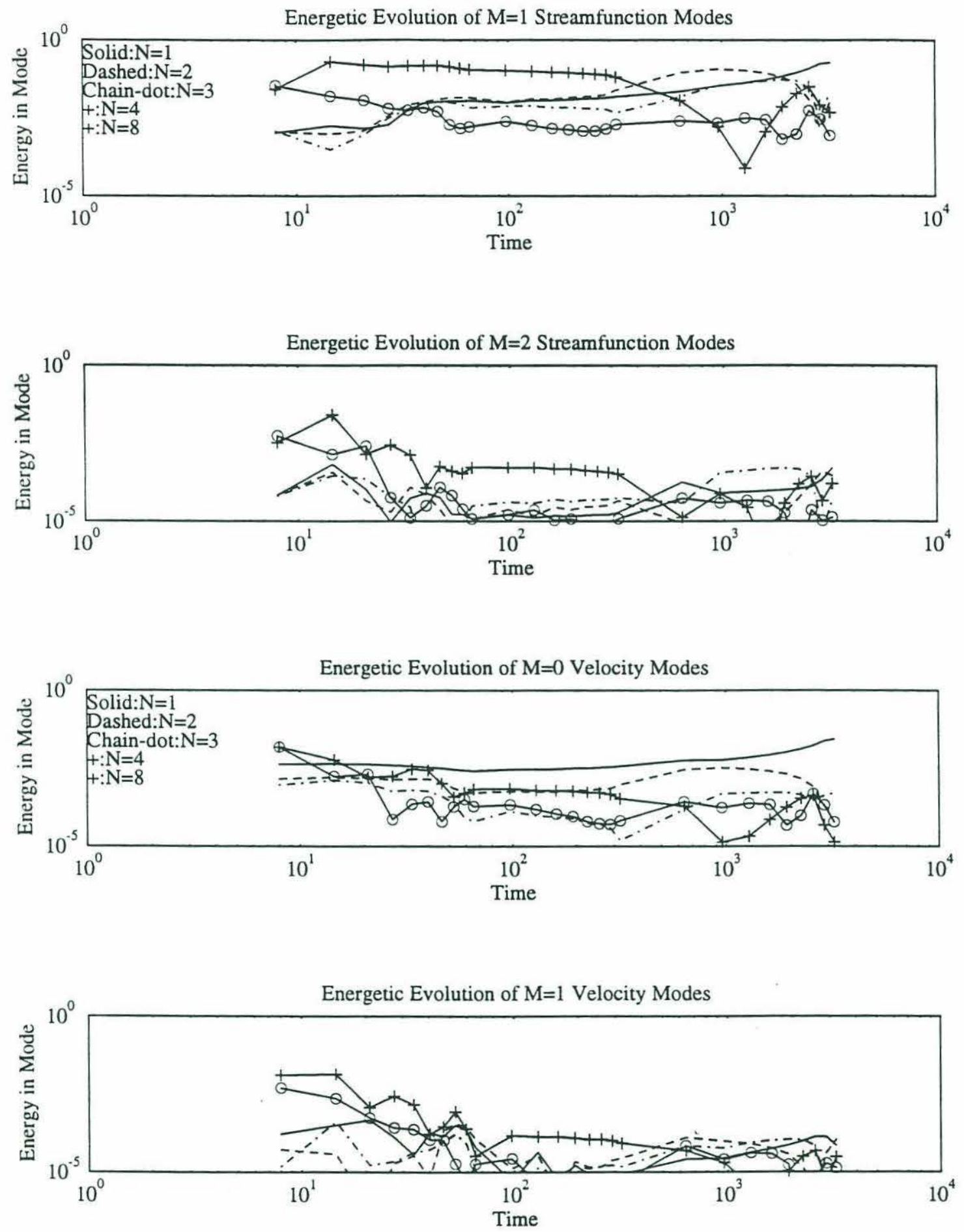

Figure 4.10: Energetic evolution of the Fourier modes for a domain width of 32. $\mathrm{La}=.01, \mathrm{Ri}=1, \mathrm{D}=2$ assuming a surface shear of 1 and monochromatic waves. (a) Streamfunction, $M=1, N=1$ (solid), 2 (dashed),3, (chain-dot) 4 (+), and 8 (o). (b) Same as (a), but for $M=2$. (c) Alongcell velocity, $m=0$. line conventions are same as for (a). (d) Same as (c) but for M=1. 
a mode with $\mathrm{L}=4$ ( $\mathrm{n}=4$, marked with open circles) as the dominant streamfunction mode is very short, roughly 10 nondimensional time units. The time required for the wavelength of the dominant mode to double a second time (to $n=2, L=16$ marked by a dashed line) is much longer, $200-300$ nondimensional time units. The time required for the wavelength to double again (to $n=1, L=32$, marked by a solid line) is even longer, about 1500 nondimensional time units. Interestingly, the final doubling of horizontal scale when the streamfunction field goes from two pairs to one pair of cells in the domain (shown in Figure 4.4) involves energy being pumped into both wavenumbers with 1 and 3 cycles per domain. This is evidence that some sort of nonlinear coupling is involved. Although the scales of the different cells differ by a factor of 4 , the overall level of energy is fairly constant.

Figure $4.10 \mathrm{~b}$ shows the energy in the streamfunction modes with $\mathrm{m}=2$. In general, these modes have much less energy in them than the gravest $(\mathrm{m}=1)$ modes. The exception is right near the start of the run, when the shorter modes dominate (since we know from our linear stability analysis that the most unstable cells at small wavelengths tend to be trapped closer to the surface, it is not surprising that there is more energy in the $\mathrm{m}=2$ mode). Note that as time progresses, the horizontal wavelength which is dominant in the $\mathrm{m}=2$ band does not progress uniformly to lower and lower wavelength. The dominant modes are succesively $n=4, n=8, n=1, n=3, n=4$, and $n=2$. The processes involved in setting the energy level at this wavenumber band are complex and probably nonlinear.

Figure 4.10c shows the vertically gravest mode of the alongcell velocity perturbations $(m=0)$, for $n=1,2,3,4$, and 8 . The velocity perturbation of the $n=1$ mode has a constant amplitude for quite a long time. As the long-wavelength cells take over it starts to increase. Unlike the streamfunction, the strength of the velocity perturbation does have a very strong dependence on wavelength. This is consistent with the equilibrium results in equations 4-6 and 4-9 (a similar result was seen by $\mathrm{Li}$ and Garrett, 1993a). The (relatively small) increases in the eddy kinetic energy seen in Figures 4.3a and 4.4a are due to this increase in jet strength.

Figure 4.10d shows the energy in the alongcell velocity modes with $\mathrm{m}=1$. As is the case for the streamfunction modes, the second-gravest velocity modes are initially strong, then become much weaker than the gravest velocity modes. There is again no steady transfer of energy to large scales with the $n=2$ mode being succeeded by the $n=3$ as the dominant mode, followed by $n=1$ and then $n=2$ at the end. This result suggests that nonlinear interaction is important in moving energy from one scale to another. 
In summary, the results of this section are

-For nonrotating $(\mathrm{F}=0)$ fluid layers with Langmuir cells, the energy associated with the cells evolves to larger and larger horizontal scales.

-Since longer wavelengths penetrate deeper into the water column, the evolution to larger and larger horizontal wavelengths implies a deeper and deeper penetration of the cells over time.

-The evolution to large scales is initially quite fast, but then slows down with the eddy kinetic energy being constant for quasi-equilibrium periods which may be quite long (of order hundreds to thousands of nondimensional time units). This means that the initial quasiequilibrium state may be the one actually seen much of the time in the field, since both wave and wind directions vary over time, and the cells may sometimes be capped off by heat fluxes.

-When there is only one unstable mode at a given wavenumber, the vertical structure of the streamfunction and velocity fields are well described by the linear instability code.

\subsubsection{Some mechanisms for scale selection in the absence of Coriolis forces}

As has already been shown the horizontal scale determines the depth of penetration and the depth of penetration in turn determines a number of important parameters. Knowing the horizontal scale is therefore important for characterizing the cells. In Section 4.3.1 it was demonstrated that the horizontal scale for nonrotating Langmuir cells evolves over time with energy moving to larger and larger scales. This section considers the following questions

-What are the processes which govern this evolution?

-What makes the scale change proceed so slowly after some given point?

The simple truncated models introduced in section 4.2 are used to isolate relevant processes and obtain rough scalings for how fast they should operate. Although the results are inconclusive, they do isolate a candidate mechanism for future work, as well as providing a baseline against which to compare three-dimensional theories of merging. It is argued that cell merging results from a process in which vortices at long wavelength advect smaller-scale velocity perturbations, creating longerwavelength perturbations which then strengthen the long-wavelength vortices through the Craik-Leibovich wave-current interaction.

The failure of long-wavelength modes to dominate the final solution is not the result of the linear growth rates of these modes being very small. Consider, for example, the scenario presented in Figures 4.3 and 4.4 for which $\mathrm{La}=0.01, \mathrm{Ri}=0$, 
and $\mathrm{D}=2$. The initial growth rate $\gamma(8)$ of the most unstable mode for $\mathrm{L}=8$ is 0.205 , while $\gamma(16)$ (the growth rate for $\mathrm{L}=16$ ) is 0.102 . The most unstable mode for any wavenumber has a wavelength of about 1.85 and a growth rate of 0.437 . The failure of the $\mathrm{L}=16$ mode to dominate the flow fields at times of order 400 (more than 40 times $1 / \gamma(16)$ ) is not due to its linear growth rate being very small.

Examination of the actual processes which result in energy transfer between different horizontal scales is necessary to explain the selection of $\mathrm{L}=8$ as the horizontal scale which dominates the first quasiequilibrium state.

Looking at the equations of motion yields at least three possibilities which can explain the transfer of energy from one scale to another. These are: -Interactions between cells of various scales through the advection terms in the vorticity transport equation. Such interactions are known to be responsible for the cascade of energy to large scales in two-dimensional geostrophic turbulence (Salmon, 1980).

-Interactions between cells of different scales through the advection term in the velocity and density transport equations.

-Interactions between finite-amplitude cells of different scales and the mean flow and stratification, in which cells of one scale alter the flow so that it is stable to cells of a smaller scale, but unstable to cells of a larger scale. A similar process has been shown to result in scale changes in baroclinically unstable waves (Pedlosky,1981) and Rayleigh-Benard convection (Fiedler,1989). Each of these processes is considered below. It is argued that the latter two are probably responsible for the merging seen in nonrotating Langmuir cells.

One process which is involved in transferring energy to large scales in twodimensional flows is the two-dimensional enstrophy cascade first discussed by Batchelor (1969). The cascade of energy to large scales is a well-known phenomenon in two-dimensional turbulence and results from interactions between triads of waves These triad interactions require that the wavenumbers of the modes involved be summable to zero in some way. In order to transfer energy from a mode whose streamfunction is given by

$$
\psi=\psi_{2} \sin (2 \mathrm{kx}) \sin (\pi \mathrm{z} / \mathrm{D})
$$

to one which has a form

$$
\psi=\psi_{1} \cos (\mathrm{kx}) \sin (\pi \mathrm{z} / \mathrm{D})
$$

there must be a triad interaction with modes which have a horizontal wavenumber of $k$ or $3 k$ and a vertical wavenumber of $2 \pi / D$. A schematic of this process is 

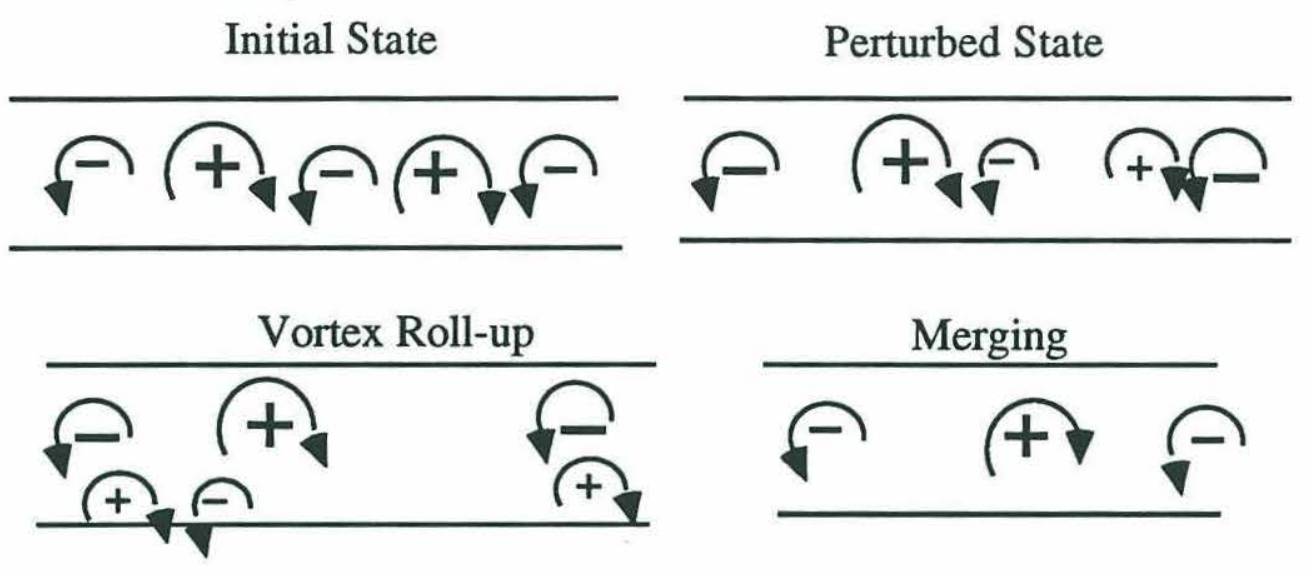

(a)

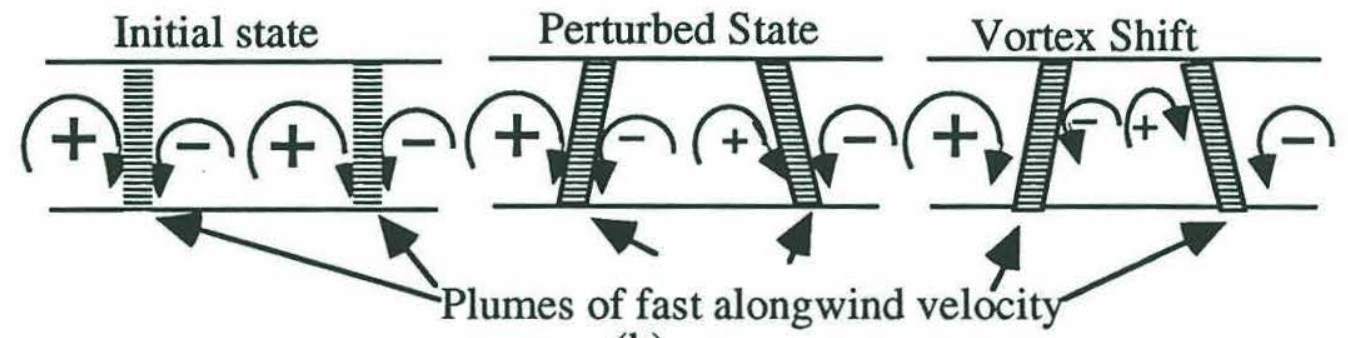

Amplitude

(b)

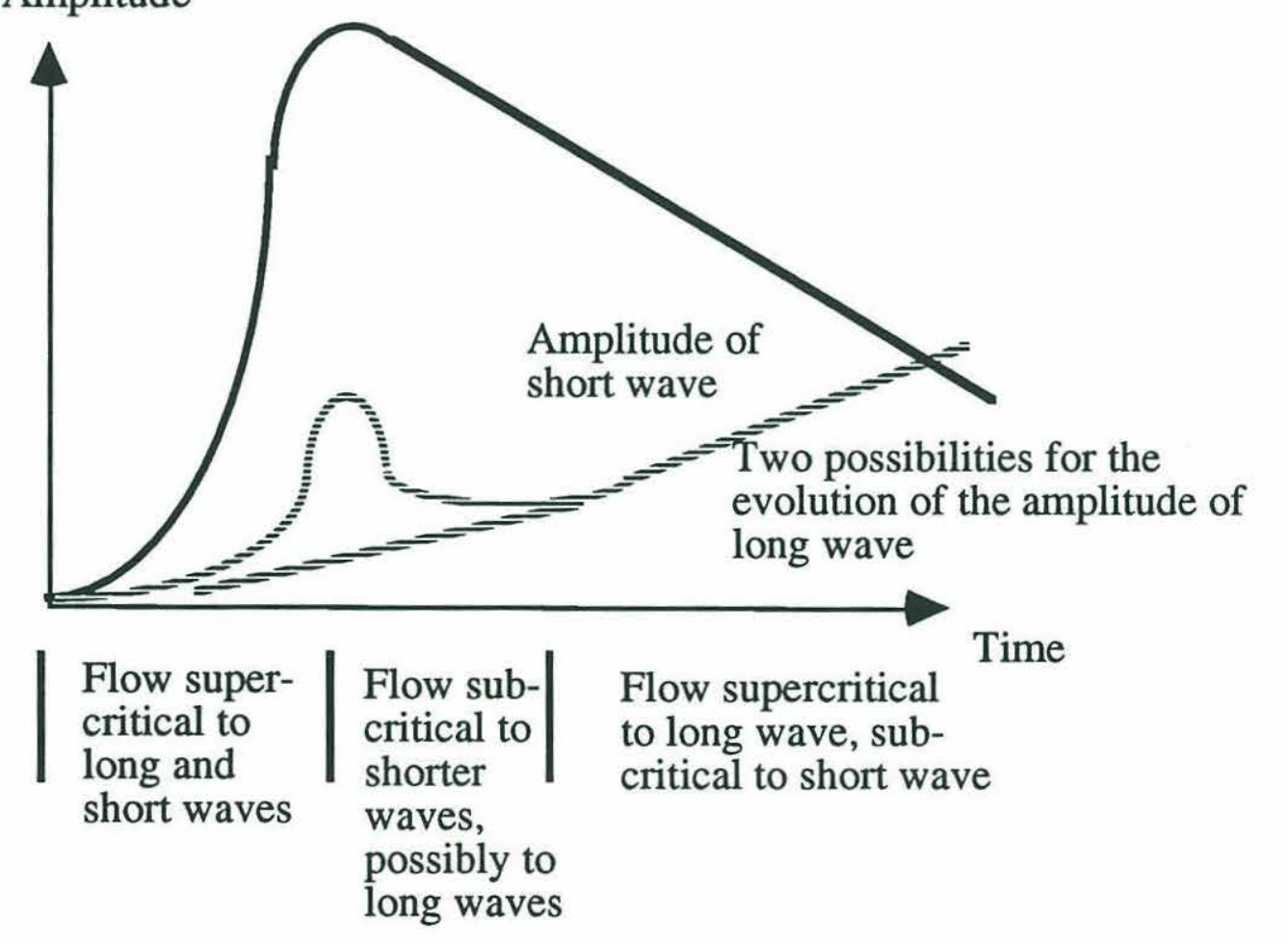

(c)

Figure 4.11: Schematics of three mechanisms for changing the cell spacing.

(a) Vorticity advection. (b) Velocity advection (c) Wave-mean flow interaction. 
shown in Figure 4.11a. Initially, there is a linear array of vortices of opposite signs. A perturbation in vorticity is added so that vortices of opposite sign are moved together, forming a pair such that one vortex becomes weaker and the other becomes stronger. The weaker vortex then rotates around the stronger one as shown. In the presence of viscosity, as the weak vortices are advected towards each other by the strong vortices, they merge, resulting in a line of vortices with twice the spacing as the original one. This process will be referred to as the vorticity advection mechanism for changing the cell scale.

There are some questions about whether or not this process could really apply to Langmuir cells ( $\mathrm{Li}$ and Garrett, 1993a). One serious objection to vorticity advection as an explanation for the change in cell spacing is that the presence of horizontal boundaries acts to stabilize a row of vortices. Rosenhead (1929) showed for an inviscid layer of fluid that when the vortex spacing was more than $1 / \sqrt{2}$ the layer depth, that a row of point vortices of alternating signs was stable to small perturbations of the vortex position. Since the cell spacings under consideration range up to 16 times the layer depth, it is somewhat unlikely that this mechanism is really responsible for Langmuir cell merging.

A second objection to the vorticity advection mechanism being really important is that it does not affect the energy balance of the largest cells. In Figure

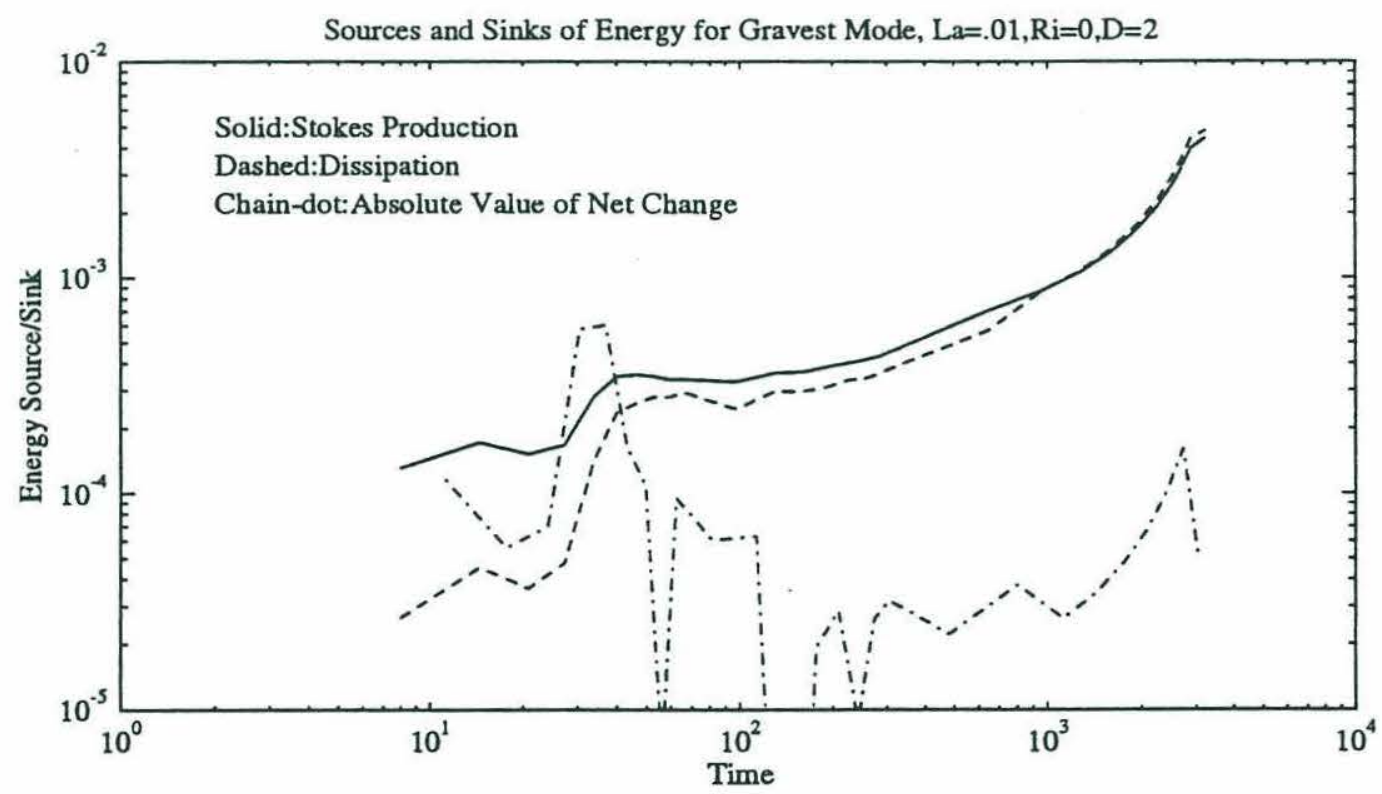

Figure 4.12: Energy balance of a long-wavelength Langmuir cell during merging. Stokes production is the solid line, dissipation the dashed line. The chain-dotted line is the net change in the energy needed to give the observed change in Figure 4.10a. 
4.10, the energy in the longest streamfunction mode went from about 0.05 to 0.2 in about 1000 nondimensional time units, implying a rate of increase of about $1.5 \mathrm{x}$ $10^{-4}$. Figure 4.12 shows the dissipation (dashed) and Stokes production (solid) for this mode during the time period $\mathrm{T}=320$ to $\mathrm{T}=3200$. The dissipation and Stokes production are much larger than the total rate of energy gain and essentially balance. This means that the advection of vorticity (the only remaining energy source term) is not a significant source of energy for this long wavelength-mode. If it were, the observed growth rate would be much larger than $1.5 \times 10^{-4}$.

A second mechanism which can change the spatial scale of Langmuir cells involves the velocity and density transport equations. Given a velocity perturbation of the form $v=v_{1} \cos \left(k_{1} x\right)$ and a streamfunction perturbation of the form $\psi^{\prime}=\psi_{1} \sin \left(k_{2} x\right) \sin (\pi z / D)$, then

$$
\mathrm{u} \frac{\partial \mathrm{v}}{\partial \mathrm{x}}+\mathrm{w} \frac{\partial \mathrm{v}}{\partial \mathrm{z}}=\frac{\mathrm{k}_{1} \pi}{2 \mathrm{D}} \psi_{1} \mathrm{v}_{1}\left(\cos \left(\left(\mathrm{k}_{1}+\mathrm{k}_{2}\right) \mathrm{x}\right)+\cos \left(\left(\mathrm{k}_{1}-\mathrm{k}_{2}\right) \mathrm{x}\right)\right) \cos (\pi \mathrm{z} / \mathrm{D})
$$

A schematic of this process is shown in Figure $4.11 \mathrm{~b}$. Initially, there is an array of vortices, shown by the + and - signs, of equal strength. Between each pair of vortices, there is a downwelling plume, with associated strong alongcell velocities. The horizontal shear in the alongcell velocity on each side of the plume results in a region of strong vorticity generation near the top surface as the result of the CraikLeibovich instability term $\frac{\partial v_{s} \partial v}{\partial z \partial x}$. Suppose that a perturbation in the vortex strength is introduced, as shown, so that the rightmost and leftmost vortices are strengthened while the middle two vortices are weakened. The result is that the plumes of fast-moving alongcell velocity tilt, so that the surface expression of the plume moves towards the weaker of the two vortices. This means that the vortex generation region shifts. As a result the vortex locations shift as well. As the plumes get closer and closer together, viscosity acts to reduce the gradient between them and the intermediate vortices become weaker. This process for changing the cell scale is referred to as the velocity advection mechanism.

Both mechanisms advanced thus far suppose that cells of different scales couple to each other directly through the advection terms in the vorticity, alongcell velocity and density equations. There is also the possibility that cells of different scales can couple to each other through the mean flow and stratification. The basic idea is that a given quasiequilibrium state is associated with a mean profile which is unstable to longer-wavelength disturbances, albeit with growth rates which are much smaller than those for the initial state. A schematic of how this process works is shown in Figure 4.11c. At early times, the mean flow is unstable to both 
short wavelength, quickly growing Langmuir cells and longer wavelength, slowly growing cells. In the first stage, both scales of cells grow, with the quickly growing short wavelength mode predominating. In terms of classical instability theory, the flow is supercritical to both long and short waves during this initial stage. As the small cells reach large amplitude they modify the mean Eulerian flow, until it is no longer supercritical to the smaller wavelengths. This mean flow is still supercritical, however, to the longer wavelength disturbance which continues to grow. Eventually, the flow becomes further modified so that it is supercritical to the long wavelength cells, but subcritical to the short wavelength cells. The cells with long wavelengths then grow while those with smaller wavelengths decay. This process for changing the cell scale is referred to as the wave-mean flow interaction mechanism for changing the cell spacing.

It is possible to model the last two processes using the two simple truncated models of the wave and mean flow.introduced in Section 4.2 The first of these truncations (T1N) is used to focus on the predicted growth rate for a doubling of the dominant wavenumber caused by the velocity advection mechanism. The second truncation (T2N) is used to consider the effectiveness of wave-mean flow interactions in changing the cell spacing.

Consider the nonlinear evolution equations for $\psi_{21}, \mathrm{v}_{21}$, and $\rho_{21}$ in truncation T1N. Letting $\mathrm{k}_{2}=\mathrm{k}$ and $\mathrm{k}_{1}=2 \mathrm{k}$, the equations become

$$
\frac{\partial \psi_{21}}{\partial \mathrm{t}}=\frac{\mathrm{k} \hat{V}_{\mathrm{z} 1}}{\mathrm{k}^{2}+\left(\pi / \mathrm{D}^{\prime}\right)^{2}} \mathrm{v}_{21}+\frac{8 \mathrm{kRi} / 3 \pi}{\mathrm{k}^{2}+\left(\pi / \mathrm{D}^{\prime}\right)^{2}} \rho_{21}-\mathrm{La}\left(\mathrm{k}^{2}+\left(\pi / \mathrm{D}^{\prime}\right)^{2}\right) \psi_{21}
$$

b) $\frac{\partial \mathrm{v}_{21}}{\partial \mathrm{t}}=\mathrm{k} \psi_{2}\left(\hat{\mathrm{v}}_{\mathrm{z} 1^{-}} \frac{\pi}{8 \mathrm{D}^{\prime} \mathrm{v}_{01}}-\frac{3 \pi}{8 \mathrm{D}^{\prime}} \mathrm{v}_{02}+\frac{3 \pi}{4 \mathrm{D}^{\prime}} \mathrm{v}_{1}\right)-\mathrm{La}\left(\mathrm{k}^{2}+\left(\pi / 4 \mathrm{D}^{\prime}\right)^{2}\right) \mathrm{v}_{21}$

(4-13c) $\frac{\partial \rho_{21}}{\partial \mathrm{t}}=\mathrm{k} \psi_{2}\left(\hat{\mathrm{P}}_{0 \mathrm{z}}-\frac{\pi}{8 \mathrm{D}^{\prime}} \rho_{01}-\frac{3 \pi}{8 \mathrm{D}^{\prime}} \rho_{02}+\frac{3 \pi}{4 \mathrm{D}^{\prime}} \rho_{11}\right)-\mathrm{La}\left(\mathrm{k}^{2}+\left(\pi / 4 \mathrm{D}^{\prime}\right)^{2}\right) \rho_{21}$

This system of equations can be solved for the largest linear eigenvalue. Letting as in Chapter $2, \gamma_{\text {diff } 1}=\mathrm{La}\left(\mathrm{k}^{2}+\left(\pi / \mathrm{D}^{\prime}\right)^{2}\right) \sqrt{\mathrm{k}^{2}+\left(\pi / 4 \mathrm{D}^{\prime}\right) 2 / \mathrm{k}}$

$$
\gamma^{\mathrm{T} 1 \mathrm{~N}}=-\mathrm{La}\left(\mathrm{k}^{2}+5 \pi^{2} / 8 \mathrm{D}^{\prime 2}\right)+
$$

$$
\begin{aligned}
& \left(\left(\mathrm{k}^{2}+5 \pi^{2} / 8 \mathrm{D}^{\prime 2}\right)^{2}+\frac{\mathrm{k}^{2}}{\mathrm{k}^{2}+\left(\pi / \mathrm{D}^{\prime}\right)^{2}}\left(\hat{\mathrm{Vs}}_{\mathrm{z} 1}\left(\hat{\mathrm{V}}_{\mathrm{z} 1}-\frac{\pi}{8 \mathrm{D}^{\prime} \mathrm{v}_{01}}-\frac{3 \pi}{8 \mathrm{D}^{\prime}} \mathrm{v}_{02}+\frac{3 \pi}{4 \mathrm{D}^{\prime}} \mathrm{v}_{21}\right)\right.\right. \\
& \left.\left.-\mathrm{Ri}\left(\hat{\mathrm{P}}_{0 \mathrm{z}}-\frac{\pi}{8 \mathrm{D}^{\prime}} \rho_{01}-\frac{3 \pi}{8 \mathrm{D}^{\prime} \rho_{02}}+\frac{3 \pi}{4 \mathrm{D}^{\prime}} \rho_{21}\right)-\gamma_{\text {diff } 1}^{2}\right)\right)^{1 / 2}
\end{aligned}
$$

In Chapter 2, the growth rate in the absence of nonlinearity was shown to be:

$(4-15) \gamma^{\mathrm{T} 1}=-\mathrm{La}\left(\mathrm{k}^{2}+\left(5 \pi / 8 \mathrm{D}^{\prime}\right)^{2}\right)$

$$
\pm \sqrt{\mathrm{La}^{2}\left(\mathrm{k}^{2}+\left(5 \pi / 8 \mathrm{D}^{\prime}\right)^{2}\right)+\frac{\mathrm{k}^{2}}{\mathrm{k}^{2}+\pi^{2} / \mathrm{D}^{\prime 2}}\left(\hat{\mathrm{V}}_{\mathrm{z} 1} \hat{\mathrm{V}}_{\mathrm{z} 1}-\left(\frac{8}{3 \pi}\right)^{2} \mathrm{Ri}_{0 \mathrm{z}}\right)-\gamma_{\text {diff }}^{2}\left(\mathrm{k}^{2}+\left(\pi / 4 \mathrm{D}^{\prime}\right)^{2} / \mathrm{k}^{2}\right)} \text {. }
$$


Long-wavelength Langmuir cells growing on an equilibrium field have a growth rate which resembles (4-15) but with two modifications. The first modification of the growth rate is due to long-wavelength cells advecting the shorter-wavelength velocity and density jets, resulting in production of structure at longer wavelengths. In physical space, this corresponds to the velocity advection mechanism discussed earlier in this section. The second is that the depth-weighted horizontally averaged velocity shear $\hat{\mathrm{V}}_{\mathrm{z} 1}$ and density stratification $\hat{\mathrm{P}}_{0 \mathrm{z}}$ are replaced with $\hat{\mathrm{V}}_{\mathrm{z} 1}-\frac{\pi}{8 \mathrm{D}^{\prime}} \mathrm{v}_{01}-\frac{3 \pi}{8 \mathrm{D}^{\prime}} \mathrm{v}_{02}$ and $\hat{\mathrm{P}}_{0 z}-\frac{\pi}{8 \mathrm{D}^{\prime}} \mathrm{\rho}_{01}-\frac{3 \pi}{8 \mathrm{D}^{\prime}} \mathrm{\rho}_{02}$ respectively. This corresponds to the effect of changes in the mean flow, and is the mathematical signature of the wave-mean flow interaction mechanism. The short-wavelength cells reduce the mean shear and stratification, resulting in a mean profile which is more stable to Langmuir cells. Suppose that the terms, $v_{11}, \rho_{11}, v_{01}, v_{02}, \rho_{01}, \rho_{02}$ are given by their equilibrium values in equation (4-6) Recalling the earlier definitions that $\gamma_{C L 1}=\sqrt{\hat{V}_{s_{z 1}} \hat{\mathrm{V}}_{\mathrm{z} 1}}$ and $N_{1}=\frac{8}{3 \pi} \sqrt{\mathrm{Ri}_{0 \mathrm{P}}}$ one may also define for notational convenvience $\gamma_{\text {diff } 2}=\mathrm{La}\left(4 \mathrm{k}^{2}+\left(\pi / \mathrm{D}^{\prime}\right)^{2}\right), \mathrm{G}_{1}=\frac{\mathrm{k}^{2}+5 \pi^{2} / 8 \mathrm{D}^{\prime 2}}{\mathrm{k}^{2}+\left(\pi / \mathrm{D}^{\prime}\right)^{2}}, \mathrm{G}_{2}=\frac{\mathrm{k}^{2}+\left(\pi / 4 \mathrm{D}^{\prime}\right)^{2}}{\mathrm{k}^{2}}$ and $\mathrm{G}_{3}=\frac{4 \mathrm{k}^{2}+\left(\pi / 4 \mathrm{D}^{\prime}\right)^{2}}{4 \mathrm{k}^{2}}$. Then

$$
\hat{V}_{\mathrm{z} 1}\left(\hat{\mathrm{V}}_{\mathrm{z} 1}-\frac{\pi}{8 \mathrm{D}^{\prime} \mathrm{v}_{00}}-\frac{3 \pi}{8 \mathrm{D}^{\prime}} \mathrm{v}_{01}\right)=\gamma_{\mathrm{CL} 1}^{2} \frac{\mathrm{G}_{3} \gamma_{\mathrm{diff}}^{2}}{\gamma_{\mathrm{CL} 1}^{2}-\mathrm{N}_{1}^{2}}
$$

so that the the growth rate is given by

$$
\operatorname{Ri}\left(\hat{\mathrm{P}}_{0 \mathrm{z}}-\frac{\pi}{8 \mathrm{D}^{\prime}} \rho_{00}-\frac{3 \pi}{8 \mathrm{D}^{\prime}} \rho_{01}\right)=\mathrm{N}_{1}^{2} \frac{\mathrm{G}_{3} \gamma_{\text {diff } 2}^{2}}{\gamma_{\mathrm{CL} 1}^{2}-\mathrm{N}_{1}^{2}}
$$

$$
\begin{aligned}
& \gamma^{\mathrm{T} 1 \mathrm{~F}}=-\mathrm{G}_{1} \gamma_{\text {diff }} \\
& +\sqrt{\left(\mathrm{G}_{1} \gamma_{\mathrm{diff}}\right)^{2}+\frac{\mathrm{k}^{2}}{\mathrm{k}^{2}+\left(\pi / \mathrm{D}^{\prime}\right)^{2}}\left(\mathrm{G}_{3} \gamma_{\mathrm{diff} 2}^{2}+\frac{3 \pi \gamma_{\mathrm{diff}}}{4 \mathrm{kD} D^{\prime}} \sqrt{\gamma_{\mathrm{CL} 1}^{2}-\mathrm{N}_{1}^{2}-\mathrm{G}_{3} \gamma_{\mathrm{dif} 2}^{2}-\mathrm{G}_{2}} \gamma_{\text {diff }}^{2}\right)}
\end{aligned}
$$

When the initial state is highly unstable the stratified Craik-Leibovich instability parameter is much larger than diffusive scales $\sqrt{\gamma_{\mathrm{CL} 1}^{2}-\mathrm{N}_{1}^{2}}$ » $\mathrm{G}_{(1,2,3)} \gamma_{\text {diff. In this }}$ case, becomes very small, and the growth rate asymptotes to

$$
\gamma^{\mathrm{T} 1 \mathrm{~N} \sim} \sqrt{\frac{3 \pi \mathrm{k}}{4 \mathrm{D}^{\prime} \mathrm{k}^{2}+\mathrm{D}^{\prime}\left(2 \pi / \mathrm{D}^{\prime}\right)^{2}} \gamma_{\mathrm{diff} 2} \sqrt{\gamma_{\mathrm{CL} 1}^{2}-\mathrm{N}_{1}^{2}}}
$$

so that the velocity advection mechanism is more important for driving instability than is the mean flow. For $k \ll \pi / D^{\prime}(4-18)$ becomes 


$$
\gamma^{\mathrm{T} 1 \mathrm{~N} \sim} \sqrt{\frac{3 \mathrm{kD}^{\prime}}{4 \pi} \gamma_{\text {diff } 2} \sqrt{\gamma_{\mathrm{CL} 1}^{2}-\mathrm{N}_{1}^{2}}}=\sqrt{\frac{3 \mathrm{kD}^{\prime}}{4 \pi} \gamma_{\text {diff } 2} \gamma_{\mathrm{CLS} 1}}
$$

So that the growth rate, instead of scaling as the Craik-Leibovich instability parameter, scales as the geometric mean of the Craik-Leibovich instability parameter and a characteristic diffusive decay scale. For strongly forced cells, this implies a nonlinear growth rate much slower than the linear growth rate.

Thus, given a nonrotating fluid layer containing finite-amplitude, depthlimited Langmuir cells with spacing $L$ at equilibrium, the initial pattern is unstable to cells with twice the wavelength and the same depth of penetration. The longer wavelength cells grow as a result of a process in which

1. Advection of the shorter wavelength alongcell velocity jets by the longer wavelength cells produces alongcell jets with a longer wavelength

2. These jets act to reinforce the longer wavelength cell via the Craik-Leibovich instability mechanism.

Although truncation T1N may be appropriate for cells which are limited in depth (small L or large $\mathrm{Ri}$ ), it not appropriate for cases where the cells fill the domain and the velocity jets are basically constant from top to bottom (large La, L). Truncation $\mathrm{T} 2 \mathrm{~N}$ is more appropriate to examine these cases. Assume that the initial state is given by equations 4-9. Then the equations for $\psi_{2}$ and $v_{2}$ are

$$
\begin{gathered}
\frac{\partial \psi_{2}}{\partial \mathrm{t}}=\frac{\mathrm{k}_{2} \hat{\mathrm{V}}_{\mathrm{z} 2}}{\mathrm{k}_{2}^{2}+(\pi / \mathrm{D})^{2}} \mathrm{v}_{2}-\mathrm{La}\left(\mathrm{k}_{2}^{2}+(\pi / \mathrm{D})^{2}\right) \psi_{2} \\
\frac{\partial \mathrm{v}_{2}}{\partial \mathrm{t}}=\mathrm{k}_{2} \psi_{2}\left(\hat{\mathrm{V}}_{\mathrm{z} 2}-\frac{\pi}{\mathrm{D}} \mathrm{v}_{0}\right)-\mathrm{La} \mathrm{k}_{2}^{2} \mathrm{v}_{2}
\end{gathered}
$$

As noted earlier in this chapter, the only mechanism for scale change in this set of equations is wave-mean flow interaction. The growth rate of the most unstable mode is then given by

$$
\sqrt{\gamma^{\mathrm{La} 2}\left(\mathrm{k}_{2}^{2}+\frac{\pi^{2}}{2 \mathrm{D}^{2}}\right)^{2}+\frac{\mathrm{k}_{2}^{2}}{\mathrm{k}_{2}^{2}+\pi^{2} / \mathrm{D}^{2}}\left(\hat{\mathrm{V}}_{\mathrm{z} 2}\left(\hat{\mathrm{V}}_{\mathrm{z} 2}-\frac{\pi}{\mathrm{D}^{\mathrm{V}}} \mathrm{v}_{0}\right)-\mathrm{La}^{2}\left(\mathrm{k}_{2}^{2}+\pi^{2}+\mathrm{D}^{2}\right)^{2}\right)}
$$

As in Section 4.2, $\gamma_{\mathrm{CL} 2}=\sqrt{\hat{\hat{V}_{\mathrm{z} 2}} \hat{\mathrm{V}}_{\mathrm{z} 2}}$, and $\gamma_{\mathrm{diff} 2}=\mathrm{La}\left(\mathrm{k}_{1}^{2}+\pi^{2} / \mathrm{D}^{2}\right)$. If $\mathrm{G}_{4}=\frac{\mathrm{k}_{2}^{2}+\pi^{2} / 2 \mathrm{D}^{2}}{\mathrm{k}_{2}^{2}+\pi^{2} / \mathrm{D}^{2}}$, and $\gamma_{\text {diff } 2 \_}=\mathrm{La}\left(\mathrm{k}_{2}^{2}+\pi^{2} / \mathrm{D}^{2}\right)$ then substituting the value of $\mathrm{v}_{0}$ from equation (4-9) yields: 


$$
\gamma^{\mathrm{T} 2 \mathrm{~N}}=-\mathrm{G}_{4} \gamma_{\text {diff } \_2}+\sqrt{\mathrm{G}_{4}^{2} \gamma_{\text {diff } \_2} 2^{2}+\frac{\mathrm{k}_{2}^{2}}{\mathrm{k}_{2}^{2}+\pi^{2} / \mathrm{D}^{2}}\left(\gamma_{\text {diff2 }}{ }^{2}-\gamma_{\text {diff } \_2^{2}}\right)}
$$

This solution predicts that given an initial pattern of equilibrated cells with horizontal wavenumber $\mathrm{k}_{1}$, only cells for which $\gamma_{\text {diff2 }}>\gamma_{\text {diff2_2 }}$ should be unstable. This implies that only cells with a longer wavelength can grow. If $\mathrm{k}_{1}, \mathrm{k}_{2} \ll \pi / \mathrm{D}$, then (4-22) asymptotes to

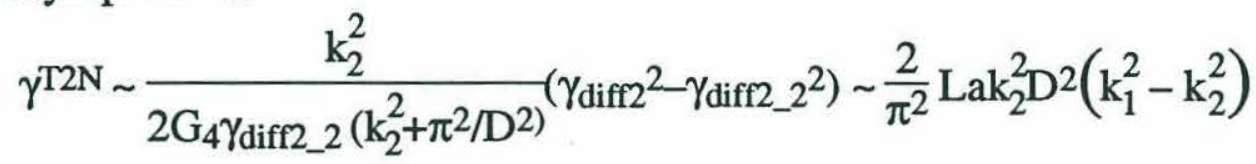

This will be very much smaller than even $\gamma_{\text {diff2 }}$ which is in turn smaller than $\gamma_{C L 2}$. Thus if the forcing is very strong, so that the characteristic diffusive decay is much less than the Craik-Leibovich instability parameter, then wave-mean flow interaction is much weaker than velocity advection in causing scale changes.

Table 4.1 shows linear and nonlinear growth rates predicted by these mechanisms for $\mathrm{La}=0.01, \mathrm{Ri}=0, \mathrm{D}=2$ for cells of wavelength $\mathrm{L}$. The time taken in the finite difference code for cells of length $L=8$ to take over from cells of length 4 was about 10 nondimensional time units, while that for $L=16$ to take over from $\mathrm{L}=8$ was roughly 400 nondimensional time units and that for $\mathrm{L}=32$ to take over was about 2000 nondimensional time units. The growth rates shown are 1) the linear growth rate predicted by the spectral instability code, assuming a fully developed flow without Langmuir cells 2 ) the linear growth rate predicted by truncation $\mathrm{T} 1$ in the absence of cells. 3 ) the linear growth rate predicted by truncation T1N assuming fully developed cells with wavelength 2L. 4) the linear growth rate predicted by truncation $\mathrm{T} 2$ in the absence of cells. 5) the linear growth rate predicted by truncation $\mathrm{T} 2 \mathrm{~N}$ assuming fully developed cells with wavelength 2L. In general:

- The linear growth rates for an initial flow with no cells agree fairly well. - The nonlinear growth rates are smaller than the linear growth rates, and the growth rates do decrease at larger and larger wavelengths. For truncation T1 the growth rates are in general only slightly smaller (10-30\%), whereas for T2 they are much smaller.

- The nonlinear growth rates predicted by truncation T1N (which includes extremely efficient velocity advection) are quite a bit larger than those actually seen, while those predicted by $\mathrm{T} 2 \mathrm{~N}$ (which only allow wave-mean flow interaction) are quite a bit smaller. 


\begin{tabular}{|l|l|l|l|l|l|}
\hline $\mathrm{L}$ & $\gamma(20)$ & $\gamma^{\mathrm{T} 1}$ & $\gamma^{\mathrm{T} 1 \mathrm{~N}}$ & $\gamma^{\mathrm{T} 2}$ & $\gamma^{\mathrm{T} 2 \mathrm{~N}}$ \\
\hline 8 & 0.205 & 0.179 & 0.132 & 0.218 & 0.007 \\
\hline 16 & 0.102 & 0.092 & 0.068 & 0.115 & $5 \times 10^{-4}$ \\
\hline 32 & 0.045 & 0.040 & 0.031 & 0.054 & $4 \times 10^{-5}$ \\
\hline
\end{tabular}

Table 4.1: Growth rates for a mode of length $\mathrm{L}$ assuming $\mathrm{La}=.01, \mathrm{Ri}=0, \mathrm{D}=2$. $\gamma(20)$ :linear growth rate from spectral instability code assuming no cells initially. $\gamma^{\mathrm{T} 1}$ : linear growth rate from truncation T1 assuming no cells initially. $\gamma^{\mathrm{T} 1 \mathrm{~N}}$ : linear growth rate from truncation $\mathrm{T} 1 \mathrm{~N}$ assuming fully developed cells with wavelength 2L. $\gamma^{\mathrm{T} 2}$ : linear growth rate from truncation T2 assuming no cells initially. $\gamma^{\mathrm{T} 2 \mathrm{~N}}$ : linear growth rate from truncation T2N assuming fully developed cells with wavelength $2 \mathrm{~L}$.

Neither truncation presented here exactly captures the physics involved in cell merging. Nonetheless, they both provide important physical insight into the relative strengths of two processes potentially responsible for this phenomenon. Given strongly forced cells, both truncations show that the wave-mean flow interaction will be very weak in producing changes in cell size. Truncation T1 shows that if the structure of the alongcell velocity plumes falls off with depth in the right way, advection of these plumes is very effective in producing cascades of energy to large scales. On the other hand, truncation T2 shows that if the plumes do not have any structure with depth the growth of longer-scale instability is very small. In reality, the structure of the velocity plumes lies somewhere in between their representations in truncations $\mathrm{T} 1$ and $\mathrm{T} 2$, and so the growth rate of a mode of length $\mathrm{L}$ driven by velocity advection is between those predicted by $\mathrm{T} 1$ and $\mathrm{T} 2$.

The results of this section have a number of implications for numerical simulations of Langmuir circulation.

-In the absence of Coriolis forces, the final structure is a function of the domain size. This has worrisome implications for applying the results of a particular model to the real ocean. However, there is the interesting caveat that for large enough domains it takes a very long time to get to the final state and that there are quasiequilibrium states along the way which may be more suitable for comparison with field data. This means that one can run a numerical model of Langmuir cells which is much wider than it is deep and have some reasonable expectation of being able to use the results. Models of Langmuir circulation which simply seek to converge on the final equilibrium state will miss these intermediate quasiequilibrium states which may actually be the states of interest. 
-Fully developed cells with a given wavelength $\mathrm{L}$ and depth of penetration $\mathrm{D}$ ' will slow down the growth of cells at a longer wavelength when

1. The stratified Craik-Leibovich instability parameter for depth $\mathrm{D}^{\prime}$ is much larger than the characteristic decay scale of disturbances of that size ( $\mathrm{Ra}_{\mathrm{CLS}}$ is small).

2. The disturbances at longer wavelength have a similar depth of penetration.

The stratified Craik-Leibovich instability parameter serves as an upper bound for the rate at which vortices can merge. Again, this means that a good understanding of the dependence of the Craik-Leibovich instability parameter and characteristic diffusive decay rate on depth helps to understand the development of larger and larger scales.

-The details of cell evolution have quite a strong dependence on the ratio between the Craik-Leibovich instability parameter and the characteristic diffusive decay scale. When this ratio is large, the flow will be modified to such an extent that only advection of small-scale plumes by larger-scale cells will be able to cause cell merging.

\subsection{Scale Selection in the Presence of Coriolis Force}

\subsubsection{Results from the Finite-Difference Code}

We now turn to the question of Langmuir cells in the presence of Coriolis forces. Chapter 3 demonstrated that the presence of Coriolis forces can strongly modify the instability characteristics of the cells. In particular, the Coriolis force was shown to result in crosswind shears which limited the growth of longwavelength cells. This section demonstrates that the Coriolis force can modify the equilibrium properties as well.

Figure 4.13 shows contours of perturbation streamfunction (the horizontally averaged crosstream flow has been removed) for $T=300,600,1500$, and 2700 for $\mathrm{La}=\mathrm{F}=0.01$. At very long times (up to $\mathrm{T}=2700$, which is more than four pendulum days) the energy containing eddies retain a wavelength of 64/3. There are no hints, as was the case in the nonrotating cases, of cells getting squeezed together as time progresses. The merging process has been arrested by the presence of Coriolis forces.

Although increasing $\mathrm{F}$ from 0 to 0.01 suppresses the transfer of energy to large scales, the exact point at which such transfer stops has a complicated 
dependence on $\mathrm{F}$. This can be seen by increasing the Langmuir number and Coriolis parameter together, so that the initial current profile does not change. Figure 4.14a shows the streamfunction at a time $\mathrm{T}=1500$ for $\mathrm{La}=\mathrm{F}=0.05$. The pattern of streamfunction presented here is quite different qualitiatively from that for $\mathrm{La}=\mathrm{F}=0.01$. There is no single dominant wavelength in this case. Examination of a series of contour plots of streamfunction shows that this is due to the fact that the pair of cells between $x=40$ and $x=50$ is merging. This case takes much longer to come to equilibrium than $\mathrm{La}=\mathrm{F}=0.01$, and the equilibrium state has a longer wavelength.

If $\mathrm{La}$ and $\mathrm{F}$ are increased yet further, the picture changes again. Figure 4.14b shows contours of perturbation streamfunction for $\mathrm{La}=\mathrm{F}=0.1$ at time $\mathrm{T}=1500$, corresponding to doubling the Langmuir number and Coriolis parameter relative to Figure 4.14a. This time the increase in Coriolis parameter and $\mathrm{La}$ seems to have strongly suppressed the cascade of energy to large scales. The dominant wavelength is now 64/7. Examination of the flow field at various earlier times shows that the structure at this time is essentially constant. Even if the mean structure (determined by $\mathrm{La} / \mathrm{F}$ ) is kept constant, there is no simple dependence of cell spacing on $\mathrm{La}$ and $\mathrm{F}$.

If $\mathrm{La}$ is increased, but $\mathrm{F}$ is kept constant the mean structure in the absence of cells changes, and the transfer of energy to large scales is suppressed. Figure $4.14 \mathrm{c}$ and $4.14 \mathrm{~d}$ show contours of perturbation streamfunction for $\mathrm{F}=0.01$, with $\mathrm{La}=0.05$ and 0.1 respectively. As $\mathrm{La}$ increases the field changes from having three pairs of cells in the domain ( $\mathrm{La}=\mathrm{F}=0.01$, Figure 4.13) to four pairs of cells which might be showing signs of slow merging (Figure 4.14c) to four pairs of cells which look very even and show basically no signs of merging (Figure 4.14d). A number of other runs were done for different values of $\mathrm{La}$ and $\mathrm{F}$. The general pattern shown in Figure 4.14 was found in all of these runs. In general, the larger La, the sooner the evolution to large scales broke down.

Such simple dependence was not found for F. Increasing F while keeping La constant was found in some cases to increase the cell spacing, and in others to decrease the cell spacing at equilibrium. The behavior parallels that for the instability, where increasing F made the low wavenumbers more unstable by increasing the effect of Ekman instability, but also reduced the effect of the CraikLeibovich wave-current interaction by increasing the angle between the Stokes 

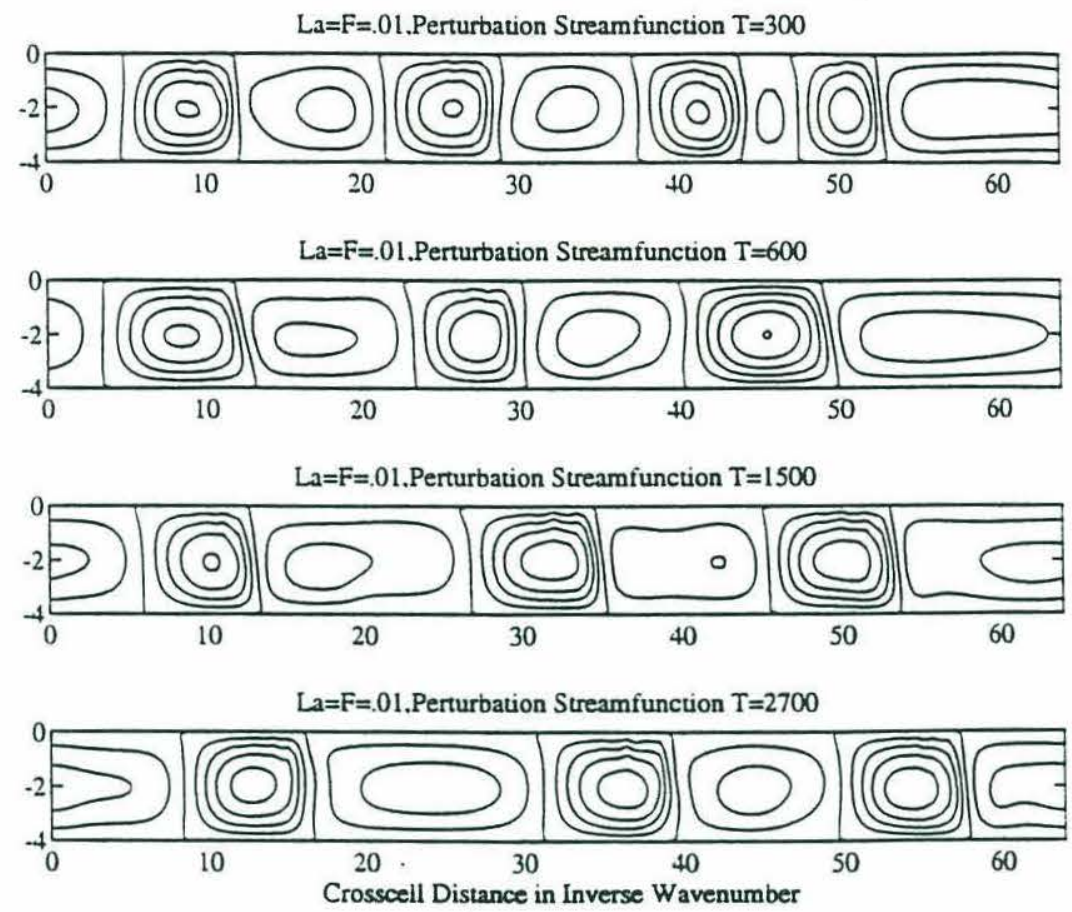

Figure 4.13:Equilibrium states of unstratified, rotating Langmuir circulations. All plots are contours of perturbation streamfunction for $\mathrm{La}=\mathrm{F}=0.01$. Contour interval is 0.1 . (a) $\mathrm{T}=300$ (b) $\mathrm{T}=600$ (c) $\mathrm{T}=1500$ (d) $\mathrm{T}=2700$.
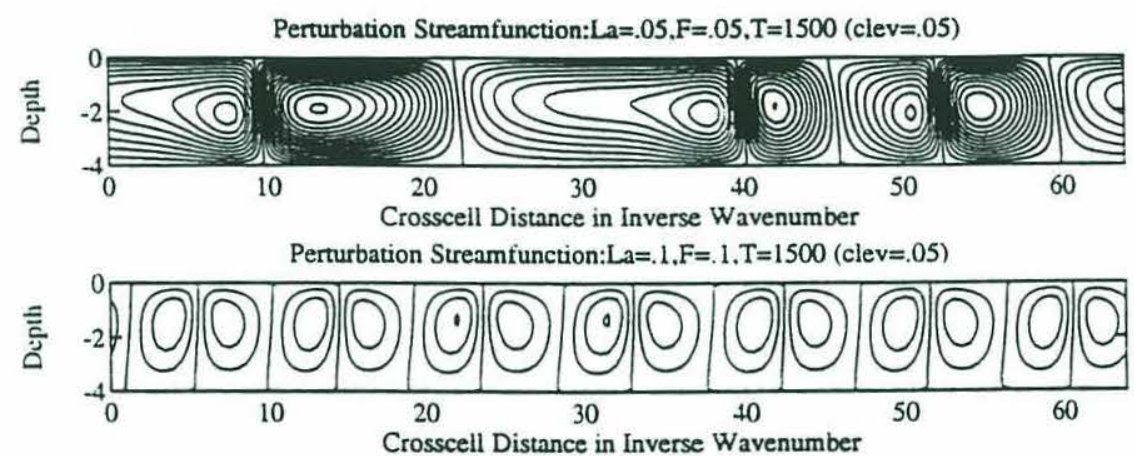

Perturbation Streamtunction: $\mathrm{La}=.05 . \mathrm{F}=.01, \mathrm{~T}=1500$ (clev=.1)
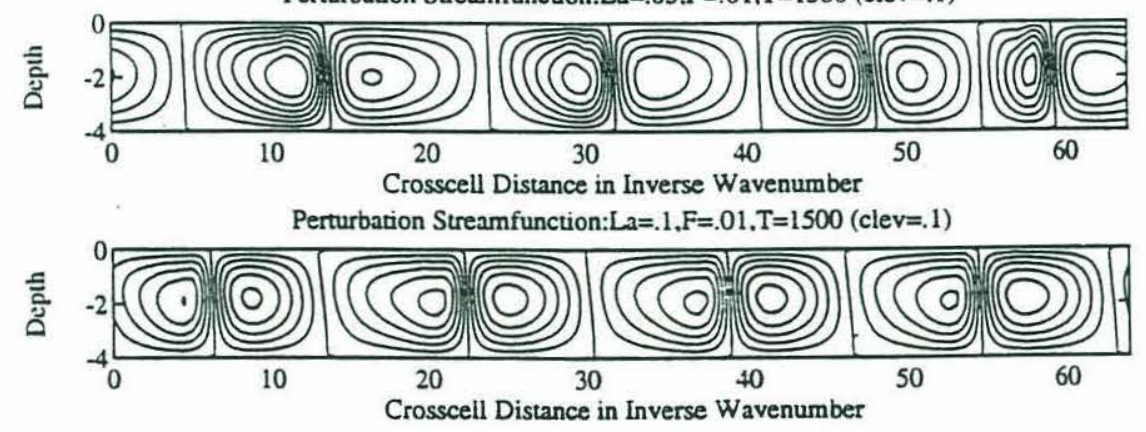

Figure 4.14: Quasiequilibrium states of unstratified, rotating, Langmuir circulations. All plots are contours of perturbation streamfunction. (a) $\mathrm{La}=\mathrm{F}=0.05$, $\mathrm{T}=1500$ (b) $\mathrm{La}=\mathrm{F}=0.1, \mathrm{~T}=1500$. (c) $\mathrm{La}=0.05, \mathrm{~F}=0.01, \mathrm{~T}=1500$ (d) $\mathrm{La}=0.1, \mathrm{~F}=0.01$, $\mathrm{T}=1500$. 


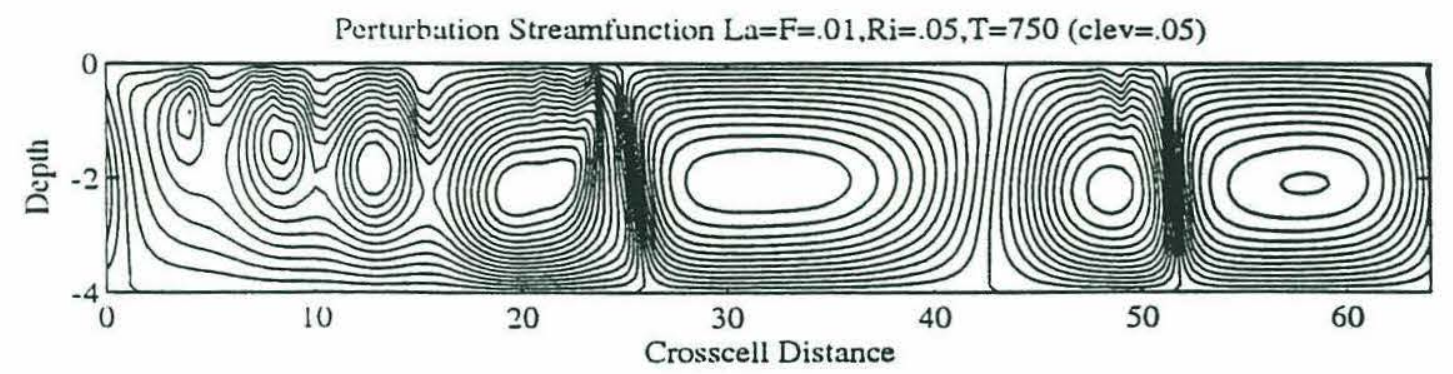

(a)

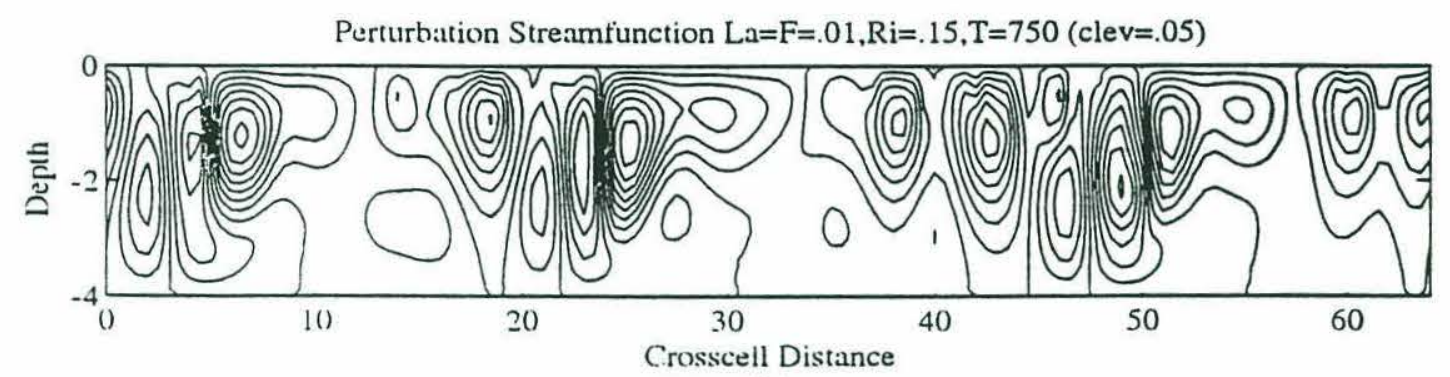

(b)

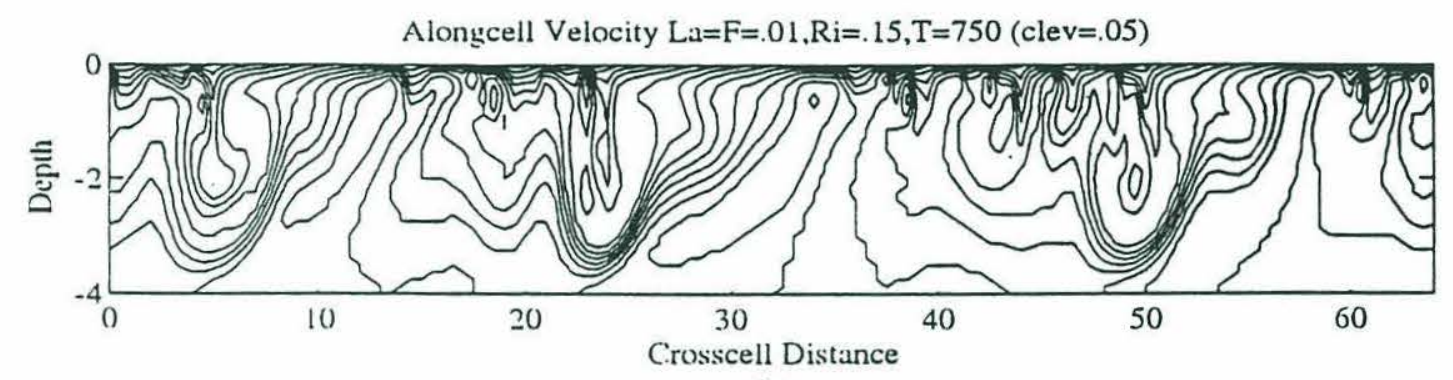

(c)

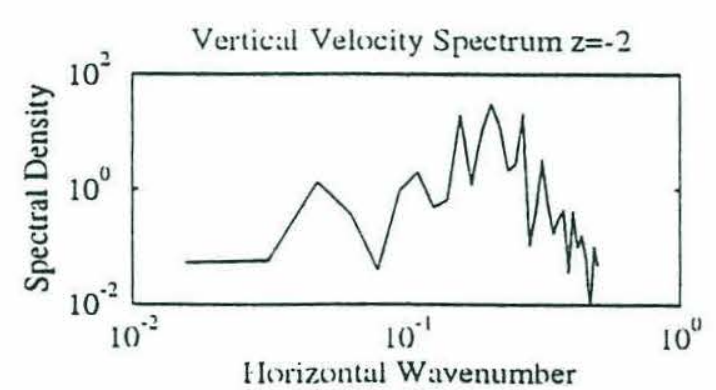

(d)

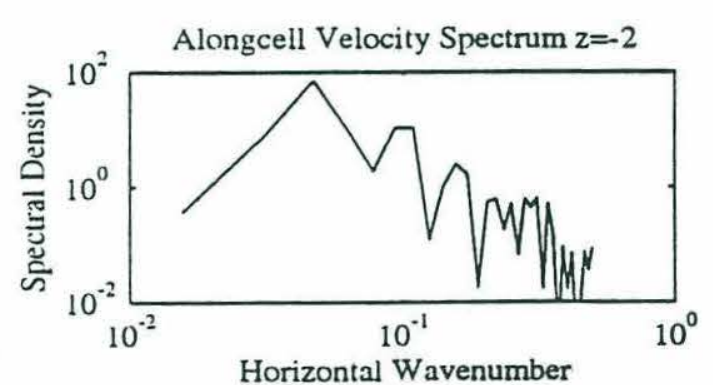

(e)

Figure 4.15: Equilibrium states of stratified, rotating Langmuir circulations. (a) Perturbation streamfunction. $\mathrm{La}=\mathrm{F}=0.01, \mathrm{Ri}=0.05, \mathrm{~T}=750$ (b) Perturbation streamfunction, $\mathrm{La}=\mathrm{F}=0.01, \mathrm{Ri}=0.05, \mathrm{~T}=750$. (c) Alongcell velocity, $\mathrm{La}=\mathrm{F}=0.01$, $\mathrm{Ri}=0.15, \mathrm{~T}=750$. (d) Vertical velocity spectrum, $\mathrm{La}=\mathrm{F}=0.01, \mathrm{Ri}=0.15, \mathrm{~T}=750$, at a depth $\mathrm{z}$ of -2 . (e) Alongcell velocity spectrum, $\mathrm{La}=\mathrm{F}=0.01, \mathrm{Ri}=0.15, \mathrm{~T}=750$ at a depth of -2 . 
drift and the Eulerian shear and created crosscell shears which suppressed the instability.

The presence of stratification complicates matters further. Figure $4.15 \mathrm{a}$ shows contours of streamfunction for $\mathrm{La}=0.01, \mathrm{~F}=0.01, \mathrm{Ri}=0.05$. The stratification in this case is dynamically weak compared with the Craik-Leibovich instability mechanism, but it produces a change in the observed pattern. The regular pattern of Figure 4.13 has been replaced by a pattern in which there are some hints of smaller cells (notice the multiple downwelling zones on the leftmost vortex) riding atop larger cells. Figure 4.15b shows the perturbation streamfunction for $\mathrm{La}=\mathrm{F}=0.01, \mathrm{Ri}=0.15$ at a time $\mathrm{T}=750$. Figure $4.15 \mathrm{c}$ shows the alongcell velocity for the same case. There are now multiple scales of cells. The presence of stratification also limits the transfer of energy to larger scales.

In the presence of multiple scales of cells, the question of which scale dominates a particular field depends on the field chosen. Figure $4.15 \mathrm{~d}$ shows a spectrum of vertical velocity and $4.15 \mathrm{e}$ a spectrum of alongcell velocity vs. wavenumber (in cycles/nondimensional length) at the middle of the layer. The vertical velocity at this depth is dominated by wavenumbers of $0.15-0.3$ corresponding to lengths of 3-6 while the alongcell velocity is dominated by longer wavelengths.

To summarize, in the presence of rotation -The evolution of cell spacing to low wavenumbers is halted. Steady equilibrium states are found for which the merging of cells is suppressed.

-The exact spacing at which the evolution stops is a function of $\mathrm{La}, \mathrm{F}$, and stratification. In general, increasing La seems to cause the dominant wavelength to decrease (as long as we are not in a regime where increasing La causes a highwavenumber cutoff). The exact dependence on $\mathrm{F}$ and stratification is less clear.

\subsubsection{Discussion}

The physical processes which lead to the results of the last subsection are at present unclear. This subsection explores three possibilities which could lead to interrupting the transfer of energy to large scales. Two of these are shown to have potential to interrupt the cascade. The results are speculative, however, as a rigorous theory for explaining the interruption of cell merging by Coriolis forces has not been developed. 
As with the nonrotating case there are a number of possibilities for how the interruption of the cascade to long wavenumbers takes place. Three of these are listed below:

1. Stabilization of the low wavenumber modes. If the low-wavenumber mode has a very small linear growth rate, it may not be able to build up much energy.

2. Breaking of a resonance condition in frequency. In the presence of Coriolis force, the linearly unstable modes have complex eigenvalues, (they are traveling waves). In order for resonant transfer of energy to occur, a triad of such waves must have

$$
\begin{aligned}
& \mathrm{k}_{1}+\mathrm{k}_{2}=\mathrm{k}_{3} \\
& \sigma_{1}+\sigma_{2}=\sigma_{3}
\end{aligned}
$$

where $\mathrm{k}_{1,2,3}$ are the wavenumbers of the linear modes and $\sigma_{1,2,3}$ are the frequencies. Breaking this resonance could result in sharply reducing the efficiency of velocity and vorticity advection in producing changes in the scale of the cells.

3. Breaking a resonance condition in space. Consider a simple model in which we have three modes, each with an associated streamfunction and velocity perturbation field. Suppose further that these fields have the horizontal dependence

$$
\begin{gathered}
\psi_{1,2,3} \sim \sin \left(\mathrm{k}_{1,2,3} \mathrm{x}\right) \\
\mathrm{v}_{1,2,3} \sim \cos \left(\mathrm{k}_{1,2,3} \mathrm{x}+\delta_{1,2,3}\right)
\end{gathered}
$$

Suppose $\mathrm{k}_{1}+\mathrm{k}_{2}=\mathrm{k}_{3}$ and consider what terms are generated in the Jacobians of the various equations by interactions between modes 1 and 2 . - Vorticity: $\mathrm{J}(\psi, \Omega)$ generates products of the form $\sin \left(\mathrm{k}_{1}, \mathrm{x}\right) \cos \left(\mathrm{k}_{2} \mathrm{x}\right)$ and $\cos \left(\mathrm{k}_{1}, \mathrm{x}\right) \sin \left(\mathrm{k}_{2} \mathrm{x}\right)$. Each of these terms is capable of giving rise to a term which goes as $\sin \left(k_{3} x\right)$.

- Alongcell velocity: $\mathrm{J}(\psi, \mathrm{v})$ generates products of the form $\sin \left(\mathrm{k}_{1} \mathrm{x}\right) \sin \left(\mathrm{k}_{2} \mathrm{x}+\delta_{2}\right)$ and $\cos \left(\mathrm{k}_{1} \mathrm{x}+\delta_{1}\right) \cos \left(\mathrm{k}_{2} \mathrm{x}\right)$. Each of these terms is capable of giving rise to a term which goes as $\cos \left(\mathrm{k}_{3} \mathrm{x} \pm \delta_{1,2}\right)$. If $\delta_{1}, \delta_{2} \neq \delta_{3}$, then the nonlinear advection of alongcell velocity forces a linear mode which is out of phase with that forced by the advection of vorticity. Blocking resonant transfer in space would therefore limit the effectiveness of velocity advection in changing the scale of the dominant mode.

It is easy to show that the first of these three mechanisms is not the answer. Table 4.2 shows the real and imaginary parts of the eigenvalues corresponding to the linearly most unstable mode for $\mathrm{La}=\mathrm{F}=0.05,0.075$, and 0.1 , for $\mathrm{L}=16,8$, and 5.67. The last two of these $(\mathrm{La}, \mathrm{F})$ pairs give two pairs of cells in the model box at 
Most Unstable Mode, $\mathrm{F}=.1, \mathrm{La}=.1, \mathrm{~L}=8$

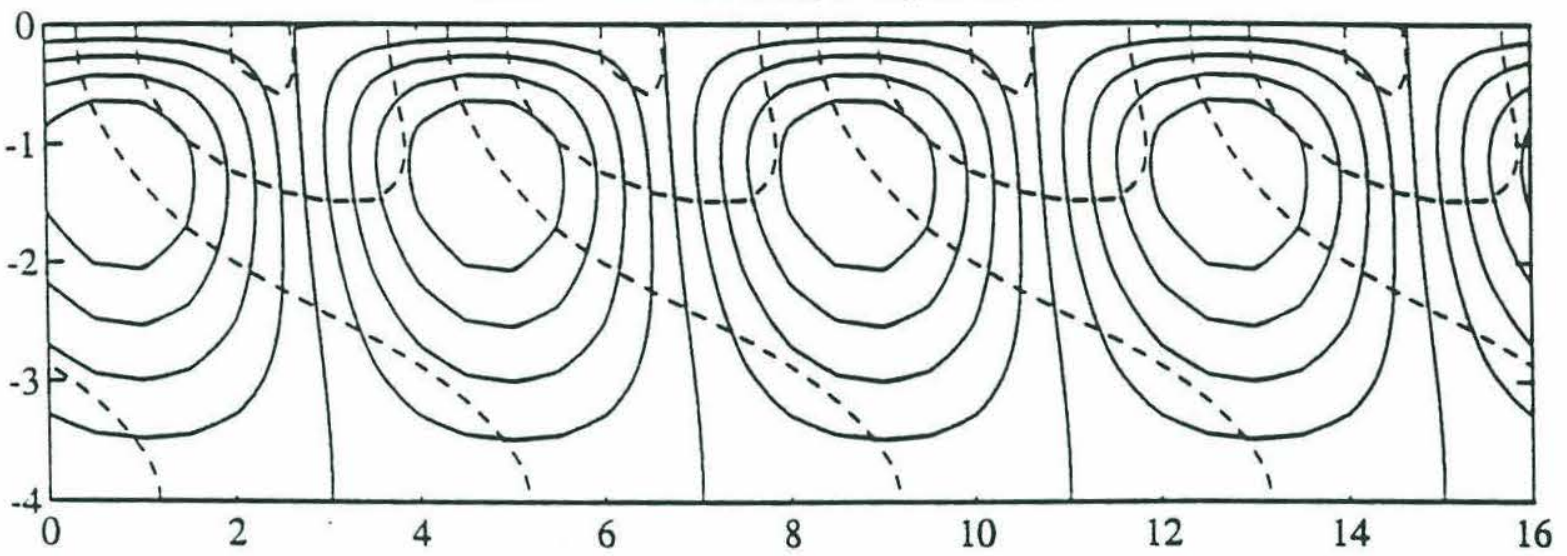

(a)

Most Unstable Mode, $\mathrm{F}=.1, \mathrm{La}=.1, \mathrm{~L}=16$

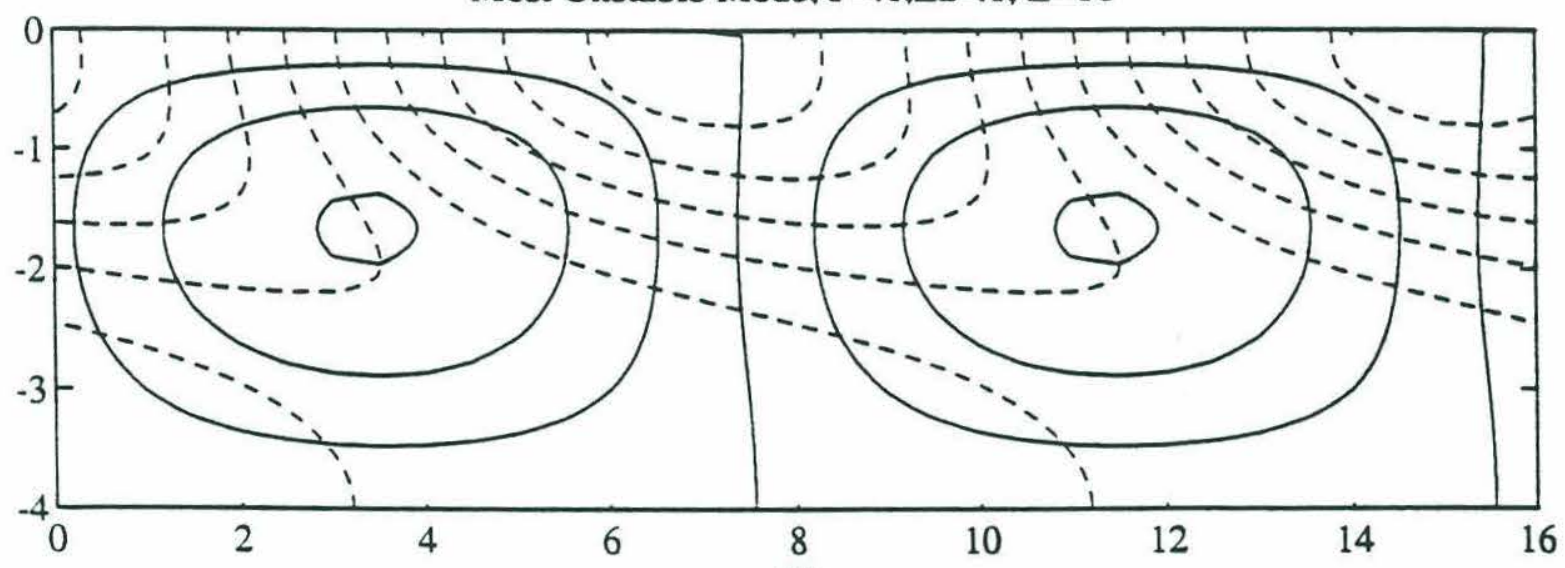

(b)

Figure 4.16: Breaking the resonance between modes of different scales in space. Streamfunction (solid lines) and alongcell velocity (dashed lines) associated with the most unstable mode for $\mathrm{F}=.1, \mathrm{La}=.1$, surface shear $=1$, monochromatic waves aligned with the wind and cells oriented 15 degrees to the right of the wind. (a) $\mathrm{L}=8$. (b) $\mathrm{L}=16$. 
equilibrium, while the first one gives only a single pair of cells. The lowest horizontal wavenumber has an unstable eigenvalue for all three cases. The value of this growth rate is large enough so that the failure of the long-wavelength mode to dominate the flow field at nondimensional times of order 1000 is not explicable through the growth rate being too small.

\begin{tabular}{|l|l|l|l|l|l|l|}
\hline $\mathrm{La}, \mathrm{F}$ & $\gamma, \mathrm{L}=16$ & $\sigma, \mathrm{L}=16$ & $\gamma, \mathrm{L}=8$ & $\sigma, \mathrm{L}=8$ & $\gamma, \mathrm{L}=5.67$ & $\sigma, \mathrm{L}=5.67$ \\
\hline .05 & .055 & -.141 & .102 & -.256 & .115 & -.385 \\
\hline .075 & .037 & -.153 & .059 & -.261 & .045 & -.385 \\
\hline .1 & .011 & -.164 & .021 & -.267 & .021 & -.387 \\
\hline
\end{tabular}

Table 4.2: Real and imaginary parts of the eigenvalues of the most unstable for three cases where $E k=1$.

By constrast, decoupling of the frequency resonance is a possibility for explaining why the gravest unstable mode does not dominate the solution. Table 4.2 shows that the complex part of the eigenvalues for the three cases change slightly as $\mathrm{La}$ and $\mathrm{F}$ increase. The complex part of the eigenvalue given $\mathrm{L}=16$ changes by about $15 \%$, while that for $\mathrm{L}=8$ changes about $4 \%$. Thus interactions which were resonant for $\mathrm{La}=\mathrm{F}=0.05$ may no longer be resonant for $\mathrm{La}=\mathrm{F}=0.075$.

The third possibility, decoupling of the resonance term in space, is illustrated in Figure 4.14. The streamfunction field (solid lines) and alongcell velocity field (dashed lines) are shown for $\mathrm{F}=\mathrm{La}=0.1$ for $\mathrm{L}=8$ and 16 . For $\mathrm{L}=8$, (top) the downstream velocity jets are not located exactly along the center of the downwelling, but are offset to one side. For $\mathrm{L}=16$ (bottom) the downwelling and the jet are basically aligned. This offset is the result of the Coriolis force. In the absence of Stokes' drift and mean shear, the Coriolis force creates velocity jets which are in phase spatially with the streamfunction, instead of out of phase, as is the case with Craik-Leibovich instability. In Section 4.3 it was argued that advection of the alongcell velocity is the most likely mechanism for causing transfer of energy between cells with long wavelengths. Breaking the resonance in space interferes with this mechanism, making it a likely candidate for explaining the failure of cells to transfer energy to large wavelengths in the presence of Coriolis forces. 


\subsection{Conclusions}

The importance of Langmuir cells as a transport mechanism within the mixed layer depends both on their depth of penetration and the ratio of the strength of the forcing driving the cells to the dissipation damping them. In Chapters 2 and 3 these quantities were shown to be closely linked to the horizontal scale of the cells. This chapter has investigated the physics which set this horizontal scale. The results are far from complete, but they suggest a number of further avenues of research.

In the absence of Coriolis forces, cell spacing increases over time, in accordance with theories of two-dimensional turbulence. This evolution to large scales is very slow, however, and for times corresponding to dimensional times of order days, there are intermediate quasi-equilibrium states. The increase in spatial scale appears to be due to a process involving advection of the small-scale velocity and density perturbations by large-scale cells, creating large scale perturbations. These in turn reinforce the large-scale cells through the Craik-Leibovich mechanism.

In the presence of Coriolis forces, the energy transfer stops. Stratification further limits the cell spacing. It is suggested that the limitation of the horizontal length scale is due to the Coriolis force interfering with the velocity advection mechanism. The cascade of energy to large scales is shown to be affected by the Langmuir number and scaled Coriolis parameter-but not necessarily in a simple way.

There are several implications to this work. The first is that, in general, the cell spacing is not dominated by the fastest-growing mode over all wavelengths, but by a mode with a much longer wavelength which may well fill the mixed layer. Since the fastest-growing mode over all wavelength is often one with a very short wavelength and small depth of penetration, this is good news for observationalists. It means one has some hope of measuring the important wavelengths at some equilibrium or quasiequilibrium state.

A second important implication to this work is that the depth of penetration of Langmuir cells can be limited over time periods of days, even when cells with longer wavelengths (and hence deeper penetration) than those observed are unstable. The limitation of cell depth is closely linked to the limitation of the horizontal wavelength. The cells are generally wider than they are deep. If the scaled Coriolis parameter is sufficiently large (so that the Coriolis parameter is 
large or the waves are small). Langmuir cells may be limited to depths of order the Ekman depth. In Chapters 6, 7, and 8 it will be shown that during two field experiments the cell depth of penetration is not limited for unstratified mixed layers, but that it can be limited when stratification is included.

A third important implication is that the stratified Craik-Leibovich instability parameter and diffusive decay rate are important quantities to estimate in the field, since they give insight into the relative importance of various nonlinear processes as well as insight into the linear instability problem. In Chapters 6 and 7, the estimation of these parameters is carried out for two field experiments.

A final important implication of this work is that it shows that a finitedifference code can give some very important information about the evolution of Langmuir cells which will not be gained from other approaches, such as linear instability theory, or simply choosing an equilibrium state with a given wavenumber.

One major limitation of the runs described here is their general failure to produce multiple scales of cells. A number of observers have reported seeing small-scale cells embedded within bigger cells and sonar observations seem to support this picture (Farmer and Zedel, 1991). Although a few cases with stratification and Coriolis force did produce multiple scales of cells, these were exceptional. The reason for this failure to reproduce observation is probably due to the lack of mechanisms within the model to erase the large-scale cells and to reseed the mixed layer with smaller-scale cells. This question is addressed in more detail in Chapter 8.

The question of what actually causes the transfer of energy to large scales has not been adequately investigated. It is suggested that the most likely mechanism involves the advection of small-scale plumes by large-scale cells and the subsequent reinforcement of these cells through the Craik-Leibovich forcing mechanism. In some simple truncations this mechanism is more important than wave-mean flow interactions in causing changes in cell scaling. While these results are highly suggestive, they are not rigorous proofs that velocity advection is in fact responsible for changing the cell scaling. Resolution of the exact process involved would help to answer the question of whether or not it is the interruption of this process by the presence of Coriolis forces and stratification that result in the limitation of the equilibrium cell spacing. 


\section{Chapter 5: The Velocity and Density Structure of Fluid Layers with Finite Amplitude Langmuir Circulations}

\subsection{Introduction}

The first chapter of this thesis set forth the hypothesis that vortices driven by the Craik-Leibovich wave-current interaction mechanism with vertical scales comparable to the oceanic mixed layer are the principal transport mechanism for velocity and density within that layer. This conceptual picture contrasts with one in which near-surface mixing is accomplished by homogeneous small-scale turbulence. The major problem with testing this hypothesis is that the concentration of the forcing near the upper boundary makes it difficult to define a characteristic scale for the forcing analogous to the mean stratification for Rayleigh-Benard convection. As a result, it is difficult to define an analogue to the Rayleigh number which would determine whether or not a layer mixed only by small-scale turbulence should become unstable to Langmuir circulations and whether or not these circulations should play a role in density and buoyancy transport.

Chapters 2 and 3 showed that for infinitesimally strong disturbances, the analogue to the stratification for Rayleigh-Benard convection was the stratified Craik-Leibovich instability parameter $\gamma_{C L S}$. This parameter is a function of the vertical structure of the cells (see page 39 for a derivation):

$$
\gamma_{C L S}^{2}(\mathrm{D})=\frac{4}{D^{2}}\left(\int_{-D}^{0} F(z) \frac{\partial v_{s}}{\partial z} d z \int_{-D}^{0} F(z) \frac{\partial V}{\partial z} d z-\int_{-D}^{0} G(z) \frac{g \partial \rho}{\rho \partial z} d z\right)
$$

where $\mathrm{v}_{\mathrm{s}}$ and $\mathrm{V}$ are the Stokes drift and Eulerian velocity parallel to the alongcell axis, $\mathrm{z}$ is the vertical axis and $\rho$ is the density. The functions $\mathrm{F}(\mathrm{z})$ and $\mathrm{G}(\mathrm{z})$ are weighting functions which depend on the boundary conditions and are proportional to the nonlinear flux of momentum and buoyancy carried by the most unstable mode at a given horizontal wavenumber. If $\gamma_{\text {diff }}$ is the characteristic diffusive decay scale for the particular mode then the stratified Craik-Leibovich Rayleigh number ( $\mathrm{Ra} C L S$ ) is defined as

$$
\text { RacLS }=\gamma_{\text {CLS }}^{2} / \gamma_{\text {diff }}^{2}
$$

In Chapters 2 and 3 it was shown that the necessary condition for an idealized surface layer to be unstable to Langmuir cells is that

$$
\gamma_{\text {CLS }}>\gamma_{\text {diff }} \sigma
$$


where $\sigma$ is the frequency with which crosscell shear tilts the cells (for a formal definition see pages 75-76).

One complication of this result is that $\gamma_{C L S}, \gamma_{\text {diff, and }} \sigma$ all depend on the geometry of the cells, so that understanding the spatial scale of the dominant mode is important. In Chapter 4 it was shown that in the absence of Coriolis forces, the dominant mode evolves to larger and larger crosscell spacing, resulting in deeper and deeper penetration over time. This evolution to large scales can be very slow, however, so that stratification may effectively limit the depth of penetration in nature (even though it does not do so mathematically). The presence of Coriolis forces halts the cascade of energy to horizontal scales, and as a result limits the depth of penetration of the cells at equilibrium.

In this chapter it is assumed that the horizontal and vertical scale of the cells is known. The effect of fully developed cells on the velocity and density structure of idealized surface layers is then considered. When RaCLS» 1:

-The cells replace small-scale diffusion as the primary transport mechanism for momentum and density.

-The vertical shear of the horizontally varying horizontal velocity scales as $\gamma_{C L S}$ rather than as the mean shear.

The approach taken is as follows. Section 5.2 reviews the equations of motion and suggests how to derive an approximate theory for their solution in the absence of Coriolis forces (for which closed-form solutions are more easily found). Section 5.3 develops this approximate theory and compares the predicted momentum flux, density flux and vertical shear of horizontal velocity to solutions from the finite difference code introduced in Chapter 4 and documented in Appendix C. Section 5.4 considers the velocity and density structure in the presence of Coriolis forces. Section 5.5 considers the implications of these results for dynamical modelling of the mixed layer.

\subsection{Equations of Motion and Methods of Solution}

The physical scenarios considered in this chapter are identical to those considered in Chapter 4. A schematic showing these scenarios is given in Figure 4.1 (page 94). The equations of motion are repeated from Chapter 4 below.

$$
\frac{\partial \Omega}{\partial \mathrm{t}}+\left(\mathrm{u}+\mathrm{u}_{\mathrm{s}}\right) \frac{\partial \Omega}{\partial \mathrm{x}}+\mathrm{w} \frac{\partial \Omega}{\partial \mathrm{z}}=\mathrm{F} \frac{\partial}{\partial \mathrm{z}}\left(\mathrm{v}+\mathrm{v}_{\mathrm{s}}\right)+\frac{\partial \mathrm{v}_{\mathrm{S}} \partial \mathrm{v}}{\partial \mathrm{z}}+\mathrm{R} \mathrm{R} \frac{\partial \rho}{\partial \mathrm{x}}+\mathrm{La} \nabla^{2} \Omega
$$




$$
\frac{\partial \mathrm{v}}{\partial \mathrm{t}}+\left(\mathrm{u}+\mathrm{u}_{\mathrm{s}}\right) \frac{\partial \mathrm{v}}{\partial \mathrm{x}}+\mathrm{w} \frac{\partial \mathrm{v}}{\partial \mathrm{z}}=-\frac{\partial \mathrm{p}}{\partial \mathrm{y}}-\mathrm{F}\left(\mathrm{u}+\mathrm{u}_{\mathrm{s}}\right)+\mathrm{La} \nabla^{2} \mathrm{v}
$$

$$
\frac{\partial \rho}{\partial t}+\left(u+u_{s}\right) \frac{\partial \rho}{\partial x}+w \frac{\partial \rho}{\partial z}=L_{a} \nabla^{2} \rho
$$

$$
\begin{aligned}
& \Omega=\nabla^{2} \psi \\
& \frac{\partial \psi}{\partial \mathrm{x}}=-\mathrm{w} \quad \frac{\partial \psi}{\partial \mathrm{z}}=\mathrm{u} \\
& \mathrm{La}=\frac{\mathrm{ve}_{\mathrm{e}}}{\mathrm{a}^{2} \sigma} \quad \mathrm{F}=\frac{\mathrm{f}}{\mathrm{k}_{\mathrm{w}}^{2} \mathrm{a}^{2} \sigma} \quad \mathrm{Ri}=\frac{\mathrm{N}^{2}}{\mathrm{k}_{\mathrm{w}}^{4} \mathrm{a}^{4} \sigma^{2}} \\
& \mathrm{k}_{\mathrm{w}}^{-1}(\mathrm{x}, \mathrm{y}, \mathrm{z})=(x, y, z) \\
& \left(\left(\mathrm{k}_{\mathrm{w}} \mathrm{a}\right) 2 \frac{\sigma}{\mathrm{k}_{\mathrm{w}}} \mathrm{u}, \mathrm{u}_{\mathrm{s}}, \mathrm{v}, \mathrm{v}_{\mathrm{S}}, \mathrm{w}\right)=\left(u, u_{s}, v, v_{\mathrm{s}}, w\right) \\
& \frac{1}{\mathrm{k}_{\mathrm{w}}^{2} \mathrm{a}^{2} \sigma} \mathrm{t}=t^{\prime}
\end{aligned}
$$

In these equations $\mathrm{k}_{\mathrm{w}}, \mathrm{a}$, and $\sigma$ are the wavenumber, amplitude and frequency of the driving waves, $v_{e}$ the eddy viscosity, $N$ the buoyancy frequency, $f$ the Coriolis parameter, and $\mathrm{u}_{\mathrm{S}}$ and $\mathrm{v}_{\mathrm{S}}$ the Stokes Drift. La represents the Langmuir number, which is a scaled eddy viscosity or inverse Reynolds number. $\mathrm{Ri}$ is the square of the scaled buoyancy frequency and $\mathrm{F}$ the scaled Coriolis parameter. Equations (5-4a-e) are for dimensionless variables, with equations (5-4g-i) showing how these variables are converted to dimensional (italicized) form. The boundary conditions on the velocity and density are

$$
\left.\operatorname{La} \frac{\partial \mathrm{v}}{\partial \mathrm{z}}\right|_{\mathrm{z}=0}=\frac{\tau}{\rho} \cos \alpha
$$

$$
\left.\operatorname{La} \Omega\right|_{\mathrm{z}=0}=-\frac{\tau}{\rho} \sin \alpha
$$

$$
\left.\operatorname{La} \frac{\partial \mathrm{v}}{\partial \mathrm{z}=0}\right|_{\mathrm{z}=-\mathrm{D}}=0
$$

$$
\left.\operatorname{La} \frac{\partial \rho}{\partial z}\right|_{z=0}=1
$$




$$
\left.\rho\right|_{\mathrm{z}=-\mathrm{D}}=-\mathrm{D}
$$

where $\alpha$ is the angle of orientation of the cells relative to the wind and D is the depth of the fluid layer.

The shortcomings of these equations as a realistic idealization of the oceanic surface layer have been explored in great detail in the last four chapters. A detailed discussion of the assumptions made in these equations may be found in Chapter 1 and in Appendix A. As in Chapter 4, two cases are considered, one for which $F=\alpha=0$ and $\partial p / \partial y=\tau / D$, the other for which $\partial p / \partial y=0$ and $\alpha, F \neq 0$.

The focus in this chapter is on determining the velocity and density structure given a field of cells at equilibrium. In order for the mixed layer to be at equilibrium, a number of balances must hold. These are derived as follows. Let the velocity, vorticity density fields be given by a horizontally averaged part and a horizontally varying part:

$$
\begin{gathered}
(\Psi, v, \rho)=(\Psi, V, P)(z, t)+\left(\psi^{\prime}, v^{\prime}, \rho^{\prime}\right)(x, z, t) \\
u(x, z, t)=U(z, t)+u^{\prime}(x, z, t)=\frac{\partial}{\partial z}\left(\Psi(z, t)+\psi^{\prime}(x, z, t)\right) \\
w(x, z, t)=w^{\prime}(x, z, t)=-\frac{\partial}{\partial x}\left(\Psi(z, t)+\psi^{\prime}(x, z, t)\right) \\
\Omega(x, z, t)=\frac{\partial^{2}}{\partial z^{2}}\left(\Psi(z, t)+\psi^{\prime}(x, z, t)\right)+\frac{\partial^{2}}{\partial x^{2}} \psi^{\prime}(x, z, t)
\end{gathered}
$$

If the overbar symbol - is defined to denote horizontal averaging then

$$
\mathrm{U}(\mathrm{z}, \mathrm{t})=\overline{\mathrm{u}(\mathrm{x}, \mathrm{z}, \mathrm{t})} \quad \mathrm{V}(\mathrm{z}, \mathrm{t})=\overline{\mathrm{v}(\mathrm{x}, \mathrm{z}, \mathrm{t})} \quad \mathrm{P}(\mathrm{z}, \mathrm{t})=\overline{\rho(\mathrm{x}, \mathrm{z}, \mathrm{t})}
$$

At equilibrium, the horizontally averaged vorticity, velocity and density fields are given by the following balances:

$$
\begin{gathered}
\frac{\partial^{2}}{\partial z^{2}} \overline{u^{\prime} w^{\prime}}-F \frac{\partial}{\partial z}\left(V+v_{s}\right)=L a \frac{\partial^{3}}{\partial z^{3}} U \\
\frac{\partial}{\partial z^{v^{\prime} w^{\prime}}}+F\left(U+u_{s}\right)=-\frac{\partial p}{\partial y}+L a \frac{\partial^{2}}{\partial z^{2}} V \\
\frac{\partial}{\partial z^{\prime} \rho^{\prime}}=L a \frac{\partial^{2}}{\partial z^{2}} P
\end{gathered}
$$


Equation (5-5c) may be integrated from the base of the layer (at which point w' is zero) to give an equation for the density flux. If $\mathrm{F}=0$ both equations $(5-8 \mathrm{a}, \mathrm{b}) \mathrm{can}$ also be integrated to yield equations for the momentum flux. In this case:

$$
\begin{gathered}
-\overline{u^{\prime} w^{\prime}}+\mathrm{La} \frac{\partial U}{\partial z}=0 \\
-\overline{v^{\prime} w^{\prime}+L a} \frac{\partial V}{\partial z}=L a \frac{\partial V_{0}}{\partial z} \\
-\overline{\rho^{\prime} w^{\prime}}+L a \frac{\partial P}{\partial z}=L a \frac{\partial P_{0}}{\partial z}
\end{gathered}
$$

where $V_{0}$ and $P_{0}$ are the velocity and density profiles, respectively, in the absence of Langmuir cells. In the absence of the Coriolis force the steady-state momentum and density flux are independent of whether or not cells are present. The cells replace eddy diffusion due to small-scale turbulence as the major mechanism carrying the fluxes of momentum and density.

As in Chapter 2, balance equations for the eddy kinetic energy in the crosscell direction, eddy kinetic energy in the alongcell direction, and density variance may also be derived. These are:

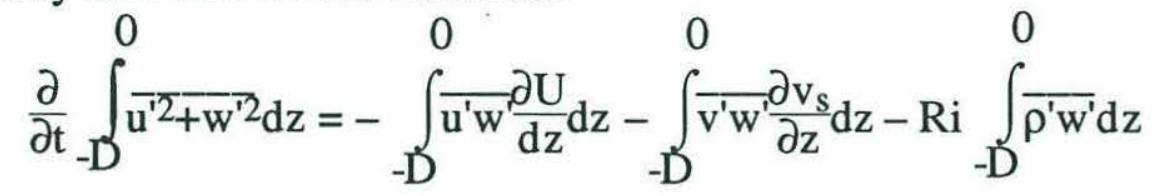

$$
-F \int_{-D}^{0} \overline{u^{\prime} v^{\prime}} d z-L a \int_{D}^{0} \frac{\partial u^{\prime} 2}{\partial x}+\frac{\partial u^{\prime} 2}{\partial z}+\frac{\partial w^{\prime 2}}{\partial x}+\frac{\partial w^{\prime 2}}{\partial z} d z
$$

$$
\begin{gathered}
\frac{\partial}{\partial t} \int_{-D}^{0} \overline{v^{\prime 2}} d z=\int_{-D}^{0} \frac{v^{\prime} w}{d} \frac{\partial v}{d z} d z+F \int_{-D}^{0} \overline{u^{\prime} v^{\prime}} d z-L a \int_{-D}^{0} \frac{\partial v^{\prime} 2}{\partial x}+\frac{\partial v^{\prime 2}}{\partial z} d z \\
\frac{\partial}{\partial t} \int_{-D}^{0} \frac{0}{\rho^{\prime 2}} d z=\int_{-D}^{0} \frac{\partial}{\rho^{\prime} w^{\prime}} \frac{\partial P}{d z} d z-L a \int_{-D}^{\frac{\partial \rho^{\prime}}{\partial x}+\frac{\partial \rho^{\prime} 2}{\partial z} d z}
\end{gathered}
$$

At equilibrium, the energy balance is

$$
\frac{\partial}{\partial t} E_{c c}=P_{c c}+P_{\text {stokes }}-E_{\text {rot }}-B_{\text {trans }}-\varepsilon_{c c}=0
$$




$$
\frac{\partial}{\partial t} E_{a c}=P_{d c}+E_{\text {rot }}-\varepsilon_{a c}=0
$$

where $\mathrm{E}_{\mathrm{cc}, \mathrm{ac}}$ are the energies associated with flow in the crosscell and alongcell directions respectively. $\mathrm{P}_{\text {stokes }}$ is the Stokes production (the work done by the waves on the cell vortices). $\mathrm{P}_{\mathrm{cc}, \mathrm{ac}}$ are the shear production terms caused by the nonlinear shear stress working against the crosscell and alongcell shears, respectively. $E_{\text {rot }}$ is the transfer of energy from crosscell to alongcell via the Coriolis force. $\mathrm{B}_{\mathrm{trans}}$ is the buoyancy transport. $\varepsilon_{\mathrm{cc} \text {,ac }}$ are the dissipation terms associated with the crosscell velocities and alongcell velocity respectively.

In Chapter 2, the energy balance equations were used to extract the characteristic frequencies associated with the important physical processes. In the absence of rotation, one can combine the equations for momentum and density balance with those for energy balance and obtain equations depending solely on the nonlinear amplitude of the cells. To do this one must solve for:

1. The shape of the horizontally varying streamfunction, velocity and density perturbation associated with the equilibrium cells.

2. The amplitude of these streamfunction, velocity, and density perturbations. Three methods for doing this are given below:

Method I: Assume that the shapes of the alongcell velocity, vorticity, and density perturbations are given by linear instability theory, but that the relationship between their amplitudes is not.

$$
\begin{aligned}
v^{\prime} & =v_{1} V(z) e^{i k x} \\
\rho^{\prime} & =\rho_{1} \rho(z) e^{i k x} \\
\psi^{\prime} & =\psi_{1} \psi(z) e^{i k x}
\end{aligned}
$$

This methodology is similar to the finite-amplitude convection results of Malkus and Veronis (1958).* Their paper assumed that the shape and relationship between the density and streamfunction perturbation of the finite amplitude modes was given by the neutrally stable solution at the critical Rayleigh number. For cells oriented in alongwind direction in the absence of Coriolis force the assumption that the relationship between streamfunction, density, and alongcell velocity perturbations is given by the neutrally stable solution is equivalent to demanding that

$$
\frac{\partial v_{S} \partial v}{\partial z \partial x}+\operatorname{Ri} \frac{\partial \rho}{\partial x}+L a \nabla 2 \Omega=0
$$

\footnotetext{
${ }^{*}$ It should be noted that Malkus and Veronis derived this approximation rigourously using a weakly nonlinear approach. This is not done in the present case.
} 
This is functionally equivalent to requiring that the energy balance in the crosscell direction given in equation (5-10a,12a) hold for the equilibrium cells.

This approach differs from Malkus and Veronis in that the structure functions $V(\mathrm{z}), \rho(\mathrm{z})$, and $\psi(\mathrm{z})$ and the wavenumber $\mathrm{k}$ of the unstable cells are allowed to differ from those asssociated with the neutrally stable cells at the critical Rayleigh number $\mathrm{Ra}_{\mathrm{c}}$. This is important since in the absence of rotation $\mathrm{k}$ is infinite at $\mathrm{Ra}_{\mathrm{c}}$. One further difference between this work and that of Malkus and Veronis is that the self-advective effects of the finite-amplitude cells are not solved for. Consideration of such effects is necessary in order to obtain a full description of the flow and extend the results to cases where the forcing is very strong, but is not necessary to answer the basic question addressed here.

Method II: Assume that the structure functions $V(z), \rho(z)$, and $\psi(z)$ are given by the neutrally stable solution for cells with wavenumber $\mathrm{k}$ identical to that dominating the final solution. This solution is found by increasing $\mathrm{La}$ and $\mathrm{F}$ together, until cells with wavenumber $\mathrm{k}$ are neutrally stable and then taking the structure functions predicted by the instability code for this neutrally stable solution. As a weakly nonlinear theory, this method is again similar to that of Malkus and Veronis, and is slightly more rigorous than Method I.

The effect of using this method as opposed to Method I can be seen by considering the dependence of depth of maximum vertical velocity $\mathrm{D}_{\max }$ on Langmuir number. Chapter 2 showed that if La increased while the horizontal wavenumber remained constant, $\mathrm{D}_{\max }$ also increased. The effect was most pronounced at large wavelengths, and was not as pronounced when stratification was present. This method is more cumbersome than simply using results from the instability code, and as a result it will be used in only a few cases.

Method III: Use instability theory or finite-difference code runs to guide the choice of some simple, analytic truncation. The basic idea is to use some prior information about cell shape to obtain a closed-form solution from which information about the relevant physics can be extracted.

Both of the truncations used below were introduced in Chapter 2 to look at the physics of Craik-Leibovich instability. The first truncation (T1) assumed cells with a limited penetration depth $\mathrm{D}^{\prime}$ and identical vertical structures for the density and alongcell velocity perturbations. It is used in cases where stratification limits the depth of penetration of the cells. This truncation predicts 
the growth rate and depth at which the maximum vertical velocity occurs for infinitesimally strong cells.

$$
\begin{array}{cc}
\psi^{\prime}=\psi_{1} \sin \left(\pi z / D^{\prime}\right) e^{i k x} & z>-D^{\prime} \\
\left(v^{\prime}, \rho^{\prime}\right)=\left(v_{1}, \rho_{1}\right) \cos \left(\pi z / 2 D^{\prime}\right) e^{i k x} & z>-D^{\prime} \\
\left(\psi^{\prime}, v^{\prime}, \rho^{\prime}\right)=0 & z<-D^{\prime}
\end{array}
$$

The second truncation (T2) was for cells which had a long enough wavelength so that the velocity perturbation was relatively constant with depth and the effect of stratification was negligible ( $\mathrm{La}$ and or cell spacing $\mathrm{L}$ large).

$$
\begin{gathered}
\psi^{\prime}=\psi_{1} \sin (\pi z / D) e^{i k x} \\
v^{\prime}=v_{1} e^{i k x}
\end{gathered}
$$

As in Chapter 2, these truncations are special cases of Method I which give useful insights into the physics determining the evolution of Langmuir cells. It is worth noting that the structure functions in T2 are identical to those for the neutrally stable solution at the critical Rayleigh number (as used by Malkus and Veronis).

\subsection{Velocity and Density Transport Accomplished by Finite-Amplitude Cells} in Non-rotating Mixed Layers

\subsubsection{Theoretical Results from Methods II and III}

When the cells are oriented in the alongwind direction and there is no Coriolis force $(\mathrm{F}=0)$, the streamfunction and velocity/density perturbations $\left(\psi^{\prime}, \mathrm{v}^{\prime}\right.$ $\left.\rho^{\prime}\right)$ are out of phase. Suppose then that:

$$
\begin{aligned}
& v^{\prime}=v_{1} V(z) \cos (k x) \\
& \rho^{\prime}=\rho_{1} \rho(z) \cos (k x) \\
& \psi^{\prime}=\psi_{1} \psi(z) \sin (k x)
\end{aligned}
$$

with the normalization of the structure functions $V, \psi, \rho$ given (as in Chapter 2) by

$$
\frac{2}{\mathrm{D}} \int_{-\mathrm{D}}^{0}|(V, \psi, \rho)|^{2} \mathrm{dz}=1
$$

Then the crosscell energy balance then yields the following equation:

$$
\begin{gathered}
\psi_{1} \mathrm{v}_{1} \frac{\mathrm{k}}{2} \int_{-\mathrm{D}}^{0} \psi(\mathrm{z}) V(\mathrm{z}) \frac{\partial \mathrm{v}_{\mathrm{s}}}{\partial \mathrm{z}} \mathrm{dz}+\operatorname{Ri} \psi_{1} \rho_{1} \frac{\mathrm{k}}{2} \int_{-\mathrm{D}}^{0} \psi(\mathrm{z}) \rho(\mathrm{z}) \mathrm{dz}= \\
\frac{\psi_{1}^{2} \mathrm{La}}{2} \int_{-\mathrm{D}}^{0}\left(\frac{\partial^{2} \psi}{\partial \mathrm{z}^{2}}\right)^{2}+2 \mathrm{k}^{2}\left(\frac{\partial \psi}{\partial \mathrm{z}}\right)+\mathrm{k}^{4} \psi^{2} \mathrm{dz}
\end{gathered}
$$

Following Chapter 2, define 
$(5-22 a)$

$$
\beta=\frac{2}{D} \int_{-D}^{0} \psi(z) \rho(z) d z
$$

0

$(5-22 b)$

$$
\hat{\mathrm{V}}_{\mathrm{z}}=\frac{2}{\mathrm{D}} \int_{-\mathrm{D}} \psi(\mathrm{z}) V(\mathrm{z}) \frac{\partial \mathrm{v}_{\mathrm{s}}}{\partial \mathrm{z}} \mathrm{dz}
$$

$(5-22 c)$

$$
\mathrm{K}_{\psi}^{4}=\frac{2}{\mathrm{D}} \int_{-\mathrm{D}}^{0}\left(\frac{\partial^{2} \psi}{\partial \mathrm{z}^{2}}\right)^{2}+2 \mathrm{k}^{2}\left(\frac{\partial \psi}{\partial \mathrm{z}}\right)^{2}+\mathrm{k}^{4} \psi^{2} \mathrm{dz}
$$

Dividing out common terms yields

$$
\mathrm{kV}_{\mathrm{V}_{\mathrm{z}} \mathrm{v}_{1}}+\mathrm{Ri} \beta \mathrm{k} \rho_{1}=\mathrm{La} \mathrm{K}{ }_{\psi}^{4} \psi_{1}
$$

The alongcell variance balance yields

$$
\psi_{1} v_{1} \frac{\mathrm{k}}{2} \int_{-\mathrm{D}}^{0} \psi(\mathrm{z}) V(\mathrm{z}) \frac{\partial \mathrm{V}}{\partial \mathrm{z}} \mathrm{dz}=\frac{\mathrm{La} \mathrm{v}_{1}^{2}}{2} \int_{-\mathrm{D}}^{0}\left(\frac{\partial V}{\partial z}\right)^{2}+\mathrm{k}^{2} V^{2} \mathrm{dz}
$$

Solving for $\frac{\partial \mathrm{V}}{\partial \mathrm{z}} \mathrm{using}(5-8)$ yields

(5-25) $\psi_{1} \mathrm{v}_{1} \frac{\mathrm{k}}{2} \int_{-\mathrm{D}}^{0} \psi(\mathrm{z}) V(\mathrm{z})\left(\frac{\partial \mathrm{V}_{0}}{\partial \mathrm{z}}-\frac{\mathrm{k}}{2 \mathrm{La}} \psi_{1} \mathrm{v}_{1} \psi(\mathrm{z}) V(\mathrm{z})\right) \mathrm{dz}=\frac{\mathrm{La} \mathrm{v}_{1}^{2}}{2} \int_{-\mathrm{D}}^{0}\left(\frac{\partial V}{\partial \mathrm{z}}\right)^{2}+\mathrm{k}^{2} V^{2} \mathrm{dz}$

Letting

$$
\begin{aligned}
& \hat{\mathrm{V}}_{\mathrm{z}}=\frac{2}{\mathrm{D}} \int_{-\mathrm{D}}^{0} \psi(\mathrm{z}) V(\mathrm{z}) \frac{\partial \mathrm{V}_{0}}{\partial \mathrm{z}} \mathrm{dz} \\
& \alpha_{\mathrm{V}}=\frac{2}{\mathrm{D}} \int_{-\mathrm{D}}^{0}(\psi(\mathrm{z}) V(\mathrm{z}))^{2} \mathrm{dz} \\
& \mathrm{k}_{\mathrm{v}}^{2}=\frac{2}{\mathrm{D}} \int_{-\mathrm{D}}^{0}\left(\frac{\partial V}{\partial \mathrm{z}}\right)^{2}+\mathrm{k}^{2} V^{2} \mathrm{dz}
\end{aligned}
$$

allows for the equlibrium amplitude of the alongcell velocity perturbation $\mathrm{v}_{1}$ to be solved for in terms of the streamfunction amplitude $\psi_{1}$.

$$
\mathrm{v}_{1}=\frac{\mathrm{k}_{\mathrm{z}} \psi_{1}}{\mathrm{La} \mathrm{k}_{\mathrm{v}}^{2}+\mathrm{k}^{2} \alpha_{\mathrm{v}} \psi_{1}^{2} / 2 \mathrm{La}}
$$

Similarly, defining 


$$
\begin{aligned}
& \alpha_{p}=\frac{2}{D} \int_{-D}^{0}(\psi(z) \rho(z))^{2} d z \\
& k_{\rho}^{2}=\frac{2}{D} \int_{-D}^{0}\left(\frac{\partial \rho}{\partial z}\right)^{2}+k^{2} \rho^{2} d z
\end{aligned}
$$

the density perturbation amplitude $\rho_{1}$ is given by:

$$
\rho_{1}=\frac{\mathrm{k} \beta \mathrm{P}_{0 z} \psi_{1}}{\mathrm{La} \mathrm{k}_{\rho}^{2}+\mathrm{k}^{2} \alpha_{\rho} \psi_{1}^{2} / 2 \mathrm{La}}
$$

Substituting into (5-23) gives the following quadratic equation in $\psi_{1}$.

$$
\text { (5-30) } \begin{aligned}
\frac{\mathrm{k}^{4} \alpha_{\mathrm{v}} \alpha_{\rho}}{4 \mathrm{La}^{2}} \psi_{1}^{4}+ & \left(\frac{\mathrm{k}^{2}}{2}\left(\alpha_{\rho} \mathrm{k}_{\mathrm{v}}^{2}+\alpha_{\mathrm{v}} \mathrm{k}_{\rho}^{2}\right)-\frac{\mathrm{k}^{4}}{2 \mathrm{La}^{2} \mathrm{~K}_{\psi}^{4}}\left(\alpha_{\rho} \hat{\mathrm{V}}_{\mathrm{z}} \hat{\mathrm{V}}_{\mathrm{z}}+\alpha_{\mathrm{v}} \mathrm{RiP}_{0 \mathrm{z}} \beta^{2}\right)\right) \psi_{1}^{2}+ \\
& \mathrm{La}^{2} \mathrm{k}_{\rho}^{2} \mathrm{k}_{\mathrm{v}}^{2}-\frac{\mathrm{k}^{2}}{\mathrm{~K}_{\psi}^{4}}\left(\mathrm{k}_{\rho}^{2} \hat{\mathrm{V}}_{\mathrm{z}} \hat{\vartheta}_{\mathrm{z}}+\mathrm{k}_{\mathrm{v}}^{2} \mathrm{RiP}_{0 \mathrm{z}} \beta^{2}\right)=0
\end{aligned}
$$

which gives a solution for the streamfunction amplitude. The resulting formula is quite complicated and difficult to interpret. However, it is possible to find simplified solutions by making certain assumptions. Two such solutions are explored below.

The first simple solution of equation (5-30) can be found as $\mathrm{k}$ goes to 0 . In Chapter 4 it was shown that for nonrotating cells energy cascades towards long wavelengths over time, and that the result is steadily deeper penetration into the mixed layer over time. The vertical structure of the velocity and streamfunction perturbation reflects that of the most unstable mode with wavelength equal to the dominant cell spacing. As $\mathrm{k} \rightarrow 0$, the vertical structure of the alongcell velocity perturbation asymptotes to a constant.

$$
\begin{gathered}
V(\mathrm{z}) \rightarrow 1 / \sqrt{2} \\
\mathrm{k}_{\mathrm{v}}^{2} \rightarrow \mathrm{k}^{2}
\end{gathered}
$$

while the density perturbation continues to have some vertical structure since the density is fixed on the bottom boundary. The streamfunction perturbation likewise retains vertical structure. Then as $\mathrm{k}$ goes to 0 , (5-32)

$$
\mathrm{k}_{\mathrm{V}} / \mathrm{K}_{\psi} \rightarrow 0 \quad \mathrm{k}_{\mathrm{V}} / \mathrm{k}_{\mathrm{\rho}} \rightarrow 0
$$

Then as $\mathrm{k}$ goes to zero, the quadratic equation (5-30) tends to:

$$
\frac{\mathrm{k}^{4} \alpha_{\mathrm{v}} \alpha_{\rho}}{4 \mathrm{La}^{2}} \psi_{1}^{4}+\frac{\mathrm{k}^{2}}{2} \alpha_{\mathrm{v}} \mathrm{k}_{\rho}^{2} \psi_{1}^{2}+\mathrm{La}^{2} \mathrm{k}_{\rho}^{2} \mathrm{k}_{\mathrm{v}}^{2}-\frac{\mathrm{k}^{2} \mathrm{k}_{\rho}^{2}}{\mathrm{~K}_{\psi}^{4}} \hat{\mathrm{V}}_{\mathrm{z}} \hat{\mathrm{V}}_{\mathrm{z}}=0
$$


Solving this and applying the relationships in (5-32) yields

$$
\psi_{1}=\frac{1}{K_{\psi}^{2}} \sqrt{\frac{2}{\alpha_{v}}\left(\hat{V}_{\mathrm{z}} \hat{V}_{\mathrm{z}}-\mathrm{La}^{2} \mathrm{~K}_{\psi}^{4} \mathrm{k}_{\mathrm{v}}^{2} / \mathrm{k}^{2}\right)}
$$

substituting in (5-27) yields an equation for the velocity perturbation amplitude.

$$
\mathrm{v}_{1}=\frac{\mathrm{LaK}_{\psi}^{2}}{\mathrm{kV} \hat{s}_{\mathrm{z}}} \sqrt{\frac{2}{\alpha_{\mathrm{V}}}\left(\hat{\mathrm{V}}_{\mathrm{z}} \hat{\mathrm{V}}_{\mathrm{z}}-\mathrm{La}^{2} \mathrm{~K}_{\psi}^{4} \mathrm{k}_{\mathrm{v}}^{2} / \mathrm{k}^{2}\right)}
$$

This solution is also the correct one for $\mathrm{Ri}=0$. For equilibrium solutions which have a long enough wavelength so that they "feel" the bottom strongly, the effect of stratification vanishes. This parallels the result for instability.

The horizontally averaged alongcell shear is given by

$$
\frac{\partial \mathrm{V}}{\partial \mathrm{z}}=\frac{\partial \mathrm{V}_{0}}{\partial \mathrm{z}}-\frac{1}{\alpha_{\mathrm{V}} \hat{\mathrm{V}}_{\mathrm{z}}}\left(\hat{\mathrm{V}}_{\mathrm{z}} \hat{\mathrm{V}}_{\mathrm{z}}-\mathrm{La}^{2} \mathrm{~K}_{\psi}^{4} \mathrm{k}_{\mathrm{v}}^{2} / \mathrm{k}^{2}\right)
$$

As in previous chapters let $\gamma_{C L}=\sqrt{\hat{\mathrm{V}}_{\mathrm{Z}} \widehat{V}_{\mathrm{z}}}, \gamma_{\text {diff }}=\mathrm{La} \mathrm{K}_{\psi}^{2} \mathrm{k}_{\mathrm{v}} / \mathrm{k}$. Then if

$$
\mathrm{Ra}_{\mathrm{CL}}=\gamma_{\mathrm{CL}}^{2} / \gamma_{\text {diff }}^{2}
$$

the horizontally averaged shear is

$$
\frac{\partial \mathrm{V}}{\partial \mathrm{z}}=\frac{\partial \mathrm{V}_{0}}{\partial \mathrm{z}}-\frac{\hat{\mathrm{V}}_{\mathrm{Z}}}{\alpha_{\mathrm{V}}}\left(1-\gamma_{\text {diff }}^{2} \gamma_{\mathrm{CL}}^{2}\right)=\frac{\partial \mathrm{V}_{0}}{\partial \mathrm{z}}-\frac{\hat{\mathrm{V}}_{\mathrm{Z}}}{\alpha_{\mathrm{V}}}\left(1-1 / \mathrm{Ra}_{\mathrm{CL}}\right)
$$

Substituting for $\alpha_{\mathrm{v}}$, equation (5-38) becomes

$$
\frac{\partial \mathrm{V}}{\partial \mathrm{z}}=\frac{\partial \mathrm{V}_{0}}{\partial \mathrm{z}}-\frac{\hat{\mathrm{V}}_{\mathrm{z}}}{\alpha_{\mathrm{V}}}=\frac{\partial \mathrm{V}_{0}}{\partial \mathrm{z}}-\frac{\int_{-\mathrm{D}}^{0} \psi(\mathrm{z}) V(\mathrm{z}) \frac{\partial \mathrm{V}_{0}}{\partial \mathrm{z}} \mathrm{dz}}{\int_{-\mathrm{D}}^{0} \psi(\mathrm{z})^{2} V(\mathrm{z})^{2} \mathrm{dz}} \psi(\mathrm{z}) V(\mathrm{z})\left(1-1 / \mathrm{Ra}_{\mathrm{CL}}\right)
$$

When the wave-current forcing is strong, Langmuir cells erase that part of the initial shear which projects on the nonlinear momentum flux. The cells replace small-scale diffusion as the principal transport mechanism for momentum over their depth of penetration.

Truncation T2 is a special case of these results. Suppose the Stokes drift and Eulerian velocity are given by the Fourier expansion

$$
\begin{aligned}
& \mathrm{v}_{\mathrm{s}}(\mathrm{z})=\mathrm{v}_{\mathrm{s} 0}+\mathrm{v}_{\mathrm{s} 1} \cos (\pi \mathrm{z} / \mathrm{D})+\ldots \\
& \mathrm{v}_{0}(\mathrm{z})=\mathrm{V}_{00}+\mathrm{V}_{01} \cos (\pi \mathrm{z} / \mathrm{D})+\ldots
\end{aligned}
$$


When the functional forms from equations (5-19) are substituted into the formalism developed above,

$$
\hat{\mathrm{V}}_{\mathrm{z} 2}=\frac{\sqrt{2}}{\mathrm{D}} \int_{-\mathrm{D}}^{0} \frac{\partial \mathrm{v}_{\mathrm{s}}}{\partial \mathrm{z}} \sin (\pi \mathrm{z} / \mathrm{D}) \mathrm{dz}=\frac{\pi}{\mathrm{D} \sqrt{2}} \mathrm{v}_{\mathrm{s} 1}
$$

$$
\hat{\mathrm{V}}_{\mathrm{z} 2}=\frac{\sqrt{2}}{\mathrm{D}} \int_{-\mathrm{D}}^{0} \frac{\partial \mathrm{V}_{0}}{\partial \mathrm{z}} \sin (\pi \mathrm{z} / \mathrm{D}) \mathrm{dz}=\frac{\pi}{\mathrm{D} \sqrt{2}} \mathrm{~V}_{01}
$$

When the cells are strongly forced (RaCL2»1) they erase the first nonconstant component of the Fourier expansion of the velocity.

The second set of simple solutions is for $V(z)=\rho(z)$ (velocity and density structure functions identical). Within the framework of equations (5-4), this is only approximately true unless the Stokes drift profile is linear with depth and the boundary conditions for density and alongcell velocity are the same. Nonetheless, as in Chapters 2 and 3 this idealization provides useful insight into the effect of stratification on equilibrium Langmuir cells. If the alongcell velocity and density perturbations have the same structure, then $\mathrm{k}_{\mathrm{V}}=\mathrm{k}_{\rho}, \alpha_{\mathrm{V}}=\alpha_{\rho}$. Letting $-\mathrm{N}^{2}=\mathrm{Ri} \beta^{2} \mathrm{P}_{0 \mathrm{z}}$ the solution becomes

$(5-42 a) \psi_{1}=\frac{1}{K_{\psi}^{2}} \sqrt{\frac{2}{\alpha_{\mathrm{V}}}\left(\hat{\mathrm{V}}_{\mathrm{z}} \hat{\mathrm{V}}_{\mathrm{z}}+\mathrm{Ri} \beta^{2} \mathrm{P}_{0 \mathrm{z}}-\mathrm{La}^{2} \mathrm{~K}_{\psi}^{4} \mathrm{k}_{\mathrm{v}}^{2} / \mathrm{k}^{2}\right)}=\frac{1}{\mathrm{~K}_{\psi}^{2}} \sqrt{\frac{2}{\alpha_{\mathrm{V}}}\left(\gamma_{\mathrm{CL}}^{2}-\mathrm{N}^{2}-\gamma_{\text {diff }}^{2}\right)}$

$$
\begin{aligned}
& \mathrm{v}_{1}=\frac{\mathrm{LaK}_{\psi}^{2} \hat{V}_{\mathrm{z}}}{\mathrm{k}\left(\gamma_{\mathrm{CL}}^{2}-\mathrm{N}^{2}\right)} \sqrt{\frac{2}{\alpha_{\mathrm{V}}}\left(\gamma_{\mathrm{CL}}^{2}-\mathrm{N}^{2}-\gamma_{\text {diff }}^{2}\right)} \\
& \rho_{1}=\frac{\mathrm{LaK}_{\psi}^{2} \beta \mathrm{P}_{0 \mathrm{z}}}{\mathrm{k}\left(\gamma_{\mathrm{CL}}^{2}-\mathrm{N}^{2}\right)} \sqrt{\frac{2}{\alpha_{\mathrm{V}}}\left(\gamma_{C L}^{2}-\mathrm{N}^{2}-\gamma_{\text {diff }}^{2}\right)}
\end{aligned}
$$

The momentum and density fluxes associated with this solution are

$$
-\overline{v^{\prime} w^{\prime}}=\frac{L a \hat{V}_{z}}{\alpha_{\mathrm{v}}}\left(1-\gamma_{\text {diff }}^{2} d\left(\gamma_{C L}^{2}-N^{2}\right)\right)
$$




$$
-\overline{\rho^{\prime} w^{\prime}}=\frac{L a \beta P_{0 z}}{\alpha_{v}}\left(1-\gamma_{\text {diff }}^{2}\left(\gamma_{C L}^{2}-N^{2}\right)\right)
$$

Defining $\gamma_{C L S}=\sqrt{\gamma_{C L}{ }^{2}-N^{2}}$ the condition for the cells to transport a large fraction of the momentum and buoyancy flux within the mixed layer is then just that

$$
\mathrm{Ra}_{\mathrm{CLS}}=\gamma_{\mathrm{CLS}}^{2} / \gamma_{\text {diff }}^{2} \gg 1
$$

The condition that RaCLS be large is not the same as demanding that the growth rate of the unstable modes be large compared with the diffusive decay rate. In Chapter 2, it was shown that when (5-44) is satisfied

$$
\gamma \sim \frac{\mathrm{k}}{\sqrt{\mathrm{k}^{2}+\pi^{2} / \mathrm{D}^{\prime 2}}} \gamma_{\text {CLS }} \text {. }
$$

When $\mathrm{k}$ is small compared with $\pi / \mathrm{D}$ ' the growth rate is quite small compared with $\gamma_{C L S}$, so that it need not be much larger than $\gamma_{\text {diff. }}$

We now turn to the question of the vertical shear of the horizontally varying horizontal current associated with the cells. This quantity will be referred to as the perturbation shear. When the forcing is strong, (RaCLS»0), the simple models predict that the perturbation shear in the crosscell direction $\omega_{\mathrm{CC}}$ will be given by

$$
\omega_{\mathrm{CC}} \sim \psi_{1} \frac{\partial^{2}}{\partial \mathrm{z}^{2}} \psi(\mathrm{z})
$$

One can get a better sense of what this means by considering the form predicted by truncation $\mathrm{T} 1$ (used for $\mathrm{La}$ and $\mathrm{L}$ small, or stratification strong).

$$
\begin{aligned}
\omega_{\mathrm{CC}} \sim & \frac{1}{\mathrm{~K}_{\psi}^{2}} \sqrt{\frac{2}{\alpha_{\mathrm{V}}}\left(\gamma_{\mathrm{CL}}^{2}-\mathrm{N}^{2}-\gamma_{\text {diff }}^{2}\right) \frac{\partial^{2}}{\partial \mathrm{z}^{2}} \psi(\mathrm{z})} \\
= & \frac{\gamma_{\mathrm{CLS}} \pi^{2} / \mathrm{D}^{\prime 2}}{\mathrm{k}^{2}+\pi^{2} / \mathrm{D}^{\prime 2}} \sqrt{\frac{2}{\alpha_{\mathrm{V}}}(1-1 / \mathrm{RaCLS})} \sin \left(\pi \mathrm{z} / \mathrm{D}^{\prime}\right)
\end{aligned}
$$

For long-wavelength cells $\mathrm{k}$ is much smaller than $\pi / \mathrm{D}^{\prime}$, while for small wavelengths, $\mathrm{kD}$ ' is of order 1 (Chapters 2 through 4 ). This means that the horizontal shear scales as $\gamma_{C L S}$, where $\gamma_{C L S}$ is appropriately defined for the penetration depth of the cells producing the shear. Chapter 4 showed that for many cases, the dominant cell structure consisted of cells whose crosscell spacing was quite a bit larger than their depth of penetration. For such cells, the shear predicted by equation (5-47) will not change substantially as the wavenumber decreases due to cell merging.

A similar scaling of the shear can be predicted on energetic grounds by assuming a local energy balance. Taking the dimensional energy balance 
equation for crosscell flow, and assuming that $\omega_{\mathrm{CC}}$ is a characteristic scale for the shear associated with perturbations (rather than with the mean flow) then

$$
-\overline{v^{\prime} w^{\prime}} \frac{\partial v_{\mathrm{s}}}{\mathrm{dz}}+\frac{\mathrm{g}}{\rho^{\prime} \mathrm{w}^{\prime}} \sim-v_{\mathrm{e}} \omega_{\mathrm{CC}}^{2}
$$

where $v_{\mathrm{e}}$ is the eddy viscosity. But in the presence of strongly forced cells, the cells carry momentum and density fluxes of the same order as the viscous fluxes in the absence of cells $\left(v_{\mathrm{e}} \frac{\partial \mathrm{V}}{\mathrm{dz}}\right.$ for momentum and $v_{\mathrm{e}} \frac{\partial \rho}{\mathrm{dz}}$ for density) so that

$$
v_{\mathrm{e}} \omega_{\mathrm{CC}}^{2} \sim v_{\mathrm{e}}\left(\frac{\partial \mathrm{v}_{\mathrm{S}}}{\mathrm{dz}} \frac{\partial \mathrm{V}}{\mathrm{dz}}+\frac{\mathrm{g} \partial \rho}{\rho \mathrm{dz}}\right) \sim \mathrm{v}_{\mathrm{e}} \gamma_{\mathrm{CLS}}^{2}
$$

When the eddy viscosity cancels out, $\omega_{C C}$ scales as $\gamma_{C L S}$. This is different than the scaling which would be expected if shear instability was balancing dissipation. In such a case the equation (5-48) would be replaced by

$$
-\overline{v^{\prime} w} \frac{\partial \mathrm{V}}{\mathrm{dz}}+\frac{\mathrm{g}}{\rho \rho^{\prime} w^{\prime}} \sim-v_{\mathrm{e}} \omega_{\mathrm{CC}}^{2}
$$

leading to a characteristic scale for horizontally varying shear which would go as

$$
\omega_{\mathrm{CC}} \sim \sqrt{\left(\frac{\partial V}{\partial z}\right)^{2}-\mathrm{N}^{2}}
$$

To summarize, approximate models of finite-amplitude Langmuir circulation in the absence of Coriolis forces predict that when the wave-current interaction forcing, corrected for buoyancy effects, is much stronger than the characteristic diffusive decay scale $\left(\gamma_{\text {CLS }}^{2} \gamma_{\text {diff }}^{2}=\mathrm{Ra}_{\mathrm{CLS}}{ } 1\right)$ :

-Langmuir cells are the principal transport mechanism within the mixed layer. -The perturbation crosscell shear is proportional to $\gamma_{\text {CLS. }}$.

These predictions are independent of whether the growth rate of the most unstable mode is larger than the characteristic diffusive decay scale and are relatively insensitive to the actual value of the Langmuir number.

\subsubsection{Testing the Model Predictions for Momentum and Density Transport}

The model predictions derived above are approximate solutions, since they are not complete descriptions of the flow field. This subsection tests the predictions of the finite-amplitude theory against solutions from a finite-difference code. In a number of cases the cells penetrate over the full depth of the layer. 
Three such cases are listed below, two which represent relatively good agreement between the approximate theory and actual model runs and one for which the agreement shows some systematic differences from the approximate theory.

Table 5.1 lists the three cases, giving the layer depth, the Langmuir number, the growth rate of the linearly most unstable mode with wavelength equal to the dominant cell spacing and $\gamma_{\mathrm{CL}}, \gamma_{\mathrm{diff}}$ and $\mathrm{Ra} \mathrm{CL}$. The estimates of the important scales given by truncation $\mathrm{T} 2\left(\gamma_{\mathrm{CL} 2}, \gamma_{\mathrm{diff} 2}\right.$ and $\left.\mathrm{Ra}_{\mathrm{CL} 2}\right)$ are also shown for comparison.

\begin{tabular}{|l|l|l|l|l|l|l|l|l|l|}
\hline Case & $\mathrm{D}$ & $\mathrm{La}$ & $\gamma$ & $\gamma_{\mathrm{CL}}$ & $\gamma_{\text {diff }}$ & $\mathrm{RaCL}$ & $\gamma_{\mathrm{CL} 2}$ & $\gamma_{\text {diff2 }}$ & RaCL2 \\
\hline 1 & 2 & 0.05 & 0.118 & 0.492 & 0.154 & 6.16 & 0.396 & 0.154 & 6.61 \\
\hline 2 & 2 & 0.1 & 0.045 & 0.455 & 0.308 & 2.18 & 0.396 & 0.308 & 1.65 \\
\hline 3 & 4 & 0.01 & 0.145 & 0.432 & 0.033 & 173.1 & 0.232 & 0.008 & 841 \\
\hline
\end{tabular}

Table 5.1: Scales for forcing and dissipation in finite-amplitude Langmuir cells. Three cases are shown. D is the layer depth, La the Langmuir number, $\gamma$ the growth rate of the most unstable mode with wavelength equal to that of the dominant cell spacing, $\gamma_{C L}$ the Craik-Leibovich instability parameter, $\gamma_{\text {diff }}$ the diffusive decay scale, $\mathrm{Ra}_{\mathrm{CL}}$ the Craik-Leibovich-Rayleigh number predicted using the cell structure given by the most unstable mode. $\gamma_{\mathrm{CL} 2}, \gamma_{\mathrm{diff} 2}$ and RaCL2 are the equivalent quantities predicted by Truncation $\mathrm{T} 2$.

Case 1: Layer depth $D=2$, Langmuir number $L a=0.05$. For this case $\gamma_{C L}>\gamma_{\text {diff }}>\gamma$ for all three approximate representations of the flow field. Since $\mathrm{RaCL}_{\mathrm{CL}}$ is quite a bit larger than 1, the cells are predicted to transport a lot of momentum. Figure 5.1a shows the horizontally averaged downstream velocity profile averaged over nondimensionalized times $\mathrm{T}=200-400$ (about 12-24 hours after the start of the run). The solid lines show the results of the finite-difference code runs. The chaindotted line in Figure 5.1a shows the velocity profile in the absence of cells. The dashed line shows the prediction of the mean velocity profile assuming the perturbations to have a shape given by the instability code, but amplitudes allowed to vary freely. The open circles show the prediction using truncation T1 (cells fill the layer and feel the bottom boundary), while the crosses show the predictions using truncation T2 (cells need not fill the layer and do not feel the bottom boundary).

The horizontally-averaged velocity profile for an idealized mixed layer with finite-amplitude Langmuir cells is much less sheared than the profile without 


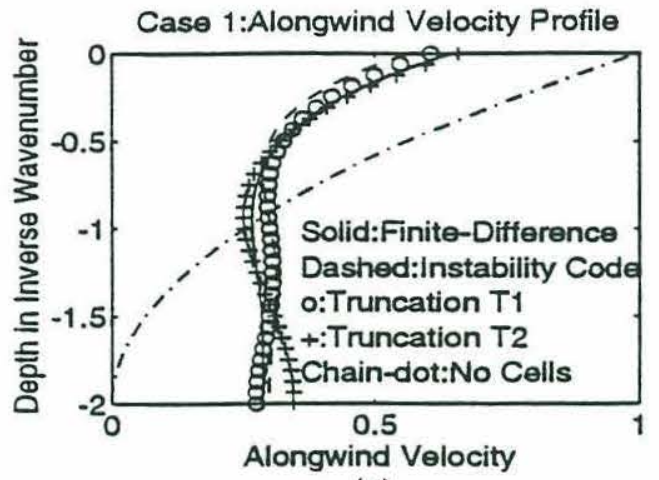

(a)

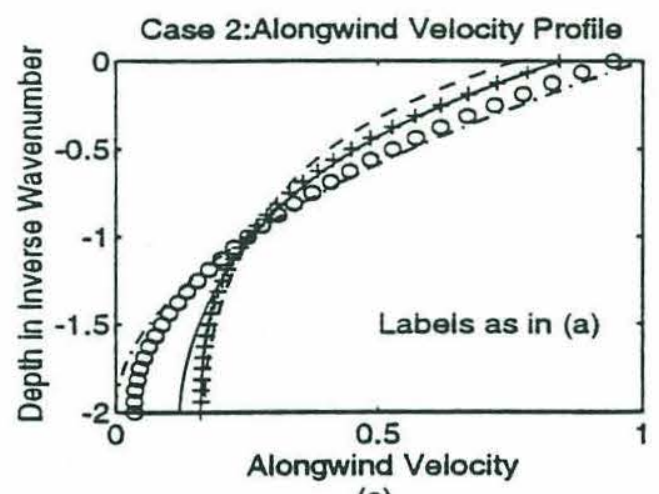

(c)

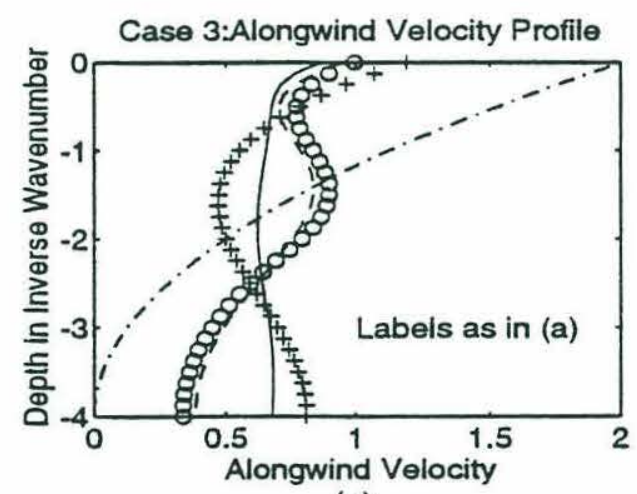

$(\Theta)$

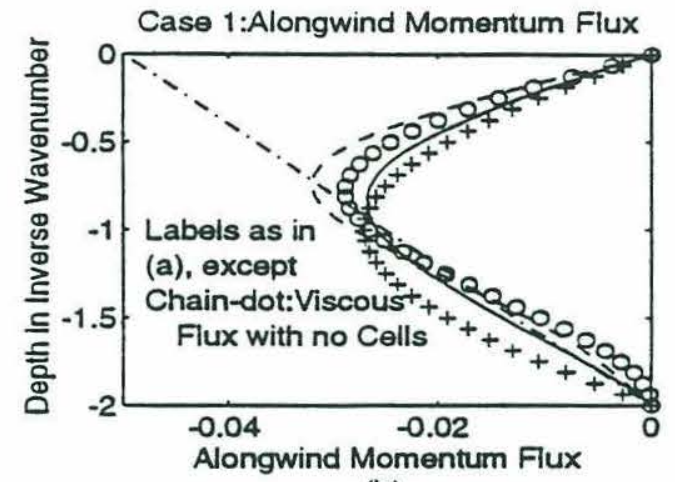

(b)

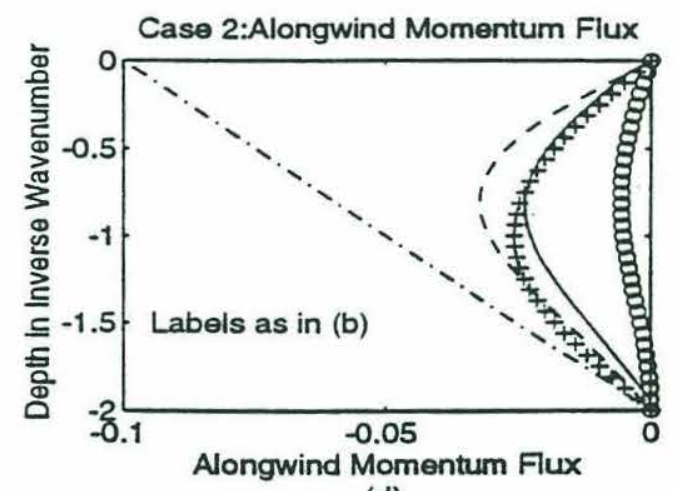

(d)

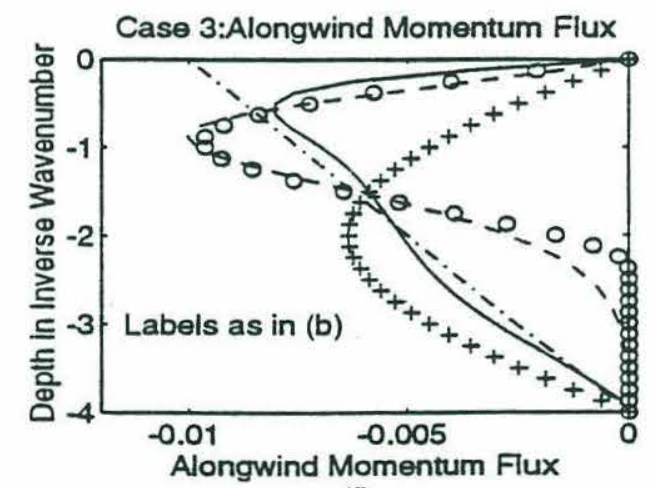

(f)

Figure 5.1: Alongwind velocity in an unstratified layer with finite-amplitude Langmuir cells. All cases have nondimensional surface Eulerian shear of 1 and monochromatic waves. (a) Horizontally averaged alongwind velocity, $\mathrm{La}=0.05$, $\mathrm{D}=2$. Solid: Finite-difference code. Dashed: Solution from equation (5-30) assuming cell structure given by instability code. 0 : Solution assuming cell structure described by Truncation T1 +: Solution assuming cell structure described by Truncation T2. Chain-dotted line is velocity profile in absence of cells. (b) Vertical flux of alongwind momentum $\mathrm{La}=0.05, \mathrm{D}=2$. Labels same as in (a), except that chain-dotted line is the momentum flux carried by viscous stresses in the absence of cells. (c) Same as (a) but for $\mathrm{La}=0.1, \mathrm{D}=2$. (d) Same as (b) but for $\mathrm{La}=0.1, \mathrm{D}=2$. (e) Same as (a) but for $\mathrm{La}=0.01, \mathrm{D}=4$. (f) Same as (b) but for $\mathrm{La}=0.01, \mathrm{D}=4$. 
Langmuir cells. All three approximate representations of the velocity and streamfunction perturbations capture this homogenization of the interior to first order, although there are some qualitative differences between the various predicted profiles. The profile predicted by the finite-difference code and truncation $\mathrm{T} 2$ are essentially identical down to $\mathrm{z}=-1.5$, at which point $\mathrm{T} 2$ shows slightly more upwind velocity than the finite-difference code.

The chain-dotted line in Figure 5.1b shows the total stress at equilibrium, normalized by the surface stress $\left.\mathrm{La} \frac{\partial \mathrm{V}}{\partial \mathrm{z}}\right|_{\mathrm{z}=0}$. The approximate theories do a good job at predicting both the magnitude and the structure of the stress, with the theory based on the instability code and truncation T1 capturing the location of the maximum slightly better than truncation $\mathrm{T} 2$, but slightly overestimating the magnitude of the momentum transport.

Case 2: $\mathrm{D}=2, \mathrm{La}=0.1$. The dominant spacing is $\mathrm{L}=8$ and the cells penetrate from top to bottom. Once again $\gamma_{C L}>\gamma_{\text {diff }}>\gamma$. Now however, $\gamma_{C L}$ is of the same order as $\gamma_{\text {diff }}$ so that the stress carried by the cells should be a great deal smaller than in Case 1 . This is the case. Figures $5.1 \mathrm{c}$ and $5.1 \mathrm{~d}$ show the horizontally averaged velocity and alongwind momentum flux for this case. The velocity range is slightly reduced (of order $20 \%$ ) in the presence of cells, but the general character of the flow does not change.

Two of the three approximate theories (that based on the instability code and Truncation $\mathrm{T} 2$ ) do well at predicting the equilibrium velocity profile, indicating that the finite-amplitude cells are well-approximated by these simplified representations. Because the cells do in fact penetrate from top to bottom with little vertical structure in the velocity perturbation, Truncation T1 turns out not to be a very good representation of the flow field. As a result it underpredicts the effective Rayleigh number and thus underpredicts the stress carried by the cells.

Case 3: $\mathrm{D}=4, \mathrm{La}=0.01$. The dominant spacing is 16 and the cells penetrate from top to bottom. Figures 5.1e and 5.1f parallel 5.1a and b for this scenario. Since $\gamma_{C L}>\gamma \gamma_{\text {diff }}$ the theory predicts that the cells carry almost all the stress in the layer. Figure 5.1f shows that the cells do carry most of the stress. As a result, there is very little shear in the layer at equilibrium except for a very thin layer near the surface.

This case provides another example of how approximate theories fail when the shape of the equilibrium mode is incorrectly chosen. In this case, there are two 
unstable modes and the final equilibrium state is a mixture of the two. The two approximate theories which suppose the cells to be given by the most unstable mode alone fail to capture the shape of the cells and the depth to which they penetrate, as noted in Chapter 4. Note that the simple truncation T1 does an extremely good job at approximating the shape of the most unstable mode predicted by the instability code, as was generally found to be the case in Chapter 2. Truncation T2, however, comes closest to approximating the actual flow field. The difference between the observed and predicted shear profiles has a structure that looks like $\cos (2 \pi \mathrm{z} / \mathrm{D})$, a mode which is not included in the truncations which would represent self-advection by the cells.

The condition that the Craik-Leibovich instability parameter be much larger than the diffusive decay scale gives at least a qualitative prediction of when Langmuir cells are capable of transporting a great deal of momentum within the mixed layer. As $\mathrm{Ra}_{\mathrm{CL}}$ decreases, the cells transport less and less momentum. Quantitatively, the comparison between theory and data is sometimes superb (Figure 5.1a) and sometimes less good (Figure 5.1e). Analysis of why this might be the case shows that the comparisons are in general best when there is only one unstable mode at a given wavenumber. This will in general be true at moderate values of Rayleigh number (of order 1-100).

\begin{tabular}{|l|l|l|l|l|l|l|l|l|l|}
\hline Case & La & $\mathrm{Ri}$ & $\gamma$ & $\gamma_{\text {CLS }}$ & $\gamma_{\text {diff }}$ & RaCLS & $\gamma_{\text {CLS1 }}$ & $\gamma_{\text {diff1 }}$ & RacLS1 \\
\hline 4 & 0.05 & 0.2 & 0.022 & 0.460 & 0.090 & 26.0 & 0.377 & 0.065 & 33.3 \\
\hline 5 & 0.1 & 0.2 & 0.002 & 0.356 & 0.237 & 2.25 & 0.257 & 0.182 & 2.01 \\
\hline 6 & 0.025 & 0.05 & 0.106 & 0.391 & 0.072 & 29.8 & 0.330 & 0.054 & 37.6 \\
\hline
\end{tabular}

Table 5.2: Scales for forcing and dissipation in finite-amplitude Langmuir cells in stratified surface layers. Three cases are shown. La is the Langmuir number, Ri the Richardson number, $\gamma$ the growth rate of the most unstable mode with wavelength equal to that of the dominant cell spacing (16 in all three cases), $\gamma_{\mathrm{CL}}$ the Craik-Leibovich instability parameter, $\gamma_{\text {diff }}$ the diffusive decay scale, $\mathrm{RaCL}$ the Craik-Leibovich-Rayleigh number. The estimates of these last three parameters given by Truncation $\mathrm{T} 1$ are shown for comparison.

The presence of stratification limits the depth of penetration of the cells for at least some finite time. As a result, Truncation T1 is a more appropriate truncation to use. This is illustrated in Figure 5.2. In order to compute the profiles shown, the depth of penetration D' was assumed to be that of the most unstable mode with the observed cell spacing. As noted already, there are problems with 
this approach. Three cases are shown, one for which the qualitative agreement is quite good but the detailed structure is different, and another two for which the agreement between the approximate theory and the full model is excellent. Table 5.2 gives the Langmuir number, Richardson number, growth rate of the most unstable mode and the CL instability parameter $\gamma_{\mathrm{CL}}$, characteristic diffusive decay rate $\gamma_{\text {diff }}$ and stratified Craik-Leibovich Rayleigh number RacLS for the three cases. Estimates of these parameters given by truncation T1 ( $\gamma_{\mathrm{CL} 1}, \gamma_{\mathrm{diff} 1}$, and $\mathrm{Ra}_{\mathrm{CLS}}$ ) are also shown. In general, truncation $\mathrm{T} 1$ does a good job at estimating the approximate size of RaCLS.

Case 4: Layer depth $\mathrm{D}=4, \mathrm{La}=0.01, \mathrm{Ri}=0.2$. For this case $\gamma_{\mathrm{CLS}}$ 》 $\gamma_{\text {diff }}>\gamma$. The finite-amplitude theory predicts that the finite-amplitude cells transport a great deal of momentum and density. Figure 5.2a shows the horizontally averaged velocity profile, $5.2 \mathrm{~b}$ the horizontally averaged density, and $5.2 \mathrm{c}$ the vertical flux of alongwind momentum. The solid lines show the finite-difference code runs, the chain-dotted lines the profiles in the absence of Langmuir cells (density, alongcell velocity, and vertical flux of alongcell velocity carried by small-scale diffusion). The results of the approximate theory developed earlier in this section are shown by the dashed lines (assuming cell structure to be given by the most unstable mode from the full instability code) and o-marks (assuming cell structure to be given by truncation $\mathrm{T} 1$, cells limited in penetration depth).

The prediction made by the approximate theory that the cells alter the velocity and density profile over the depth of penetration is accurate. The presence of stratification results in the creation of a shallow mixed layer with a depth of about 1.5. At some depths, the fluxes carried by the cells exceed those carried in the absence of cells by small-scale diffusion. As a result, there are reversals in the shear and density stratification. The quantitative agreement between the approximate theories and the full finite-difference code is extremely good. A mixed layer is created even though the growth rate of the cells with dominant wavelength is smaller than the characteristic diffusive decay scale for the mixed layer.

Case 5: $\mathrm{D}=4, \mathrm{La}=0.1, \mathrm{Ri}=0.2$. This time $\gamma_{\mathrm{CLS}}>\gamma_{\text {diff }} \gg \gamma$. The velocity profile, momentum transport and density flux are shown in Figure 5.2d,e, and $\mathrm{f}$ respectively. For this case the diffusive term is of the same order of magnitude as the instability parameter. As a result, the total transport of momentum and density 


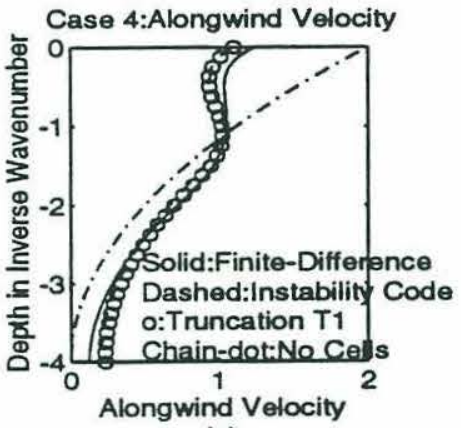

(a)

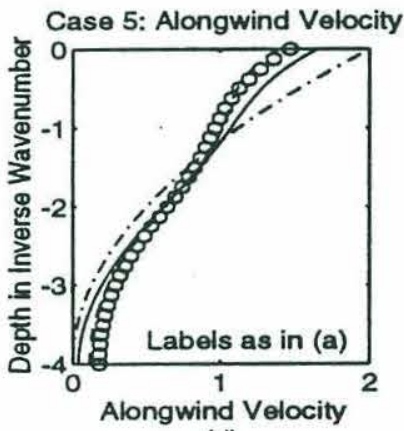

(d)

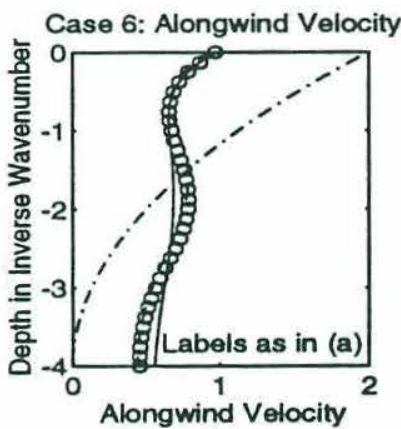

(g)

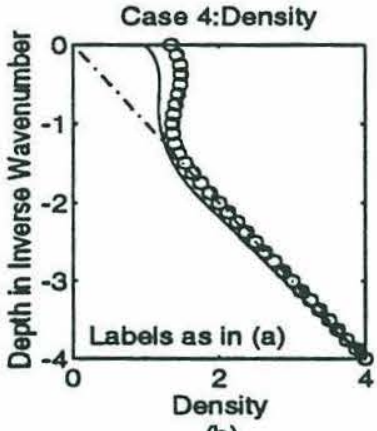

(b)

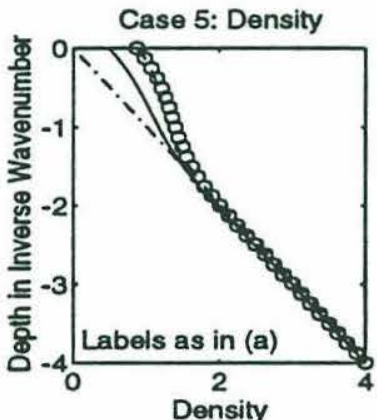

(e)

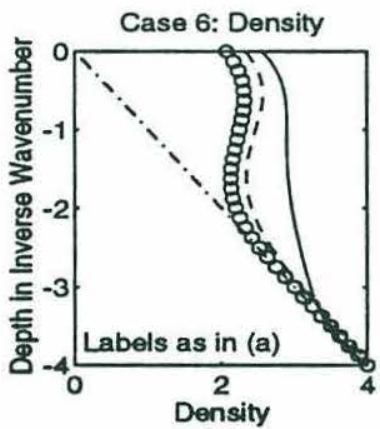

(h)

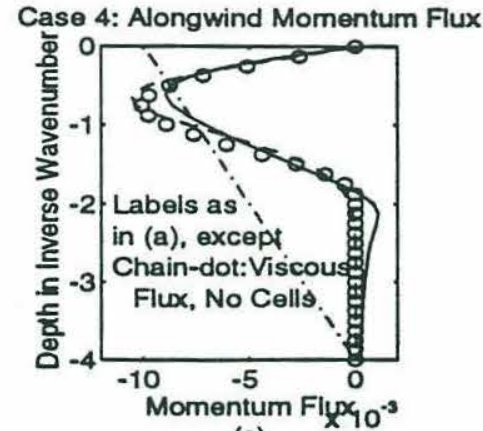

(c)

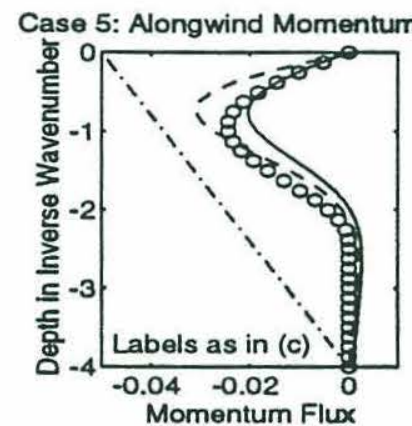

(f)

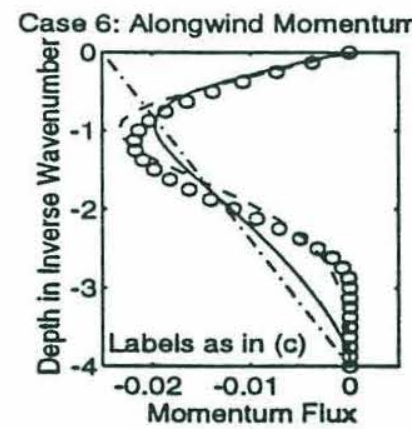

(i)

Figure 5.2: Velocity and density structure in a stratified layer with finiteamplitude Langmuir cells. All cases have surface Eulerian shear of 1 and monochromatic waves. (a) Horizontally averaged alongwind velocity, $\mathrm{La}=0.05$, $\mathrm{Ri}=0.2, \mathrm{D}=4$. Solid: Finite-difference code. Dashed: Solution from equation (5-30) assuming cell structure given by instability code. o: Solution assuming cell structure described by Truncation T1. +: Solution assuming cell structure described by Truncation T2. Chain-dotted line is velocity profile in absence of cells. (b) Density. $\mathrm{La}=0.05, \mathrm{Ri}=0.2, \mathrm{D}=4$. Labels same as in (a), except that chaindotted line is the density in the absence of cells. (c) Vertical flux of alongwind momentum $\mathrm{La}=0.05, \mathrm{D}=2$. Labels same as in (a), except that chain-dotted line is the momentum flux carried by viscous stresses in the absence of cells. (d) Same as (a) but for $\mathrm{La}=0.1, \mathrm{Ri}=0.2, \mathrm{D}=4$. (e) Same as (b) but for $\mathrm{La}=0.1, \mathrm{Ri}=0.2, \mathrm{D}=4$. (f) Same as (c) but for $\mathrm{La}=0.1, \mathrm{Ri}=0.2, \mathrm{D}=4$. (g) Same as (a) but for $\mathrm{La}=0.025$, $\mathrm{Ri}=0.05, \mathrm{D}=4$. (h) Same as (b) but for $\mathrm{La}=0.025, \mathrm{D}=4$. (i) Same as (c) but for $\mathrm{La}=0.025, \mathrm{Ri}=0.05, \mathrm{D}=4$. 
decreases relative to Case 4. Again the theoretical result of equation (5-30) seems to produce not only an excellent qualitative result, but also a excellent quantitative prediction of the mean velocity and density structure. The theory does seem to overestimate the fluxes slightly but the shape and magnitude of the flux profile are well reproduced. The cells seem to have come to equilibrium despite the fact that the growth rates predicted by the instability code are very small.

Case 6: $\mathrm{D}=4, \mathrm{La}=0.025, \mathrm{Ri}=0.05$. In this case $\gamma_{\mathrm{CLS}}>\gamma>\gamma_{\text {diff. }}$ The cells penetrate over most of the depth of the water column. The approximate theory does a good job at predicting the velocity structure but not such a good job at the density. The reason is apparently that the density perturbation is quite poorly represented by the theory (it turns out to be constant over most of the depth with a narrow boundary layer starting at a depth of $z=-3.5$ ). As a result, the density flux is underestimated. In general, the cells carry most of the velocity and density flux over all but the surface region.

The solutions in Figures 5.1 and 5.2 were based on the most unstable modes for cells with the observed wavelength (Method I, above). It is worthwhile to briefly consider solutions based on the neutrally stable solution. For small

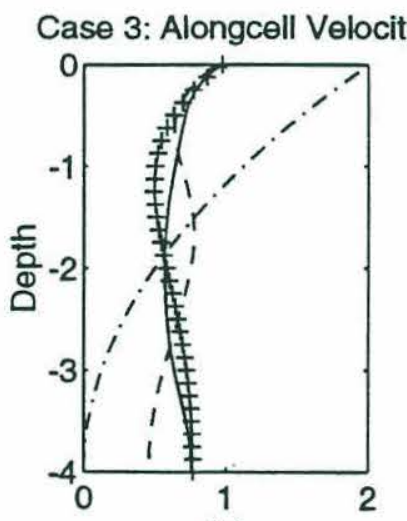

(a)

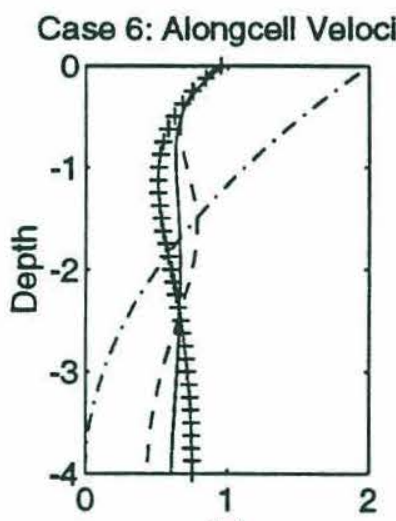

(b)

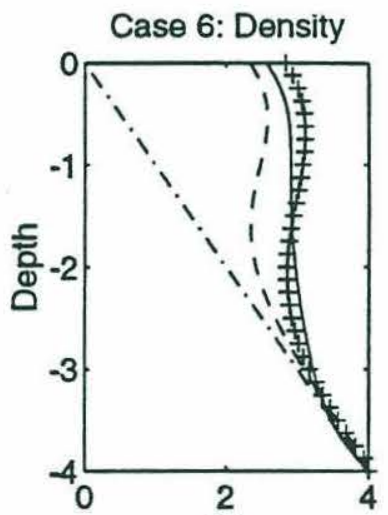

(c)

Figure 5.3: Effect of using different methods of estimating the structure functions when RaCLS is large. Solid lines are solutions from finite-difference code. Chain-dotted lines are solutions in the absence of Langmuir cells. Dashed lines are solutions from equation (5-30) assuming cell structure given by most unstable mode at dominant wavelength, + marks are solutions from (5-30) assuming cell structure given by the neutrally stable mode at the dominant wavelength. (a) Mean Alongcell Velocity: Case 3 (Figure 5.1e) $\mathrm{La}=0.01, \mathrm{Ri}=0.0$, $\mathrm{D}=4$. (b) Mean Alongcell Velocity: Case 6 (Figure 5.2g), $\mathrm{La}=0.025, \mathrm{Ri}=0.05$, $\mathrm{D}=4$. (c) Mean Density: Case 6 (Figure 5.2h). 
values of RacLS, this method asymptotes to Method 1. When RaCLS is large, however, the structure of the neutrally stable solution may be very different from the most unstable mode. Figure 5.3 shows predictions made using the neutrally stable solution for two cases when RaCLS was large, Cases 3 (RaCL somewhere between 150 and 900 ) and 6 ( $R a C L S$ about 30 ). Figure 5.3a shows the mean alongcell velocity profile predicted by Method I (dashed lines) and Method II (+ marks) for Case 3. The solid lines show the solutions from the finite-difference code and the chain-dotted lines show the solutions in the absence of Langmuir cells. Method II appears to do a slightly better job at predicting the horizontally averaged velocity structure than Method I, reflecting the fact that the cells at equilibrium have a deeper penetration than the most unstable mode. Figure 5.3b repeats 5.3a for Case 6. Here the finite-difference code splits the difference between Methods I and II. Figure 5.3c, however, shows that Method II produces a slightly better prediction of the horizontally-averaged density profile in the presence of fully developed Langmuir cells. In general, the differences between Methods I and II are small, except as $\mathrm{Ra}_{\mathrm{CLS}}$ becomes very large.

\subsubsection{Testing the Model Predictions for Shear Scaling}

We now turn to the question of how the vertical shear of the horizontally varying horizontal current (the perturbation shear) scales in the presence of finiteamplitude Langmuir cells. The six case studies from section 5.3.2 are used as test cases. In general, the truncated models did not do a very good job at predicting the shape of the shear, since they did not capture the curvature of the unstable modes. This is hardly surprising since the theory developed in Sections 5.2 and 5.3 is primarily sensitive to the shape of the streamfunction, not necessarily its second derivative. For this reason this section concentrates on comparing the approximate theory which uses the cell shape given by the instability code with the results of the finite-difference code.

The three unstratified cases are considered first. Figure 5.4 shows the shear in the crosswind (left column) and alongwind (right column) direction for Cases 1 ( $\mathrm{La}=0.05, \mathrm{D}=2$, top row), 2 ( $\mathrm{La}=0.1, \mathrm{D}=2$, middle row), and 3 ( $\mathrm{La}=0.01, \mathrm{D}=4$, bottom row).

Case 1: For this case, it has been established that $\mathrm{Ra}_{\mathrm{CL}}$ is roughly 6 (the critical value being 1 ). The approximate nonlinear theory captures a number of the 


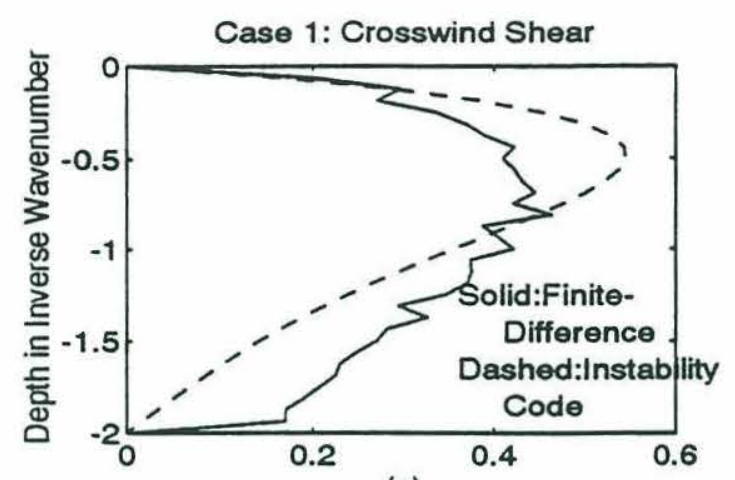

(a)

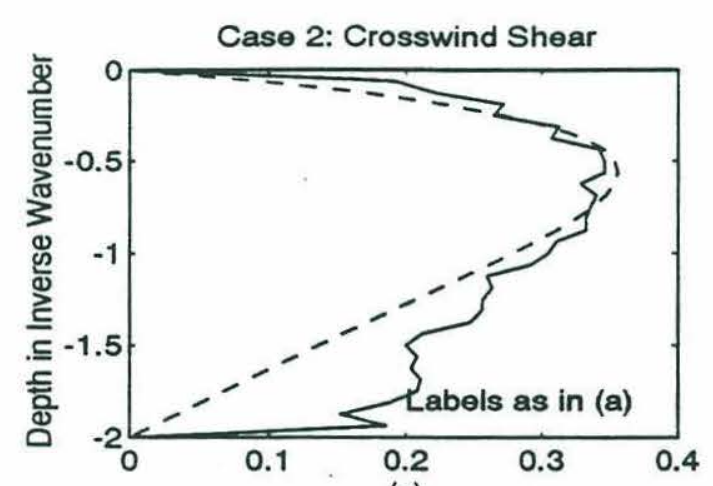

(c)

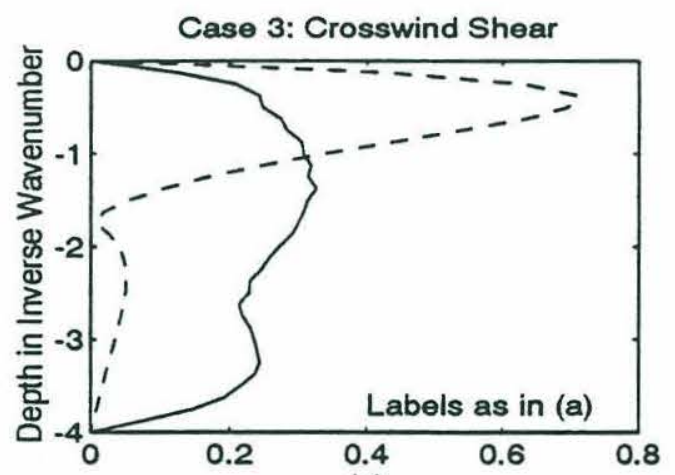

(ө)

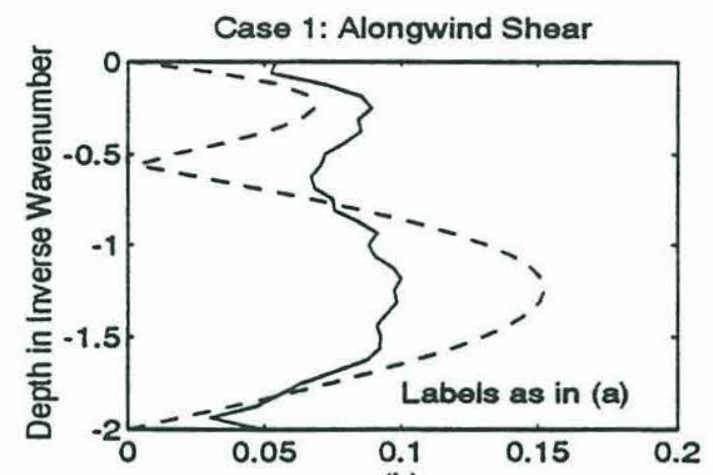

(b)

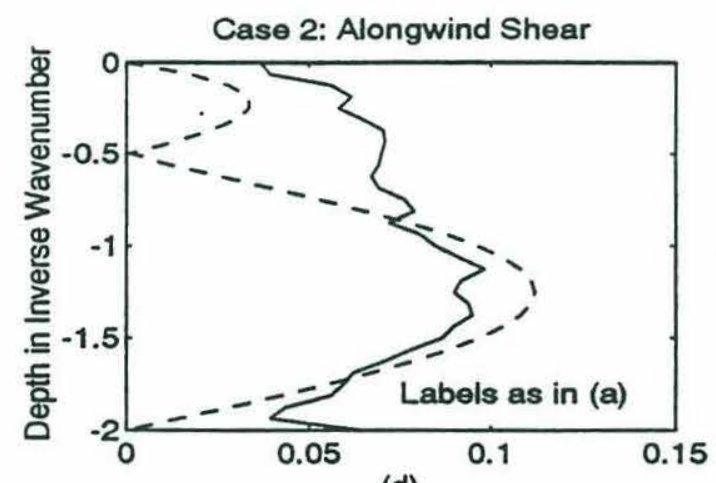

(d)

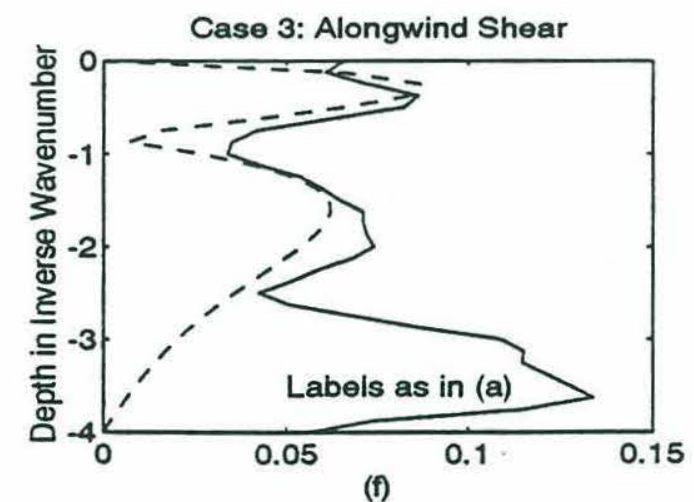

(f)

Figure 5.4: Horizontally varying shear in an unstratified layer with finiteamplitude Langmuir cells. All cases have surface Eulerian shear of 1 and monochromatic waves. Solid: Finite-difference code. Dashed: Solution from equation (5-30) assuming cell structure given by instability code. (a) Standard devation of the crosswind shear, $\mathrm{La}=0.05, \mathrm{D}=2$. (b) Standard deviation of the alongwind shear, $\mathrm{La}=0.05, \mathrm{D}=2$. (c) Standard deviation of the crosswind shear, $\mathrm{La}=0.1, \mathrm{D}=2$. (d) Standard devation of the alongwind shear, $\mathrm{La}=0.1, \mathrm{D}=2$. (e) Standard deviation of the crosswind shear, $\mathrm{La}=0.01, \mathrm{D}=4$. (f) Standard deviation of the alongwind shear, $\mathrm{La}=0.01, \mathrm{D}=4$. 


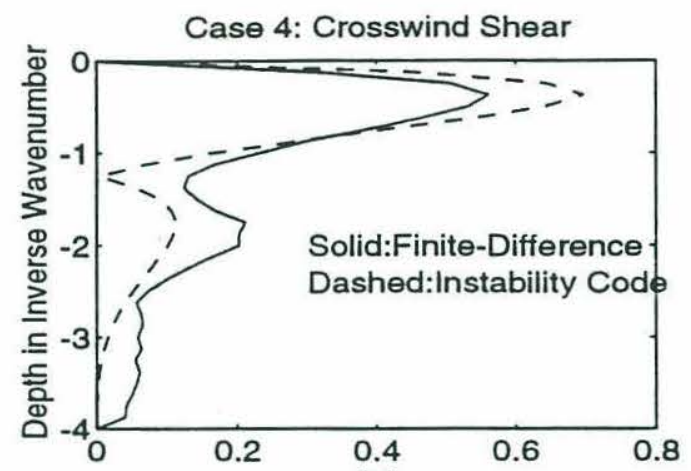

(a)

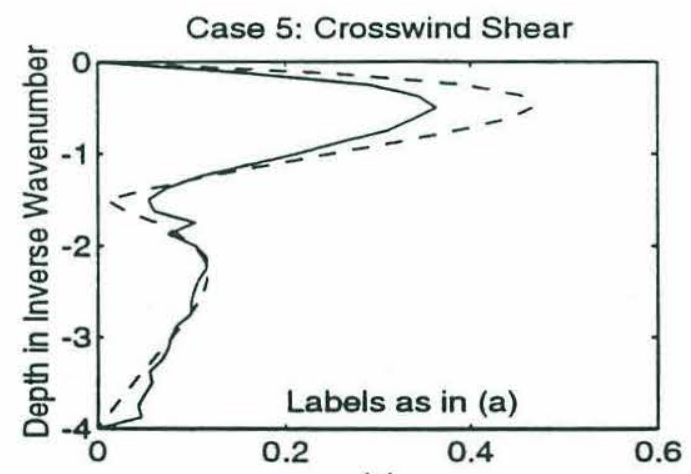

(c)

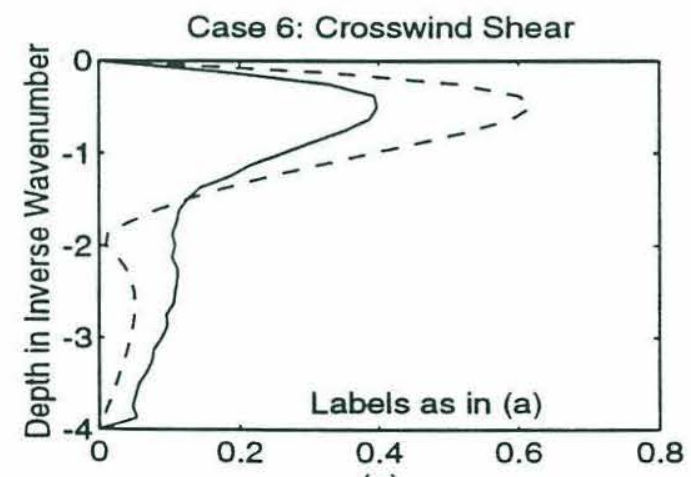

(e)

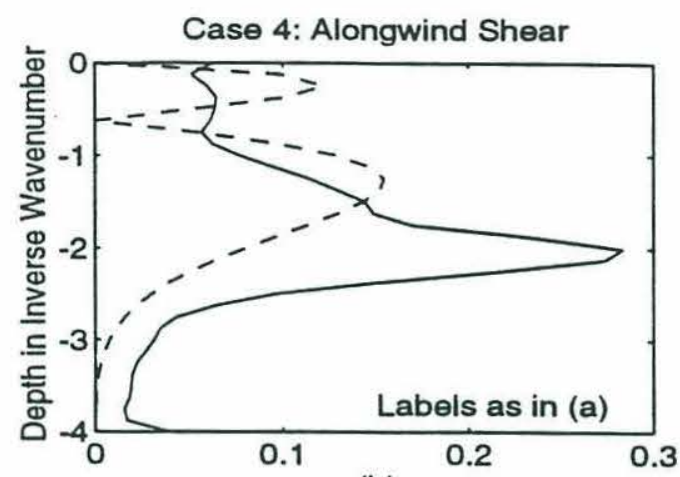

(b)

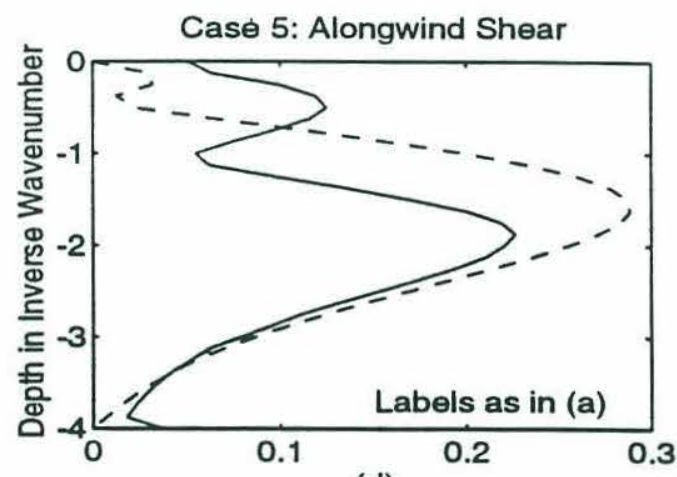

(d)

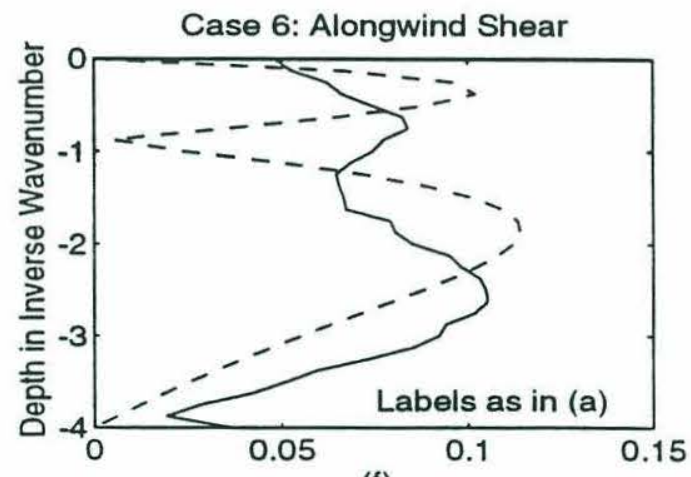

(f)

Figure 5.5: Horizontally varying shear in a stratified layer with finite-amplitude Langmuir cells. All cases have surface Eulerian shear of 1 and monochromatic waves. Solid: Finite-difference code. Dashed: Solution from equation (5-30) assuming cell structure given by instability code. (a) Standard deviation of the crosswind shear, $\mathrm{La}=0.05, \mathrm{Ri}=0.2, \mathrm{D}=4$. b) Standard deviation of the alongwind shear, $\mathrm{La}=0.05, \mathrm{Ri}=0.2, \mathrm{D}=4$. (c) Standard deviation of the crosswind shear, $\mathrm{La}=0.1, \mathrm{Ri}=0.2, \mathrm{D}=4$. (d) Standard deviation of the alongwind shear, $\mathrm{La}=0.1$, $\mathrm{Ri}=0.2, \mathrm{D}=4$. (e) Standard deviation of the crosswind shear, $\mathrm{La}=0.025, \mathrm{Ri}=0.05$, $\mathrm{D}=4$. (f) Standard deviation of the alongwind shear, $\mathrm{La}=0.025, \mathrm{Ri}=0.05, \mathrm{D}=4$. 
qualitative features of the shear seen in the finite-difference code. The overall magnitude of the shear in both directions is captured to within about $30 \%$ as is the fact that the crosswind shear is much more energetic than the alongwind shear. The rough locations of the maxima in the shear are also reproduced by the theory. However the shear in the finite-difference code "fills in" the places where the approximate theory predicts no shear at all.

Case 2: For this case $\mathrm{RaCL}_{\mathrm{CL}}$ is about 2. Again the approximate theory does a good job at reproducing the order of magnitude and the location of the maximum for both the alongwind and crosswind shears. Although the Langmuir number is twice that for Case 1, the amplitude of the crosswind shear only changes by $20 \%$. There is, once again, more shear away from the maximum in the finite-difference code than there is in the model.

Case 3: For this case RaCL is very large, of order $200-800$ and there is more than one linearly unstable mode. Despite this fact, the standard deviation of the crosswind shear is only $25 \%$ larger than for Case 1 . Figure 5.1 showed that the instability code did a poor job at predicting the structure of the momentum transport for this case. The same is true for the shear.

Figure 5.5 repeats Figure 5.4 for cases $4(\mathrm{La}=0.05, \mathrm{Ri}=0.2, \mathrm{D}=4$, top row), $5(\mathrm{La}=0.1, \mathrm{Ri}=0.2, \mathrm{D}=4$, middle row $)$, and $6(\mathrm{La}=0.025, \mathrm{Ri}=0.05, \mathrm{D}=4$, bottom row) in which stratification was important in determining the cell structure. The results parallel those for the unstratified cases. In general, the approximate theory overestimates the level of the crosswind shear by about $20 \%$ and captures the location of the shear maximum. The level of the alongwind perturbation shear is also captured with reasonably fidelity, but the vertical structure of the perturbation shear is much less well captured.

\subsubsection{Conclusions}

The primary results of this section are as follows -For surface layers without Coriolis forces in which RaCLS is large, and Langmuir cells are thus strongly forced, the cells replace small-scale turbulent diffusion as the most important mechanism for momentum and density transport. As a result 
the shear and stratification over depths where the cells are important are sharply reduced.

-When only one unstable mode is allowed for the wavelength which dominates at equilibrium, relatively simple finite-amplitude models of cell structure give good predictions for the horizontally averaged velocity and density structure, given RaCLS between 1 and 100 .

-For RaCLS between about 5-100, the level of the vertical shear of the horizontal velocity scales as $\gamma_{\text {CLS }}$. This result is not strongly dependent on the Langmuir number if the cells are strongly forced. The detailed structure of the shear is not well explained by the finite-amplitude theories.

These major results and their implications are considered on more detail in section 5.5.

A number of points of subsidiary interest are raised by these runs. One of the more interesting ones is that the instability code does give, for moderate values of RaCLS, a prediction of the depth to which the cells can mix in a viscous, stratified surface layer. The depth of the mixing is not only dependent on the stratification, Stokes drift profile, and Eulerian shear profile, but also on the Langmuir number. This may be seen by looking at Cases $4(\mathrm{La}=0.05, \mathrm{Ri}=0.2)$ and $5(\mathrm{La}=0.1, \mathrm{Ri}=0.2)$ in Figure 5.2. Increasing La causes a decrease in the strength of the momentum and density flux, but increases the depth to which mixing occurs. The physics behind this increase, the result of a tradeoff between minimizing the diffusive decay scale and maximizing the Craik-Leibovich instability parameter, are explained in Chapter 2. Such an increase would not be predicted by Method II, which would assume the same cell structure for both cases. The use of Method I, while less rigorous and accurate at high values of $\mathrm{Ra}_{\mathrm{CLS}}$, has some advantages at lower values.

\subsection{Finite-Amplitude Cells in Rotating Surface Layers}

\subsubsection{Linking the Nonrotating Solutions to Rotating Solutions}

The theory developed in section 5.3 can give certain insight into the dynamics of Langmuir cells in mixed layers with Coriolis forces. One of the problems of working with such layers is that one cannot integrate the equations for horizontally averaged momentum momentum at equilibrium

$$
\frac{\partial}{\partial z} \overline{v^{\prime} w^{\prime}}+F\left(\bar{u}+u_{s}\right)=L a \frac{\partial 2 \bar{v}}{\partial z^{2}}
$$




$$
\frac{\partial}{\partial z} \overline{u^{\prime} w^{\prime}}-F\left(\bar{v}+v_{s}\right)=L a \frac{\partial^{2} \bar{u}}{\partial z^{2}}
$$

(where the overbar denotes horizontal averaging) to solve for the shear directly. Two alternatives would be:

1. Using Green's functions to solve for the shear profile in terms of the amplitudes of the finite-amplitude modes.

2. Simplifying the equations yet further by making some assumptions about the final flow.

The first approach is more rigorous, but has the drawback that it does not result in closed form solutions which permit simple insight into the physics governing the final flow. Since one major reason to develop an approximate theory is precisely to obtain such solutions the second method is used below.

Suppose that RaCLS, calculated using the Ekman spirals in the presence of waves derived in Chapter 3, is large. Suppose further that $\mathrm{u}_{\mathrm{s}}$ is small (the cells are oriented roughly parallel with the wind and waves). Then if the cells are the major mechanism for transporting momentum, equation (5-52a) can be replaced with

$$
\frac{\partial}{\partial z} \overline{v^{\prime} w^{\prime}}+F \bar{u}=0
$$

Assuming a slab-like mixed layer, in which the cells account for most of the momentum transport, (5-53) can be integrated to obtain

$$
\overline{\mathrm{v}^{\prime} \mathrm{w}^{\prime}}=\overline{\mathrm{Fu}}(\mathrm{z}+\mathrm{D})=\mathrm{La}(\mathrm{z}+\mathrm{D}) / \mathrm{D}
$$

But this is exactly the stress profile for the nonrotating case. This suggests that in the presence of strongly forced cells, the solution for the horizontally averaged velocity and density structure in a rotating mixed layer will largely be given by that for a nonrotating mixed layer, with the addition of an offset term to account for the wave return flow and the Ekman transport. The perturbation shear in such cases would be expected to scale as in the nonrotating layer, since essentially the same dynamics hold. This would imply, however, that RaCL for the infinitesimal disturbances is not the correct RaCL for the fully developed flow.

Another set of cases where the nonrotating solutions can be used to gain insight into the mean flow and shear is when $\mathrm{La} / \mathrm{F}$ is large. While the solution derived above was basically derived by throwing away the diffusive term, the solution for these cases is derived by essentially neglecting the Coriolis force. For these cases the initial profile is identical to that for the nonrotating case with an offset to account for the Ekman transport and wave return flow. 
Velocity: $\mathrm{La}=\mathrm{F}=.01$ No Waves or Cells

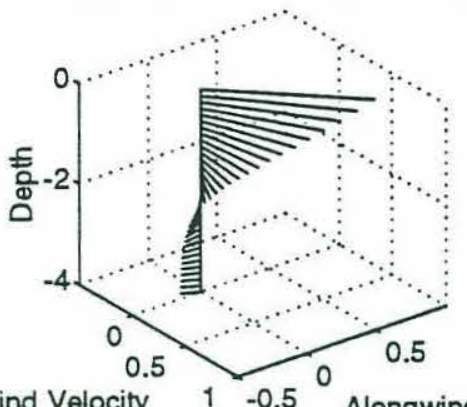

(a)

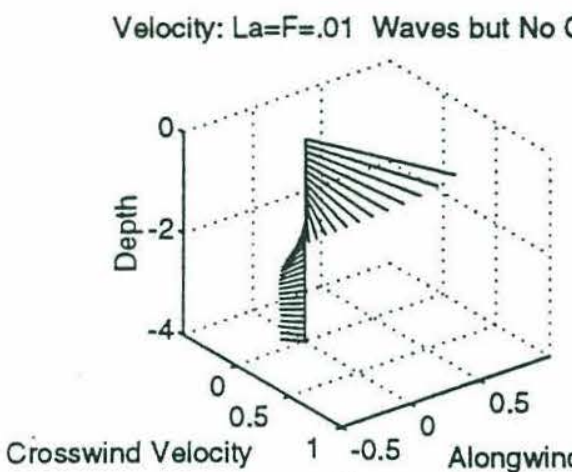

(c)
Shear: $\mathrm{La}=\mathrm{F}=.01$, No Waves or Cells

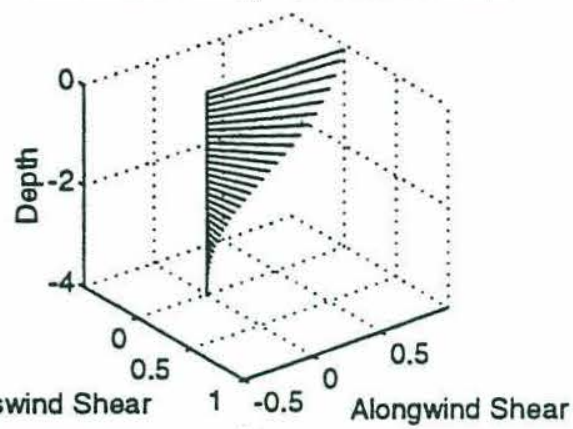

(b)

Shear: $\mathrm{L} a=F=.01$, Waves but No Cells

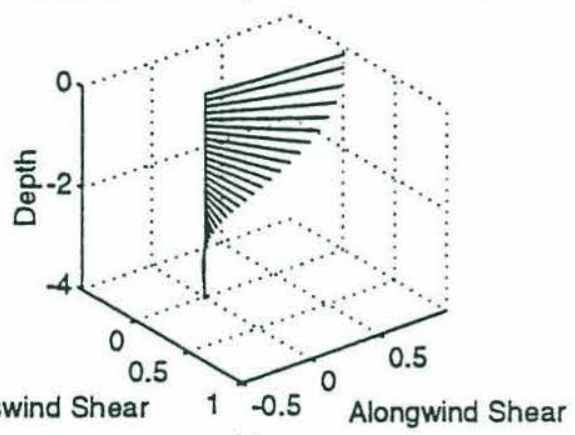

(d)

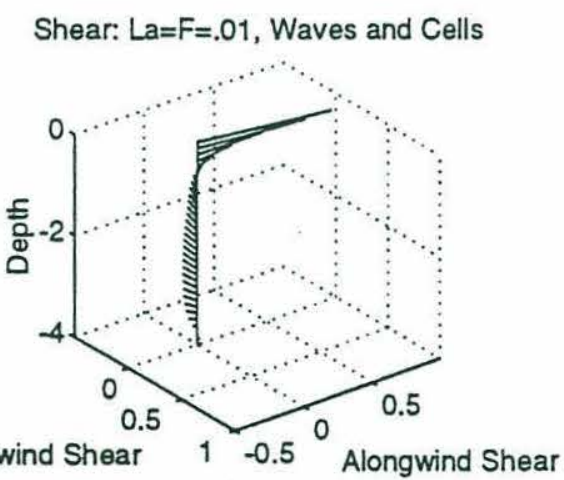

(f)

Figure 5.6: Horizontally averaged velocity and shear in an unstratified layer in the presence of Coriolis forces. All cases shown here have $\mathrm{La}=\mathrm{F}=0.01$ and a surface Eulerian shear of 1 . (a) Velocity in the absence of waves and cells. (b) Shear in the absence of waves and cells. (c) Velocity in the presence of waves but with no cells. (d) Shear in the presence of waves but with no cells. (e) Velocity in the presence of waves and finite-amplitude Langmuir cells. (f) Shear in the presence of waves and finite-amplitude Langmuir cells. 
Both of the arguments outlined above are approximate and sketchy. Somewhat surprisingly, however, they turn out to be valid. This is demonstrated in the following section.

\subsubsection{Momentum and Density Transport in Rotating Surface Layers}

In Section 5.3 it was shown that the necessary condition for Langmuir cells to replace small-scale diffusion as the dominant transport mechanism within a mixed layer with no Coriolis forces is that RaCLS defined using the structure of the linearly unstable mode with the dominant cell spacing be large. This section uses the finite-difference code to argue that the same condition holds for surface layers in the presence of Coriolis forces.

Consider a scenario with $\mathrm{La}=\mathrm{F}=0.01(\mathrm{Ek}=1)$, a layer depth $\mathrm{D}=4$, monochromatic waves and a surface Eulerian shear of 1 . In Chapter 4 it was shown that a steady equilibrium for this scenario was reached in which the cell wavelength was 21.33. Using the current profile in the absence of cells and the structure of the most unstable mode for this wavelength, one can estimate the important scales for this case. The results are shown in the top row of Table 5.3. The Rayleigh number of the most unstable mode with a wavelength of 21.33 is 13.8 , indicating that the cells could transport a good deal of momentum.

Figure 5.6 shows three-dimensional vector plots of the mean velocity and shear for this scenario (the velocity is averaged over an inertial period as well as horizontally). The top row shows the velocity and shear predicted for an Ekman spiral in the absence of waves. The center row shows the velocity and shear predicted for an Ekman spiral in the presence of waves, but not Langmuir cells. The presence of waves introduces a slight upwind shift of the velocity, but does not greatly modify the shear. The bottom row shows the horizontally-averaged Ekman spiral and shear in the presence of waves and cells. The current and shear structures are quite different in the presence of cells. As might be expected from the nonrotating results, the finite-amplitude cells erase the alongcell shear over most of the domain. At depth there is a hint of upwind shear, with water near the base of the surface layer moving more quickly in the downwind direction than water in the middle of the layer. The solution in the presence of finite-amplitude cells shows not only some upwind shear but also crosswind shears which result in the water at depth moving more to the right of the wind than water near the surface. This behavior is discussed in more detail later on in this section. 


\begin{tabular}{|l|l|l|l|l|l|}
\hline $\mathrm{La}=\mathrm{F}$ & $\mathrm{Ri}$ & $\mathrm{L}$ & $\gamma_{\text {CLS }}$ & $\gamma_{\text {diff }}$ & RaCLS \\
\hline 0.01 & 0 & 21.3 & 0.314 & 0.085 & 13.8 \\
\hline 0.025 & 0 & 16 & 0.300 & 0.127 & 5.6 \\
\hline 0.05 & 0 & 21.3 & 0.249 & 0.172 & 2.1 \\
\hline 0.075 & 0 & 8 & 0.335 & 0.300 & 1.2 \\
\hline 0.1 & 0 & 9.2 & 0.294 & 0.306 & 0.9 \\
\hline 0.01 & 0.05 & 16 & 0.253 & 0.087 & 10.8 \\
\hline 0.01 & 0.015 & 8 & 0.231 & 0.102 & 5.1 \\
\hline 0.01 & 0.5 & 2 & 0.388 & 0.406 & 0.9 \\
\hline 0.01 & 1.0 & Stable & Stable & Stable & Stable \\
\hline
\end{tabular}

Table 5.3: Estimates of the critical parameters for five cases with identical shear and Stokes drift profiles but different values of Langmuir number $\mathrm{La}=\mathrm{F}$ the scaled Coriolis parameter. $\mathrm{Ri}$ is the Richardson number. $\mathrm{L}$ is the wavelength of the dominant mode. The Craik-Leibovich instability parameter $\gamma_{C L S}$, the characteristic diffusive decay scale $\gamma_{\text {diff }}$, and the Craik-Leibovich Rayleigh number RaCLS are calculated using the most unstable mode with length $\mathrm{L}$.

Increasing the Langmuir number and the scaled Coriolis parameter together while keeping the shear profile constant decreases the strength of the momentum transport carried by the cells (physically, changing La and $\mathrm{F}$ together corresponds to changing the wave amplitude). In Table 5.3 it can be seen that as $\mathrm{La}=\mathrm{F}$ increases RacLS drops. Figure 5.7 illustrates the corresponding drop in the momentum transport by showing profiles of the mean shear, averaged over the final inertial period of each of the unstratified runs reported in Table 5.3. As RacLS drops the shear approaches that in the absence of cells. Again, the CraikLeibovich Rayleigh number computed from the linearly unstable cells is a good diagnostic of whether or not Langmuir cells make an important contribution to keeping the mixed layer mixed.

Stratification also reduces the momentum transport. Figure 5.8 shows the effect of increasing $\mathrm{Ri}$ on the scenario with $\mathrm{La}=\mathrm{F}=0.01$. As noted in Table 5.3, the effect of increasing the stratification is to cause the diffusive decay scale to increase as the cells become trapped closer and closer to the surface. Figure 5.8a shows the shear profile for $\mathrm{Ri}=0$, Figures $5.8 \mathrm{~b}-\mathrm{d}$ illustrate how the horizontallyaveraged shear approaches that in the absence of cells as Ri increases and RaCLS decreases. Note that even a small amount of stratification $(\mathrm{Ri}=0.05$, a case shown 


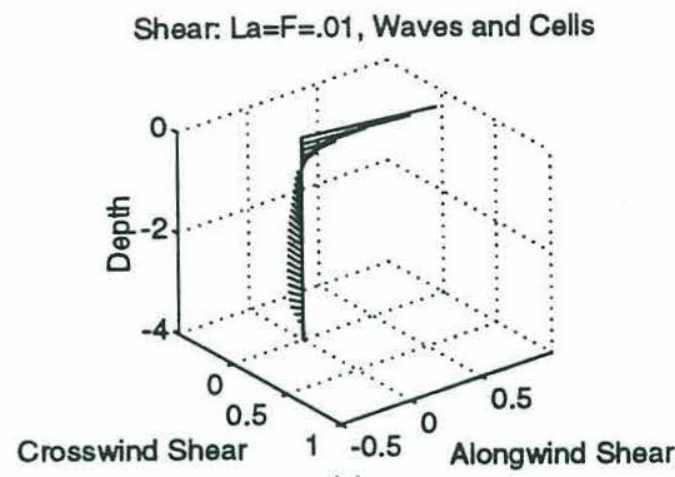

(a)

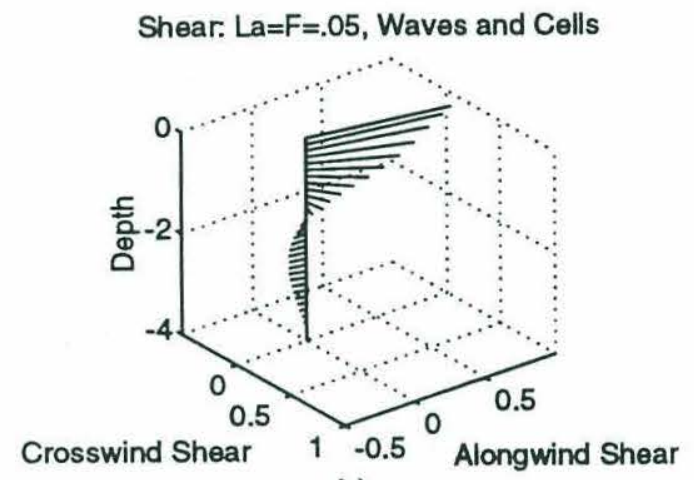

(c)

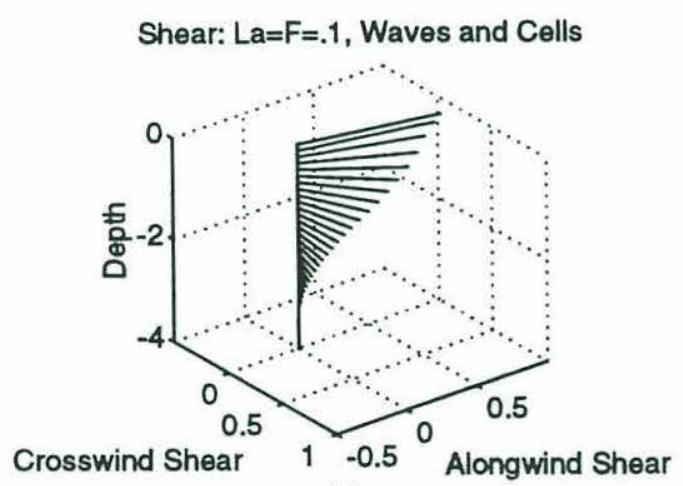

(e)

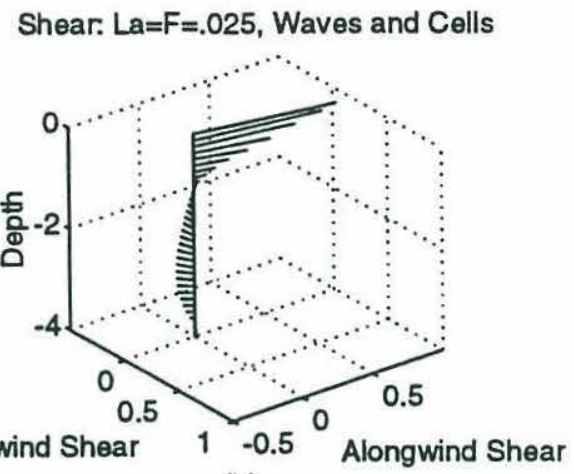

(b)

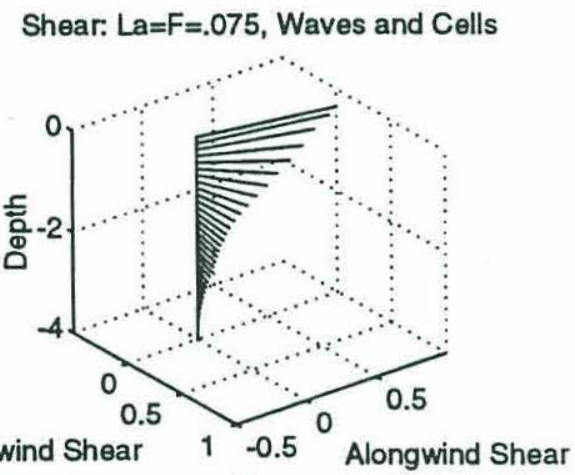

(d)

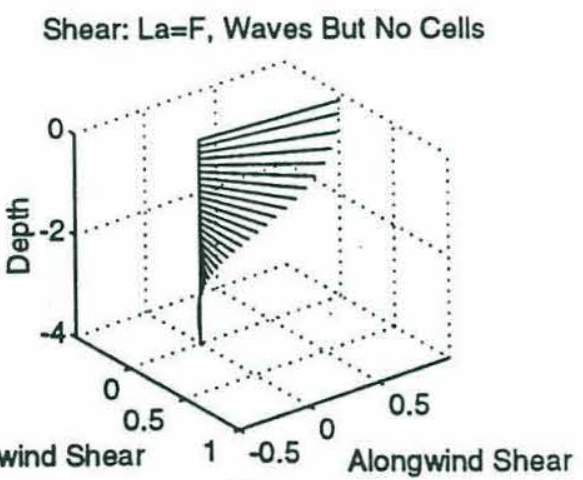

(f)

Figure 5.7: Horizontally averaged shear in an unstratified surface layer in the presence of finite-amplitude Langmuir cells. This figure demonstrates how increasing $\mathrm{La}$ (and thus decreasing RaCLS) results in reducing the momentum transport. All cases shown here have monochromatic waves and a surface Eulerian shear of 1. (a) $\mathrm{La}=\mathrm{F}=0.01$. (b) $\mathrm{La}=\mathrm{F}=0.025$. (c) $\mathrm{La}=\mathrm{F}=0.05$. (d) $\mathrm{La}=\mathrm{F}=0.075$. (e) $\mathrm{La}=\mathrm{F}=0.1$. (f) $\mathrm{La}=\mathrm{F}$, No cells present. 


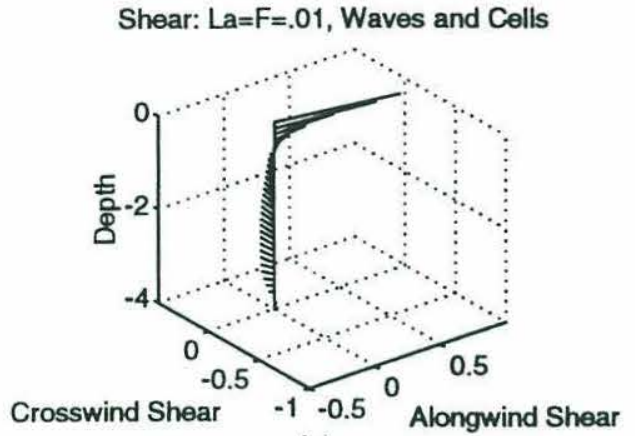

(a)
Shear: $\mathrm{La}=\mathrm{F}=.01, \mathrm{Ri}=0.05$, Waves and Cells

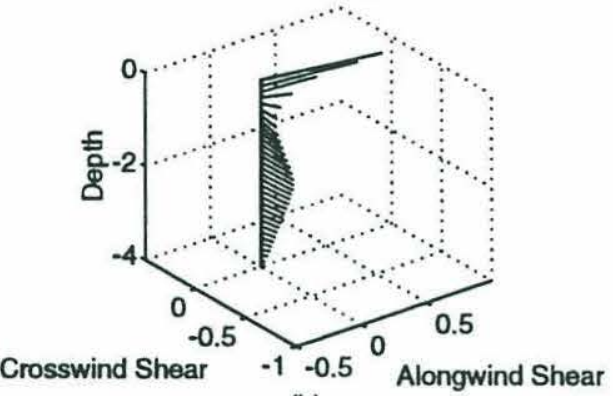

(b)
Shear: $\mathrm{La}=\mathrm{F}=.01, \mathrm{Ri}=0.15$, Waves and Cells

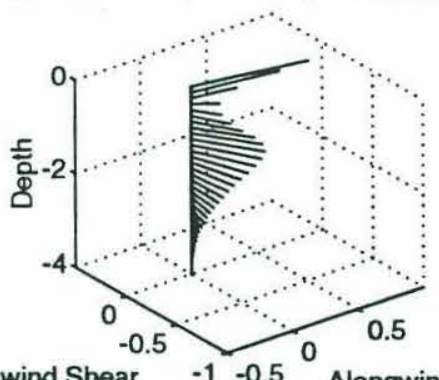

(c)
Shear: $\mathrm{La}=\mathrm{F}=.01, \mathrm{Ri}=0.5$, Waves and Cells

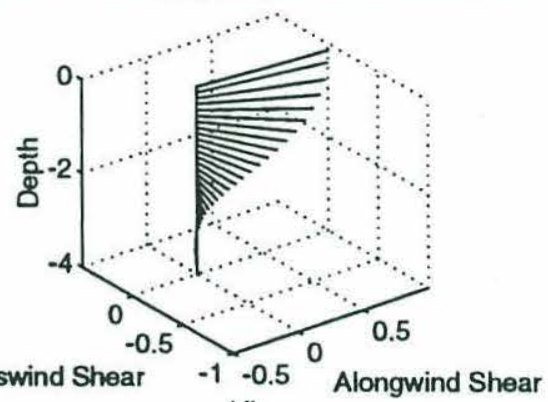

(d)

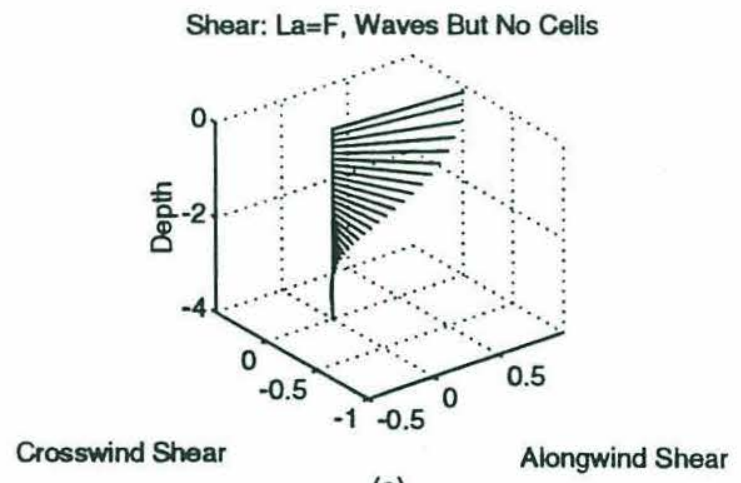

(e)

Figure 5.8: Horizontally averaged shear in a stratified surface layer in the presence of finite-amplitude Langmuir cells. This figure demonstrates how increasing $\mathrm{Ri}$ (and thus decreasing RacLS) results in reducing the momentum transport. All cases shown here have monochromatic waves, a surface Eulerian shear of 1 , and $\mathrm{La}=\mathrm{F}=0.01$. (a) $\mathrm{Ri}=0.0$. (b) $\mathrm{Ri}=0.05$. (c) $\mathrm{Ri}=0.15$. (d) $\mathrm{Ri}=0.5$. (e) No cells present. 
in Figure 4.15a) results in changing the shear below a nondimensional depth of 1.5 from lying to the left of the wind to lying to the right of the wind. For $\mathrm{Ri}=0.15$ (shown in Figure 4.15b-e) the shear below a depth of about 2 is basically identical to that in the absence of cells, while above that depth the cells transport momentum and density.

Another way of suppressing the momentum transport is to increase $\mathrm{F}$ while keeping La constant. Increasing F reduces the Ekman depth, and thus traps the unstable modes closer and closer to the surface, increasing $\gamma_{\text {diff }}$ and decreasing $\mathrm{Ra}_{\mathrm{CL}}$. Figure 5.9 demonstrates this effect. Three-dimensional shear stick plots are shown for $\mathrm{La}=0.01, \mathrm{~F}=0.025$ (top row), 0.05 (middle row), and 0.075 (bottom row). The left-hand column shows the shear in the absence of cells, while the right-hand column shows the shear in the presence of cells. One can compute RaCLS using the structure of the most unstable mode predicted by the instability code given the dominant wavelength from the finite-difference code. The resulting RaCLS is 7.3 for $\mathrm{F}=0.025,2.5$ for $\mathrm{F}=0.5$, and 1.3 for $\mathrm{F}=0.075$. As expected, for $\mathrm{F}=0.025$ the shear is essentially erased by the finite-amplitude cells, which take over from the small-scale viscosity the task of transporting the momentum. For $\mathrm{F}=0.05$ the effect of the cells is still present but much weaker, and for $\mathrm{F}=0.075$ it is negligible.

This section concludes by arguing that the mean structure of a surface layer with Coriolis forces can sometimes be predicted by looking at the structure of a surface layer without Coriolis forces. Figure 5.10a and b compare the mean velocity structure for two idealized surface layers, one with $\mathrm{F}=0$ (denoted by open circles) and the other with $\mathrm{F}=0.01$ (denoted by solid lines), given waves which are monochromatic and parallel to the wind and a surface Eulerian shear of 1. Figure $5.10 \mathrm{a}$ shows the mean alongwind velocity profile from two runs where $\mathrm{D}=4$, the surface shear is 1 and $\mathrm{La}=0.01$ (the solution for $\mathrm{F}=0$ is offset so that the two profiles have the same mean). Figure $5.10 \mathrm{~b}$ shows the perturbation shear in the crosswind direction from the two runs. This is a very strongly forced case. The vertical structure of both the velocity profile and the perturbation shear is very similar for the rotating and nonrotating cases, as predicted at the start of this section. This is despite the fact, noted in Chapter 4, that the presence of rotation suppresses cell merging. As noted in Section 5.3, the level of the perturbation shear produced by long-wavelength cells is relatively insensitive to the length scale responsible for the forcing. This result supports the hypothesis advanced at the beginning of this section that when a rotating surface layer is unstratified and 


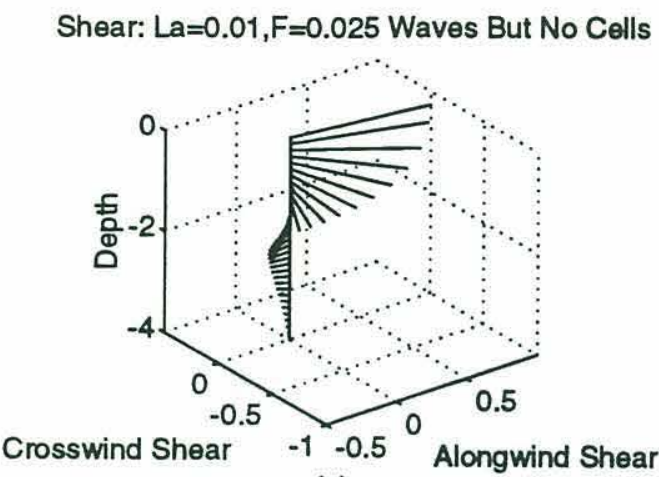

(a)

Shear: $L a=0.01, F=0.05$, Waves But No Cells

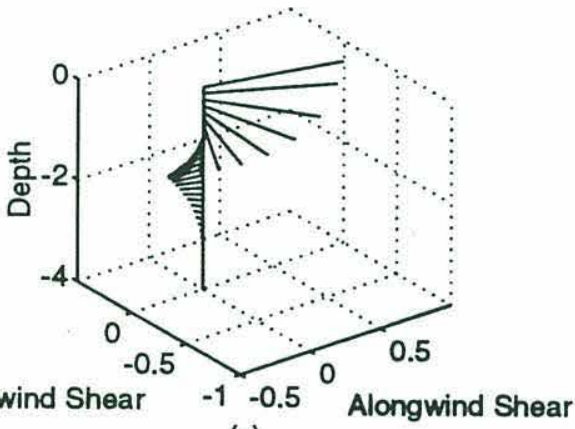

(c)

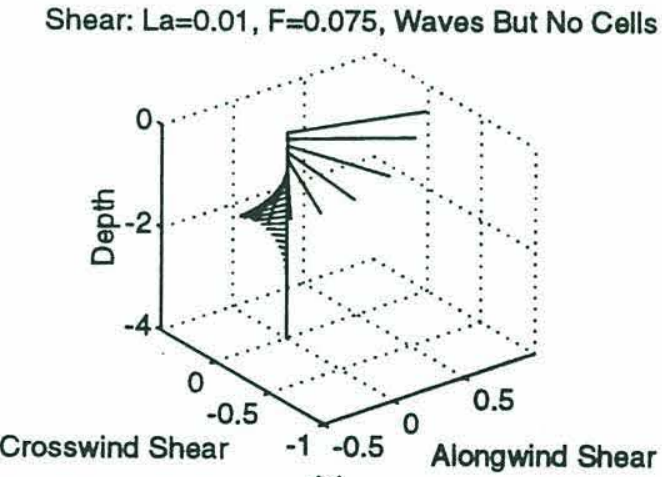

(e)

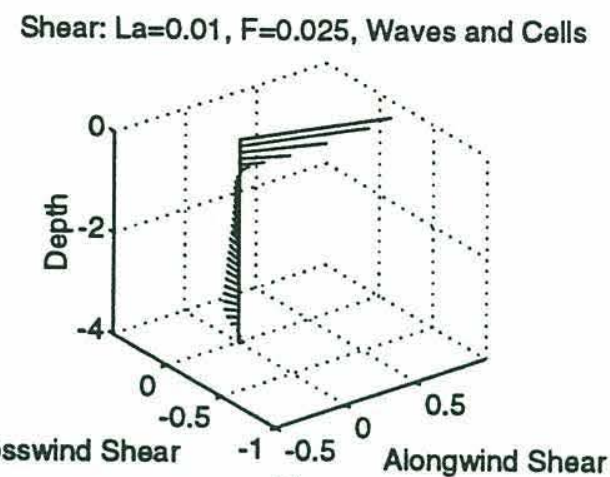

(b)

Shear: $\mathrm{La}=.01, \mathrm{~F}=0.05$, Waves and Cells

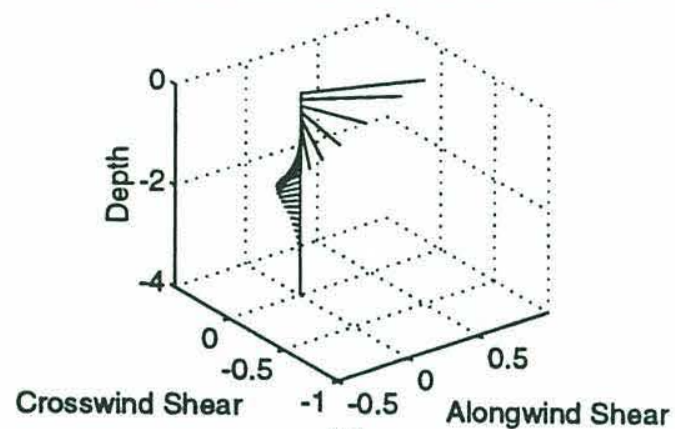

(d)

Shear: $L a=0.01, F=0.075$, Waves and Cells

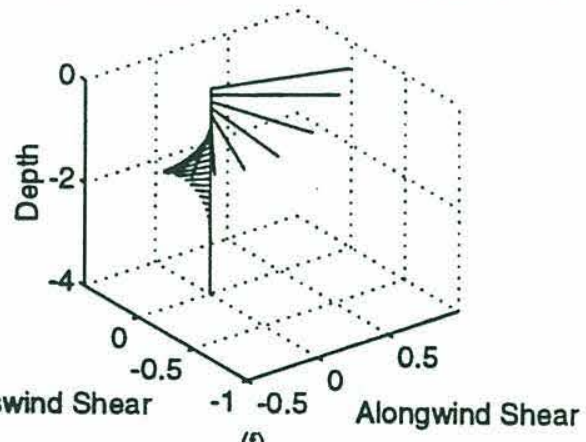

(f)

Figure 5.9: Horizontally averaged shear in an unstratified surface layer in the presence of finite-amplitude Langmuir cells. This figure demonstrates how increasing $\mathrm{F}$ results in trapping the shear closer to the surface, decreasing $\mathrm{Ra}_{\mathrm{CL}}$ and thus decreasing the momentum flux. All cases shown here have monochromatic waves, a surface Eulerian shear of 1 and $\mathrm{La}=0.01$. (a) Shear with waves but no cells, $F=0.025$. (b) Shear with finite-amplitude cells, $F=0.025$. (c) Shear with waves but no cells, $F=0.05$. (d) Shear with finite-amplitude cells, $F=0.05$. (e) Shear with waves but no cells, $F=0.075$. (f) Shear with finiteamplitude cells, $\mathrm{F}=0.075$. 


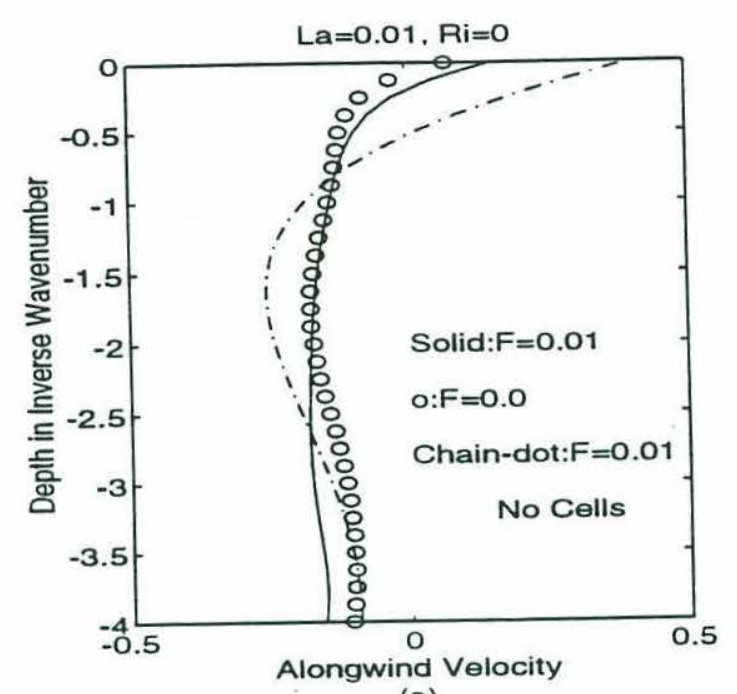

(a)

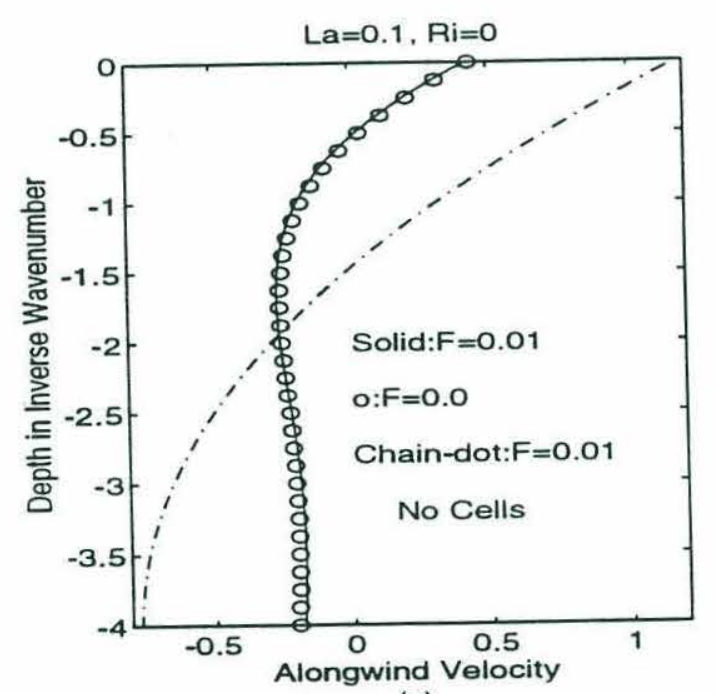

(c)

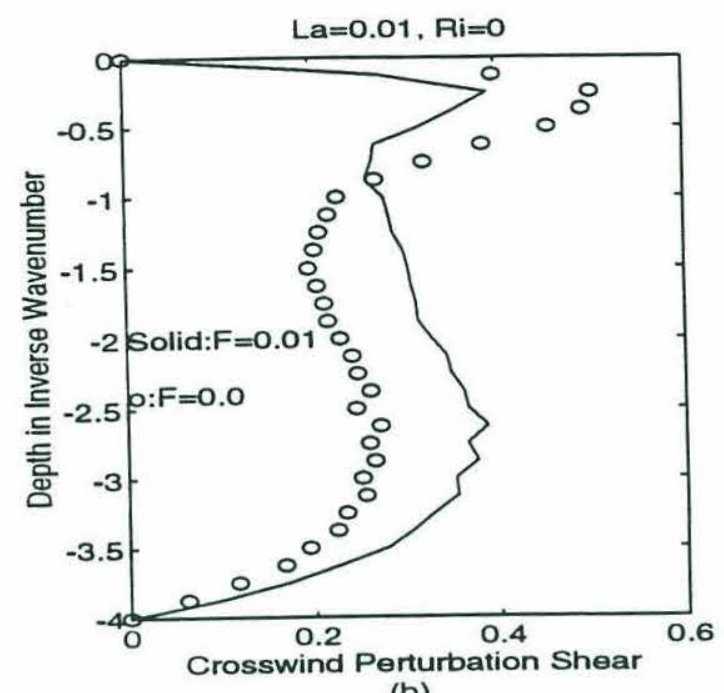

(b)

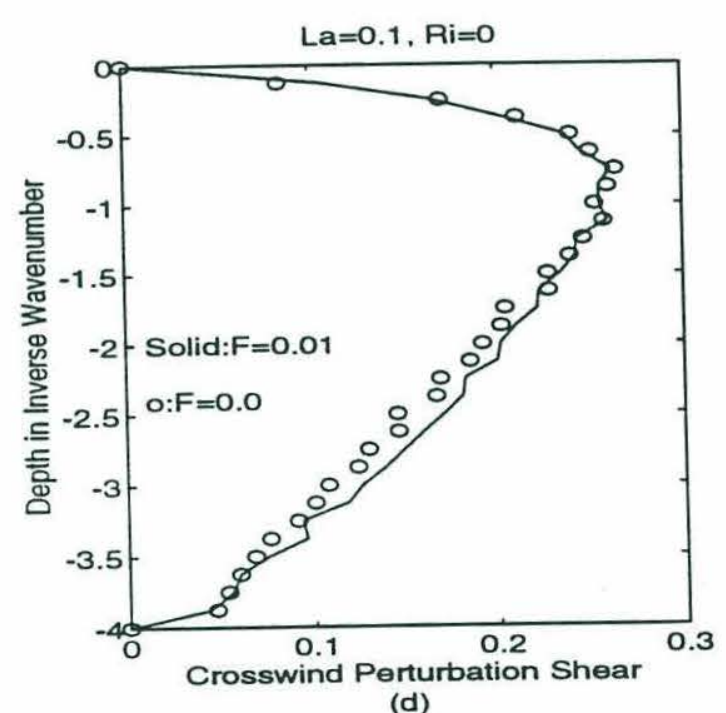

(d)

Figure 5.10: Comparing runs with rotation with runs without rotation. All cases shown are unstratified with monochromatic waves and a surface Eulerian shear of 1. Results for $F=0.01$ are shown as solid lines, results for $F=0$ as open circles. Velocity in the absence of cells is shown by chain-dotted line. Velocity for $F=0$ has been offset for sake of comparison. (a).Horizontally averaged alongwind velocity. $\mathrm{La}=0.01$. (b) Perturbation crosswind shear, $\mathrm{La}=0.01$. (c) Horizontally averaged alongwind velocity, $\mathrm{La}=0.1$. (d) Perturbation crosswind shear $\mathrm{La}=0.1$. 
$\mathrm{Ra}_{\mathrm{CL}}$ is large, the final velocity profile and vertical structure of the perturbation shear resemble those in the absence of rotation.

Figures $5.10 \mathrm{c}$ and $5.10 \mathrm{~d}$ repeat Figures 5.10 and $\mathrm{b}$ for $\mathrm{La}=0.1, \mathrm{Ri}=0$. For these cases $\mathrm{Ra}_{\mathrm{CL}}$ is estimated to be 7.8. In this case the initial velocity profile in the presence of rotation is essentially identical to that in the absence of rotation with an offset to account for the wave return flow and Ekman transport. In Chapter 4, it was shown that the solutions for this scenario in the presence of rotation are qualitatively somewhat different from those in the absence of rotation, with the cells in the presence of rotation showing a distinct handedness (cf. Figure 4.13). Nonetheless, the vertical structure of the perturbation shear and horizontally averaged alongwind velocity are essentially identical to that in the absence of rotation. Thus in the two limits where $\mathrm{Ra}_{\mathrm{CL}}$ is large and Ek is large, the nonrotating scaling for shear seems to hold.

\subsubsection{Discussion of the Rotating Results}

The most important results from this section parallel those for the nonrotating case. Cells which are strongly forced transport large amounts of density and create horizontally varying shears which are the same order of magnitude as the mean shears in the absence of cells. The most important implications of these results will be discussed in detail in the final section of this chapter. At this point one result of interest is highlighted.

The creation of horizontally averaged crosscell shears was seen in one case presented in this chapter (Figure 5.4) for which RaCL was large. It is interesting to note that similar behavior was observed in a number of model runs (including some with $\mathrm{F}=0$ ) when $\mathrm{RaCL}$ of the final state was larger than 100-200. The creation of such shears has not been studied in detail, since the analysis of their formation involves an even more detailed stability analysis. At present there is reason to believe that the creation of such crosswind shears is similar to that seen by Krishnamurti and Howard (1981) in lab experiments involving RayleighBenard convection. The mechanism by which such shears form was discussed in Howard and Krishnamurti (1986). It involves the tilting of cells by the mean crosscell shear. The tilted cells then have correlated crosscell velocities $u^{\prime}$ and vertical velocities w'. The nonlinear stress u'w' carried by these cells reinforces the crosscell shear which then tilts the cells yet more. An equilibrium is reached when u'w' $\frac{\partial \mathrm{U}}{\partial \mathrm{z}}$ balances $\mathrm{La}\left(\frac{\partial \mathrm{U}}{\partial \mathrm{z}}\right)^{2}$. 


\subsection{Conclusions}

In Chapters 2 and 3 the presence of Craik-Leibovich instability in mixed layers with stratification and Coriolis force was shown to depend on the stratified Craik-Leibovich instability parameter being larger than diffusive decay, tilting due to crosscell shear, and the Coriolis frequency. This chapter demonstrates that when the cells are strongly forced:

- They replace small-scale diffusion as the principal transport mechanism for velocity and density. The result is to homogenize the velocity structure in the mixed layer in the alongcell direction relative to that predicted by assuming smallscale mixing alone. In some cases, the cells produce upwind shears at depth.

- There are spatially varying shears within the layer which go (roughly) as the stratified Craik-Leibovich instability parameter.

Simple truncations which approximate the vertical structure of the velocity, density, and streamfunction perturbations are capable of giving excellent predictions of the horizontally averaged velocity and density structure in the presence of finite-amplitude cells as long as RaCLS is of order 1-30 times the critical value.

These results have a number of implications for observing cells in oceanic surface layers. Firstly, they point to the importance of the velocity and density profile in the absence of cells for understanding the finite-amplitude solution in the presence of cells. These profiles are determined by the external forcing and by the strength of the small-scale mixing.

A second important implication of these results relates to how the cells come to equilibrium. The basic cycle leading to Craik-Leibovich instability is as follows:

1. The vertical velocity associated with the cells acts on the vertical shear of alongcell velocity to produce horizontal perturbations in alongcell velocity. 2 . The perturbations in the alongcell velocity reinforce the cells through the Craik-Leibovich instability mechanism.

In the theory developed above the finite-amplitude cells reduce the vertical shear of the alongcell velocity. This limits the size of the perturbations in alongcell velocity which are produced by the cells. This in turn limits the magnitude of the vorticity in the cells themselves.

The weak dependence of the perturbation shear on the Langmuir number and RaCLS is also an important point, since in the field the Langmuir number is 


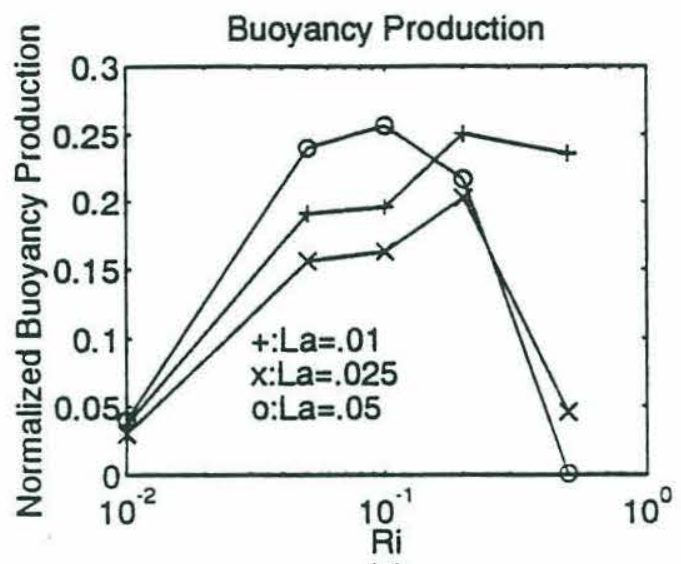

(a)

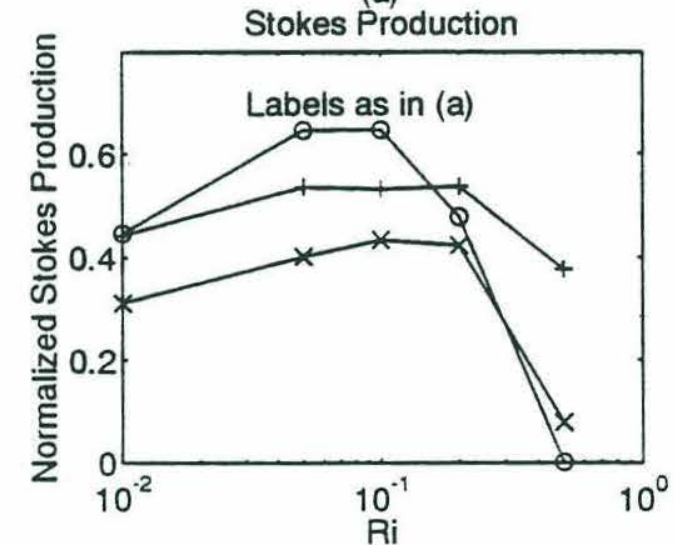

(c)

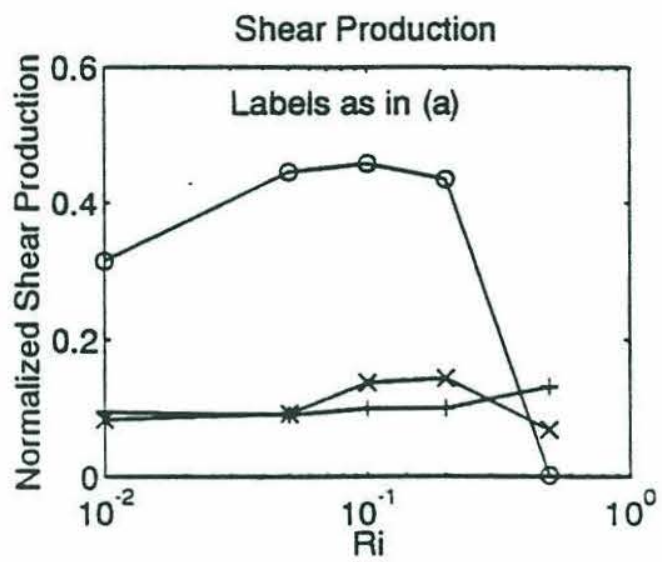

(b)

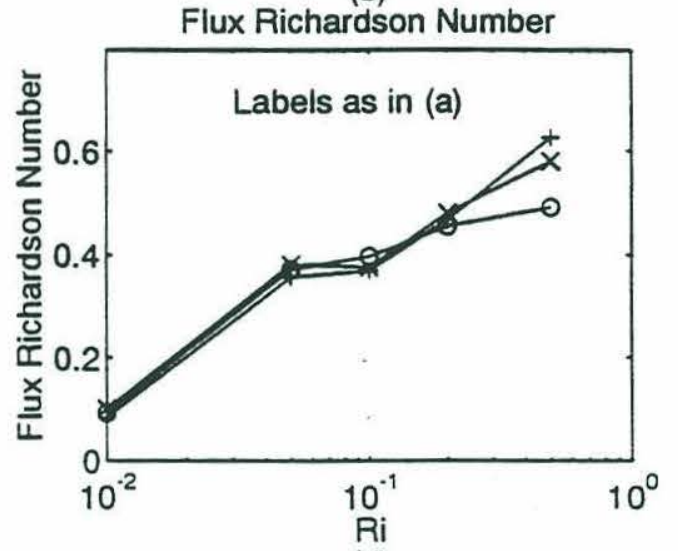

(d)

Figure 5.11: Energy balance for finite-amplitude Langmuir cells. All cases shown are for monochromatic waves, a surface Eulerian shear of 1 and a layer depth of 4 . Horizontal axis is Ri. Three different values of La are shown, 0.01 (denoted by +), 0.025 (denoted by $\mathrm{x}$ ), and 0.05 (denoted by o). (a) Buoyancy transport nondimensionalized by surface stress times the surface Stokes drift. (b) Shear production nondimensionalized by surface stress times the surface Stokes drift. (c) Stokes production nondimensionalized by surface stress times the surface Stokes drift. (d) Flux Richardson number (defined as buoyancy transport over Stokes production). 
extremely difficult to estimate. Examination of truncated, finite-amplitude solutions shows that a number of other parameters such as the kinetic energy , alongcell velocity perturbation, and vertical velocity depend strongly on the cell spacing and Langmuir number. The fact that the shear is insensitive to the exact value of the Langmuir number makes it a good candidate for an index of cell strength which can be compared with theory. This is done in Chapters 6 and 7 with marked success. A related implication of importance, made in Chapters 2 and 3 but which deserves to be reiterated here, is that the key shears for determining cell dynamics are not those right at the surface but rather those at depths where the cells transport momentum and density.

Instability codes and truncated models which capture the cell structure are able to predict RaCLS. This means that such models can not only be used to test whether the water column is unstable to Langmuir cells, but whether or not the unstable cells will replace small-scale diffusion as the primary transport mechanism when they grow to finite-amplitude.

A final implication of these results is the introduction of a new source of energy for turbulent mixing within the oceanic mixed layer. Earlier sections of this chapter showed that when $\mathrm{Ra}_{\mathrm{CL}}$ is large, the momentum transport is a large fraction of $\left.\mathrm{La} \frac{\partial \mathrm{V}}{\partial \mathrm{z}}\right|_{\mathrm{z}=0}$. This in turn means that the Stokes production scales as the surface stress times the surface Stokes drift.

$$
\left.\mathrm{P}_{\text {stokes }} \sim \int_{-\mathrm{D}} \mathrm{La} \frac{\partial \mathrm{V}}{\partial \mathrm{z}}\right|_{\mathrm{z}=0} \frac{\partial \mathrm{v}_{\mathrm{s}}}{\partial \mathrm{z}} \mathrm{dz}=\left.\mathrm{v}_{\mathrm{S}} \mathrm{La} \frac{\partial \mathrm{V}}{\partial \mathrm{z}}\right|_{\mathrm{z}=0}=\tau * \mathrm{v}_{\mathrm{S}}(\mathrm{z}=0)
$$

Figures $5.11 \mathrm{a}-\mathrm{c}$ show the buoyancy production, shear production in the alongcell direction and Stokes production normalized by $\tau * \mathrm{v}_{\mathrm{S}}(\mathrm{z}=0)$ as a function of $\mathrm{La}$ and $\mathrm{Ri}$ from the nonrotating numerical model runs. The Stokes production is indeed a large fraction (up to 60\%) of $\tau * \mathrm{v}_{\mathrm{S}}(\mathrm{z}=0)$ ), and is in general as large, or larger than the shear production. This is especially true at low $\mathrm{La}$ (large $\mathrm{RaCL}$ ) when the cells are strongly forced. How big is this energy source term in the real world? Wu (1975) argued that the surface drift due to waves was about $5-13 \%$ of the total surface drift current (of order $1-2 u_{*}$ ). While Wu's results are likely to underestimate the size of the Stokes drift since they contain only wind waves and not swell waves, this would mean that $\tau * v_{s}(z=0)$ would be a small fraction (of order $10 \%)$ of the total turbulent kinetic energy production $\tau * \mathrm{v}(\mathrm{z}=0)$. This means that the presence of Langmuir cells does not greatly alter either the overall level 
of the small-scale turbulent kinetic energy or the dissipation. However, dissipation due to wall-layer shear would be expected to fall off as

$$
\varepsilon(z) \sim v^{\prime} w^{\prime} \frac{\partial v}{\partial z}
$$

which, for a logarithmic layer, falls off as $1 / z$. Dissipation due to Langmuir cells, on the other hand, would be expected to be concentrated in the downwelling plumes, and so to persist to fairly great depths. Thus, while Langmuir cells might play a small role setting the peak levels of dissipation or turbulent kinetic energy, they could play an important role in setting the structure and level of the dissipation and turbulent kinetic energy at greater depths.

Although the Stokes production due to Langmuir cells is potentially quite a small player in the total turbulent kinetic energy budget of the upper ocean, it is potentially quite important when the density transport is concerned. Figure 5.11d shows the Flux Richardson number

$$
\mathrm{Ri}_{\mathrm{f}}=\frac{\mathrm{B}_{\text {trans }}}{\mathrm{P}_{\text {stokes }}}
$$

Up to $60 \%$ of the Stokes production can go into buoyancy transport. This is much larger than the $8-12 \%$ which is associated with three-dimensional turbulent mixing (Linden, 1981; Park, Whitehead and Gnanadesikan, 1994) in stratified fluids. (It should be noted that this value is an upper bound. Within a boundary mixed layer, Park et al. found efficiencies which were closer to $4 \%$ ). Thus even if the cells only make up $15 \%$ of the total turbulent kinetic energy production, they could still account for the majority of the turbulent transport of density. Denman and Miyake (1973) showed that the entrainment fluxes associated with the yearly cycle of mixing at ocean station Papa were of order pu* and Davis et al., (1981) found that an energy flux of about $0.4 \mathrm{\rho u}^{3} *$ was necessary to account for observed mixed layer deepening during the MILE experiment. The density transport due to Langmuir circulation is of the same order of magnitude. In Chapter 7 two days during the Surface Waves Processes Program when this energy source may have played a role in mixed layer deepening are considered.

Some of the questions that these model runs raise which have not been answered and must be left for future investigation are listed below:

-Is there some optimum flow towards which the mixed layer tends in the presence of finite-amplitude cells? A number of investigators (Malkus and Veronis, 1958 and Foster, 1969 among them) have proposed that the equilibrium flow in Rayleigh-Benard convection is that which maximizes density transport. Is there 
some analogous quantity for Langmuir circulations? For example, is the final flow chosen so as to maximize momentum transport?

-Can we develop a method of predicting the mean flow given finite-amplitude cells in rotating mixed layers where $\mathrm{Ra}_{\mathrm{CL}}$ is not so large that one can use the nonrotating solutions?

-What determines the presence of mean crosscell shears? 


\section{Chapter 6: Langmuir Circulation during the Mixed Layer Dynamics Experiment}

\subsection{Introduction}

As stated in the first chapter of this thesis, there are at present two different conceptual views of how the oceanic mixed layer is maintained. One view supposes that it is primarily stirred by eddies which have dimensions small with respect to those of the layer. A second view supposes that the layer is mixed by structures with horizontal and vertical dimensions comparable to the mixed layer depth which may have some associated coherent pattern. This thesis argues that when diffusion is small enough large eddies driven by the Craik-Leibovich wavecurrent interaction mechanism (Langmuir cells) replace small-scale diffusion as the main mixing mechanism within the mixed layer.

Chapters 2 through 5 of this thesis assumed that wave-current interaction was responsible for driving Langmuir cells and showed how to derive the equivalent of the buoyancy frequency and Rayleigh number which characterize the buoyant convection problem. Given Langmuir cells of a given size in the presence of Coriolis forces and stratification, it was shown that the analogue of the stratification is the Craik-Leibovich instability parameter $\left(\gamma_{C L S}\right)$ and that an analogue of the Rayleigh number (RaCLS), could also be defined. When RaCLS was large (so that the cells were strongly forced by the Craik-Leibovich instability mechanism), an idealized surface layer was unstable to Langmuir cells. When the resulting cells grew to finite amplitude, they replaced small-scale turbulence as the dominant mechanism for momentum and density transport within this surface layer. When RacLs was large, the horizontally varying velocity shear associated with the cells scaled roughly as $\gamma_{\text {CLS }}$.

This chapter and the following one take a different approach to the problem. They begin by demonstrating that eddies which are large in comparison to the mixed layer are important in determining the velocity and density structure and then try infer their driving mechanism. It is found that:

-When Langmuir cells are present, the mean mixed layer drift carries them across fixed strings of current meters, producing time-varying velocity shears at superinertial (1-30 cph) frequencies.

-In the presence of elevated levels of high-frequency shear, the subinertial response of the mixed layer to surface forcing differs from that predicted by two standard models. 
Assuming the cells to be driven by the Craik-Leibovich mechanism allows: -Prediction of the level of the high frequency shear.

-Explanation for the failure of standard models to predict the velocity or density structure of the upper layer.

This chapter uses data from the Mixed Layer Dynamics Experiment (MILDEX), while the next chapter uses data from the Surface Waves Processes Program (SWAPP).

The Mixed Layer Dynamics Experiment main cruise provides a good opportunity for testing the main hypothesis. Measurements of surface forcing, waves, temperature, salinity, turbulence, and horizontal and vertical currents were all made during the cruise. Most importantly, the current measurements were made from the Research Platform FLIP as it drifted freely. In such a deployment mode FLIP is extremely stable, so that it is possible to obtain measurements which are at a constant depth while minimizing contamination by wave motion.

A number of papers have been published which use the MILDEX dataset. Smith, Weller, and Pinkel (1987, henceforth SWP) compared Doppler sonar data with data from current meters to construct a picture of Langmuir cells during a storm on November 9th and 10th. Weller and Price (1988, henceforth WP) published the most complete summary of the evidence for Langmuir circulations in the current meters. Paduan et al., (1989) reported on the amplitude of near inertial motions during MILDEX, demonstrating that the response of a mixed layer model to wind forcing depended on the pre-existing velocity structure. To date, there has been no detailed examination of the relationship between the level of the high-frequency shear and Langmuir cell strength. Additionally, the response of the mixed layer during the experiment has not been studied in detail for sub-inertial frequencies. This chapter addresses these questions.

The approach taken is as follows. Section 6.2 outlines the MILDEX experiment, presenting the oceanographic and meteorological background for the experiment. Section 6.3 links the strength of Langmuir cells to the level of the high-frequency shear. Section 6.4 examines the near-surface current response during MILDEX during time periods of differing cell strength, Section 6.5 argues that the level of the high frequency shear is consistent with wave-current interaction being responsible for driving the cells. Section 6.6 demonstrates that the velocity profile produced by assuming small-scale mixing is unstable to Langmuir cells. Section 6.7 provides a discussion of the results. 

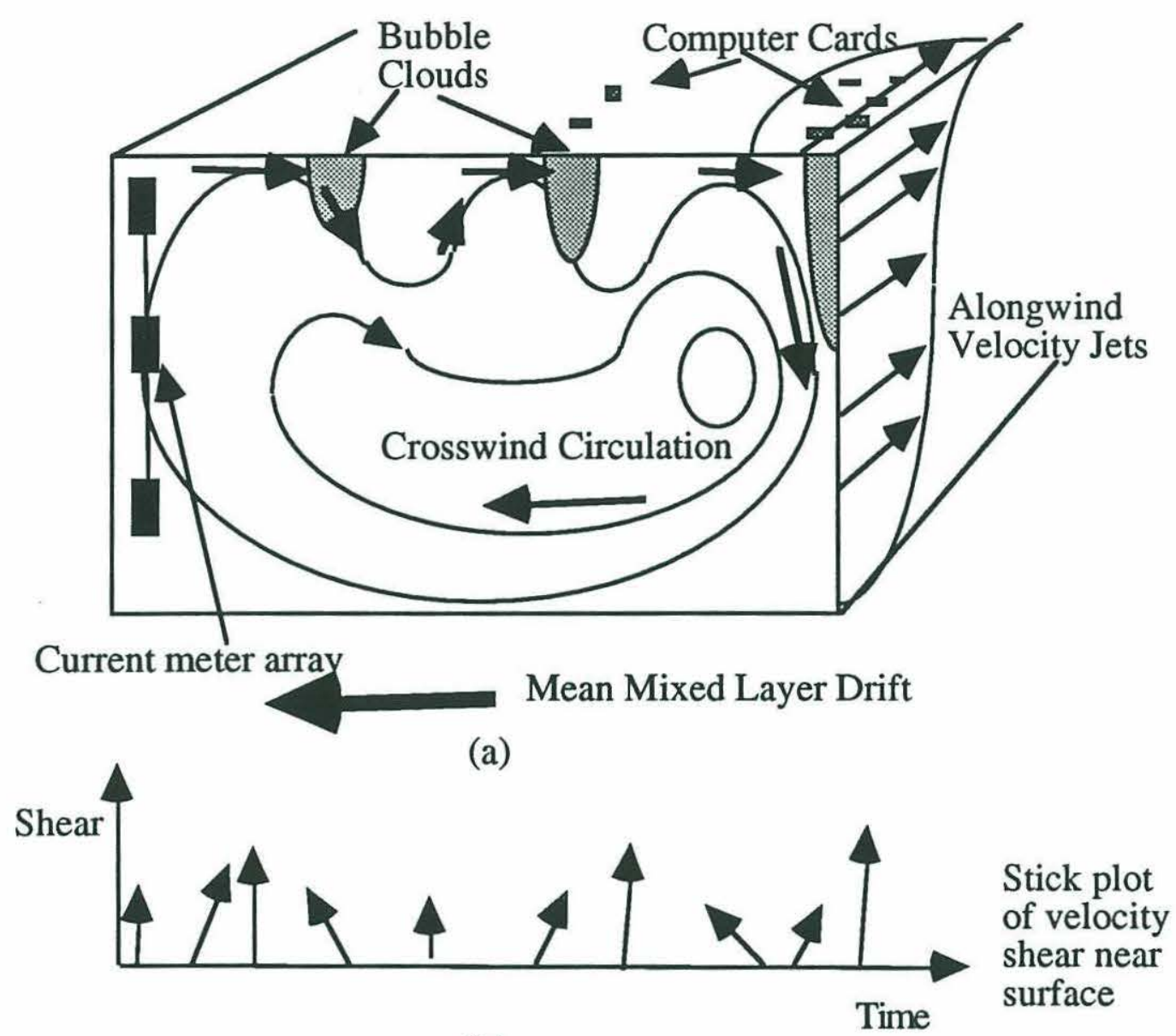

(b)

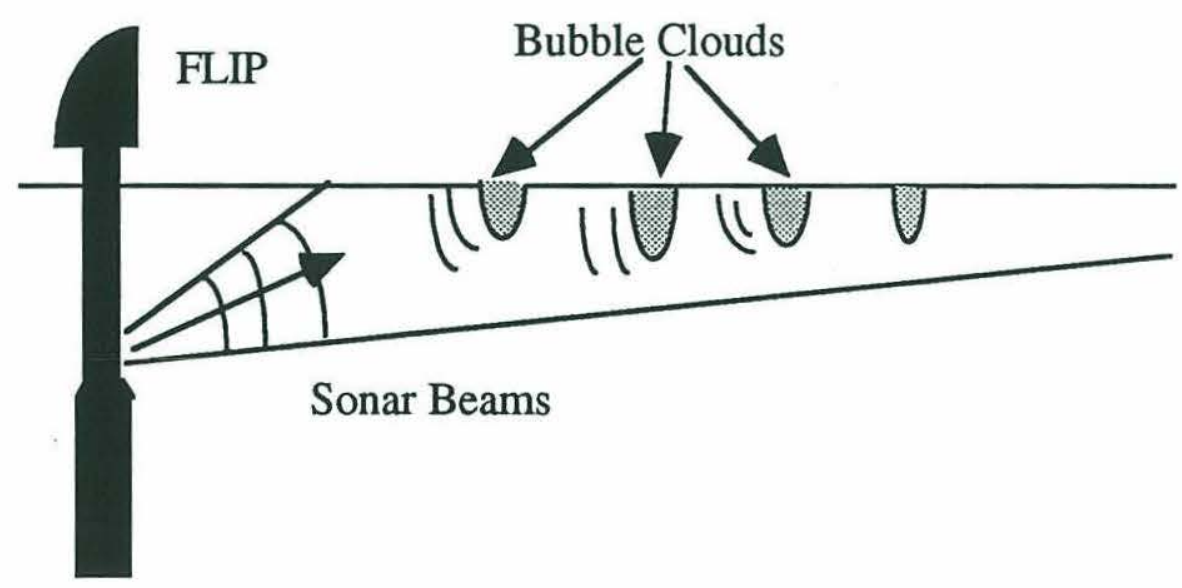

(c)

Figure 6.1: Schematic of measurement approach during MILDEX. (a) Velocities, bubble clouds, and surface convergence of cards associated with cells. (b) Current shears seen near surface as the result of cells drifting across current meter array. (c) Sonars scattering sound off of bubble clouds 


\subsection{The MILDEX Dataset}

\subsubsection{Overview and Instrumentation}

The Mixed Layer Dynamics Experiment main cruise took place in 1983 off the coast of Southern California. Participating vessels were the Research Platform FLIP, $R / V$ Acania and the $R / V$ Wecoma . This chapter primarily uses data taken from FLIP, the exception being longwave radiation and microstructure data taken aboard the Acania and a hydographic survey made from the Wecoma.

Figure 6.1 shows a summary schematic of the measurement strategies used to look at Langmuir cells during MILDEX. The cells organize surface drifters into windrows, and bubbles into plumes (Figure 6.1a). These plumes scatter sound from Doppler sonars, producing high-backscatter regions which persist over time (Figure 6.1c). The bubble plumes are also associated with strong alongwind jets of velocity. In the presence of a mean mixed layer drift, FLIP moves relative to these features, so that Doppler sonars see persistent features which seem to move along the beam, and current meters see time-varying currents and shears as they pass through the cells (Figure 6.1b).

During the MILDEX main cruise, FLIP drifted within a $50 \mathrm{~km}$ radius of $34^{\circ} \mathrm{N}, 126^{\circ} \mathrm{W}$, approximately $600 \mathrm{~km}$ due west of Point Conception. Figure $6.2 \mathrm{a}$ shows a map, taken from Weller and Price (1988), showing the experiment site and FLIP's drift. Figure 6.2b is taken from Smith et al, (1987) and shows the general layout of FLIP for MILDEX. In all, three booms were deployed during the experiment, a short $10 \mathrm{~m}$ boom canted about 45 degrees to the left of the heading (forward boom), a 15 meter boom off to port (port boom), and a 15 meter boom aft of FLIP (aft boom). The angle of the platform relative to the wind was maintained by a thruster at a depth of 15 meters. Six doppler sonars were deployed on FLIP's hull, two of which were set up to look at Langmuir circulation. Figure 6.2c, also taken from Smith et al., 1987, shows a planview of FLIP, showing the locations of the various measurements along the booms, and the angles along which the two Doppler sonars were deployed.

Because of its great draft when vertical, FLIP has a natural period larger than those associated with surface waves. As a result, the platform is very stable even in energetic wave fields. This means that instruments suspended from the booms do not move large distances in the vertical, as as is the case for instruments suspended from a surface float. 
(a) FLIP DRIFT TRACKS - DEC 82, MAY 83, OCT 83

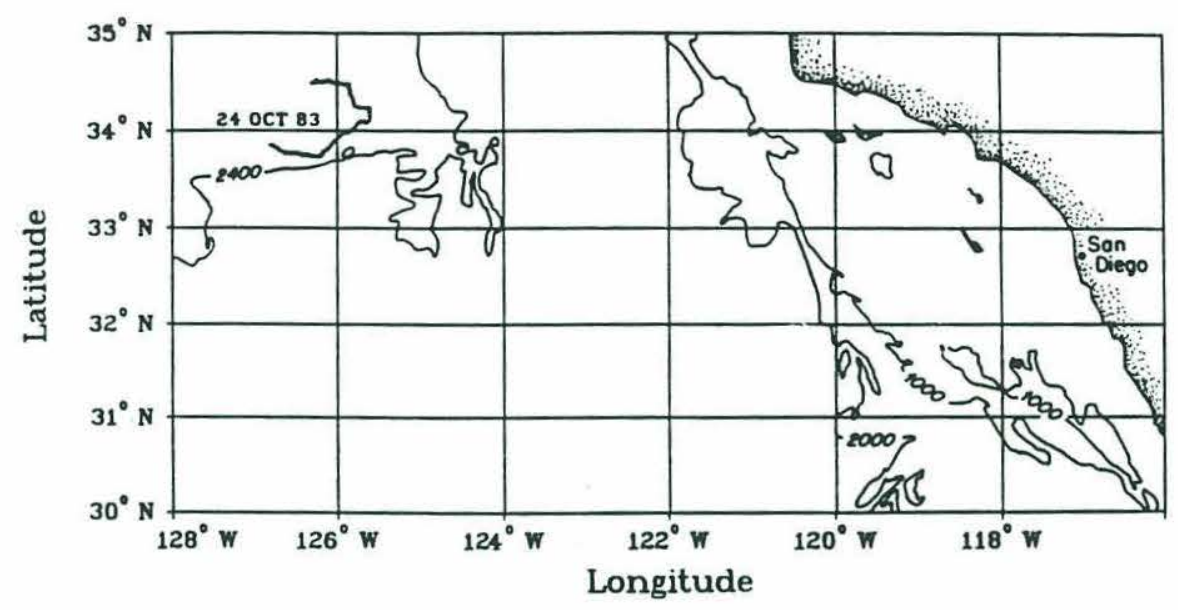

(b)

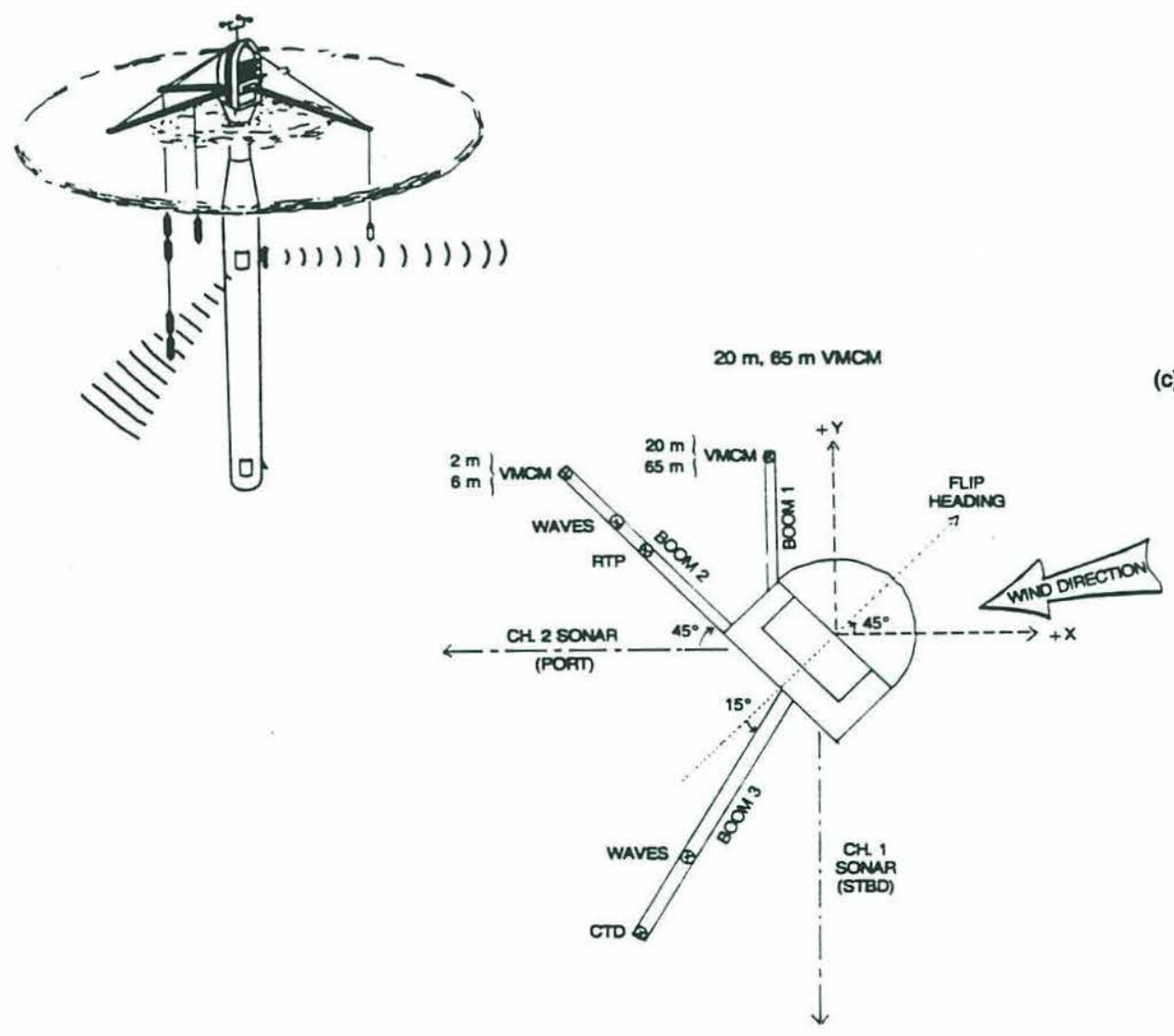

Figure 6.2: (a) The location of the Mixed Layer Dynamics Experiment, from Weller and Price, 1988. (b) The Floating Instrument Platform during MILDEX, showing the setup of the instrumentation from Smith et al., 1987. (c) Planview of FLIP during MILDEX main cruise showing location of instrumentation along the booms and orientation of the sonars. 
A large number of environmental measurements were made from FLIP during MILDEX. Two resistance wires were deployed from the port and aft booms to measure wave height. These measurements agreed well in general, and were in close agreement when they were used to calculate the Stokes drift (calculated Stokes drifts from the two wavestaffs were within $1 \mathrm{~cm} / \mathrm{s}$ most of the time). Meteorological measurements were made from the mast on FLIP. Incoming shortwave radiation, air temperature, sea surface temperature, wind speed and direction, and relative humidity were measured using a Vector-Averaging Wind Recorder (VAWR) package. Wind stress was calculated from these measurements using the formula of Large and Pond (1981), latent and sensible heat fluxes using the formulae of Large and Pond (1982), and net shortwave by using a surface albedo of 0.06 (Payne, 1972). Net longwave radiation was calculated by a combination of bulk formula estimates (List, 1972) and interpolated incoming longwave data from the $R / V$ Acania.

Water velocities were measured during MILDEX by a number of current meters suspended off the booms. Two strings of Vector Measuring Current Meters (VMCMs) were used. These current meters were designed to measure small mean flows in the presence of waves (Weller, 1978). They accomplish this by using propellors which are large compared with eddy-shedding parts of the current meter and which have a cosine response to flows. The first of the two current meter strings consisted of three VMCMs which were fixed at nominal depths of 2, 6.5 and 12 meters. The second string had four instruments which were profiled through the water column during the first part of the cruise from October 25 to November 3rd and fixed at nominal depths of 20, 35, 50 and 65 meters from November 3rd to November 14. Additionally, a new instrument, known as the Real-Time Profiler (RTP) was deployed midway down the port boom. This instrument measured vertical as well as horizontal velocities, temperatures, and conductivity. The RTP was profiled over the water column during some time periods and held fixed at various depths during other time periods. All the VMCMs averaged over periods of one minute before writing to tape. The RTP averaged over a period of 14.025 seconds (1/256th of an hour).

The data from the RTP and the VMCMs was binned, averaged, and interpolated in time and space. The top two bins covered 0-3 and 3-7.5 meters respectively, with the remaining bins covering 5 meter intervals down to a depth of 170 meters. Each bin contained a 1-hour average in time. The binned data was used to calculate the response to wind forcing. 
A thruster on FLIP's hull at a nominal depth of 15 meters was used to maintain a constant orientation relative to the wind. This thruster was found to produce a narrow jet with speeds up to $60 \mathrm{~cm} / \mathrm{s}$ which contaminated the fixed VMCM at 12 meters. The velocity signal due to the thruster needed to be removed in order for the binned data to be useful in calculating the mixed layer response to surface forcing. This was done by hand, taking times when the velocity at 12 meters was grossly different from the velocities around it and interpolating across the contaminated depth. It is not thought that the thruster contaminated the VMCMs at 6.5 and $20 \mathrm{~m}$. Evidence for this conclusion is presented in Appendix E.

At a number of times during the experiment, surface drifters (computer cards during the day, bags of florescent dye during the night) were deployed from FLIP. If Langmuir cells were strong, these drifters lined up into rows. These measurements gave a crude sense of when Langmuir cells were present and an order of magnitude estimate at certain times for the spacings and crosscell velocities associated with some small scales of cells.

One of the more striking techniques which showed the effect of Langmuir cells during MILDEX were Doppler sonar measurements carried out by Jerome Smith and Robert Pinkel of Scripps. Microscopic bubbles (with diameters of 20$400 \mu \mathrm{m}$ ) are generated by wave breaking and organized by Langmuir cells into clouds many meters deep which are roughly aligned with the wind. When a pulse of sound is propagated through the near-surface layer, these clouds show up as regions of high scattering. The Doppler shift associated with bubble motion is used infer the velocity with which the bubbles are being advected. Because scattering is a strong function of bubble density, the returns are dominated by the regions with the most bubbles. On short time scales (1-2 seconds), the Doppler velocities can be used to track surface waves (Pinkel and Smith, 1987). If the data is averaged over longer periods of time the velocities due to the surface waves can be removed and those due to Langmuir circulation may be recovered. During MILDEX the two upward-looking sonars on FLIP produced 3-minute averages of the backscatter and velocity from a range bin approximately 11.25 meters long along the beam. One of these sonars looked across the wind and was dominated by surface scattering over a range of 600-1400 meters. The other looked alongwind and was dominated by features within the mixed layer at depths of 20-35 meters for the first 800 meters or so. Details of the sonars deployed during MILDEX are given in SWP. 
Lastly, microstructure measurements were made from the Acania using a tethered free-fall vehicle (Lueck, 1988, unpublished). The shears at small scales were measured and used to produce profiles of the near-surface dissipation. The instrumentation and data processing followed closely that of Yamazaki and Lueck (1987).

\subsubsection{Review of Meteorological and Oceanographic Conditions during MILDEX}

A summary of the meteorological and oceanographic conditions during the experiment is shown in Figures 6.3 and 6.4. The first of these figures shows meteorological variables measured from the VAWR on FLIP and from the current meter at 2 meters depth. From top to bottom the fields shown are the wind speed in $\mathrm{m} / \mathrm{s}$, the wind direction (towards which the wind is blowing) in degrees, the incoming shortwave radiation in $\mathrm{W} / \mathrm{m}^{2}$, the barometric pressure in $\mathrm{mb}$, the air temperature in degrees $\mathrm{C}$ and the water temperature in degrees $\mathrm{C}$. As outlined above, the measured meteorological quantities were used to calculate the heat and momentum fluxes using bulk formulae. The fluxes and some measures of the oceanographic response to them are shown in Figure 6.4. From top to bottom, the fields shown are the zonal wind stress in $\mathrm{Pa}$, meridional wind stress in $\mathrm{Pa}$, total heat flux in $\mathrm{W} / \mathrm{m}^{2}$, temperature difference between the current meters at 2 and $6.5 \mathrm{~m}$ depth, and significant wave height in $\mathrm{m}$.

During the first week of the experiment (October 23-30th) the winds were fairly low (with the exception of October 28th). The upper part of the surface layer became stratified during the day and mixed during the night. On October 31 st and November 1st the winds blew from the north, the temperature dropped and strong latent and sensible cooling led to heat losses of $200 \mathrm{~W} / \mathrm{m}^{2}$. The upper 6.5 meters did not restratify during these two days. November 2nd-6th saw a return to milder conditions. Interestingly, the wave height increased sharply on November 2 nd despite the lack of wind, indicating a swell propagation event. The near-surface temperature difference showed some indications of frontal activity on November 3rd, with a temperature inversion despite very little surface cooling. On November 6th there was another episode of cold air blowing from the north, with strong resultant latent and sensible cooling leading to heat losses up to $300 \mathrm{~W} / \mathrm{m}^{2}$. During November 8th the wind slackened, then reversed direction as a strong low pressure system moved over the experiment site. During 


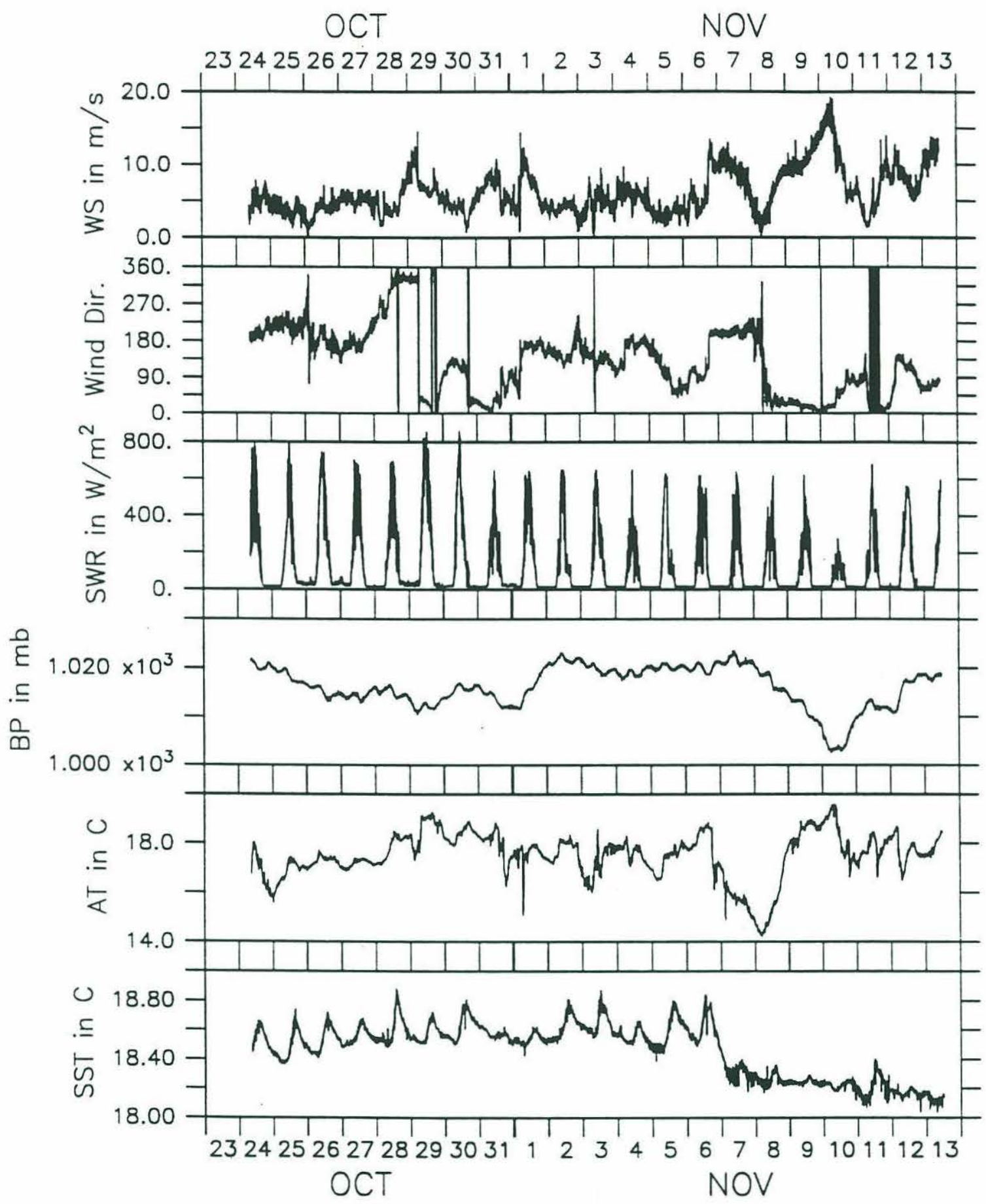

MILDEX:Meteorological Variables Recorded from FLIP

Figure 6.3: Meteorological fields measured from FLIP during MILDEX main cruise. From top to bottom, the fields are, wind speed in $\mathrm{m} / \mathrm{s}$, wind direction (towards) in degrees, incoming shortwave radiation in $\mathrm{W} / \mathrm{m}^{2}$, barometric pressure in $\mathrm{mb}$, air temperature in $\mathrm{C}$, water temperature in $\mathrm{C}$. 


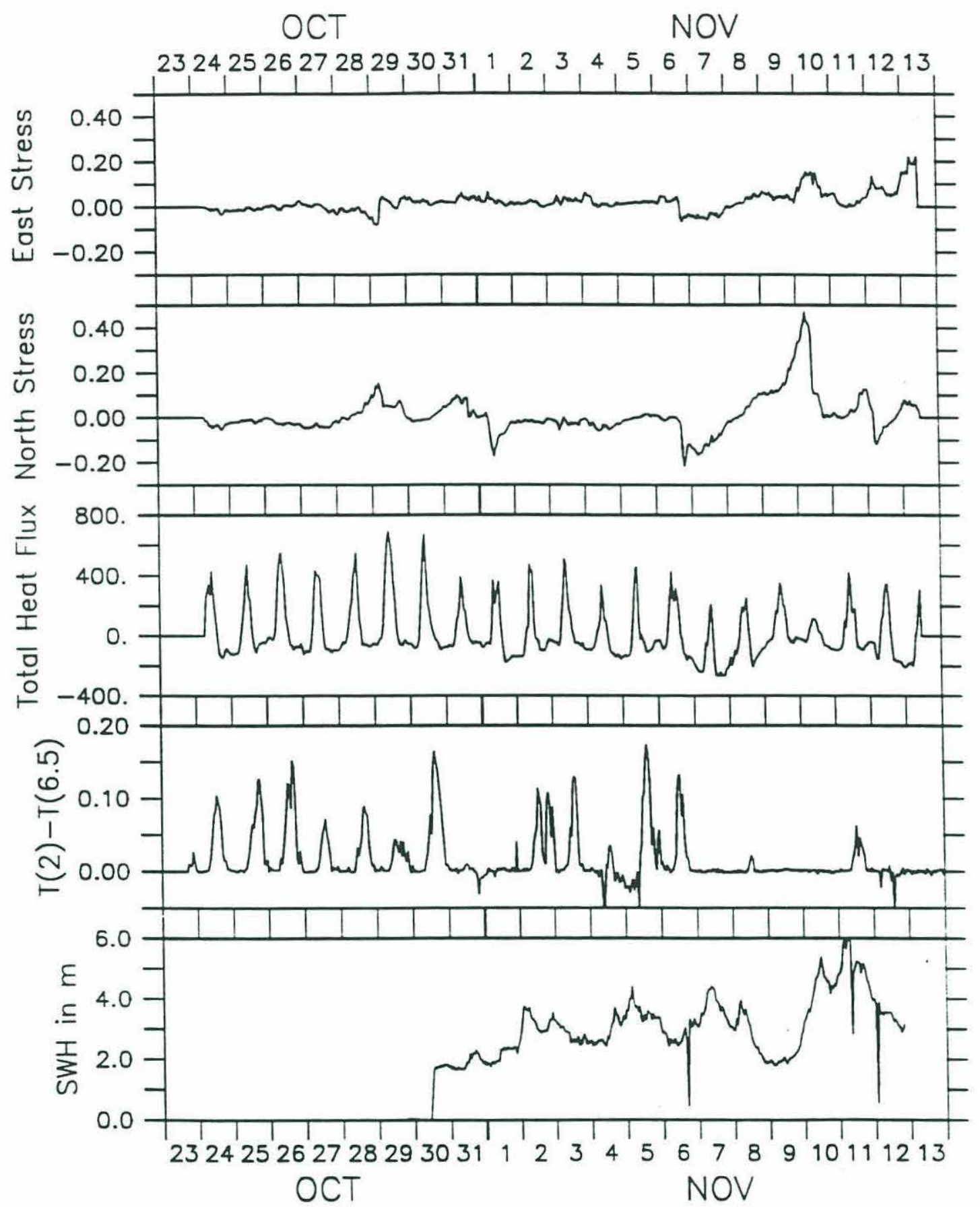

MILDEX:Meteorological and Oceanographic Background

Figure 6.4: Meteorological and oceanographic conditions during MILDEX. All variables are hourly-averages. Fluxes are computed from bulk formulae as noted in the text. Significant wave height is the mean height of the highest $1 / 3$ of the waves. From top to bottom, East stress in $\mathrm{Pa}$, North stress in $\mathrm{Pa}$, Total Heat Flux in $\mathrm{W} / \mathrm{m}^{2}$, Temperature difference in $\mathrm{C}$ between current meters at 2 and 6.5 meters, Significant wave height in meters from wave staffs. 
the early morning hours of November 10th, the winds reached their maximum strength for the entire experiment. Sustained wind speeds of $19.2 \mathrm{~m} / \mathrm{s}$ were seen, leading to wind stresses of $0.5 \mathrm{~Pa}$. Wave heights approached $6 \mathrm{~m}$. In the wake of the low, wave heights continued to be high during much of the 10th and 11th, falling off slightly on November 12th as another period of cold winds from the north began.

In order to understand the subinertial response during MILDEX, the importance of frontal activity must be considered. The heat balance during MILDEX was affected both by atmospheric forcing and by fronts. Figure $6.5 \mathrm{a}$ shows contours of the temperature smoothed over 12 hours to eliminate the effects of variability associated with the semidiurnal tide. The contours are every 1 degree in the main thermocline (below temperatures of $18 \mathrm{C}$ ), and every 0.1 degrees in the mixed layer (18.2 $\mathrm{C}$ and above). Figure $6.5 \mathrm{~b}$ shows the cumulative heat flux (solid), integrated heat content, defined as

$$
H(D)=\int_{z=-D}^{0} \rho c_{p} T(z) d z
$$

for $\mathrm{D}=20$ meters (dashed) and 40 meters (chain-dotted), computed using the 12 hour averaged temperature.

During the experiment, the depth of the top of the main thermocline was relatively constant at about 40 meters. The fact that the integrated heat flux disagrees with the heat content above this depth is evidence for frontal activity. The rise in heat content on October 26 corresponded to a warm intrusion between depths of 20 and 40 meters. The temperature anomaly associated with this intrusion was quite small, of order 0.1-0.2 degrees $C$. The divergence of the integrated heat flux and the heat content curves on October 29th was the result of the movement of a mass of slightly colder water over the site. Again the temperature anomaly was small, of order 0.1-0.2 degrees. During the following week, both the heat content and integrated heat flux were fairly flat, indicating a regime in which diurnal warming is balanced closely by nighttime cooling. The upper 10 meters of the water column saw some frontal activity during this time (note in particular the persistent stratification of the upper water column during the nights of November 3rd and 5th) in Figure 6.4, but the fronts had very small amplitude, of order $0.05 \mathrm{C}$.

During November 6th, there was a sharp drop in the heat content and integrated heat flux associated with passage of a cold air mass over the site. The 


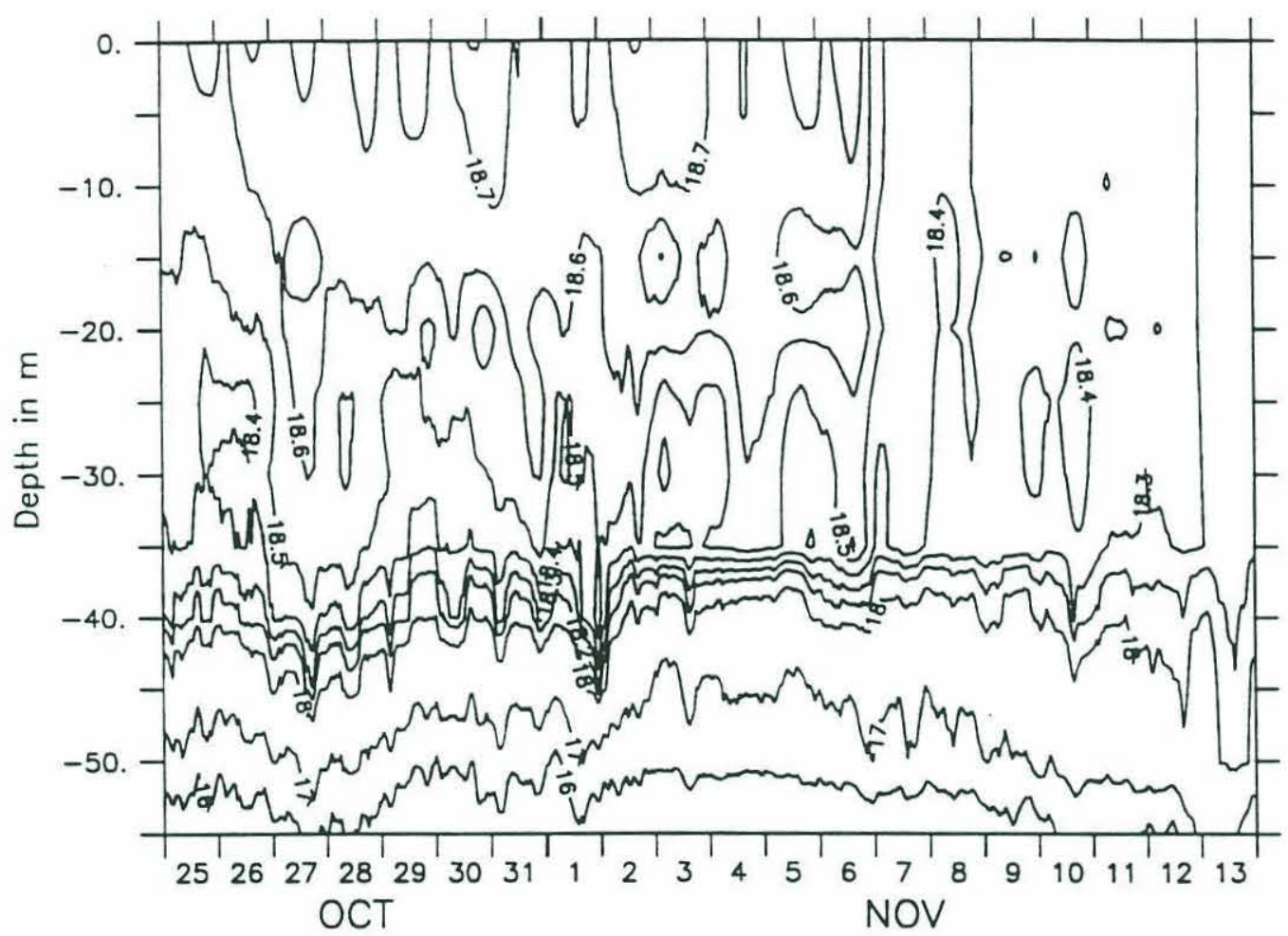

MILDEX:Upper Ocean Temperature (6 Hr. Running Mean)

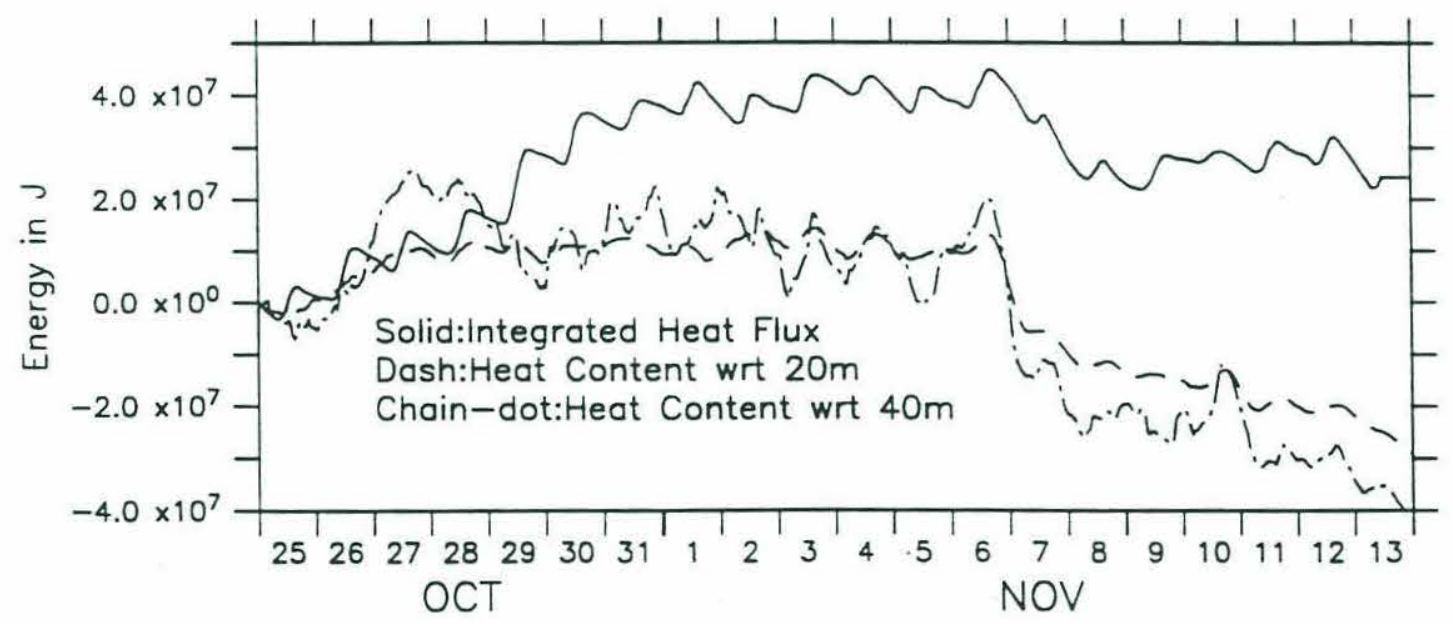

MILDEX Heat Balance

Figure 6.5: Temperature structure and heat balance during MILDEX. Top: Contour plot of temperature. Contours are 1.0 degrees below 18 degrees $C$ (in the main thermocline), and 0.1 degrees from 18.2-19 $\mathrm{C}$ (within the mixed layer). Bottom: Integrated heat flux (solid) and change in heat content from the start of the experiment relative to reference depths of $20 \mathrm{~m}$ (dashed) and $40 \mathrm{~m}$ (chaindotted) as explained in equation (6-4). 
heat content dropped about twice as far as the integrated heat flux, indicating the presence of a second cold intrusion. The magnitude of this cold intrusion was about $0.2 \mathrm{C}$.

In general, the temperature anomalies associated with these frontal events were not associated with significant salinity anomalies. From the beginning to the end of the cruise, profiling CTD measurements made by Pinkel and Smith of Scripps showed the salinity within the mixed layer to have decreased by only about 0.1 PSU. About half of this freshening occurred during the frontal intrusion on November 6th, compensating the cooling. The magnitudes of the salinity and temperature changes associated with the fronts are consistent with a survey made from the Wecoma (Paduan, pers. comm.) which showed temperature changes of about $0.2 \mathrm{C}$ and salinity anomalies of about $0.05 \mathrm{PSU}$ with spatial scales of tens of kilometers. Using the thermal wind relationship, the shear associated with these fronts is calculated to have been smaller than $0.001 \mathrm{~s}^{-1}$. This value is smaller than the shear signals associated with the wind-forced response.

\subsection{Indices of Langmuir Circulation Strength during MILDEX}

\subsubsection{From Dopplers to Shear}

In order to measure the effect of Langmuir cells, it is first necessary to characterize their strength. This section examines measures of Langmuir cell strength during MILDEX, connecting the presence of spatially and temporally coherent roll vortices aligned close to the wind with enhanced levels of superinertial shear.

As noted in Section 6.2, Doppler sonars are particularly well suited to detect the spatially and temporally coherent structures associated with bubble plumes. SWP demonstrated that such structures were present during MILDEX, concentrating on an eight-hour period from 2200 PST November 9th to 0600 PST November 10th. Their results, reviewed below, demonstrate the existence of persistent velocity structures closely tied to concentrations of scatterers.

Figure 6.6 shows high-pass filtered sonar data taken from Figures 12-16 of SWP. The color contour plots from these figures have been converted by handtracing into "skeletonized" plots showing the locations of persistent features. The top panel (6.6a) shows data from the alongwind ( $\mathrm{x}$ ) beam, which pointed aft from FLIP, sampling depths of 20-40 meters over the first 800-1000 meters before the beam dipped into the main thermocline. Maxima in intensity (solid lines) and 


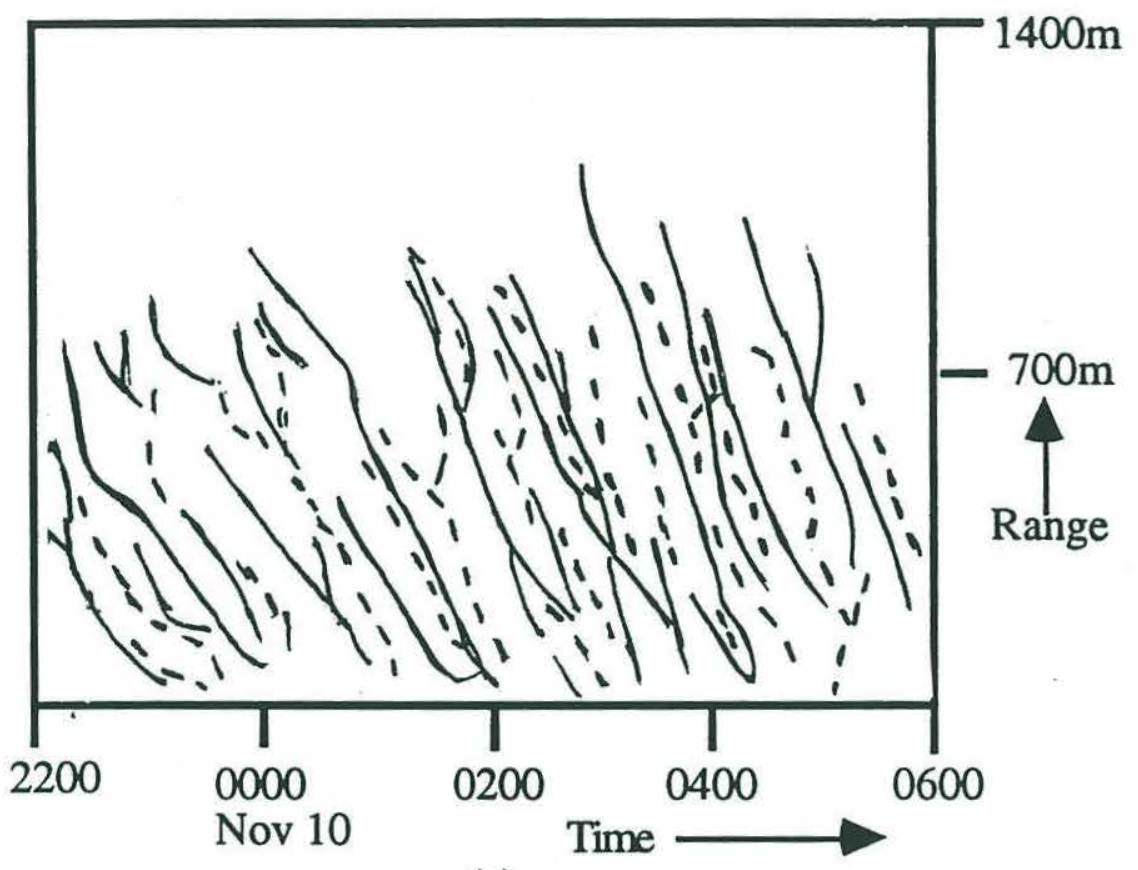

(a)

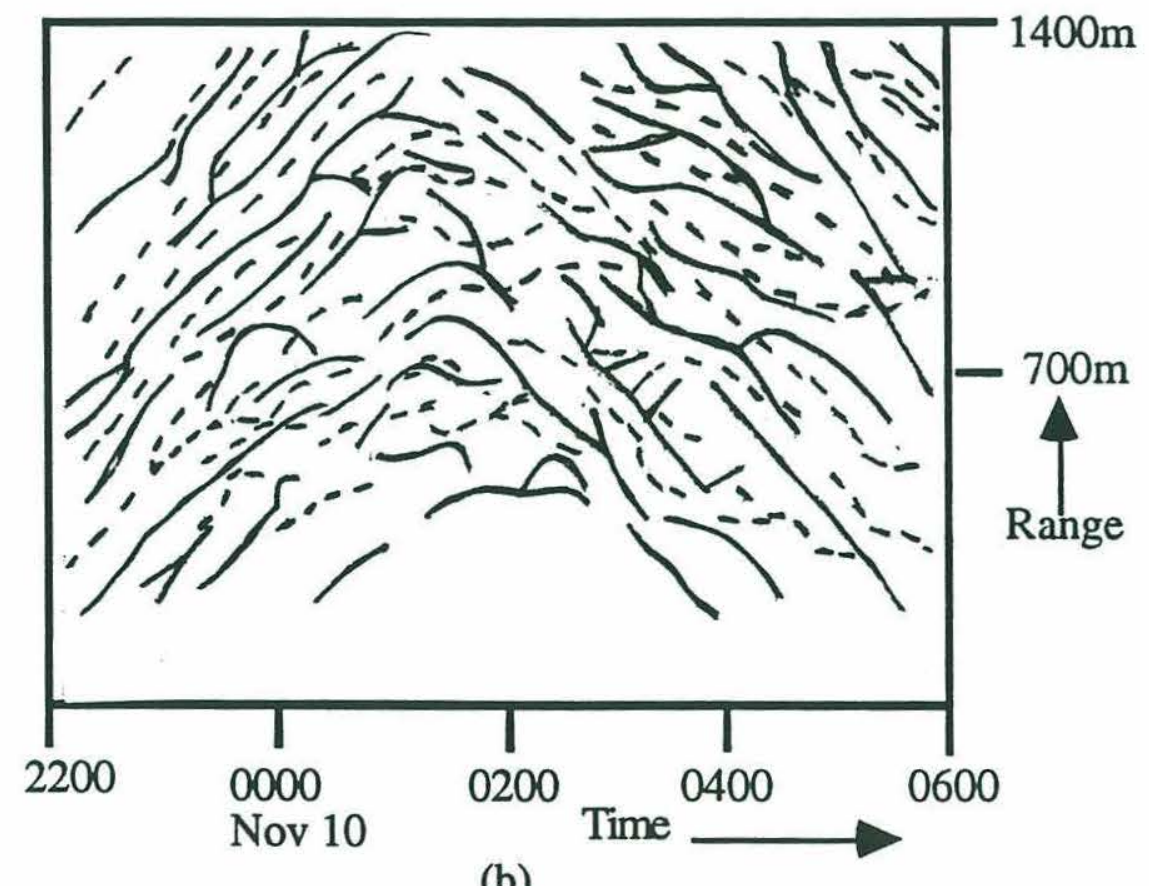

(b)

Figure 6.6: Skeletonized Plots of Intensity and Velocity Structure from Doppler Sonars. Plots are hand-drawn from Figures 12-16 of Smith et al., 1987. (a) Data from the alongwind beam. Solid lines are maxima in intensity, dashed lines minima in alongwind velocity. (b) Data from crosswind beam. Solid lines are maxima in intensity, dashed lines maxima in divergence in alongbeam velocity. 
minima in velocity along the beam (dashed lines) were subjectively drawn by hand from color contour plots. The plot shows that features were seen in both the intensity and velocity fields with roughly the same spacing and translation velocity relative to FLIP. Additionally, the relative phasing of the velocity and intensity features was such that alongwind velocity minima were out of phase with intensity maxima. This is consistent with a conceptual picture in which the cells create plumes of bubbles associated with jets of water moving more quickly in the alongwind direction.

Figure $6.6 \mathrm{~b}$ shows data from the crosswind (y) beam, which was dominated by surface returns at ranges from $700-1400 \mathrm{~m}$. The solid lines are, as before, regions of intensity maxima, representing centers of bubble clouds. The dashed lines in this case are minima in $\partial \mathrm{V}_{\mathrm{y}} / \partial \mathrm{y}$, regions where the surface velocity was divergent. Again, the features in intensity and convergence had roughly the same horizontal wavelength and translation velocity relative to FLIP. Furthermore, regions of divergence were out of phase with regions where bubbles collected, a picture consistent with the presence of Langmuir cells.

The spatial structure of the scatterers is also consistent with their being due to Langmuir circulation. The bands of scatterers had a much longer wavelength in the alongwind beam (of order 500-700 meters) than they did in the crosswind beam (of order 100 meters). This means that the features were elongated in the alongwind direction. During the time that this data was taken, FLIP was moving through the mixed layer with an average velocity of about $12 \mathrm{~cm} / \mathrm{s}$ in the downwind direction (less at the beginning of the time period, more at the end). Because of this movement, features which persisted in the crosswind beam for up to two hours at a time must have been at least 700 meters in length, a result consistent with that inferred from the alongwind sonar.

Jets of alongwind velocity similar to those seen in the Doppler sonars were also detected by the VMCMs and RTP. These jets were linked to strong downwelling events. Figure 6.7 demonstrates this by presenting time series of horizontal and vertical velocity from $0715-1050$ PST, November 10 , just after the time period studied in SWP. During this time period the thruster was turned off. The time series are from the RTP which was parked at a depth of 28 meters. Alongwind velocity is shown in the top panel (6.7a), crosswind velocity in the middle panel (6.7b) and vertical velocity in the bottom panel (6.7c). A number of strong alongwind jets occur at the same time as strong downwelling events during this time period. 


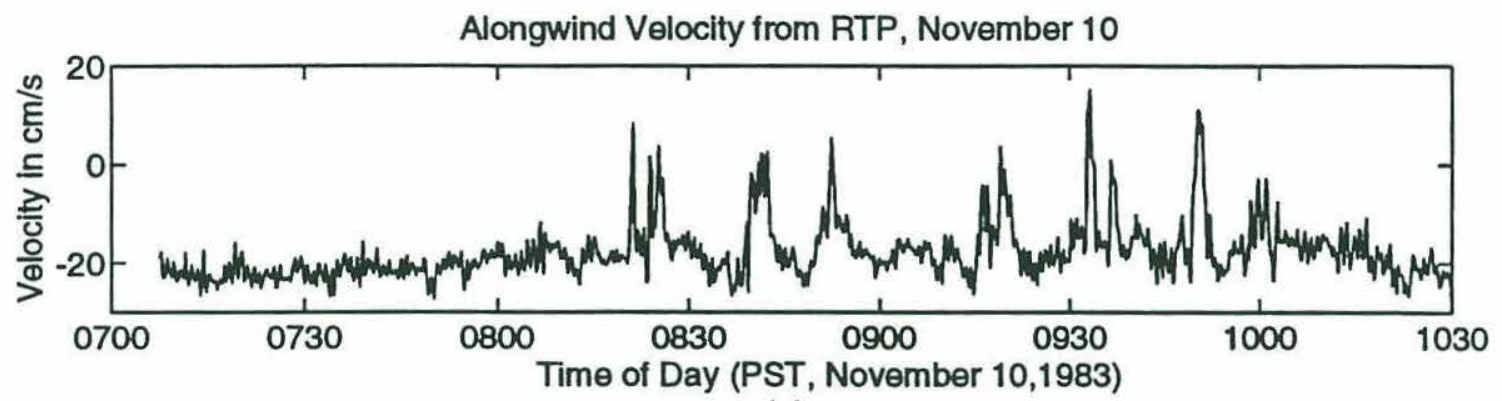

(a)

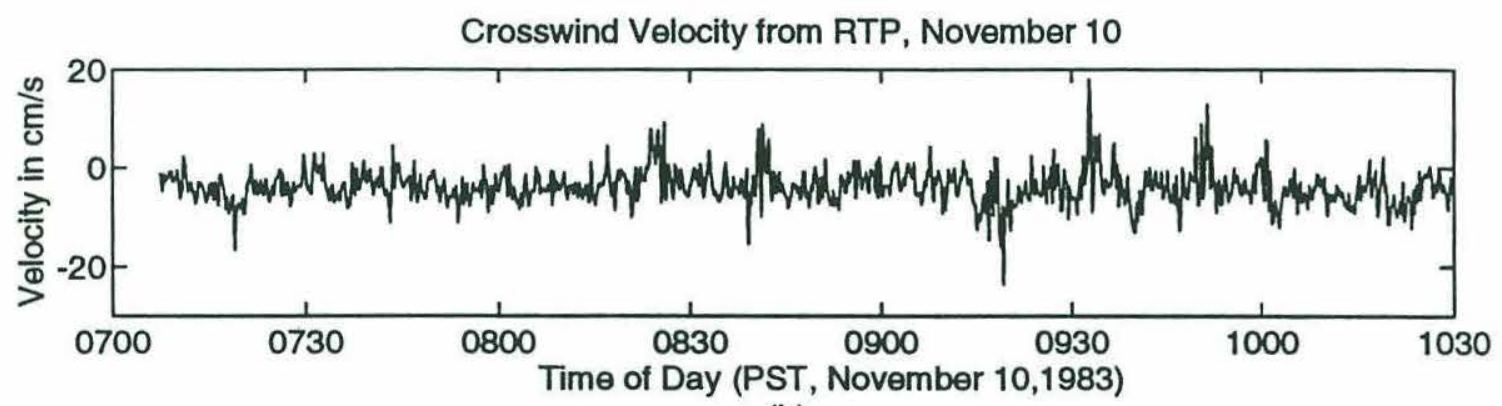

(b)

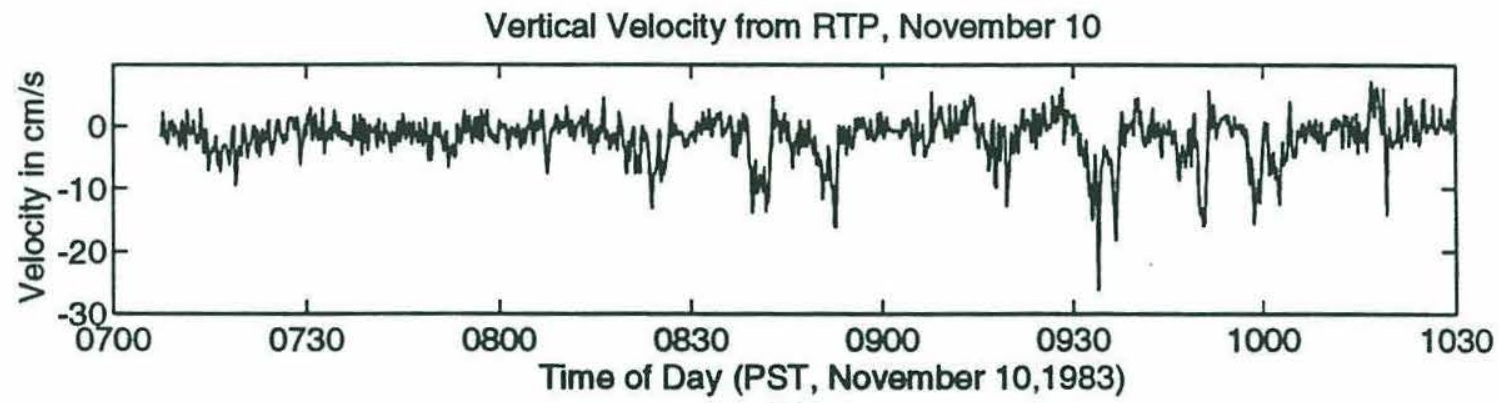

(c)

Figure 6.7: Velocity data from the Real-Time Profiler showing the relationship between the downwind jets and strong downwelling. Time shown is 0715-1050 PST on November 10. Depth is $28 \mathrm{~m}$. (a) Alongwind Velocity. (b) Crosswind Velocity. (c) Vertical Velocity. 
In WP, it was demonstrated that on days when Langmuir circulation was strong, scatterplots of the near surface shear showed "upwind" velocity shears, times when the water at 6.5 meters appeared to be moving faster in the downwind direction than water at 2 meters. These shears can be linked to the velocity jets and downwelling seen by the RTPs. Figure 6.8 shows spectra of velocity from the RTP and shear from the top two current meters during the time (0715-1050 PST, November 10) shown in Figure 6.7. The solid lines are the spectral density, the dashed lines show the $95 \%$ confidence level. The short length of the time series means that the confidence levels were in general nearly as large as the signal, implying that caution should be used when making detailed comparisons between the various signals. Figure $6.8 \mathrm{a}$ and $\mathrm{b}$ show spectra of downwind and vertical velocity respectively from the RTP. Both signals showed clear peaks at frequencies of 2-6 cph, with a secondary plateau from $10-20 \mathrm{cph}$. Coherence (not shown) between the two signals was excellent in both of these frequency bands, dropping off outside them.

Figures $6.8 \mathrm{c}$ and $\mathrm{d}$ show spectra of the shear from the top two current meters $(2$ and $6.5 \mathrm{~m})$ in the alongwind and crosswind directions. The alongwind shear was fairly flat while the crosswind shear retained many of the same broad qualitative features (a peak from 1-5 cph, a plateau at 10-20 cph) as the RTP velocities. Both the $2-6.5 \mathrm{~m}$ crosswind shear and the RTP velocities are peaked in a band from 1-6 cph, although the crosswind shear has a slightly different structure from the RTP velocities. This agreement is surprisingly good given the fact, (noted in Chapter 5), that the velocity structure of fully developed Langmuir cells is highly dependent on cell spacing. The alongwind shear spectrum is quite different from the crosswind shear spectrum even though the integrated amplitude is very similar.

It is instructive to compare these signals to a time when the wave field was energetic, but Langmuir cells do not appear to have been strong. Figures $6.8 \mathrm{e}$ and f show spectra alongwind and crosswind shear respectively for 1600-2400 PST on November 4. During this period, the significant wave height was between 4 and 5 meters, nearly as large as during 0715-1050 PST on November 10. Despite the fact that the waves were high, the spectral levels for velocity and shear are much lower (by a factor of 10) than those corresponding to the period when cells were strong. Note that the time-varying shear is lower across almost the entire frequency band for both crosswind and alongwind shear. This means that wave aliasing cannot be solely responsible for generating the shear signals. 


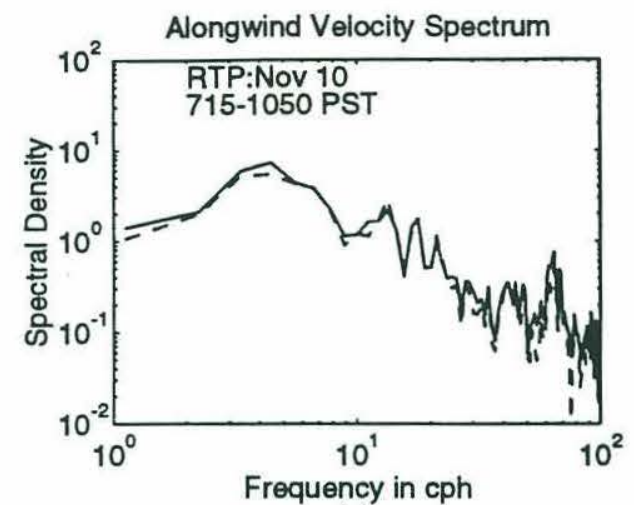

(a)

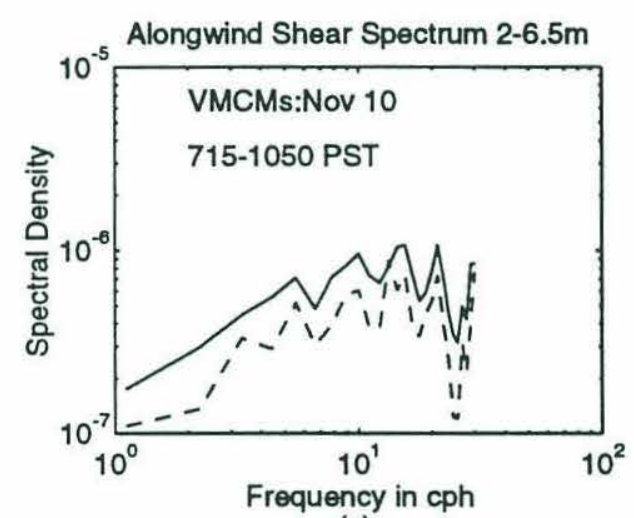

(c)

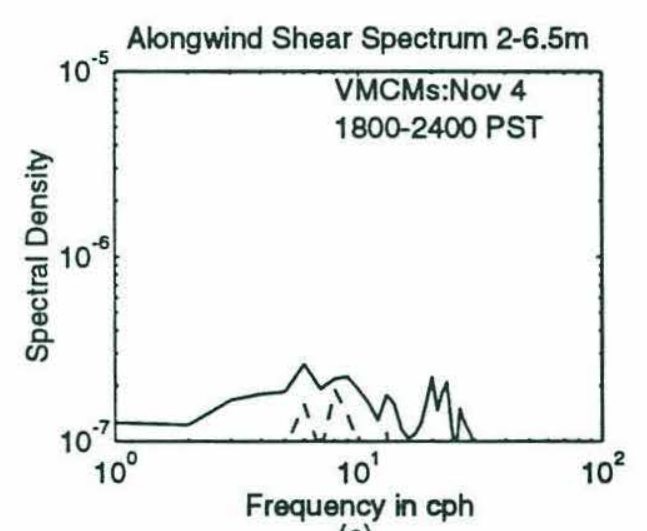

(e)

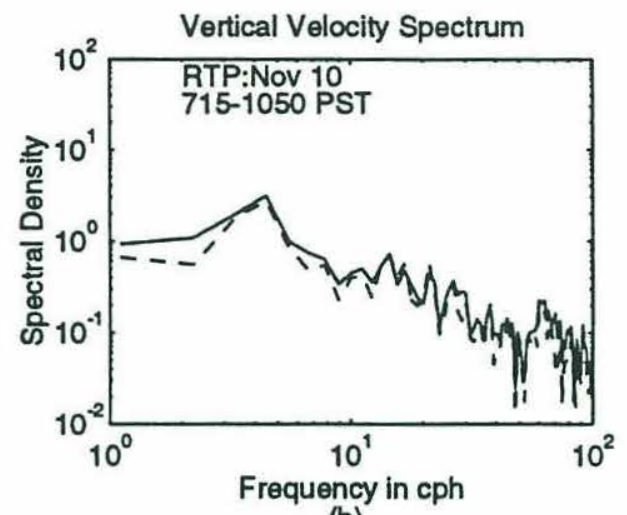

(b)

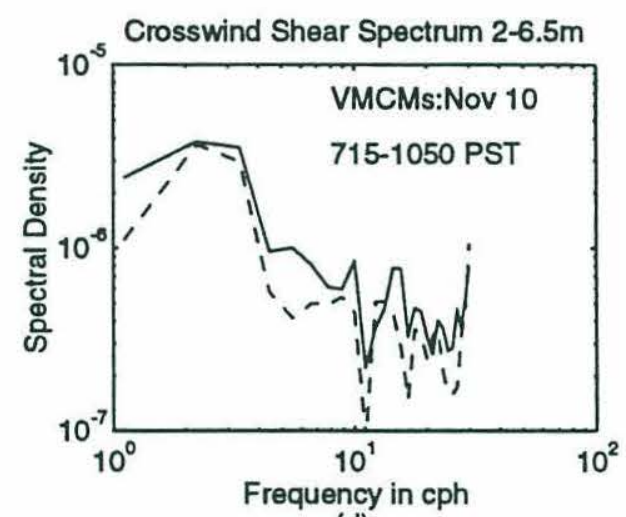

(d)

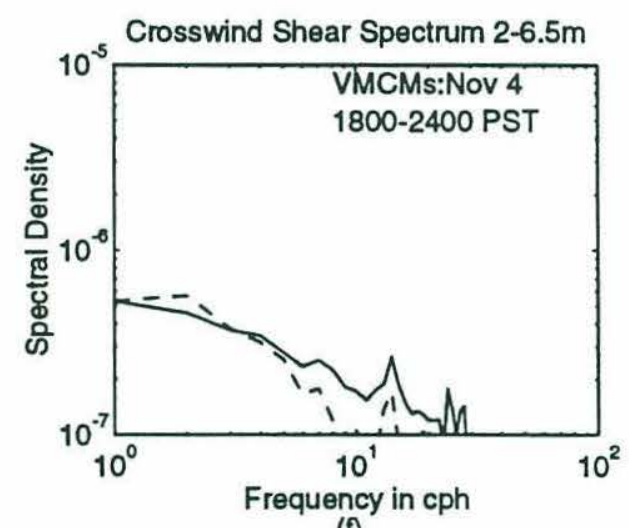

(f)

Figure 6.8: Current and shear spectra in the presence and absence of Langmuir cells.Dashed lines are confidence interval. (a) Spectrum of alongwind velocity from the RTP, 0715-1050 PST, November 10. (b) Spectrum of vertical velocity from the RTP, 0715-1050 PST, Novmeber 10. (c) Spectrum of alongwind velocity shear from the VMCMs at 2 and 6.5m, 0715-1050 PST, November 10.

(d) Spectrum of crosswind velocity shear from the VMCMs at 2 and 6.5m, 07151050 PST, November 10. (e) Spectrum of alongwind velocity shear from the VMCMs at 2 and 6.5m, 1600-2400 PST, November 4. (f) Spectrum of crosswind velocity shear from the VMCMs at 2 and 6.5m, 1600-2400 PST, November 4. 
To summarize then, during MILDEX there is evidence of persistent structures with much broader extent in the alongwind direction than in the crosswind direction (Doppler sonars) involving surface convergences (Doppler sonars) which are in phase with alongwind jets (Dopplers, VMCMs) of velocity and strong downwelling events (RTP). These structures were associated with shear in the 1-30 cph band (VMCMs).

\subsubsection{High-Frequency Shear as an Index of Cell Strength}

We have established a number of measures of cell strength during MILDEX. Unfortunately, it is only possible to compute indices of cell strength spanning the length of the cruise from the current meters. Two logical signals are the amplitude of time-varying current or shear in the 1-30 cph band. As noted earlier, such variability would be the result of any mean drift in the mixed layer sweeping the cells past the current meter strings. If the cells had more than one scale, as has been suggested by many observers, large-scale cells would also have advected small-scale cells past the current meters.

The high-frequency shear and velocity were extracted as follows. Hourlong segments of data from the VMCMs were rotated relative to the wind and the trend was removed. The standard deviation of the current and shear was then used to compute a measure of the strength of the high-frequency variability (1-30 $\mathrm{cph}$ ) associated with the passage of cells. Figure 6.9 shows the results when this procedure was applied to the current at $2 \mathrm{~m}$ and $6.5 \mathrm{~m}$ and the shear between $2 \mathrm{~m}$ and $6.5 \mathrm{~m}, 6.5 \mathrm{~m}$ and $20 \mathrm{~m}$, and $20 \mathrm{~m}$ and $35 \mathrm{~m}$. The current and shear in the alongwind direction are shown by the solid lines, the crosswind current and shear by the dashed lines. The signals were very similar at all depths and did not show significant differences between the crosswind and alongwind direction. The amplitude of the shear signal fell off with depth, being strongest near the surface. There were "noise floors" beneath which the high-frequency shears and currents never seemed to drop. The level of these noise floors was consistent with the error calculations presented in Appendix D.

As already stated, there are two different physical regimes during MILDEX. During the early part of the cruise (October 26-November 6), the mixed layer was dominated by diurnal restratification with only a few time periods with strong variability. These periods were relatively short in duration (of order a few hours) and occurred mostly at night. During the latter part of the cruise 

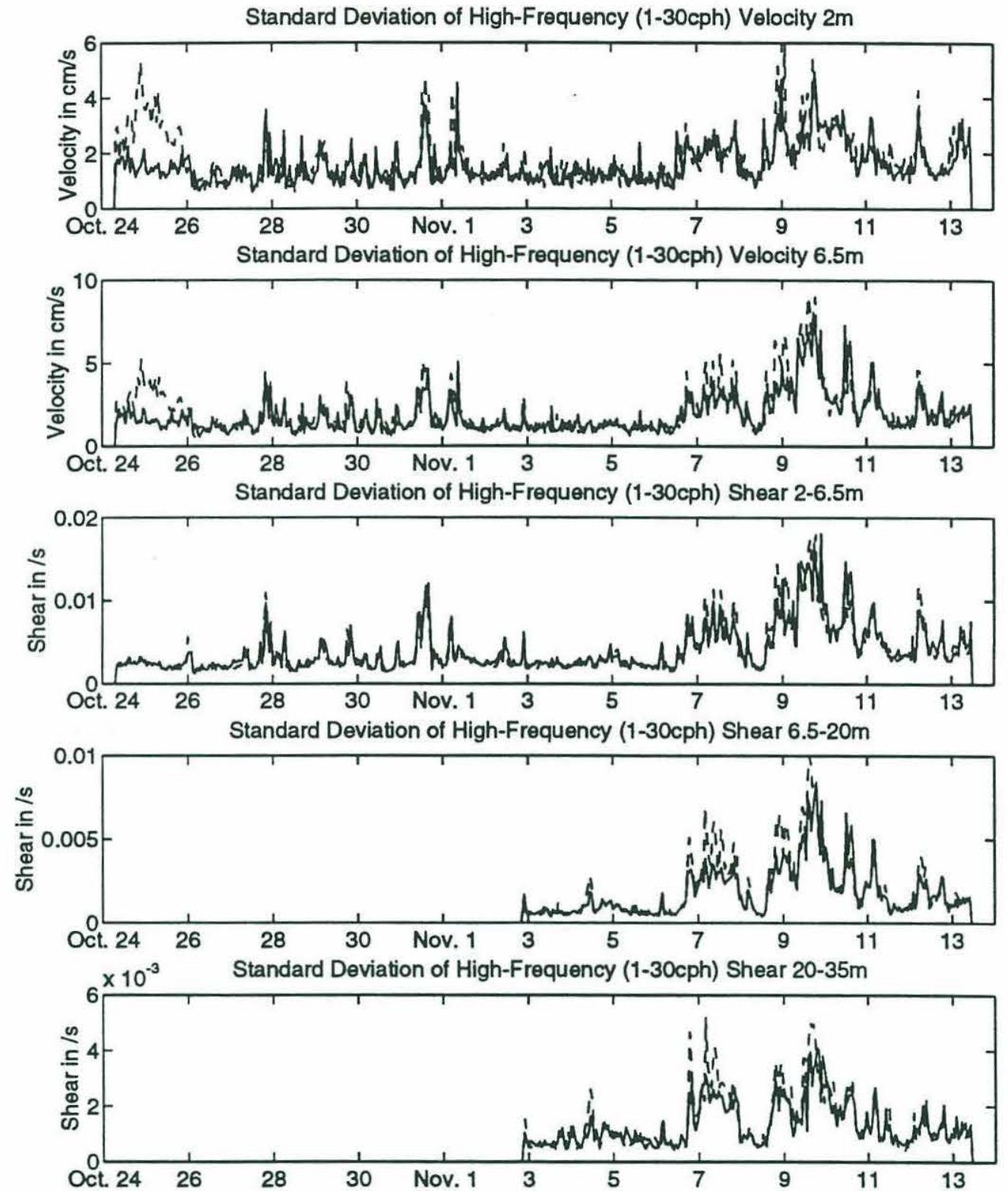

Figure 6.9: Time-varying current and shear during MILDEX. In all cases, solid lines indicate alongwind velocity or shear, dashed lines crosswind velocity or shear. From top to bottom. Standard deviation of the detrended velocity at $2 \mathrm{~m}$. Standard Deviation of the detrended velocity at $6.5 \mathrm{~m}$. Standard deviation of the detrended velocity shear between 2 and $6.5 \mathrm{~m}$. Standard deviation of the detrended velocity shear between 6.5 and 20 meters. Standard deviation of the detrended velocity shear between 20 and 35 meters. 
(November 6-13) when the forcing was stronger and the mixed layer was deeper, there were a number of strong, persistent rises in the level of the high-frequency current and shear. While these high-shear periods were related to the strong wind events mentioned earlier, the relationship is not simple, as the levels of the highfrequency current and shear showed a much more spiky signal than either the wind speed or the wave height.

The high frequency shear is chosen as an index of cell strength for a number of reasons. First, the errors introduced by wave aliasing are less important for high-frequency shear (Appendix D). Additionally, as argued in Chapter 5, the characteristic scale of the shear associated with the cells provides information about the forcing which drives the cells.

The high-frequency shear has an amplitude much larger than that associated with wave aliasing. This does not mean, however, that it is necessarily the result of Langmuir cells. It is not clear a priori that the $1-30 \mathrm{cph}$ frequency band captures the right range of wavelengths for Langmuir circulation. In order to address this question a technique to estimate the energy in a wavelength band from 10-200 m (assuming a frozen-field approximation) was developed. For a 2-hour time period, the mean velocity was computed and rotated into alongwind and crosswind directions. Assuming a frozen field of cells oriented parallel with the wind, the crosswind velocity is a measure of the speed at which cells are advected past FLIP. If $k$ is the wavenumber of the cells and $U_{a d}$ is the advection velocity then

$$
\sigma=\mathrm{k} \mathrm{U}_{\mathrm{ad}}
$$

is the frequency at which one might expect to see velocity fluctuations corresponding to cells of a given horizontal wavenumber. From Figure 6.6 it is clear that simply taking the crosswind velocity will not give the right encounter rate, since the cells may be oriented at some small angle to the wind. Three possible orientations of cells were allowed, 15 degrees to the left of the wind, parallel with the wind, and 15 degrees to the right of the wind, and the crosscell advection velocity was computed for each one. $U_{a d}$ was then taken as the maxima of the three possible crosscell advection velocities. The spectrum of the shear was calculated, and the variance in a frequency band associated with spatial scales of 15-200 meters was extracted. This method is termed the "LC Bandpass".

The shear between 2 and $6.5 \mathrm{~m}$ due to disturbances with wavenumbers of 10-200 meters wavelength was computed using this method. The result is shown 


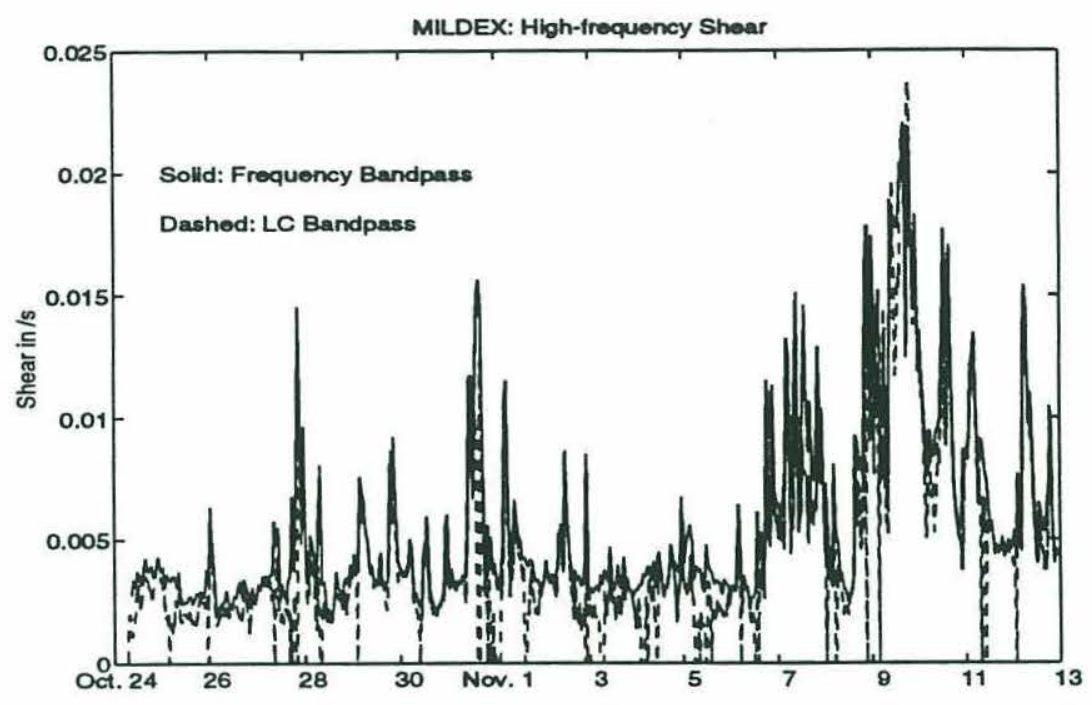

Figure 6.10: Demonstration that high-frequency bandpass captures a range of wavelengths associated with Langmuir cells. Solid line is standard deviation of hourly detrended velocity shear between 2 and $6.5 \mathrm{~m}$. Dashed line is LC bandpass technique discussed in the text.

by the dashed line in Figure 6.10. The solid line shows the simple frequency bandpass. The LC bandpass created a signal with an envelope essentially identical with the frequency band-pass with drop outs due to low crosswind advection. The overall character of the signal was not significantly changed. The overall agreement between the two signals supports the use of the highfrequency shear as a measure of Langmuir circulation, since it offers yet more evidence that the frequency band from 1-30 $\mathrm{cph}$ is affected by structures with the right wavelengths to be Langmuir circulation.

How does the high-frequency shear compare with previously published measurements of Langmuir circulation strength? Figure 6.11a shows the highfrequency shear over the entire experiment and Figure $6.11 \mathrm{~b}$ shows an index of cell strength based on alignment times for surface drifters and vertical velocities seen by the RTP presented in WP. Figure 6.11c shows the wind stress and Figure 6.11d the significant wave height. The indices of cell strength presented here agree in that on days with large high-frequency shears there are also large downwelling and fast alignment of cards. It is difficult, however, to link changes in strength in the card or vertical velocity index to those seen in the highfrequency shear. 


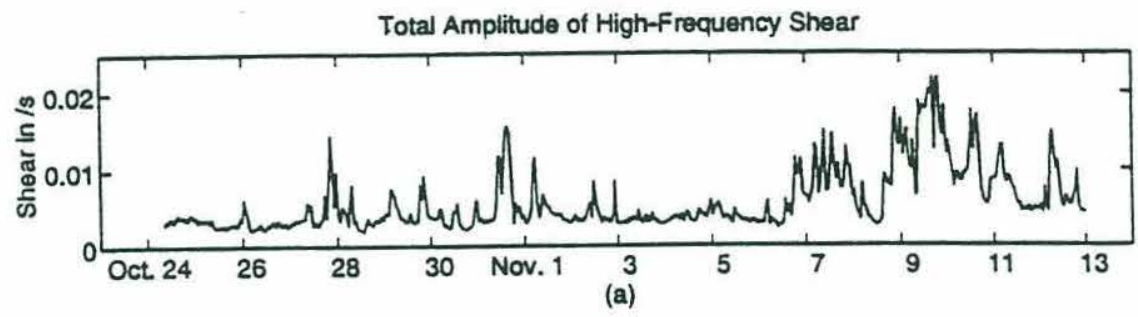

Card/Vertical Velocity Index (Weller and Price, 1988)
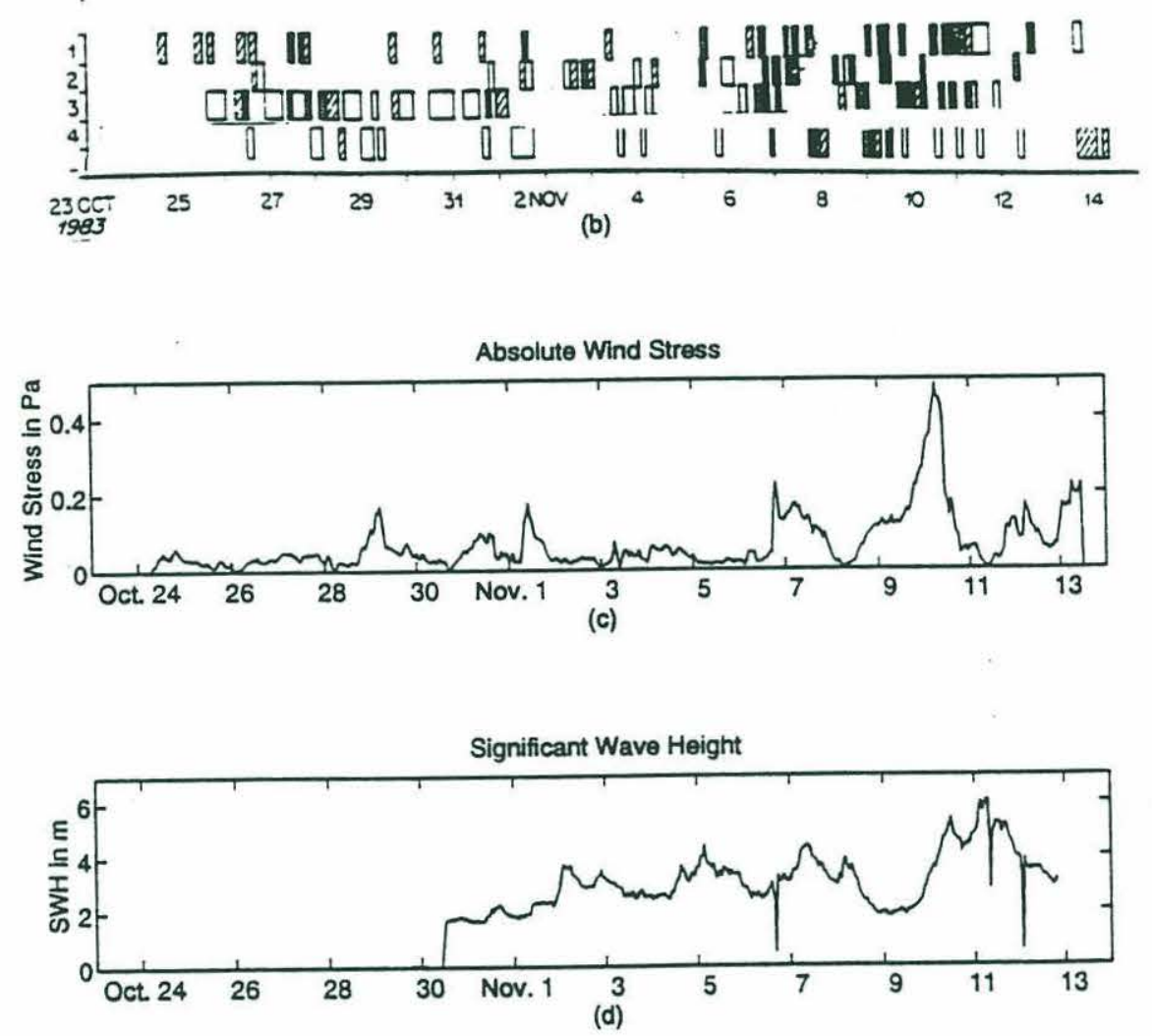

Figure 6.11: Cell Strength during MILDEX (a) High frequency shear (standard deviation of hourly detrended velocity shear between 2 and $6.5 \mathrm{~m}$ ) over the course of the whole cruise. (b) Card and vertical velocity index (from Weller and Price,1988). Intensity of Langmuir circulation at a given depth is summarized as a function of time (horizontally) and depth (vertically) by the shading inside the boxes. Boxes indicate periods of observation at a given depth, level 1 is the surface, 2 is 0 to $15 \mathrm{~m} 3$ is $15-30 \mathrm{~m} .4$ is depths below $30 \mathrm{~m}$. For level 1 , surface drifter observations were quantized by the length of time needed for an initially scattered distribution to form into organized lines; black indicates alignment within 1-2 minutes, closely spaced slanted lines indicate alignment took up to 10 minutes, horizontal lines indicate alignment took 30 minutes to an hour and the most widely spaced lines indicate alignmen took more than an hour. For RTP observations at the various depths the same shading scale is used with the shades (darkest first) corresponding to downwelling speeds of $>25 \mathrm{~cm} / \mathrm{s}, 15-25 \mathrm{~cm} / \mathrm{s}, 5-15 \mathrm{~cm} / \mathrm{s}$ and more than 0 but less than $5 \mathrm{~cm} / \mathrm{s}$. (c) Absolute value of wind stress in Pa. (d) Significant wave height in $\mathrm{m}$. 
Section 6.3.1 showed that the presence of high-frequency shear was related to the presence of Langmuir cells as measured by the Doppler sonars. An attempt was made to make a more quantitative comparison using a subjective index of cell strength based on the Doppler sonar data. This index was constructed as follows. Sonar images during the intensive period studied in SWP were examined in 2-hour segments. During each two-hour segment an index of the cell strength (varying from 0-4) was assigned based on the strength of the coherent velocity structures in the crosswind beam. Figure $6.12 \mathrm{a}$ and $\mathrm{b}$ show the level of the high-frequency shear and the subjctive sonar index during the passage of the low pressure system on November 8,9 and 10. The indices do not agree particularly well, especially as regards the timing of the maximum cell strength. This result is presented as a cautionary example of the difficulty in constructing a consistent picture of cell strength.

To summarize, it has been shown that the presence of Langmuir cells in the mixed layer during MILDEX was broadly correlated with enhanced shear variability in a frequency band from 1-30 cph. Within this frequency band, the upper part of the mixed layer was far from slab-like. The standard deviation of this high frequency shear is a measure of the strength of the cells. As such, however, it did not agree particularly well with the wind stress wave height, or a subjective measure of cell activity based on the Doppler sonar images.
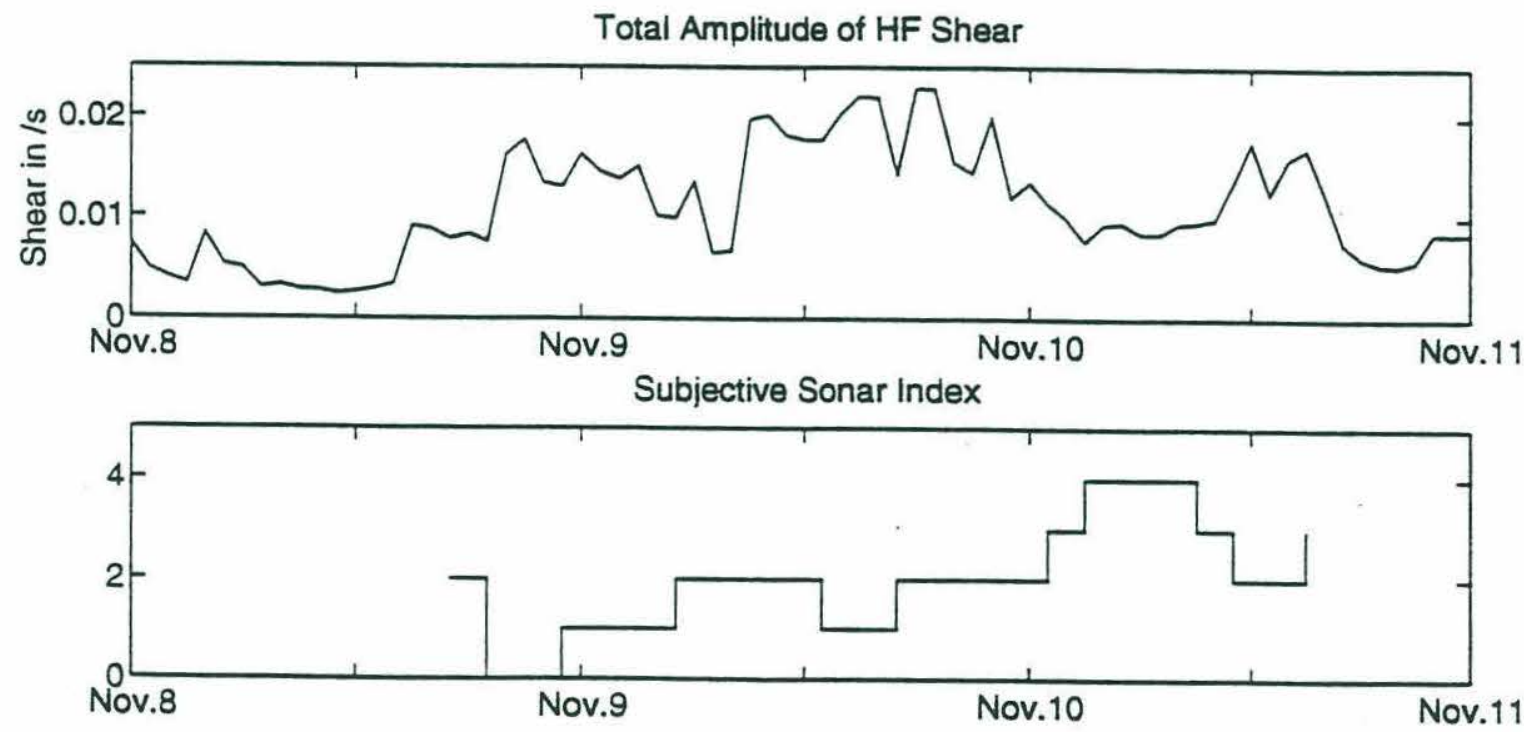

Figure 6.12: Comparison of high-frequency shear and subjective sonar index. Top: Total amplitude of high frequency shear (standard deviation of hourly detrended shear 2-6.5m). Bottom: Subjective sonar index. 


\subsection{Effects of Langmuir Cells on the Velocity and Density Structure}

\subsubsection{Models of Oceanic Response to Wind and Buoyancy Forcing}

Having derived an index of Langmuir cell strength, we now turn to the question of when and how the cells affect the horizontally averaged structure of the upper ocean. As noted earlier in this chapter there are at present two competing conceptual pictures of how the mixed layer is mixed. In the first of these conceptual pictures the processes maintaining the mixed layer have the same scale as that layer. Up to the present point, models within this framework have taken the mixed layer to be a slab, well-mixed with respect to all scalar and vector quantities (Pollard et al., 1973; Denman and Miyake, 1973; Price et al., 1986, henceforth PWP). The depth of the mixed layer changes as the result of buoyancy forcing and shear instability and/or isotropic turbulence produced at the upper boundary and transported to the mixed layer base by unspecified processes. The processes maintaining the slab have not been specified.

This section uses the slab model presented by Price,Weller, and Pinkel (1986) as a baseline against which to compare the observed the response. The PWP model has been verified on a number of occasions (PWP, 1986; Stramma, et al., 1986; Price, Weller, Bowers, and Briscoe, 1987) and is used operationally by the U.S. Navy. The model has two regimes in which active mixing occurs. In the "mixed layer" regime, the upper part of the water column is treated as a perfectly mixed slab which becomes shallow as the result of surface heating or freshwater input and deepens as the result of shear (Kelvin-Helmholtz) instability or buoyant convection. Below this layer is a transition layer which mixes so as to maintain a local Richardson number greater than 0.25 . In the transition layer, mixing occurs only between neighboring grid points and so is small-scale in its character.

A second conceptual picture of how the mixed layer is maintained is a pure small-scale mixing picture, exemplified by the work of Mellor and Yamada (1974). In this picture, mixing is driven by Kelvin-Helmholtz and Rayleigh-Taylor (buoyant) instabilities which have small (order tens of centimeter) scales. The Mellor-Yamada models produce fields of conservative scalar quantities like temperature and salinity which are similar to those given by the PWP model, with the difference that they allow for mean gradients within the well-mixed portions of the surface layer. This section uses a level 2 Mellor-Yamada model, similar to that of Klein and Coste (1984). This model will be referred to as the MY2 model. It assumes a balance between local production and dissipation: 


$$
-\overline{\mathrm{u}^{\prime} \mathrm{w}} \frac{\partial \mathrm{U}}{\partial \mathrm{z}}-\overline{\mathrm{v}}^{\prime} \mathrm{w} \frac{\partial \mathrm{V}}{\partial \mathrm{z}} \quad-\frac{\mathrm{g}}{\rho^{\prime} \mathrm{w}} \quad=\quad \varepsilon
$$

\section{Shear Production + Buoyancy Production $=$ Dissipation}

The nonlinear transports and dissipation are then parameterized in terms of the eddy viscosity, eddy diffusivity, mean shear, and stratification, and the viscosity and diffusivity are solved for. The energy balance does not include a term for wave forcing corresponding to the Craik-Leibovich mechanism.

Because mixing within the MY2 model is accomplished by eddy diffusivity and viscosity, the model produces a horizontally averaged velocity profile which is quite different from the PWP model. As shown in Chapter 8, a conceptual picture in which Langmuir circulations are responsible for maintaining the mixed layer can narrow the gap between the slab models and small-scale mixing models by allowing Langmuir cells to homogenize the velocity structure within the mixed layer produced by small-scale mixing.

\subsubsection{The Near-Surface Shear and Stratification during MLDEX}

We begin our analysis of the low-frequency response by looking at time series of the near-surface shear and stratification during MILDEX. Figure 6.13 shows the temperature difference between 2 and 10 meters computed from the hourly-binned data. The solid line shows the result from data, the dashed line the

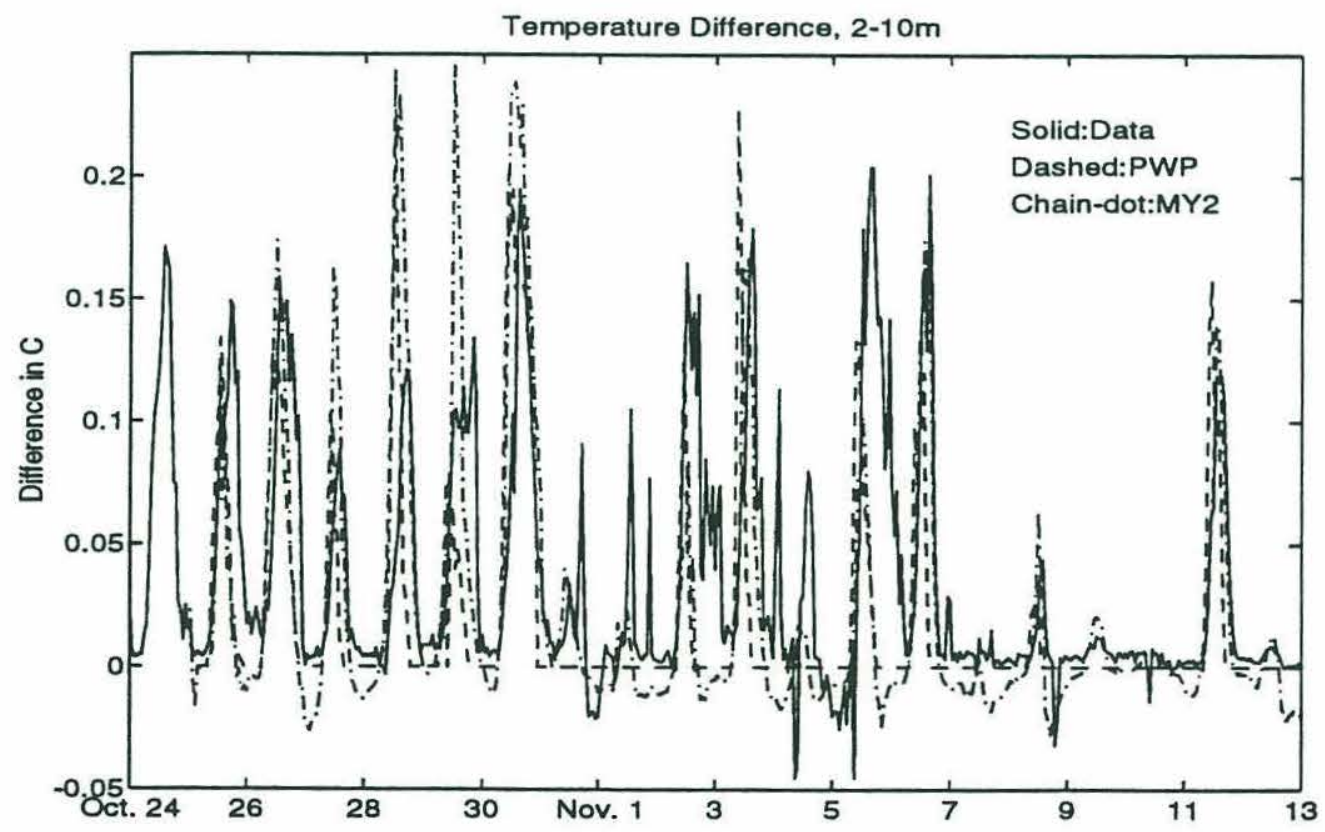

Figure 6.13: Temperature difference between 2 and 6.5m. Solid: Data. Dashed: PWP model. Chain-dotted: Mellor-Yamada Model. 
result from the PWP model, and the chain-dotted line the result of the MY2 model. The three curves were generally in good agreement. When the data showed diurnal restratification, both of the models did too. In general, the amplitude of the diurnal restratification was quite well captured, but there were a few exceptions. On October 28th both the PWP and MY2 models overpredicted the diurnal restratification by a factor of about 2 . On October 30, the PWP model underpredicted the temperature difference, while the MY2 model overpredicted it. On November 1st and 6th, the models underpredicted the stratification, which appeared to be associated with frontal activity.

In general, the two models did relatively well at reproducing the observed temperature stratification. The velocity structure is another matter altogether.

Figure $6.14 \mathrm{a}$ and $6.14 \mathrm{~b}$ show the velocity difference between 2 and 10 meters in the alongwind and crosswind directions respectively. There were major differences between the observed velocity and that predicted by the models. A time of particular interest is November 7-11, where the PWP model predicted no shear between 2 and 10 meters and the MY2 model predicted shear in the downwind direction. The data in fact shows the water at depth moving more strongly upwind and to the right of the wind than the water at the surface.

The presence of such upwind shear is an indicator of mixing accomplished by large-scale processes. In a small-scale mixing picture, shear is the result of local "eddy viscosity" producing mixing proportional to a mean velocity gradient. Upwind shear implies that the eddy viscosity must be negative, something which can only occur in the presence of organized, nonlocal mixing.

The presence of upwind shear was related to the presence of highfrequency shear, while strong downwind shear was related to stratification. Figure 6.15a shows the shear along an axis 45 degrees to the right of the wind, Figure $6.15 \mathrm{~b}$ shows the the standard deviation of the shear between 2 and $6.5 \mathrm{~m}$ while $6.15 \mathrm{c}$ shows the temperature difference between 2 and $10 \mathrm{~m}$. Strong upwind shears occurred on October 31 and each day between November 6 and 12. Additionally, there were episodes of downwind shear on October 24, 26, 27 , 28, November 2,3 and 4.

The strong low-frequency upwind shears were correlated with the elevated levels of high-frequency shear while the downwind shears were correlated with diurnal restratification. Figure $6.15 \mathrm{~d}$ shows a scatterplot of the level of high frequency shear versus the velocity difference along an axis 45 degrees to the right of the wind. Times when the high-frequency shear was large 


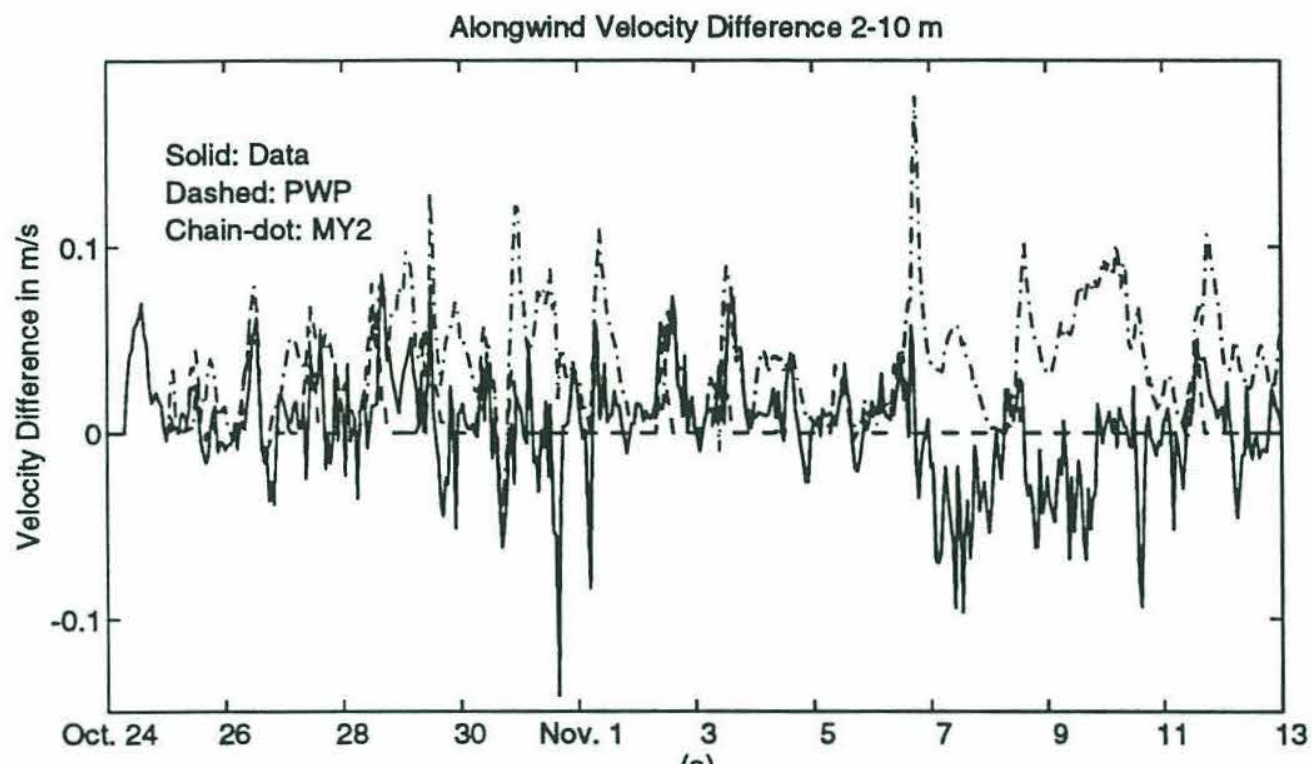

(a)

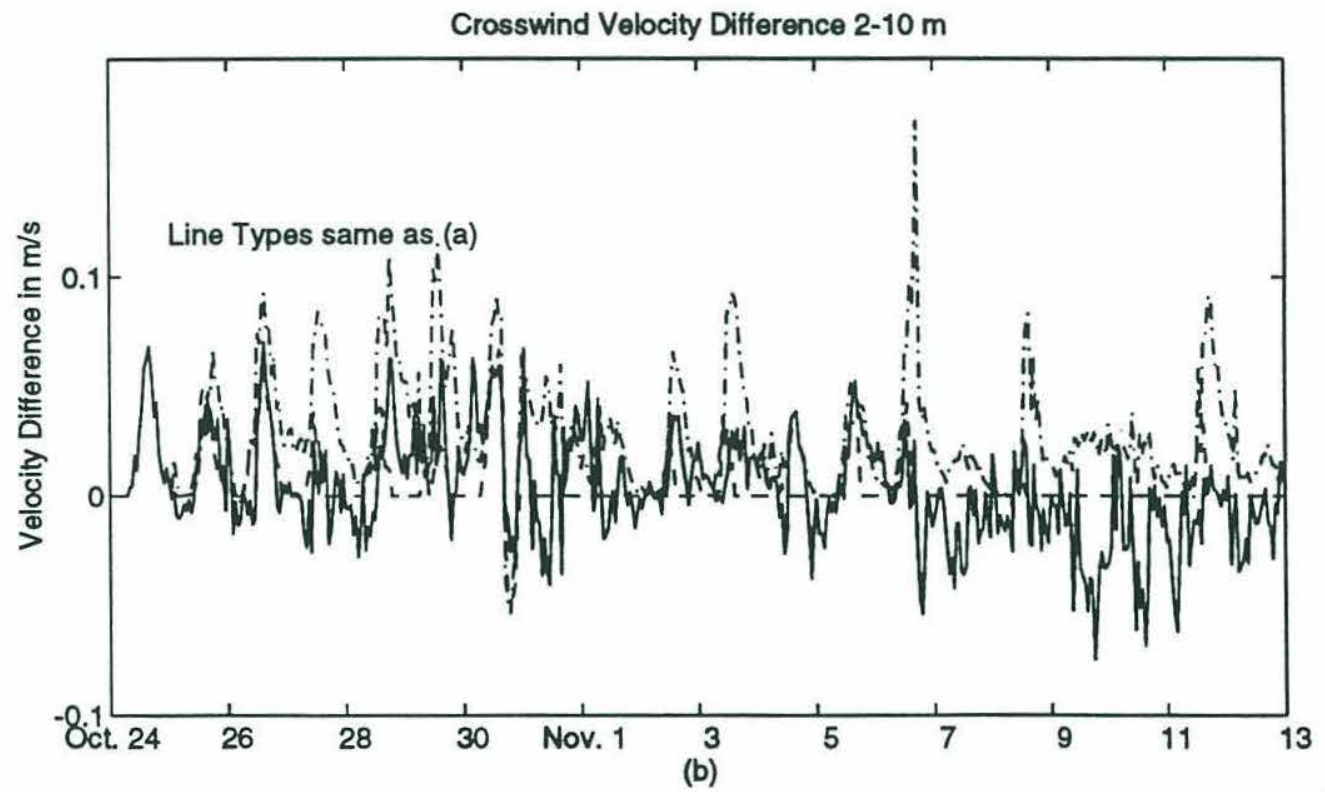

Figure 6.14: Velocity Difference between 2 and 10 meters in $\mathrm{m} / \mathrm{s}$. Solid: Data. Dashed: PWP model. Chain-dotted: MY2 Model. (a) Alongwind velocity. (b) Crosswind velocity. 


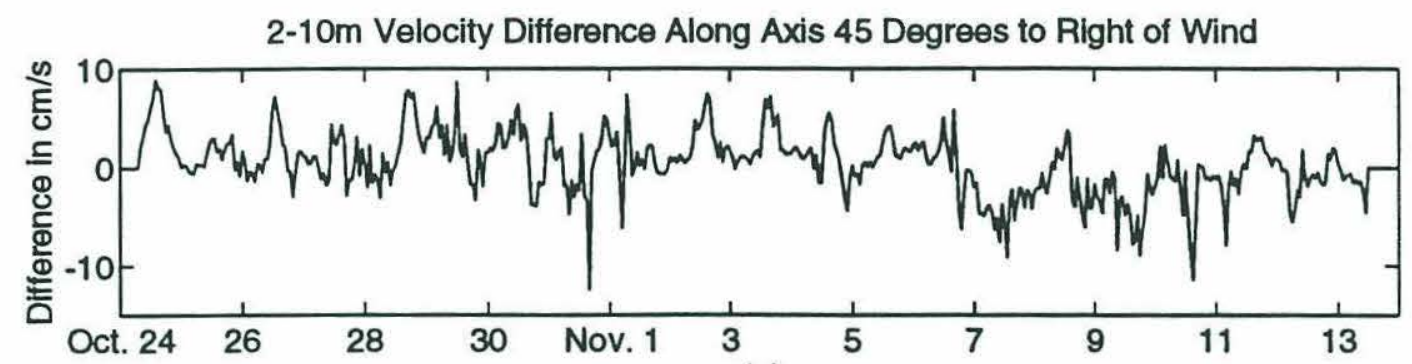

(a)

Standard Deviation HF Shear 2-6.5m

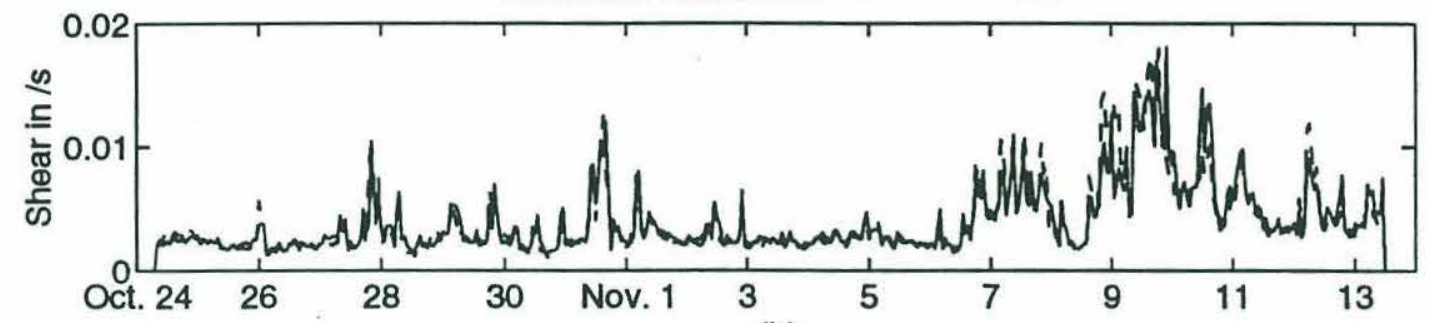

(b)

Temperature Difference 2-10m

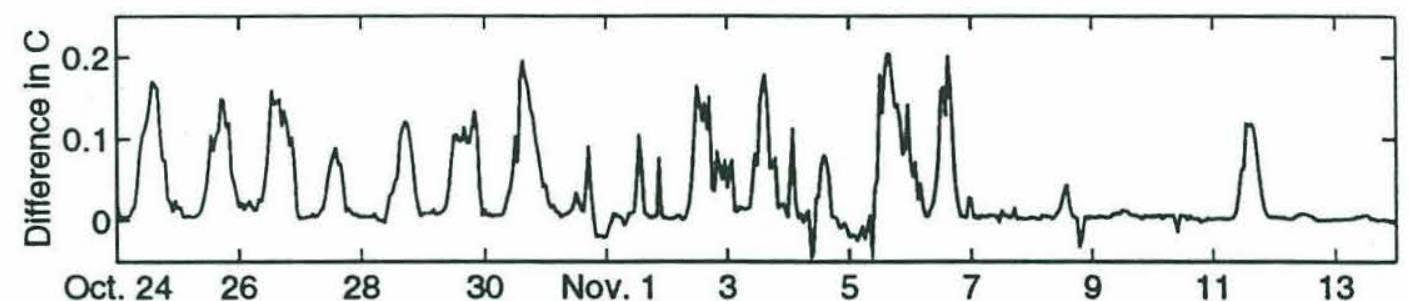

(c)

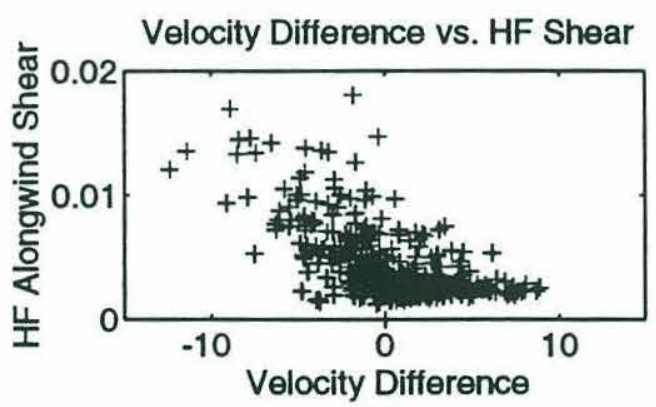

(d)
Velocity Difference vs. Temp. Difference

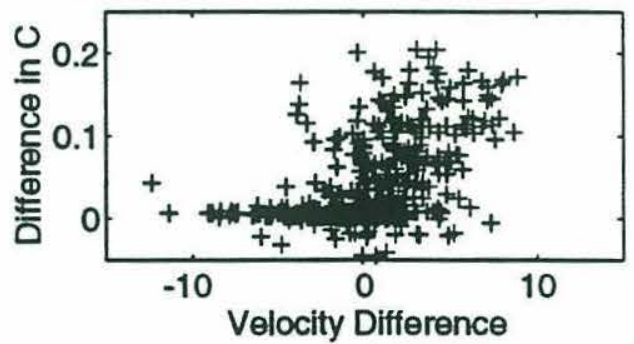

(e)

Figure 6.15: Occurence of upwind shear is related to presence of high-frequency shear. (a) $2-10 \mathrm{~m}$ velocity Difference along axis 45 degrees to the right of the wind. (b) Standard deviation of the detrended shear between 2 and $6.5 \mathrm{~m}$, solid is alongwind,dashed is crosswind. (c) Temperature Difference between 2 and 10 meters. (d) $2-10 \mathrm{~m}$ velocity difference in axis 45 degrees to right of wind vs. level of alongwind high-frequency shear. (e) $2-10 \mathrm{~m}$ velocity difference along axis 45 degrees to right of wind vs. $2-10 \mathrm{~m}$ temperature difference. 
corresponded to times when there was more upwind shear (negative values of velocity difference). Figure 6.15 e shows temperature difference between 2 and $10 \mathrm{~m}$ plotted against the velocity difference along an axis 45 degrees to the right of the wind. When there was strong temperature stratification, there was generally a corresponding downwind shear (positive velocity difference).

In summary, neither a small-scale mixing model or a slab model accurately captured the velocity structure in the top 10 meters during MILDEX when Langmuir cells were strong, even though both models did a reasonable job at capturing the temperature structure. The following subsection studies how this difference is reflected in the low-frequency $(0.01-0.1 \mathrm{cph})$ response of the mixed layer to wind forcing.

\subsubsection{The Ekman Response during MILDEX}

In order to attack the question of the Ekman response during MILDEX, the first and second parts of the experiment are considered separately. During the first part of the experiment (October 25-November 5), the mixed layer was dominated by the cycle of daytime heating and nightime cooling described in PWP. Langmuir cells, as measured by cards and current meters appear to have been weak. During the second part of the experiment (November 6-14) the mixed layer is fairly deep and cells were strong. This section examines the Ekman response to surface forcing during these two periods and compares it to that predicted by the MY2 and PWP models.

In order to properly characterize the wind-driven part of the flow, it is necessary to separate the wind-driven flow from the mean geostrophic flow associated with mesoscale features. This is done by choosing a reference depth, below which the response to the local stress (as opposed to the curl or gradient of that stress) is taken as zero.

Davis et al. (1981) proposed a spectral model for extracting the Ekman response. They asssumed that the velocity profile might be modelled by the relationship

$$
\hat{\mathrm{U}}(\mathrm{z}, \omega)=\mathrm{S}(\mathrm{z}, \omega) \hat{\tau}(\omega)
$$

where $\hat{\tau}$ represents the Fourier transform of the complex stress vector $\tau_{x}+i \tau_{y}$ and $\hat{\mathrm{U}}$ represents the Fourier transform of the complex velocity vector $\mathrm{u}+\mathrm{iv}$ relative to some reference depth. Within this model, the reference depth $\mathrm{z}_{\text {ref }}$ is computed as 
that depth which maximizes the coherence between velocities above that depth and the wind stress.

An alternative methodology is to simply rotate the velocity into a coordinate system referenced to the wind direction and to average over time. This method picks out the velocity response which is in phase with the most energetic components of the wind. Within this framework, the reference depth is chosen as that which gives the most nearly correct value for the magnitude of the Ekman transport. The magnitude of this transport can be predicted using a mixed layer model (which has to close the momentum budget within its domain). One problem with this method is that it convolves the velocity response across many different forcing frequencies. It has the advantage, however, of being applicable to relatively short data sets (as short as a single inertial period).

In order to isolate the effect of Langmuir circulation, the experiment is divided into a time period where cells were not strong (Period 1), and a time period where cells were strong (Period 2). The 14 inertial periods from 0200 PST October 25 to 1400 PST November 5th are denoted as Period 1. Choosing this interval avoids effects from the cold front which passed through the experiment site on November 6. Period 2 covers five inertial periods starting at 1500 PST on November 8.

During Period 1, diurnal restratification was strong. Figure 6.16 presents the Ekman response during this period given a reference depth of 35 meters for the data (top row), PWP model (middle row) and Mellor-Yamada model (bottom row) using the methodology of Davis et al. (1981). The left-hand column shows the coherence between velocity relative to 35 meters and wind stress. Only contours above 0.3 are shown. The $90 \%$ confidence level, (computed by taking 100 Gaussian white noise time series of the same length and applying the same processing) is 0.32 , the $95 \%$ level 0.40 . The right-hand column shows the structure of the velocity response for a frequency band centered at $-0.01 \mathrm{cph}$ as a function of depth.

During Period 1, the velocity relative to 35 meters was significantly coherent with the surface stress over a range of frequencies from about $-0.05 \mathrm{cph}$ to $0.05 \mathrm{cph}$ and over depths down to 20 meters. Maximum coherence was found at low frequencies, in a band centered around $-0.01 \mathrm{cph}$. There was a drop in coherence near the inertial frequency (not surprisingly, since inertial oscillations need not be coherent with the wind stress). The transfer function at $-0.01 \mathrm{~Hz}$ 

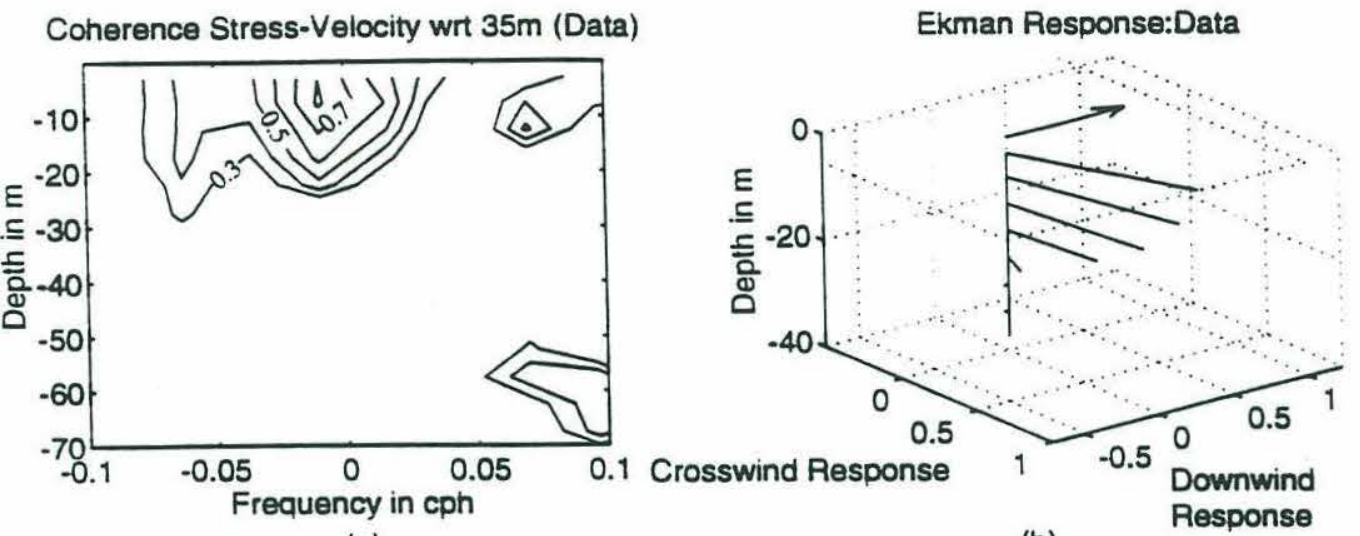

(a)

(b)

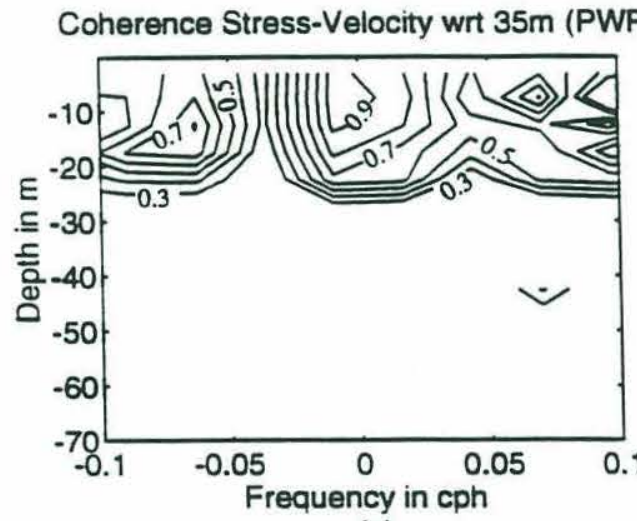

(c)

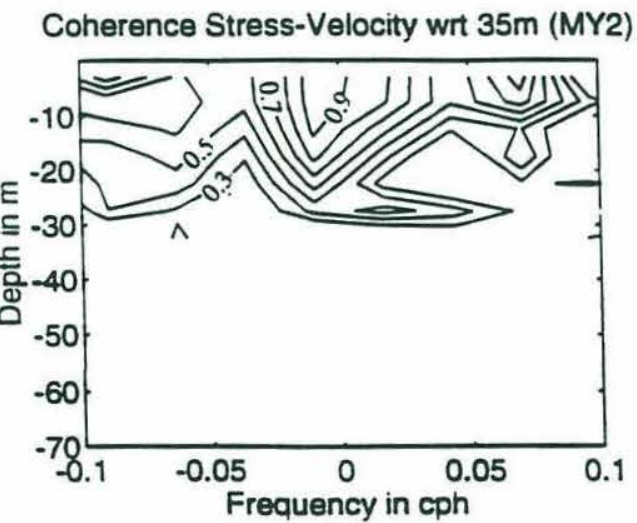

(e)

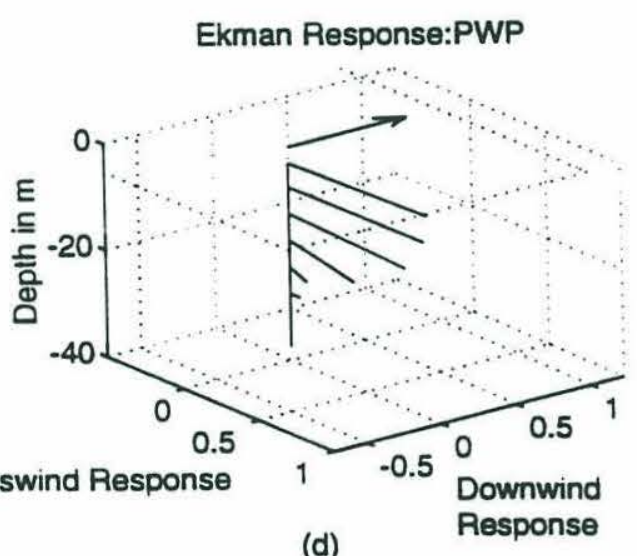

(d)

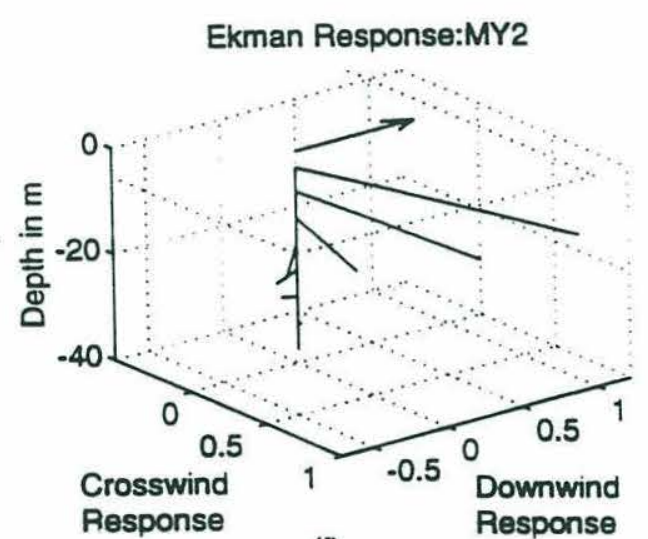

(f)

Figure 6.16: Velocity response relative to 35 meters during Period 1 computed by spectral method. $95 \%$ confidence level is 0.35 . (a) Coherence between wind stress and observed velocity. (b) Transfer function for frequency band with maximum coherence (centered at $-0.01 \mathrm{cph}$ ) between wind stress and observed velocity. (c) Coherence between wind stress and PWP velocity. (d) Transfer function for frequency band with maximum coherence (centered at $-0.01 \mathrm{cph}$ ) between wind stress and PWP velocity. (e) Coherence between wind stress and MY2 velocity. (f) Transfer function for frequency band with maximum coherence (centered at $-0.01 \mathrm{cph}$ ) between wind stress and MY2 velocity. 
between wind stress and velocity showed a response off to the right of the wind. The response was concentrated in the upper 20 meters of the water column.

Both the PWP and MY2 models did a reasonable job at reproducing the observed coherence, although both models predicted significantly more coherence at superinertial frequencies and the PWP model predicted less coherence at the inertial frequency. The PWP model produced a low-frequency Ekman response similar to the data, though it was more to the right of the wind near the surface. The MY2 model's low frequency Ekman response was more strongly sheared than the data or the PWP model. As a result, the surface velocity was about $50 \%$ larger than the data and oriented more in the crosswind direction.

The picture changes when shear rather than velocity is examined. Figure 6.17 shows the coherence between the wind stress and local shear and the structure of the coherent shear during Period 1 for the data and the two models. The coherence is shown in the left-hand column. The data showed low coherence near the surface with high coherence near the mixed layer base at 25 meters. In this case the PWP model performed significantly better than the MY2 model in reproducing the observed coherence. The MY2 model showed strong coherence at low frequencies all the way up to the surface. In fact, the highest coherences in the MY2 model occurred near the surface. The PWP model, with a slab-like nearsurface layer did not show such coherence. This point is considered in more detail in 6.5.5.

The shear response during Period 1 is shown in the right-hand column of Figure 6.17. Once again, the PWP model comes closer than the MY2 model to reproducing the near-surface shear. In both the data and the PWP model the shear was small and off to the right of the wind near the surface. In both the model and data the shear increased with depth, turning slightly to the right of the wind reaching a maximum around 10 meters. The data showed slightly more downwind shear than the PWP model, but the differences were not glaringly obvious. The MY2 model, on the other hand showed a concentration of shear near the surface, decreasing and turning to the right with depth as in the classical Ekman spiral.

The normalized Ekman transport $\mathrm{T}_{\mathrm{ek}}$ during Period 1 may be computed by integrating the transfer functions shown in Figure 6.16 down to a given depth.

$$
T_{\text {ek }}=\int_{z_{\text {int }}}^{0} \rho f S(\omega, z) d z
$$




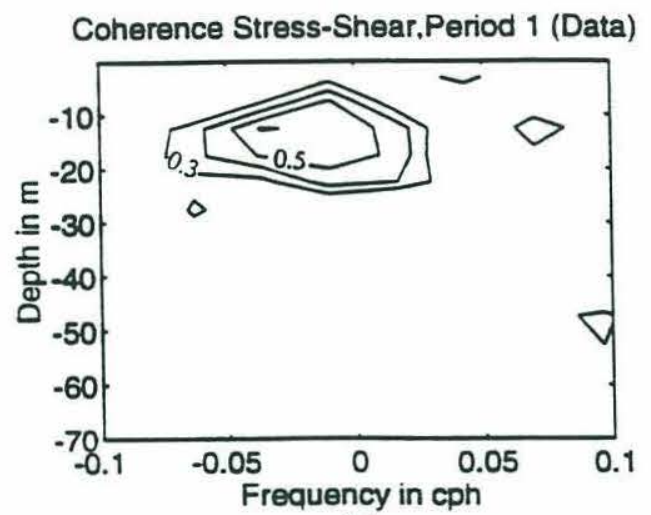

(a)

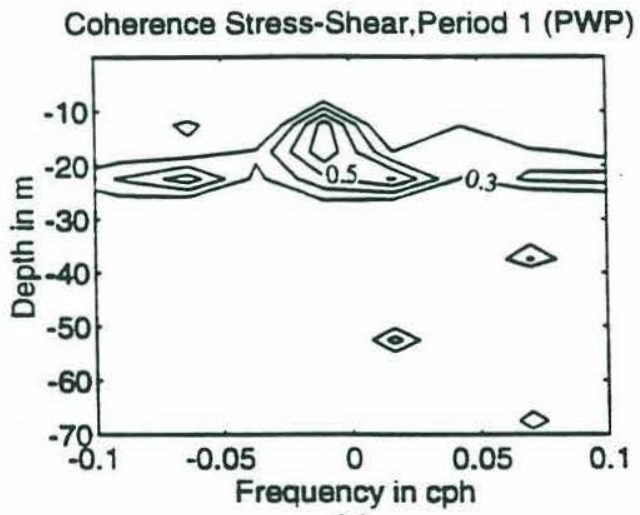

(c)

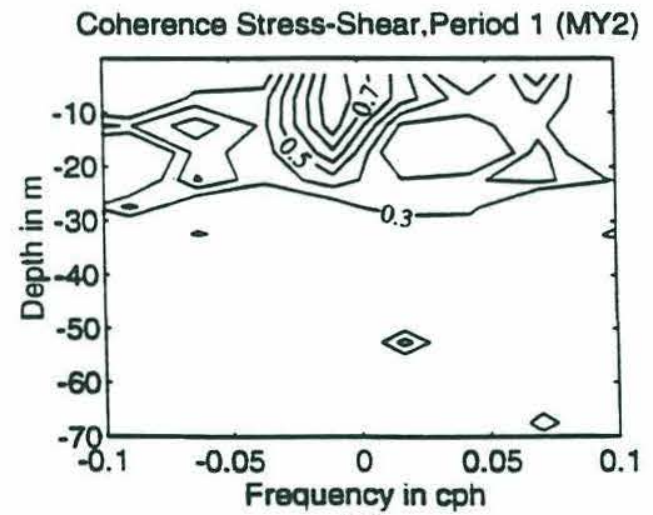

(e)

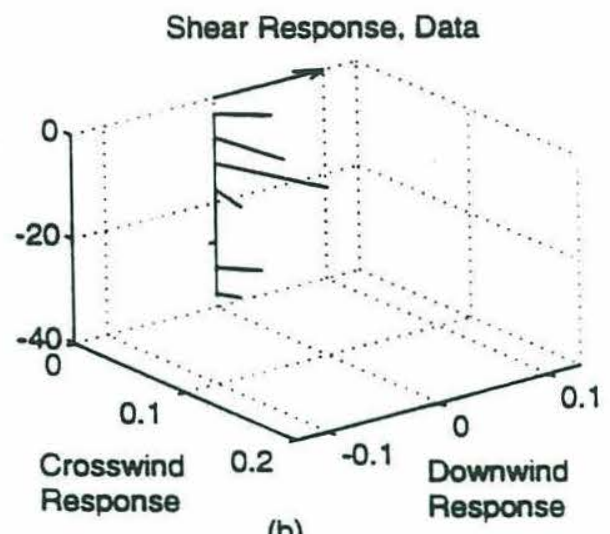

(b)

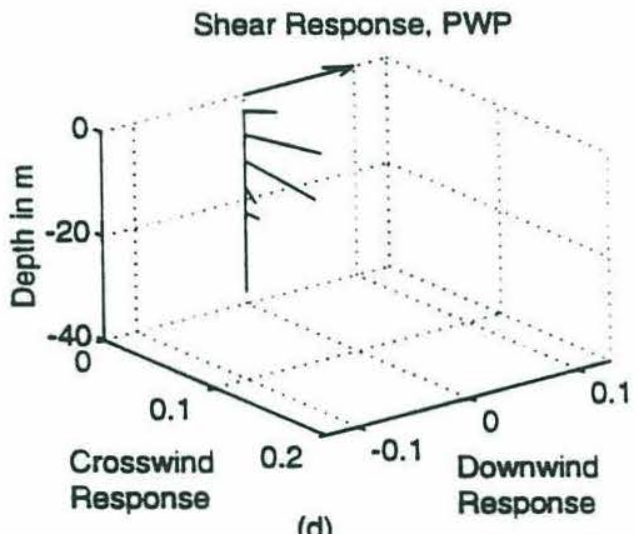

(d)

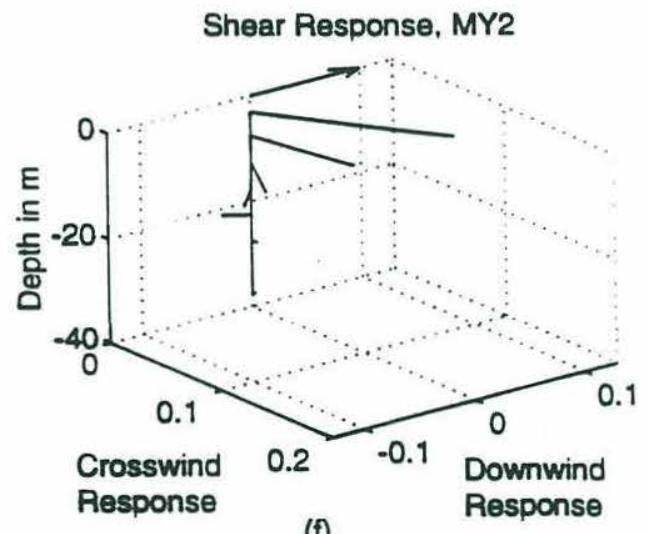

(f)

Figure 6.17: Shear response to surface wind stress during Period 1 computed by spectral method.. (a) Coherence between wind stress and observed shear. (b) Transfer function for frequency band with maximum coherence (centered at $-0.01 \mathrm{cph}$ ) between wind stress and observed shear. (c) Coherence between wind stress and PWP shear. (d) Transfer function for frequency band with maximum coherence (centered at $-0.01 \mathrm{cph}$ ) between wind stress and PWP shear. (e) Coherence between wind stress and MY2 shear. (f) Transfer function for frequency band with maximum coherence (centered at $-0.01 \mathrm{cph}$ ) between wind stress and MY2 shear. 


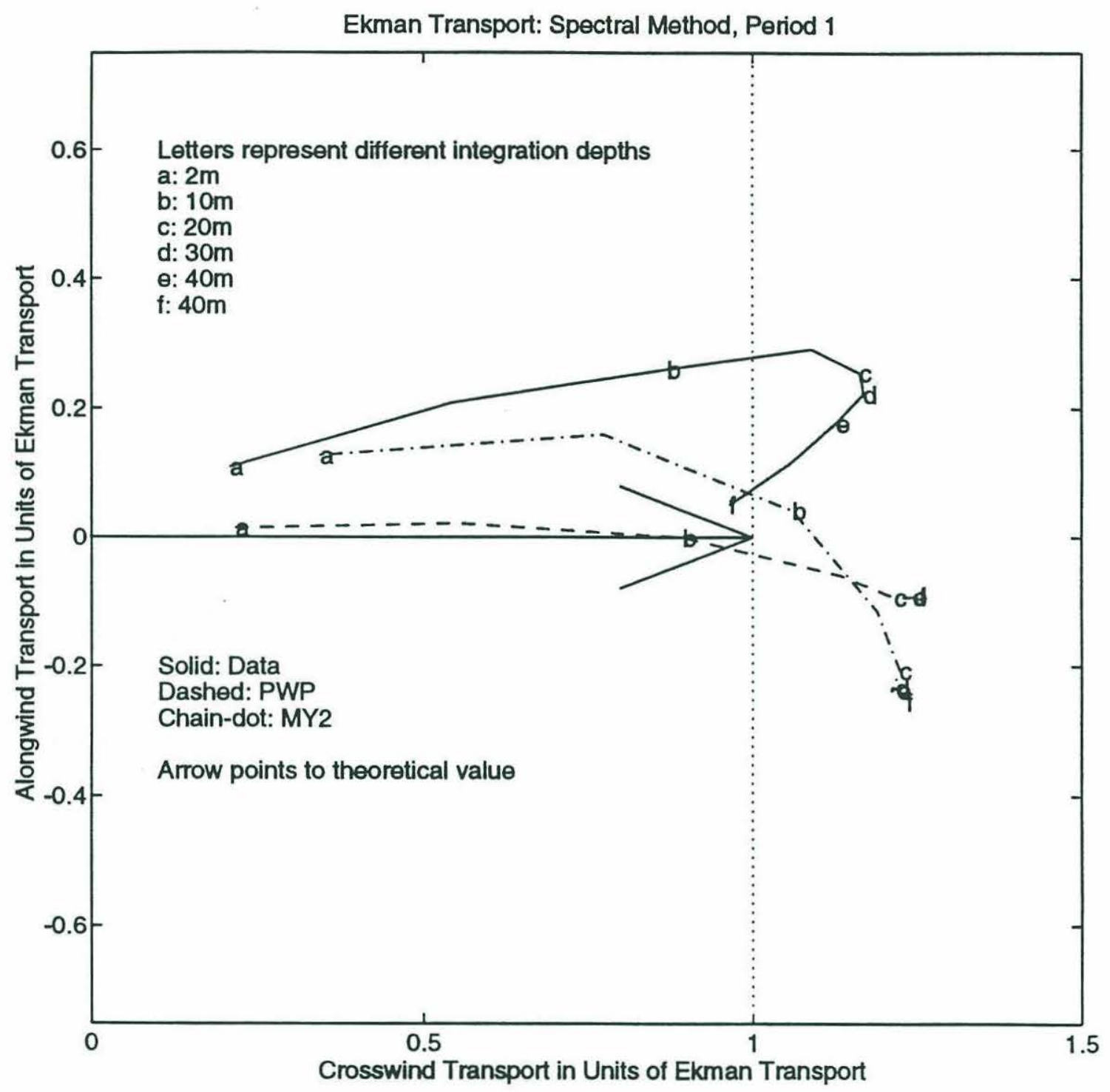

Figure 6.18: Ekman transport calculated by integrating the spectral transfer function $S(\omega, z)$ calculated using a reference depth of 35 meters from the surface to a depth $\mathrm{z}=\mathrm{z}_{\text {int }}$ for $\omega=-0.01 \mathrm{cph}$. (Equation 6-11) Values shown are normalized by the theoretical value of Ekman transport. Solid lines are using the observed transfer function, dashed lines that predicted by the PWP model, chain-dotted lines that predicted by the MY2 model. Letters represent different values of $\mathrm{z}_{\text {int }}$. $(\mathrm{a}=2 \mathrm{~m}, \mathrm{~b}=10 \mathrm{~m}, \mathrm{c}=20 \mathrm{~m}, \mathrm{~d}=30 \mathrm{~m}, \mathrm{e}=40 \mathrm{~m}, \mathrm{f}=50 \mathrm{~m})$. Arrow points to theoretical value of transport ( 1 in the crosswind direction, 0 in the alongwind direction). 
Essentially, $\mathrm{z}_{\mathrm{ref}}$ is chosen so as to extract the wind-driven response while $\mathrm{z}_{\text {int }}$ gives some measure of the depth to which there is structure in that response. If the response is modal, an appropriate $\mathrm{z}_{\mathrm{ref}}$ might occur at a zero-crossing of the mode while $z_{\text {int }}$ would be deeper in the water column. The results of the calculation outlined in (6-11) are shown in Figure 6.18 as a function of $z_{\text {int }}$. The solid line represents the integration of the transfer function from data from the surface downward, the dashed line the PWP model, and the chain-dotted line the MY2 model. The letters a through $f$ show different values of $z_{\text {int }}(a=2 m, b=10 m$, $c=20 \mathrm{~m}, \mathrm{~d}=30 \mathrm{~m}, \mathrm{e}=40 \mathrm{~m}, \mathrm{f}=50 \mathrm{~m})$. The theoretical Ekman transport is shown by the arrow. The model transfer functions show essentially no change when $z_{\text {int }}$ is greater than 20 meters, indicating the transport is entirely carried at depths of 20 meters and above. The data, however, does show some change when $z_{\text {int }}$ is greater than the reference depth. As the coherence between current and wind stress is low for these depths, the agreement may be fortuitous, but it is indicative of the difficulty in making good estimates of the Ekman transport.

If the three transfer functions are integrated down to 30 meters, the transport in the data is $120 \%$ of the theoretical value and 79 degrees to the right of the wind. The PWP and MY2 models both give transports which are also about 25\% larger than the theoretical value and are 94 and 100 degrees to the right of the wind respectively. The models do not give a result which agrees with theory exactly because of inertial oscillations and because the model output has been degraded to the same resolution as the data. As such the models provide a rough estimate of the inherent error in measuring the Ekman transport given the available spatial and temporal resolution. The final transports agree to within $20 \%$.

The above calculation demonstrates that the Ekman transport during the first part of MILDEX may be recovered by using the spectral method of Davis et al. (1981). A second method, involving orienting relative to the wind and averaging is now considered. In this method, one begins by choosing a reference depth and then looking at the response.

During Period 1, the mean rotated and averaged transport from the models was very close to the theoretical value when reference depths below 30 meters were chosen. The data showed its best agreement with the theory and the models when the reference depth was chosen to be $40 \mathrm{~m}$. This was quite close to the 35 meters chosen as the reference depth by the coherence criterion of Davis et al. (1981). As in the spectral model, most of the transport was carried above 20 


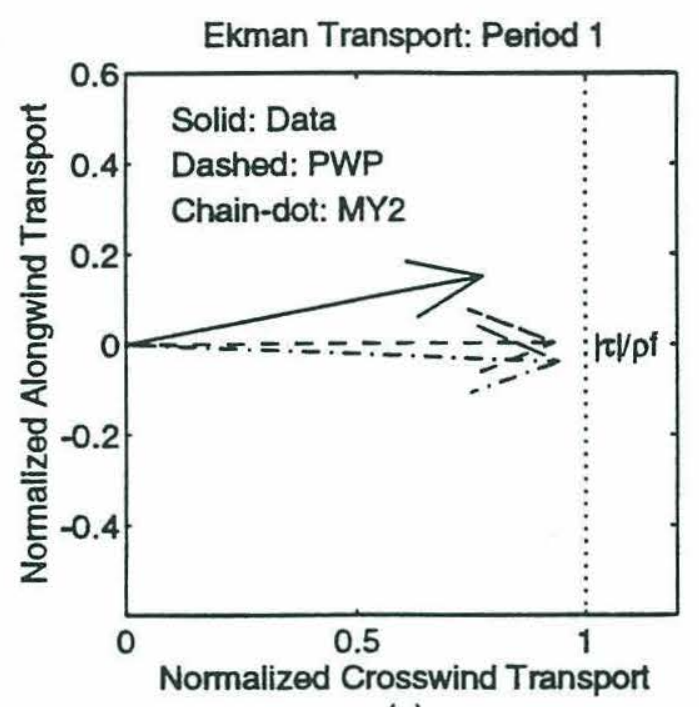

(a)

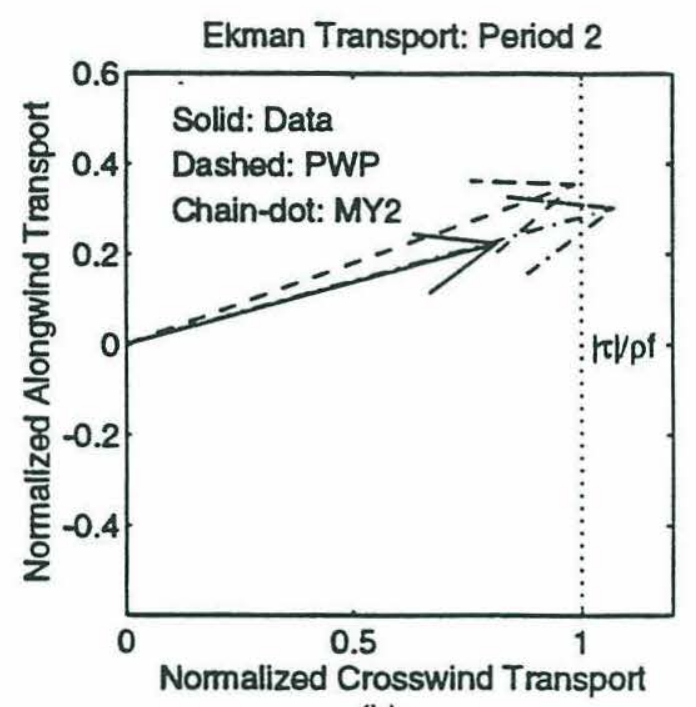

(b)

Figure 6.19: Ekman transports computed by orienting the velocity relative to the wind and averaging. Transports are normalized by $|\tau| / \rho f$ where $|\tau|$ is the mean stress over each time period. Theoretical value is 1 in the crosswind, 0 in the alongwind direction. (a) Period 1 (Wind and Langmuir cells weak). (b) Period 2 (wind and cells strong).

meters depth and the transport in the data is slightly smaller and more downwind than in the models. The Ekman transports for this period are shown in Figure 6.19a. The transports predicted by orienting the current relative to the wind and averaging are in close agreement with the naive prediction. The observed transport is slightly larger in the alongwind direction than predicted, as was the case for the spectral model.

The Ekman transports for Period 2 are shown in Figure 6,19b. During Period 2, the transport in the models was slightly larger than the naive theory would predict and was somewhat downwind. The data came closest to reproducing this transport when a reference depth of 60 meters was chosen. The transport was $85 \%$ of the theoretical value in the data, $104 \%$ in the PWP model and $111 \%$ in the MY2 model. The data and both models showed the transport to be oriented about 70 degrees to the right of the wind.

The agreement between the predicted and observed transport is interesting since the theory developed in Chapters 3 and 5 predicted that there should be an Eulerian return flow associated with the waves. A strong upwind transport associated with this flow is not seen. Instead, the data shows a flow which has a larger downwind transport than is predicted by the models. 


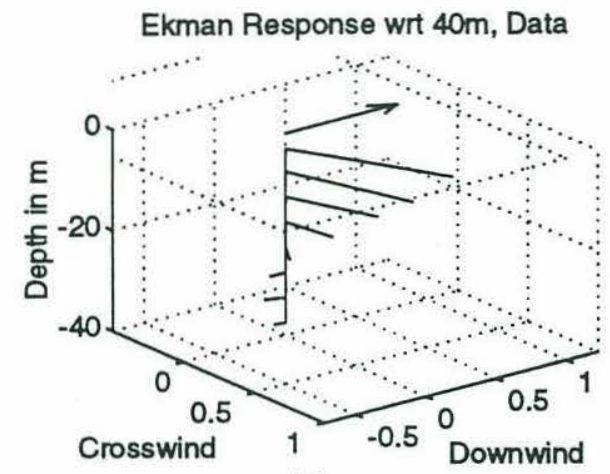

(a)

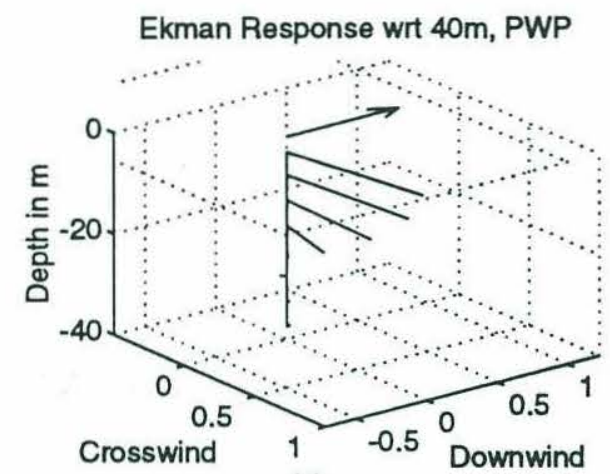

(c)

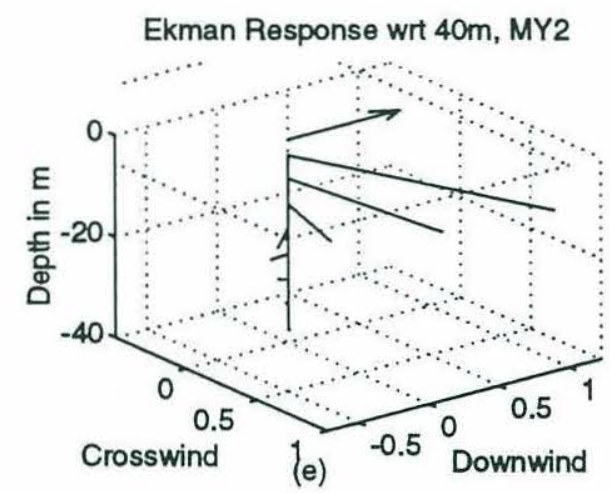

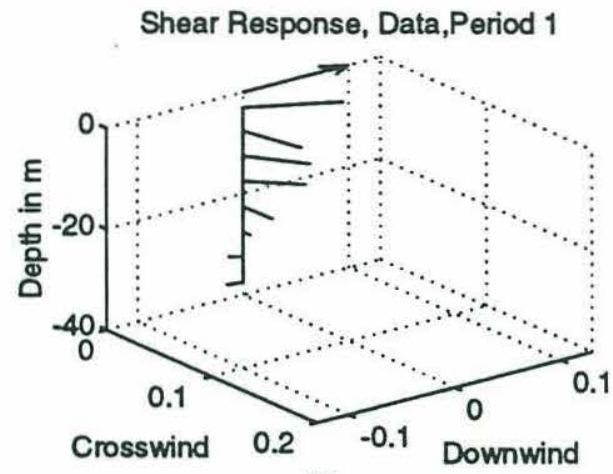

(b)

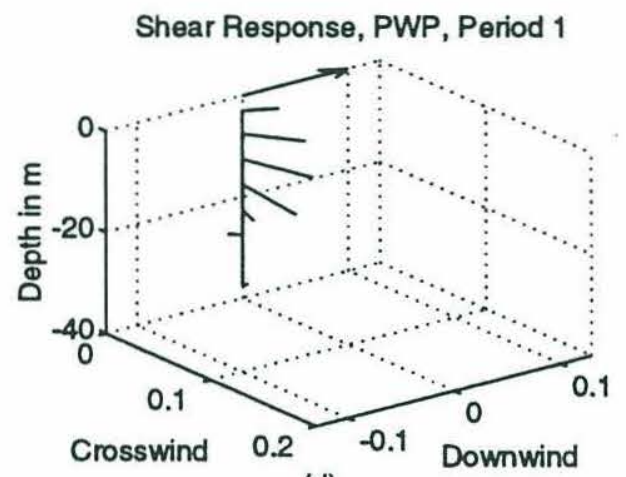

(d)

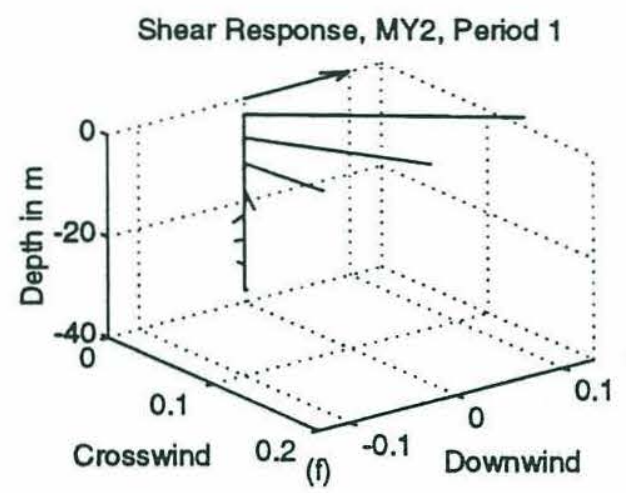

Figure 6.20: Velocity response relative to 40 meters and shear response as a whole during Period 1 computed by orienting relative to wind and averaging. (a) Velocity response, Data. (b) Shear Response, Data. (c) Velocity response, PWP. (d) Shear response, PWP. (e)Velocity Response, MY2. (f) Shear response, MY2. 
Figure 6.20 shows the velocity and shear responses during Period 1 computed by orienting the velocity and shear profiles relative to the wind and averaging. The reference depth for velocity was taken to be 40 meters. The lefthand column shows the velocity response and the right-hand column the shear response. Comparison between these response functions and those computed by spectral methods shows that the two methods are almost identical for Period 1 (though there are some small differences which account for the difference in transport calculated using the two methods).

Having built a case for rotating the velocity relative to the wind and averaging, we now turn to later in the experiment when Langmuir cells were strong. During this time, the high-frequency shear shows evidence of very strong cells. Because this time period represents such a short record it was not possible to obtain results of high enough significance using spectral methods. The response was computed using the method of rotating the velocity relative to the wind and averaging. The results of this procedure are shown in Figure 6.21. The velocity response during Period 2 relative to a reference depth of 60 meters is shown in the left-hand column of Figure 6.21. Again the results are shown for the data and the two models, with 6.21a showing the response from data, $6.21 \mathrm{c}$ from the PWP model, and 6.21e from the Mellor-Yamada model. During Period 2, the velocity structure was again better characterized by the PWP model than the MY2 model. However, in this case there were clear differences between the PWP model and data. The observed velocity response exhibited a subsurface maximum at depths of 5-10 meters, while the PWP model predicted a slab down to a depth of 25 meters. The MY2 model, as before, showed the velocity response as being concentrated near the surface, with a surface response 4 times as large as that seen in the data and 1.7 times as large as that seen in the PWP model.

An additional difference between the data and the models is that the velocity response seems to penetrate more deeply into the water column in the data. This can also be seen by looking at the transport calculation used to calculate the reference depth. Whereas the models converged on a transport given a reference depth of about 45 meters, in the data it was necessary to integrate down to 60 meters to get close to the right value for the transport. Since the top of the main thermocline was at 40 meters during MILDEX, it appears that the observed velocity response did penetrate into the thermocline. 


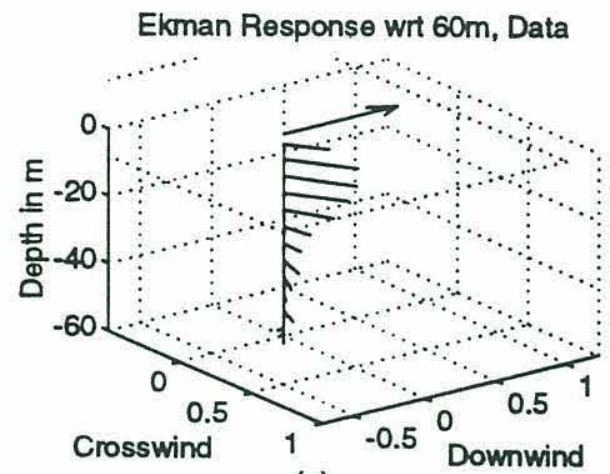

(a)

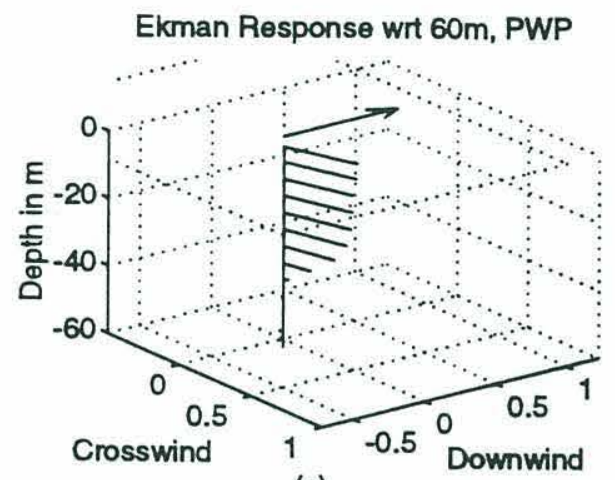

(c)

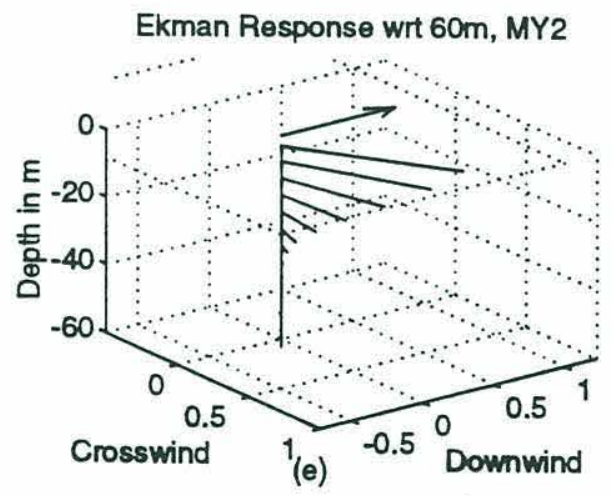

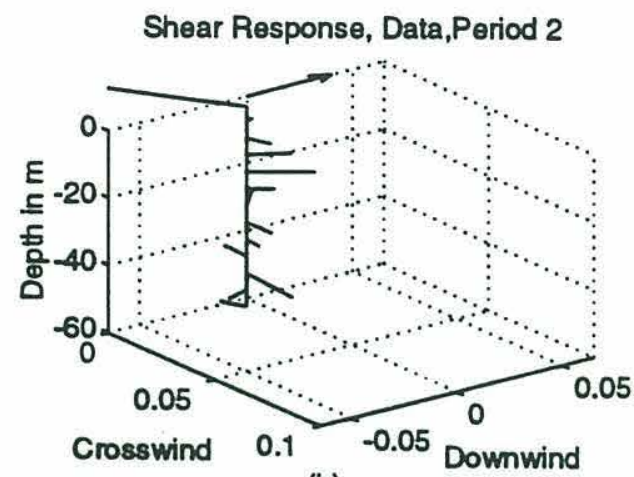

(b)

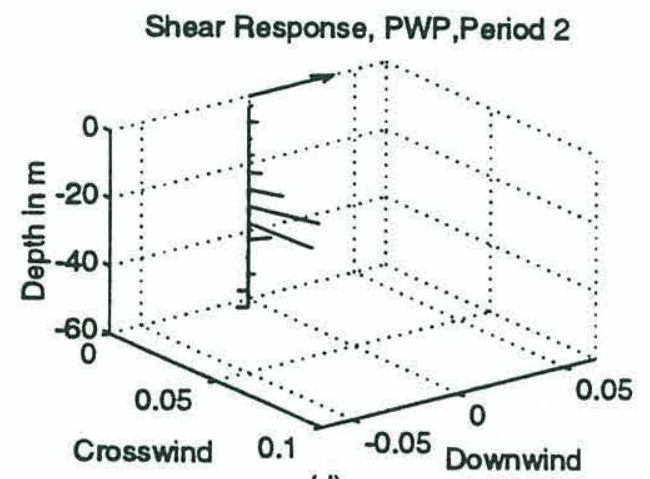

(d)

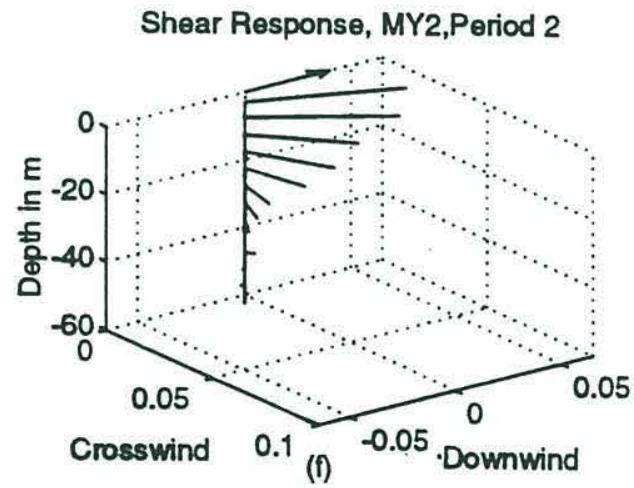

Figure 6.21:Velocity response relative to 60 meters and shear response as a whole during Period 2 computed by rotating relative to the wind and averaging. (a) Velocity response, Data (b) Shear Response, Data. (c) Velocity response,PWP. (d) Shear response PWP. (e)Velocity Response, MY2 (f) Shear response MY2. 
The shear response during Period 2 is shown in the right-hand column of Figure 6.21. Here the differences between the data and the two models are most clearly shown. The data showed strong upwind shear near the surface, crosswind shear at a depth of 12 meters, downwind shear at about 20 meters and shear which rotates below that. The PWP model showed almost all shear concentrated at depths of 30-40 meters, near the main mixed-layer base. The MY2 model showed large shears throughout the mixed layer which rotated clockwise with depth in classical Ekman spiral form. The shears were surface-concentrated, but it is notable that the shear at 25 meters in the MY2 model was almost as large as the shears at 25 meters in the PWP model. There are major disagreements between the three pictures of mixing presented here.

In summary, the mean Ekman transport during both Periods 1 and 2 was off to the right of the wind and was relatively close to theoretical values. The vertical distribution of the Ekman transport was more similar to that predicted by a slab model than that predicted by a Mellor-Yamada model. The structure of the Ekman response was affected by the presence of Langmuir circulation. When the cells were strong, the velocity response had a maximum in the mixed layer interior, and may have penetrated into the main thermocline. The shear response was radically different in the presence of Langmuir circulation. The mixed layer was far more sheared near the surface than predicted by a slab model but less sheared at depths of 5-20 meters than predicted by a Mellor-Yamada model.

\subsubsection{Discussion}

The results from MILDEX are in better agreement with a conceptual picture in which Langmuir cells stir the surface mixed layer then one in which small-scale mixing is responsible for momentum and buoyancy transport. When the cells were energetic they resulted in the creation of mean shears within the mixed layer, and perhaps in a greater penetration of the surface momentum input than predicted by either of the standard mixing models.

An interesting point is the low coherence between the shear within the mixed layer and the surface stress seen during MILDEX. This lack of coherence is predicted by the PWP model but not by the Mellor-Yamada model, even during times when the cells were weak. This phenomenon occurs because the coherence between two signals drops when the dependence of one signal on the other is nonlinear, and particularly when it is not monotonic. An example is the 
PWP model given a fixed surface heating. Consider a fixed depth $\mathrm{z}_{\mathbf{f}}$, well above the seasonal thermocline, but below the depth at which most solar radiation is absorbed. At low wind stresses, mixing does not occur and the shear at this depth is zero. As the wind stress increases, the transition layer penetrates deeper into the water column and the shear at $\mathrm{z}=\mathrm{z}_{\mathrm{f}}$ increases. However, if the wind stress increases enough so that the mixed layer depth is greater than $\mathrm{z}_{\mathrm{f}}$, the shear at $\mathrm{z}=\mathrm{z}_{\mathrm{f}}$ vanishes. Thus in the PWP model, if the stress to the east increases, the vertical shear of the eastward velocity will not necessarily increase.

Similarly, if Langmuir cells stir the mixed layer, the mean shear does not depend monotonically on the wind stress. Given low values of wind stress and waves, so that cells are weak, the shear is aligned with the wind and presumably increases with the wind stress. If the wind stress increases so that the Langmuir cells take over from small-scale diffusion, the shear within the mixed layer may reverse direction (as occurred near the surface during MILDEX), or vanish (as occurred in the middle of the water column). If such non-monotonic behavior occurs frequently at a given depth, the wind stress and shear become incoherent.

For the Mellor-Yamada model on the other hand, given a constant buoyancy forcing, increasing the stress increases the shear. At large values of wind stress, the shear scales as the friction velocity. As a result, the coherence between the shear and surface stress is high. The fact that this did not occur during MILDEX is another indication that the physics of the MY2 model were insufficient to explain the observed velocity structure.

Before bringing this section to a close, the effect of Langmuir cells on the density field will be touched on. Both models did a reasonable job at modelling the temperature stratification. In Figure 6.13 the amplitude of the diurnal warming relative to 10 meters predicted by the models differed substantially from the data on only two days (October 27th and 28th). Although the level of the high frequency shear was elevated during the night of October 27th, there was little difference between the mixed layer depth seen in the data and that predicted by the two models. During daylight hours on the 27th and 28th, there was no evidence that Langmuir cells were in fact present. Whether or not Langmuir cells had an effect on the density structure in MILDEX remains an open question, but the answer is apparently that such effects were not significant.

To summarize, the velocity structure of the mixed layer during MILDEX was characterized by the following phenomena.

1. High-frequency shear within the mixed layer. 
2. Coherent velocity structures which were much longer in the alongwind than the crosswind direction.

3. A low-frequency velocity response which was far less sheared than that predicted by a model which assumed mixing accomplished by small-scale eddies. 4. A low-frequency velocity response with more shear within the mixed layer than was predicted by a mixing model which parameterizes the mixed layer as a homogeneous slab.

These phenomena are consistent with a conceptual picture in which the mixed layer is stirred by Langmuir circulation. As demonstrated in Chapter 5, Langmuir cells can remove some of the low-frequency shear associated with small-scale mixing by actively transporting momentum and density throughout the mixed layer. However, when the cells are very strong, they can produce crosswind or even upwind shears.

Sections 6.6 and 6.7, discuss the question of whether the Craik-Leibovich theory, which models the cells as being driven by wave-current interaction, can account for the phenomena we have seen above. The level of the high-frequency shear is considered in 6.6, and the presence of cellular structures and the breakdown of small-scale mixing is treated in 6.7 .

\subsection{Understanding Langmuir Cell Variability During MILDEX: Evidence for Wave-Current Interaction}

Section 6.3 argued that the amplitude of the high-frequency shear during MILDEX was an indicator of cell strength. If the cells are driven by wave-current interaction the level of the high frequency shear (according to Chapter 5) should go as the stratified Craik-Leibovich instability parameter $\gamma_{\text {CLS }}$. Chapters 2-5 considered how to calculate $\gamma_{C L S}$ for a wide variety of situations. In all of them, however, the velocity and density profile in the absence of Langmuir cells was known. When working with field data, one must estimate the velocity and density structure in the absence of Langmuir cells from the velocity and density structure in the presence of Langmuir cells. In particular, the eddy viscosity and eddy diffusivity must be estimated, a non-trivial exercise. Section 6.5

demonstrated that during times when cells were strong, the shear across the top two current meters was 135 degrees to the left of the wind rather than along the wind direction. Thus even the topmost current meter pair, which one would hope would capture a near-surface shear layer, did not do so. 
One simple way of estimating the eddy viscosity is to assume that near the surface

$$
v_{\mathrm{e}} \frac{\partial \overrightarrow{\mathrm{u}}}{\partial \mathrm{z}}=\frac{\vec{\tau}}{\rho} \Rightarrow v_{\mathrm{e}}=\vec{\tau} \bullet \frac{\partial \overrightarrow{\mathrm{u}}}{\partial \mathrm{z}} /\left|\frac{\partial \overrightarrow{\mathrm{u}}}{\partial \mathrm{z}}\right|^{2}
$$

This may be a reasonable approximation at depths where Langmuir cells are not strong. It is not necessarily such a good approximation at depths where they are strong. Chapter 5 showed that when the Craik-Leibovich instability parameter is much larger than the characteristic diffusive decay scale, finite-amplitude Langmuir cells are capable of altering the shear over a large portion of the layer, causing it to reverse direction at some depths and change from downwind to crosswind in some cases. During MILDEX such reversals in the mean were linked with the presence of strong high-frequency shear. Under such circumstances a better approximation is for

$$
v_{\mathrm{e}}\left|\frac{\partial \overrightarrow{\mathrm{u}}}{\partial \mathrm{z}}\right|=\frac{|\vec{\imath}|}{\rho} \Rightarrow v_{\mathrm{e}}=|\vec{\tau}| / \rho\left|\frac{\partial \overrightarrow{\mathrm{u}}}{\partial z}\right|
$$

so that the absolute value of the shear is proportional to the absolute value of the stress. As in Chapters 2-5, the turbulent Prandtl number is assumed to be 1 (eddy diffusivity = eddy viscosity). The eddy viscosity computed from equation (6-13) is shown by the solid line in Figure 6.22.

One check on the size of the viscosity estimated from the data is to compare it with that obtained from the Mellor-Yamada model. The eddy viscosity from the Mellor-Yamada Level 2 model between the depths of 2 and 5 meters is shown by the dashed line in Figure 6.22. The two curves agree fairly well, the correlation of the $\log$ (eddy viscosity) being 0.50 . The eddy viscosity predicted by (6-13) is generally within a factor of 3 of that predicted by the model, even as the overall level of the viscosity varies over two orders of magnitude. There are, of course, many small-scale differences which prevent the correlation from being even higher.

A second check on the size of the eddy viscosity may be derived from some unpublished dissipation data of Lueck (1988). As noted above, dissipation profiles were taken during three periods on the 8th and 9th of November. If the measured dissipation at some depth is due only to shear production, then if $\omega$ is a characteristic frequency for the shear, the dissipation should go as

$$
\varepsilon \sim v_{\mathrm{e}} \omega^{2}
$$

If $\omega$ is given by the mean shear, an estimate for the size of $v_{e}$ can be obtained As with the previous estimate, this one will also be biased somewhat high, since there 


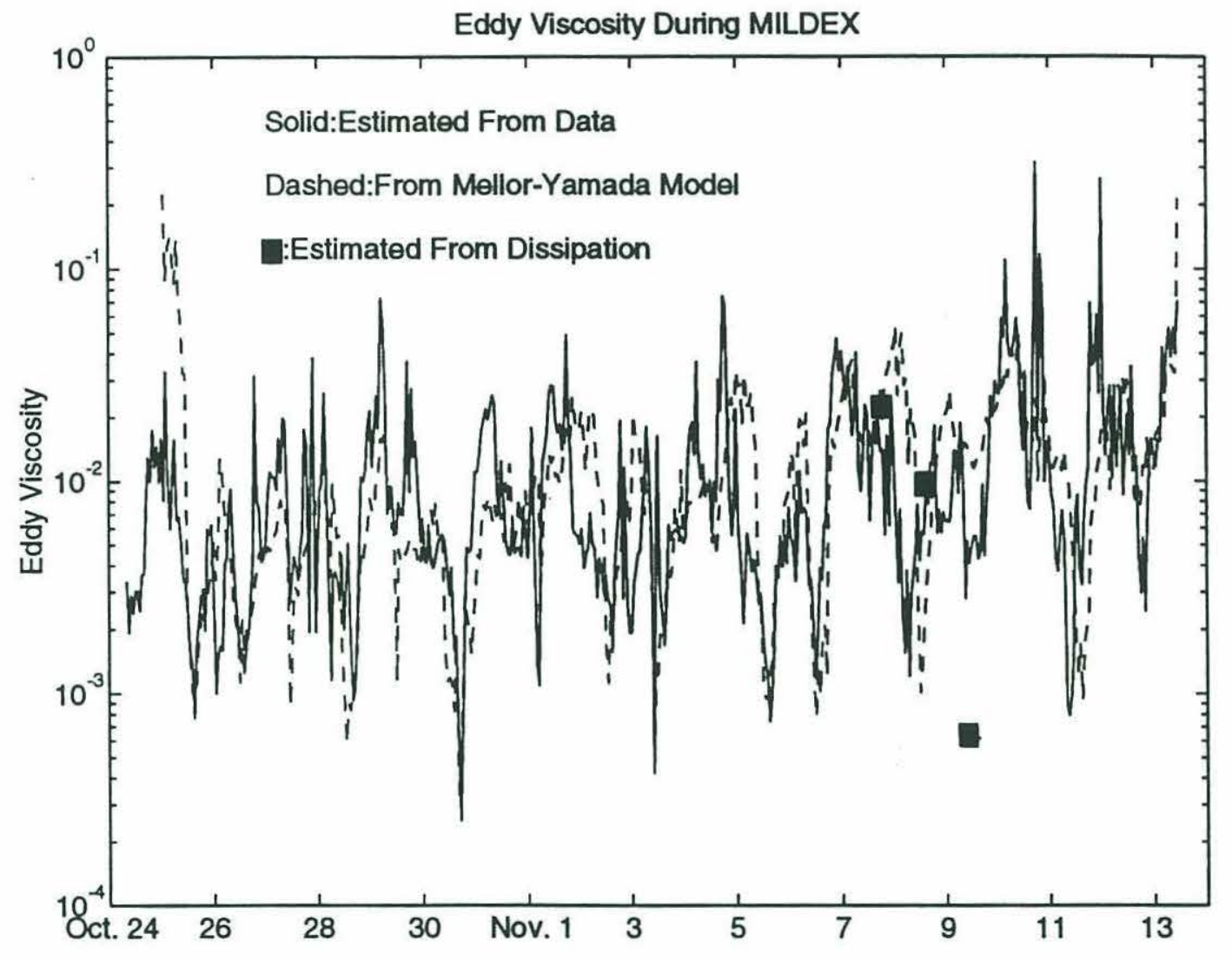

Figure 6.22: Estimated eddy viscosity during MILDEX. Solid lines are estimated from data (equation 6-13), dashed lines taken from Mellor-Yamada, Level 2 model. The solid squares are estimated from Lueck (1988). 
is no provision for turbulence driven by buoyant overturning or by wave-current interaction. Eddy viscosities were computed for three time periods during MILDEX. The results are shown by the squares in Figure 6.22. The first two values agree fairly well with the estimates from the data and MY2 model. The third value is quite a bit lower than either the data or the MY2 model. To some extent, the low value may be blamed on undersampling (only 12 profiles were used in its calculation, so that if mixing was patchy, the profiler might never have gotten into a turbulent patch).

It is unlikely that either of the methods used to estimate the eddy viscosity seriously underestimate the diffusive decay scale, and thus overestimate RacLs. This lowers the chance that the theory will predict Langmuir cells when none would actually occur, but raises the possibility that it might predict the cells to be weak when they are actually strong (RaCLS may be underestimated).

In order to compute the Craik-Leibovich instability parameter, the Stokes drift shear and the stratification in the absence of Langmuir cells need to be estimated. The Stokes drift can be computed from the time series of wave height at the wavestaff.

$$
\mathrm{v}_{\mathrm{S}}(\mathrm{z})=\int_{0}^{\infty} \omega^{3}|\mathrm{a}(\omega)|^{2} / \mathrm{g} d \omega
$$

where $a(\omega)=\int_{0}^{\infty} \eta(t) e^{-i \omega t} d t$. Figure $6.23 a$ shows the Stokes drift shear between 2 and 6.5 meters during MILDEX. The Stokes drift shear has a noticably different structure than the significant wave height (Figure 6.4). In many ways, it resembles the wind stress.

Estimating the stratification in the absence of cells also poses a challenge. Obviously in the presence of near-surface temperature gradients one can let

$$
\mathrm{N}_{\mathrm{T}}^{2}=g \alpha \frac{\partial \mathrm{T}}{\partial \mathrm{z}}
$$

where $\mathrm{g}$ is gravity and $\alpha$ is the coefficient of thermal expansion. If the gradient is being mixed away, the problem is more difficult. Using the estimated eddy viscosity, one can estimate the temperature stratification required to carry a heat flux of the same value as the surface heat flux.

$$
\mathrm{N}_{\mathrm{pre}}^{2}=\frac{\mathrm{g} \alpha \mathrm{Q}}{\rho c_{\mathrm{p}} v_{\mathrm{e}}}
$$

where $Q$ is the heat flux, $\rho$ the density, and $c_{p}$ the specific heat. Figure $6.23 \mathrm{~b}$ shows a time series of $\mathrm{N}^{2}$ computed from (6-16) (solid) and (6-17) (dashed). There 


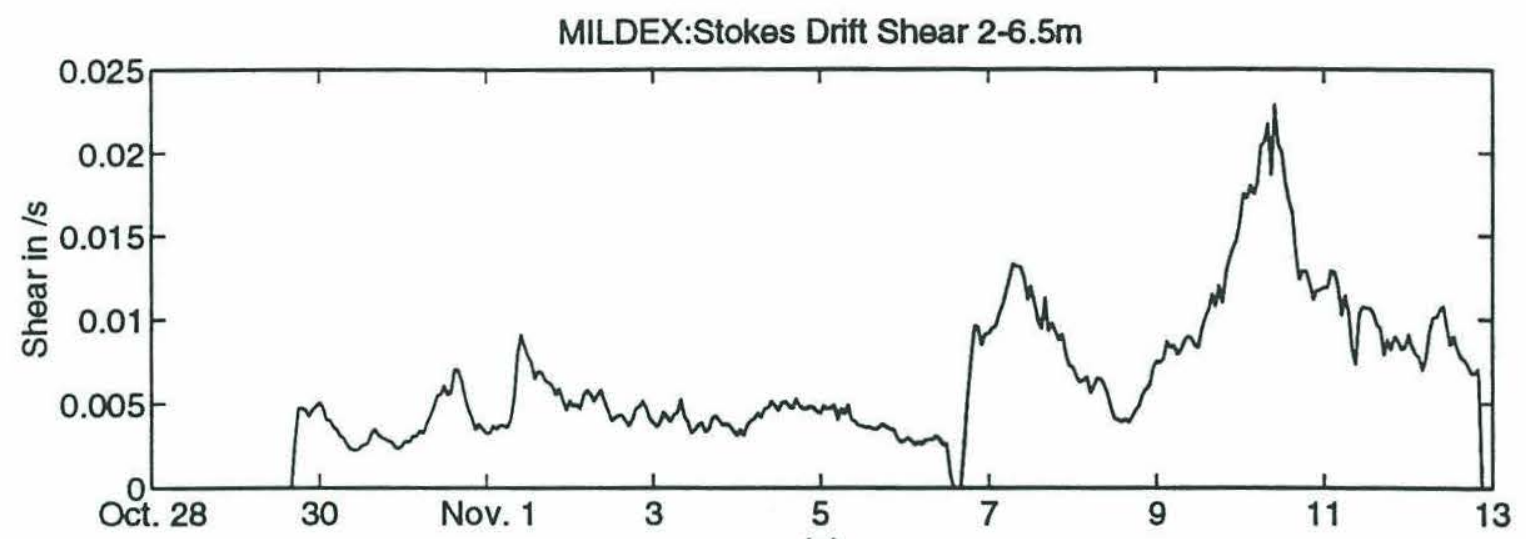

(a)

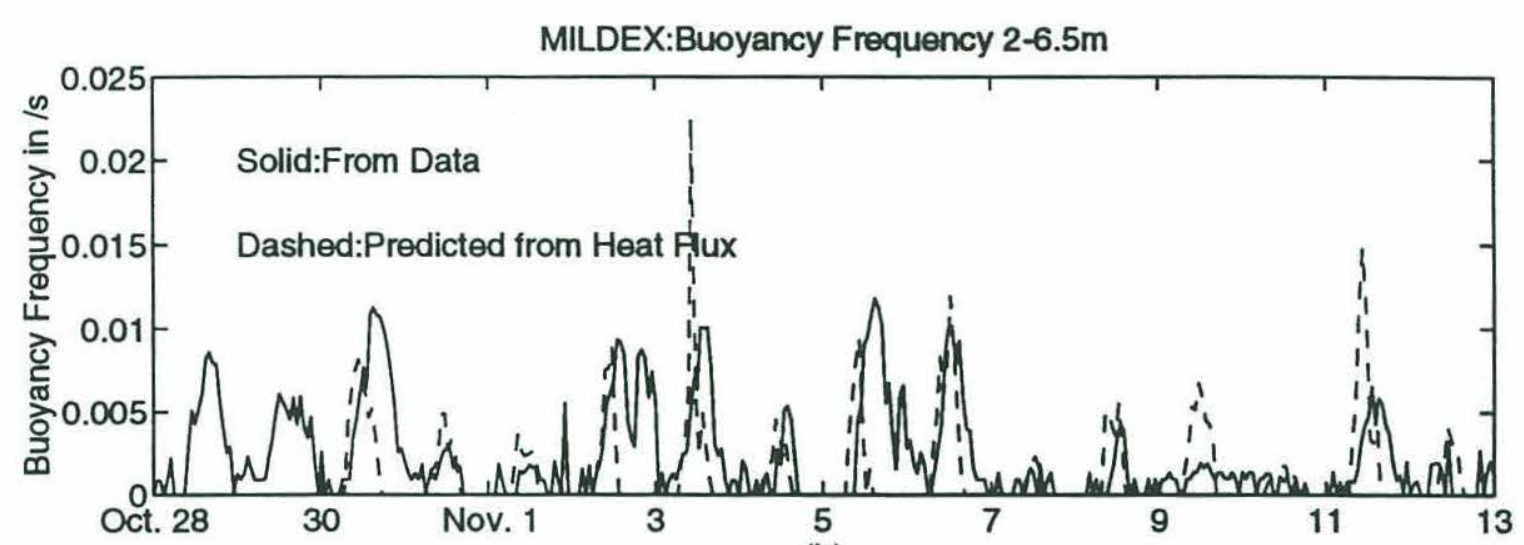

(b)

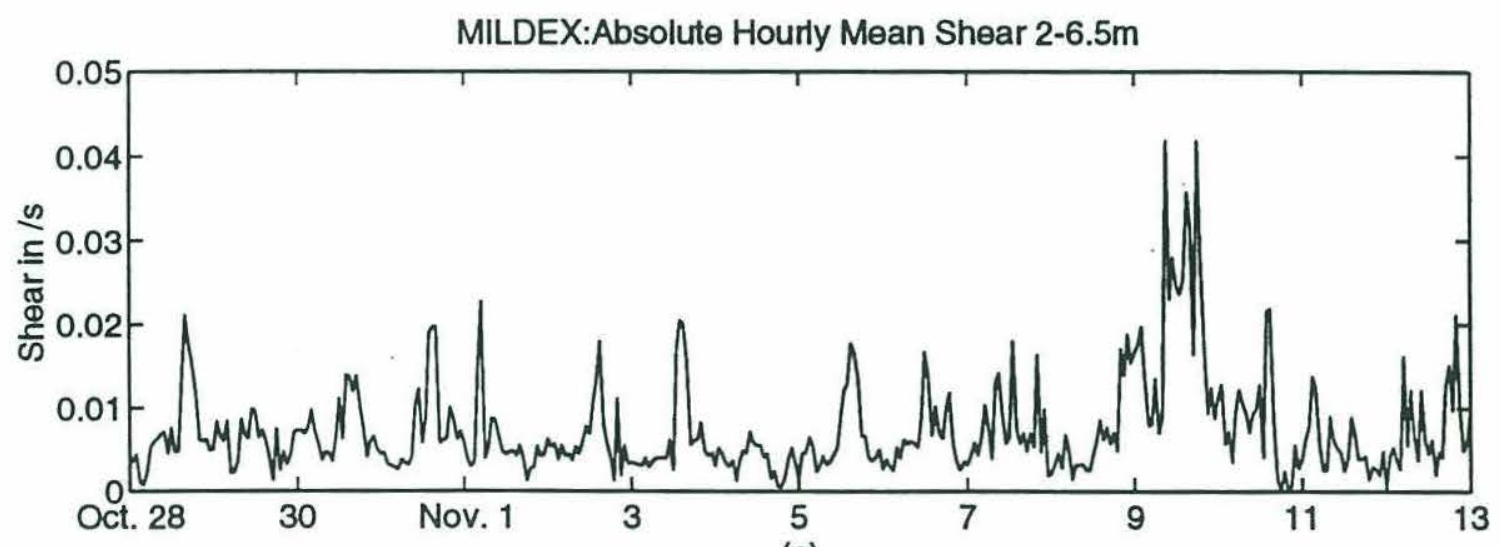

(c)

Figure 6.23: Frequency scales which go into making the estimate of the CraikLeibovich instability parameter. (a) Stokes drift shear between 2 and $6.5 \mathrm{~m}$.

(b) Buoyancy frequency from equation 6-20 (solid) and 6-21 (dashed).

(c) Absolute value of the hourly mean shear between 2 and 6.5 meters. 
are some times when the two agree very well. Only on October 30, November 4th and November 12th does the predicted value exceed the observed value. In what follows $\mathrm{N}^{2}$ is taken as the maximum of (6-16) and (6-17) so as to capture cases when the Langmuir cells might be transporting buoyancy.

All the quantities needed to put together a rough estimate of the CraikLeibovich instability parameter have been discussed. As noted in Section 6.1, this parameter measures the strength of the wave-current interaction in driving the cells. Since this work focuses on the shear near the surface, the relevant CraikLeibovich instability parameter is one for cells which are relatively small, roughly 12 meters depth. From Chapter 2, the characteristic Stokes drift shear for driving such cells is found at roughly 4 meters. Then if $\omega$ is a characteristic size for the high-frequency shear, it is proposed that:

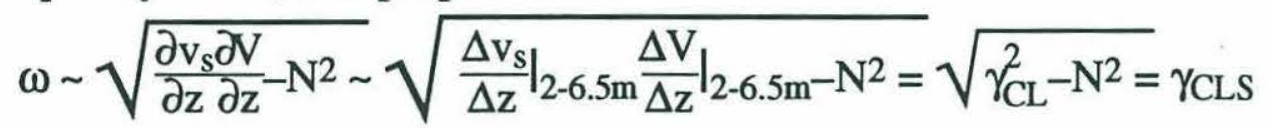

where the shears and stratification are defined across 2 and 6.5 meters and $\mathrm{N}^{2}$ is calculated as the maximum of (6-16) and (6-17). This shear scaling is clearly analogous to that in Chapter 5, with the key difference that the Stokes drift and Eulerian shears are not weighted over the depth of the mixed layer.

Figure 6.24a shows the stratified Craik-Leibovich instability parameter calculated over the top 10 meters during the experiment Figure $6.24 \mathrm{~b}$ shows the diffusive decay rate and $6.24 \mathrm{c}$ shows the ratio of the two. Figure $6.24 \mathrm{~d}$ shows the level of the high-frequency shear. The mixed layer during MILDEX should often have been unstable to Langmuir circulations, with the Craik-Leibovich instability parameter being much larger than the diffusive decay scale most of the time. However, the Craik-Leibovich instability parameter was often quite small, so that the shears associated with Langmuir cells might not necessarily have risen above the background noise. The Craik-Leibovich instability parameter reproduces most of the major features in the high-frequency shear. The events on October 31, November 6-8th, and November 8-11 are all captured quite well by the Craik-Leibovich instability parameter. The instability parameter captures the temporal variability during and after the passage of the low pressure system on November 9-11, showing episodic bursts of activity during this time period, a signal also found in the high-frequency shear.

The predicted and observed amplitudes of the high-frequency shear during MILDEX compare fairly well quantitatively as well as qualitatively. Table 6.1 shows the correlations between the high-frequency shear from the current meters 


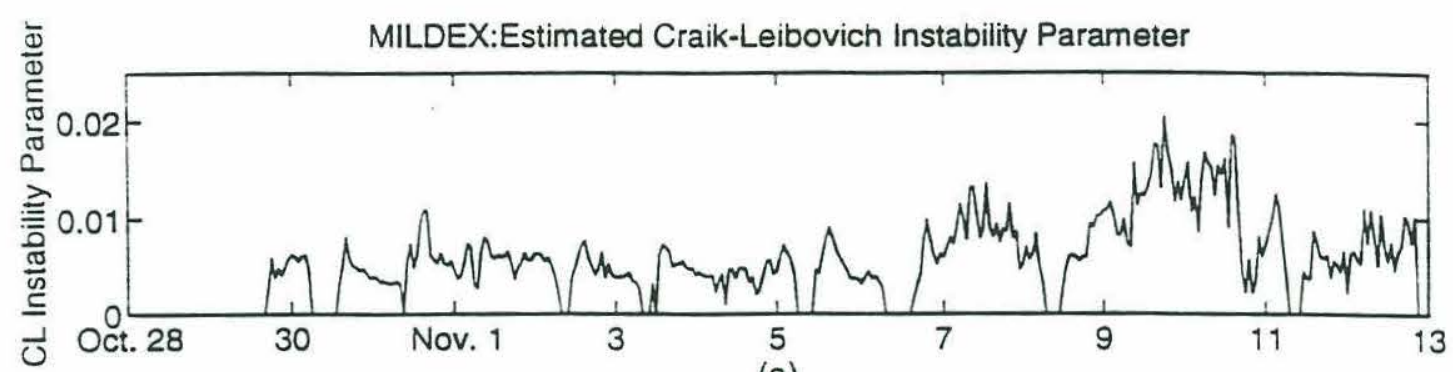

(a)

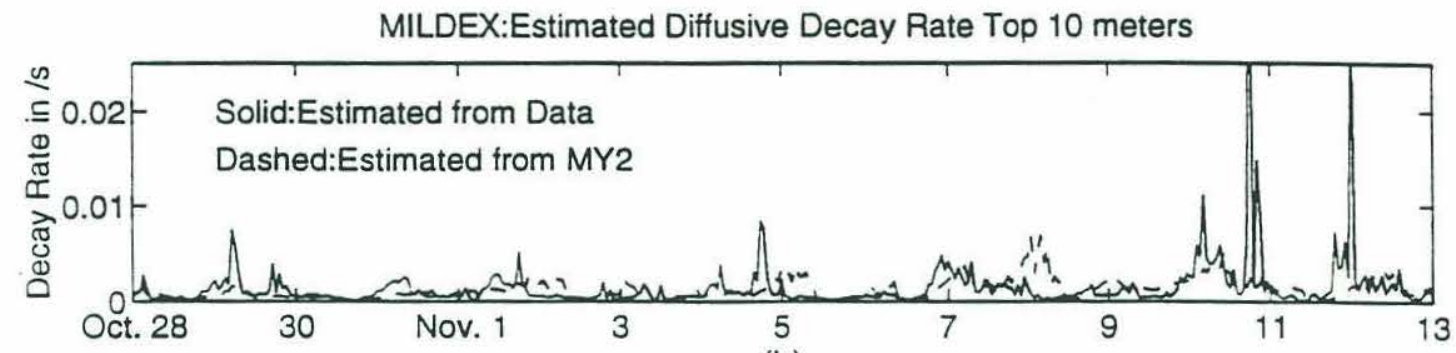

(b)

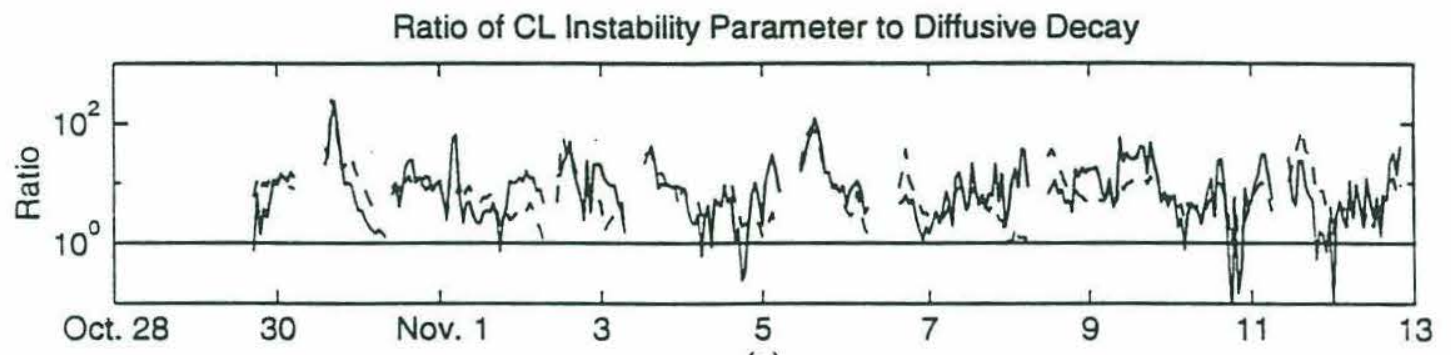

(c)

High-Frequency (1-30cph) Alongwind Shear 2-6.5m

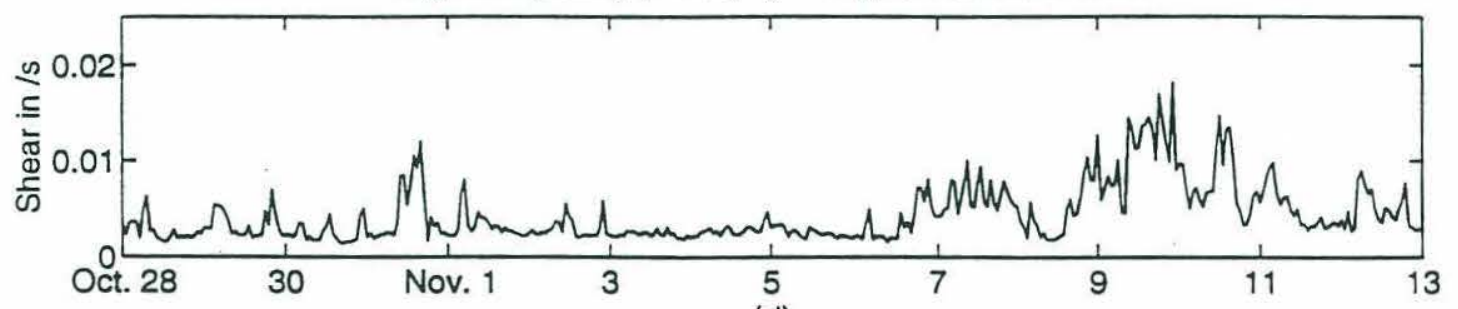

(d)

Figure 6.24: Craik-Leibovich instability and high-frequency shear during MILDEX. (a) Estimate of stratified Craik-Leibovich instability parameter (solid using data, dashed Mellor-Yamada) (b) Diffusive decay scale over the top 10 meters (solid using data, dashed Mellor-Yamada). (c) Ratio of Craik-Leibovich instability parameter to diffusive decay scale. (solid using data, dashed MellorYamada) (d) High-frequency shear (standard deviation of the hourly detrended shear between 2 and $6.5 \mathrm{~m}$ ) 


\begin{tabular}{|c|c|}
\hline Field & Correlation \\
\hline Wind Stress $\tau$ & .5448 \\
\hline Significant Wave Height & .1362 \\
\hline Eulerian Shear $\frac{\partial \mathrm{V}}{\partial \mathrm{z}}$ & .6825 \\
\hline Stokes Drift Shear $\frac{\partial \mathrm{v}_{\mathrm{s}}}{\mathrm{z}}$ & .6007 \\
\hline$\gamma_{\mathrm{CL}}=\sqrt{\frac{\partial \mathrm{v}_{\mathrm{s}}}{\partial \mathrm{V}} \frac{\mathrm{V}}{\partial \mathrm{z}}}$ & .8396 \\
\hline$\gamma_{\mathrm{CLS}}=\sqrt{\frac{\partial \mathrm{v}_{\mathrm{S}}}{\partial \mathrm{V}} \frac{\partial}{\partial \mathrm{z}}-\mathrm{N}^{2}}$ & .7931 \\
\hline$\gamma_{\text {shear }}=\sqrt{\frac{\partial \mathrm{V}^{2}}{\partial \mathrm{z}}-\mathrm{N}^{2}}$ & .6294 \\
\hline
\end{tabular}

Table 6.1: Correlation of the high-frequency shear between 2 and 6.5 meters on various parameters.

at 2 and $6.5 \mathrm{~m}$ and the wind stress,wave height, Stokes Drift shear, Eulerian shear, and various combinations thereof. The $98 \%$ confidence level for significance is 0.089 . The time-varying shear is clearly strongly correlated with a number of related parameters. The correlation is highest for $\gamma_{C L}$ and $\gamma_{C L S}$. In reality, it is hard to differentiate between these two cases. Both $\gamma_{C L}$ and $\gamma_{C L S}$ are superior to indices based on the Eulerian shear alone for determining the strength of the high-frequency shear.

The fact that a simple scaling based on Craik-Leibovich instability does such a good job at predicting the amplitude of the high-frequency shear has a two important implications. The first is that it supports the claim that the signals seen during MILDEX were in fact due to Langmuir circulation. It is difficult to postulate another mechanism which would produce shear variability which is so well described by a parameter involving both mean shear and Stokes drift shear. The second is that it supports the idea of the Craik-Leibovich instability mechanism as playing an important role in the generation of Langmuir cells in the field. Although both the estimation of the Craik-Leibovich instability parameter and the shear associated with the cells are rather crude, the fact that the two correlate so well is an extremely significant result.

In conclusion, there is hard quantitative evidence that Langmuir cells during MILDEX are driven by wave-current interaction. The fact that the amplitude of the high-frequency shear scales better with a crude estimate of the 
Craik-Leibovich instability parameter than with the mean value of the shear is particularly encouraging. On the one hand, it is evidence against shear instability or Kelvin-Helmholtz instability being responsible for the presence of near-surface shear. Additionally, it provides confidence that the thruster is not causing both the increase in the high frequency shear as well as the low-frequency shear reversal, since one would expect a closer relationship between the two were this true.

\subsection{The Breakdown of the Mellor-Yamada Model: Further Evidence for Wave-Current Interaction}

Section 6.5 used the level of the high frequency shear to argue for CraikLeibovich instability as a driving mechanism for Langmuir cells. This section uses the failure of the Mellor-Yamada model to capture the velocity structure as evidence for the importance of Craik-Leibovich instability.

Section 6.4 showed that a model which only includes small-scale mixing fails to capture the observed velocity structure during MILDEX. Bulk mixing models which treat the mixed layer as a slab mixed by large eddies do a better job at characterizing the velocity structure, even though they do not succeed in capturing the shear structure. This section argues that the MY2 model fails to produce a mixed layer which is suffiiciently slab-like because it does not account for Langmuir cells driven by the Craik-Leibovich instability mechanism. By combining the results of the last section with the instability codes developed in Chapters 2 and 3, it is shown that the Mellor-Yamada model produces a mean Ekman response which is unstable to Langmuir cells driven by wave current interaction.

We begin by considering the Ekman response predicted by the MellorYamada model during Period 2, shown in Figure 6.21e and $\mathrm{f}$. The velocity and shear structure within the mixed layer are very similar to that predicted by Ekman's (1905) simple spiral solution. The MY2 model computes the eddy viscosity at each time step, and the mean between 2 and $5 \mathrm{~m}$ over Period 2 is approximately $0.017 \mathrm{~m}^{2} / \mathrm{s}$. When this value is used to predict the velocity response over the depth of the mixed layer the result is one which agrees surprisingly well with the averaged response. Figure 6.25 a shows the velocity response relative to 60 meters from the MY2 model (solid), and assuming an eddy viscosity of $0.0168 \mathrm{~m}^{2} / \mathrm{s}$ (dashed). The two responses are almost identical. 

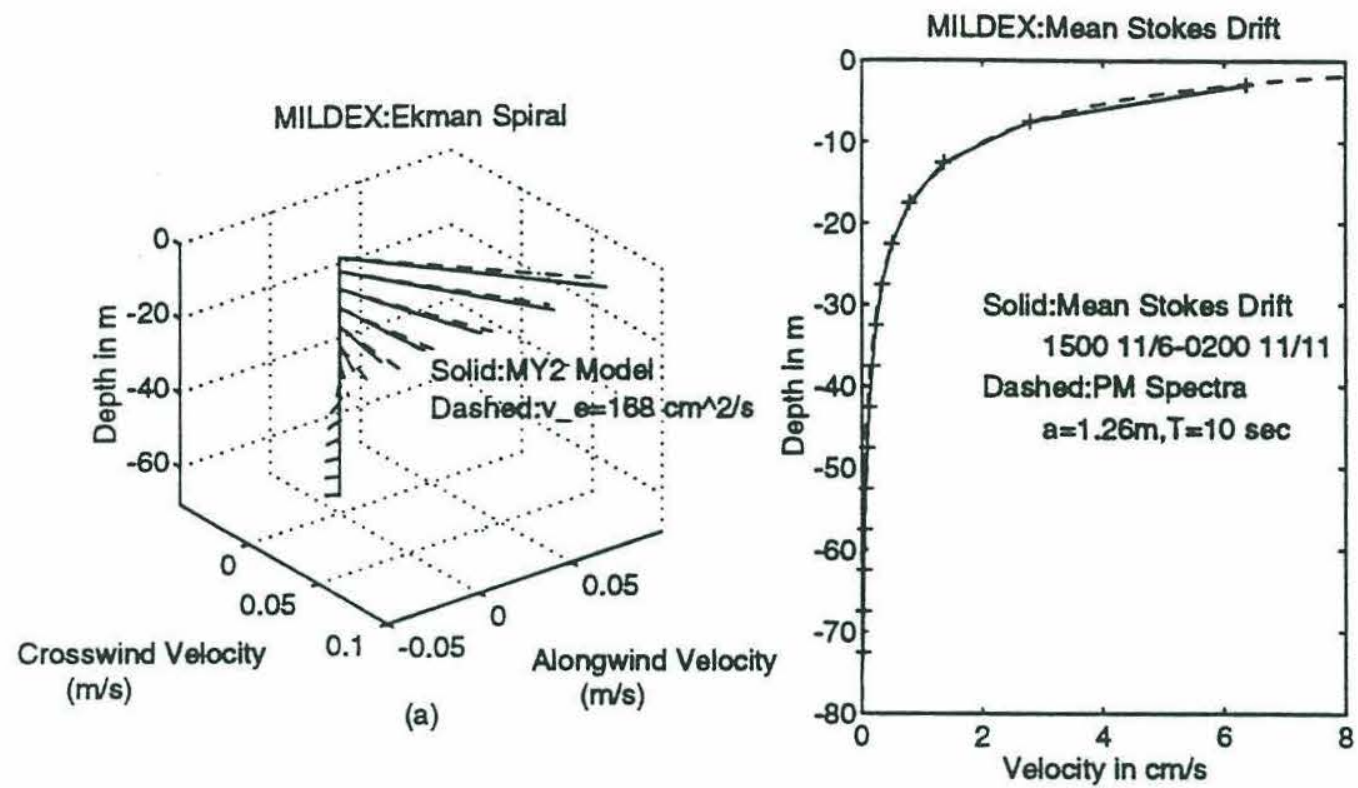

(b)

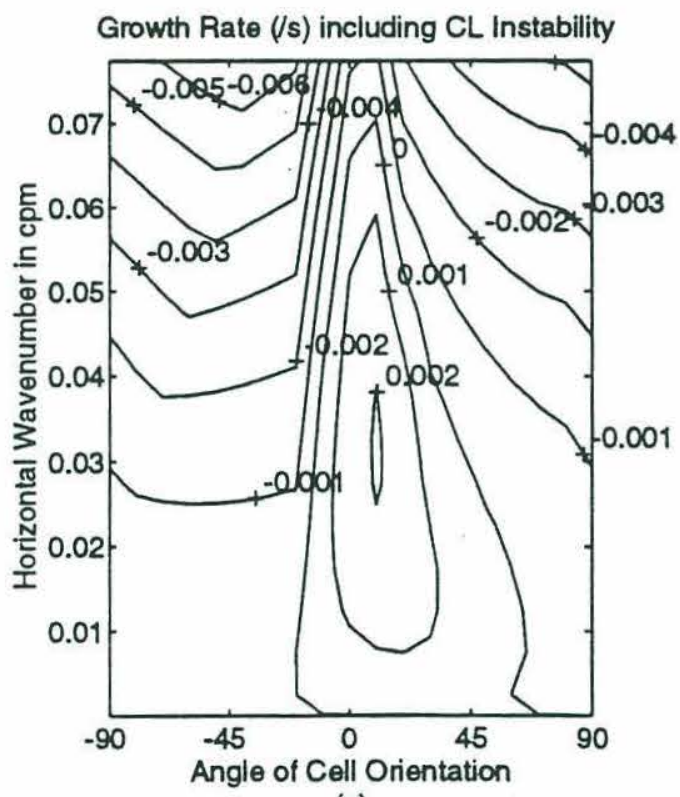

(c)

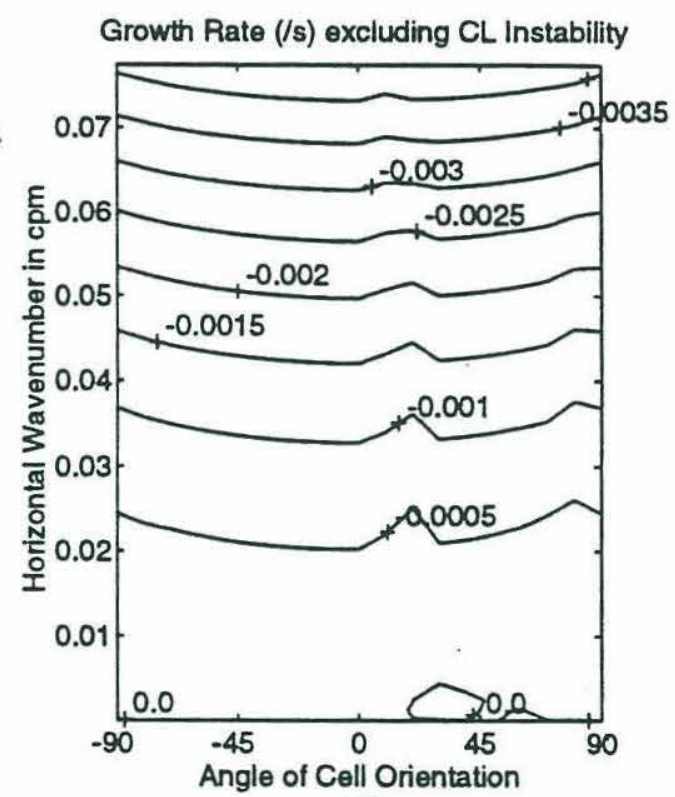

(d)

Figure 6.25: Instability of the Mellor-Yamada Ekman response. (a) Ekman Response relative to 60 meters during MILDEX Period 2. Solid lines are MY2 model, dashed lines Ekman spiral computed using an eddy viscosity of 0.0168 $\mathrm{m}^{2} / \mathrm{s}$. (b) Mean Stokes drift profile during Period 2. Solid is from data, dashed is a Pierson-Moskowitz spectrum with rms amplitude of $1.26 \mathrm{~m}$ and peak period of 10 seconds. (c) Growth rate of most unstable mode in $\mathrm{s}^{-1}$ for Period 2 Ekman response (assuming surface stress of $0.13 \mathrm{~Pa}$ ) when effects of waves are included. (d) Growth rate of most unstable mode in $\mathrm{s}^{-1}$ for Period 2 Ekman response (assuming surface stress of $0.13 \mathrm{~Pa}$ ) when effects of waves are NOT included. 
The averaged Stokes drift during Period 2 can by modelled by a PiersonMoskowitz spectrum which has a peak period $\mathrm{T}_{\text {peak }}$ of 10 seconds and an rms wave amplitude of $1.26 \mathrm{~m}$. This implies a characteristic length scale $\mathrm{L}$ of $25 \mathrm{~m}$ (the inverse wavenumber of a deep-water surface gravity wave with a period of 10 s). This in turn implies a Langmuir number of

$$
\mathrm{La}=\frac{v_{\mathrm{e}} \mathrm{T}_{\text {peak }}}{2 \pi \mathrm{a}^{2}}=0.017
$$

and a scaled Coriolis frequency

$$
\mathrm{F}=\frac{\mathrm{fT}_{\text {peak }} \mathrm{L}^{2}}{8 \pi^{3} \mathrm{a}^{2}}=0.051
$$

where $\mathrm{f}$ is the dimensional Coriolis frequency. By taking the mean absolute stress over this time period $(0.131 \mathrm{~Pa})$, one can calculate a mean, nondimensionalized surface shear. These values, together with a mixed layer depth of 43 meters were used to run the spectral instability codes introduced in Chapters 2 and 3 which are documented in Appendix B. The results were then converted to dimensional units.

Figure $6.25 \mathrm{c}$ shows contours of the growth rate in $\mathrm{s}^{-1}$ of the most unstably growing mode as a function of wavenumber in $\mathrm{cpm}$ and angle of cell axis relative to the wind.The fastest-growing cells have a wavelength of 25-50 meters, an angle of orientation slightly to the right of the wind, and grow on time scales of 400 seconds. In the absence of waves (Figure 6.25d) there are no unstably growing cellular structures with wavelengths less than 200 meters. Those that are unstable are very weakly so, with growth rates of the same size as the Coriolis frequency.

In the presence of waves the Ekman layer produced by a local mixing model is unstable to Langmuir circulation. The unstable disturbances are oriented close to the wind, with relatively small spacing compared with the mixed layer depth, and very large growth rates. This then, is a possible explanation for the failure of the MY2 model to capture the velocity structure during MILDEX.

In the absence of waves (and thus Craik-Leibovich instability) the Ekman layer produced by local mixing is very stable to two-dimensional disturbances. Insofar as the cells observed in MILDEX were two-dimensional, the failure of the the MY2 model to capture the velocity structure is evidence that Craik-Leibovich instability (rather than Kelvin Helmholtz or Ekman instability) is responsible for driving the cells which then homogenize the mixed layer. 


\subsection{Conclusions}

The Mixed Layer Dynamics Experiment provides an opportunity to examine both the effects and dynamics of Langmuir cells. The results, though somewhat rough, are extremely promising. During MILDEX, the mixed layer was maintained by processes with time scales which were fast in comparison with the Coriolis frequency. Langmuir cells, which have spatial scales similar to the mixed layer depth and mix on time scales fast in comparison with the Coriolis frequency, were shown to be present by both sonars and current meters during one period of the experiment, and by the current meters at a number of other times. The presence of Langmuir cells was shown to affect both the level of the high frequency shear and the structure of the low- frequency response to wind forcing. The shear structure of a mixed layer with strong cells was not captured either by a bulk model which treats this layer as a slab (PWP) or by a model where the mixing occurs as the result of purely local processes (Mellor-Yamada). Instability calculations show that the latter fact can be explained as the result of the current profile produced by local mixing processes alone being unstable to roll vortices driven by wave-current interaction. The level of the high frequency shear varies over time in a manner which is also consistent with the cells being driven by wave-current interaction.

While the evidence developed in this chapter is promising, it should be emphasized that the agreement between theory and data is far from exact. Two differences which stand out are:

1. The presence of strong, low-frequency upwind shears near the surface. Such shears were not seen at equilibrium in any of the model solutions presented during Chapters 4 and 5. In Chapter 8 it is shown that they do not appear for finite-difference code solutions given the mean conditions during MILDEX. 2. The Ekman transport was close to that predicted by mixed layer models which did not include surface gravity waves. There is no evidence of an Eulerian return flow associated with the surface gravity waves.

These differences are discussed in more detail in Chapter 8.

This work has a number of important implications. The fact that the mixed layer does appear to be mixed by large eddies is a validation of the large-eddy mixing approach taken by many authors. However, this work points out some shortcomings of that approach, particularly as regards the assumption that the mixed layer is perfectly mixed with regard to all scalar and vector quantities. 
Another shortcoming of existing slab models is that they do not include effects of the wave field. As seen in this chapter, the velocity structure in the oceanic surface layer is coupled to the wave field. Wave fields which result in large Stokes drift shears drive Langmuir cells and cause mixing. If, as argued above, the driving mechanism for this mixing is wave-current interaction, this implies that there is an energy source for the cells which goes as the Stokes drift multiplied by the wind stress. As noted in Chapter 5, this energy source will in general be smaller than those sources associated with turbulence production. However, it may well be a very efficient means of mixing density and as such may play a disproportionate role in maintaining the slab-like structure of the mixed layer.

The MILDEX experiment has a number of weaknesses. The most prominent of these is the lack of any independent quantitative measure of cell strength over the course of the experiment. The lack of correspondence between cell strength and wind stress and wave height on November 9th and 10th leads to some interesting questions about the potential role of wave breaking in suppressing cells. It would be reassuring if the Doppler sonar data collected during the experiment could be analyzed to give objective measures of cell strength which could be compared with the VMCMs. Another major weakness of the MILDEX experiment is the fact that at least some of the data was contaminated by the thruster. A third weakness of the MILDEX data set is the relatively low temporal and spatial resolution. In particular, the lack of infomation about the shear structure very close to the surface leads to major questions about the way in which the eddy viscosity was estimated.

Some of these shortcomings have been addressed in the dataset studied in the following chapter, which was collected during the Surface Waves Processes Program (SWAPP). During this experiment, many of the same measurements were made as during MILDEX, but with higher spatial and temporal resolution. As will be seen, although some of the details differ, the picture offered by SWAPP reinforces the broad outlines suggested by MILDEX. 


\section{Chapter 7: Langmuir Circulation during the Surface Waves Processes Program}

\subsection{Introduction}

Chapter 6 examined the velocity structure of a mixed layer in which Langmuir cells were strong. The structure was more consistent with a conceptual picture in which mixing is carried out by motions with the same vertical scale as the layer than one in which mixing is carried out by small-scale instabilities with spatial scales of centimeters. In contrast, however, to standard large-eddy mixing models which treat the mixed layer as a homogeneous slab, the mixed was sheared at both high (1-30 cph) and low frequencies (0.01-0.1cph). These shears were argued to be associated with the presence of Langmuir cells. Using results from Chapters 2-5 of this thesis, evidence was presented that the cells were driven by the wave-current interaction mechanism of Craik and Leibovich (1976).

The measurements made during MILDEX had a number of shortcomings, however. Primary among these were the lack of spatial and temporal resolution, the contamination of some of the velocity measurements by a thruster, and the lack of an independent time series measuring Langmuir circulation strength. This chapter, looks at another experiment, the Surface Waves Processes Program (SWAPP). The general setup of the instrumentation during SWAPP was similar to that during MILDEX, but with higher temporal and spatial resolution. In addition indices of cell strength based on Doppler sonars and computer cards were available for extended time periods during the experiment. Many of the techniques developed in Chapter 6 will be used to look at the behavior of Langmuir circulations during SWAPP. The general conclusions of the MILDEX experiment hold for SWAPP as well, although some of the details are different. In particular, the Langmuir cells observed during SWAPP were weaker than those during MILDEX. In contrast to MILDEX, the Research Platform FLIP was moored during SWAPP. The result is that platform motion and FLIP's wake are more important than in MILDEX, where their effect was small. The thruster, however, does not contaminate the velocity measurements as it did during MILDEX.

The SWAPP experiment has already been discussed in a number of papers. A summary of the overall experiment is given in Weller et al., (1990). Weller and Plueddemann (1994, henceforth WP94) studied the subinertial response to surface forcing during SWAPP. They extracted the Ekman response and demonstrated the 
presence of shear within the unstratified mixed layer. Plueddemann et al. (1994,henceforth PEA94) examined a number of the measurements of Langmuir cells made during the experiment. They demonstrated that one can combine different types of measurements (computer cards, current meters, and Doppler sonars) to produce a picture of coherent structures within the mixed layer which match the conceptual picture we have of Langmuir cells. Some of the Doppler sonar measurements are reported separately in Smith (1993). This chapter draws on these papers as it summarizes the results of the experiment.

The structure of this chapter is as follows. Section 7.2 presents the instrumentation used during SWAPP and gives an overview of the background meteorological and oceanographic conditions during the experiment. Section 7.3 expands on the work of PEA94, documenting the high-frequency $(1-30 \mathrm{cph})$ shear as a measure of Langmuir cell strength. Section 7.4 expands on the work of WP94, looking at the dependence of the low-frequency response on the presence of cells. During times when the cells are strong, the velocity structure of the mixed layer is more sheared than predicted by a slab model, but less sheared than predicted by a Mellor-Yamada model. Additionally, on two days when the cells are present the mixed layer fails to restratify as predicted by the models. Section 7.5 demonstrates that the scaling for the level of the high-frequency shear derived in Chapter 6 holds during SWAPP as well as during MILDEX, supporting the idea that the cells are driven by wave-current interaction. Section 7.6 presents results from the instabilty codes derived in Chapter 3 and documented in Appendix B to show that on two days, the current and temperature profile predicted by the Mellor-Yamada model is unstable to Langmuir cells. Section 7.7 demonstrates that the Craik-Leibovich mechanism could provide sufficient energy to explain the failure of the mixed layer to restratify on two days. Section 7.8 concludes this chapter.

\subsection{The SWAPP Field Experiment}

\subsubsection{Instrumentation}

The SWAPP main field experiment took place off the California coast in February and March of 1990. As in MILDEX, the experiment was again centered around the Research Platform FLIP, which was taut-moored at $35.08 \mathrm{~N}, 127.59 \mathrm{~W}$, approximately 200 miles northwest of San Diego and in close proximity to the MILDEX experiment site. Participating in the experiment were the R/P FLIP, the USNS Navajo, and the Canadian Survey Ship Parizeau. Current meter, profiling 


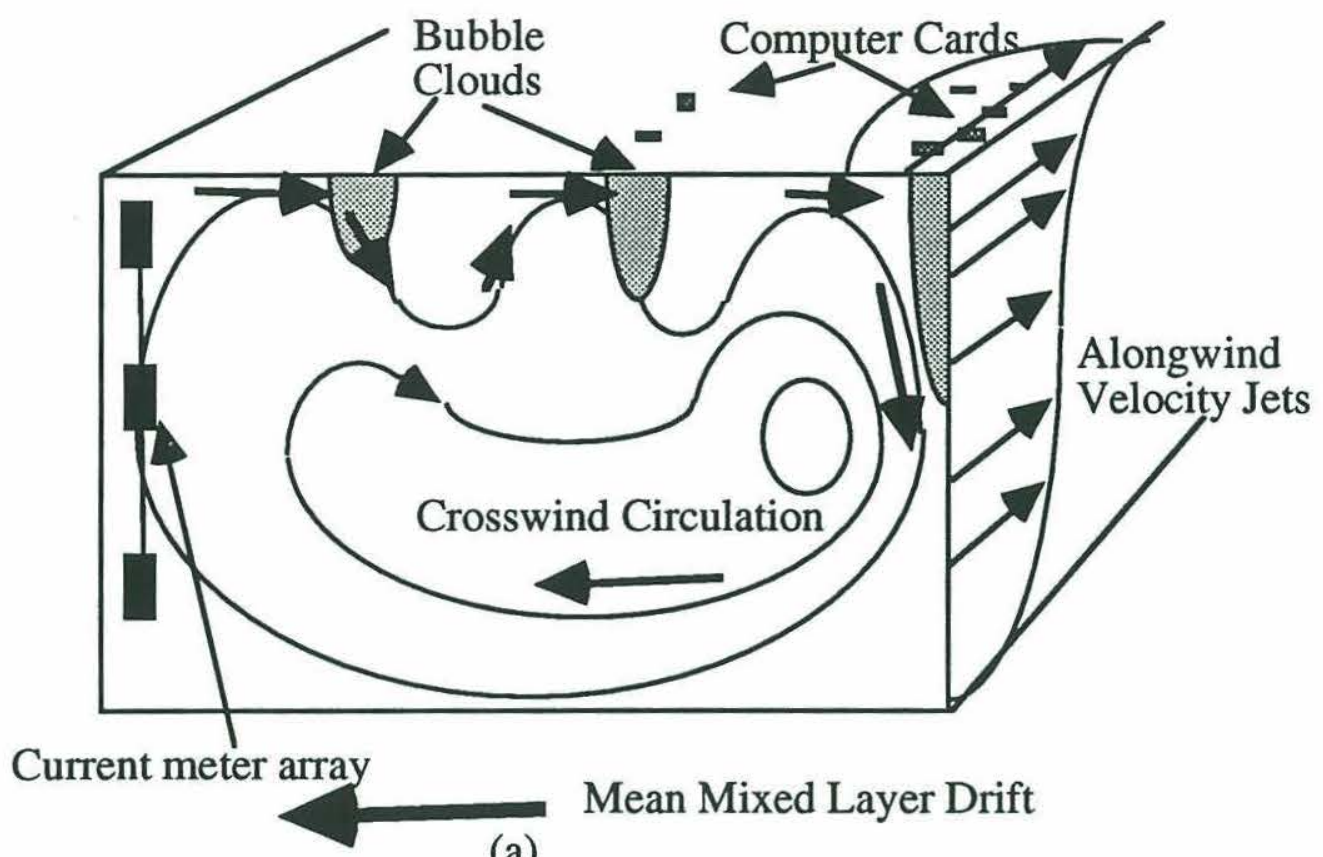

(a)

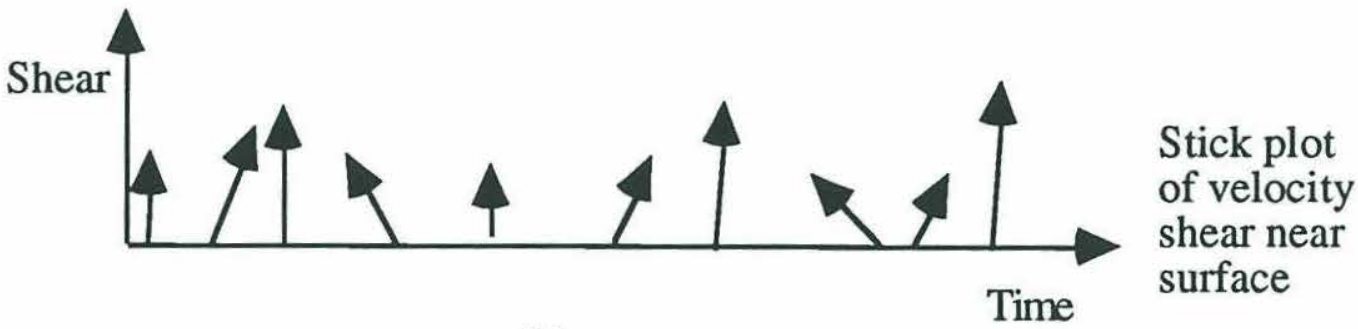

(b)

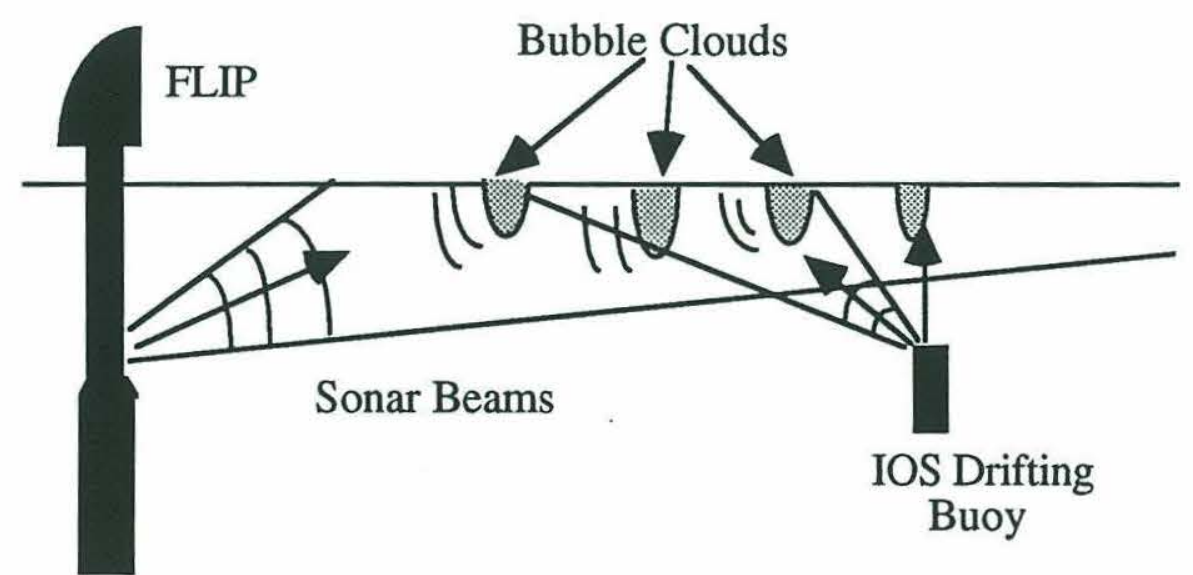

(c)

Figure 7.1: Schematic of measurement approach during SWAPP. (a) Velocities, bubble clouds, and surface convergence of cards associated with cells. (b) Current shears seen near surface as the result of cells drifting across current meter array. (c) Sonars scattering sound off of bubble clouds. 
CTD, and Doppler sonar measurements were made from FLIP as shown in the schematic in Figure 7.1c. An acoustics drifter was deployed from the Parizeau. Profiles of turbulence were made from the Parizeau as well as from the launch Slicker. A mesoscale survey of the temperature structure was carried out from the Navajo.

The experimental strategy during SWAPP was basically identical to that during MILDEX. As shown in Figure 7.1a, in the presence of Langmuir cells surface drifters (i.e computer cards) are organized into rows and bubbles are organized into plumes. The bubble plumes are good scatterers of sound and may be detected using sonars. During SWAPP, both sidescan sonars deployed from FLIP and upward looking sonars from the IOS drifting instrument SUSY were used to image the plumes (Figure 7.1c). As the mixed layer moves relative to FLIP, the bubble clouds are advected past the platform and the velocity structures associated with the cells result in time-varying velocity and shears. These are measured by the current meters suspended from FLIP's booms (Figure 7.1b).

We begin the detailed description of the measurements made during SWAPP by considering the measurements made from FLIP. Figure 7.2 shows a planview of FLIP, illustrating the measurements made during the cruise. Three booms were deployed from FLIP, one jutting out from the port side of the vessel at approximately right angles to FLIP's direction of orientation (port boom), one on the port side pointing directly aft (aft boom), and one pointing slightly to the starboard side of the vessel (starboard boom).

Wind speed and direction, air and sea surface temperature, incoming shortwave and longwave radiation, and precipitation were all measured from FLIP. Except for sea surface temperature and precipitation, all other measurements were made from the mast at a height of $28.9 \mathrm{~m}$. Sea surface temperature and all the other meteorological variables were measured by a Vector Averaging Wind Recorder (VAWR) every 56.25 seconds. Precipitation was measured 15 meters out along the port boom. Details of the measurements and corrections made to the dataset for shifts in calibration are given in WP94.

Water velocities and temperatures were measured at a number of depths throughout the mixed layer using three types of instruments. This chapter will use data from only two of these types, the Vector Measuring Current Meters (VMCM) described in Chapter 6. Two strings of these current meters were deployed. A long string with VMCMs at $2.25,4.5,6.75,9.0,11.25,15.75,20.25,24.75,33.75$, $41.0,53.25,70.5,100$, and $132.25 \mathrm{~m}$, and an RTP at $29.25 \mathrm{~m}$ was deployed from 


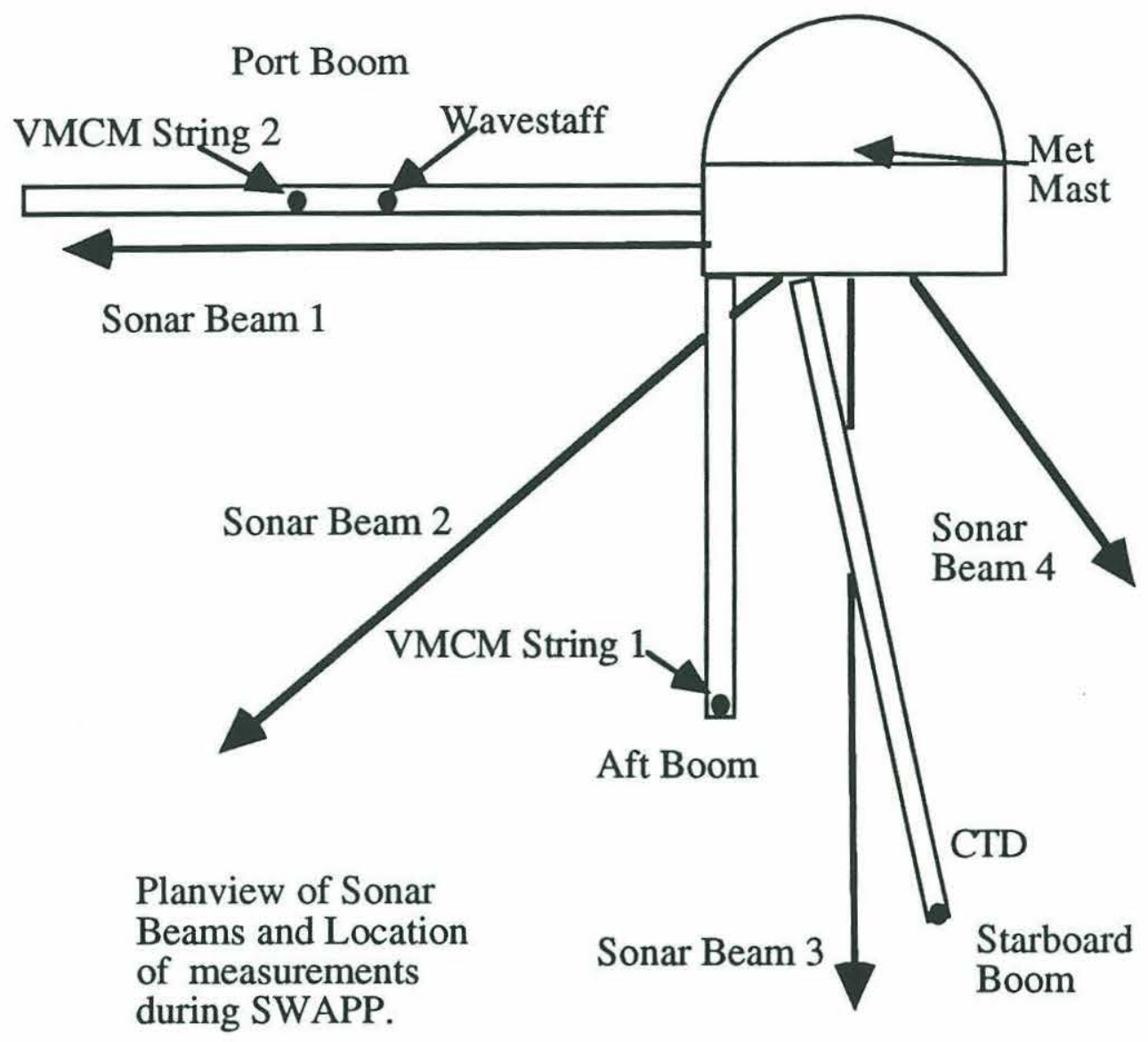

Figure 7.2: Planview of sonar beams and location of various measurements during SWAPP main cruise.

the aft boom. A shorter string with VMCMs at 2.25, 6.75, 11.25, 20.15 and $41.3 \mathrm{~m}$ was deployed off of the port boom. The VMCMs were sampled at a rate of once every 2 seconds.

During SWAPP, FLIP was moored using a three-point mooring. This created two sources of potential contamination for the current meter measurements. The first of these was that the platform was found to move in phase with the waves. The error introduced in velocity measurements due to such motion is studied in Appendix D and is shown to be a small fraction of the overall variance and shear. The second source of error is that large velocities relative to FLIP's hull caused the shedding of eddies in a relatively narrow wake which would alternately contaminate one or the other of the two current meter strings. The wake was found not to affect the time-averaged velocity structure when averaging periods of 15 minutes or more were used. However, it did cause enhanced variance in the 10-30 cph frequency band. In the real-time displays aboard FLIP, the wake 
was easily detectable, as the character of the shear in the two current meter strings was clearly different (only one string at a time was affected). As a result, by taking the minimum of the variance between the two strings, the effect of the wake could be removed when calculating the level of the high-frequency (1-36 cph) shear.

The fact that FLIP was moored means that the relative velocities measured from the platform are much closer to being identical to the absolute velocities than those measured during MILDEX. WP94 show that FLIP did move on its mooring, with velocities of order a few $\mathrm{mm} / \mathrm{s}$ and frequencies near the inertial frequency. As shown in Appendix D, during SWAPP FLIP did rotate in phase with the surface gravity waves, as well as moving on its mooring with a period of about 100 seconds. For purposes of this chapter, it is assumed that the velocities which vary with periods longer than 100 seconds are absolute velocities. In order to isolate the wind-forced response however, it is still necessary to choose a reference level to eliminate the effect of subinertial flows.

Temperature and salinity were also measured from FLIP using a profiling CTD system. The CTD was profiled off the starboard boom from the surface down to a depth of 150 meters. CTD data above $10 \mathrm{~m}$ was found to exhibit effects from bubbles and was not used.

A four-beam Doppler-sonar system was deployed from FLIP. The setup of the sonars is similar to that deployed in MILDEX, with the critical difference that the sonar range was quite a bit smaller (only 450 meters as opposed to 1400 meters during MILDEX). The short range of the sonars made it impossible to detect the long-wavelength alongwind features associated with Langmuir cells-only the beams in the crosswind direction gave reliable indices of cell strength. Details of the sonar setup are given in Smith (1993) and PEA 94. Smith (1993) used data from the side-scan sonars to construct an index of the crosswind velocity associated with the cells. He did this by the following process:

1. Averaging the velocity returns over 1 minute to filter the effect of the surface waves.

2. Taking the spatial Fourier transform of the velocity along each beam.

3. Forming the cross-spectrum of the Fourier coefficients at time $t$ with those at time $t+\Delta t$. and averaging over one hour. This process isolates features which have some temporal persistence of at least $\Delta \mathrm{t}$ (in this case taken as 1 minute) and a reasonably constant phase speed over the averaging period. The coefficients $\mathrm{C}$ are complex numbers whose phase is proportional to the amount by which the features 
have been advected over the delay time. If $U_{a d}$ is the advection velocity along the beam the phase shift is proportional to $\mathrm{kU}_{\mathrm{ad}}$.

4. Computing the lagged cross-correlation $\mathrm{D}$ in wavenumber of the temporally lagged cross-spectra. This selects out features in wavenumber space that have similar advection velocities. The phases are rescaled and wavenumber bands are chosen so that only advection velocities less than $0.8 \mathrm{~m} / \mathrm{s}$ contribute significantly to the cross-correlation. (Details of how this is done are given in Smith,1993).

The fourth root of $\mathrm{D}$ is a measure of the velocity contained in temporally persistent, spatially coherent structures which propagate along the beam at speeds less than $0.8 \mathrm{~m} / \mathrm{s}$. Smith (1993) refers to this velocity as the "doubly-lagged" velocity and uses it as a measure of the velocity associated with the Langmuir cell vortices. It is referred to below as the "sonar LC velocity amplitude".

Waves were measured off the port boom using a wavestaff which sampled at $4 \mathrm{~Hz}$, off the starboard boom by an three-wire wave gauge array and by the Doppler sonars. This work concentrates on measurements made with the wavestaff on the port boom, since this was the dataset most readily available. Time series of wave height from this instrument were used to compute the vertical profile of the Stokes drift.

A group from Institute of Ocean Sciences, Victoria, BC used side-scan sonar deployed from a drifting buoy to track bubble clouds as they drifted over the instrument. These observations were correlated with backscatter measurements from an inverted echo-sounder to show that the features observed in the side-scan sonar corresponded to plumes of bubbles.

Turbulence measurements were made using a profiler which was dropped from the Parizeau or from the launch Slicker when the weather was calm enough to permit deployment. The instrument used was the IOS Fast Light Yo-Yo II (FLY II) described in Crawford and Gargett (1988), which uses airfoil shear probes to measure the shear on small scales. These measurements are then used to infer the the dissipation of eddy kinetic energy. The measurements used here are reported in Crawford (1992).

\subsubsection{Meteorological and Oceanographic Background}

We begin our examination of the SWAPP data set with a summary of the meteorological and oceanographic conditions during the experiment. Figure 7.3 shows a series of weather maps for the west coast of North America during the 

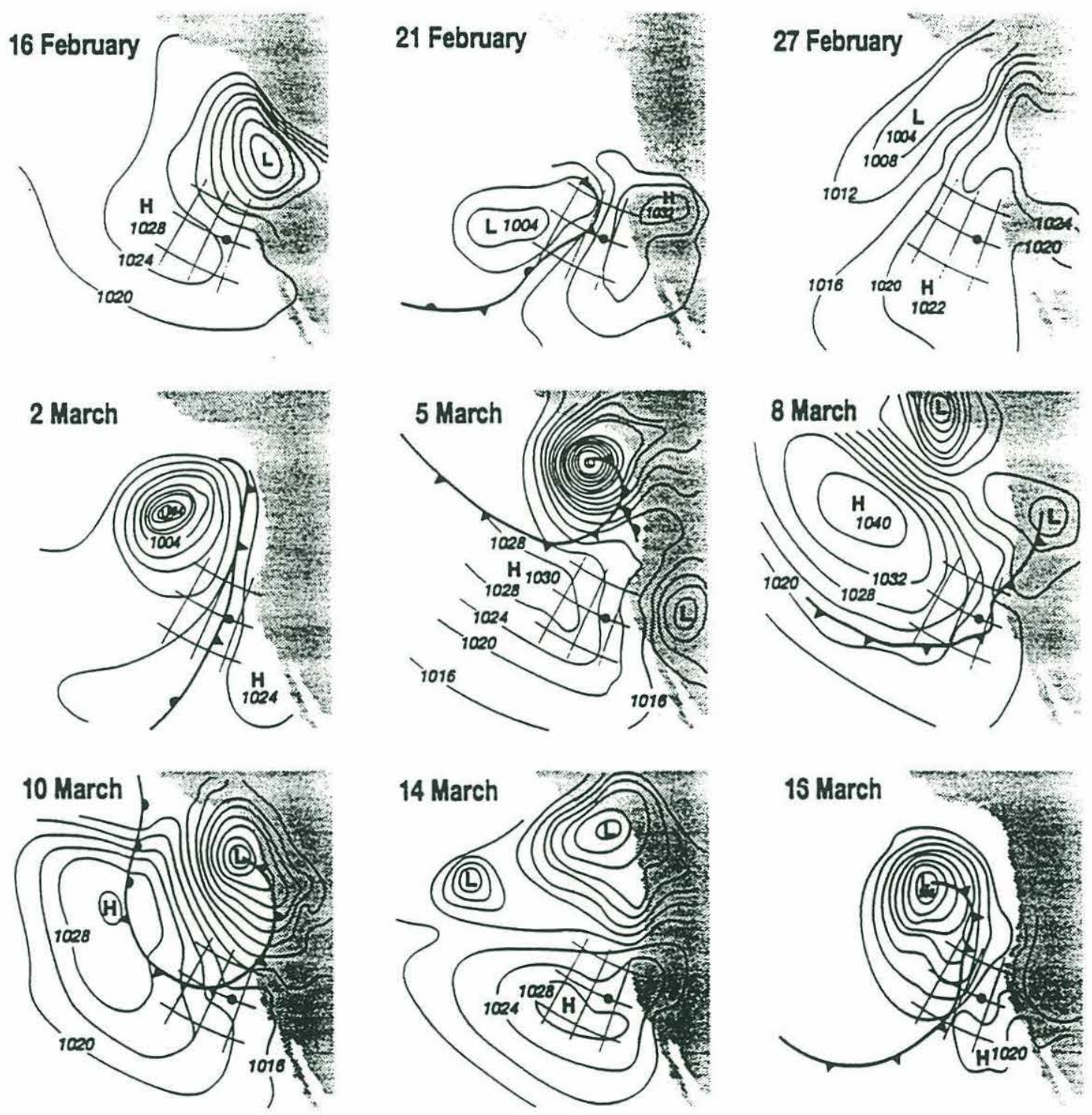

Figure 7.3: Summary weather maps of the meteorology during SWAPP from Weller and Plueddemann, 1994. FLIP's position is marked with $a \bullet$. 
course of the experiment. The meteorological conditions during SWAPP were largely determined by slow-moving high pressure systems which formed over the North Pacific and moved onto land over a time scale of about 2 weeks. The first of these highs formed in mid-February and moved onto the continent about March 2. The second high formed about March 4 and moved onto land around March 16th. Fast-moving low pressure systems tracked around the edges of these quasistationary highs. During most of SWAPP, the experiment site was located on the flanks of the high pressure systems. However, during the intervals between the passage of the highs, (March 2, March 16) two low pressure systems did pass near the site.

The background meteorology during SWAPP is summarized in Figures 7.4 and 7.5. Figure 7.4 shows the wind speed and direction, barometric pressure, relative humidity, sea surface temperature, air temperature, and cumulative precipitation. Figure 7.5a shows the wind stress in the east-west (solid) and northsouth (dashed) directions. Figure $7.5 \mathrm{~b}$ shows the total heat flux computed from a combination of measured fluxes (incident shortwave and longwave radiation) and bulk formulae (latent heat flux, sensible heat flux, and outgoing longwave radiation). Figure $7.5 \mathrm{c}$ shows the significant wave height, and $7.5 \mathrm{~d}$ shows the Brunt-Vaisala frequency calculated from the temperature difference between 2.25 and $4.5 \mathrm{~m}$.

The time series of wind shows five events, occurring on March 1st and 2nd (Event 1), March 4th and 5th (Event 2), March 8-10 (Event 3), March 11-13 (Event 4), and March 16-17 (Event 5). Events 1 and 5 are associated with the passage of lows to the north of the experiment site, while events 2, 3, and 4 are the result of the flanks of the high pressure system shifting over the experiment site. As the leading edge of the high moves over the site, the winds intensify in a southerly direction bringing colder, dryer air from the north. The result is large latent and sensible heat fluxes, leading to the large negative values of heat flux on the nights of March 5, 8, and 11. By contrast, wind events 1 and 5 are not associated with strong heat losses- although heating due to shortwave radiation does decrease during wind event 1 .

The surface gravity wave field shows a somewhat sawtooth response to these wind events, building up quickly and then dying off slowly. The slow die-off of the waves after wind events $2,3,4$ is due to the long fetch associated with the flanks of the high pressure system combined with the fact that the wind does not change direction as the high pressure system shifts position. 


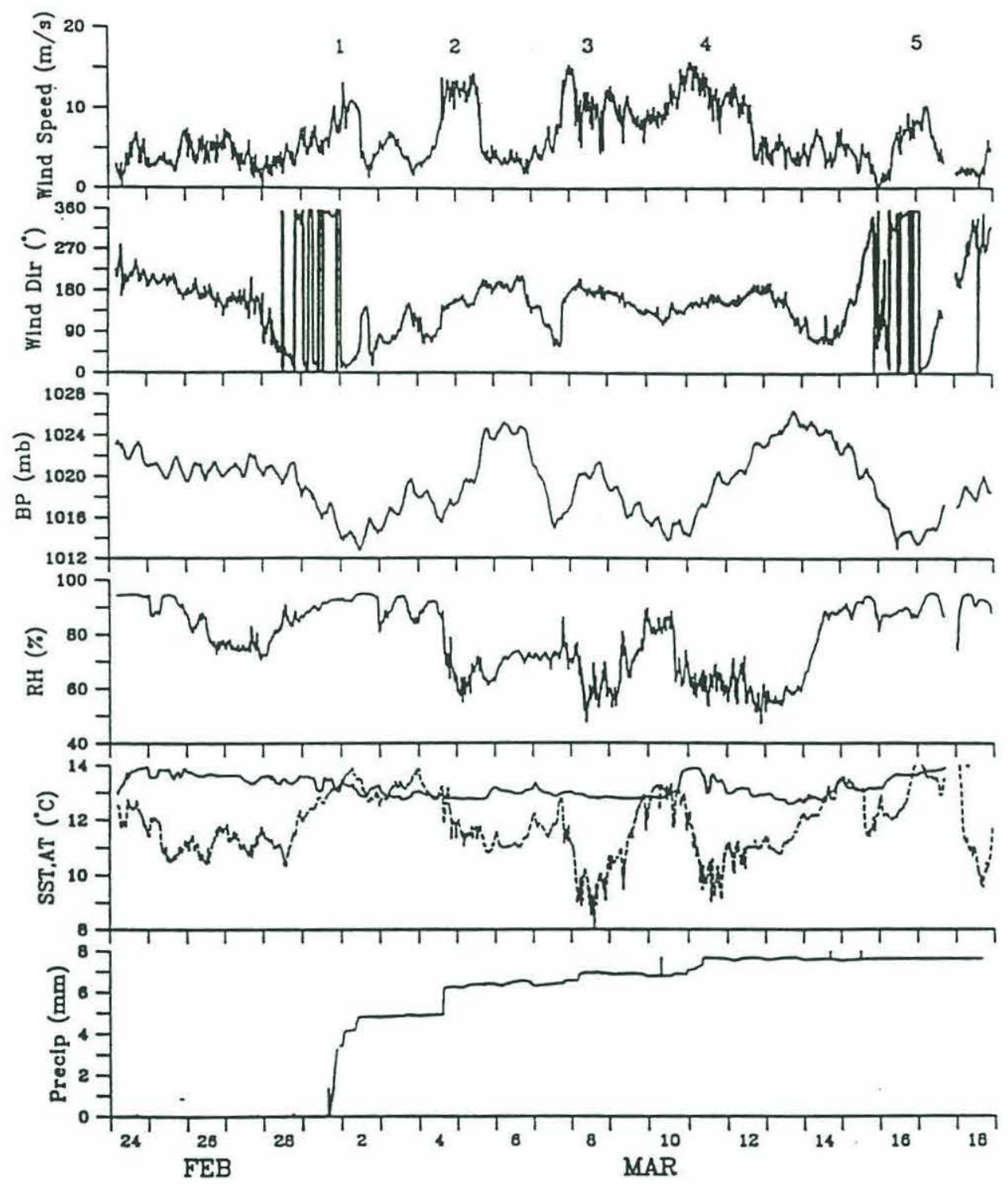

Figure 7.4: Time series of meteorological variables recorded from FLIP during SWAPP from Weller and Plueddemann, 1994. From top to bottom, wind speed in $\mathrm{m} / \mathrm{s}$, wind direction (towards) in degrees, barometric pressure in mb, relative humidity in percent, sea temperature (solid) and air temperature (dashed), and cumulative precipitation in $\mathrm{cm}$ are shown. Data plotted are 60 minute running means of original one minute data. The five wind events mentioned in the text are labelled 1 through 5 . 

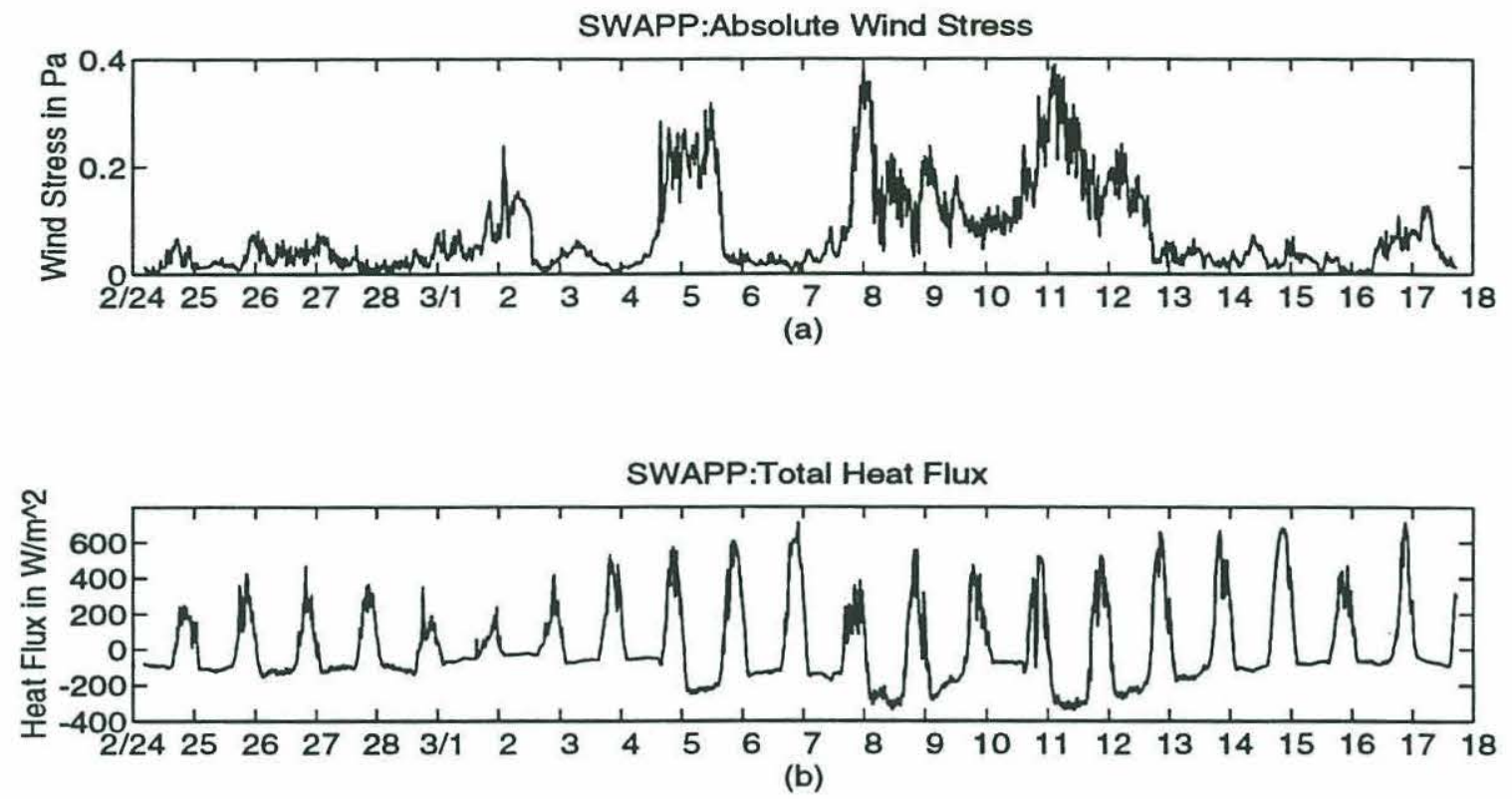

SWAPP:Significant Wave Height (WHOI Wavestaff)

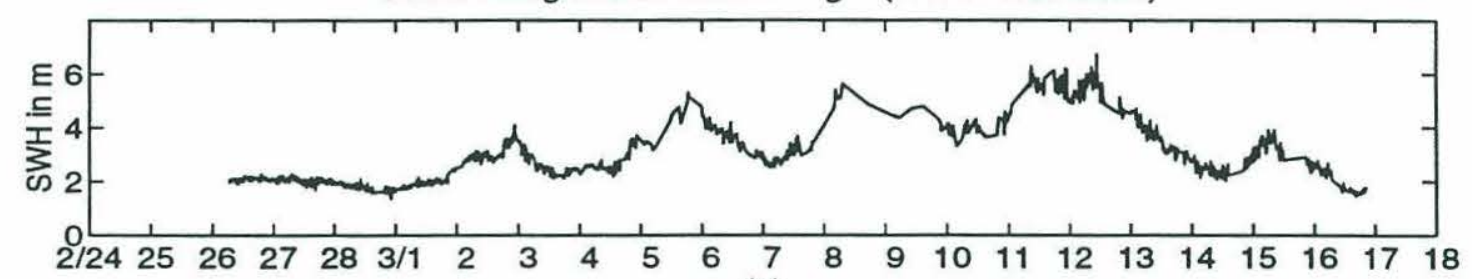

(c)

SWAPP:Buoyancy Frequency 2.25-4.5m

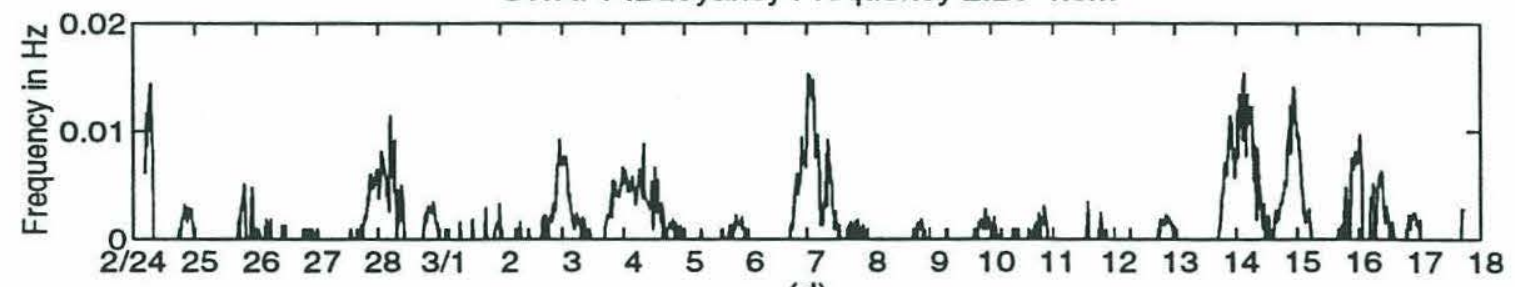

(d)

Figure 7.5: Time series of (from top to bottom) wind stress magnitude in $\mathrm{Pa}$, heat flux in $\mathrm{W} / \mathrm{m}^{2}$, significant wave height in $\mathrm{m}$ calculated using the WHOI wavestaff, and buoyancy frequency calculated from the temperature gradient between 2.25 and $4.5 \mathrm{~m}$. Each data point represents an average over 15 minutes. 


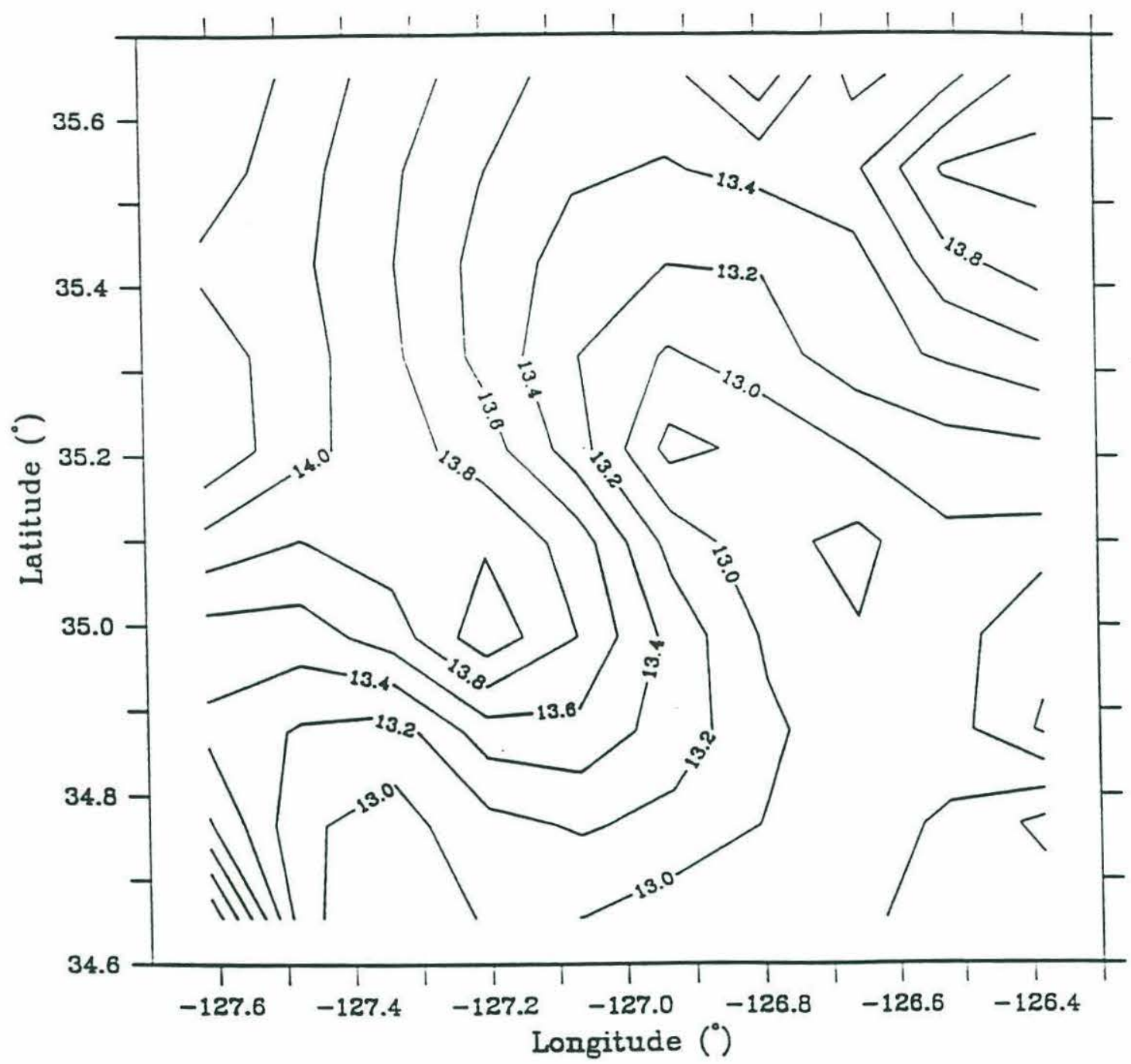

Figure 7.6: Contour plot of surface temperatures from mesoscale XBT survey conducted from USNS Navajo during a 30 hour period after FLIP was moored at $35 \mathrm{~N}, 127 \mathrm{~W}$. Temperatures are representative of the mean mixed layer temperature. Plot is taken from Weller and Plueddemann (1994), Data is reported in Gnanadesikan (1990). 
During SWAPP, FLIP was moored in the middle of a frontal system. Figure 7.6 shows the results of an XBT survey made from the Navajo, (reported fully in Gnanadesikan, 1990). As seen from the isotherms, FLIP was moored in the middle of a strong temperature front, with warm $(>14.0 \mathrm{C}$ ) water to the northwest and cooler ( $<12.8 \mathrm{C}$ to the southeast). Over the course of the experiment, the front moved past FLIP a number of times. However, during the most of the cruise the temperature anomaly associated with this front was almost totally compensated by salinity within the mixed layer. Figure 7.7 illustrates this fact. Figure $7.7 \mathrm{a}$ is a mesh plot of temperature from the CTD data over a depth range of $10-70 \mathrm{~m}$. During the early part of the experiment (from February 25 until March 1st) there is strong frontal activity in the vicinity of FLIP and the temperature over the top 70 meters varies by of order $0.5 \mathrm{C}$. Later on in the experiment, (around March 11th) the entire top 70 meters warms by almost 1.5 degrees, then cools around March 13th, then warms again, with some vertical structure being again evident. The density changes associated with this movement of the warm and cold water masses across the experiment site would be of order $0.4 \mathrm{~kg} / \mathrm{m}^{3}$ in the absence of salinity compensation. Figure $7.7 \mathrm{~b}$ shows the density at a depth of $30 \mathrm{~m}$ calculated from the CTD data. The strong changes in temperature in the upper part of the water column are not reflected in changes in density, which only changes by of order $0.05 \mathrm{~kg} / \mathrm{m}^{3}$.

Because of salinity compensation, the strong shears that would be expected to be associated with the frontal structure seen in Figure 7.6 were not seen over the top forty meters of the water column. If the shear is given by the thermal wind relation and salinity compensates approximately $80 \%$ of the temperature anomaly, then based on the XBT survey the shears within the mixed layer associated with the presence of the front are of order $0.001 \mathrm{~s}^{-1}$. This is a small signal in comparison with the shears of interest. The movement of the front also apparently does not introduce strong stratification into the mixed layer above depths of 40 meters or so. Figure 7.7c shows the density difference between 10 and $40 \mathrm{~m}$. Again, the changes seen are about $0.05 \mathrm{~kg} / \mathrm{m}^{3}$, corresponding to a buoyancy frequency of 3.5 $\mathrm{x} 10^{-3} \mathrm{~s}^{-1}$. This is smaller than the stratification due to diurnal warming. Below about 40 meters, however, frontal dynamics are potentially important in determining the stratification. 


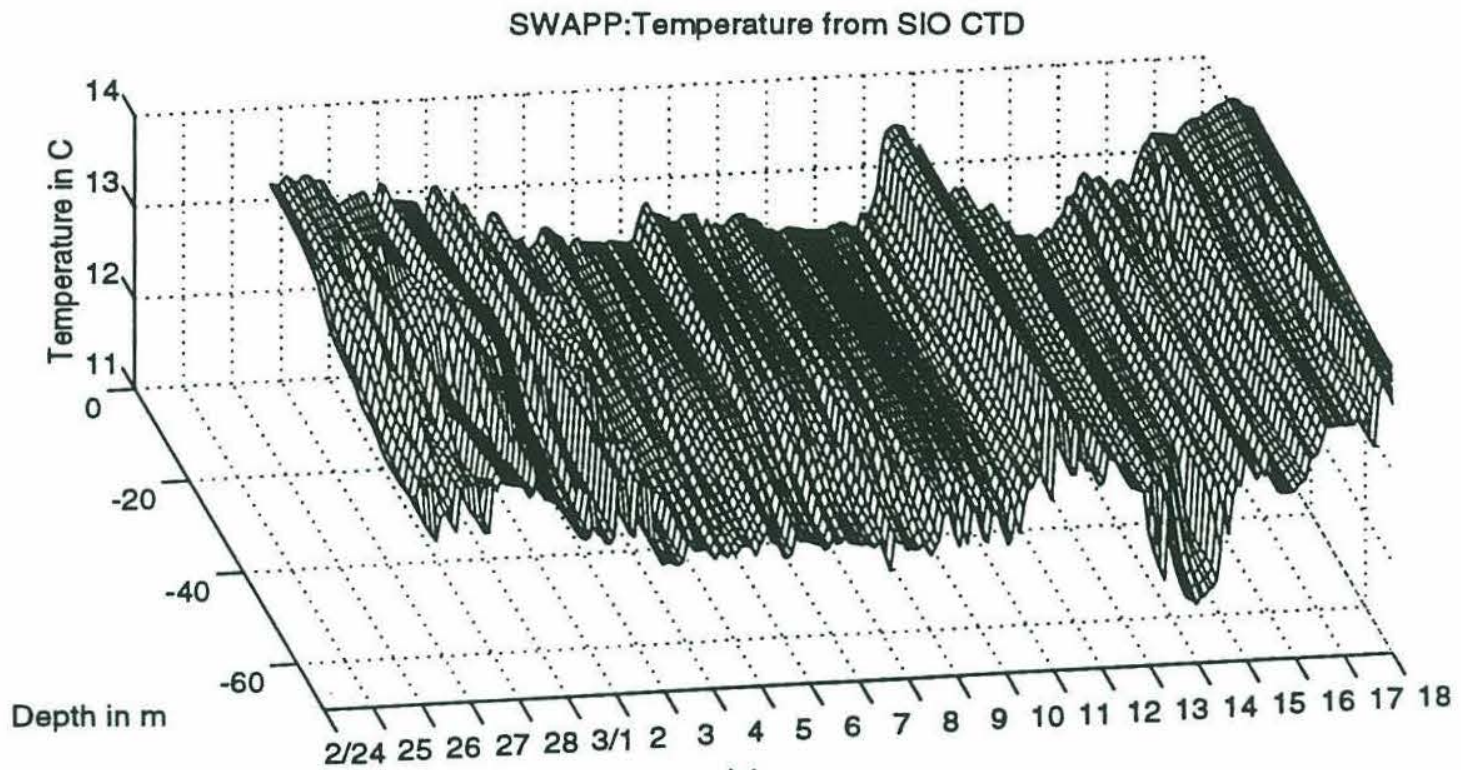

(a)

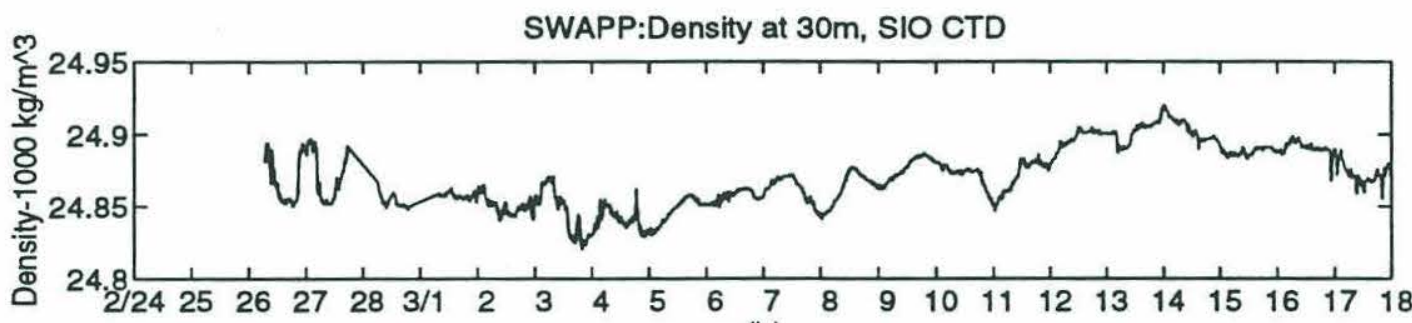

(b)

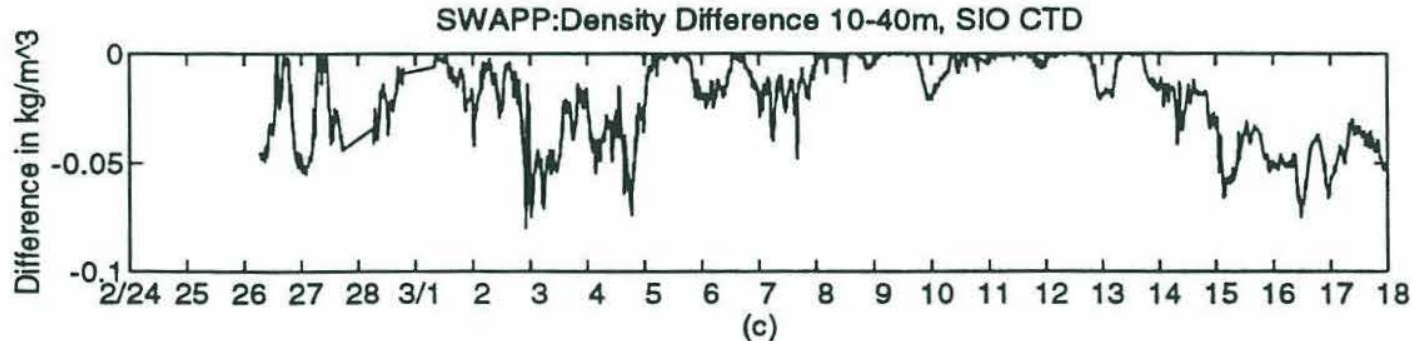

Figure 7.7: Summary of density structure within the mixed layer during SWAPP.

(a) Mesh plot of temperature over 10 to 70 meters depth from the SIO CTD.

(b) Time series of density $-1000 \mathrm{~kg} / \mathrm{m}^{3}$ at 30 meters calculated from the SIO

CTD. (c) Time series of the density difference between 10 and 40 meters in $\mathrm{kg} / \mathrm{m}^{3}$. 


\subsection{Measurements of Langmuir Cells made during SWAPP: A synopsis of measurements of Cell Strength}

One weakness of the MILDEX dataset studied in Chapter 6 is that, except for the time period intensively studied by Smith et al, 1987, there is only qualitative evidence that Langmuir cells were present in the mixed layer. This means that there is no objective way to distinguish the Langmuir cell indices derived in Chapter 6 from possible noise terms such as thruster contamination.

In contrast, PEA94 combine evidence from computer card deployments, doppler sonars, and current meters to come up with a time series of cell strength for the entire SWAPP experiment. They find that at times when cells were strong - Computer cards at the surface are aligned into rows.

-Bubbles also are aligned into rows, producing regions of high backscatter in the sidescan sonars. The IOS and SIO sonars both see the clouds and report similar structures.

-These rows of bubbles are associated with plumes of bubbles penetrating 5-15 meters into the water column by the IOS sonars. Zedel and Farmer (1991) showed that similar plumes during the Ocean Storms experiment were linked to downwelling.

-The rows of bubbles are also correlated with convergence in the crosswind velocity in the SIO sonar.

-When the SIO sonars show strong convergent velocities enhanced levels of highfrequency (1-36 cph) current and shear are seen in the current meters.

The last of these results represents the author's contribution to PEA94. Because of limited space it was not possible to give a full development of the difference in character between those times when Langmuir cells were present and those when they were not, and to rigourously characterize the long-time variation of cell strength. These shortcomings are addressed in this section. A period of 18 days $(0000 \mathrm{Z}$ February $26-0000 \mathrm{Z}$ March 16$)$ is considered during which data were available from both strings of current meters, the SIO Doppler sonar, and the wavestaff.

The high-frequency velocity and shear during SWAPP has a different character when Langmuir cells are present. Figure 7.8a shows a six-hour time series of current difference between 2.25 and $4.5 \mathrm{~m}$ during a calm period on February 28th. During this time, the surface stress was very small (about $0.01 \mathrm{~Pa}$ ) the temperature difference between the current meters at 2.25 and 4.5 meters was 
SWAPP:2.25-4.5m Shear in $\mathrm{s}^{-1}$, Langmuir Cells Weak

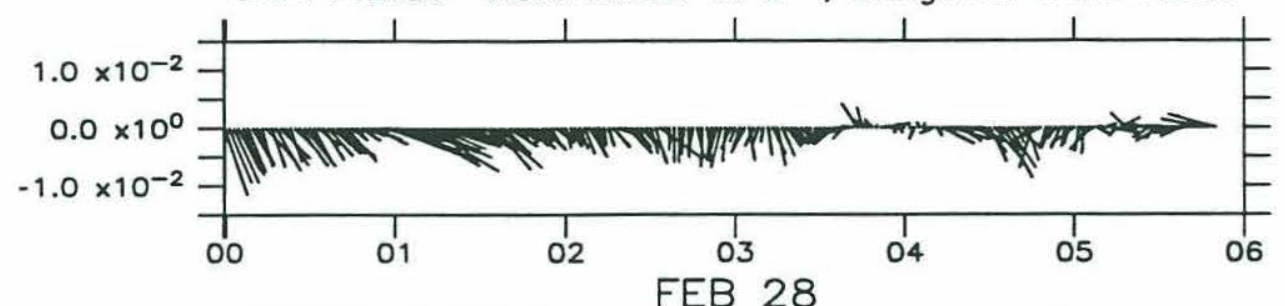

FEB 28

SWAPP:4.5-6.75m Shear in $\mathrm{s}^{-1}$, Langmuir Cells Weak

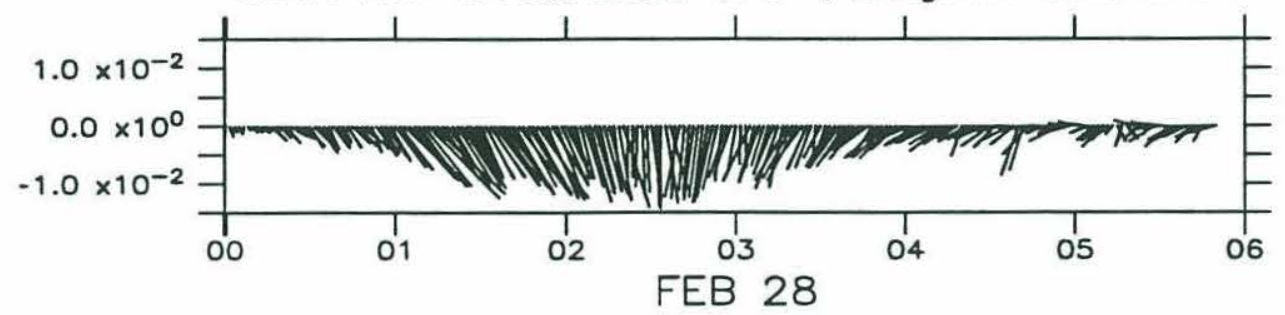

SWAPP:2.25-4.5m Shear in $\mathrm{s}^{-1}$, Langmuir Cells Strong

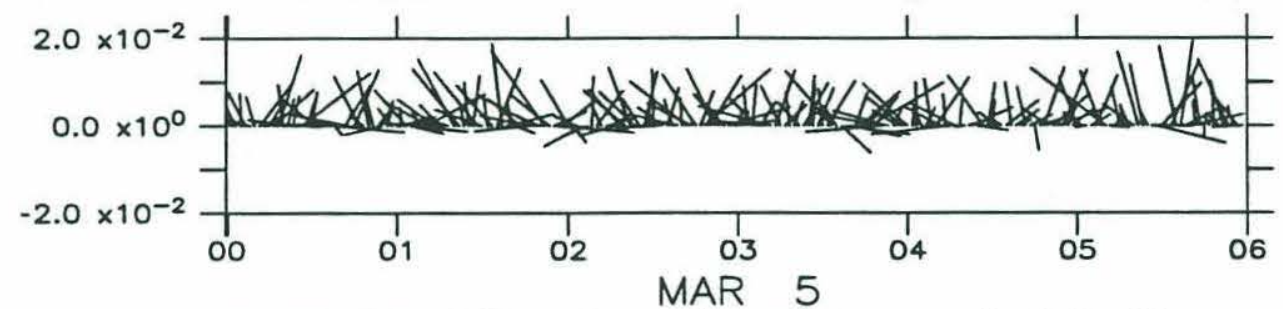

SWAPP:4.5-6.75m Shear in $\mathrm{s}^{-1}$, Langmuir Cells Strong

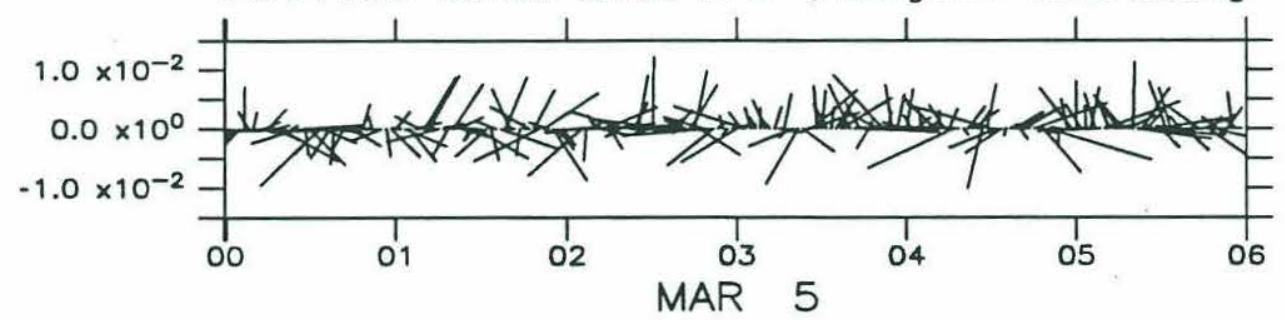

Figure 7.8: Example of how Langmuir cells change the character of the nearsurface shear. Wind points the the +y direction. Each stick represents a 100 second average. Top two panels show the shear between $2.25 \mathrm{~m}$ and $4.5 \mathrm{~m}$ and $4.5 \mathrm{~m}$ and $6.75 \mathrm{~m}$ from $0000 \mathrm{Z}-0600 \mathrm{Z}$ on February 28th when the upper portion of the water column was stratified. Bottom two panels show the shear between 2.25 and $4.5 \mathrm{~m}$ and $4.5 \mathrm{~m}$ and $6.75 \mathrm{~m}$ from $0000 \mathrm{Z}-0600 \mathrm{Z}$ on March 5th when the upper portion of the water column was well-mixed and Langmuir cells were strong. 
Alongwind Shear Spectrum, 00-07Z 2/28

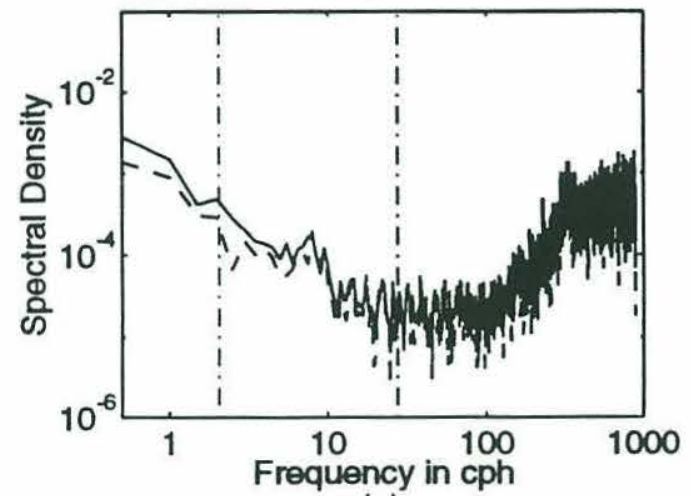

(a)

Alongwind Shear Spectrum, $00-07 Z$ 3/5

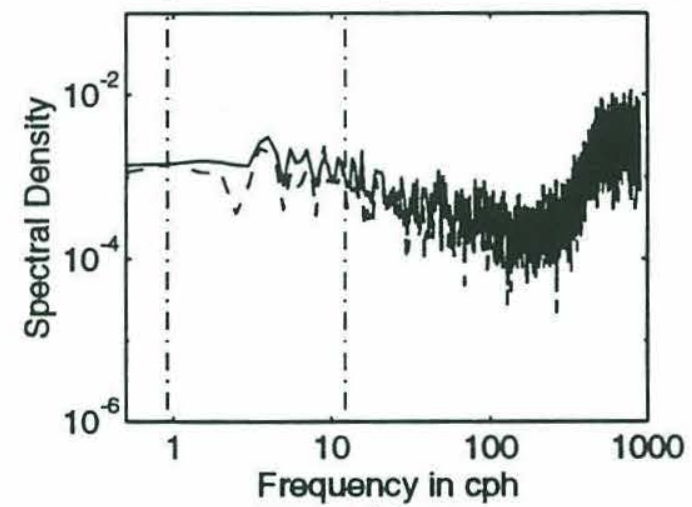

(c)

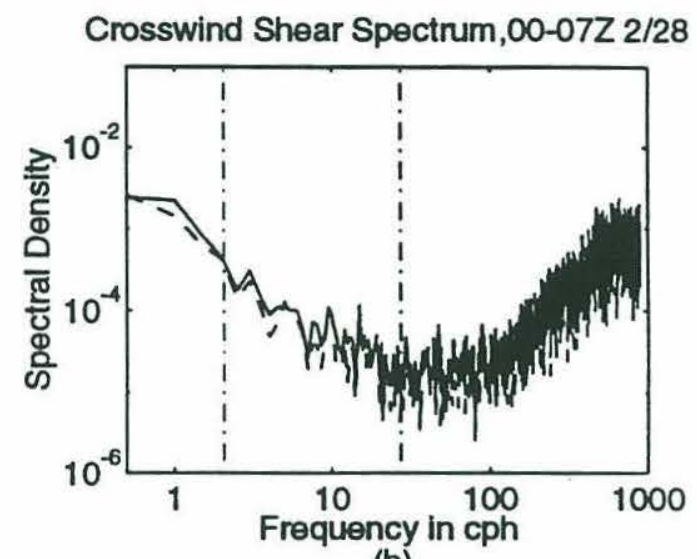

(b)

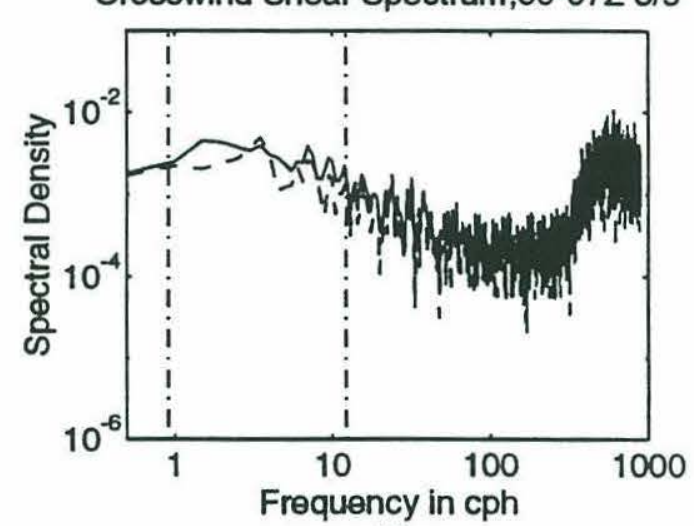

(d)

Figure 7.9: Spectra of shear between 2.25 and $4.5 \mathrm{~m}$ from two time periods: 0000 0700Z, February 28th (cells weak) and 0000-0700Z, March 5th (cells strong). Time series were divided into six overlapping two-hour intervals, spectra were computed for each interval and averaged to form the spectra shown above. The solid lines show the spectra, the dashed lines the confidence interval. The chaindotted lines show the limits of a frequency band corresponding to crosswind advection of features with wavelengths of $15-200 \mathrm{~m}$. (a) Alongwind shear, February 28th. (b) Crosswind shear, February 28th. (c) Alongwind shear, March 5th. (d) Crosswind shear, March 5th. 
$0.05 \mathrm{C}$ and the sonar LC velocity amplitude was $2.4 \mathrm{~cm} / \mathrm{s}$. Figure $7.8 \mathrm{~b}$ shows the same picture for the current meters at 4.5 and $6.75 \mathrm{~m}$. The shear varies smoothly with little variability on periods less than an hour or so. The shear is also oriented opposite to the wind direction, with water at depth moving faster in the downwind direction than water near the surface. Figure $7.8 \mathrm{c}$ shows the current difference between 2.25 and 4.5 meters and $7.8 \mathrm{~d}$ the difference between 4.5 and 6.75 meters for $0000 \mathrm{Z}-0600 \mathrm{Z}$ on March 4th, when the wind stress averaged $0.23 \mathrm{~Pa}$ and the cells were strong. The sonar velocity LC amplitude for this time period is $6.8 \mathrm{~cm} / \mathrm{s}$ and the upper part of the water column is unstratified. Near the surface the shear is oriented in the downwind direction and shows a great deal more variability than on February 28th. Between 4.5 and $6.75 \mathrm{~m}$, there is very little discernable mean signal and a good deal of variability with periods shorter than one hour.

Figure 7.9 shows spectra of the velocity shear from the two periods shown in Figure 7.8. Two-hour segments of data were taken, oriented relative to the wind, and Fourier-transformed. The solid lines show the spectral density of the velocity and the chain-dotted lines the confidence level. Figure 7.9a shows the spectrum of the crosswind velocity and $7.9 \mathrm{~b}$ the alongwind velocity for $0000 \mathrm{Z}-0700 \mathrm{Z}$ on February 28th for the shear over $2.25-4.5 \mathrm{~m}$. Figure $7.9 \mathrm{c}$ and $7.9 \mathrm{~d}$ show the spectra of crosswind and alongwind velocity respectively for $0000 \mathrm{Z}-0700 \mathrm{Z}$ on March 5th. When the mixed layer is strongly forced there is enhanced variability in the shear over a frequency band from 2 to $50 \mathrm{cph}$.

As noted in the last chapter, by assuming that the Langmuir cells have spacings from 15-200 meters and are oriented parallel to the wind, one can estimate the frequency with which convergence zones pass over the current meter array. If $\mathrm{U}_{\mathrm{cw}}$ is the velocity in the crosswind direction then the frequency band in which cells of such wavelengths contribute to the variance is given by

$$
\mathrm{U}_{\mathrm{cw}} / 200 \mathrm{~m}<\mathrm{f}<\mathrm{U}_{\mathrm{cw}} / 15 \mathrm{~m}
$$

The dashed lines in Figure 7.9 show the limits of this frequency band, given the mean $\mid \mathrm{U}_{\mathrm{cw}} \mathrm{l}$ over the six two-hour periods which went into making up the spectrum. The frequency band which would correspond to wavelengths of the right size for Langmuir cells exhibits enhanced variance when the cells are strong.

During SWAPP, times when Langmuir cells are strong correspond to times when the shear and current variance are elevated in a band between the inertial frequencies and wave frequencies. Chapter 6 showed that during MILDEX the overall level of the shear provided a measure of the cell strength which compared well with theory. Figure 7.10 shows a time series of the standard deviation of the 

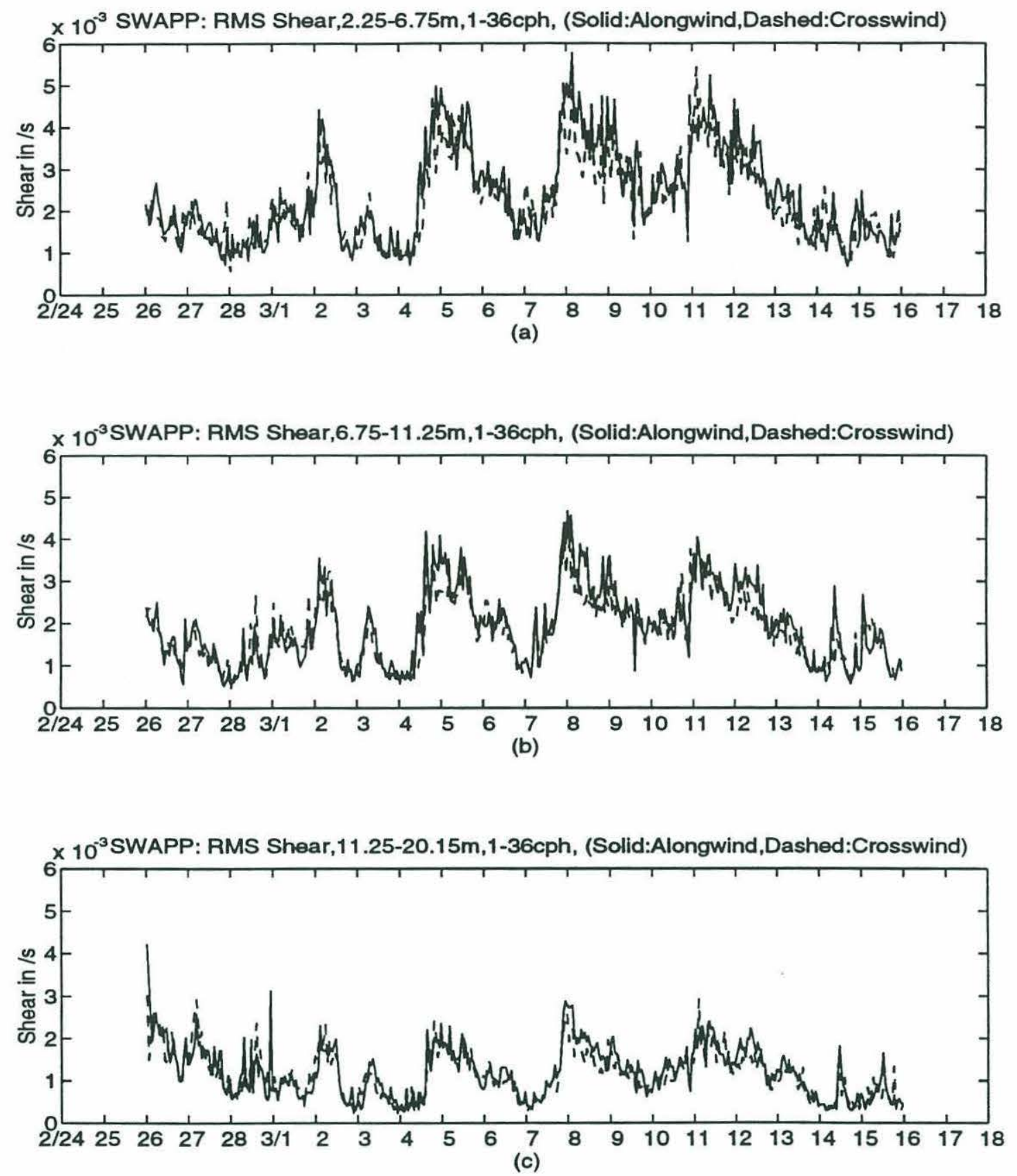

Figure 7.10: Shear standard deviation in 1-36 cph frequency band. Data shown is minimum from the two current meter strings. Solid is alongwind shear, dashed is crosswind shear. (a) $2.25-6.75 \mathrm{~m}$ (b) $6.75-11.25 \mathrm{~m}$ (c) $11.25-20.15 \mathrm{~m}$. 


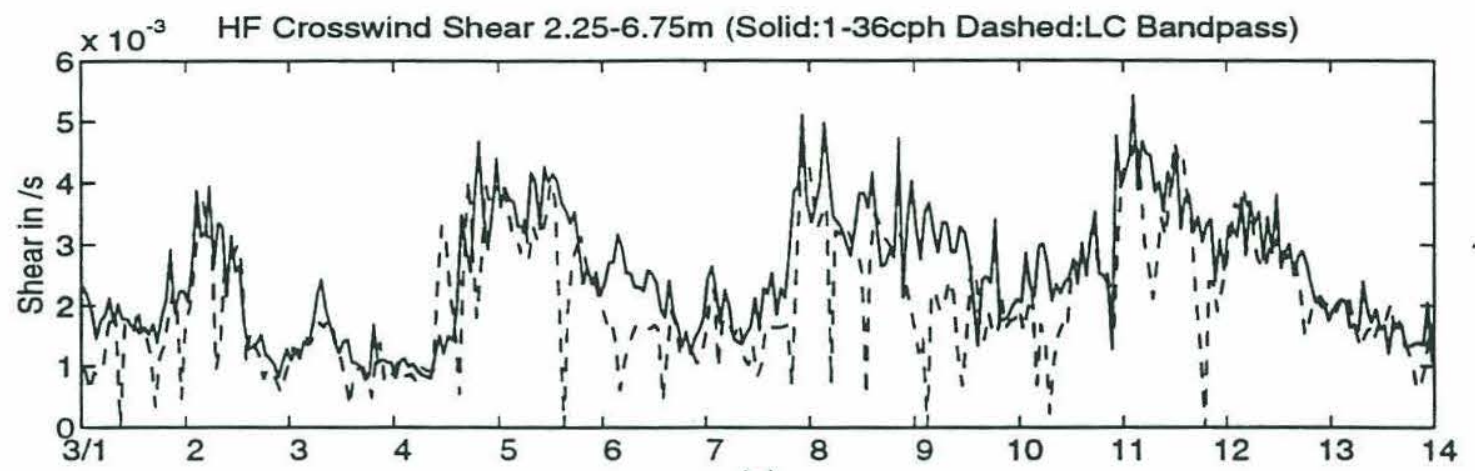

(a)

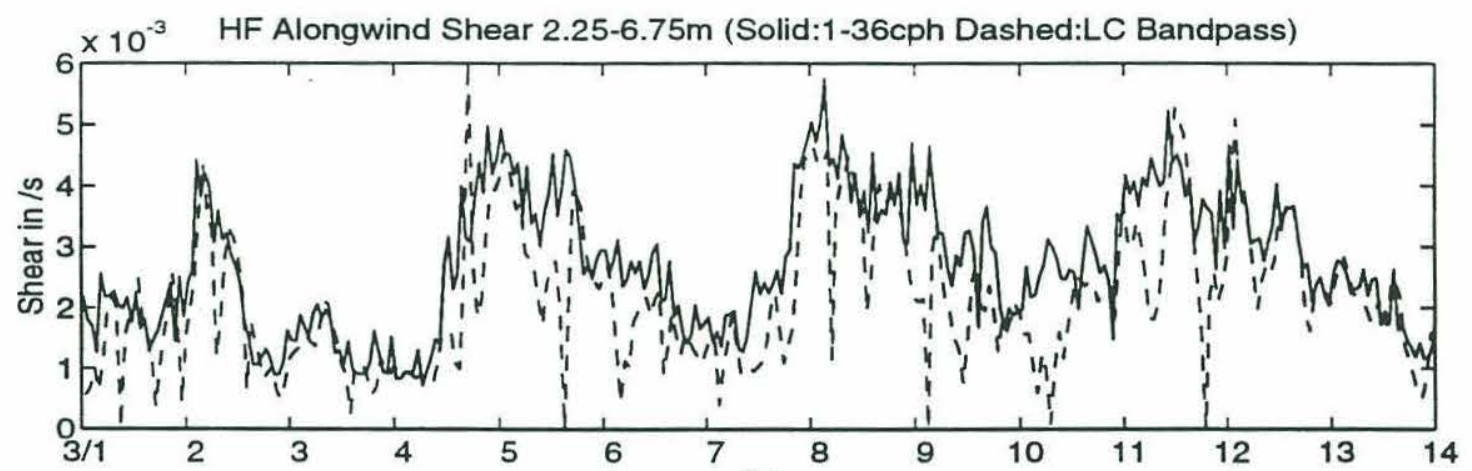

(b)

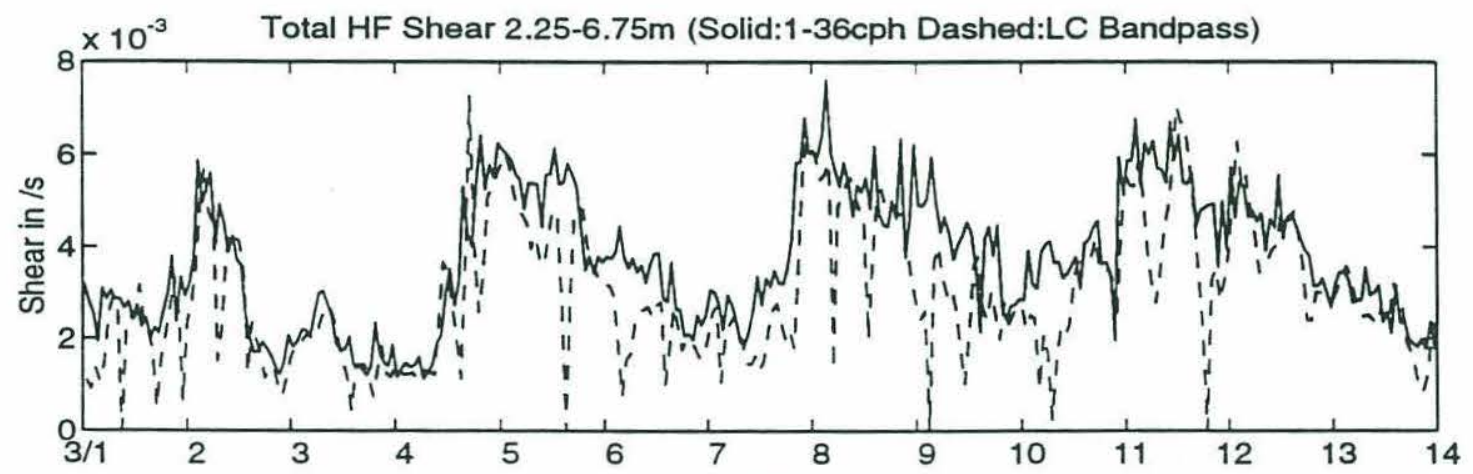

(c)

Figure 7.11: Demonstration that high-frequency (1-36 cph) shear corresponds to the right wavelength band for Langmuir cells. Solid lines are standard deviation of high-frequency (1-36 cph) shear. Dashed lines are standard deviation of shear in a frequency band chosen to correspond to features with spatial scales of $15-200 \mathrm{~m}$ being advected past the current meters in the crosswind direction (LC Bandpass). (a) 2.25-6.75m crosswind shear. (b) $2.25-6.75 \mathrm{~m}$ Alongwind shear. (c) $2.25 \mathrm{~m}-$ $6.75 \mathrm{~m}$ total shear amplitude. 
shear in a frequency band from 1-36 cph over the course of SWAPP for three depth pairs, 2.25 and $6.75 \mathrm{~m}, 6.75 \mathrm{~m}$ and $11.25 \mathrm{~m}$ and 11.25 and $20.75 \mathrm{~m}$. The data shown is the minimum of the standard deviations seen in the two strings of current meters, since when one current meter string was in the wake of FLIP it showed a much higher variance than the one which was not in the wake.

During the time periods shown in Figure 7.9 assuming a frozen field of cells means that the frequency band of 1-36 cph corresponds to a wavenumber band of the right general size for Langmuir cells. We now consider if that result is generally true. The "LC Bandpass" was constructed as follows:

1. For a two-hour period the velocity in the crosswind direction was computed.

2. Using equation (7-1) the frequency band corresponding to crosswind wavelengths of 15 to 200 meters was computed.

3 . The standard deviation of the crosswind and alongwind velocity and velocity shear in this frequency band was computed.

Results of this calculation are shown in Figure 7.11, expanded to show the days when Langmuir cells were strongest. As noted in Chapter 6, using this method ignores the possibility that large scale cells may be advected by small-scale cells, and that the cells may not be oriented parallel to the wind. The solid lines represent the standard deviation computed by using the pure frequency bandpass, the dashed lines the result of using a frequency bandpass based on cell wavelength denoted as the "LC Bandpass" in the figure. The results agree well on most days, but there are sometimes "drop-outs" when the crosswind advection velocity was too small. The results support the idea that the frequency band of $1-36 \mathrm{cph}$ is the right one to use to capture features the size of Langmuir cells. Because using the LC Bandpass would introduce spikiness into the time series, making correlation with other indices difficult and because of the caveats given above, the pure frequency bandpass was chosen as an index of cell strength.

The amplitude of the shear resembles, but does not exactly track, the wind and waves. Figure 7.12a shows the absolute value of the wind stress and 7.12b shows the significant wave height. Figure $7.12 \mathrm{c}$ shows the standard deviation of the high-frequency shear between 2.25 and $6.75 \mathrm{~m}$. The high-frequency shear tracks the wind on most days, except March 5th and 12th, the days immediately following wind events 2 and 4 respectively.

The level of the high-frequency shear agrees very well with the sonar LC velocity amplitude as a measure of cell strength. Figure 7.12d shows the sonar LC velocity amplitude. The correlation between the high-frequency shear amplitude 


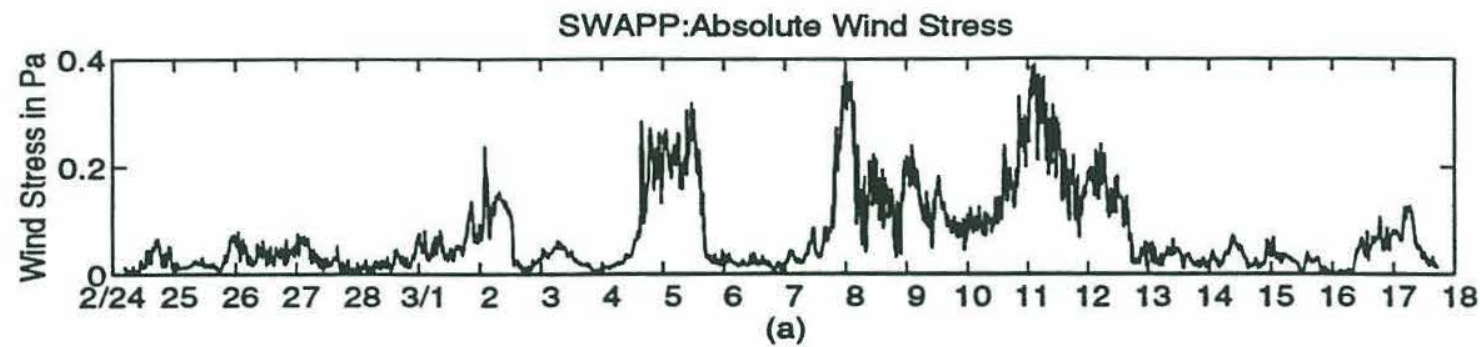

SWAPP:Significant Wave Height

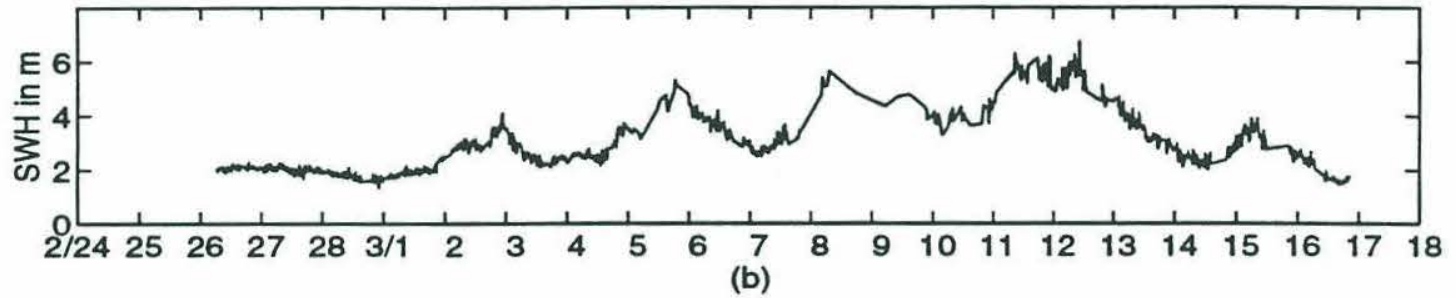

SWAPP:HF Shear $2.25-6.75 \mathrm{~m}$

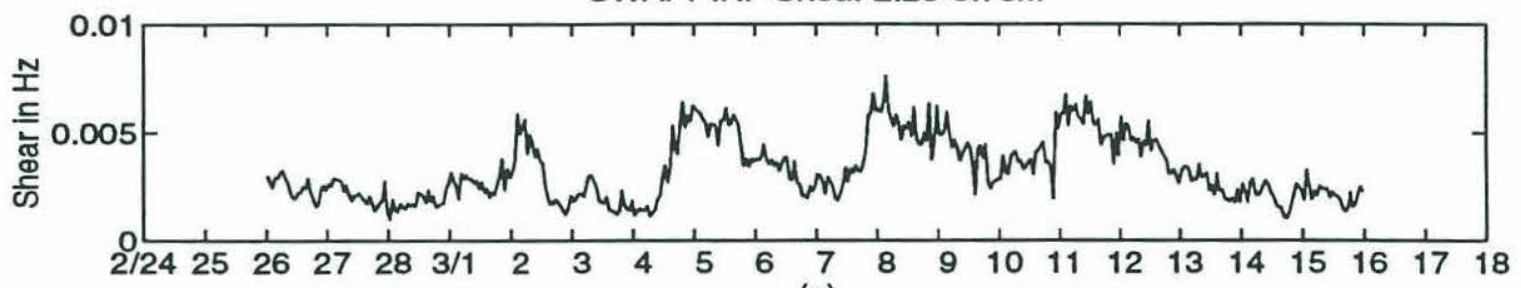

(c)

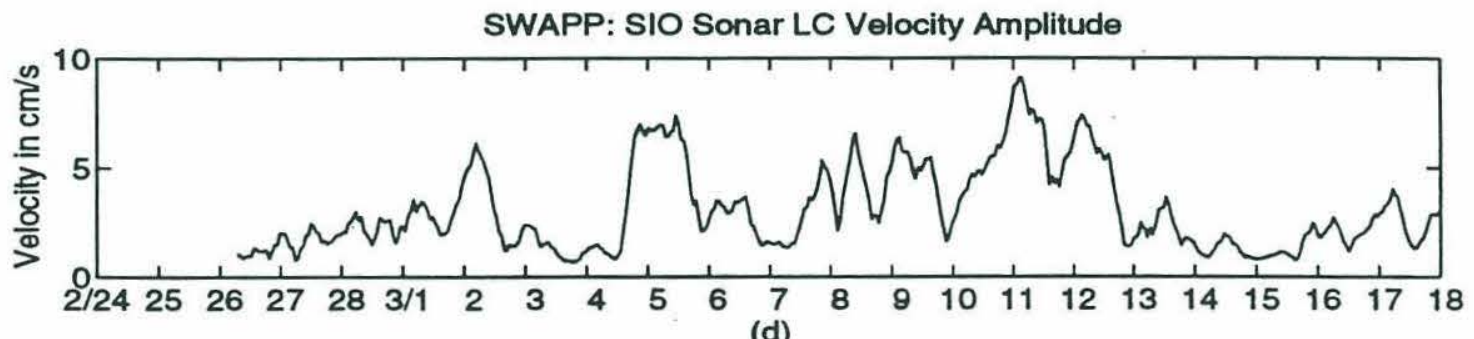

Figure 7.12: Langmuir cell strength and surface forcing. (a) Absolute magnitude of the wind stress in $\mathrm{Pa}$. (b) Significant wave height in $\mathrm{m}$. Data shown courtesy of J. Smith. (c) High-frequency (1-36 cph) shear between 2.25 and 6.75 meters in $\mathrm{s}^{-1}$. (d) Sonar LC velocity amplitude in $\mathrm{cm} / \mathrm{s}$ from SIO Doppler sonars. Data is reported in Smith (1993) and Plueddemann et al. (1994). 
and the sonar LC velocity amplitude is 0.81 . Note that the sonar velocity amplitude is also elevated on March 5th and 12th, when the wind is low but the waves are high.

To summarize, then, the high-frequency vertical shear of the horizontal current gives a measure of Langmuir circulation which is consistent with measures drawn from SIO doppler sonar measurements. Band-passing the cells to isolate a frequency band corresponding to wavelengths of order 15-200 meters does not produce substantial changes in the velocity or shear time series, indicating that the frequency band chosen corresponds to disturbances with the right order of magnitude spacing to be Langmuir cells.

\subsection{Low-Frequency Response to Surface Forcing during SWAPP}

\subsubsection{Time Series}

Having derived an index of the Langmuir cell strength, we now consider how the low-frequency response of the horizontally averaged velocity and density structure differs from standard models of mixed layer dynamics when the cells are strong. As in Chapter 6, the observed response is compared to that predicted by two models. The first of these is the PWP model of Price et al., (1986), which parameterizes the mixed layer as a slab in which mixing occurs completely and instantaneously over the whole layer. Implicit in this model is the idea of large eddies driven by shear instability which mix the entire mixed layer. The second model is a Mellor-Yamada Level 2 model which parameterizes mixing in terms of a local eddy viscosity, implicitly assuming eddies which are small in comparison to the scale of the diffusive features. (A Mellor-Yamada Level 2.5 model was used in earlier runs but did not produce significantly different results).

We begin to examine the low-frequency response by looking at the temperature difference. Figure 7.13a shows the temperature difference between the current meters at 2.25 and 11.25 meters. Figure $7.13 \mathrm{~b}$ shows time series of the temperature difference predicted by the PWP (solid) and MY2 (dashed) models. Each day that the mixed layer restratifies as a result of solar heating, the temperature difference between 2.25 and 11.25 meters exhibits a positive spike with an amplitude of a few tenths of a degree. Figure 7.13c shows two indices of Langmuir cell strength scaled so as to be on the same vertical axis. The solid lines show the high-frequency shear over 2.25-6.75 meters multiplied by 1000 and the 


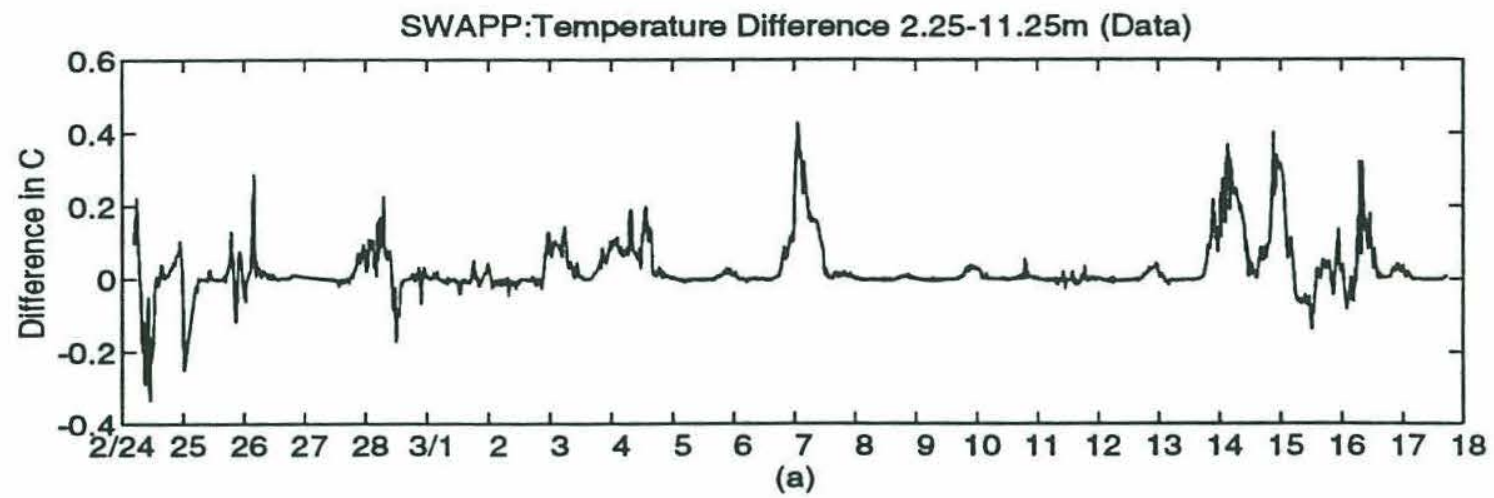

SWAPP:Temperature Difference 2.25-11.25m (Solid:PWP Model, Dashed:MY2 Model)

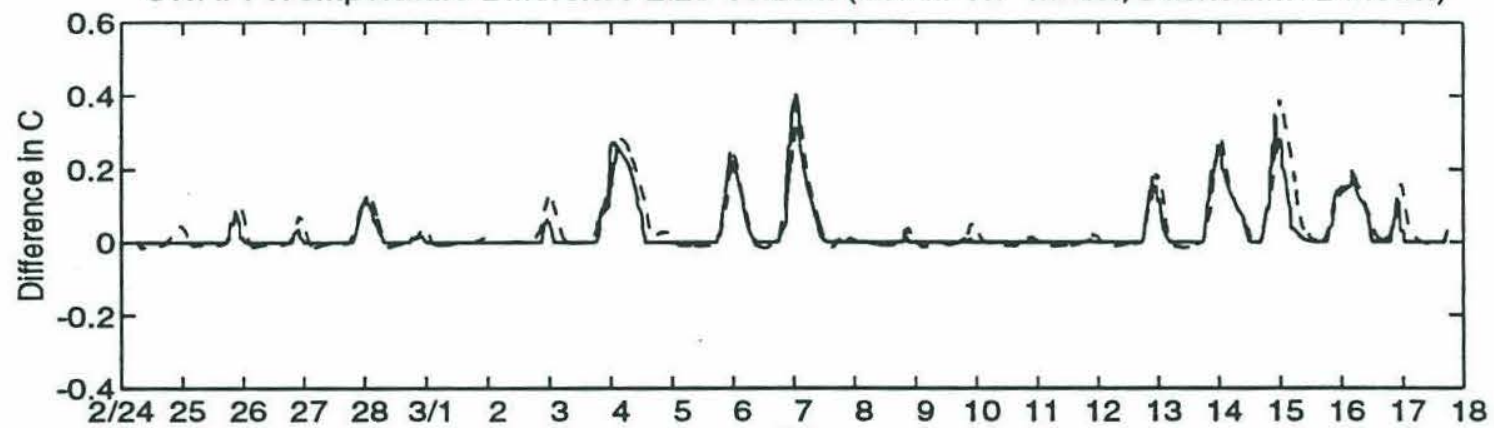

(b)

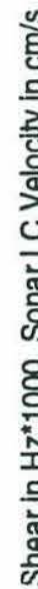

10

SWAPP:Two Indices of Langmuir Cell Strength

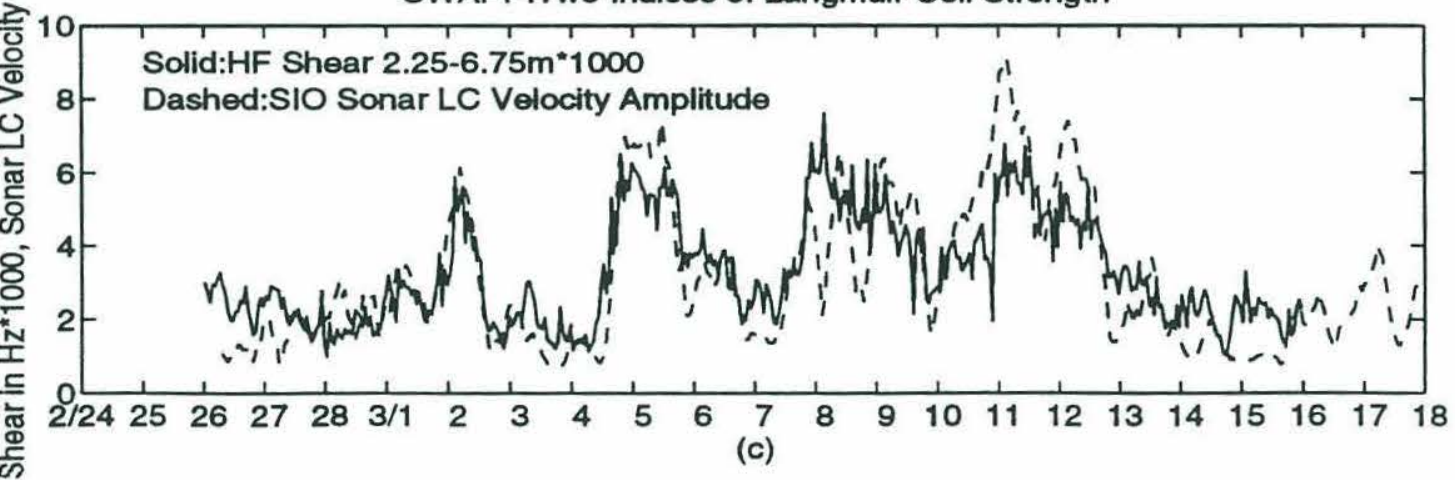

Figure 7.13: Linking diurnal restratification to Langmuir cell strength. (a) Temperature difference between 2.25 and $11.25 \mathrm{~m}$ from data. (b) Temperature difference between 2.25 and $11.25 \mathrm{~m}$ from models. Solid: PWP. Dashed: MY2. (c) Two indices of cell strength. Solid: HF shear amplitude for current meters at 2.25 and $6.75 \mathrm{~m} \mathrm{in} \mathrm{s}^{-1}$ multiplied by 1000 . Dashed: Sonar LC velocity amplitude. 
dashed lines the SIO sonar LC velocity amplitude. One of the most striking features of the data is the failure of the mixed layer to restratify on March 5th and 12th. Both the PWP and MY2 models predict such restratification, but the data shows a layer which is well mixed down to 11.25 meters. As can be seen by comparing with Figure 7.13c, the failure to restratify is linked to the presence of Langmuir cells. On both March 5th and 12th, the sonar velocity index and highfrequency shear are elevated. With the exception of March 5th and 12th, both models reproduce the observed temperature stratification to first order, capturing the magnitude of the observed restratification to first order.

Even when they disagree with the data, the PWP and MY2 models show similar temperature structures. This is not the case for velocity. Figure 7.14 shows the alongwind velocity difference between $2.25 \mathrm{~m}$ and $11.25 \mathrm{~m}$ Figure $7.14 \mathrm{a}$ and $\mathrm{b}$ show the observed alongwind velocity as solid lines, with the alongwind velocity difference predicted by the PWP model overlaid as the dashed line in Figure 7.14a and that for the MY2 model in Figure 7.14b. Figures 7.15a and b repeat 7.14a and $\mathrm{b}$ for the crosswind velocity. The MY2 model agrees very well with the data early on (February 24-26th) and late (March 14-17th) in the experiment, when diurnal restratification is strong and Langmuir cells are weak. On March 1-2nd, 4-5th, and 7th-13th when the winds and Langmuir cells are strong, however, the velocity differences predicted by the MY2 model are much larger than observed.

\subsubsection{The Ekman Response during SWAPP}

Chapter 6 , showed that by rotating the velocity into a coordinate system referenced to the wind and averaging over time the low-frequency Ekman response could be recovered. In this section the same methodology is applied to the SWAPP dataset. As noted earlier, in order to recover the wind-driven response, it is necessary to reference the velocities to some depth so as to eliminate signals associated with fronts and eddies. Using methods identical to those used in Chapter 6, a reference depth of 70 meters was chosen. This depth is right at the top of the main thermocline, so that choosing it as a reference level reduces the effect from fronts within the thermocline. As noted in Section 7.2, frontal structures above the main thermocline depth are not associated with strong shears, since the temperature and salinity signals compensate each other to a large extent.

We begin by considering the dataset as a whole. Figure 7.16 shows the velocity relative to $70 \mathrm{~m}$ (left-hand column) and the shear response (right-hand 

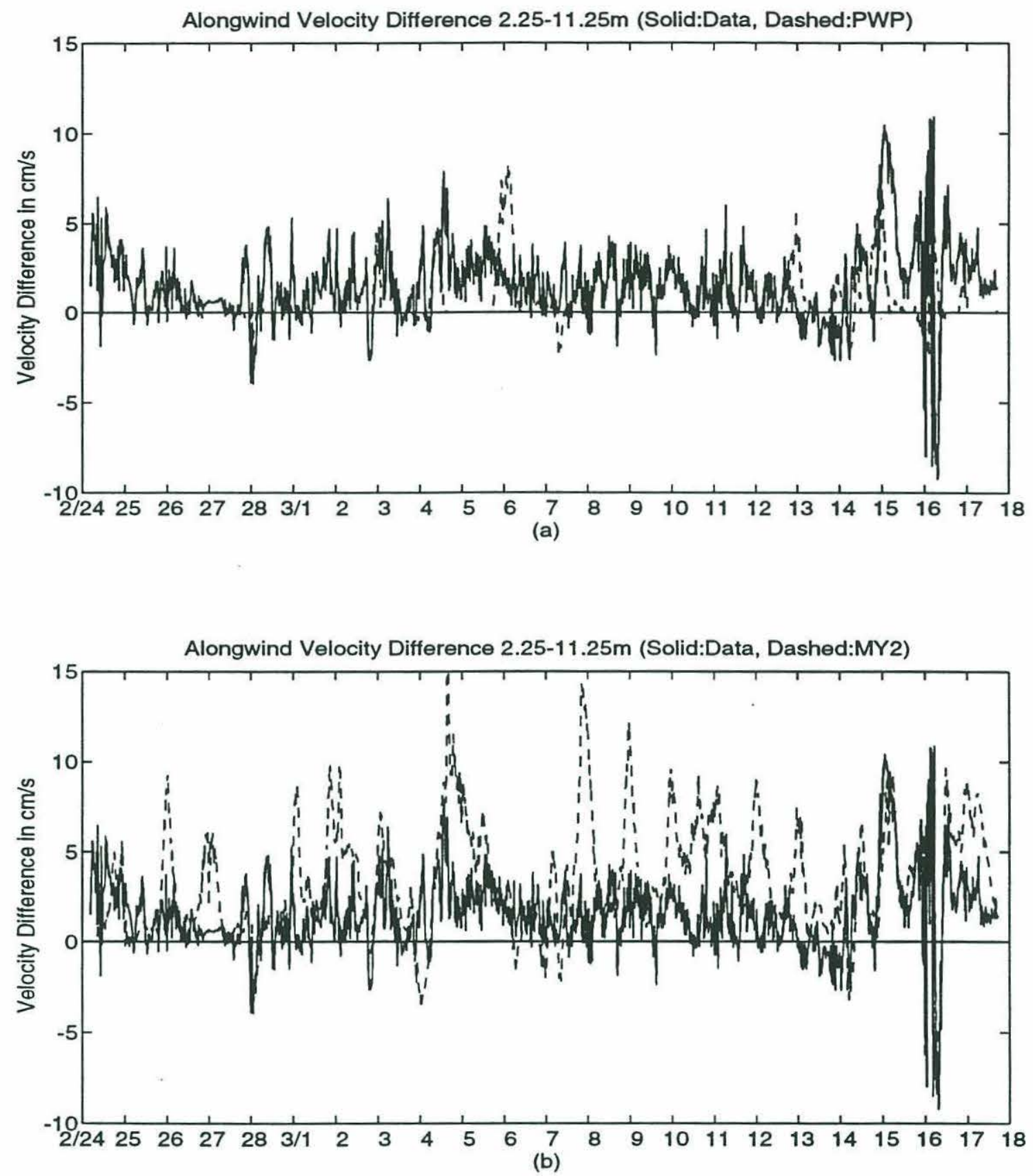

Figure 7.14: Velocity structure during SWAPP. (a) Mean alongwind velocity difference $2.25-11.25 \mathrm{~m}$. Solid line is data, dashed is PWP model. (b) Mean alongwind velocity difference $2.25-11.25 \mathrm{~m}$. Solid line is data, dashed is MY2 model. 

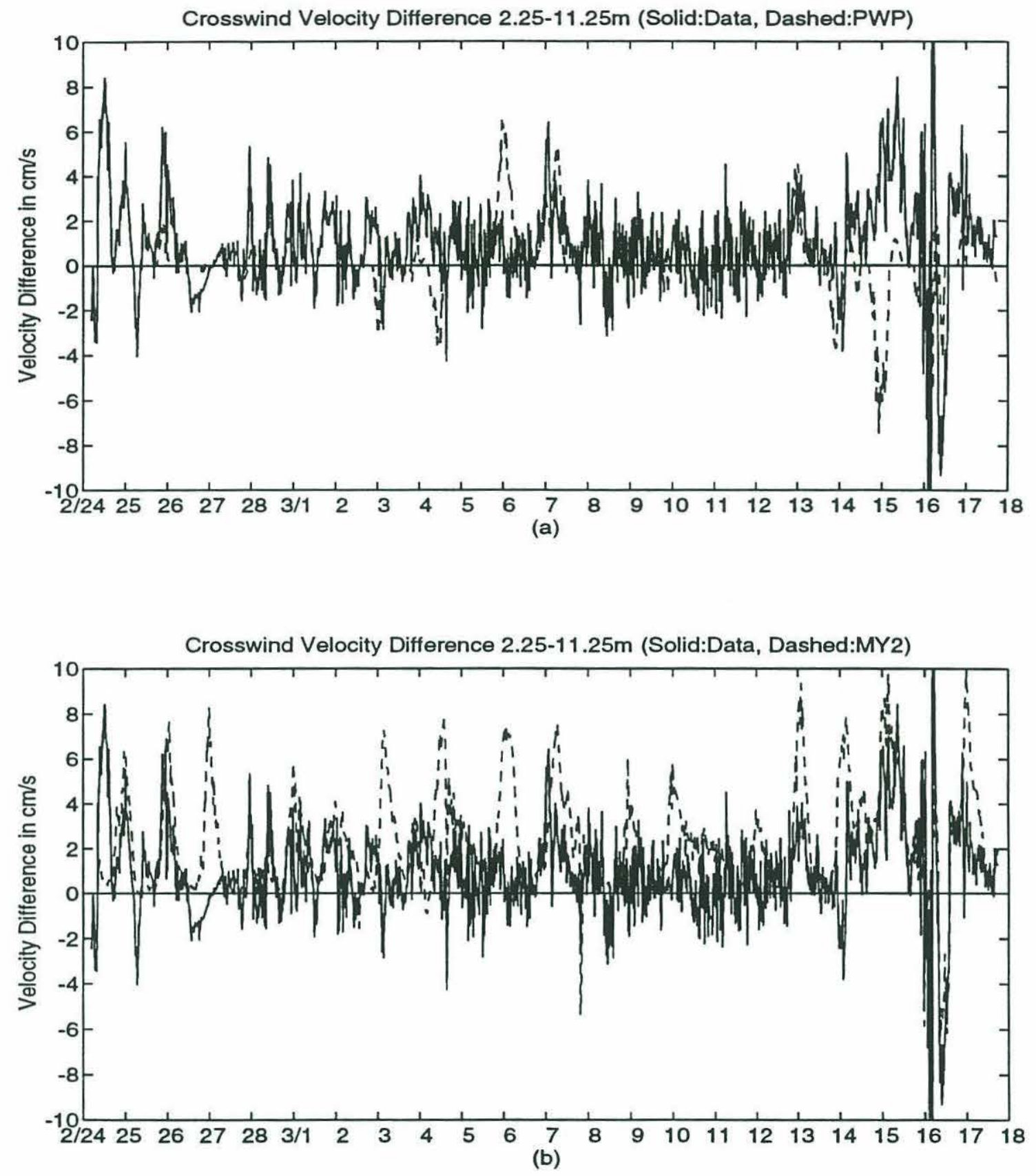

Figure 7.15: Velocity structure during SWAPP. (a) Mean crosswind velocity difference $2.25-11.25 \mathrm{~m}$. Solid line is data, dashed is PWP model. (b) Mean crosswind velocity difference $2.25-11.25 \mathrm{~m}$. Solid line is data, dashed is MY2 model. 
column). The top row shows the response calculated from data, the middle row the response from the PWP model and the bottom row the response calculated from the MY2 model. Each stick corresponds to a depth at which current meter measurements are made, and so the measurements are closely spaced near the surface, and widely spaced at depth.

There are clear differences between the observed and modelled responses. As was the case during MILDEX, the MY2 model predicts a velocity response which looks like an Ekman spiral, relatively closely trapped to the surface, with most transport occuring above 25 meters depth. The top 10 meters are quite strongly sheared. The PWP model, on the other hand, predicts a flat spiral, with small shears (of order $10^{-3} \mathrm{~s}^{-1}$ ) throughout the top 50 meters. The data lies somewhere in between. The deep velocities are closer to that predicted by the PWP model than the MY2 model. There is considerably more shear within the upper 20 meters than predicted by the PWP model, but much less than predicted by the MY2 model.

The difference between the models is not primarily the result of different responses on days when the mixed layer restratifies. This may be seen by looking at a time period when Langmuir cells were strong. Figure 7.17 parallels 7.16 for a time period from $0000 \mathrm{Z}$ on March 4th until $1715 \mathrm{Z}$ on March 12th. During this time period, Langmuir cells were strong on all but one day (March 6th) which was also the only day on which the mixed layer restratified. The response is extremely similar to that derived for the experiment as a whole. The observed velocity structure looks like a combination of the PWP and Mellor-Yamada models. Again, there is shear near the surface in both the data and Mellor-Yamada model, but very little in the PWP model. The near-surface shear is only about half as large in the data as predicted by the MY2 model. At depths below about 10 meters, the shear seen in the data is far smaller and less clearly downwind than that predicted by the MY2 model. Comparing these results to the time series in Figure 7.8 results in a similar picture, in which the shear near the surface is essentially downwind and large, but at deeper levels the mean shear is smaller and its signal is essentially drowned out by strong time-varying shears.

It is more difficult to interpret these results than those during MILDEX. During MILDEX, the structure of the near-surface velocity response was far more similar to that given by the PWP model, than the MY2 model. The lack of shear in the mixed layer interior was used to argue that the mixed layer during MILDEX was mixed on time scales fast compared with an Ekman period, presumably by 

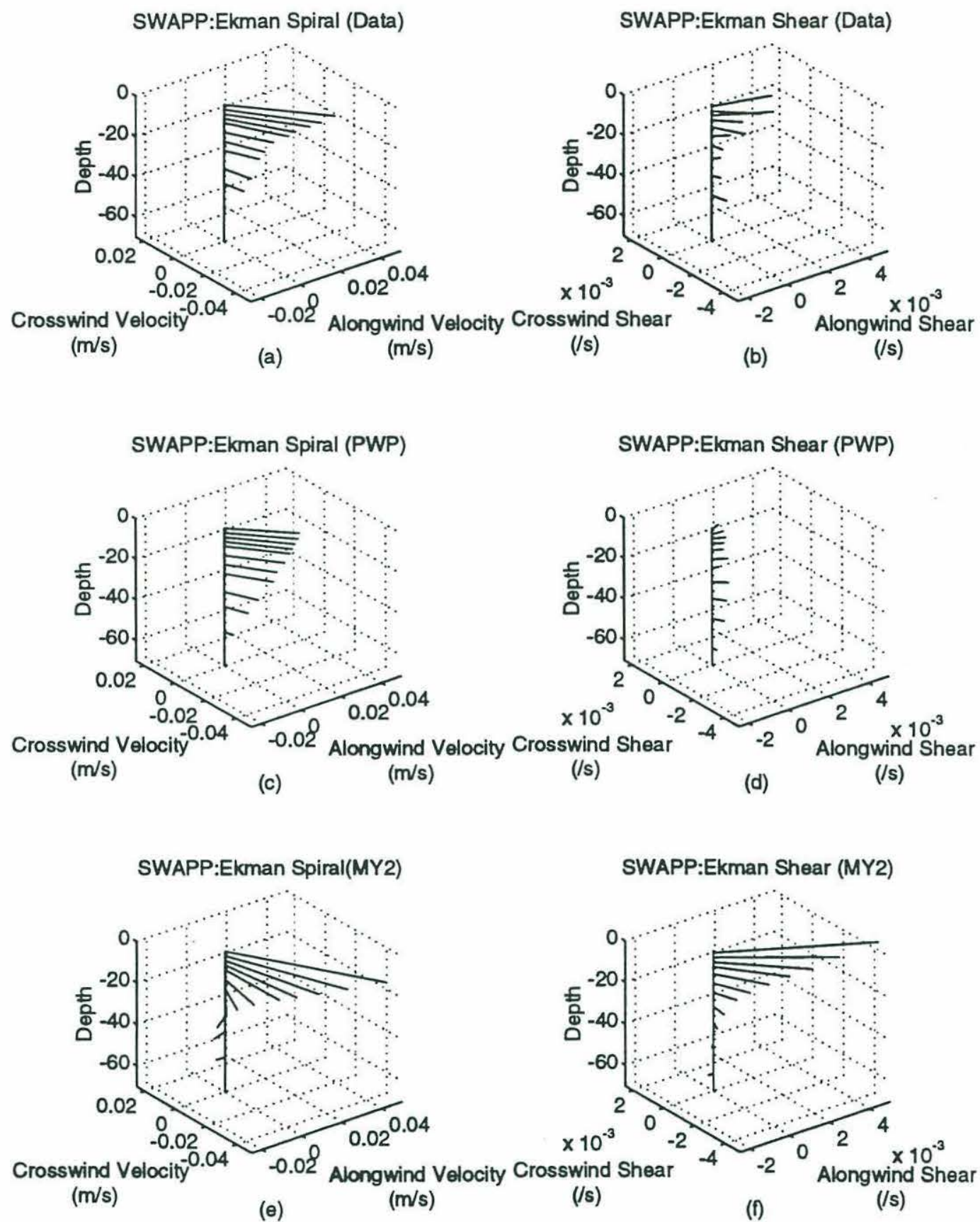

Figure 7.16: Ekman response for SWAPP for models and data. Velocities are relative to $70 \mathrm{~m}$ in $\mathrm{ms}^{-1}$, shear in $\mathrm{s}^{-1}$, depth in $\mathrm{m}$. Velocity vectors are shown at $2.25,4.5,6.75,9.0,11.25,15.75,20.25,29.0,41.0,50.0$, and $70.0 \mathrm{~m}$. Shear vectors are shown at depths halfway between current meter depths. (a) Ekman spiral for data. (b) Shear relative to the wind in /s for data. (c) Ekman spiral for PWP model (d) Shear relative to the wind in /s for PWP. (e) Ekman spiral for MY2 model. (f) Shear relative to the wind in /s for MY2 model. 


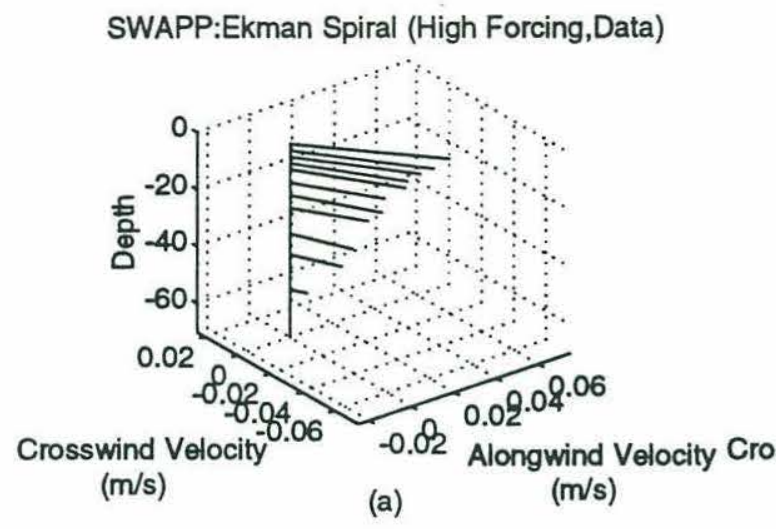

SWAPP:Ekman Shear (High Forcing,Data)
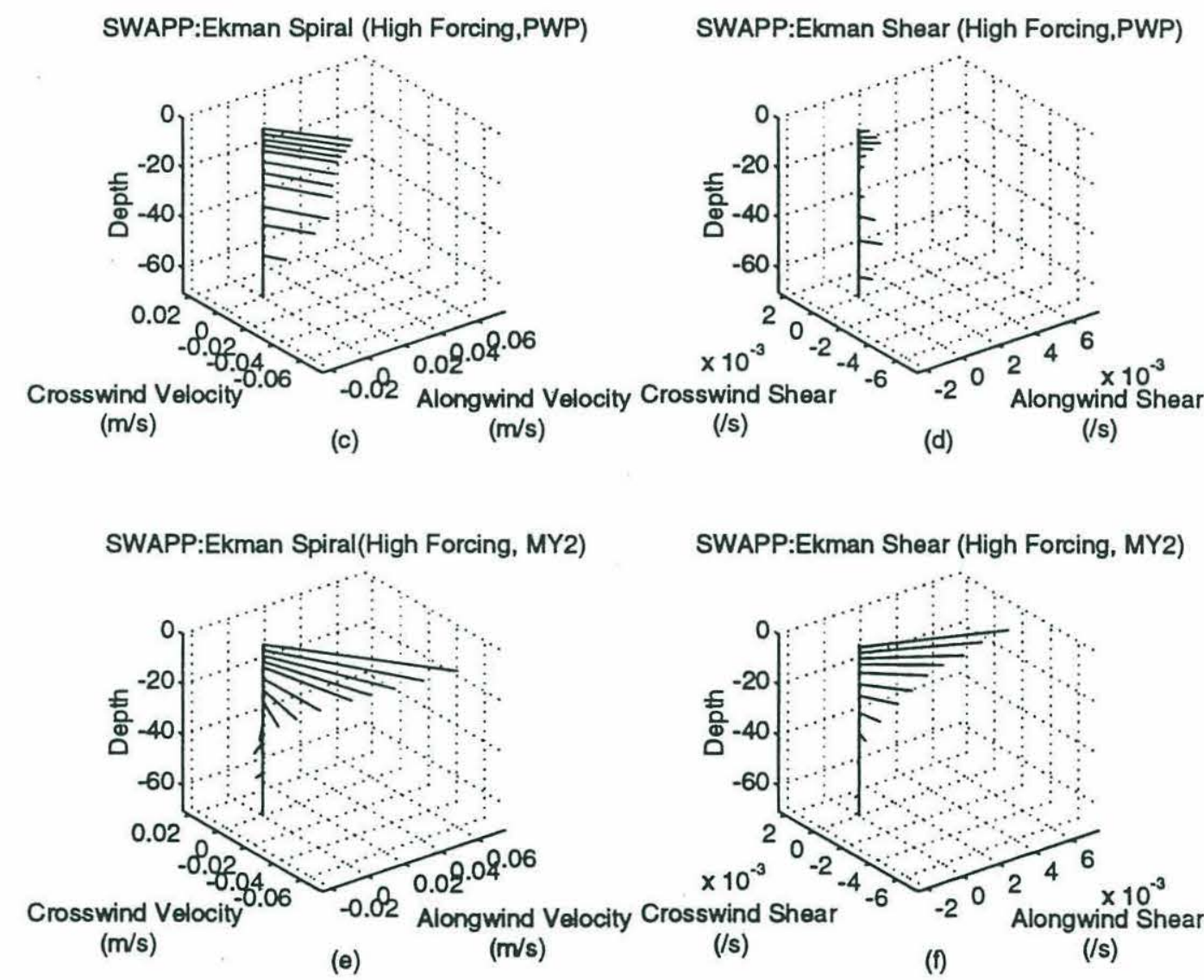

Figure 7.17: Ekman response for SWAPP high-forcing period (0000Z March 4th$1715 \mathrm{Z}$ March 12th) for models and data. Velocities are relative to $70 \mathrm{~m} \mathrm{in} \mathrm{ms}^{-1}$, shear in $\mathrm{s}^{-1}$, depth in $\mathrm{m}$. Velocity vectors are shown at $2.25,4.5,6.75,9.0,11.25$, $15.75,20.25,29.0,41.0,50.0$, and $70.0 \mathrm{~m}$. Shear vectors are shown at depths halfway between current meter depths. (a) Ekman spiral for data. (b) Shear relative to the wind in /s for data. (c) Ekman spiral for PWP model (d) Shear relative to the wind in /s for PWP. (e) Ekman spiral for MY2 model. (f) Shear relative to the wind in /s for MY2 model. 
large eddies. During SWAPP, the picture which emerges is more ambiguous. There is evidence for large-scale eddies which mix the surface layer quickly, homogenizing the mixed layer below the surface and thus reducing the shear relative to the Mellor-Yamada model. However, there is also evidence for a nearsurface layer which is strongly sheared. In this near-surface shear layer small, slowly mixing eddies would also play a role in the momentum transport. A similar sheared surface layer was seen in the finite-difference code runs in Chapter 5.

The transport associated with all these current spirals is in close agreement and off to the right of the wind. Once again there is no sign of the upwind transport expected as a result of the Eulerian return flow balancing the Stokes drift. This issue is discussed in more detail in Chapter 8.

\subsubsection{Summary of Observational Results}

The experimental results are summarized as follows:

-The existence of Langmuir circulations is demonstrated by the presence of coherent backscatter structures in sidescan and upward-looking sonars. The presence of these structures is correlated with spatially coherent, temporally persistent velocity signals in the sonars. These in turn are linked to the presence of high-frequency (1-36 cph) signals in the velocity and shear seen by VMCMs. -On two occasions, the mixed layer fails to restratify immediately after a wind event when the waves were high but the wind stress was small. This enhanced mixing is not seen in either the PWP or Mellor-Yamada models.

-Within the mixed layer there is persistent low-frequency shear which is smaller than that predicted by the Mellor-Yamada model, and larger than that predicted by the PWP model.

The remainder of this chapter concentrates on explaining the long-time variability of the high-frequency shear, the failure of the mixed layer to restratify when the surface forcing was weak but the waves were high, and the breakdown of the MY2 model. The last question is also studied using a finite-difference code in the next chapter.

\subsection{Does the theory explain temporal variability in cell strength?}

There are a number of possible physical interpretations for the long-time variability in the two indices (sonar velocity amplitude and high-frequency shear) 
which were defined as being representative of Langmuir cell strength in Section 7.3. Three such possibilities are listed below

1. The variability seen in the current meters and sonars is due to turbulence associated with a flat plate (see Robinson, 1991 for a review). If this were true, the mean shear $|\partial \mathrm{V} / \partial z|$ and high-frequency shear $\omega$ would scale as the friction velocity.

2. The variability is due to Kelvin-Helmholtz instability. As outlined in Chapter 6, this would imply that the level of the high-frequency shear would scale as

$$
\omega=\sqrt{\mid \partial \mathrm{V} / \partial \mathrm{zl}^{2}-\mathrm{N}^{2} \sim} \sqrt{\Delta \mathrm{V}^{2} / \Delta \mathrm{z}^{2}-\max \left(\mathrm{g} \alpha \frac{\partial \mathrm{T}}{\partial \mathrm{z}} \cdot \frac{\mathrm{g} \alpha \mathrm{Q}}{\rho c_{\mathrm{p}} \mathrm{v}_{\mathrm{e}}}\right)}
$$

where $\partial \mathrm{V} / \partial \mathrm{z}$ is the mean Eulerian shear, $\mathrm{N}$ is the buoyancy frequency, $\Delta \mathrm{V}$ is the Eulerian current difference across the top two current meters, $\Delta \mathrm{z}$ is the distance between these two current meters,g, the gravitational constant, $\alpha$ the coefficient of thermal expansion, and $v_{\mathrm{e}}$ is the eddy viscosity. The eddy viscosity may be estimated either from data (as $\tau \Delta z / \Delta \mathrm{V}$ ), or from the Mellor-Yamada model. 3. The variability is due to Craik-Leibovich instability. Based on the results in Chapter 5, it was argued in Chapter 6 that the level of the high-frequency shear would scale as

$$
\omega \sim \gamma_{C L S} \sim \sqrt{I \partial \mathrm{V} / \partial \mathrm{z} \mid \frac{\partial \mathrm{v}_{\mathrm{s}}}{\partial \mathrm{z}}-\mathrm{N}^{2} \sim} \sqrt{\Delta \mathrm{V} / \Delta \mathrm{z} \frac{\partial \mathrm{v}_{\mathrm{s}}}{\partial \mathrm{z}}-\max \left(\mathrm{g} \alpha \frac{\partial \mathrm{T}}{\partial \mathrm{z}} \cdot \frac{\mathrm{g} \alpha \mathrm{Q}}{\rho c_{\mathrm{p}} v_{\mathrm{e}}}\right)}
$$

where $\mathrm{v}_{\mathrm{s}}$ is the Stokes' drift computed from the nondirectional wave spectrum,

The scaling of the shear with the Craik-Leibovich instability parameter was shown to hold only if the equivalent of the Rayleigh number for stratified CL instability

$$
\operatorname{Ra}_{\mathrm{CLS} S}=\frac{\gamma_{\mathrm{CLS}}^{2}}{\gamma_{\text {diff }}^{2}} \sim \frac{\gamma_{\mathrm{CLS}}^{2}}{\left(2 v_{\mathrm{e}} \pi^{2} / \mathrm{D}^{2}\right)^{2}}
$$

(where $\mathrm{D}$ is a depth of penetration for the cells), was large. In order to argue that the cells are in fact driven by wave current interaction, it is necessary to show to show that RaCLS is large when the cells are strong.

The validity of the estimate of RaCLS is highly dependent on the assumption that the shear near the surface is proportional to the ratio of the wind stress over the eddy viscosity. We can check that the estimate of the viscosity is reasonable by comparing it to that predicted by the MY2 model. This is done in Figure 7.18a. There is a general qualitative agreement between the two estimates of viscosity, the difference is generally only about a factor of 2-4 while the viscosity itself varies by several orders of magnitude. The correlation between the two curves is 

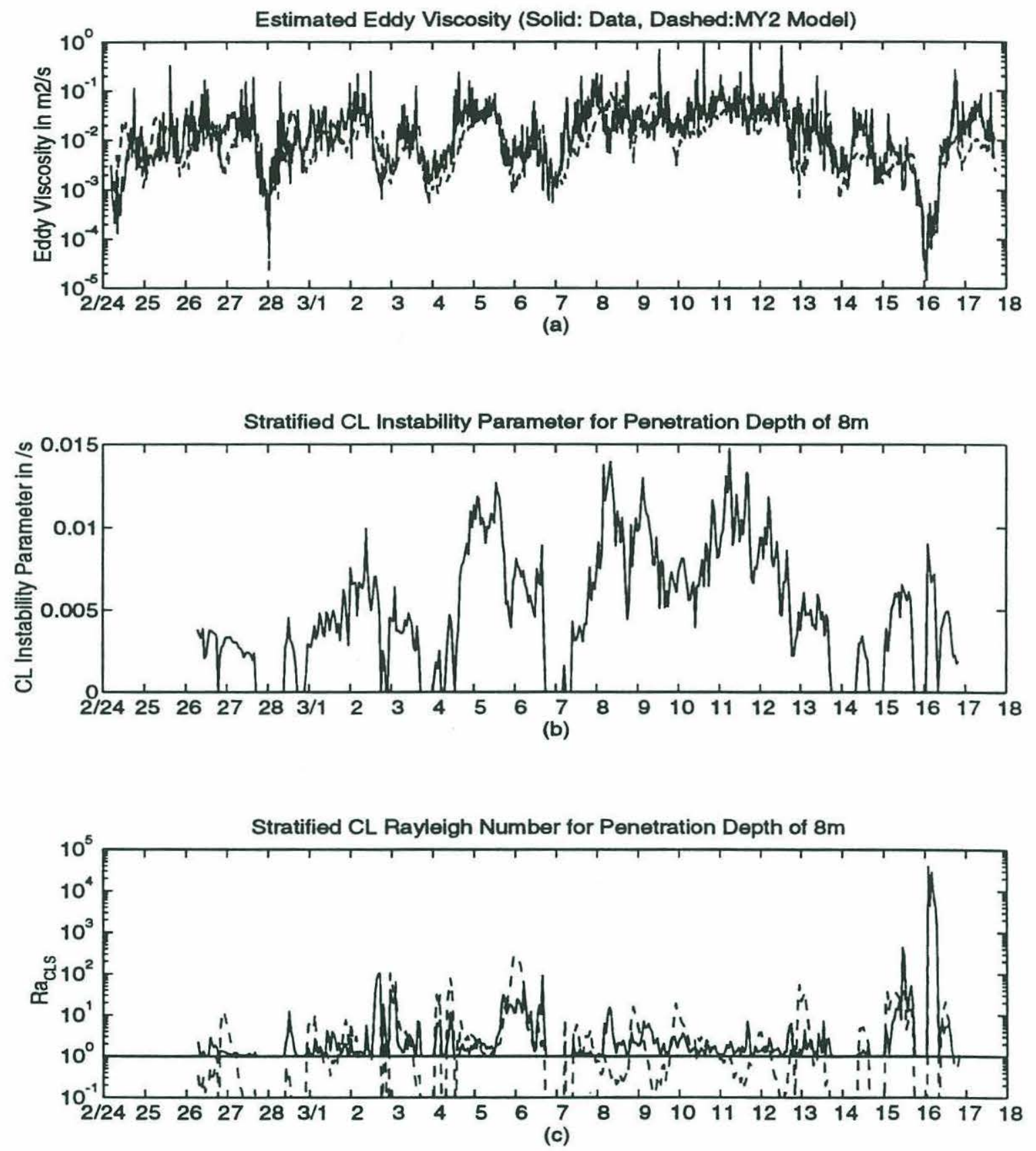

Figure 7.18: Estimate of the supercriticality of Langmuir cells during SWAPP. (a) Eddy viscosity estimated from data (solid) and MY2 model (dashed). (b) Stratified CL instability parameter from equation (7-3). (c) RacLS from equation (7-4) using eddy viscosity estimated from data (solid) and MY2 model (dashed). 
0.72 . The viscosity predicted by the MY2 model is generally smaller than that predicted by the data (no surprise considering that the MY2 model overpredicts the near-surface shear). This means that estimating RaCLs from data may result in an underestimate. Thus the theory may predict the non-existence of cells when they are actually present, but it is unlikely to predict that cells exist when they do not. Taking the Stokes drift shear and Eulerian shear across 2.25 and $4.5 \mathrm{~m}$, one can show, in a method parallel to that used in Chapters 2 and 5, that this corresponds to the appropriate stratified Craik-Leibovich instability parameter for a depth of approximately $8 \mathrm{~m}$. Figure $7.18 \mathrm{~b}$ shows the stratified Craik-Leibovich instability parameter and 7.18c RaCLS. With only a few exceptions, RaCLS is greater than 1 for most of the experiment. As shown in Chapter 5, this indicates that the highfrequency shear ought to scale as $\gamma_{\text {CLS }}$ and that the cells are involved in momentum and density transport within the mixed layer.

Given that RaCLS is large, the high-frequency shear should scale as $\gamma_{C L S}$. This is in fact the case. Figure 7.19 shows time series of the unstratified CraikLeibovich instability parameter, the stratified Craik-Leibovich instability parameter, the friction velocity, and the Kelvin-Helmholtz instability parameter compared with the observed band-passed shear. The variability of the band-passed shear is well captured by both the stratified Craik Leibovich instability and by the friction velocity. There are some differences. On days when restratification does occur the sonar velocity amplitude and high-frequency shear are still nonzero despite the fact that the stratified Craik-Leibovich instability parameter is zero. On February 28th, for example, during a time period when the upper portion of the water column is stratified, the high frequency shear is $0.0015 \mathrm{~s}^{-1}$, and the sonar velocity amplitude is $2.8 \mathrm{~cm} / \mathrm{s}$. This gives an estimate of the "noise floor" inherent in each of these measurements of cell strength. On March 7th and 8th at the start of wind event 3 , the Craik-Leibovich instability parameter rises more slowly than the friction velocity, sonar velocity amplitude, and high-frequency shear. This may be because the cells initially mix away much of the near-surface shear, thus leading to an underestimate of the CL instability parameter. In general, however, the agreement between the Langmuir cell strength and the stratified CraikLeibovich instability parameter is still good. A more quantitative comparison of cell strength and forcing functions is given below. 


LC Strength (Solid:HF Shear Dashed:Sonar LC Velocity Amplitude/1000)
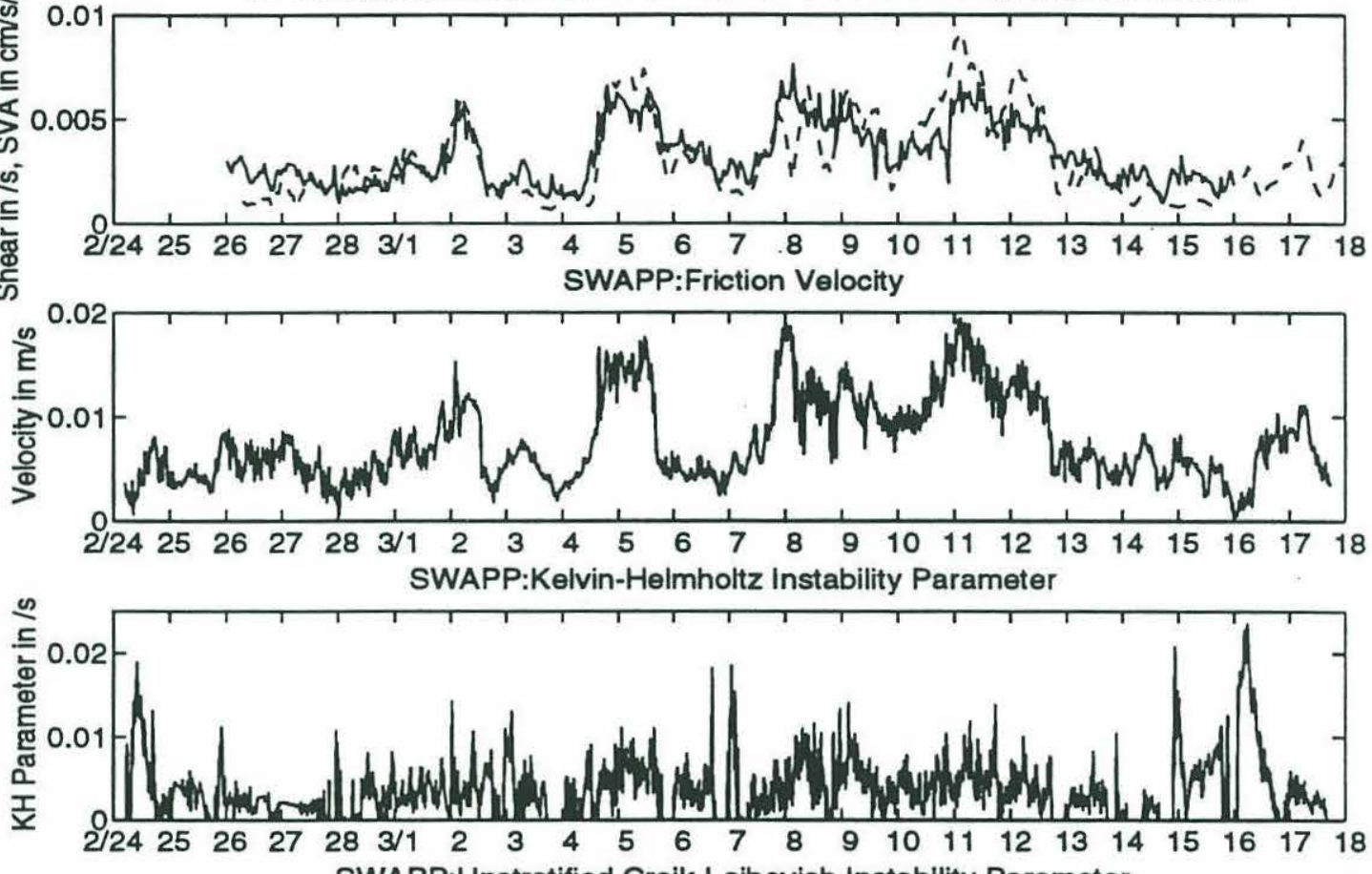

SWAPP:Unstratified Craik-Leibovich Instability Parameter
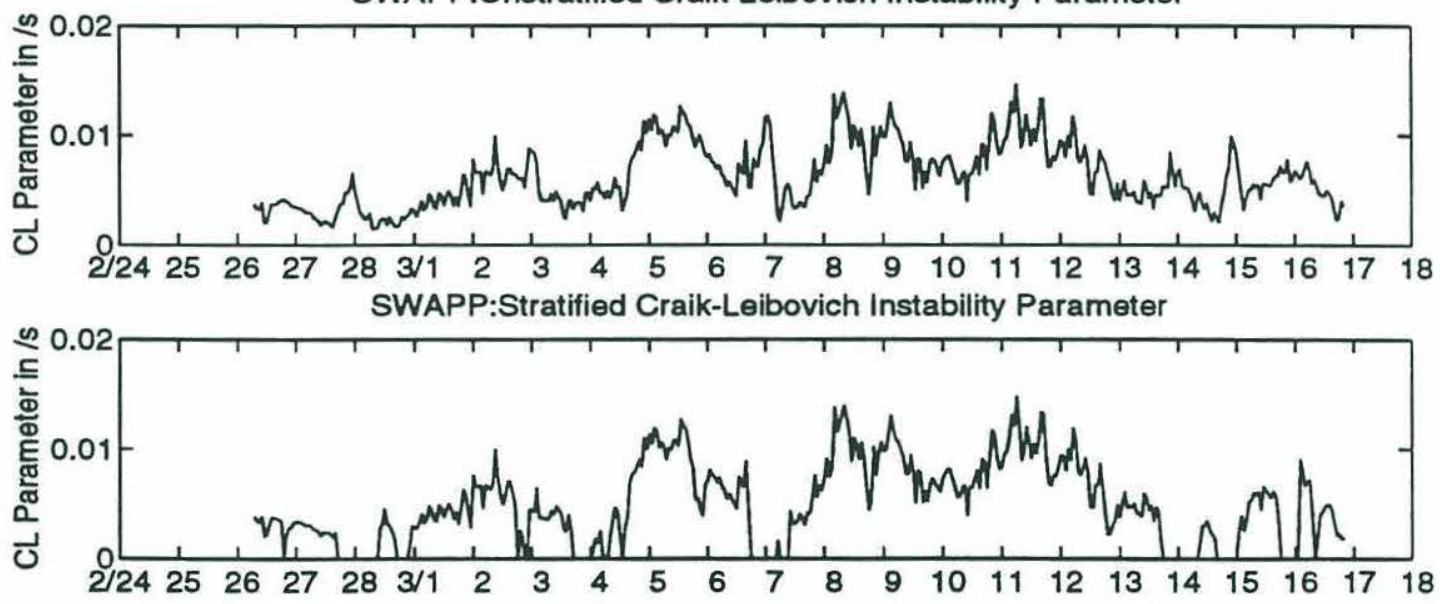

Figure 7.19: Forcing functions for Langmuir cells. (a) Two indices of cell strength. Solid is total HF shear between 2.25 and $6.75 \mathrm{~m}$, dashed is sonar velocity amplitude/1000. (b) Friction velocity in $\mathrm{m} / \mathrm{s}$. (c) $\gamma_{\mathrm{KH}}:$ Kelvin-Helmoholtz instability parameter from equation (7-2). (d) $\gamma_{C L}$ : Unstratified Craik-Leibovich instability parameter from equation (7-3) (assuming $\mathrm{N}^{2}=0$ ). (e) $\gamma_{C L S}$ : Stratified CraikLeibovich instability parameter from equation (7-3). 


\begin{tabular}{|l|l|l|}
\hline & $\begin{array}{l}\text { Sonar LC Velocity } \\
\text { Amplitude }\end{array}$ & Band-Passed Shear \\
\hline Absolute Shear 2.25-4.5m & 0.05 & 0.05 \\
\hline$\gamma_{\mathrm{KH}}$ & 0.42 & 0.43 \\
\hline Significant Wave Height & 0.65 & 0.74 \\
\hline$\gamma_{\mathrm{CL}}$ & 0.67 & 0.72 \\
\hline $2.25 \mathrm{~m}$ Stokes Drift & 0.74 & 0.84 \\
\hline$\gamma_{\mathrm{CLS}}$ & 0.80 & 0.82 \\
\hline Friction Velocity & 0.85 & 0.82 \\
\hline Stokes Drift Shear 2.25-4.5m & 0.83 & 0.88 \\
\hline
\end{tabular}

Table 7.1: Correlation between various forcing functions and two measures of Langmuir cell strength. Note that the correlation between the two measures of cell strength is 0.81 .

Table 7.1 shows the correlation coefficients between the cell strength (as given by sonar LC velocity amplitude and band-passed shear) and various quantities. It should be recalled that the correlation between these independent measurements of cell strength is 0.81 , which serves as a rough upper bound for how well any theoretical index of cell strength could be expected to agree with an observational index of cell strength. The correlation is highest for the stratified Craik-Leibovich instability parameter, Stokes drift shear and friction velocity. As predictors of cell strength these three quantities are essentially indistinguishable. The indices of cell strength are not well correlated with the absolute value of the Eulerian shear. The Kelvin-Helmholtz instability parameter is somewhat better correlated with the cells strength than is the raw shear, but is still significantly less well correlated than the friction velocity and Craik-Leibovich instability parameter.

The results support the physical interpretation that the cell strength as measured by sonars and current meters is the result of the Craik-Leibovich instability mechanism. Although the correlation between the cell strength and friction velocity is high, the physical mechanism which yields such a scaling also implies that the mean shear should be logarithmic (and as such should scale as the friction velocity). This was not found to be the case in the data. The natural scaling for Kelvin-Helmholtz instability was also found not to give a high correlation with 
the cell strength. The fact that the Stokes drift shear correlates highly with the Langmuir cell strength explains in large part the fact that the Craik-Leibovich instability parameter does a much better job at explaining the observed variability than the Kelvin-Helmholtz instability parameter. Apart from the Craik-Leibovich theory, there is no independent mechanism which would explain the high levels of correlation. These facts support the hypothesis that the cells are driven by the Craik-Leibovich instability mechanism.

\subsection{Instability of the Predicted Current Spiral to Langmuir Circulation:}

\subsubsection{Instability of the Mean Structure}

The failure of the MY2 model to capture the observed shear structure during SWAPP, while not as spectacular as that during MILDEX, is still striking. One possible explanation is that the MY2 model systematically underestimates the turbulent production, and thus the eddy viscosity, near the surface at times when the mixed layer is unstratified. A second possibility is that the current spiral predicted by the model is unstable to Langmuir cells as a result of the CL wavecurrent interaction mechanism. Chapter 6 demonstrated that the mean profile predicted by the MY2 model during MILDEX was strongly unstable to Langmuir cells. This analysis is now repeated, demonstrating that the same conclusion holds during SWAPP.

We will focus on a time period referred to in Section 7.4 as the "highforcing period", comprising 10 inertial periods from $0000 \mathrm{Z}$ on March 4th until $1715 \mathrm{Z}$ on March 13th. During this time period Langmuir cells were strong on all but one day (March 6th) and the top $11.25 \mathrm{~m}$ of the water column remained unstratified on all but that same day. Figure 7.20a shows the mean Stokes drift during this time period, and demonstrates that it may be well-approximated by a Pierson-Moskowitz spectrum with a peak period of $11.2 \mathrm{~s}$ and an rms wave amplitude of $1.4 \mathrm{~m}$ (corresponding to a mean significant wave height of $4 \mathrm{~m}$ ). Figure 7.20b shows the mean Ekman spiral predicted by the MY2 model during this time period (solid), compared to that predicted by taking the mean MY2predicted eddy viscosity of $235 \mathrm{~cm}^{2} / \mathrm{s}$ (dashed). The fact that the two spirals agree relatively well means that this mean viscosity can be used to calculate the Langmuir number $\mathrm{La}=\mathrm{ve}_{\mathrm{e}} / \mathrm{a}^{2} \sigma=0.021$ where $\mathrm{a}$ is the rms wave amplitude and $\sigma$ the wave frequency. The scaled Coriolis frequency is 0.0736 . The mean nondimensional shear is 4.4 . 


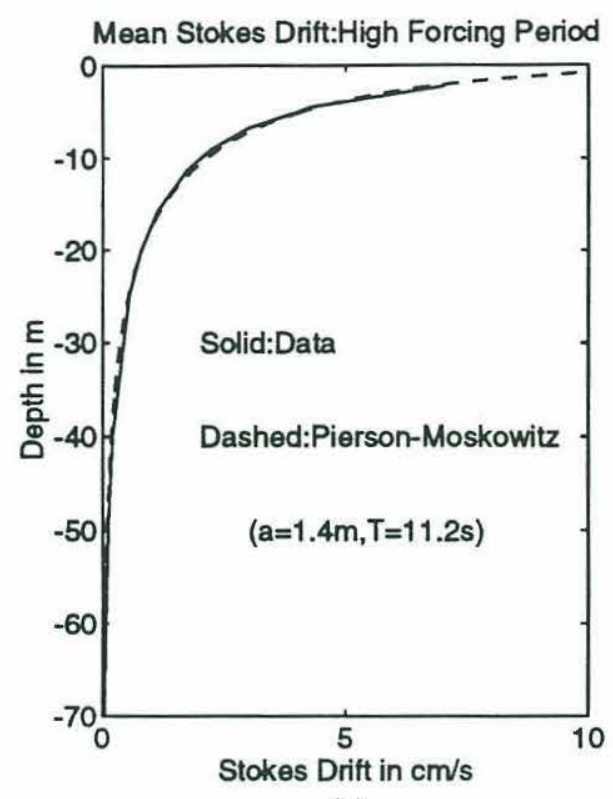

(a)

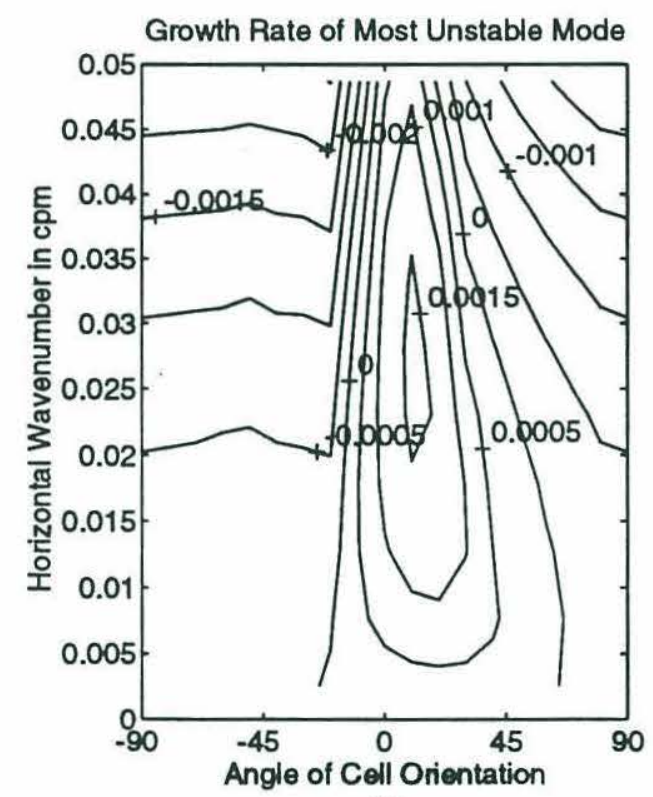

(c)

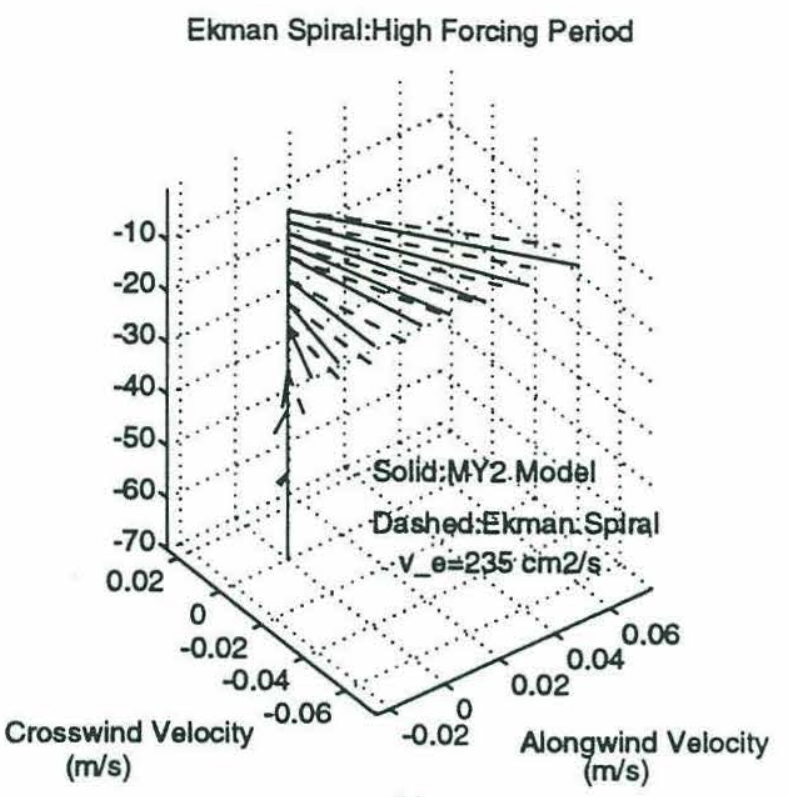

(b)

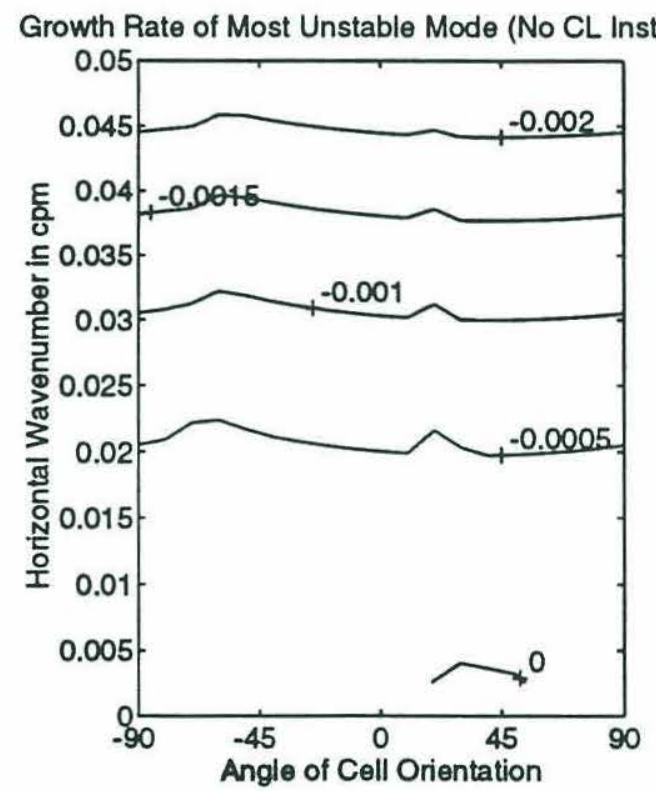

(d)

Figure 7.20: Instability of the Mean Ekman Spiral predicted by the MY2 model during high-forcing period (0000Z March 4th-1715Z March 13th). (a) Mean Stokes drift. Solid:Data. Dashed:Stokes drift for Pierson-Moskowitz spectrum with peak period of $11.2 \mathrm{sec}$ and rms amplitude of $1.4 \mathrm{~m}$. (b) Mean current spiral. Solid:predicted from MY2 model. Dashed: Ekman spiral given mean eddy viscosity predicted from MY2 model and mean wind stress. (c) Contours of growth rate of the most unstable two-dimensional mode in $\mathrm{s}^{-1}$ as a function of angle of cell orientation relative to the wind and waves and horizontal wavenumber in cpm. (d) Contours of growth rate of the most unstable twodimensional mode with no CL instability present. 
The instability code introduced in Chapter 3 and documented in Appendix $B$ is used to calculate the growth or decay rate of the fastest growing (or slowest decaying) two-dimensional mode at a given wavenumber and angle of cell orientation. By calling a mode two-dimensional we mean that there is a direction along which its structure does not vary, thus distinguishing it from the threedimensional mixing events associated with wall layers. Figure 7.20c shows the growth rate of the most unstable mode when waves are included, so that the mean shear is modified as indicated in Chapter 3 and the Craik-Leibovich instability mechanism is operative. The angle of cell orientation relative to the wind is shown along the horizontal axis and the crosscell wavelength in cpm is shown along the vertical axis. Figure 7.20d shows the growth rate of the most unstable mode when there are no waves and thus no Stokes drift.

In the presence of waves, the instability code predicts that the mean Ekman spiral predicted by the MY2 model should be unstable with growth rates exceeding $1.5 \times 10^{-3} \mathrm{~s}^{-1}$. The maximum growth rate is $1.62 \times 10^{-3} \mathrm{~s}^{-1}$, implying a time scale for the fastest growing modes of about 10 minutes. This is far shorter than the time needed to set up an Ekman spiral, implying that such a spiral would become unstable to Langmuir cells and thus would not be seen. In the absence of waves, however, the instability code predicts that all modes sampled should be stable. This result, which parallels that obtained for MILDEX in Chapter 6, offers an explanation for why the MY2 model fails to predict the shear during both experiments.

\subsubsection{Three Case Studies}

In section 7.5 it was shown that the Craik-Leibovich instability parameter usually tracked the friction velocity during SWAPP. We now turn to a time period, March 5th, when this was not the case. Section 7.3 showed that during this time period the mixed layer failed to restratify, even though such restratification was predicted by both the PWP and MY2 models. The failure of the mixed layer to restratify when Langmuir cells were present offers a chance to determine the mechanism driving the cells.

Three 12-hour periods are considered, each corresponding to 1200Z-2400Z, on March 4th, 5th and 6th. During the first of these time periods the wind and cells were strong. During the second time period, the wind was weak, but the 
waves continued to be strong and the cells were still strong. During the third time period, the waves and wind were much weaker and mixed layer restratified.

The time-averaged velocity structure, heat flux, and wave conditions on each of these three days were used to estimate the parameters for driving the equations. It should be stressed that using the time-averaged structure smooths out variations in the buoyancy forcing, near-surface shear, Stokes drift, and eddy viscosity which might result in the mixed layer being stable to Langmuir cells for part of the averaging period, and unstable for the remainder of that period. The results should thus be taken as an indication of the tendency of the current profile to become unstable, not necessarily as a prediction of the growth rate at a specific time.

Table 7.2 shows the parameters used as input to the instability code for each of these three days. The mean stress was sharply different on the three days, with the largest winds on March 4th, smaller wind stresses on the 5th, and still smaller stresses on March 6th. On the other hand, the Stokes drift is twice as large on March 5th as it is on March 4th and 6th. The mean heat flux is roughly the same on all three days. The maximum growth rate for modes on March 4th and 5th is

\begin{tabular}{|c|c|c|c|}
\hline & March 4th & March 5th & March 6th \\
\hline Peak Frequency $\left(\mathrm{s}^{-1}\right)$ & 0.70 & 0.63 & 0.63 \\
\hline Wave Amplitude (m) & 0.96 & 1.60 & 1.09 \\
\hline $2.25 \mathrm{~m}$ Stokes Drift $(\mathrm{cm} / \mathrm{s})$ & 4.8 & 10.6 & 5.2 \\
\hline Mean Surface Stress $(\mathrm{Pa})$ & 0.161 & 0.086 & 0.018 \\
\hline Mean Heat Flux $\left(\mathrm{W} / \mathrm{m}^{2}\right)$ & 197 & 199 & 251 \\
\hline $\begin{array}{l}\text { Mean Eddy Viscosity ( } \mathrm{m}^{2} / \mathrm{s} \text { MY2 } \\
\text { Model) }\end{array}$ & 0.0123 & 0.0140 & 0.0073 \\
\hline Langmuir Number (La) & 0.020 & 0.009 & 0.01 \\
\hline Nondimensional Surface Shear & 8.42 & 2.32 & 1.98 \\
\hline Richardson Number (Ri) & 3.28 & 1.00 & 11.20 \\
\hline Scaled Coriolis Parameter $(\mathrm{F})$ & 0.054 & 0.033 & 0.070 \\
\hline $\begin{array}{l}\text { Maximum Growth Rate with CL } \\
\text { Instability }\left(\mathrm{s}^{-1}\right)\end{array}$ & $3.7 \times 10^{-3}$ & $3.2 \times 10^{-3}$ & $1.8 \times 10^{-4}$ \\
\hline
\end{tabular}

Table 7.2: Parameters used as input to the instability code, and the output of the instability code. 


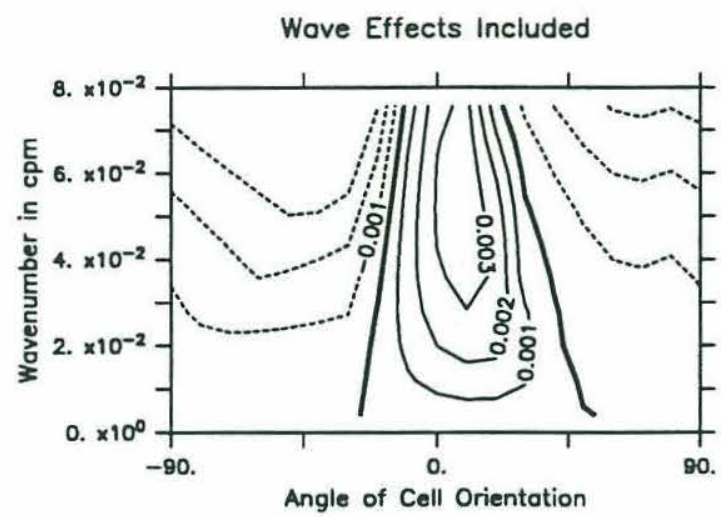

(a)

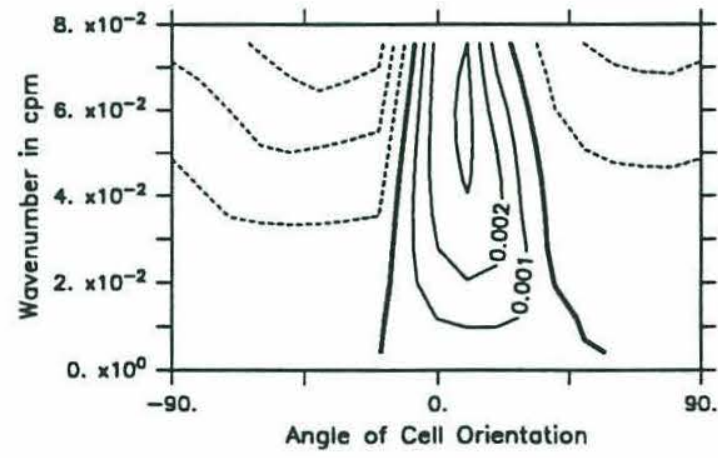

(c)

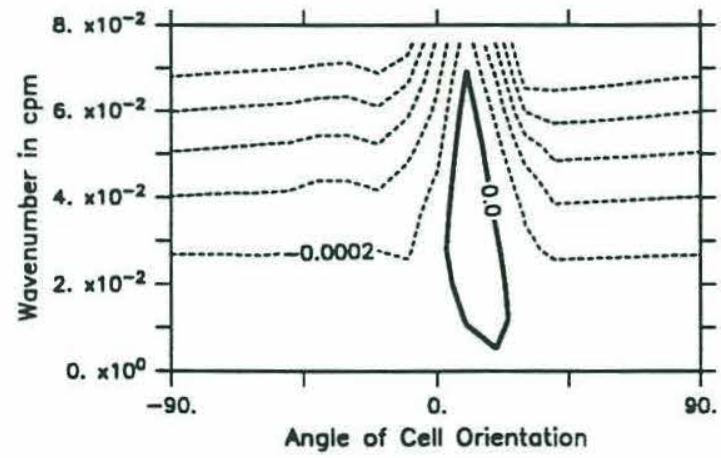

(e)
Wove Effects Not Included

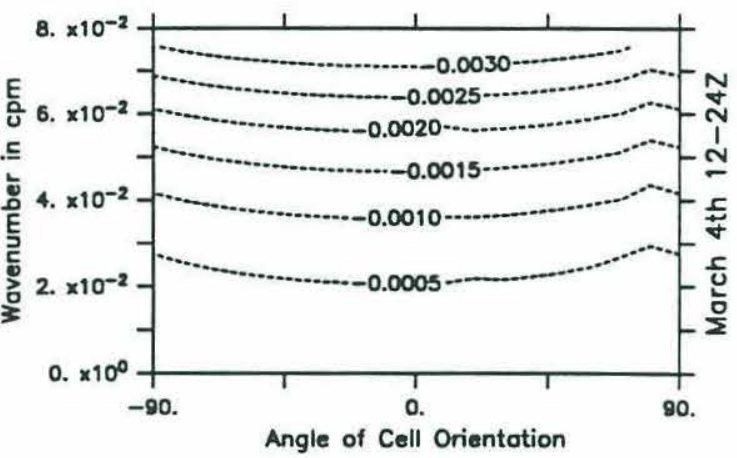

(b)

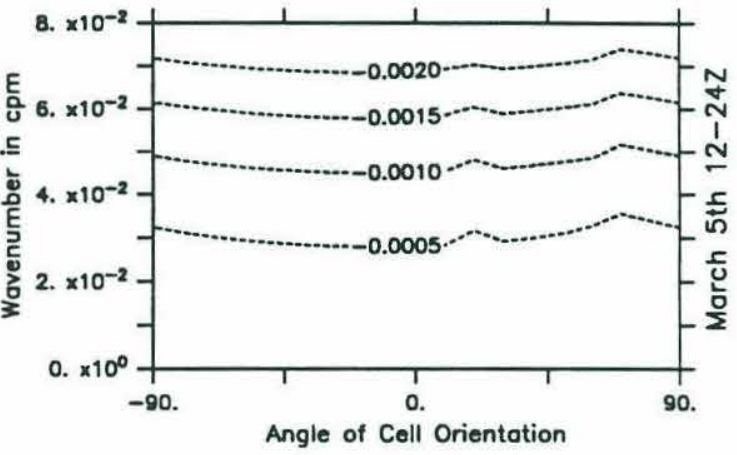

(d)

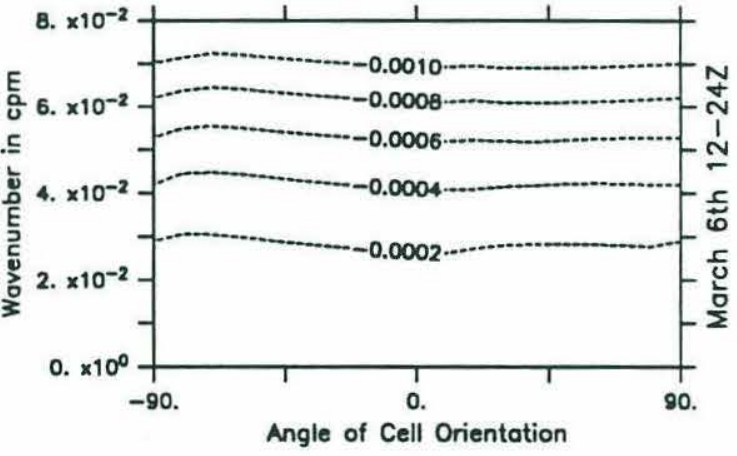

(f)

Figure 7.21: Three Case Studies of Instability during SWAPP. All plots show contours of the most unstable growth rate in $\mathrm{s}^{-1}$ as a function of angle of cell orientation relative to the wind and waves and horizontal wavenumber in cpm. (a) Growth rates for March 4th, with effects of waves included. (b) Growth rates for March 4th, without effects of waves included.(c) Growth rates for March 5th, with effects of waves included. (d) Growth rates for March 5th, without effects of waves included.(e) Growth rates for March 6th, with effects of waves included. (f) Growth rates for March 6th, without effects of waves included. 
essentially identical. The time scale required for cells to grow on these two days is of order 10 minutes. On March 6th the growth rate of the most unstable mode is more than an order of magnitude smaller.

Figure 7.21 shows contours of the growth rate for each of the three 12 hour periods on March 4-6 as a function of horizontal wavenumber and angle of cell orientation. The top row shows growth rates for March 4th, the middle row growth rates for March 5th, and the bottom row growth rates for March 6th. The left-hand column shows the growth rate in the presence of waves while right-hand column shows the growth rate in the absence of waves. The most important result is that in the absence of waves, the profile is basically stable. In the presence of waves and thus Craik-Leibovich instability, there are unstable rolls. Again the presence of waves strongly destabilizes the Ekman spiral.

The second most important result is that on March 4th and 5th there is strong instability, with cells growing on scales of 5 minutes. On March 6th, the instability is much weaker, by a factor of about 20 . This argues that the waves would have destabilized the predicted profile on March 5th, but not on March 6th, when the growth rates are comparable to the inertial frequency. Additionally, on March 6th, the diffusive decay rate for a mixed layer depth of $12 \mathrm{~m}$ is roughly the same size as the largest growth rates. This means that the Craik-Leibovich Rayleigh number is small, so that the cells cannot modify the Ekman spiral significantly even if they do grow. The maximum growth rates in all three cases occur for horizontal wavelengths of about $20 \mathrm{~m}$ and the axis of orientation of the most unstable cells is slightly to the right of the wind.

On a number of occasions, then, the viscosity predicted by the MY2 model produces a velocity and density profile which is itself unstable to Langmuir circulations driven by wave-current interaction. In the absence of waves, the predicted structure is stable to two-dimensional disturbances. The degree to which the predicted structure is unstable mirrors the strength of Langmuir circulations. On a day when the maximum growth rates are of the same order as the Coriolis frequency, the mixed layer restratifies.

\subsection{Could wave-current interaction produce enough energy to prevent diurnal restratification?}

Sections 7.4 and 7.5 showed that during both March 5th and 12th estimates of the stratified Craik-Leibovich instability parameter and the diffusive decay scale indicated that the cells were strongly forced (RaCLS»1). Section 7.6 showed that 
during March 4th and 5th the current profile predicted by the MY2 model should have been unstable. These conclusions are now extended to test one of the predictions of Chapter 5, namely that if RaCLS»1, the Craik-Leibovich mechanism will provide an energy source as large as $0.25 \tau * v_{s}(\mathrm{z}=0)$ for transporting density within the mixed layer. The temporal variability of this energy source explains why the mixed layer restratified on March 6th and 13th but not March 5th and 12th

We begin by considering sources of energy which could be responsible for mixed layer deepening and evaluating whether or not they could explain the failure of the mixed layer to restratify on March 5th and 12th.

-Buoyant convection: This mechanism is included in the PWP and MY2 models and is the result of unstable buoyancy flux at the surface leading to convective deepening. During the days when the mixed layer fails to restratify, the heat flux is stabilizing, so that this cannot explain the observations.

-Shear Production:This mechanism, included in the PWP and MY2 models, assumes local turbulence production by Kelvin-Helmholtz instability. The fact that both of these models restratify on March 5th and 12th indicates that this mechanism cannot explain the observations unless the surface stress is grossly in error.

-Near-Surface Turbulence: Studies made in turbulent wall layers indicate that the rate of turbulence production goes as $\mathrm{u}^{3} *$. A number of studies have attempted to use this surface-produced turbulence to deepen the mixed layer (Denman, 1973; Davis et al., 1981). The constant of proportionality required to explain the observed mixed layer evolution in these studies was found to be small, of order 0.4-1.0 This is a viable candidate for explaining the failure of the mixed layer to restratify on March 5th since it is not included in the PWP model.

-Stokes Production: Chapter 5 showed that Langmuir cells have the ability to transport density within the mixed layer. The energy source was shown to be of order $0.25 \mathrm{u}^{2} * \mathrm{v}_{\mathrm{s}}(\mathrm{z}=0)$. This is also a viable candidate for explaining the failure of the mixed layer to restratify.

-Wave breaking: Breaking waves would result in deepening the mixed layer in two ways. The turbulence produced by breaking waves will directly deepen a very shallow mixed layer. Additionally, the momentum input from the waves to the mixed layer will enhance the Kelvin-Helmholtz instability mechanism. Since the waves were high on both March 5th and 12th, wave breaking is a third possibility for explaining the lack of restratification on these two days. 
We begin the analysis of the enhanced mixing on March 5th and 12th by considering whether wave breaking could have enhanced the Kelvin-Helmholtz mechanism. In the presence of wave breaking, the near surface stress should have increased. During March 5th, however, the momentum budget within the top 13.5 meters can be closed with only slight adjustments to the stress.

Suppose the Ekman transport is carried above a given depth D. Then if $U$ and $\mathrm{V}$ are the transport with geostrophic contributions removed by referencing relative to some depth

$$
\frac{\partial}{\partial t} \mathrm{U}+\mathrm{iV}=-\mathrm{iF}(\mathrm{U}+\mathrm{iV})+\frac{\tau_{\mathrm{x}}+\mathrm{i} \tau_{\mathrm{y}}}{\rho}
$$

Inserting the observed transport and stress into the right-hand side and integrating over time yields

$$
\mathrm{U}_{\mathrm{p}}+\mathrm{iV} \mathrm{p}=\int_{0}^{\mathrm{t}}-\mathrm{iF}\left(\mathrm{U}\left(\mathrm{t}^{\prime}\right)+\mathrm{iV}\left(\mathrm{t}^{\prime}\right)\right)+\frac{\tau_{\mathrm{x}}\left(\mathrm{t}^{\prime}\right)+\mathrm{i} \tau_{\mathrm{y}}\left(\mathrm{t}^{\prime}\right)}{\rho} d \mathrm{t}^{\prime}
$$

If the actual stress is very different from that produced by the bulk formulae, $\mathrm{U}_{\mathrm{p}}+\mathrm{iV}_{\mathrm{p}}$ will diverge from $\mathrm{U}+\mathrm{iV}$ and the size of the correction needed to bring the two curves back together gives an estimate of the size of the error in the stress.

Consider March 5th. Figure 7.22 shows the predicted and observed transport over the top $13.5 \mathrm{~m}$. A reference depth of $70 \mathrm{~m}$ was used to eliminate the geostrophic components. The east transport matches its predicted value very well (Figure 7.22a) but the north transport diverges (7.22b). In order to bring the curves back together, a mean stress of $0.005 \mathrm{~Pa}$ was applied to the south $(7.22 \mathrm{c})$. This gives an estimate of the error in the stress, which is less than $10 \%$ of the observed stress of $0.057 \mathrm{~Pa}$. Given the error in the measurements in the mean current, the agreement is extremely good. It implies that wave breaking did not significant amounts of momentum to the mixed layer. Figure $7.22 \mathrm{~d}$ shows the temperature difference between 11.25 and 15.75 meters. Large temperature differences indicate when the diurnal mixed layer was shallower than 15.75 meters.

A similar analysis was done for March 12th-13th. Again the mean correction to the wind stress required to bring the predicted and observed northsouth transports together is $0.005 \mathrm{~Pa}$, less than $10 \%$ of the bulk-formula-derived stress of $0.063 \mathrm{~Pa}$. Once again, this means that the wind stress is quite close to the real momentum flux during the time period in question, so that wave-breaking does not significantly alter the momentum balance within the mixed layer. 

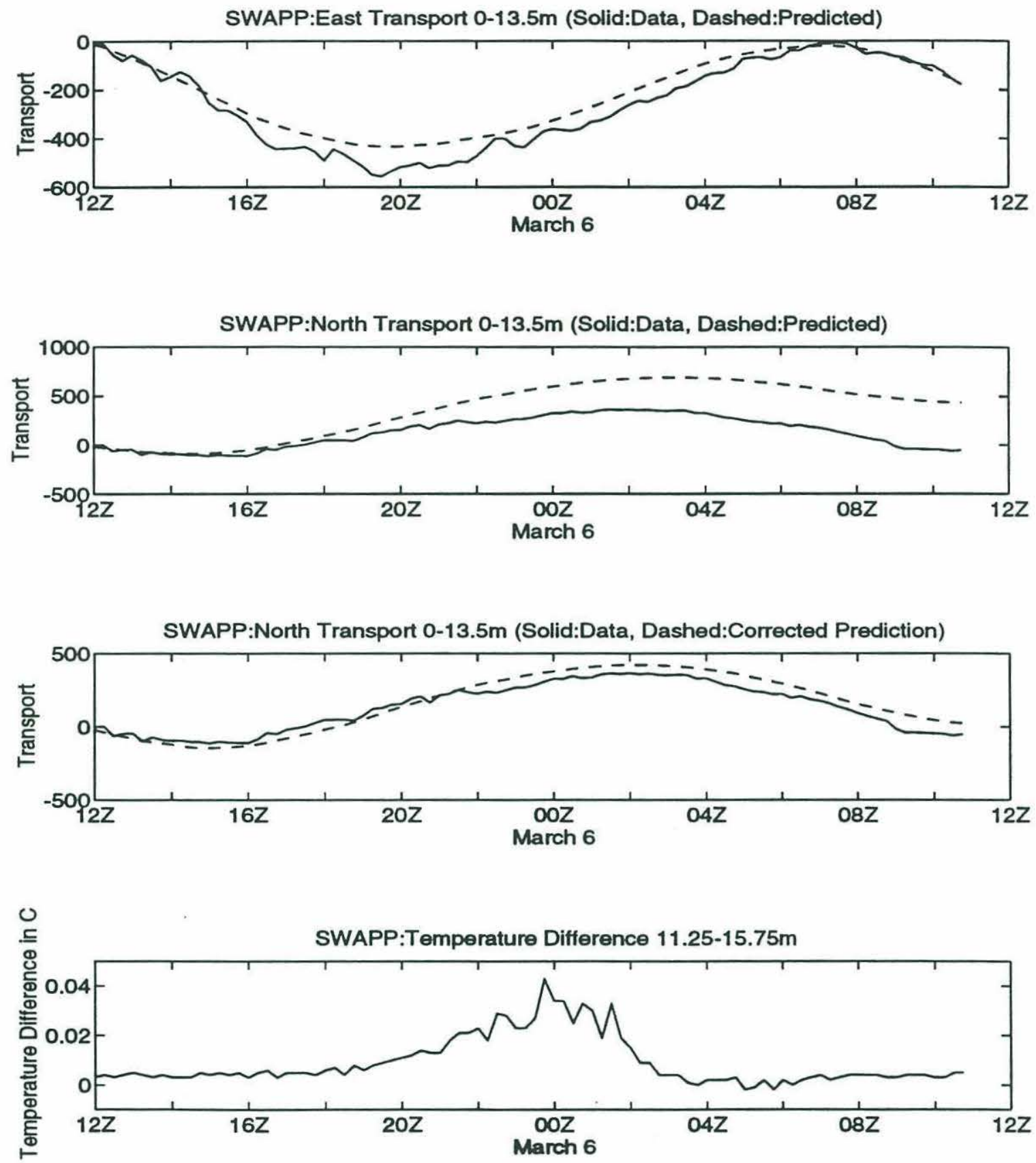

Figure 7.22: Closing the momentum balance in the upper 13.5m during SWAPP. (a) East transport on March 5th. Solid line is observed transport, dashed line is predicted from equation (7-6). (b) North transport on March 5th. Solid line is observed transport, dashed line is predicted from equation (7-6).(c) North transport on March 5th. Solid line is observed transport, dashed line is predicted from equation (7-6) with stress to the south increased by $0.005 \mathrm{~Pa}$. (d) Temperature difference 11.25-15.75. 
Therefore, wave breaking does not enhance Kelvin-Helmholtz instability during March 5th and 12th.

We now turn to the question of whether or not Stokes production or turbulent kinetic energy production could be responsible for the enhancement in mixing. Figure 7.23 compares the energy needed to mix the surface density flux down to a depth of 11.25 meters over two three day periods March 4-7 (in 7.19a) and March 11-14 (in 7.19b). compared with a reasonable value for turbulent production $\left(u_{*}^{3}\right)$ and Stokes production. Because the range of values is so large, the cube roots of these three energy sources in are presented. The solid line is

$$
\mathrm{u}_{\mathrm{CL}}=\left(0.25 \mathrm{u}_{*}^{2} \mathrm{u}_{\mathrm{s}}(\mathrm{z}=2.25 \mathrm{~m})\right)^{1 / 3}
$$

and is an estimate of the energy available from Stokes production. (The Stokes drift at $2.25 \mathrm{~m}$ is used because the Stokes drift right at the surface is strongly affected by high-frequency waves which were not measured. In Chapters 2-5, it was argued that such waves were not important to the dynamics well away from the surface). The dashed line is the friction velocity. The chain-dotted line is the cube root of the energy flux which is either needed to mix the incoming buoyancy flux down to a depth of $11.25 \mathrm{~m}$ (for times of net heating) or released by mixing the surface buoyancy flux down to $11.25 \mathrm{~m}$ (for times of net cooling). This quantity is referred to as $\mathrm{u}_{\text {buoy. }}$. When $\mathrm{u}_{\text {buoy }}$ is greater than zero, energy needs to be added to the mixed layer to mix the surface flux down to 11.25 meters. When $u_{b u o y}$ is negative, turbulent convection down to 11.25 provies an energy souce for mixing.

The mixed layer failed to restratify on March 5th and 12th. but did restratify on March 6th and 13th. Figure 7.23, shows that during both March 5th and 12, u* is of the same order as $u_{b u o y}$, but $u_{C L}$ is much larger than $u_{b u o y}$. Thus Stokes production would provide more than enough energy to mix down to $11.25 \mathrm{~m}$ while turbulent production would provide only just enough. On the other hand, on March 6th and 13th, both $\mathrm{u}_{\mathrm{CL}}$ and $\mathrm{u} *$ are of the same order as $\mathrm{u}_{\mathrm{buoy}}$. If surface turbulent production is the mechanism for mixed layer deepening on March 5th and 12th one cannot explain why the mixed layer does restratify on March 6th and 13th. However, if Craik-Leibovich instability is responsible, there is plenty of available energy on March 5th and 12th, but only barely enough on March 6th and 13th.

It is of course possible that wave breaking could be responsible for altering the mixing depth on March 5th and 12th directly, even if it does not affect the momentum balance. If this were true, the estimate of turbulence production as going as $u^{3}$ underestimates the true dissipation. Unfortunately, rigorous estimates 


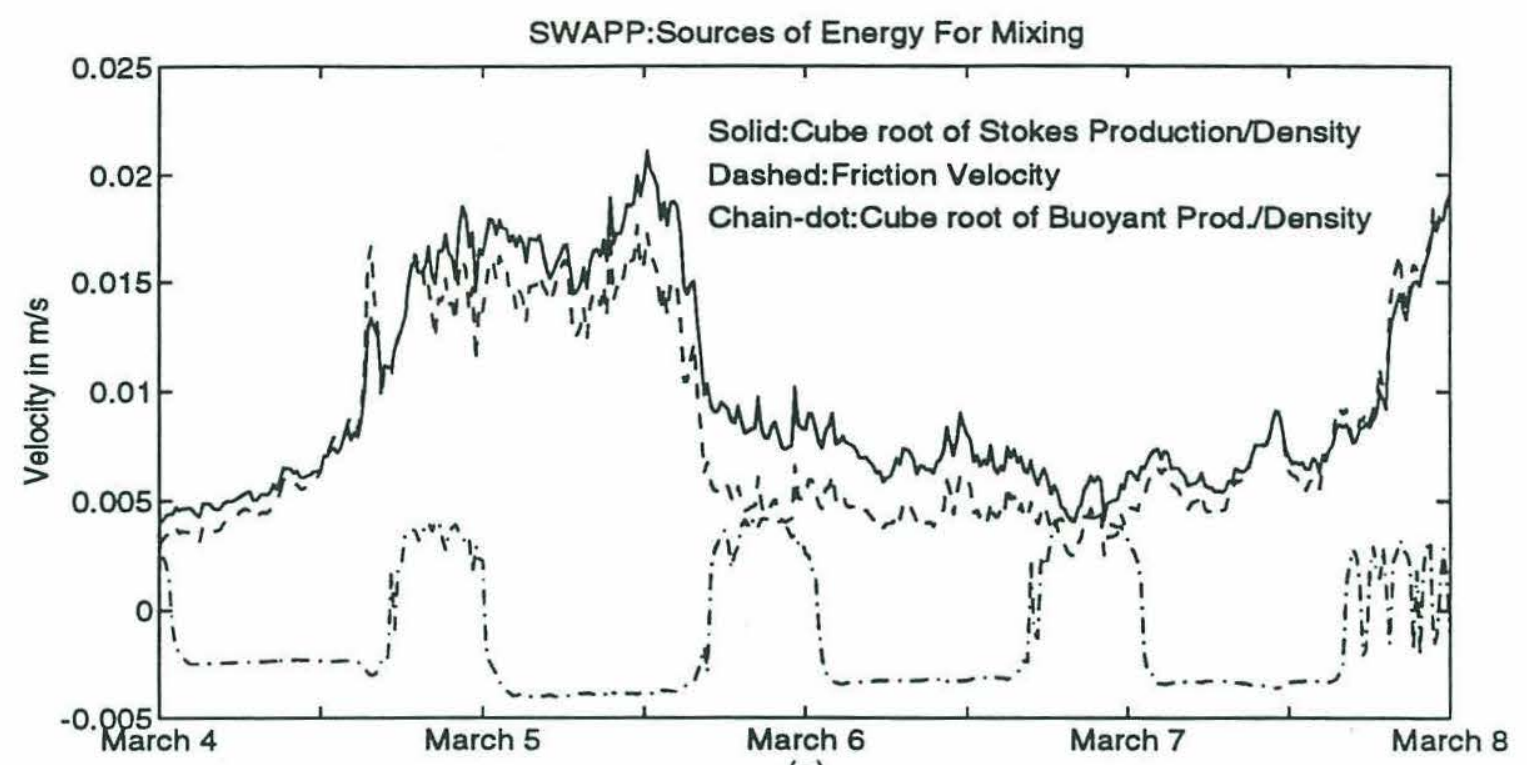

(a)

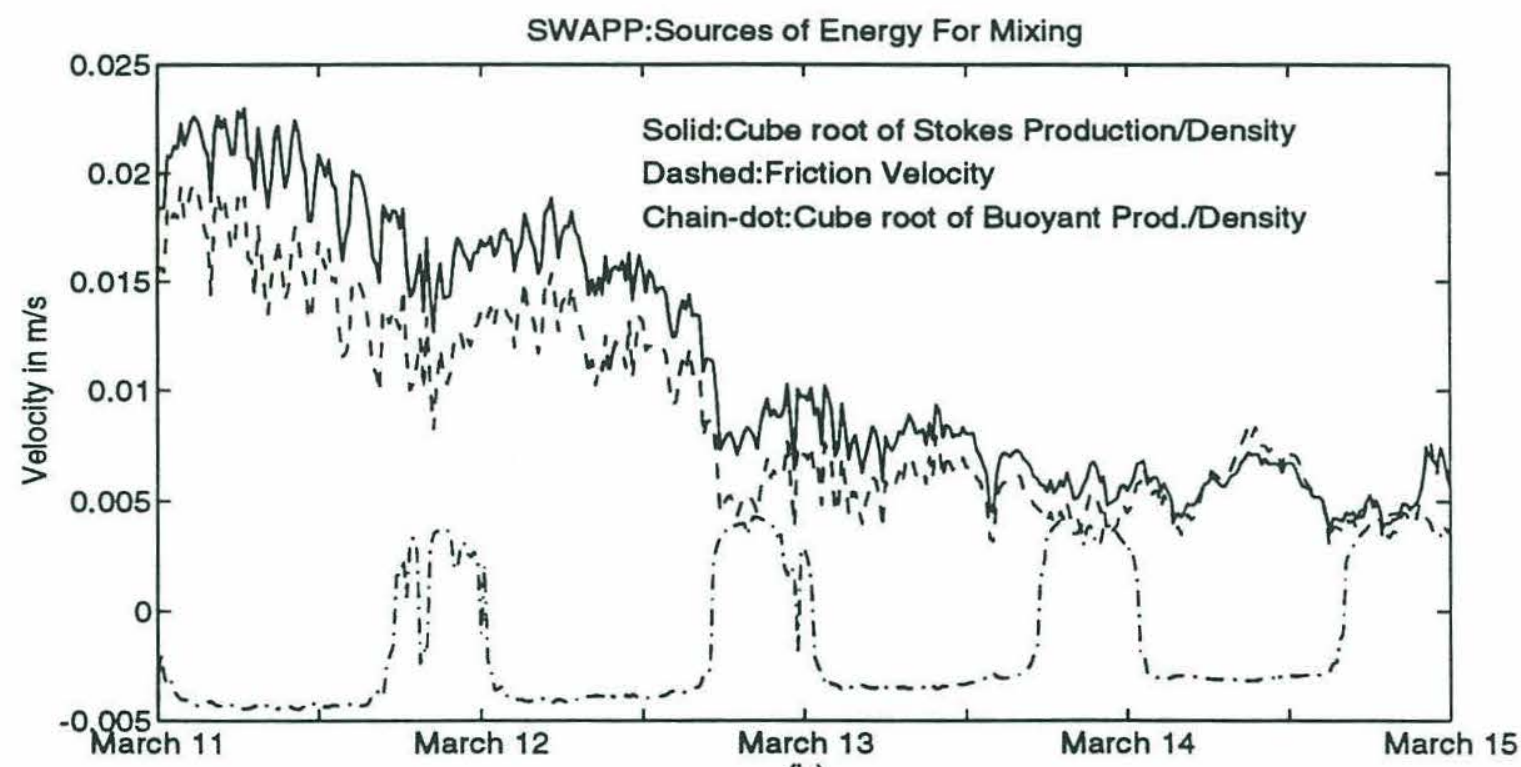

(b)

Figure 7.23: Sources of energy for mixing during two periods during SWAPP. Solid lines show the cube root of the Stokes production, dashed lines the friction velocity, and chain-dotted lines the buoyancy production which is the result of mixing the observed surface heat flux down to $11.25 \mathrm{~m}$. (a) March 4-8th. The mixed layer restratifies on March 6th, but not on March 4th, 5th and 7th. PWP and MY2 models predict restratification on 5th and 6th. (b) March 11-15th. The mixed layer restratifies on March 13th and 14th, but not on March 11th and 12th. PWP and MY2 models predict restratification on 12th, 13th and 14th. 
of the turbulent dissipation are not available. However, visual inspection of plots of turbulent dissipation in Crawford's (1992) preliminary report on the SWAPP dataset does provide some information about the level of turbulence on March 12th. On this day, the launch Slicker was deployed from 2230-2400Z and made about 20 profiles within the mixed layer. During this time period the mean friction velocity was $0.64 \mathrm{~cm} / \mathrm{s}$. The measurements showed dissipation levels which rarely exceeded $10^{-3} \mathrm{~cm}^{2} / \mathrm{s}^{3}$. Assuming an average dissipation rate of $10^{-3} \mathrm{~cm}^{2} / \mathrm{s}^{3}$ (a high value considering the data) over this depth range implies that the total dissipation over 5-15 m was less than $1 \mathrm{~cm}^{2} / \mathrm{s}^{3} \sim 4 \mathrm{u}^{3}$. Assuming $20 \%$ of the total turbulent production to go to density transport and $80 \%$ to dissipation, this implies that only about of order $u^{3} *$ worth of density transport was occurring, just the size of the turbulent production we considered earlier in this section. But as we have already seen, assuming the density transport to be carried by turbulent production does not explain the increased levels of transport seen on March 5th and 12th relative to March 6th and 13th.

The failure of the mixed layer to restratify on March 5th and 12th, days when both the sonars and current meters saw evidence of Langmuir circulations, supports the hypothesis that the cells are driven by wave-current interaction. A rough estimate of the energy flux due to the CL mechanism (the Stokes production) which would be expected to go to density transport reveals that wavecurrent interaction could provide more than enough energy to explain the observed mixing. The Stokes production was significantly larger on days when the mixed layer failed to restratify, thus explaining why the mixed layer responded differently to surface forcing on days with nearly identical wind and buoyancy forcing but different wave conditions.

\subsection{Conclusions and Implications}

The SWAPP experiment supports the hypotheses that the oceanic mixed layer is stirred by large cellular vortices which are driven by the Craik-Leibovich wave-current interaction mechanism. There are three pieces of direct evidence for this conclusion:

-The presence of high-frequency (1-36 cph) shear whose presence is linked to strongly forced Langmuir cells and whose amplitude scales as the stratified CraikLeibovich instability parameter. 
-The failure of the mixed layer to restratify on two days when the waves were high but the wind was low. CL instability provides sufficient energy on both days to explain the failure of the mixed layer to restratify.

-The breakdown of the Mellor-Yamada Level 2 in explaining the observed shear profile. The predicted profile is strongly unstable to two-dimensional roll vortices when the CL instability mechanism is present, but not when it is absent.

Although these pieces of evidence are promising, the comparison between theory and data is still far from complete. The actual structure of the lowfrequency shear response, the cell spacing, and the perturbation shear have not been rigorously compared with solutions from the full finite-difference code. These comparisons are made in Chapter 8 for some of the cases studied in this chapter as well as during the MILDEX experiment. 


\section{Chapter 8: Finite-Amplitude Langmuir Circulation during MILDEX and SWAPP}

\subsection{Introduction}

This thesis hypothesizes that the oceanic mixed layer is stirred by Langmuir cells driven by wave-current interaction which are of the same spatial scale as the layer rather than by small-scale diffusion. Chapters $2-5$ developed a theoretical framework within which this idea could be tested. Chapters 6 and 7 examined the velocity and density structure during two field experiments in the light of this theory. Three points of consistency with the principal hypotheses were found. These were:

1. Small mean shears in the interior of the mixed layer.

2. Instability of the velocity profile predicted by small-scale mixing to roll vortices.

3. Scaling of the level of the high-frequency shear consistent with wave-current interaction driving the cells.

Although these results provided powerful support for the main hypotheses, a detailed comparison between theory and data given fullydeveloped cells was not made. This chapter makes this comparison with the finitedifference code used during Chapters 4 and 5. The focus is on:

-The horizontally-averaged velocity and shear structure in the presence of finiteamplitude Langmuir cells.

-The spatial structure of the fully-developed cells.

-The horizontally varying velocity and shear structure in the presence of cells.

As expected, the finite-amplitude cells replace small-scale diffusion as the primary mechanism for momentum and density transport through much of the layer. The details of both the horizontally varying and horizontally averaged structure, however, differ from observations. Possible reasons for these differences are considered at the end of the chapter.

\subsection{Equations of Motion and Methods of Solution}

The equations of motion used in the finite-difference code are identical to those used in Chapter 5 when the wind stress is balanced by the Coriolis force.

$$
\frac{\partial \Omega}{\partial \mathrm{t}}+\left(\mathrm{u}+\mathrm{u}_{\mathrm{s}}\right) \frac{\partial \Omega}{\partial \mathrm{x}}+\mathrm{w} \frac{\partial \Omega}{\partial \mathrm{z}}=\mathrm{F} \frac{\partial}{\partial \mathrm{z}}\left(\mathrm{v}+\mathrm{v}_{\mathrm{s}}\right)+\frac{\partial \mathrm{v}_{\mathrm{s}} \partial \mathrm{v}}{\partial \mathrm{z} \partial \mathrm{x}}+\mathrm{Ri} \frac{\partial \rho}{\partial \mathrm{x}}+\mathrm{La} \nabla^{2} \Omega
$$




$$
\begin{gathered}
\frac{\partial v}{\partial t}+\left(u+u_{s}\right) \frac{\partial v}{\partial x}+w \frac{\partial v}{\partial z}=-F\left(u+u_{s}\right)+L a \nabla^{2} v \\
\frac{\partial \rho}{\partial t}+\left(u+u_{s}\right) \frac{\partial \rho}{\partial x}+w \frac{\partial \rho}{\partial z^{2}}=L a \nabla^{2} \rho
\end{gathered}
$$

$$
\begin{array}{ccc}
\frac{\partial \psi}{\partial \mathrm{x}}=-\mathrm{w} & \frac{\partial \psi}{\partial \mathrm{z}}=\mathrm{u} \\
\mathrm{La}=\frac{\mathrm{ve}_{\mathrm{e}}}{\mathrm{a}^{2} \sigma} \quad \mathrm{F}=\frac{\mathrm{f}}{\mathrm{k}_{\mathrm{w}}^{2} \mathrm{a}^{2} \sigma} \quad \mathrm{Ri}=\frac{\mathrm{N}^{2}}{\mathrm{k}_{\mathrm{w}}^{4} \mathrm{a}^{4} \sigma^{2}} \\
\\
\mathrm{k}_{\mathrm{w}}^{-1}(\mathrm{x}, \mathrm{y}, \mathrm{z})=(x, y, z) \\
\left(\mathrm{k}_{\mathrm{w}} \mathrm{a}\right)^{2} \frac{\sigma}{\mathrm{k}_{\mathrm{w}}}\left(\mathrm{u}, \mathrm{u}_{\mathrm{s}}, \mathrm{v}, \mathrm{v}_{\mathrm{s}}, \mathrm{w}\right)=\left(u, u_{s}, v, v_{s}, w\right) \\
\frac{1}{\mathrm{k}_{\mathrm{w}}^{2} \mathrm{a}^{2} \sigma} \mathrm{t}=t
\end{array}
$$

In these equations $\mathrm{k}_{\mathrm{w}}, \mathrm{a}$, and $\sigma$ are the wavenumber, amplitude and frequency of the driving waves. $v_{\mathrm{e}}$ is the eddy viscosity, $\mathrm{N}$ the buoyancy frequency, $\mathrm{f}$ the Coriolis parameter, and $\mathrm{u}_{\mathrm{S}}$ and $\mathrm{v}_{\mathrm{S}}$ the Stokes Drift. La is the Langmuir number, which is a scaled eddy visocity or inverse Reynolds number. $\mathrm{Ri}$ is the square of the scaled buoyancy frequency and $\mathrm{F}$ is the scaled Coriolis parameter. Equations (8-1a-e) are for dimensionless variables, with equations (8-1g-i) showing how these variables are converted to dimensional (italicized) form. The boundary conditions on the velocity and density are.

$$
\begin{gathered}
\left.\mathrm{La} \frac{\partial \mathrm{v}}{\partial \mathrm{z}}\right|_{\mathrm{z}=0}=\frac{\tau}{\rho} \cos \alpha \\
\left.\mathrm{La} \Omega\right|_{\mathrm{z}=0}=-\frac{\tau}{\rho} \sin \alpha \\
\left.\mathrm{La} \frac{\partial \mathrm{v}}{\partial \mathrm{z}}\right|_{\mathrm{z}=0}=\left.\Omega\right|_{\mathrm{z}=-\mathrm{D}}=0 \\
\left.\mathrm{La} \frac{\partial \rho}{\partial \mathrm{z}}\right|_{\mathrm{z}=0}=1 \\
\left.\rho\right|_{\mathrm{z}=-\mathrm{D}}=-\mathrm{D}
\end{gathered}
$$

where $\alpha$ is the angle of orientation of the cells relative to the wind and D is the depth of the fluid layer. The shortcomings of these equations as a realistic idealization of the oceanic surface layer are explored in great detail in Chapters 
2-5 and in Appendix A. These equations are solved using the finite-difference code introduced in Chapter 4 and outlined in Appendix C.

As part of the analyses of both the MILDEX and SWAPP datasets, the mean Langmuir number, scaled Coriolis parameter, Stokes drift and surface shear were computed. The current profiles obtained by using these parameters to force a one-dimensional balance (as in Chapter 3) were shown to be unstable to Langmuir circulations. This chapter focusses on three time periods where this analysis was done:

1. 1500 PST November 6th -0200 PST November 11th during MILDEX (referred to in Chapter 6 as Period 2). Cells were strong during almost all of this period. 2. $0000 \mathrm{Z}$ March 4th $-1715 \mathrm{Z}$ March 13th during SWAPP. Again, during this period the cells were strong on all but one day (March 6th) which was the only day on which the upper part of the water column restratified.

3. 1200Z March 5th - 0845Z March 6th. During this time period, both the PWP and MY2 models predicted restratification which was not seen in the data.

The parameters were used to force the finite-difference code for each of these three cases are shown in Table 8.1. The results from the runs are presented in Sections 8.3 and 8.4.

\begin{tabular}{|l|l|l|l|}
\hline & $\begin{array}{l}\text { MILDEX: } \\
\text { November 6-11 }\end{array}$ & $\begin{array}{l}\text { SWAPP: } \\
\text { March 4-13 }\end{array}$ & $\begin{array}{l}\text { SWAPP: } \\
\text { March 5 }\end{array}$ \\
\hline Time Scale & $630 \mathrm{sec}$ & $900 \mathrm{sec}$ & $380 \mathrm{sec}$ \\
\hline Length Scale & $25 \mathrm{~m}$ & $31 \mathrm{~m}$ & $25 \mathrm{~m}$ \\
\hline Velocity Scale & $4.0 \mathrm{~cm} / \mathrm{s}$ & $3.5 \mathrm{~cm} / \mathrm{s}$ & $6.5 \mathrm{~cm} / \mathrm{s}$ \\
\hline La & 0.017 & 0.021 & 0.009 \\
\hline $\mathrm{F}$ & 0.051 & 0.074 & 0.033 \\
\hline Ri & 0.0 & 0.0 & 1.0 \\
\hline Surface Shear & 4.8 & 4.4 & 2.3 \\
\hline Layer Depth & 2 & 2.5 & 2 \\
\hline
\end{tabular}

Table 8.1: Nondimensional parameters input to the finite-difference code to predict momentum and density structure during SWAPP and MILDEX.

During SWAPP the waves had a somewhat lower frequency than during MILDEX, possibly as a result of the long fetches associated with the flanks of the high-pressure system. Since the time scale $T=1 /\left(k_{w} a\right)^{2} \sigma=g^{2} / a^{2} \sigma^{5}$ which is used to 
nondimensionalize the problem is strongly dependent on $\sigma$, the $10 \%$ difference in frequency results in a large difference in $\mathrm{T}$. As seen below, this makes the effect of stratification more important. Another difference between SWAPP and MILDEX was the difference in the depth of the main thermocline. These differences are reflected in the different cell strengths and structures seen during the two experiments.

There are some problems with using averaged forcing functions to predict the averaged response. The shear in a long-term average may occur at times when the surface layer is stratified and the cells are weak. Using this shear to drive the cells is not consistent. Additionally, given the fact that the forcing changes over time scales of days, the mixed layer may actually come to equilibrium. An attempt to minimize the first effect was made by limiting the analysis to periods when the mixed layer was generally deep and Langmuir circulations were strong. The second effect is a general problem with looking at an equilibrium state.

During all preceeding chapters, the upper boundary condition with respect to velocity was taken to be constant with respect to the crosscell direction. In Section 8.5 this assumption is relaxed and the surface shear allowed to vary in an attempt to explain the failure of the models to produce a range of cells. The upper boundary condition on density is also allowed to vary to simulate the effect of diurnal buoyancy forcing.

\subsection{The Horizontally-Averaged Structure of the Mixed Layer \\ 8.3.1 The MILDEX Experiment}

Chapters 6 and 7 showed that in the presence of strong Langmuir cells, the horizontally-averaged velocity and shear structure differed from that predicted by either a slab model or small-scale turbulence model. In both experiments, the velocity structure was more slab-like than predicted by the small-scale turbulence model, but near the surface the velocity response was strongly sheared. This section shows that a layer with finite-amplitude Langmuir cells has a qualitatively similar structure.

The horizontally-averaged response for Period 2 (1500 PST November 6 0200 PST November 11) during MILDEX is shown in Figures 8.1 and 8.2. Figure 8.1 shows the velocity response during this time period, from data (8.1a), from the PWP model (8.1b), from the MY2 model (8.1c), from equations (8-1) assuming no cells (8.1d) and from the finite-difference code assuming fully-developed cells 


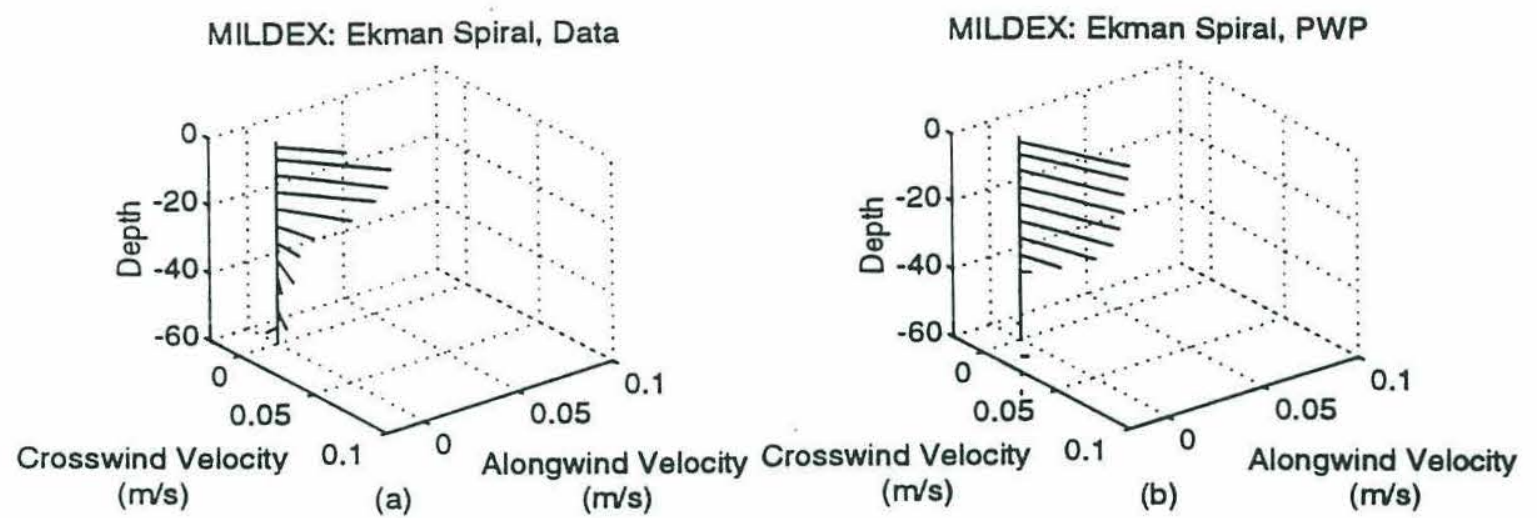

MILDEX: Ekman Spiral,MY2 Viscosity

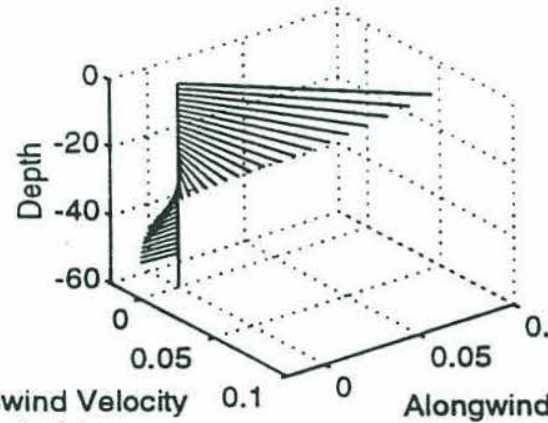

$(\mathrm{m} / \mathrm{s})$ (c)
0.1 $(\mathrm{m} / \mathrm{s})$
MILDEX: Ekman Spiral, Waves and Cells

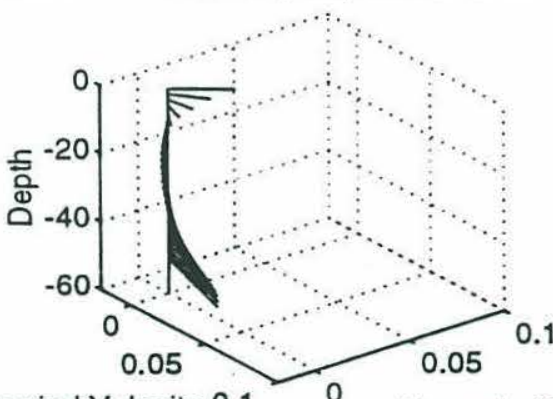

Crosswind Velocity 0.1 (e)
Alongwind Velocity Crosswind Velocity 0.1 $(\mathrm{m} / \mathrm{s})$

$(\mathrm{m} / \mathrm{s})$
MILDEX: Ekman Spiral, Waves but No Cells

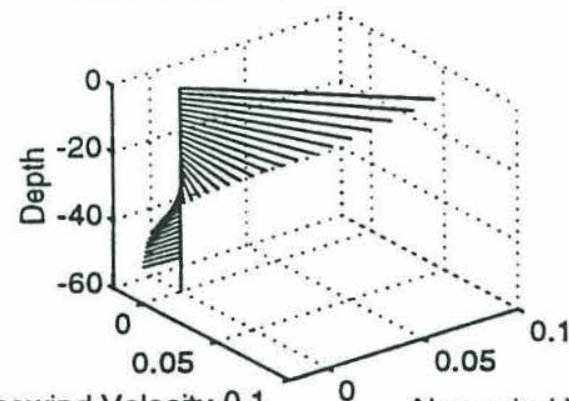

(d)
MILDEX: Ekman Spiral, Waves and Cells

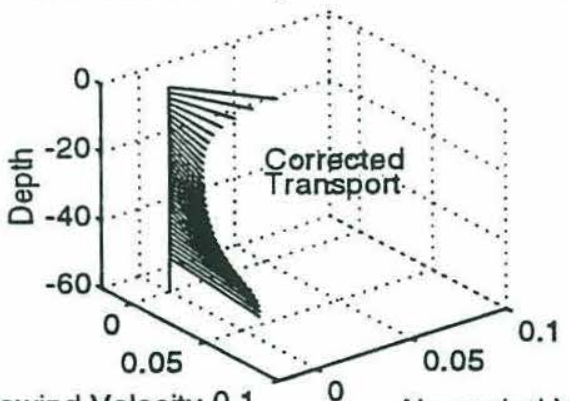

(f)
Alongwind Velocity

$(\mathrm{m} / \mathrm{s})$

Figure 8.1: Ekman spiral during MILDEX, November 6-11. (a) Observed velocity relative to 65 meters. (b)-(f) Predicted velocity: (b) from PWP model, (c) from MY2 model, (d) using mean MY2 viscosity in the presence of waves but without cells, (e) from finite-difference code given finite-amplitude Langmuir cells, and (f) from finite-difference code given finite-amplitude Langmuir cells with transport corrected to agree with data. 
MILDEX:Ekman Shear (/s), Data

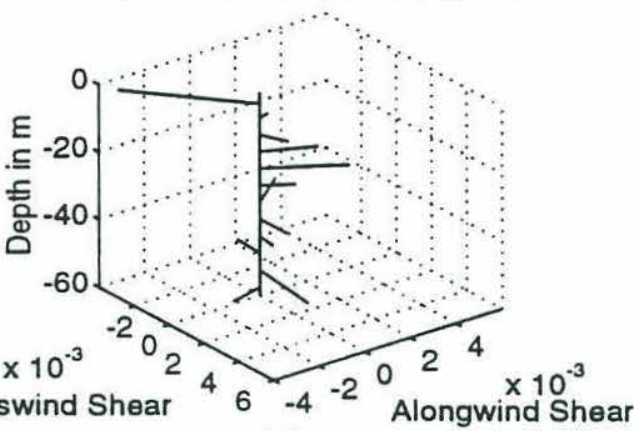

(a)

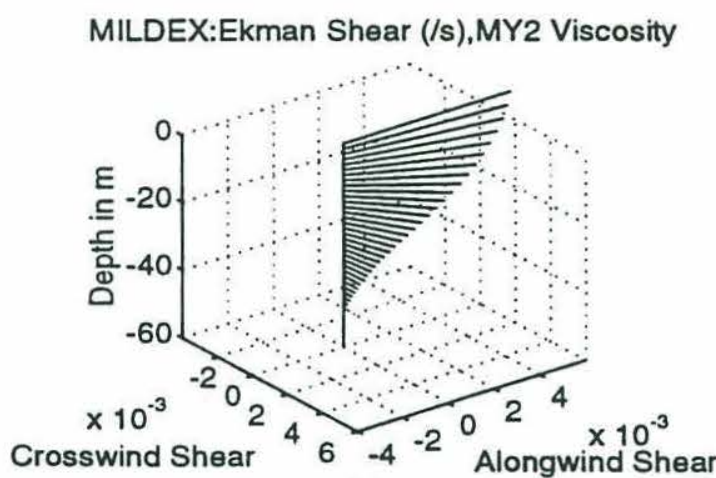

(c)
MILDEX:Ekman Shear (/s), PWP

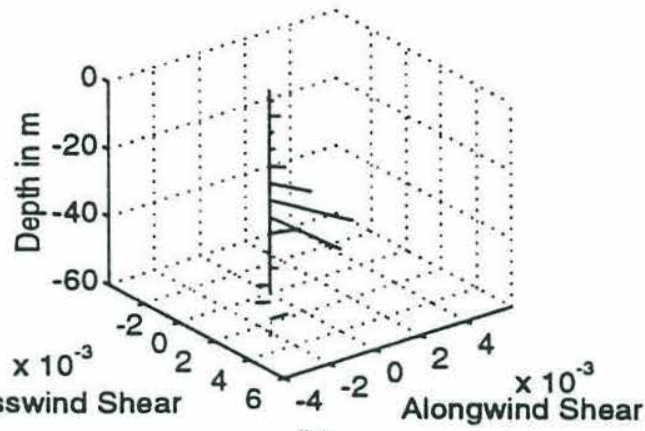

(b)

MILDEX:Ekman Shear (/s), Waves and Cells

MILDEX:Ekman Shear (/s), Waves and Cells ( $\mathrm{R}=0.5)$

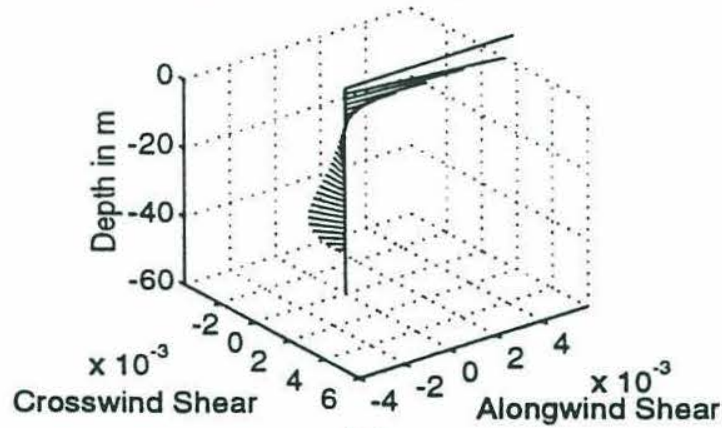

(e)

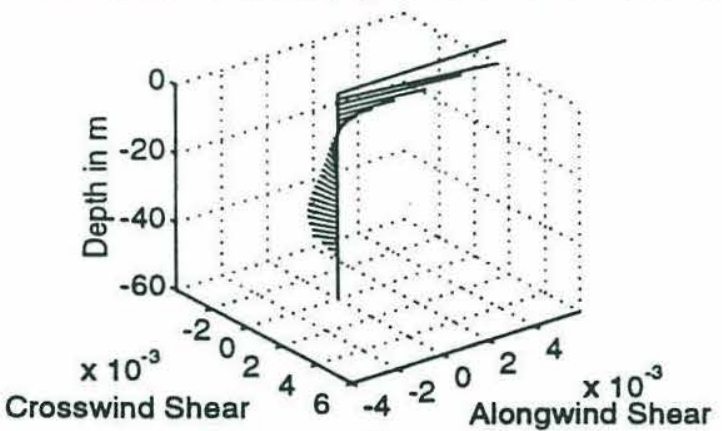

(f)

Figure 8.2: Ekman Shear during MILDEX, November 6-11. (a) Observed shear. (b)-(f) Predicted shear: (b) from PWP model, (c) from MY2 model, (d) using mean MY2 viscosity in the presence of waves but without cells, (e) from finitedifference code given finite-amplitude Langmuir cells, and (f) from finitedifference code given a stabilizing heat flux of $60 \mathrm{~W} / \mathrm{m}^{2}(\mathrm{Ri}=0.5)$. 
(8.1e). Over most of the water column, the velocity in the presence of cells is slightly upwind. The transport resulting from integrating the velocity profile is also upwind, in contrast to the observed transport, which is slightly downwind (see Figure 6.19b). Some of the difference between the observed and the modelled velocity profiles presented here stems from the difference in the mean velocity. Figure $8.1 \mathrm{f}$ corrects for this effect by offsetting the velocity profile predicted by the finite difference code so that the total transport agrees with that seen in the data. Figures 8.2 a-e repeat $8.1 \mathrm{a}$-e for the shear instead of the velocity. The shear is a measure of the momentum transport carried by small-scale diffusion. When the shear is small, little small-scale diffusive transport occurs.

The velocity and shear profiles in the presence of finite-amplitude cells show a number of common features with the data. Both show strong shear near the surface, $(<5 \mathrm{~m})$ with little shear in the middle of the water column $(5-20 \mathrm{~m})$. Below that the finite-difference code predicts more shear again, but in the opposite direction from that predicted by the Mellor-Yamada model. Within the finite-difference code the cells are not very important near the surface but replace small-scale diffusion as the primary transport mechanism at depths below 5-10m.

Although the shear is strong near the surface in both the data and the model, the observed shear is upwind, with water at depth moving faster in the downwind direction than water near the surface. This is the opposite direction from the shear seen in the finite-difference code runs and predicted by the MellorYamada model. Insofar as it is believed that the upwind shear is linked to the presence of cells (as argued during Chapter 6) the finite-difference code does not capture these cells or the momentum transport which they accomplish.

As a result of the cell momentum transport, the mean velocity profile in the finite-difference code is much more homogeneous in the alongcell direction than it is in the absence of cells. The presence of cells does not only produce homogenization, however. Looking at a hodograph of the currents from above, the Mellor-Yamada model predicts that the current vectors rotate clockwise around the mean value as one goes deeper in the water column. The finitedifference code predicts that the current vectors will rotate the other way. The reason is that there is strong crosswind transport at the base of the layer. This is in contrast to the observed response in Figure 8.1a which shows a decrease in the velocity associated with the Ekman spiral as the mixed layer base is approached.

While there are a number of possible explanations for the enhanced crosswind velocities at depth, one natural supposition is that the observed fall off 
in velocity is the result of interaction with the thermocline. The finite-difference results presented above assume no stratification, with the bottom boundary being essentially taken as a hard boundary below which mixing could not penetrate. Given the inability of the finite-difference code to allow for a nonconstant diffusive coefficient, the only way to test the sensitivity of these results to the presence of a thermocline is to force a constant heat flux through the domain. This was done assuming a temperature difference of $0.03 \mathrm{C}$ across the layer, corresponding to a stabilizing heat flux of $60 \mathrm{~W} / \mathrm{m}^{2}$ and a Richardson number of 0.5. The resulting shear profile is shown in Figure 8.2f. The presence of stratification does not change the shear or velocity profile substantially. Although stratification probably does play some role in explaining the large deep crosswind velocities modelled during MILDEX, it is most likely not the primary explanation.

\subsubsection{The SWAPP Experiment}

Over the time period from $0000 \mathrm{Z}$ March 4th to $1715 \mathrm{Z}$ March 13th during SWAPP, Langmuir cells appear to have been strong with the exception of one day. Finite-difference code runs were computed using the mean forcing functions and diffusion during this time period as well. The results in the absence of stratification are quite similar to those seen during MILDEX. Figure 8.3 repeats 8.2 for the horizontally-averaged shear seen during SWAPP. Once again, the finite-difference code predicts a near-surface shear layer, as seen in the data and in contrast to the PWP model. The finite-difference code also predicts that the alongwind shear is essentially erased at depths below about $10 \mathrm{~m}$. The observations do show some alongwind shear at this depth, though the amplitude of this shear is much smaller than that predicted by the MY2 model. Finally, at depths below about 30 meters, the finite-difference code predicts shear which is off to the left of the wind. As during MILDEX, the velocity profile (shown in Figure 8.4a) also exhibits large crosswind velocities at depth and the velocity rotates counterclockwise around the mean value.

In contrast to MILDEX, however, these results are quite sensitive to the presence of stratification. The sensitivity was tested by assuming a temperature difference across the layer of $0.05 \mathrm{C}$ (corresponding to a heat flux of about 60 $\mathrm{W} / \mathrm{m}^{2}$ as in MILDEX). Because the time scale $\mathrm{T}$ for the evolution of the cells is longer during SWAPP than during MILDEX, the effective Richardson number during SWAPP is 1.0, twice that during MILDEX. The results of including 
SWAPP:Shear (/s), Data

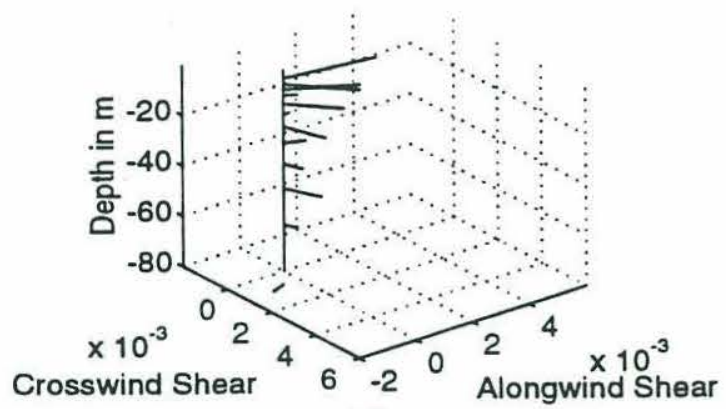

(a)

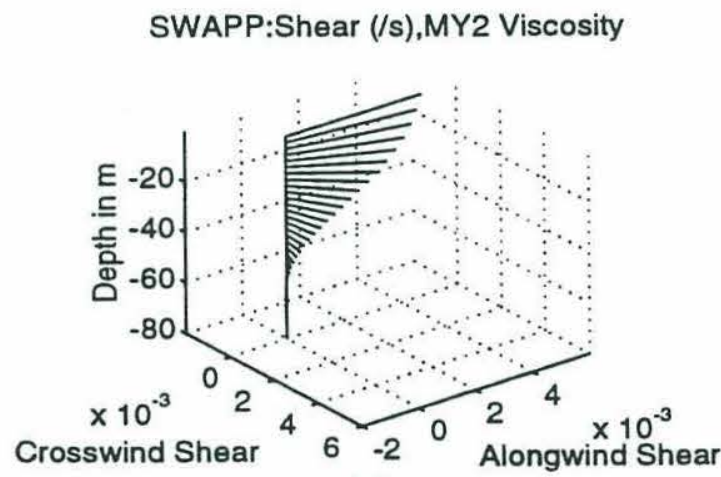

(c)
SWAPP:Shear (/s), PWP

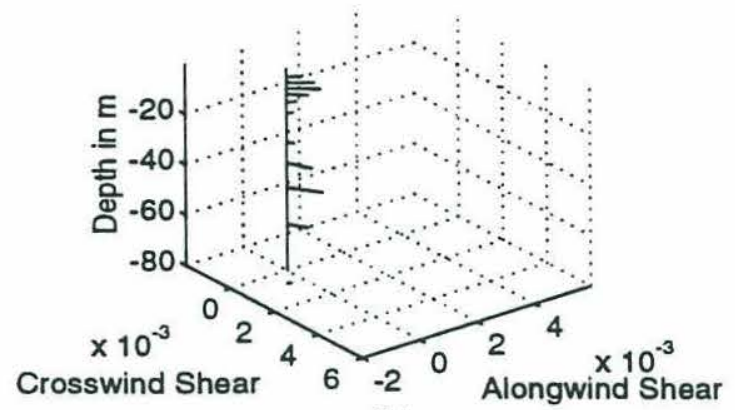

(b)

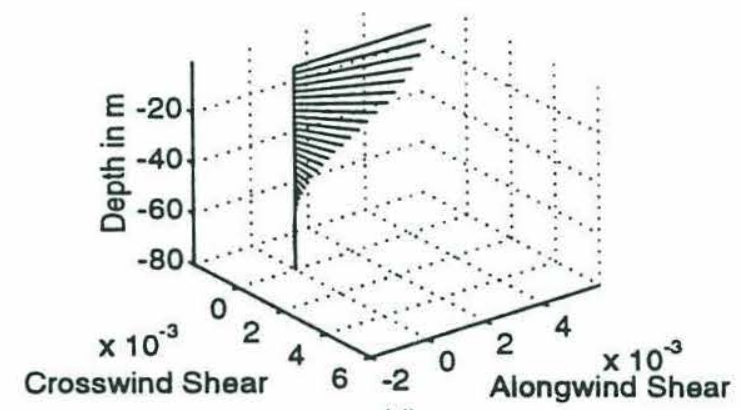

(d)

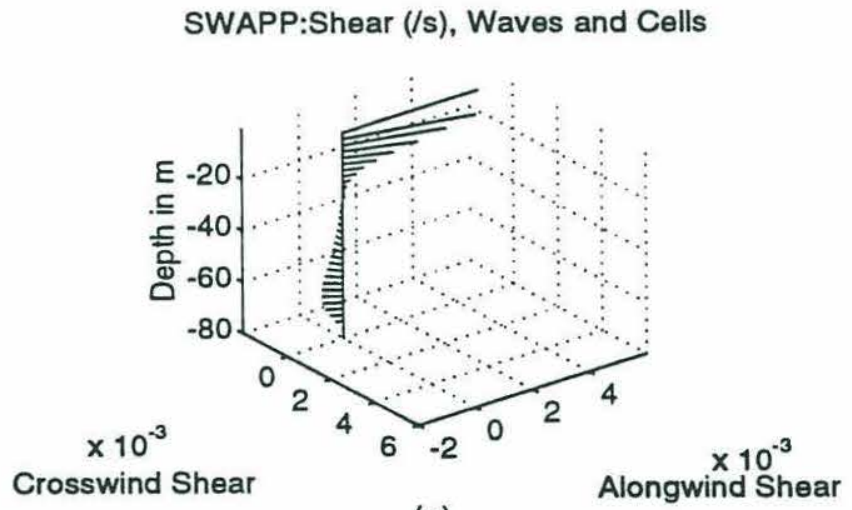

(e)

Figure 8.3: Mean shear during SWAPP, March 4th-13th. (a) Observed shear. (b)-(f) Predicted shear: (b) from PWP model, (c) from MY2 model, (d) using mean MY2 viscosity in the presence of waves but without cells, and (e) from finitedifference code given finite-amplitude Langmuir cells. 
SWAPP:Ekman Spiral (m/s), Ri=0.0

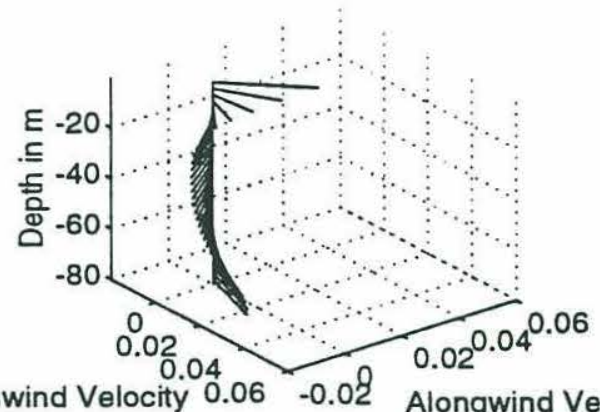

(a)
SWAPP:Ekman Shear (/s), Ri $=0.0$

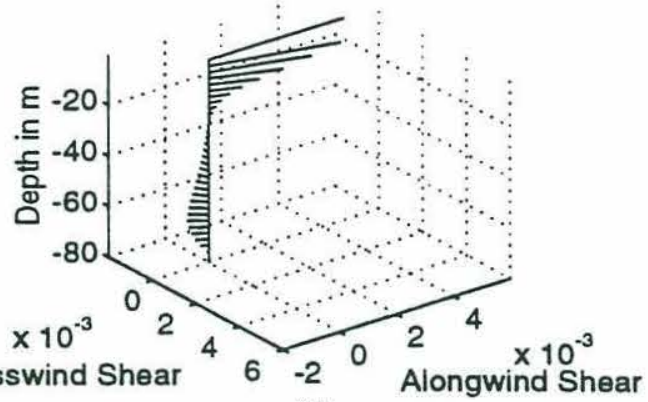

(b)
SWAPP:Ekman Spiral $(\mathrm{m} / \mathrm{s}), \mathrm{Ri}=1.0$

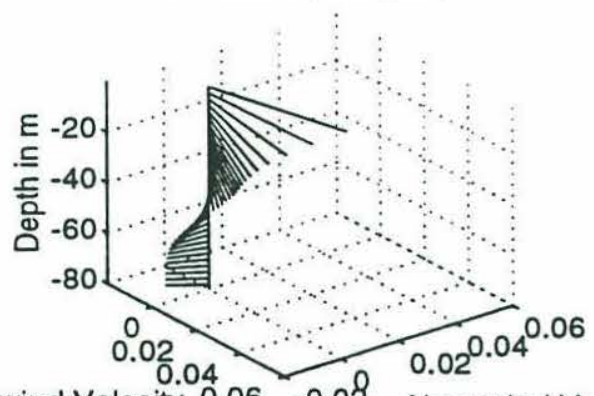

(c)
SWAPP:Ekman Shear $(\mathrm{m} / \mathrm{s}), \mathrm{Ri}=1.0$

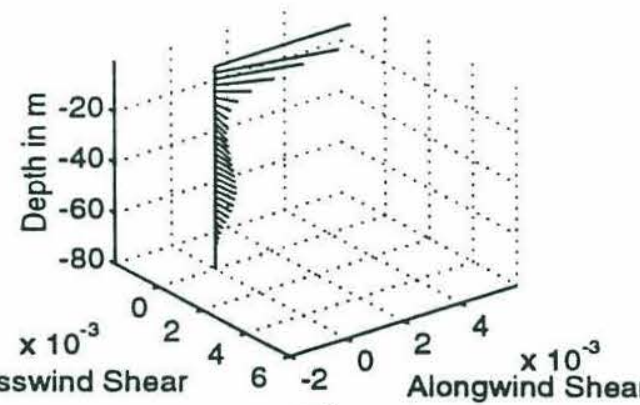

(d)

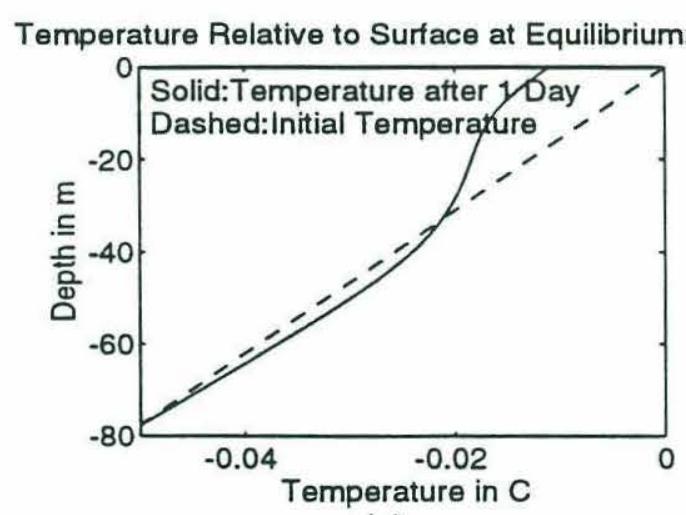

(e)

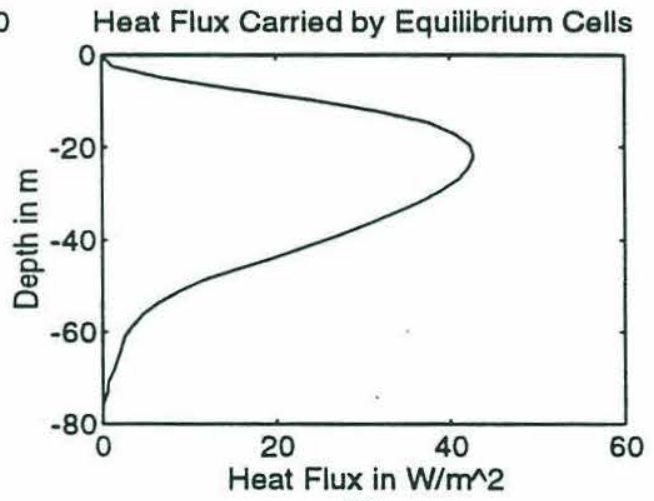

(f)

Figure 8.4: Sensitivity of the results during SWAPP to stratification. (a)-(d) Predicted velocities $(\mathrm{m} / \mathrm{s})$ and shears (/s) from finite difference code. (a) Mean velocity: $R i=0.0$. (b) Mean shear $R i=0.0$. (c) Mean velocity $R i=1.0$. (d) Mean shear: $\mathrm{Ri}=1.0$. (e) Horizontally-averaged temperature structure $\mathrm{Ri}=1.0$. Solid line is structure at end on one day, dashed line initial condition. (f) Heat flux in W/m² at equilibrium $\mathrm{Ri}=1.0$. 
stratification are shown in Figure 8.4. Figures $8.4 \mathrm{a}$ and $\mathrm{b}$ show the velocity and shear respectively in the absence of stratification $(\mathrm{Ri}=0.0)$, while Figures $8.4 \mathrm{c}$ and $\mathrm{d}$ show the velocity and shear in the presence of stratification. Figures $8.4 \mathrm{e}$ shows the temperature profile after the finite-difference code has evolved for 1 pendulum day and $8.4 \mathrm{f}$ shows the heat flux accomplished by the equilibrium cells.

In the presence of stratification, the cell depth of penetration is limited to about 40 meters. The cells reduce the temperature difference between the surface and 40 meters from about $0.025 \mathrm{C}$ to about $0.01 \mathrm{C}$. Interestingly, during SWAPP the maximum observed mixed layer depths (defined as the depth at which the temperature was $0.01 \mathrm{C}$ colder than the surface) were about 40 meters, while the PWP and MY2 models both predicted mixing down to the main thermocline at 70

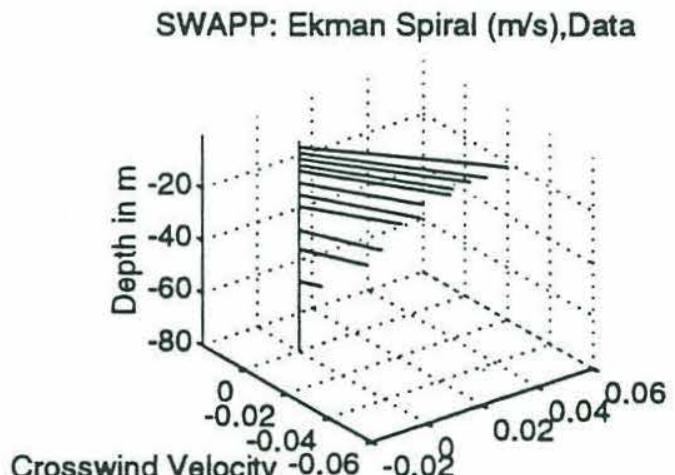

(a) Alongwind Velocity
SWAPP: Ekman Spiral (m/s), MY2

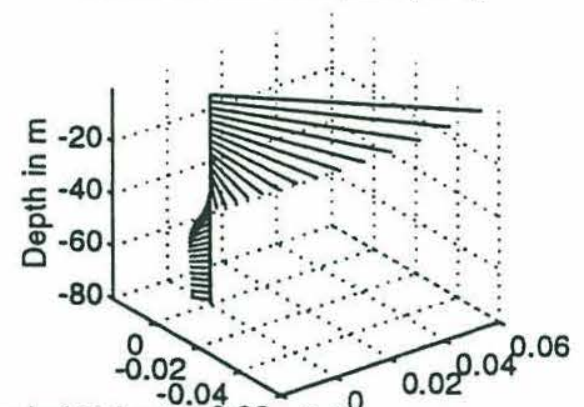

(b)

SWAPP: Ekman Spiral (m/s), Cells,Corrected Transport

Crosswind Velocity

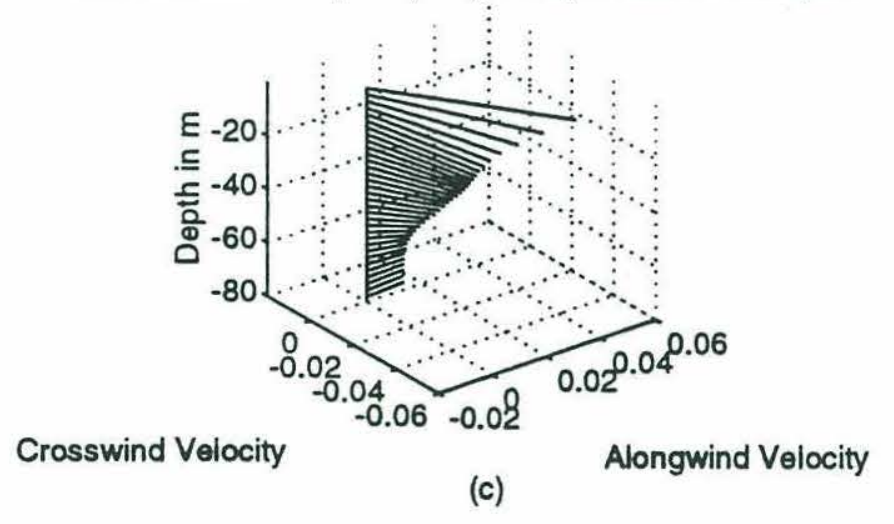

(c)
Alongwind Velocity
Alongwind Velocity

Figure 8.5: Velocity Structure during SWAPP. (a) Observed relative to 70 meters. (b) Predicted from the MY2 model. (c) Predicted from the finite-difference code assuming stratification $(\mathrm{Ri}=1.0)$ and with the mean velocity corrected so that the transport agrees with the data. 
meters. The agreement of the finite-difference code and data in this respect may be fortuitous, but is nonetheless encouraging.

The reduction in the depth over which the cells penetrate is reflected in the shear and velocity structure. The shear is now more or less in the alongcell direction down to a depth of about 40 meters, though it is very small below a depth of about 10 meters. The velocity structure reflects this fact. Figure 8.5 shows the velocity structure predicted during SWAPP given stratification compared with the observed velocity structure where the finite-difference code has been adjusted to give the same transport as the data. In the absence of cells (Figure 8.5b) small-scale mixing is not very strong, so that there is a strong intensification of the velocity near the surface. In the presence of cells, the velocity profile is extremely close to that seen in the data when the transport is corrected. For the mean conditions during SWAPP, imposing stratification limits the depth to which the cells penetrate within the mixed layer and gives a realistic velocity structure.

In the model runs just presented, stratification was included in an ad hoc way. We now turn to a time period during which it is known that buoyancy flux was important. During March 5th, the mixed layer failed to restratify in the presence of waves and Langmuir cells as predicted by the PWP and MY2 models. In Chapter 7, it was demonstrated that the mean Ekman spiral predicted by the MY2 model during March 5th was strongly unstable to Langmuir cells. The equilibrium state of the finite-difference code in the presence of fully developed Langmuir cells is now considered.

Figure 8.6a shows the mean Ekman spiral relative to the wind on March 5th from data. The Ekman spiral was calculated by orienting the currents relative to the wind and averaging over a pendulum day. Figure $8.6 \mathrm{~b}$ shows the prediction of the PWP model, Figure 8.6c the prediction of the MY2 model. Both models predict surface intensification of the velocity with lots of shear in the top 10 meters. The data, on the other hand show a profile which is much more wellmixed. This difference reflects the fact that the top 10 meters was erroneously predicted to restratify by the models.

The finite-difference code predicts that Langmuir cells will in fact mix the upper portion of the water column. Figure $8.6 \mathrm{~d}$ shows the predicted velocity structure from the finite-difference code assuming finite-ampliude cells. Figure 8.6e shows the mean temperature stratification after 1 day. Figure $8.6 \mathrm{f}$ shows the heat flux in $\mathrm{W} / \mathrm{m}^{2}$ predicted by the finite-difference code to be carried by the cells 
SWAPP:Ekman Spiral (Data), 3/5

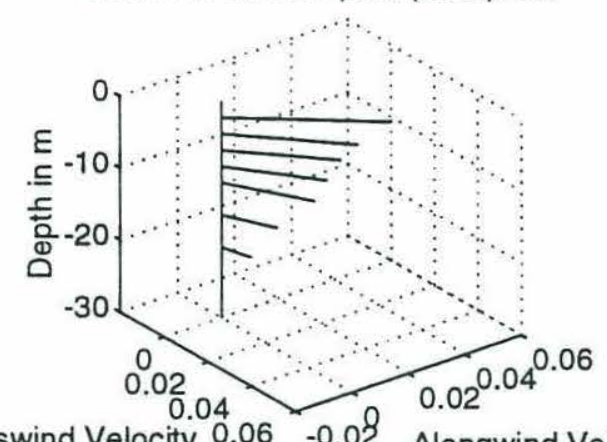

(a)

(c)

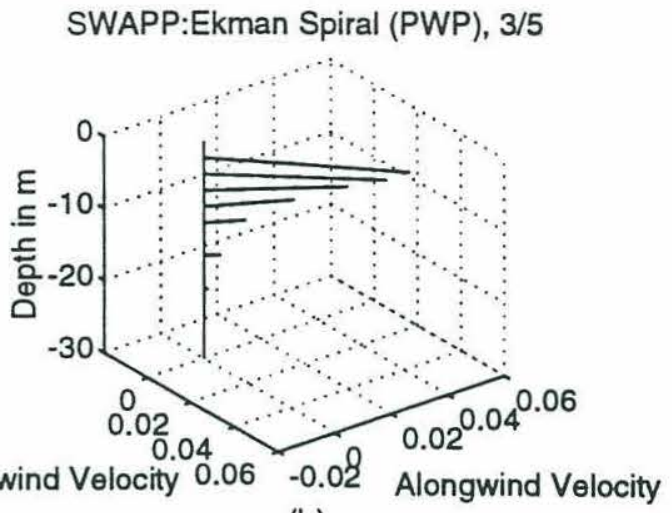

(b)

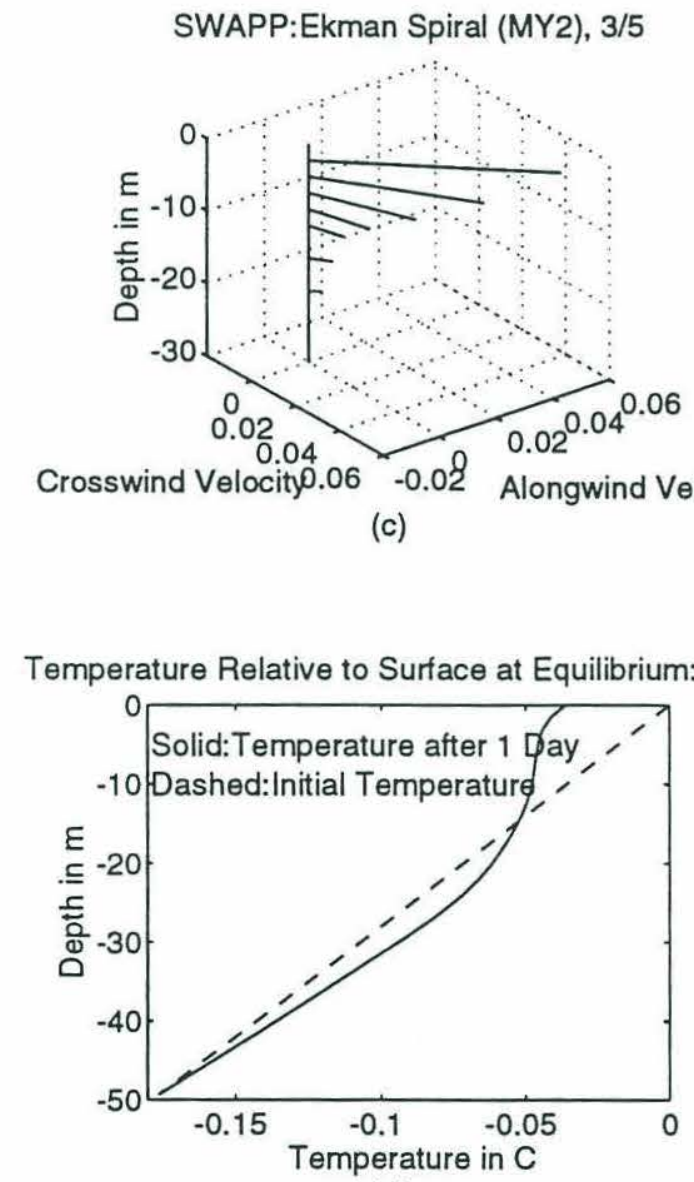

(e)

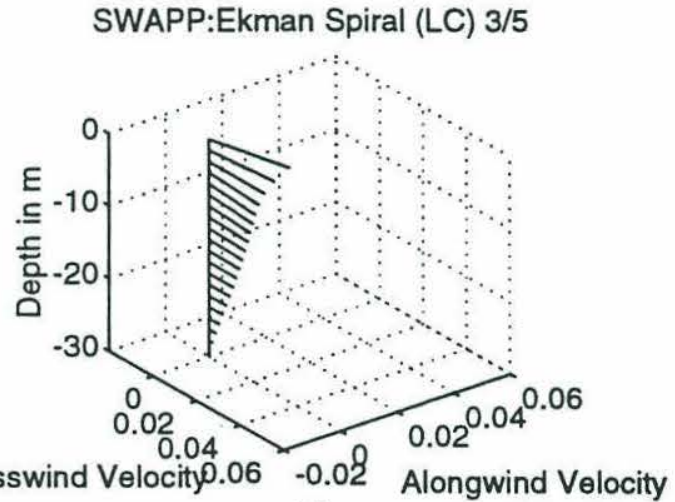

(d)

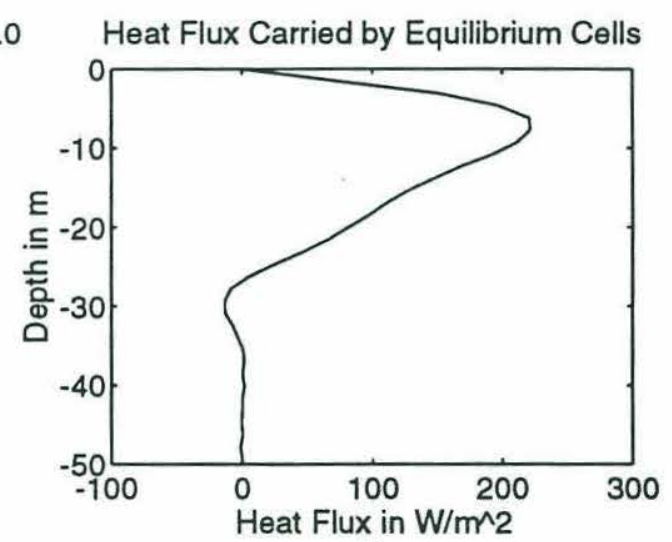

(f)

Figure 8.6: Velocity and Density structure on March 5th. (a) Observed velocity structure. (b)-(d) Predicted velocities (m/s). (b) From the PWP model. (c) From the MY2 model. (d) From the finite-difference code assuming finite-amplitude cells. (e) Horizontally averaged temperature structure. Solid line is structure at end on one day, dashed line initial condition. (f) Heat flux in $\mathrm{W} / \mathrm{m}^{2}$ at equilibrium $\mathrm{Ri}=1.0$. 
at equilibrium. The mean heat flux carried by the cells reaches $200 \mathrm{~W} / \mathrm{m}^{2}$, a remarkably large value to be sustained in the absence of strong surface winds. This result again illustrates the importance of including wave-current interaction as an energy source for stirring in the surface layer.

The presence of finite-amplitude cells changes the structure of the mixed layer on March 5th, although the change mostly occurs in the upper 20 meters of the water column. The model with finite-amplitude cells shows a mixed layer with a depth of approximately 25 meters, about twice the depth actually seen during March 5th. The mixing is very strong, carrying heat fluxes of approximately 200 $\mathrm{W} / \mathrm{m}^{2}$ in the mean. Langmuir cells are clearly sufficient to explain the failure of the mixed layer to restratify on March 5th during SWAPP.

\subsubsection{Discussion}

In Chapters 6 and 7, the current profile predicted by the Mellor-Yamada model, which assumes mixing on scales which are small compared with the mixed layer, was shown to be unstable to Langmuir cells when surface gravity waves are added to the picture. The size of the growth rates was an indication that the Craik-Leibovich Rayleigh number (RaCLS) was large. According to the theory developed in Chapter 5. this means that the cells should be more important for the transport at equilibrium than small-scale diffusion.

This section has verified the prediction that the finite-amplitude cells take over the transport of momentum and density within the interior of the mixed layer. As a result the mean shear within the mixed layer is far smaller than predicted by small-scale mixing - a result consistent with the two field experiments. Near the surface, the finite-difference code runs predict a remnant shear layer, in which small-scale turbulence is still responsible for carrying stress.

The details of the picture are somewhat different from the data. A shear layer was observed during SWAPP, but the size of the shear was smaller than that predicted by the MY2 model. During MILDEX, a near-surface layer with strong upwind shear was seen in the data but not in the model. Strong crosswind velocities were also predicted at the base of the layer in contrast to data.

Another difference between the model and the theory is the overall transport predicted within the mixed layer. In the presence of waves, Huang's equations predict an Eulerian transport in the upwind direction, balancing the Lagrangian transport associated with the Stokes drift. This upwind transport was 


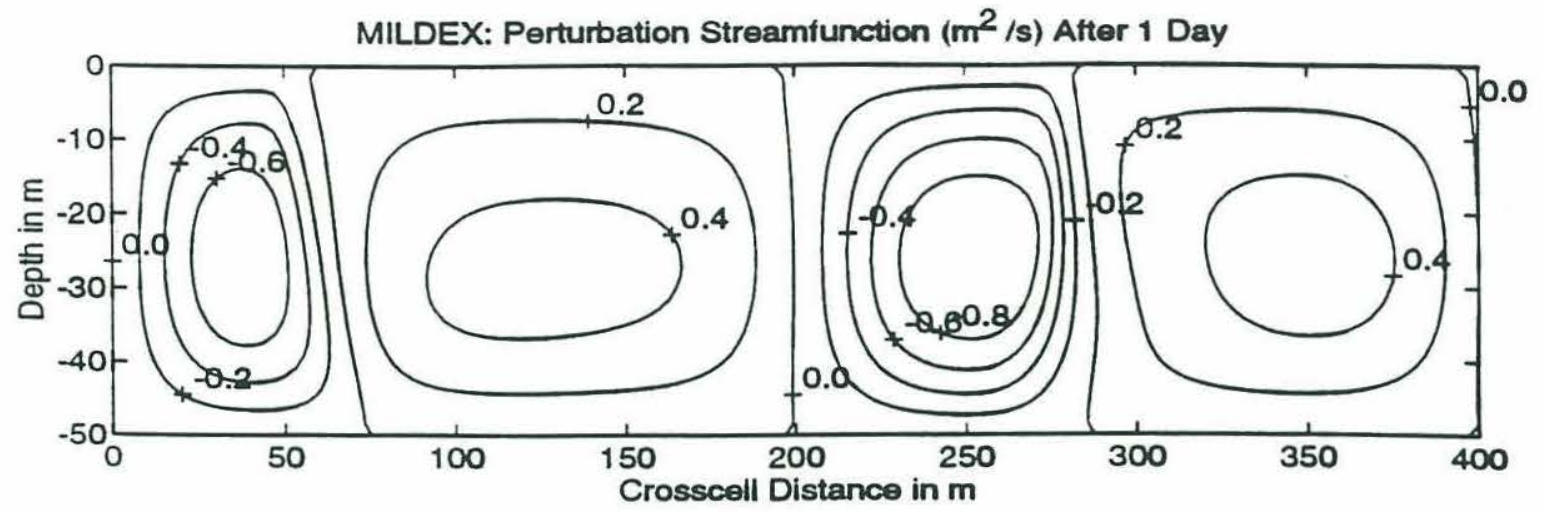

(a)

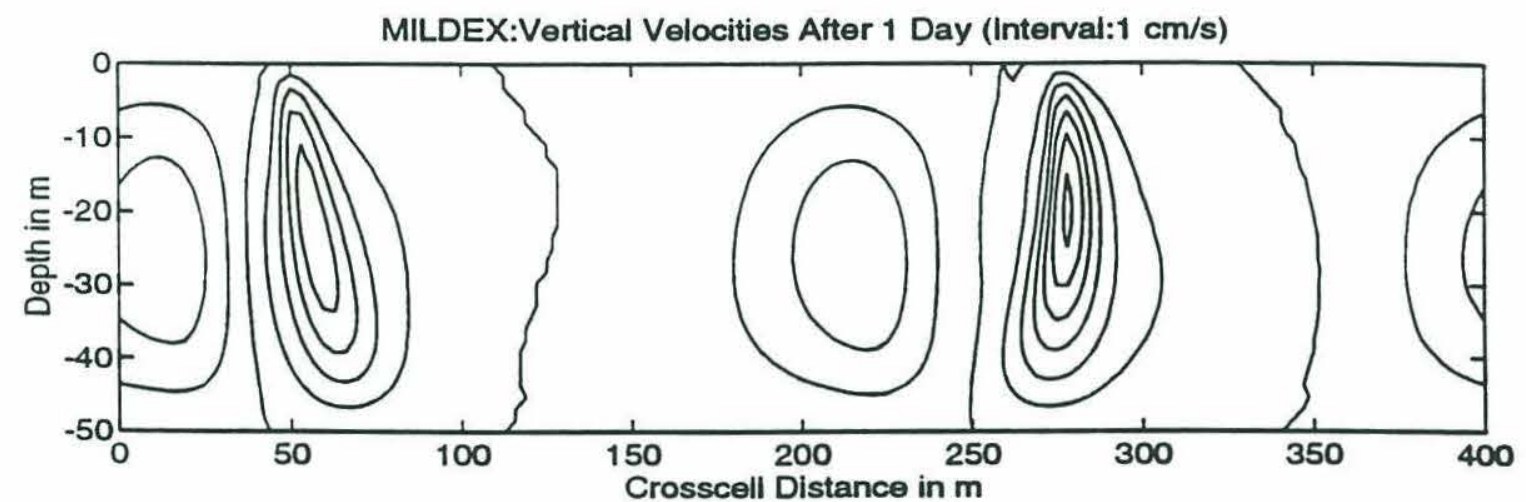

(b)

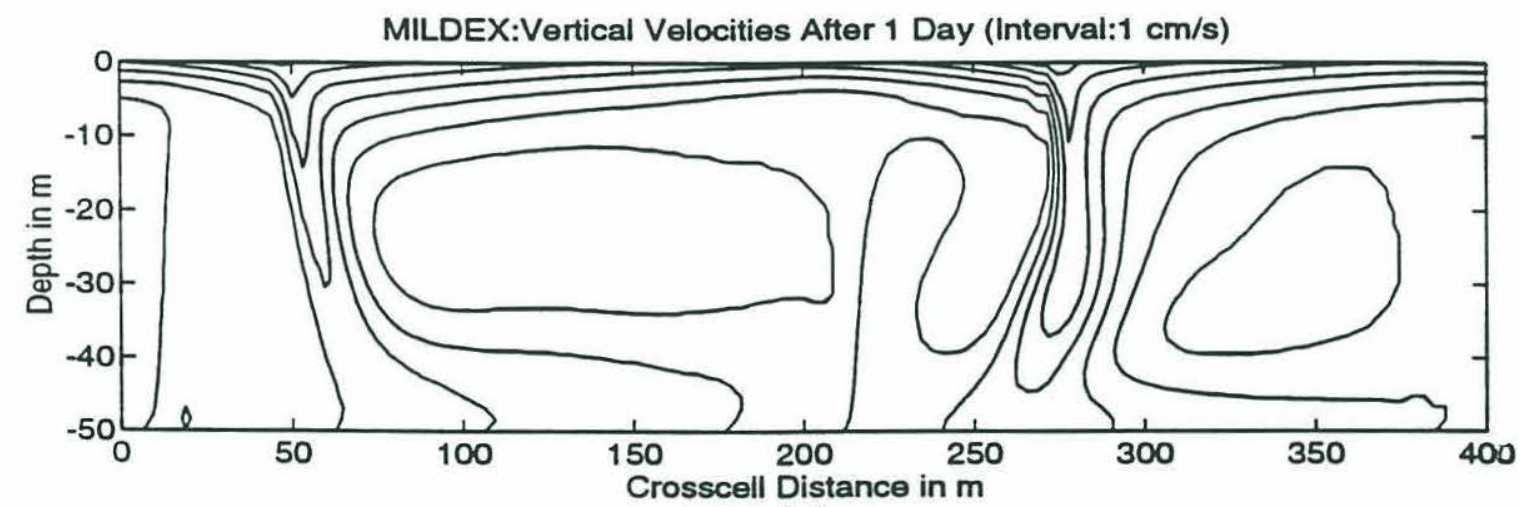

(c)

Figure 8.7: Cell structure during MILDEX. (a) Streamfunction in $\mathrm{m}^{2} / \mathrm{s}$.

(b) Vertical velocity in $\mathrm{cm} / \mathrm{s}$ (contour interval is $1 \mathrm{~cm} / \mathrm{s}$, strong velocities are downwelling). (c) Alongcell velocity in $\mathrm{cm} . \mathrm{s}$ (contour interval is $1 \mathrm{~cm} / \mathrm{s}$ ). 
not seen in either of the two experiments. The differences between theory and data are considered in more detail in the final section of this chapter.

\subsection{Horizontally and Temporally-varying Velocity and Shear during SWAPP and MILDEX}

Having considered the effect which the cells have on the mixed layer, we now turn to the question of the velocity and spatial structure of the cells themselves. Figure 8.7 shows a snapshot of the velocity structure during MILDEX after 1 day has elapsed and the model is more or less at equilibrium. Figure $8.7 \mathrm{a}$ shows the streamfunction in $\mathrm{m}^{2} / \mathrm{s}, 8.7 \mathrm{~b}$ the vertical velocity in $\mathrm{cm} / \mathrm{s}$ and $8.7 \mathrm{c}$ the alongcell velocities in $\mathrm{cm} / \mathrm{s}$.

The field of cells is dominated by a single scale, consisting of cells with an approximately 200 meter wavelength. The cells are associated with narrow plumes of downwelling approximately 25 meters across in which the vertical velocities reach $6 \mathrm{~cm} / \mathrm{s}$ and the alongcell velocities are up to $3 \mathrm{~cm} / \mathrm{s}$ larger than the mean velocity at a given depth. Both of these velocities are somewhat lower than those seen in the field. The cell spacing seen here is somewhat larger than the spacing seen during MILDEX. During the time periods when they were strongest, the cells had a spacing of about 100-140 meters (Figure 6.6). In a gross sense the model does predict cells of the right order of magnitude with respect to spacing and depth of penetration, but there is still a factor of 2 difference in the spacing.

The cell spacing predicted by the models is often quite a bit larger than that observed in the field. Figure 8.8 shows spectra of the crosswind horizontal velocity at the surface predicted by the finite-difference code. Figure $8.8 \mathrm{a}$ shows the results from SWAPP assuming the forcing to be described by the mean parameters over March 4th-13th. The solid line shows the spectrum when the layer is unstratified, the dashed line when stratification is imposed. In the absence of stratification, the velocity spectrum is dominated by very long cells, with wavelength of 500 meters. In the presence of stratification, the spectrum flattens somewhat, with a marked increase in the energy at high-wavelengths but the peak is still at wavelengths of 250 meters.

Figure $8.8 \mathrm{~b}$ shows the spectrum of surface crosswind velocity during MILDEX, again with and without stratification. In this case stratification does not changes the cell population measurably. The peak wavelength is for cells 250 meters across. Figure $8.8 \mathrm{c}$ shows the spectrum of surface crosswind velocity 


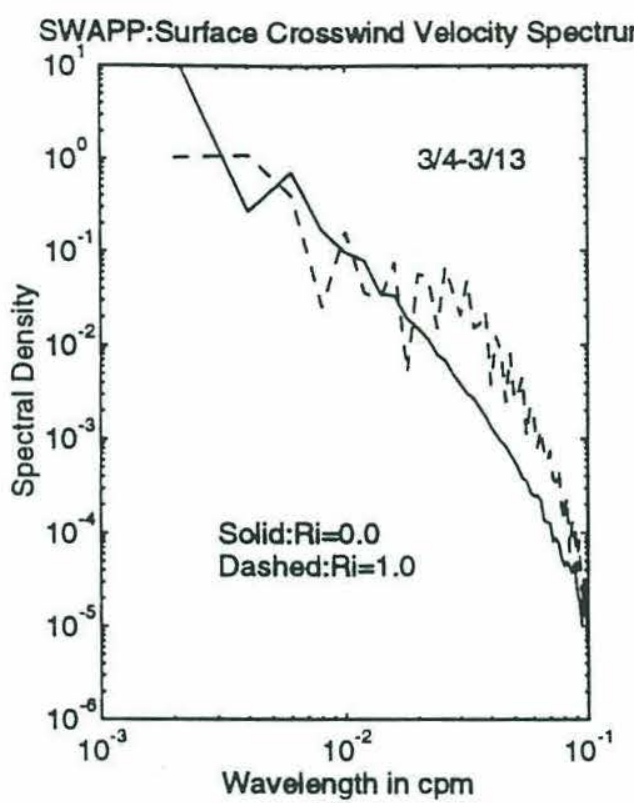

(a)

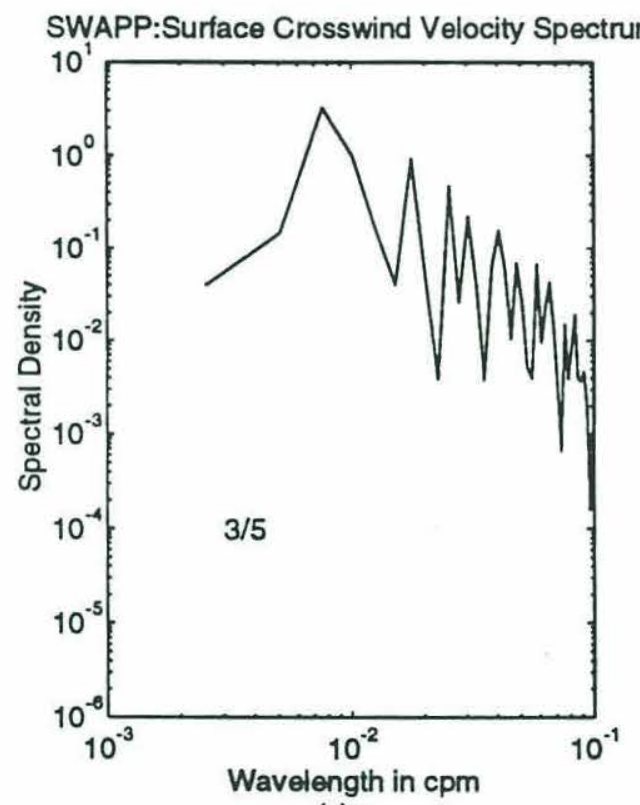

(c)

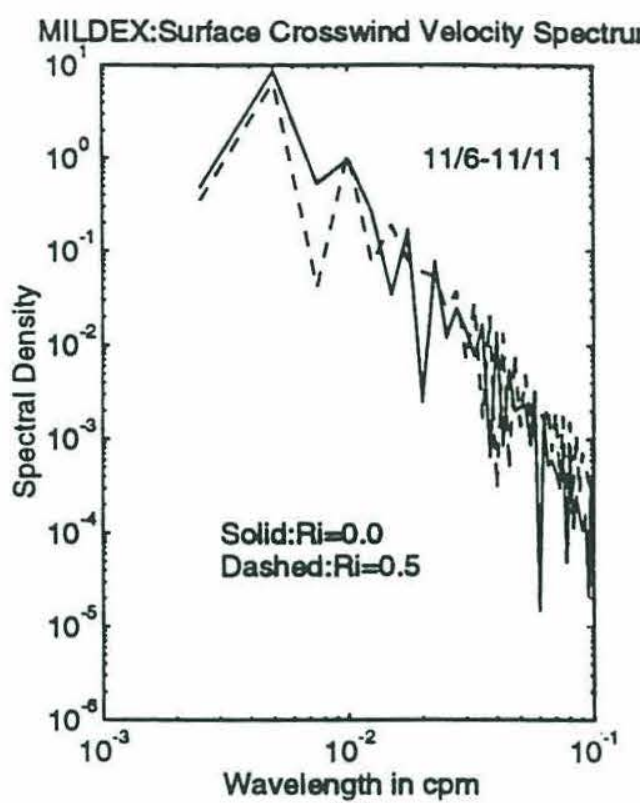

(b)

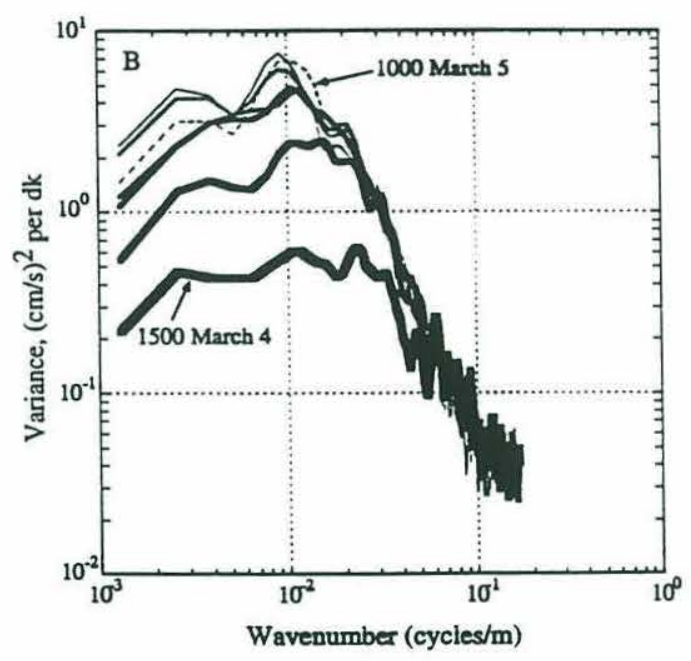

(d)

Figure 8.8: Spectra of horizontal velocities and shears during the two field experiments. (a) Crosswind velocity spectra during SWAPP from the finitedifference code assuming mean conditions from March 4th -13 th. Solid: $\mathrm{Ri}=0.0$, Dashed: $\mathrm{Ri}=1.0$ (stabilizing heat flux of $60 \mathrm{~W} / \mathrm{m}^{2}$ ). (b) Crosswind velocity spectra during MILDEX from the finite-difference code assuming mean conditions from November 6th -11 th. Solid: $R i=0.0$, Dashed: $R i=0.5$ (stabilizing heat flux of 60 $\mathrm{W} / \mathrm{m}^{2}$ ). (c) Crosswind velocity spectrum from finite-difference code during SWAPP assuming mean conditions on March 5th. (d) Spectrum of crosscell velocity from SIO sonars during SWAPP, March 4th and 5th (from Smith, 1993). 
during March 5th in SWAPP. The velocity shows a peak at about $125-160 \mathrm{~m}$, but there is also enhanced activity at higher wavelengths.

The structure of the velocity spectra during SWAPP can be compared with the SIO Doppler sonars. Figure $8.8 \mathrm{~d}$ shows spectra of the velocity seen by the sonars during SWAPP from Smith (1993). The time period shown covers a 21 hour period during which Langmuir cells were very strong on March 4th and 5th. The appropriate figure for comparison is Figure 8.8a. The spectra are noticeably peaked at a wavelength of about 120 meters, quite a bit smaller than the 250 predicted even in the presence of stratification. The peakedness is not very strong, only a factor of about 70 separates the spectral density at $0.01 \mathrm{cpm}$ and $0.1 \mathrm{cpm}$ in the data, while the model predicts a much faster falloff (about a factor of 3000). In general, the model predicts spectra with too much energy at long wavelengths and not enough at short wavelengths (even though wavelengths of 10 meters are resolved by the model and are unstable).

The vertical structure of cell velocities and shears is considered in Figure 8.9. The amplitude of the shear and velocity (defined as $\sqrt{2}$ times the standard deviation) is shown as a function of depth. The left-hand column shows the velocities, with crosscell horizontal velocities (x-direction) denoted by solid lines, alongcell horizontal velocities (y-direction) denoted by dashed lines, and vertical velocities denoted by chain-dotted lines. The right-hand column shows the shear. The solid lines are the crosscell shear, while the dashed lines are the alongcell shear. Because the results with an imposed stratification during March 4-13 in SWAPP gave a more realistic velocity profile and cell spacing they are used to compare with MILDEX and March 5th during SWAPP.

The model predicts that the strongest cells should have been seen during MILDEX, with both vertical and horizontal velocities being much larger than during SWAPP. This was in fact the case. During MILDEX large downwelling velocities (up to $20 \mathrm{~cm} / \mathrm{s}$ ) were seen, while during SWAPP they were not. The reason for the difference is apparently that the larger Stokes drift shears and smaller viscosities during MILDEX resulted in a larger effective Rayleigh number for the cells.

The highly asymmetric nature of the cells means that using the standard deviation leads to an underestimate of the peak velocities. For example, during MILDEX the peak downwelling velocities are more than $6 \mathrm{~cm} / \mathrm{s}$, while the amplitude in Figure 8.9a is only $2.5 \mathrm{~cm} / \mathrm{s}$. The upwelling velocities on the other hand, are of order $2.5 \mathrm{~cm} / \mathrm{s}$. Similar asymmetry was seen in the field data. 


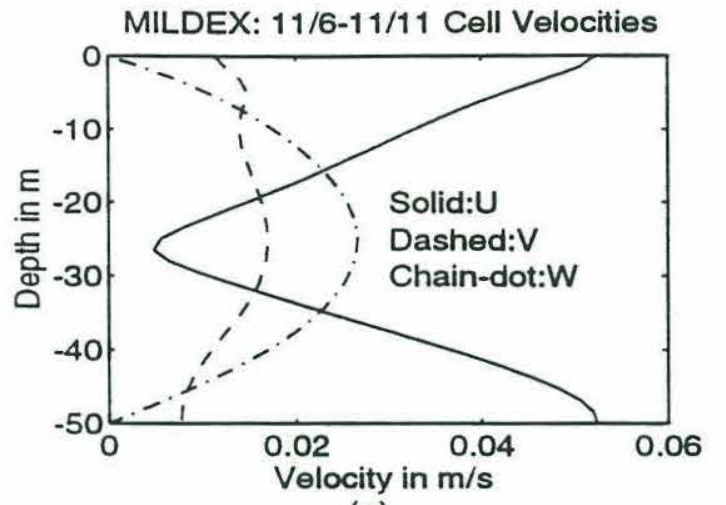

(a)

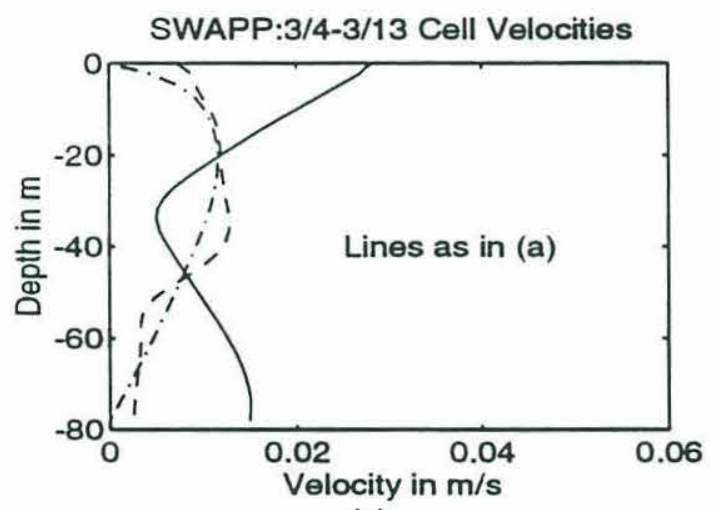

(c)

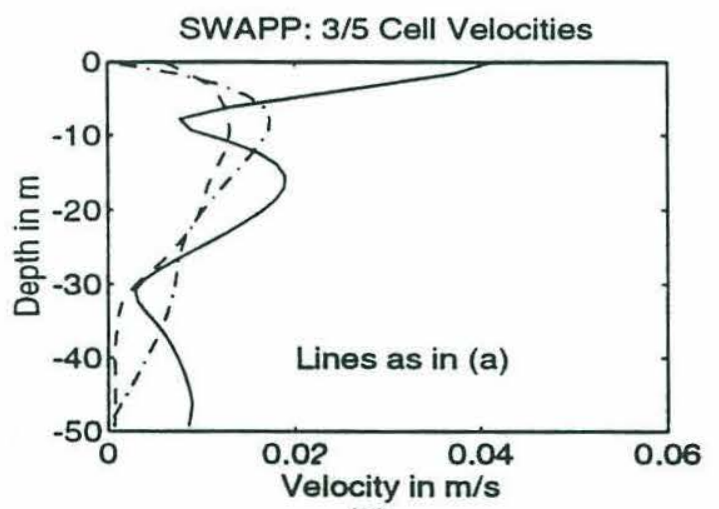

(e)
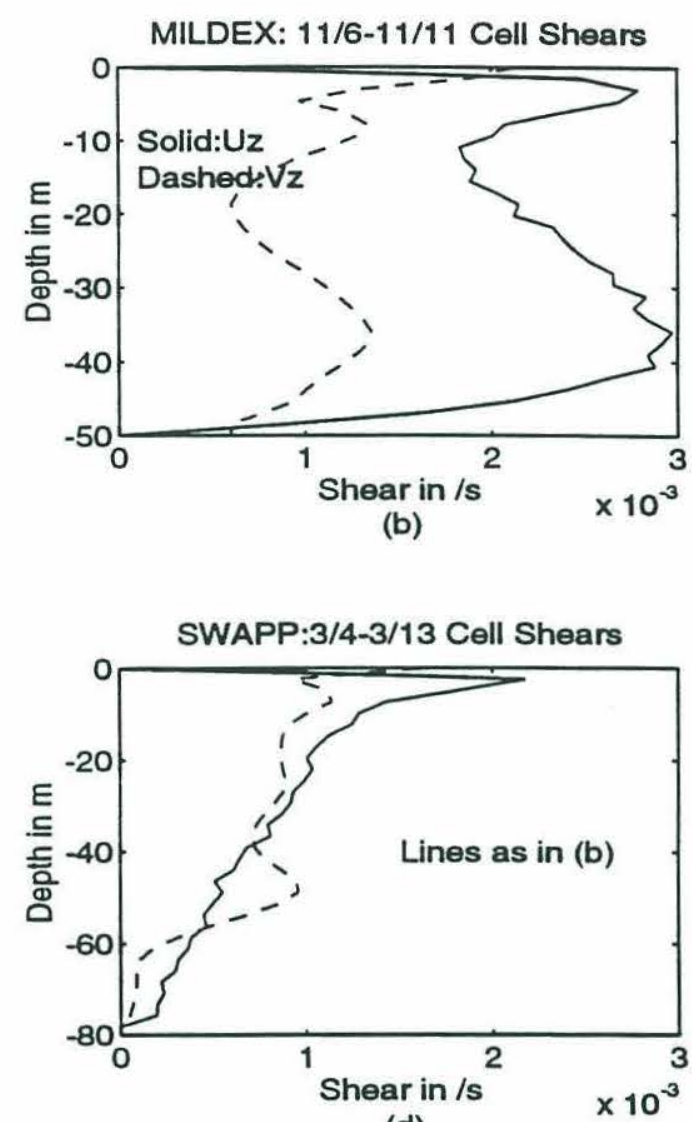

(d)

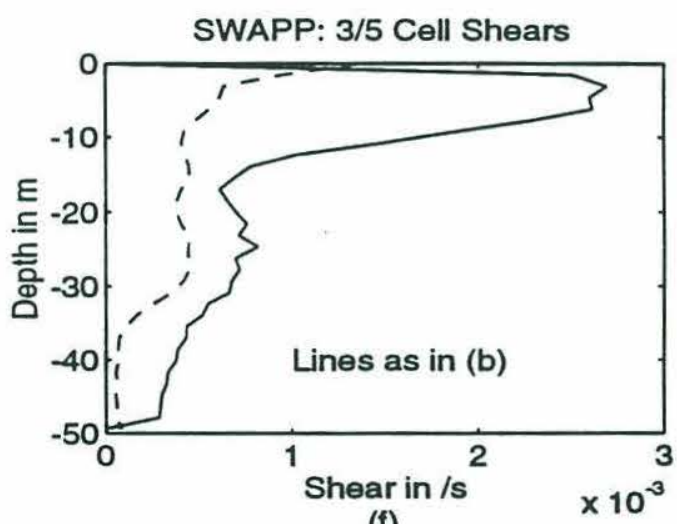

(f)

Figure 8.9: Velocity and shear structure during the two experiments. Left hand column is amplitude of the velocity fluctuation associated with the cells, with solid being the crosscell horizontal (U), dashed being the alongcell horizontal (V), and chain-dotted being the vertical (W). Right-hand column is the amplitude of the vertical shear of the horizontal current. Solid lines are the vertical shear of the crosscell current $(\partial \mathrm{u} / \partial \mathrm{z})$, dashed lines are vertical shear of alongcell current $(\partial \mathrm{v} / \partial \mathrm{z})$. (a) Velocities from MILDEX assuming mean conditions, November 6-11th. (b) Shears from MILDEX, November 6-11th. (c) Velocities from SWAPP, March 4th-13th. (d) Shears from SWAPP, March 4th-13th. (e) Velocities from SWAPP, March 5th. (f) Shears from SWAPP, March 5th. 
The difference in cell strength between SWAPP and MILDEX is not reflected in the shear. During SWAPP the perturbation shear is smaller than during MILDEX (about 30-40\%) but not as much smaller as was seen in the data. This is despite the fact that an approximate theory which assumed cells of about 10 meters depth did an excellent job at reproducing both the level and temporal variability of the high-frequency shear during both SWAPP and MILDEX. The reason for the failure of the model to reproduce the level of the perturbation shear is most likely that the short-wavelength cells which were assumed to contribute to the high-frequency shear in the field experiments are absent in the model.

\subsection{Conclusions and Discussion}

In a qualitative sense, the finite-difference code runs validate the hypothesis that Langmuir cells were more important than small-scale diffusion in homogenizing the mixed layer during SWAPP and MILDEX. As expected from the instability calculations, the finite-amplitude cells do replace small-scale diffusion as the major transport mechanism over some part of the layer. This supports the idea that Langmuir cells are the fast processes implicitly included in slab models which homogenize the mixed layer. The models also reproduce the observational result that the cells were stronger during MILDEX than during SWAPP.

In a quantitative sense, however, the agreement is not as good. The finitedifference code predicts a surface shear layer which is stronger than actually observed during SWAPP and one in which the shear is downwind rather than upwind during MILDEX. The code also predicts strong crosscell velocities near the base of the mixed layer during MILDEX and SWAPP which are not seen in reality (although adding some stratification to the SWAPP results does produce a more realistic velocity and shear profile). Lastly, the predicted mean Eulerian transport includes an component which balances the Stokes drift flow which is not seen in the data.

The difference between the predicted and observed surface shear layers may be attributed to the fact that the models underestimate the effect of cells near the surface. As seen in section 8.4 the models predict very long-wavelength, essentially monochromatic cells during MILDEX. Over the course of Chapters 2 and 3, it was demonstrated that short-wavelength cells were much more efficient than these long-wavelength cells at taking advantage of the high shears near the 
surface. The failure of the models to capture the observed horizontally averaged structure is probably linked to their failure to capture the short-wavelength cells.

The presence of crosscell velocities near the base of the mixed layer may be explained by considering the Ekman balance. For every layer contained within the surface layer, in order for a time-mean equilibrium to hold, the Coriolis force in the crosswind direction must balance the alongwind stress. If $U(z)$ is the horizontally averaged velocity in the crosswind direction,

$$
\mathrm{F} \int_{\mathrm{z}_{1}}^{\mathrm{z}_{2}} \text { Crosswind velocity } \mathrm{dz}=\text { Alongwind Stress } \mathrm{I}_{\mathrm{z}_{1}}^{\mathrm{z}_{2}}
$$

As the problem is currently cast, the alongwind stress must vanish when $\mathrm{z}=-\mathrm{D}$, so that there can be no interaction with the thermocline. This means that if the cells penetrate to depths near $\mathrm{D}$, carrying large alongwind stress, there must be strong crosswind velocities or strong shears to the left of the wind to balance this stress. Both of these are seen in the unstratified runs in MILDEX and SWAPP. The problem then is either that the bottom boundary condition on velocity is inaccurate, that the cells penetrate too close to the bottom boundary, or some combination of the two.

The remaining discrepancy between theory and data is the lack of a wave return flow in the Eulerian transport. The theory developed during Chapter 3 predicted such a return flow but it was not seen either during MILDEX (Figure 6.19) or SWAPP (Figure 7.17 also Weller and Plueddemann, 1994). There is no good explanation for this discrepancy at the present time, only speculations. One possible explanation is that the reference level for the data was improperly chosen in SWAPP and MILDEX. If the reference depth chosen was one for which there was actually wave return flow, the resulting transport would be biased in the downwind direction. This is unlikely, however, for the following reasons.

-During both SWAPP and MILDEX shifting the reference level higher in the water column would have reduced the crosswind as well as the alongwind transport. As the crosswind transport was fairly close (within 10\%) to the classical Ekman transport this makes the problem of the transport worse rather than better. -Shifting the reference level lower in the water column would require moving it well below the mixed layer into the main thermocline. In the absence of mixing, the return flow in the main thermocline should just be equal and opposite to the 


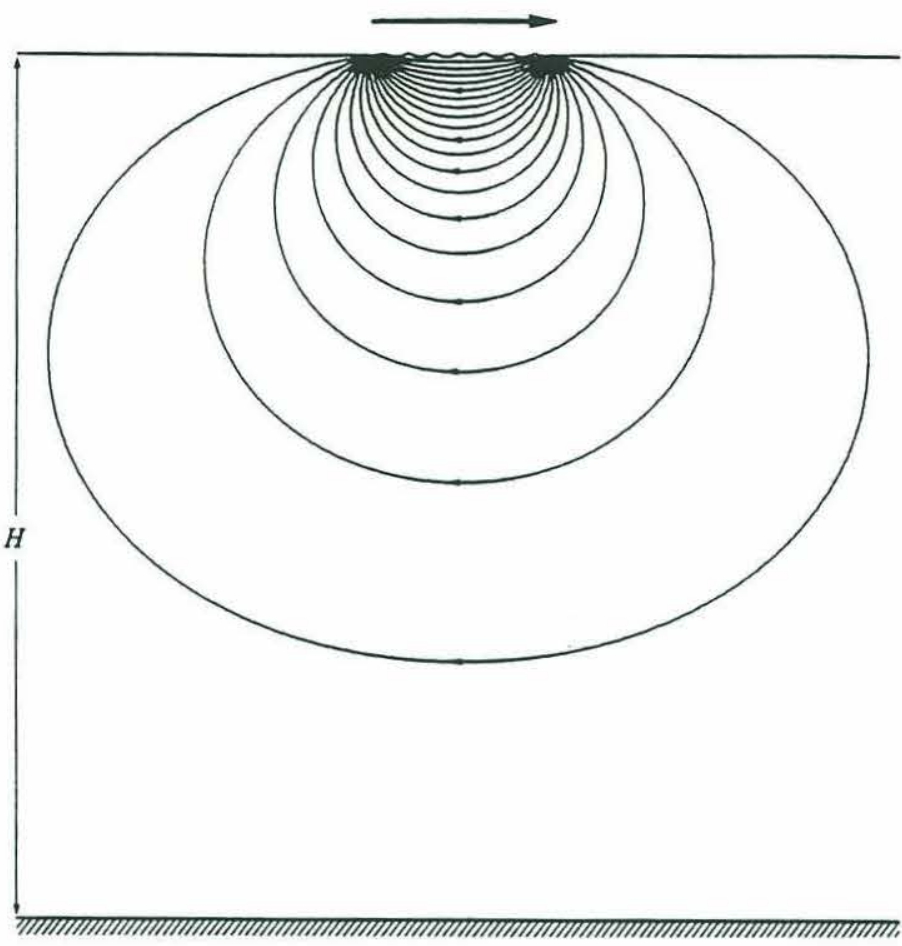

Figure 8.10: Picture of momentum associated with a finite-length wave group in deep water, from McIntyre (1981). Near-surface Stokes drift is balanced by a deep return flow. The irrotational return flow underneath the wave packet is shown for a packet of surface gravity waves propagating to the right. A third, equivalent contribution propagates with the speed of the long-wave group. 
Stokes drift (Chapter 3). For SWAPP, the return flow at $70 \mathrm{~m}$ would be less than $0.5 \mathrm{~cm} / \mathrm{s}$

-Weller and Plueddemann, 1994 applied an EOF analysis to isolate the Ekman response during SWAPP which was coherent with the wind. The structure of this response is not dependent on reference level. Since the wind and waves are correlated, this analysis should have captured the wave return flow. In fact, the transport calculated from the EOF analysis was almost exactly the classical Ekman transport.

One possible explanation why a wave return flow is not seen is that wave groups have finite-length. Figure 8.10 is taken from McIntyre (1981) and shows the structure of the momentum associated with a finite-length group of surface gravity waves. A finite-length group of gravity waves has a pressure disturbance associated with it. This pressure disturbance will force a return flow whose transport balances the Stokes drift. A third phenomenon associated with the pressure disturbance is a long-wavelength wave which propagates away from the group. The momentum associated with this long wave is the same as that associated with the Stokes drift. The upshot is that the momentum associated with a finite-length wave group may not actually be co-located with the group itself, and so neither will the return flow. This is speculation, however, as the equations for such a case have not been worked out.

Two of the major shortcomings of the finite-difference code results have been linked to the fact that they fail to reproduce the range of scales seen in the open ocean experiments. One problem is that once large-scale cells form in the models they persist. Additionally, there is no mechanism that can "reseed" the smaller scales quickly enough in order for them to grow on top of the larger cells.

Four possibilities for explaining the failure of the finite-difference code to produce multiple scales of cells are listed below.

-The models assume a surface stress which is constant spatially. In reality the surface stress is horizontally heterogeneous as the result of breaking waves, cat's paws in the wind, and so forth. Such heterogeneity might be able to provide initial perturbations for small-scale cells to grow atop the larger-scale cells. -The mean equilibrium structure forced by the average parameters has been compared to a mixed layer in which there is time-dependent forcing. If the forcing in the ocean mixed layer changes quickly enough, the mixed layer may never reach equilibrium. 
-The boundary conditions which were chosen, in which the bottom is taken as a no-stress boundary with respect to momentum but fixed with respect to flux are not realistic. They tend to favor very long-wavelength cells which do not feel the effects of stratification easily (the density perturbations are much more strongly damped than the velocity perturbation, Chapters 3 and 5).

-Since they are two-dimensional, the models neglect three-dimensional merging processes such as those studied by Thorpe (1992). These, rather than the relatively slow two-dimensional merging processes studied in Chapter 4, may be responsible for limiting both the persistence of the large-scale cells and their horizontal extent.

Of these four explanations for the failure to reproduce the cell population, only the first two are testable within the basic assumptions of Huang's equations. A few simple finite-difference code runs have been done to test whether or not the inclusion of forcing which varies over space and time can explain the failure of the models to reproduce the spatial structure of the cells.

It is possible to examine the question of spatial and temporal variability in the wind stress by changing the upper boundary condition on velocity so that $(8-4)$

$$
\mathrm{La} \frac{\partial \mathrm{v}}{\partial \mathrm{z}}=\frac{\tau}{\rho}\left(1+\delta \sin \left(2 \pi \mathrm{x} / \mathrm{L}_{\mathrm{c}}\right) \sin \left(2 \pi \mathrm{t} / \mathrm{t}_{\mathrm{c}}\right)\right)
$$

The purpose of this change is to simulate surface variability which can result in changing the horizontal velocity and shear spectrum associated with the cells. In order to do this one must first choose the time and space scales associated with the perturbations. In the field, Edson (pers. comm.) and others have found that it is necessary to average sonic anemometers $30 \mathrm{~m}$ apart for at least 30 minutes in order to obtain consistent estimates of the stress. The surface stress is allowed to vary with a dimensional time period of 30 minutes and dimensional length scale of $30 \mathrm{~m}$. (This length scale was chosen as being close to the fastest-growing mode).

Model runs made with the revised boundary conditions do not differ substantially from those made with spatially constant boundary conditions. The mean Ekman spirals are found to exhibit somewhat different shear profiles in the middle of the water column, where the shear was small to begin with, but there is no evidence at present that small-scale cells are generated given the parameter settings used to model the response during MILDEX and SWAPP.

The question of temporal variability may be considered by allowing the surface density flux to vary so that the cells are partially suppressed during the day and reappear at night. Runs made in the absence of rotation generally 
showed that unless the cells were totally erased, the long-wavelength cells dominated for all time periods. The fundamental problem is that for most cases in our two-dimensional framework, the long-wavelength cells are hard to kill off. When the boundary conditions change, they may become weaker or stronger but they do not disappear and clear the way for smaller-scale cells.

To summarize then, finite-difference code results support the idea that Langmuir cells are responsible for maintaining the mixed layer. The velocity and density profiles set up within the mixed layer by small-scale diffusion during SWAPP and MILDEX are unstable to cells. When these cells grow to finiteamplitude, they replace small-scale diffusion as the major mechanism for transport. The quantitative results are sometimes dependent on the degree to which dense water is entrained from the bottom of the mixed layer. The finite-difference code runs do not predict the cell spacing at equilibrium very well, and so do not capture the quantitative structure of the mixed layer. Inclusion of threedimensional Langmuir cells is probably necessary to capture the full quantitative structure of the mixed layer. 


\section{Chapter 9: Conclusions and Discussion}

\subsection{Summary of the Main Results}

This work examines whether Langmuir cells driven by wave-current interaction or diffusion associated with isotropic turbulence is the primary transport mechanism for momentum and density within the oceanic mixed layer. A natural response based on knowledge of Rayleigh-Benard convection is that organized convection is more important than diffusion when the characteristic scale for the forcing is larger than the characteristic scale for diffusion. However, the wave-current interaction mechanism of Craik and Leibovich is intensified near the surface, making it difficult to define a characteristic scale for the forcing. Additionally, because the forcing is so surface-intensified it is not initially obvious whether the mechanism can drive cells which penetrate over the depth of the mixed layer.

Equations for two-dimensional Langmuir cells in the presence of stratification and Coriolis forces assuming a mixed layer in which the diffusive coefficient is constant were introduced by Huang (1979). The approach taken in this thesis has been to solve these equations for the equilibrium state in the absence of cells, to calculate the instability of this state to Langmuir cells, and to consider how the finite-amplitude cells modify the velocity and density structure at equilibrium.

Chapter 2 considered the problem of instability in a layer in which the surface stress was balanced by a pressure gradient and the wind, waves, and axis of cell orientation were all parallel. Using the energy balance for unstable cells, the strength of the forcing was shown to go as (page 39):

$$
\gamma_{C L S}^{2}=\frac{4}{D^{2}}\left(\int_{-D}^{0} F(z) \frac{\partial v_{s}}{\partial z} d z \int_{-D}^{0} F(z) \frac{\partial V}{\partial z} d z-\int_{-D}^{0} G(z) \frac{g \partial \rho}{\rho \partial z} d z\right)
$$

where $\mathrm{v}_{\mathrm{s}}$ and $\mathrm{V}$ are the Stokes drift and Eulerian velocity parallel to the alongcell axis, $\mathrm{z}$ is the vertical axis and $\rho$ is the density. The functions $\mathrm{F}(\mathrm{z})$ and $\mathrm{G}(\mathrm{z})$ are weighting functions which depend on the boundary conditions and are proportional to the nonlinear flux of momentum and buoyancy carried by the most unstable mode at a given horizontal wavenumber. At the surface $F(z)=G(z)$ $=0$ since the vertical velocities associated with Langmuir cells are zero. This means that the important shears for driving Langmuir cells are not those right at 
the surface, but those at depths where v'w' is large. The square of the ratio of $\gamma_{C L S}$ to the characteristic diffusive decay scale $\gamma_{\text {diff }}$ is the stratified Craik-Leibovich Rayleigh number RaCLS.

$$
\text { RaCLS }=\gamma_{\text {CLS }}^{2} / \gamma_{\text {diff }}^{2}
$$

When RaCLS is large the growth rate was shown to go as:

$$
\gamma \sim \frac{\mathrm{k}}{\sqrt{\mathrm{k}^{2}+\left(\pi / \mathrm{D}^{\prime}\right)^{2}}} \gamma_{\mathrm{CLS}}
$$

where $\mathrm{k}$ is the horizontal wavenumber and $\mathrm{D}^{\prime}$ is the depth of penetration of the cells. Physically, the growth rate and structure of the unstable cells with a given wavelength was shown to be determined by a tradeoff between:

-Maximizing the strength of the forcing.

-Maximizing the efficiency of the forcing at reinforcing the instability, corresponding to maximizing the aspect ratio $\mathrm{D}^{\prime} / \mathrm{L}$.

-Minimizing the characteristic diffusive scale.

A principal result of this tradeoff is that long-wavelength cells penetrate more deeply into the mixed layer than shorter-wavelength, more quickly growing cells. Because the bottom boundary conditions for density and velocity were assumed to be different, at long wavelengths the effect of stratification was found to vanish. In order for the finite-amplitude cells to replace small-scale diffusion as the dominant transport mechanism over the majority of the mixed layer, such long-wavelength cells must dominate the solution at equilibrium.

Chapter 3 considered instability of an Ekman spiral in the presence of surface gravity waves. It was shown that the waves induce an Eulerian return flow whose transport balances the Stokes drift. The importance of CraikLeibovich instability depends on the Ekman number $E k=v_{e} k_{w}^{2} / f$, where $v_{e}$ is the eddy viscosity, $\mathrm{k}_{\mathrm{w}}$ is the wavenumber of the gravity waves, and $\mathrm{f}$ is the Coriolis frequency. When Ek is very small, the return flow is equal and opposite to the Stokes drift over much of the depth and wave-current interaction is less important for driving unstable roll vortices. When Ek is large, wave-current interaction is the primary driving mechanism for roll vortices. The presence of the Coriolis force was found to cause crosscell shears, which stabilized the cells at low wavenumber. In the presence of stratification, the cell penetration depth was limited by this crosscell shear.

Although Chapters 2 and 3 isolated the important physical parameters governing Langmuir cell instability, these parameters were found to depend critically on the vertical scale of the cells. This scale was in turn found to depend 
on the horizontal scale. In order to determine whether or not the cells were important, it was necessary to ascertain which horizontal scale dominated the flow at equilibrium. Chapter 4 considered this problem. It was shown that the dominant scale at equilibrium is generally larger than that associated with the fastest growing mode. In the absence of Coriolis force, the cell spacing increases without discernable limit. As a result, the cells were predicted to penetrate over the entire depth of the mixed layer at long enough times. However, the evolution of flow field to large scales is very slow after the cells establish a quasi-equilibrium state, so that in the field Langmuir cells might never have enough time to reach an equilibrium state in which they penetrate over the depth of a given mixed layer. The physical process governing cell merging was hypothesized to be one in which large-scale vortices advect smaller-scale plumes of alongcell velocity, creating velocity structure with larger wavelengths. This large-scale velocity structure then feeds back on the large-scale vortices through the Craik-Leibovich instability mechanism.

In the presence of Coriolis forces, the cascade to large horizontal scales is halted so that the cells do not penetrate over the entire surface layer. The dominant mode at equilibrium is generally one with a larger wavelength than that associated with the fastest-growing mode, but a smaller wavelength than the longest unstable mode. Some mechanisms for the interruption of the cascade were considered, but only in a speculative way,

Chapters 2 and 3 showed that when RaCLS the equilibrium layer set up by small-scale diffusion is unstable to Langmuir cells. Chapter 5 considered the effect of such cells on the velocity and density structure within the mixed layer when they grow to finite amplitude. When RaCLS is large, Langmuir cells replace smallscale diffusion as the principal vertical transport mechanism within the surface layer. Near the upper surface there is a layer within which turbulent diffusion was important but elsewhere Langmuir cells are the dominant transport mechanism. The vertical shear of the horizontal velocity was shown to scale as $\gamma_{C L S}$.

When only one linearly unstable mode is present at the dominant cell spacing, RaCLS can be estimated from the shape of the unstable mode, providing a simple way of estimating the importance of Langmuir cells as a transport mechanism. Simple truncations which capture the shape of the nonlinear momentum and density transport were also found to yield accurate predictions of the horizontally averaged velocity and density profiles at equilibrium. Thus by 
knowing the shape of the cells, one can predict their effect on the mixed layer at equilibrium.

Chapter 6 applied the theoretical results to data collected during the Mixed Layer Dynamics Experiment (MILDEX). It was demonstrated that the shear in a high-frequency band (1-30 cph) did in fact scale as $\gamma_{C L S}$, providing evidence that wave-current interaction did drive the cells. When $\gamma_{C L S}$ was large, the velocity structure within the mixed layer showed evidence of mixing accomplished quickly by large eddies rather than more slowly by small-scale eddies, in that it was far less sheared than predicted by a Mellor-Yamada model. However, the data showed shear within the isothermal mixed layer, a result not produced by a slab model which implicitly assumes homogenization of the mixed layer by large eddies.

Instability code solutions revealed that the Ekman spiral predicted by assuming small-scale mixing was strongly unstable to two-dimensional roll vortices in the presence of surface gravity waves, but not in their absence. Since such large vortices were in fact seen, this is evidence that wave-current interaction was in fact driving them.

Chapter 7 extended the results of Chapter 6 to data gathered during the Surface Waves Processes Program (SWAPP). The results from this experiment generally supported those from MILDEX, although the cells were weaker. A particularly interesting result was that $\gamma_{C L S}$ was found to predict not only the level of high-frequency shear, but the time evolution of the Langmuir cell strength measured by sonars. During SWAPP, the mixed layer failed to restratify on two days following strong wind events. This failure could be explained when wave-current interaction was presumed to drive the Langmuir cells.

Chapter 8 compared the velocity and density structure observed during SWAPP and MILDEX with solution from a finite-difference code. The finitedfference code results were found to produce mixed layers which were less sheared than those predicted by a small-scale mixing model, but more sheared than predicted by a slab model. On one day when both one-dimensional models erroneously predicted restratification, the finite-difference code predicted mixing. The picture which emerges from the two experiments is one in which Langmuir cells grow on and greatly modify the velocity and density profile established by small-scale mixing.

Quantitative disagreements between the observed structure and that predicted by the finite-difference code may be attributable to the failure of the 
two-dimensional theory to correctly predict the range of cells observed in the field. An additional discrepancy between theory and data is that the Eulerian return flow predicted in Chapter 3 was not seen in either experiment.

These results have important implications for mixed layer dynamics, and thus in turn for a number of problems of interest to a wide range of marine scientists. Section 9.2 discusses how the presence of Langmuir cells affects the dynamics of the mixed layer. Section 9.3 considers how Langmuir cells are relevant to a number of other oceanographic problems. Section 9.4 concludes this thssis by examining avenues for future research on Langmuir cells.

\subsection{The Role of Langmuir Circulations in Mixed Layer Dynamics}

This thesis has shown that Langmuir cells are an important process for maintaining mixed layers. Some results of particular importance are as follows: -Langmuir cells play an important role in determining the mean velocity structure. of the upper ocean. By homogenizing the mixed layer, they play a critical role at determining the velocity shear at the layer base. Insofar as slab models capture mixed layer evolution, they do so because Langmuir cells homogenize the mixed layer.

-Wave-current interaction serves as a source of energy for driving the cells. This means that mixed layer dynamics is coupled with surface wave dynamics.

- The cells are extremely efficient at transporting density relative to small-scale turbulence. Even if cells play a minor role in the total turbulent kinetic energy budget, they may be very important sources of energy for entrainment and mixing.

-The cells set the "cycle time" for particles to make a circuit from the surface to the base of the mixed layer and back again.

-The spatially coherent, temporally persistent velocity circulations associated with the cells mean that particles which maintain some depth will be carried into convergence zones. As a result, the velocity seen by such particles will not be representative of the mean velocity of the mixed layer as a whole.

A natural question which arises is how best to incorporate these results in operational models of the mixed layer. Two suggestions present themselves, although both have some problems. The first possibility is that the Stokes production could be added as a source of energy for mixed layer deepening within slab models. The problem with this approach is that it will overestimate the 
contribution of Langmuir cells when the mixed layer is either very weakly or very strongly stratified, when RaCLS is near 1, or when the waves are at a strong angle to the shear. Additionally, it will not improve the accuracy of the velocity structure predicted by the slab models. A second possibility is that the truncated model solutions introduced in Chapter 5 could be used to modify the velocity solutions predicted by the Mellor-Yamada model. In order to do this, however, better understanding of the dynamics governing cell spacing needs to be developed.

In some cases (as during SWAPP) Langmuir cells are responsible for creating the mixed layer rather than merely maintaining it. It is reasonable to ask whether such cases represent the rule rather than the exception, so that CraikLeibovich instability rather than small-scale shear instabilities is responsible for mixed layer creation. In this case, the depth of penetration of the cells would determine the depth of mixed layer.

One problem with framing the question in such a way is that the concept of a mixed layer is not very well defined. Insofar as it represents a region of low stratification, a mixed layer can be created by high levels of small-scale turbulence as well as by large-scale Langmuir cells. At some level this is a "chicken and egg" problem, since both Langmuir cells and small-scale turbulence reduce the stratification and both grow to larger amplitudes when the stratification is small. The turbulent diffusive coefficient during SWAPP and MILDEX was estimated at around $200 \mathrm{~cm}^{2} / \mathrm{s}, 200$ times as large as the canonical value for the thermocline. Given a one-dimensional balance, the mean stratification in the mixed layer could then be much smaller than that in the thermocline without having to invoke Langmuir cells. On the other hand, the high levels of near-surface turbulence may be due to the low stratification, which allows such turbulence to grow and persist.

Insight into which process is more important can be gained by considering the scaling of the growth rate as the eddy visocity becomes very small. From Chapters 2 and 3, the growth rate of Langmuir cells goes as:

$$
\gamma_{\mathrm{CL}} \sim \sqrt{\frac{\partial \mathrm{V} \partial \mathrm{v}_{\mathrm{S}}}{\partial \mathrm{z} \partial \mathrm{z}}-\mathrm{N}^{2}} \sim \sqrt{1 / \rho v_{\mathrm{e}}} \sqrt{\tau \cdot \frac{\partial \mathrm{v}_{\mathrm{s}}}{\partial \mathrm{z}}-\mathrm{Q} / \mathrm{c}_{\mathrm{p}}}
$$

while the growth rate of Kelvin Helmholtz instability goes as (from Howard, 1960):

$$
\gamma_{\mathrm{KH}} \sim \sqrt{\frac{1}{4}\left(\frac{\partial \mathrm{V}}{\partial \mathrm{z}}\right)^{2}-\mathrm{N}^{2}} \sim \frac{1}{\mathrm{v}_{\mathrm{e}}} \sqrt{\frac{1}{4} \tau^{2}-\mathrm{v}_{\mathrm{e}} \mathrm{Q} / \mathrm{\rho c} \mathrm{p}}
$$


As the eddy viscosity drops, the growth rate for Kelvin-Helmoholtz instability increases faster than that for Langmuir cells. If $v$ is the molecular viscosity, the condition for Craik-Leibovich instability to be more important than shear instability in creating the mixed layer is that

$$
\tau / \rho v<\frac{\partial v_{s}}{\partial z}
$$

where the Stokes drift shear is appropriately chosen for the mixed layer depth of interest. Given $\frac{\partial v_{s}}{\partial z}$ of order $0.1 \mathrm{~s}^{-1}$ (a rather large value) and a kinematic viscosity of $10^{-6} \mathrm{~m}^{2} / \mathrm{s}$, shear instability is more important than Craik-Leibovich instability for initially creating the mixed layer if wind stress is above $10^{-4} \mathrm{~Pa}$. This suggests that for very small viscosity, the surface stress is initially carried into the water column by Kelvin-Helmholtz instability rather than by Langmuir cells.

Once a region with low stratification is established, however, Langmuir cells become more important as a transport mechanism. If the turbulence is strong enough so that the eddy diffusivities are of order $0.01 \mathrm{~m}^{2} / \mathrm{s}$, Craik-Leibovich instability will be more important than Kelvin-Helmholtz instability for wind stresses less than $1 \mathrm{~Pa}$ (larger than observed during both SWAPP and MILDEX). Langmuir cells can be the dominant transport mechanism in mixed layer maintenance even if they are not the dominant process in mixed layer creation.

\subsection{Implications for Other Problems}

The conceptual picture of a mixed layer stirred by Langmuir cells has implications for a number of other problems in physical, chemical, and biological oceanography. A few of these are listed below.

-Lagrangian measurements of surface drift currents: Drifters are used to measure the Ekman flow within the mixed layer. The convergence of this flow is used to calculate the Ekman pumping which in turn drives the general circulation. Suppose an Ekman transport is $1 \mathrm{~m}^{3} / \mathrm{s}$, about the size of that seen during SWAPP and MILDEX. Measuring such transport in a 40 meter deep layer requires measuring a mean current of $2.5 \mathrm{~cm} / \mathrm{s}$.

Insofar as the velocity structure of the mixed layer is not homogeneous velocity measurements made by drifters will be biased. This work has treated three possible sources of bias.

1. The Stokes drift.

2.The vertical shear of the horizontal current. 
3. The alongwind jets associate with convergence zones.

The results from this work suggest that all of these biases are significant. The surface Stokes drifts during SWAPP and MILDEX are of order $10 \mathrm{~cm} / \mathrm{s}$. Although the presence of Langmuir cells does reduce the amount of alongwind shear in the mixed layer, a surface shear layer is predicted in which the shear is of order $0.005 \mathrm{~s}^{-1}$ and which is at least $5 \mathrm{~m}$ deep. A surface drifter will therefore have a bias of approximately $2.5 \mathrm{~cm} / \mathrm{s}$ in the alongwind direction relative to the mean mixed layer velocity. The peak velocities associated with the jets were measured as being of order $10 \mathrm{~cm} / \mathrm{s}$ during MILDEX and modelled as being of order $5 \mathrm{~cm} / \mathrm{s}$, giving a large bias in the alongwind direction. In combination these results suggest that the velocity measured by a surface drifter will be biased in the alongwind direction with respect to the mean mixed layer flow.The size of the bias is potentially large in comparison with the velocities associated with the Ekman transport. As a result estimates of Ekman pumping based on drifters will include errors which are proportional to the wind stress divergence. Moreover, droguing the drifter to some depth will not necessarily help. If the depth at which the drogue is located is above the center of the mixed layer, Langmuir cells will still carry it into convergence zones, and the velocity will still be biased in the alongwind direction.

-Oceanic Photochemistry: A number of geophysically interesting photochemical trace compounds (carbon monoxide, hydrogen peroxide, carbonyl sulfide) are produced by sunlight and consumed by biota within the surface layer. Gnanadesikan (1994, subm.) shows that the surface concentrations are strongly dependent on whether the mixing through the layer is fast or slow in comparison with the rate at which the compounds are consumed. If Langmuir cells are active the mixing will be quite fast, implying lower near-surface concentrations and airsea fluxes than predicted by Mellor-Yamada type models.

-Biological Productivity: The amount of light which phytoplankon can capture is a major factor in their productivity. It depends on the level of light and the amount of pigment which can be used to capture the light. In turn, the pigmentation of phytoplankton is a function of the amount of light to which they are exposed. (Olson et al., 1990) At depths below the e-folding scale of penetrating radiation phytoplankton maintain high levels of pigment so as to take advantage of what little light there is. Plankton near the surface, on the other hand, have much lower levels of pigmentation. Olson et al.(1990) showed that the 
picoplankton Synechococcus required many hours (of order 20-30) to adjust from high to low light levels.

In a layer where Langmuir cells are strong, phytoplankton will see rapidly varying levels of light as they are advected around the vortices. By contrast, in a mixed layer mixed by small-scale diffusion (given that the levels of turbulence are those predicted by a Mellor-Yamada type model) the level of light seen will vary essentially as the diurnal cycle. This difference in the light seen been the cells will be reflected in differences in the productivity of the cells.

-Acoustic Backscatter and Ambient Noise: The organized motions associated with the cells create curtains of bubbles The scattering from these bubble plumes has been invoked as an explanation for the degradation of active sonars in high sea states (Chapman and Harris, 1962). The oscillation of bubble plumes has been suggested as an explanation for increase in ambient noise in the $500-800 \mathrm{~Hz}$ band. These acoustic effects depend on the amount of air entrained by the cells into plumes and the spatial structure of the cells. In order to predict these phenomena, a better understanding of cell structure is necessary.

\subsection{Suggestions for Future Research}

Future work on Langmuir cells needs to take two paths; one seeking a better understanding of Craik-Leibovich instability, the other a better understanding and description of the equilibrium state. The first of these paths addresses whether Langmuir cells can "pull themselves up by their bootstraps" or as argued earlier in this chapter, small-scale diffusion provides the initial shear on which the cells grow. A theoretical study which would give useful information on this point would consider the development of a mixed layer from initial state at rest given a spatially variable viscosity dependent on the local shear and stratification. This would parallel work on large eddies in the atmospheric boundary layer, where turbulence closure models such as the Mellor-Yamada model are used to model subgrid-scale processes while eddies with spatial scales of order the mixed layer depth are modelled directly. By examining the propagation of the turbulence and Langmuir cells together it would be possible to disentangle the relative importance of the two processes in mixed layer creation.

It should be noted however, that disentangling Langmuir cells and turbulence in the field will be difficult. Low levels of turbulence are always 
present and the water column is never at rest. Good measurements of turbulent intensity are difficult to make. Additonally, in order to distinguish the cells from the energetic surface gravity wave field, it is often necessary to average over time periods which are comparable to the time scales on which the mixed layer evolves.

The second path involves clarifying the behavior of the cells once a mixed layer has been formed and the cells have reached finite amplitude. As shown in Chapter 8, the theory as currently stated fails to predict the correct cell population, with finite-difference code runs yielding (with only a few exceptions) persistent fields of cells which have only very long wavelengths. Since these cells penetrate deeply into the mixed layer, the surface shear layer is thicker and stronger than is actually seen in the data. Additionally, the theory predicts an Eulerian return flow whose volume transport balances that associated with the Stokes drift, a phenomenon not seen in the data.

These shortcomings suggest a number of directions for future theoretical work on Langmuir circulations.

- The theory needs to be extended to allow for nonconstant eddy diffusivity. This would enable a thermocline and a mixed layer to coexist within the same model domain, so that interactions between the mixed layer and thermocline in the presence of cells could be studied.

- From a practical point of view, the question of cell population is clearly important. Extension of the results of this thesis to three dimensions is a necessary step to see if three-dimensional vortex interactions (Thorpe, 1992) allow for multiple scales of cells and thus momentum transport on different vertical scales.

- The whole question of whether there should be an Eulerian return flow balancing the Stokes drift needs clarification.

- The temporal variability of cells in a mixed layer with non-constant surface forcing should be explored.

The equilibrium state also needs to be better characterized observationally. While the observations described in this thesis provide evidence that the Langmuir cells are driven by wave-current interaction and that they affect the velocity structure of the mixed layer, the case is far from complete. The cell structure remains relatively poorly characterized. The evidence for the cells actually being driven by wave-current interaction hinges on two days during SWAPP when the waves were high but the stress was low.The following experiments would help resolve these shortcomings: 
-In the field, cells have many different horizontal scales which in turn have different vertical scales. This makes it difficult to characterize the structure, since it is impossible with the present measurements to know where one is with respect to the cells. Measurements combining velocity and acoustic backscatter (or perhaps some other passive tracer such as dye) need to be made in order to obtain a better three-dimensional kinematic picture of the cell structure. The WAVES experiment on Lake Ontario (Agarwal et al., 1992) did make such measurements, but the water depth was small and the crosswind velocity low, so that it is unclear that a full three-dimensional picture of the cells can be extracted.

-A better verification of the wave-current interaction mechanism needs to be made by considering longer time series with more realizations of the forcing. Analysis of datasets collected during the 2nd and 3rd Acoustic Surface Reverberation Experiment (ASREX II and III) which include long time series (60 and 100 days respectively) of currents and directional wave spectra will help to accomplish this.

-The behavior of cells in very deep mixed layers (of order 200 meters) has not been studied. Does the level of cell activity scale as predicted by theory? Is buoyancy forcing more important? Do the cells alter the structure in ways comparable with the alterations seen in shallower mixed layers? The ASREX III dataset will provide interesting insight into these questions as it includes time periods when the mixed layer was over 200 meters deep.

In conclusion, Langmuir cells are an important phenomenon for upper ocean dynamics. It is to be hoped that future investigations into their role as agents for heat, mass, and momentum transfer in the upper ocean will resolve some of the puzzles which have plagued observational oceanographers for a large part of a century. 


\section{Appendix A: Derivation of Huang's Equations}

A diagram of the coordinate system in which we will be working is shown in Figure A.1. The $\mathrm{x}$-axis is taken to refer to the crosswave axis, the $y$-axis to the alongwave axis , and the $\mathrm{z}$-axis to the vertical axis. Using this coordinate system simplifies the representation of the boundary conditions. The equations obtained are then rotated into the coordinate system used in the text, where $\mathrm{x}$ is the crosscell, $\mathrm{y}$ the alongcell, and $\mathrm{z}$ the vertical coordinate. The derivation presented below follows the published derivations of Leibovich (1977a) and Huang (1979) with two exceptions. The first is that the consistency conditions on the irrotational pressure-driven flow are calculated and a frictional boundary layer is considered at the upper surface. These additions lead to momentum fluxes due to wave damping and Bragg scattering of the waves off of the Langmuir cells. Both effects are neglected in the Huang's equations and in the text of the thesis. This neglect is justified below.

The velocity in the $\mathrm{x}, \mathrm{y}$, and $\mathrm{z}$ axes are denoted by $\mathrm{u}, \mathrm{v}$, and $\mathrm{w}$ respectively. The vorticities in these axes are denoted by $\xi, \Omega$, and $\varsigma$. respectively.

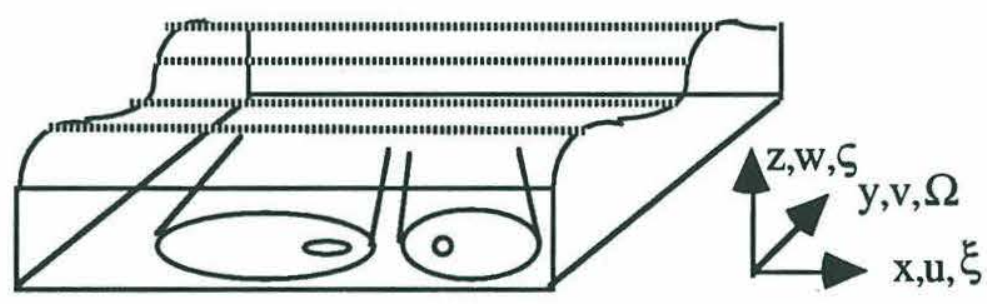

Figure A.1: Schematic of the coordinate system used in deriving the equations. Note that for the purposes of deriving the equations $\mathrm{y}$ is the alongwave and $\mathrm{x}$ the crosswave direction (rather than along- and crosscell directions).

Let $u=(u, v, w)$, (italics henceforth represent dimensional quantities). $\omega=(\xi, \Omega, \varsigma)$, and $\mathbf{i}, \mathbf{j}$, and $\mathbf{k}$, be the unit vectors in the $\mathrm{x}, \mathrm{y}$, and $\mathrm{z}$ directions. Furthermore, let $\frac{\mathrm{D}}{\mathrm{Dt}}$ be the total derivative $\frac{\partial}{\partial t}+u \frac{\partial}{\partial x}+v \frac{\partial}{\partial y}+w \frac{\partial}{\partial z}, \nabla=\left(\mathbf{i} \frac{\partial}{\partial x}+\mathbf{j} \frac{\partial}{\partial y}+\mathbf{k} \frac{\partial}{\partial z}\right)$ and $\nabla^{2}=\frac{\partial^{2}}{\partial x^{2}}$ $+\frac{\partial^{2}}{\partial y^{2}}+\frac{\partial^{2}}{\partial z^{2}}$. A further assumption, discussed in more detail in the text, is that mixing processes on scales smaller than those with which this work is concerned 
can be simply parameterized by a constant eddy viscosity $v_{e}$. Then if $\Omega_{p}$ is the planetary vorticity) the Boussinesq Equation in dimensional form becomes

$$
\frac{\mathrm{D} u}{\mathrm{D} t}+2 \Omega_{\mathrm{p}} \times \boldsymbol{u}=-\frac{1}{\rho_{0}} \nabla p+\frac{\mathrm{g} \rho}{\rho_{0}} \mathbf{k}+\mathrm{v}_{\mathrm{e}} \nabla^{2} \boldsymbol{u}
$$

The equation of continuity for incompressible flow is

$$
\text { (A-2) } \nabla * u=0
$$

and the density transport equation is

$$
\frac{\mathrm{D} \rho}{\mathrm{D} t}=v_{\mathrm{e}} \nabla^{2} \rho
$$

The kinematic surface boundary condition

$$
\frac{\mathrm{D}}{\mathrm{D} t} \eta(x, y, t)=w
$$

The dynamic surface boundary conditions require more explanation. The momentum across a surface with normal $\hat{\mathbf{n}}$ is

(A-5) Momentum transport $=(u, v, w)\left(\mathrm{u}_{\text {fluid }}-\mathrm{u}_{\text {surf }}\right) * \hat{\mathbf{n}}+\mathrm{T} * \hat{\mathbf{n}}$

where $\mathrm{u}_{\mathrm{fluid}}$ is the velocity of the fluid, $\mathrm{u}_{\text {surf }}$ the velocity of the surface and $\mathrm{T}$ is the stress tensor. At the wave surface $\mathrm{u}_{\text {surf }}=\mathrm{u}_{\text {fluid }}$. This, however, means that over a wave period

$$
u^{\prime} w^{\prime}=u^{\prime} \frac{\partial \eta}{\partial t}
$$

which is not necessarily zero.

We will be considering cases with a wavy surface, so we must solve the equation for continuity of stress. Outside the water the stress tensor is

$$
\mathrm{T}^{\mathrm{a}}=\left(\begin{array}{ccc}
-\mathrm{p}_{\mathrm{a}} & 0 & \tau_{\mathrm{x}} \\
0 & -\mathrm{p}_{\mathrm{a}} & \tau_{\mathrm{y}} \\
\tau_{\mathrm{x}} & \tau_{\mathrm{y}} & -\mathrm{p}_{\mathrm{a}}
\end{array}\right)
$$

while inside the water

$$
\mathrm{T}_{\mathrm{ij}}=\mu_{\mathrm{e}}\left(\frac{\partial \mathrm{u}_{\mathrm{j}}}{\partial \mathrm{x}_{\mathrm{i}}}+\frac{\partial \mathrm{u}_{\mathrm{i}}}{\partial \mathrm{x}_{\mathrm{j}}}\right)+\mathrm{p} \delta_{\mathrm{ij}}
$$

The normal vector is

$$
\hat{n}=\frac{(-\partial \eta / \partial x,-\partial \eta / d y, 1)}{\sqrt{1+(\partial \eta / \partial x)^{2}+(\partial \eta / \partial y)^{2}}}
$$

At the surface the normal stress must balance. This yields equations for the stress and pressure in terms of the surface shears and pressure.

$$
\text { (A-10a) } \quad p_{a}=p+\frac{2 \mu_{e}}{1-\frac{\partial \eta^{2}}{\partial x}-\frac{\partial \eta^{2}}{\partial y}}\left(\frac{\partial u}{\partial x} \frac{\partial \eta^{2}}{\partial x}+\frac{\partial v \partial \eta^{2}}{\partial y \partial y}+\left(\frac{\partial v}{\partial x}+\frac{\partial u}{\partial y}\right) \frac{\partial \eta}{\partial x} \frac{\partial \eta}{\partial y}-\frac{\partial w}{\partial z}\right)
$$


(A-10b) $\quad \tau_{x}=\mu_{e}\left(\frac{\partial u}{\partial z}+\frac{\partial w}{\partial x}\right)+$

$$
\mu_{e} \frac{2 \frac{\partial w \partial \eta}{\partial z \partial x}+\left(\frac{\partial v}{\partial x}+\frac{\partial u}{\partial y}\right) \frac{\partial \eta}{\partial y}\left(1-\frac{\partial \eta^{2}}{\partial y}+\frac{\partial \eta^{2}}{\partial x}\right)-2 \frac{\partial u}{\partial x} \frac{\partial \eta}{\partial x}\left(1-\frac{\partial \eta^{2}}{\partial y}\right)-2 \frac{\partial v}{\partial y} \frac{\partial \eta}{\partial x} \frac{\partial \eta^{2}}{\partial y}}{1-\frac{\partial \eta^{2}}{\partial x}-\frac{\partial \eta^{2}}{\partial y}}
$$

(A-10c) $\quad \tau_{y}=\mu_{e}\left(\frac{\partial v}{\partial z}+\frac{\partial w}{\partial y}\right)+$

$$
\mu_{e} \frac{2 \frac{\partial w \partial \eta}{\partial z \partial y}+\left(\frac{\partial v}{\partial x}+\frac{\partial u}{\partial y}\right) \frac{\partial \eta}{\partial x}\left(1-\frac{\partial \eta^{2}}{\partial x}+\frac{\partial \eta^{2}}{\partial y}\right)-2 \frac{\partial v}{\partial y} \frac{\partial \eta}{\partial y}\left(1-\frac{\partial \eta^{2}}{\partial x}\right)-2 \frac{\partial u}{\partial x} \frac{\partial \eta}{\partial y} \frac{\partial \eta^{2}}{\partial x}}{1-\frac{\partial \eta^{2}}{\partial x}-\frac{\partial \eta^{2}}{\partial y}}
$$

If the waves are propagating in the y direction only, these equations simplify greatly. It is this simplified form that is used below. The equations can be nondimensionalized as follows

$$
t=\sigma^{-1} \tau=\sigma^{-1} t^{\prime}+\varepsilon^{-2} \sigma^{-1} t
$$

$$
(x, y, z)=\mathrm{k}^{-1}(\mathrm{x}, \mathrm{y}, \mathrm{z})
$$

$(\mathrm{A}-11 \mathrm{c}) u=(u, v, w)=\frac{\varepsilon \sigma}{\mathrm{k}}\left(\mathrm{u}_{1}, \mathrm{v}_{1}, \mathrm{w}_{1}\right)+\frac{\varepsilon^{2} \sigma}{\mathrm{k}}\left(\mathrm{u}_{2}, \mathrm{v}_{2}, \mathrm{w}_{2}\right)+\ldots=\frac{\varepsilon \sigma}{\mathrm{k}} \mathbf{u}_{1}^{2}+\frac{\varepsilon^{2} \sigma}{\mathrm{k}} \mathbf{u}_{2}^{2}+\ldots$

$$
\begin{gathered}
p=\mathrm{p}_{\mathrm{a}}-\frac{\rho \mathrm{g}}{\mathrm{k}} \mathrm{z}+\varepsilon \frac{\rho \mathrm{g}}{\mathrm{k}} \mathrm{p}_{1}+\ldots \\
\eta=\frac{\varepsilon}{\mathrm{k}} \eta_{1}+\frac{\varepsilon^{2}}{\mathrm{k}} \eta_{2}+\ldots \\
\rho=\rho_{0}\left(1+\frac{\varepsilon^{2} \mathrm{~N}^{2}}{\sigma^{2}} \rho_{2}+\ldots\right)
\end{gathered}
$$

The derivation below makes the following assumptions.

1. The flow of order $\varepsilon$ is a field of surface gravity waves. Consistency conditions for this wave field are derived.

2. The mean flows associated with the cells are of order $\varepsilon^{2}$.

3. The cells are capable of replacing small-scale diffusion as the dominant transport mechanism for velocity. This means that

$$
\mathrm{O}\left(\frac{\varepsilon^{4} \sigma^{2}}{\mathrm{k}^{2}} \mathrm{u}_{2} \mathrm{w}_{2}\right)=\mathrm{O}\left(\mathrm{v}_{\mathrm{e}} \varepsilon^{2} \sigma \frac{\partial \mathrm{u}_{2}}{\partial \mathrm{z}}\right)
$$

But since the mean velocity shoud be at the right order of magnitude to be balanced by the Coriolis force

$$
\mathrm{O}\left(\frac{\varepsilon^{4} \sigma^{2} \partial}{\mathrm{k} \partial \mathrm{z}} \mathrm{u}_{2} \mathrm{w}_{2}\right)=\frac{\Omega_{\mathrm{p}}{ }^{*} \mathrm{k} \varepsilon^{2} \sigma}{\mathrm{k}} \mathrm{v}_{2}
$$

The cells should also be capable of being affected by density. Hence 


$$
\mathrm{g} \frac{\varepsilon^{2} \mathrm{~N}^{2}}{\sigma^{2}} \rho_{2}=\frac{\varepsilon^{4} \sigma^{2}}{\mathrm{k}} \frac{\partial \mathrm{w}_{2}}{\partial \mathrm{t}}
$$

Then the scaled Coriolis force F, Richardson number Ri, and Langmuir number $\mathrm{La}$ can then be defined as

$$
\mathrm{La}=\frac{v_{\mathrm{e}} \mathrm{k}^{2}}{\varepsilon^{2} \sigma}
$$

$$
\mathbf{F}=\frac{2 \Omega_{p}}{\varepsilon^{2} \sigma}
$$

$$
\mathrm{Ri}=\frac{\mathrm{N}^{2}}{\varepsilon^{4} \sigma^{2}}
$$

So that the full nondimensional equations with boundary conditions are

$$
\frac{\mathrm{Du}}{\mathrm{D} \tau}+\varepsilon^{2} \mathbf{F} \times \mathbf{u}=-\nabla \mathrm{p}+\varepsilon^{2} \operatorname{Ri} \rho \mathbf{k}+\varepsilon^{2} \mathrm{La}^{2} \mathbf{u}
$$

or expanding to various orders in $\varepsilon$

$$
\begin{gathered}
(\mathrm{A}-17)\left(\frac{\partial}{\partial \mathrm{t}^{\prime}}+\varepsilon^{2} \frac{\partial}{\partial \mathrm{t}}\right)\left(\varepsilon \mathbf{u}_{1}+\varepsilon^{2} \mathbf{u}_{2}+\ldots\right)+\left(\left(\varepsilon \mathbf{u}_{1}+\varepsilon^{2} \mathbf{u}_{2}+\ldots\right) * \nabla\right)\left(\varepsilon \mathbf{u}_{1}+\varepsilon^{2} \mathbf{u}_{2}+\ldots\right) \\
+\varepsilon^{2} \mathbf{F} \times\left(\varepsilon \mathbf{u}_{1}+\varepsilon^{2} \mathbf{u}_{2}+\ldots\right)=-\nabla\left(\varepsilon \mathrm{p}_{1}+\varepsilon^{2} \mathrm{p}_{2}+\ldots\right)+\operatorname{Ri} \mathbf{k} \varepsilon^{4}\left(\rho_{2}+\ldots\right) \\
+\varepsilon^{2} \mathrm{La} \nabla^{2}\left(\varepsilon \mathbf{u}_{1}+\varepsilon^{2} \mathbf{u}_{2}+\ldots\right)
\end{gathered}
$$

Because diffusion enters the equations as a perturbation, but is included in the boundary conditions, an upper boundary layer must be defined. A coordinate system is chosen in which the vertical coordinate is tied to the wave surface.

$$
\zeta=\frac{\mathrm{z}-\eta(\mathrm{x}, \mathrm{y}, \tau)}{\varepsilon}
$$

Then defining $\mathbf{u}=\mathbf{u}^{\mathrm{o}}(\mathrm{x}, \mathrm{y}, \mathrm{z}, \tau)+\mathbf{u}^{\mathrm{i}}(\mathrm{x}, \mathrm{y}, \zeta, \tau)$.

$$
\begin{gathered}
\frac{\partial \mathbf{u}}{\partial \tau}=\frac{\partial \mathbf{u}^{0}}{\partial \tau}+\frac{\partial \mathbf{u}^{\mathrm{i}}}{\partial \tau}-\frac{1}{\varepsilon} \frac{\partial \eta}{\partial t} \frac{\partial \mathbf{u}^{\mathrm{i}}}{\partial \zeta} \\
\frac{\partial \mathbf{u}}{\partial \mathrm{x}}=\frac{\partial \mathbf{u}^{\mathrm{o}}}{\partial \mathrm{x}}+\frac{\partial \mathbf{u}^{\mathrm{i}}}{\partial \mathrm{x}}-\frac{1}{\varepsilon} \frac{\partial \eta}{\partial \mathrm{x}} \frac{\partial \mathbf{u}^{\mathrm{i}}}{\partial \zeta} \\
\frac{\partial \mathbf{u}}{\partial \mathrm{z}}=\frac{\partial \mathbf{u}^{\mathrm{o}}}{\partial \mathrm{z}}+\frac{1}{\varepsilon} \frac{\partial \mathbf{u}^{\mathrm{i}}}{\partial \zeta}
\end{gathered}
$$

The last property means that one may separate the equations into outer and inner equations, where the outer equations have no dependence on $\zeta$ and the inner equations contain at least one term which varies with $\zeta$.

Lastly, the boundary conditions are applied at $\mathrm{z}=\varepsilon \eta$. This means (following conventional practice with ocean surface gravity waves) that one may expand $\mathbf{u}^{0}$ and $\mathrm{p}^{\mathrm{o}}$ in the boundary equations as follows

$$
\mathbf{u}_{\mathrm{o}} \mathrm{z}=\varepsilon \eta_{\mathrm{u}}=\mathbf{u} \mathrm{I}_{\mathrm{z}=0}+\varepsilon \eta \frac{\partial \mathbf{u}^{\mathrm{o}}}{\partial \mathrm{z}}+\ldots
$$


(A-20b)

$$
\mathrm{p}^{\mathrm{O}} \mathrm{z}_{\mathrm{z}=\varepsilon \eta}=\mathrm{p}^{\mathrm{O}} \mathrm{I}_{\mathrm{z}=0}+\varepsilon \eta \frac{\partial \mathrm{p}^{\mathrm{o}}}{\partial \mathrm{z}}+\ldots
$$

Then the external momentum equation is

$$
\begin{gathered}
(\mathrm{A}-21)\left(\frac{\partial}{\partial \mathrm{t}^{\prime}}+\varepsilon^{2} \frac{\partial}{\partial \mathrm{t}}\right)\left(\varepsilon \mathbf{u}_{1}^{\mathrm{o}}+\varepsilon^{2} \mathbf{u}_{2}^{\mathrm{o}}+\ldots\right)+\left(\left(\varepsilon \mathbf{u}_{1}^{\mathrm{o}}+\varepsilon^{2} \mathbf{u}_{2}^{\mathrm{o}}+\ldots\right) * \nabla\right)\left(\varepsilon \mathbf{u}_{1}^{\mathrm{o}}+\varepsilon^{2} \mathbf{u}_{2}^{\mathrm{o}}+\ldots\right) \\
+\varepsilon^{2} \mathrm{Fk} \times\left(\varepsilon \mathbf{u}_{1}^{\mathrm{o}}+\varepsilon^{2} \mathbf{u}_{2}^{\mathrm{o}}+\ldots\right)=-\nabla\left(\varepsilon \mathrm{p}_{1}^{\mathrm{o}}+\varepsilon^{2} \mathrm{p}_{2}^{\mathrm{o}}+\ldots\right)+\operatorname{Ri} \mathbf{k} \varepsilon^{4}\left(\rho_{2}^{\mathrm{o}}+\ldots\right) \\
+\varepsilon^{2} \mathrm{La} \nabla^{2}\left(\varepsilon \mathbf{u}_{1}^{\mathrm{o}}+\varepsilon^{2} \mathbf{u}_{2}^{\mathrm{o}}+\ldots\right)
\end{gathered}
$$

Defining $\nabla \mathrm{H}=\frac{\partial}{\partial \mathrm{x}} \mathbf{i}+\frac{\partial}{\partial y} \mathbf{j}$, the internal momentum equation is

$$
\begin{aligned}
& \text { (A-22) } \\
& \left(\frac{\partial}{\partial \mathrm{t}^{\prime}}+\varepsilon^{2} \frac{\partial}{\partial \mathrm{t}}\right)\left(\varepsilon \mathbf{u}_{1}^{\mathrm{i}}+\varepsilon^{2} \mathbf{u}_{2}^{\mathrm{i}}+\ldots\right)+ \\
& \left(\left(\varepsilon \mathbf{u}_{1}^{\mathrm{i}}+\varepsilon^{2} \mathbf{u}_{2}^{\mathrm{i}}+\ldots\right) * \nabla\right)\left(\left.\varepsilon \mathbf{u}_{1}^{\mathrm{o}}\right|_{\mathrm{z}=0}+\varepsilon^{2}\left(\left.\mathbf{u}_{2}^{\mathrm{o}}\right|_{\mathrm{z}=0}+\left.\eta_{1} \frac{\partial \mathbf{u}_{1}^{\mathrm{o}}}{\partial \mathrm{z}}\right|_{\mathrm{z}=0} \ldots\right)\right) \\
& \left.\left(\left(\left.\varepsilon \mathbf{u}_{1}^{\mathrm{o}}\right|_{z=0}+\varepsilon \mathbf{u}_{1}^{\mathrm{i}}+\varepsilon^{2}\left(\left.\mathbf{u}_{2}^{\mathrm{o}}\right|_{\mathrm{z}=0}+\mathbf{u}_{2}^{\mathrm{i}}+\left.\eta_{1} \frac{\partial \mathbf{u}_{1}^{\mathrm{o}}}{\partial \mathrm{z}}\right|_{\mathrm{z}=0} \ldots\right) \ldots\right)\right) * \nabla \mathrm{H}\right)\left(\varepsilon \mathbf{u}_{1}^{\mathrm{i}}+\varepsilon^{2} \mathbf{u}_{2}^{\mathrm{i}}+\ldots\right) \\
& \left.+\frac{1}{\varepsilon}\left(\left(\left.\varepsilon w_{1}^{o}\right|_{z=0}+\varepsilon w_{1}^{i}+\varepsilon^{2}\left(\left.w_{2}^{o}\right|_{z=0}+w_{2}^{i}+\left.\eta_{1} \frac{\partial w_{1}^{o}}{\partial z}\right|_{z=0} \ldots\right) \ldots\right)\right) \frac{\partial}{\partial \zeta}\right)\left(\varepsilon u_{1}^{i}+\varepsilon^{2} u_{2}^{i}+\ldots\right) \\
& +\varepsilon^{2} \mathbf{F} \times\left(\varepsilon \mathbf{u}_{1}^{\mathrm{i}}+\varepsilon^{2} \mathbf{u}_{2}^{\mathrm{i}}+\ldots\right) \\
& =-\nabla \mathrm{H}\left(\varepsilon \mathrm{p}_{1}^{\mathrm{i}}+\varepsilon^{2} \mathrm{p}_{2}^{\mathrm{i}}+\ldots\right)-\frac{\partial}{\partial \zeta}\left(\mathrm{p}_{1}^{\mathrm{i}}+\varepsilon \mathrm{p}_{2}^{\mathrm{i}}+\ldots\right)+\mathrm{Ri} \mathbf{k} \varepsilon^{4}\left(\rho_{2}^{\mathrm{i}}+\ldots\right) \\
& +\varepsilon^{2} \mathrm{La} \nabla^{2}\left(\varepsilon \mathbf{u}_{1}^{\mathrm{i}}+\varepsilon^{2} \mathbf{u}_{2}^{\mathrm{i}}+\ldots\right)+\mathrm{La} \frac{\partial^{2}}{\partial \zeta^{2}}\left(\varepsilon \mathbf{u}_{1}^{\mathrm{i}}+\varepsilon^{2} \mathbf{u}_{2}^{\mathrm{i}}+\ldots\right)
\end{aligned}
$$

The external and internal continuity equations at each order are

$$
\text { (A-23a) }
$$

$$
\begin{gathered}
\nabla * \mathbf{u}_{\mathrm{n}}^{\mathrm{o}}=0 \\
\nabla \mathrm{H} * \mathbf{u}_{\mathrm{n}}^{\mathrm{i}}+\frac{\partial \mathrm{w}_{\mathrm{n}+1}^{\mathrm{i}}}{\partial \zeta}+\left(\mathbf{u}_{\mathrm{n}}^{\mathrm{i}} * \nabla \mathrm{H}\right) \eta_{1}+\left(\mathbf{u}_{\mathrm{n}-1}^{\mathrm{i}} * \nabla \mathrm{H}\right) \eta_{2}+\ldots=0
\end{gathered}
$$

The kinematic surface boundary condition is

$$
\left(\frac{\partial}{\partial t^{\prime}}+\varepsilon^{2} \frac{\partial}{\partial t}\right)\left(\varepsilon \eta_{1}+\varepsilon^{2} \eta_{2}+\ldots\right)
$$$$
+\left(\left(\left.\varepsilon \mathbf{u}_{1}^{\mathrm{o}}\right|_{\mathrm{z}=0}+\left.\varepsilon \mathbf{u}_{1}^{\mathrm{i}}\right|_{\zeta=0}+\varepsilon^{2}\left(\left.\mathbf{u}_{2}^{\mathrm{o}}\right|_{\mathrm{z}=0}+\left.\mathbf{u}_{2}^{\mathrm{i}}\right|_{\zeta=0}+\left.\eta_{1} \frac{\partial \mathbf{u}_{1}^{\mathrm{o}}}{\partial \mathrm{z}}\right|_{\mathrm{z}=0}\right) * \nabla \mathrm{H}\right)\right)\left(\varepsilon \eta_{1}+\varepsilon^{2} \eta_{2}+\ldots\right)
$$

$$
-\left.\varepsilon w_{1}^{o}\right|_{z=0}-\left.\varepsilon w_{1}^{i}\right|_{\zeta=0}+\varepsilon^{2}\left(\left.w_{2}^{o}\right|_{z=0}+\left.w_{2}^{i}\right|_{\zeta=0}+\left.\eta_{1} \frac{\partial w_{1}^{o}}{\partial z}\right|_{z=0}\right)=0
$$

Given waves propagating in the $+y$ direction, the surface pressure and stress conditions are:

$(\mathrm{A}-25 \mathrm{a}) \quad \mathrm{p}_{\mathrm{a}}=\mathrm{p}_{0}+\left.\varepsilon\left(\mathrm{p}_{1}^{\mathrm{o}}+\mathrm{p}_{1}^{\mathrm{i}}\right)\right|_{\zeta=0}+\varepsilon^{2}\left(\left.\mathrm{p}_{2}^{\mathrm{o}}\right|_{\mathrm{z}=0}+\left.\mathrm{p}_{2}^{\mathrm{i}}\right|_{\zeta=0}+\left.\eta_{1} \frac{\partial \mathrm{p}_{1}^{\mathrm{o}}}{\partial \mathrm{z}}\right|_{\mathrm{z}=0}+\ldots\right)+\ldots$ 


$$
\begin{gathered}
-\left(\varepsilon \eta_{1}+\varepsilon^{2} \eta_{2}+\varepsilon^{3} \eta_{3}+\ldots\right)+\varepsilon^{2} \operatorname{La}\left(1+\left(\varepsilon \frac{\partial \eta_{1}}{\partial y}+\varepsilon^{2} \frac{\partial \eta_{2}}{\partial y}+\ldots\right)^{2}\right) * \\
\left\{-\left.2\left(\varepsilon \frac{\partial w_{1}^{o}}{\partial z}+\varepsilon^{2} \frac{\partial w_{2}^{o}}{\partial z}+\varepsilon^{2} \eta_{1} \frac{\partial^{2} w_{1}^{o}}{\partial z^{2}}+\ldots\right)\right|_{z=0}-\left.\frac{2}{\varepsilon}\left(\varepsilon \frac{\partial w_{1}^{i}}{\partial \zeta}+\varepsilon^{2} \frac{\partial w_{2}^{i}}{\partial \zeta}+\ldots\right)\right|_{\zeta=0}+\text { H.O.T. }\right\}
\end{gathered}
$$

$(\mathrm{A}-25 \mathrm{~b}) \varepsilon^{4} \tau_{\mathrm{x}}=\varepsilon^{2} \mathrm{La}\left\{\left.\left(\varepsilon \frac{\partial \mathrm{u}_{1}^{0}}{\partial \mathrm{z}}+\varepsilon \frac{\partial \mathrm{w}_{1}^{0}}{\partial \mathrm{x}}+\varepsilon^{2} \frac{\partial \mathrm{u}_{2}^{0}}{\partial \mathrm{z}}+\varepsilon^{2} \frac{\partial \mathrm{w}_{2}^{0}}{\partial \mathrm{x}}+\varepsilon^{2} \eta_{1} \frac{\partial^{2} \mathrm{u}_{1}^{0}}{\partial \mathrm{z}^{2}}+\varepsilon^{2} \frac{\partial^{2} \mathrm{w}_{1}^{0}}{\partial \mathrm{x} \partial \mathrm{z}}\right)\right|_{\mathrm{z}=0}\right.$

$$
\begin{gathered}
+\frac{1}{\varepsilon}\left(\varepsilon \frac{\partial \mathrm{u}_{1}^{\mathrm{i}}}{\partial \zeta}+\varepsilon^{2} \frac{\partial \mathrm{u}_{2}^{\mathrm{i}}}{\partial \zeta}+\ldots\right) \|_{\zeta=0}+\left.\left(\varepsilon \frac{\partial \mathrm{w}_{1}^{\mathrm{i}}}{\partial \mathrm{x}}+\varepsilon^{2} \frac{\partial \mathrm{w}_{2}^{\mathrm{i}}}{\partial \mathrm{x}}+\ldots\right)\right|_{\zeta=0} \\
+\left(\varepsilon \frac{\partial \eta_{1}}{\partial \mathrm{y}}+\varepsilon^{2} \frac{\partial \eta_{2}}{\partial \mathrm{y}}+\ldots\right)\left(\left.\varepsilon \frac{\partial \mathrm{v}_{1}^{\mathrm{o}}}{\partial \mathrm{x}}\right|_{z=0}+\left.\varepsilon \frac{\partial \mathrm{v}_{1}^{\mathrm{i}}}{\partial \mathrm{x}}\right|_{\zeta=0}+\left.\varepsilon \frac{\partial \mathrm{u}_{1}^{\mathrm{o}}}{\partial \mathrm{y}}\right|_{z=0}+\left.\varepsilon \frac{\partial \mathrm{u}_{1}^{\mathrm{i}}}{\partial \mathrm{y}}\right|_{\zeta=0}+\ldots\right)
\end{gathered}
$$

$$
\left.-\left(\frac{\partial u_{1}^{i}}{\partial \zeta}+\varepsilon \frac{\partial u_{2}^{i}}{\partial \zeta}+\ldots\right) \|_{\zeta=0}\left(\varepsilon \frac{\partial \eta_{1}}{\partial y}+\varepsilon^{2} \frac{\partial \eta_{2}}{\partial y}+\ldots\right)^{2}+\text { H.O.T. }\right\}
$$

$(\mathrm{A}-25 \mathrm{c}) \varepsilon^{4} \tau_{\mathrm{y}}=\varepsilon^{2} \mathrm{La}\left\{\left.\left(\varepsilon \frac{\partial \mathrm{v}_{1}^{\mathrm{o}}}{\partial \mathrm{z}}+\varepsilon \frac{\partial \mathrm{w}_{1}^{\mathrm{o}}}{\partial \mathrm{y}}+\varepsilon^{2} \frac{\partial \mathrm{v}_{2}^{\mathrm{o}}}{\partial \mathrm{z}}+\varepsilon^{2} \frac{\partial \mathrm{w}_{2}^{\mathrm{o}}}{\partial \mathrm{y}}+\varepsilon^{2} \eta_{1} \frac{\partial^{2} \mathrm{v}_{1}^{\mathrm{o}}}{\partial \mathrm{z}^{2}}+\varepsilon^{2} \frac{\partial^{2} \mathrm{w}_{1}^{\mathrm{o}}}{\partial \mathrm{y} \partial \mathrm{z}}\right)\right|_{\mathrm{z}=0}\right.$

$$
\begin{aligned}
& +\frac{1}{\varepsilon}\left(\varepsilon \frac{\partial v_{1}^{i}}{\partial \zeta}+\varepsilon^{2} \frac{\partial v_{2}^{i}}{\partial \zeta}+\ldots\right) \|_{\zeta=0}+\left.\left(\varepsilon \frac{\partial w_{1}^{i}}{\partial x}+\varepsilon^{2} \frac{\partial w_{2}^{i}}{\partial x}+\ldots\right)\right|_{\zeta=0} \\
& +\left(\varepsilon \frac{\partial \eta_{1}}{\partial y}+\varepsilon^{2} \frac{\partial \eta_{2}}{\partial y}+\ldots\right)\left(\varepsilon \frac{\partial w_{1}^{o}}{\partial z}-\varepsilon \frac{\partial v_{1}^{i}}{\partial y}-\varepsilon \frac{\partial v_{1}^{o}}{\partial y}+\frac{\partial w_{1}^{i}}{\partial \zeta}+\ldots\right) \\
& \left.-\left(\frac{\partial v_{1}^{i}}{\partial \zeta}+\varepsilon \frac{\partial v_{2}^{i}}{\partial \zeta}+\ldots\right)\left(\varepsilon \frac{\partial \eta_{1}}{\partial y}+\varepsilon^{2} \frac{\partial \eta_{2}}{\partial y}+\ldots\right)^{2}+\text { H.O.T. }\right\}
\end{aligned}
$$

Lastly, the density equation is given by

$$
\begin{gathered}
(\mathrm{A}-26)\left(\frac{\partial}{\partial \mathrm{t}^{\prime}}+\varepsilon^{2} \frac{\partial}{\partial \mathrm{t}}\right)\left(\varepsilon^{2} \rho_{2}+\varepsilon^{3} \rho_{3}+\ldots\right)+\left(\left(\varepsilon \mathbf{u}_{1}^{\mathrm{o}}+\varepsilon^{2} \mathbf{u}_{2}^{\mathrm{o}}+\ldots\right) * \nabla\right)\left(\varepsilon^{2} \rho_{2}+\varepsilon^{3} \rho_{3}+\ldots\right) \\
=\varepsilon^{2} \operatorname{La} \nabla^{2}\left(\varepsilon^{2} \rho_{2}+\varepsilon^{3} \rho_{3}+\ldots\right)
\end{gathered}
$$

The initial conditions is that $\mathbf{u}=\varepsilon \mathbf{u}_{1}+\varepsilon \mathbf{u}_{2}$ where $\mathbf{u}_{1}$ is a surface gravity wave field and $\mathbf{u}_{2}$ is some small initial velocity perturbation. The problem is then to solve for the evolution of the external flow field. In order to do this it is necessary to carry the expansion to $\varepsilon^{4}$, though a full solution is only necessary through the $\varepsilon^{3}$ stage. The solution procedure at each order is as follows.

1. Take the curl of the external momentum equation to obtain $\omega_{\mathrm{n}}^{0}$.

2. Take the divergence of the external momentum equation. This gives us an equation for $\mathrm{p}_{\mathrm{n}}^{\mathrm{oI}}$, the inhomogeneous part of $\mathrm{p}_{\mathrm{n}}^{\mathrm{o}}$. 
3. Solve the internal vertical momentum equation for $p_{n}^{i}$.

4. Solve the internal continuity equation for $\mathrm{w}_{\mathrm{n}}^{\mathrm{i}}$.

5. Combine the vertical momentum, surface pressure, and kinematic surface

boundary condition into a surface condition $\frac{\partial^{2} p_{n}^{o H}}{\partial t^{2}}+\frac{\partial p_{n}^{o H}}{\partial z}=$ combinations of other terms.

Solve for $\mathrm{p}_{\mathrm{n}}^{\mathrm{oH}}$.

6. Substitute $p_{n}^{o}$ into the external momentum equation, use surface pressure condition to get $\eta_{\mathrm{n}}, \mathbf{u}_{\mathrm{n}}^{\mathrm{o}}$.

7. Solve the horizontal internal momentum equations for $\mathbf{u}_{\mathrm{n}}^{\mathrm{i}}$.

8. Solve for any density variation.

\section{Solution to order $\varepsilon$.}

The equations at order $\varepsilon$ are

$$
\begin{gathered}
\frac{\partial \mathbf{u}_{1}^{\mathrm{o}}}{\partial \mathrm{t}}=-\nabla \mathrm{p}_{1}^{\mathrm{o}} \\
\frac{\partial \mathbf{u}_{1}^{\mathrm{i}}}{\partial \mathrm{t}}(\mathbf{i}, \mathbf{j})=-\nabla \mathrm{H} \mathrm{p}_{1}^{\mathrm{i}}+\mathrm{La} \frac{\partial^{2} \mathbf{u}_{1}^{\mathrm{i}}}{\partial \zeta^{2}}(\mathbf{i}, \mathbf{j}) \\
\nabla * \mathbf{u}_{1}^{\mathrm{o}}=0 \\
\frac{\partial \mathrm{w}_{1}^{\mathrm{i}}}{\partial \zeta}=0 \\
\frac{\partial \mathrm{p}_{1}^{\mathrm{i}}}{\partial \zeta}=0 \\
\frac{\partial \eta_{1}}{\partial \mathrm{t}}=\left.\mathrm{w}_{1}^{\mathrm{o}}\right|_{\mathrm{z}=0}+\left.\mathrm{w}_{1}^{\mathrm{i}}\right|_{\zeta=0} \\
\left.\mathrm{p}_{1}^{\mathrm{o}}\right|_{\mathrm{z}=0}+\left.\mathrm{p}_{1}^{\mathrm{i}}\right|_{\mathrm{z}=0}-\mathrm{g} \eta_{1}=0 \\
\frac{\partial \mathrm{u}_{1}^{\mathrm{i}}}{\partial \zeta}=\frac{\partial \mathrm{v}_{1}^{\mathrm{i}}}{\partial \zeta}=0
\end{gathered}
$$

Proceeding as outlined above yields the following solution:

1. Taking the curl of the external momentum equations yields. $\partial \omega_{1}^{0} / \partial t=0$. The flow has no order $\varepsilon$ vorticity with short-time variation. 
2.Taking the divergence of the external momentum equations yields. $\nabla^{2} \mathrm{p}_{1}^{0}=0$.

There is no inhomogenous pressure field forced by higher-order terms.

3. The internal vertical momentum equation yields $\mathrm{p}_{1}^{\mathrm{i}}=0$.

4. The internal continuity equation yields $\mathrm{w}_{1}^{\mathrm{i}}=0$.

5. The vertical momentum, surface pressure, kinematic surface boundary condition yield the dispersion relationship for surface gravity waves. In the present case, the nondimensional form of the relationship is the nondimensionalized wavelength $\lambda=\mathrm{f}^{2}$ where $\mathrm{f}$ is the nondimensionalized frequency.

6. $\mathrm{u}_{1}^{\mathrm{o}}, \mathrm{v}_{1}^{\mathrm{o}}, \mathrm{w}_{1}^{\mathrm{o}}$, and $\eta_{1}$ are the velocities associated with irrotational surface gravity waves. The equations are thus, to this order, consistent. A general form of the solution is:

(A-28a)

$$
\eta_{1}=\int_{f=0}^{\infty} a(f, t) f \sin \left(f^{2} y-f t^{\prime}+\phi(f, t)\right) d f
$$

$$
\infty
$$

$\mathrm{v}_{1}^{\mathrm{o}}=\int_{\mathrm{f}=0}^{\infty}$

$(\mathrm{A}-28 \mathrm{c})$

$$
w_{1}^{o}=\int_{f=0}^{\infty} a(f, t) f \cos \left(f^{2} y-f t^{\prime}+\phi(f, t)\right) e^{f^{2} z} d f
$$

7. $u_{1}^{i}$ and $v_{1}^{i}$ are both zero.

\section{Solution to order $\varepsilon^{2}$ :}

At order $\varepsilon^{2}$, the equations become

$$
\begin{gathered}
\frac{\partial \mathbf{u}_{2}^{o}}{\partial \mathrm{t}^{\prime}}+\left(\mathbf{u}_{1}^{\mathrm{o}} * \nabla\right) \mathbf{u}_{1}^{\mathrm{o}}=-\nabla \mathrm{p}_{2}^{\mathrm{o}} \\
\frac{\partial \mathbf{u}_{1}^{\mathrm{i}}}{\partial \mathrm{t}}(\mathbf{i}, \mathbf{j})=-\nabla \mathrm{H} \mathrm{p}_{1}^{\mathrm{i}}+\mathrm{La} \frac{\partial^{2} \mathbf{u}_{1}^{\mathrm{i}}}{\partial \zeta^{2}}(\mathbf{i}, \mathbf{j}) \\
\nabla * \mathbf{u}_{1}^{\mathrm{o}}=0 \\
\frac{\partial \mathrm{w}_{2}^{\mathrm{i}}}{\partial \zeta}=0 \\
\frac{\partial \mathrm{p}_{2}^{\mathrm{i}}}{\partial \zeta}=0
\end{gathered}
$$


(A-29f)

$$
\frac{\partial \eta_{2}}{\partial \mathrm{t}}+\left(\left.\mathbf{u}_{1}^{\mathrm{o}}\right|_{\mathrm{z}=0} * \nabla \mathrm{H}\right) \eta_{1}=\left.\mathrm{w}_{2}^{\mathrm{o}}\right|_{\mathrm{z}=0}+\left.\mathrm{w}_{2}^{\mathrm{i}}\right|_{\zeta=0}+\left.\eta_{1} \frac{\partial \mathrm{w}_{1}^{\mathrm{o}}}{\partial \mathrm{z}}\right|_{\mathrm{z}=0}
$$

$$
\mathrm{p}_{2}^{\mathrm{o}}+\left.\eta_{1} \frac{\partial \mathrm{p}_{1}^{\mathrm{o}}}{\partial \mathrm{z}}\right|_{\mathrm{z}=0}+\left.\mathrm{p}_{2}^{\mathrm{i}}\right|_{\zeta=0}-\eta_{2}=0
$$

$$
\frac{\partial u_{2}^{i}}{\partial \zeta}=-\left.\frac{\partial u_{1}^{o}}{\partial z}\right|_{z=0}-\left.\frac{\partial w_{1}^{o}}{\partial x}\right|_{z=0}=0
$$

$$
\begin{gathered}
\left.\frac{\partial v_{2}^{i}}{\partial \zeta}\right|_{\zeta=0}=-\left.\frac{\partial v_{1}^{i}}{\partial z}\right|_{z=0}-\left.\frac{\partial w_{1}^{o}}{\partial y}\right|_{z=0} \\
\frac{\partial \rho_{2}}{\partial t^{\prime}}=0
\end{gathered}
$$

These may be solved as follows:

1. Taking the curl of the external momentum equation yields. $\frac{\partial \omega_{2}^{0}}{\partial t}=0$ so that the second-order outer flow has no fast-time variation in the vorticity.

2. The outer flow is given by the second-order solution of the irrotational equations, resulting in Stokes waves, microseisms, etc. For purposes of this analysis these corrections are essentially consistency conditions on the wave field. A full analysis, however, could solve for these conditions directly.

3.-4.As at higher order the second-order vertical velocity and pressure are zero at order $\varepsilon^{2}$.

5. The surface pressure condition has the potential to produce secular terms.

None, however, appear at this order.

6. The second-order velocities on the fast time scale, are, as stated earlier, given merely by the classical second-order Stokes waves solution. For variation on the slow time scale one needs to solve to higher orders.

7.Solving the internal momentum equation given the boundary conditions yields the flow associated with a wave boundary layer. Given (A-28b), the solution for $v_{2}^{i}$. is

$(A-30) v_{2}^{i}=\int_{f=0}^{\infty}-2 a(f, t) f^{3} \sqrt{L a / f} \sin \left(f^{2} y-f t^{\prime}+\phi(f, t)+\pi / 4-\zeta \sqrt{f / 2 L a}\right) e \zeta \sqrt{f / 2 L a} d f$

8. There is no fast-time variation in the second-order density field.

\section{Solution at order $\varepsilon^{3}$ :}

At order $\varepsilon^{3}$, the equations become 
(A-31a)

$$
\begin{gathered}
\frac{\partial \mathbf{u}_{1}^{\mathrm{o}}}{\partial \mathrm{t}}+\frac{\partial \mathbf{u}_{3}^{\mathrm{o}}}{\partial \mathrm{t}^{\prime}}+\left(\mathbf{u}_{1}^{\mathrm{o}} * \nabla\right) \mathbf{u}_{2}^{\mathrm{o}}+\left(\mathbf{u}_{2}^{\mathrm{o}} * \nabla\right) \mathbf{u}_{1}^{\mathrm{o}}+\mathbf{F} \times \mathbf{u}_{1}^{\mathrm{o}}=-\nabla \mathrm{p}_{3}^{\mathrm{o}} \\
\frac{\partial \mathbf{u}_{3}^{\mathrm{i}}}{\partial \mathrm{t}}(\mathbf{i}, \mathbf{j})=-\nabla \mathrm{H} \mathrm{p}_{1}^{\mathrm{i}}+\mathrm{La} \frac{\partial^{2} \mathbf{u}_{3}^{\mathrm{i}}}{\partial \zeta^{2}}(\mathbf{i}, \mathbf{j})
\end{gathered}
$$

$$
\begin{gathered}
\nabla * \mathbf{u}_{3}^{0}=0 \\
\frac{\partial w_{3}^{i}}{\partial \zeta}=-\frac{\partial v_{2}^{i}}{\partial y}+\frac{\partial v_{2}^{i} \partial \eta_{1}}{\partial \zeta \partial y} \\
\frac{\partial p_{3}^{i}}{\partial \zeta}=0
\end{gathered}
$$

$(\mathrm{A}-31 \mathrm{f}) \frac{\partial \eta_{3}}{\partial \mathrm{t}}+\left(\left.\mathbf{u}_{2}^{\mathrm{o}}\right|_{\mathrm{z}=0}+\left.\mathbf{u}_{2}^{\mathrm{i}}\right|_{\mathrm{z}=0} * \nabla \mathrm{H}\right) \eta_{1}+\left(\left.\mathbf{u}_{1}^{\mathrm{o}}\right|_{\mathrm{z}=0} * \nabla \mathrm{H}\right) \eta_{2}+\left(\left.\eta_{1} \frac{\partial \mathbf{u}_{1}^{\mathrm{o}}}{\partial \mathrm{z}}\right|_{\mathrm{z}=0} * \nabla \mathrm{H}\right) \eta_{1}+$

$$
=\left.w_{3}^{0}\right|_{z=0}+\left.w_{3}^{i}\right|_{\zeta=0}+\left.\eta_{1} \frac{\partial w_{2}^{0}}{\partial z}\right|_{z=0}+\left.\eta_{2} \frac{\partial w_{1}^{0}}{\partial z}\right|_{z=0}+\left.\frac{\eta_{1}^{2}}{2} \frac{\partial^{2} w_{1}^{0}}{\partial z^{2}}\right|_{z=0}
$$

$(\mathrm{A}-31 \mathrm{~g}) \mathrm{p}_{3}^{\mathrm{o}}+\left.\eta_{1} \frac{\partial \mathrm{p}_{2}^{\mathrm{o}}}{\partial \mathrm{z}}\right|_{\mathrm{z}=0}+\left.\eta_{2} \frac{\partial \mathrm{p}_{1}^{\mathrm{o}}}{\partial \mathrm{z}}\right|_{\mathrm{z}=0}+\left.\frac{\eta_{1}^{2}}{2} \frac{\partial^{2} \mathrm{p}_{1}^{\mathrm{o}}}{\partial \mathrm{z}^{2}}\right|_{\mathrm{z}=0}+\left.\mathrm{p}_{3}^{\mathrm{i}}\right|_{\zeta=0}-\eta_{2}-\left.2 \mathrm{La} \frac{\partial \mathrm{w}_{1}^{\mathrm{o}}}{\partial \mathrm{z}}\right|_{\mathrm{z}=0}=0$

$$
\left.\mathrm{La} \frac{\partial \mathrm{u}_{2}^{\mathrm{o}}}{\partial \mathrm{z}}\right|_{\mathrm{z}=0}+\left.\mathrm{La} \frac{\partial \mathrm{u}_{3}^{\mathrm{i}}}{\partial \zeta}\right|_{\zeta=0}=\tau_{\mathrm{x}}
$$

(A-31i)La $\left(\left.\frac{\partial v_{2}^{o}}{\partial z}\right|_{z=0}+\left.\eta_{1}\left(\frac{\partial^{2} w_{1}^{o}}{\partial z \partial y}+\frac{\partial^{2} v_{1}^{o}}{\partial z^{2}}\right)\right|_{z=0}+\left.2 \frac{\partial \eta_{1}}{\partial y}\left(\frac{\partial w_{1}^{o}}{\partial z}-\frac{\partial v_{1}^{o}}{\partial y}\right)\right|_{z=0}+\left.\frac{\partial v_{3}^{i}}{\partial \zeta}\right|_{\zeta=0}\right)=\tau_{y}$

$$
\frac{\partial \rho_{3}}{\partial \mathrm{t}^{\prime}}=\left(\mathbf{u}_{1}^{\mathrm{o}} * \nabla\right) \rho_{2}
$$

The solution is as follows:

1. Taking the curl of the external momentum equation gives

$$
\frac{\partial \omega_{1}^{\mathrm{o}}}{\partial \mathrm{t}}+\frac{\partial \omega_{3}^{\mathrm{o}}}{\partial \mathrm{t}^{\prime}}+\left(\mathbf{u}_{1}^{\mathrm{o}} * \nabla\right) \omega_{2}^{\mathrm{o}}-\left(\omega_{2}^{\mathrm{o}} * \nabla\right) \mathbf{u}_{1}^{\mathrm{o}}-(\mathbf{F} * \nabla) \mathbf{u}_{1}^{\mathrm{o}}=0
$$

Since the first-order flow $\mathbf{u}_{1}^{0}$ developed thus far is irrotational with only fast-time variation, while the second-order flow has only slow time variation, it is entirely consistent to let $\frac{\partial \omega_{1}^{0}}{\partial t}=0$, so that the order $\varepsilon$ flow remains irrotational to all times. Letting $\omega_{2}^{0}=\left(\xi_{2}^{0}, \Omega_{2}^{0}, \varsigma_{2}^{0}\right)$ and letting $\mathbf{F}=\left(F_{\mathrm{x}}, \mathrm{F}_{\mathrm{y}}, \mathrm{F}_{\mathrm{z}}\right)$, the individual terms in equation (A-32) can be solved for. Defining $\Phi\left(f, y, t^{\prime}, t\right)=f^{2} y-f t^{\prime}+\phi(f, t)$.

(A-33a)

$$
\xi_{3}^{0}=\int_{f=0}^{\infty} \mathrm{a}(\mathrm{f}, \mathrm{t}) \mathrm{e}^{\mathrm{f}^{2} \mathrm{z}}\left(\sin \left(\Phi\left(\mathrm{f}, \mathrm{y}, \mathrm{t}^{\prime}, \mathrm{t}\right)\right) \frac{\partial \xi_{2}^{\mathrm{o}}}{\partial \mathrm{z}}-\cos \left(\Phi\left(\mathrm{f}, \mathrm{y}, \mathrm{t}^{\prime}, \mathrm{t}\right)\right) \frac{\left.\partial \xi_{2}^{\mathrm{o}}\right)}{\partial \mathrm{y}}\right) \mathrm{df}
$$




$$
\begin{aligned}
& (A-33 b) \Omega_{3}^{o}=\int_{f=0}^{\infty} a(f, t) \mathrm{f}^{2} z\left(\sin (\Phi)\left(\frac{\partial \xi_{2}^{o}}{\partial z}+f^{2} \Omega_{2}^{0}+f^{2} F y\right)-\cos (\Phi)\left(\frac{\partial \Omega_{2}^{0}}{\partial y}-f^{2} \varsigma_{2}^{o}+f^{2} F_{z}\right)\right) d f \\
& (A-33 c) \zeta_{3}^{0}=\int_{f=0}^{\infty} a(f, t) e^{f^{2} z}\left(\sin (\Phi)\left(\frac{\partial \zeta_{2}^{o}}{\partial z}-f^{2} \zeta_{2}^{0}-f^{2} F z\right)-\cos (\Phi)\left(\frac{\partial \zeta_{2}^{o}}{\partial y}-f^{2} \Omega_{2}^{0}+f^{2} F_{y}\right)\right) d f
\end{aligned}
$$

The quickly varying, order $\varepsilon$ waves tilt, compress, and stretch the slowly varying order $\varepsilon^{2}$ vorticity, to create quickly varying, $\varepsilon^{3}$ vorticity.

2. Taking the divergence of the momentum equation gives a very complicated, inhomogeneous pressure field. This field is only of subsidiary interest in this derivation, insofar as it produces secular terms. In order to illustrate how this can occur, suppose that the order $\varepsilon^{2}$ velocity field has a component with structure (A-34)

$$
\mathbf{u}_{2_{k}}^{0}=\left(0, v_{2_{k}}^{o}(\mathrm{z}) \sin (\mathrm{kx}+\mathrm{my}), \mathrm{w}_{2_{\mathrm{k}}}^{\mathrm{o}}(\mathrm{z}) \cos (\mathrm{kx}+\mathrm{my})\right)
$$

The interaction of this velocity field with the order e wave field leads to a pressure field with the structure

$$
\int_{\mathrm{f}=0}^{\infty} \frac{\mathrm{a}(\mathrm{f}, \mathrm{t})}{\sqrt{\mathrm{k}^{2}+\left(\mathrm{f}^{2}-\mathrm{m}\right)^{2}}}\{
$$

$$
\begin{aligned}
& z^{\prime}=\int_{-\infty}^{0} e^{-\sqrt{k^{2}+\left(f^{2}+m\right)^{2}}\left|z^{\prime}-z\right|}\left(\frac{\partial v_{2 k}^{o}}{\partial z^{\prime}}+\frac{\partial w_{2 k}^{o}}{\partial z^{\prime}}-m\left(v_{2 k}^{0}+w_{2 k}^{0}\right) d z^{\prime}\right) \sin (k x+m y+\Phi) \\
& \left.+\int_{z^{\prime}}^{0} e^{-\infty} \sqrt{k^{2}+\left(f^{2}-m\right)^{2}\left|z^{\prime}-z\right|}\left(\frac{\partial v_{2_{k}}^{0}}{\partial z^{\prime}}-\frac{\partial w_{2_{k}}^{0}}{\partial z^{\prime}}-m\left(v_{2_{k}}^{0}-w_{2_{k}}^{0}\right) d z^{\prime}\right) \sin (k x+m y-\Phi)\right\}
\end{aligned}
$$

The second of these integrals generates secular terms when $\left.\sqrt{k^{2}+\left(f^{2}-m\right)^{2}}\right)=f^{2}$. Essentially, this resonance condition corresponds to "Bragg scattering" of the waves off of the Langmuir cells. If the cells are oriented precisely parallel to the waves, $\mathrm{m}=0$, and the scattering condition is never met. This condition is used for all the integrations of the equations in the text, with the exception of some of those with Coriolis force.

When the waves are not parallel with the cells the calculation of this scattering is necessary for a completely rigorous integration of the equations. This is not done in this work, for the cases where it potentially applicable. The neglect of this term at this order can be justified by the same logic used by Hasselmann 
(1961) to reduce the strength of the three-wave resonant coupling interaction from $\mathrm{O}\left(\mathrm{e}^{3}\right)$ to $\mathrm{O}\left(\mathrm{e}^{4}\right)$, namely the random nature of the waves and cells.

3. As before $\mathrm{p}_{\mathrm{i}}^{3}=0$.

4. The $\varepsilon^{3}$ vertical velocity has a complicated dependence on $\zeta$. Integrating the internal continuity equation given equation (A-30) gives

$$
\begin{gathered}
w_{i}^{3}=\int_{f=0}^{\infty} 2 a(f, t) f^{2} L a \sin \left(f^{2} y-f t^{\prime}+\phi(f, t)-\zeta \sqrt{f / 2 L a}\right) e \sqrt{f / 2 L a} d f \\
+\int_{f=0}^{\infty} 2 a(f, t)^{2} f^{2} L a \sin \left(2 f^{2} y-2 f t^{\prime}+2 \phi(f, t)+\pi / 4-\zeta \sqrt{f / 2 L a}\right) e \sqrt{f / 2 L a} d f \\
+\int_{f=0}^{\infty} 2 a(f, t)^{2} f^{2} L a \sin (\zeta \sqrt{f / 2 L a}-\phi(f, t)+\pi / 4) e \zeta \sqrt{f / 2 L a} d f
\end{gathered}
$$

The first of these terms gives rise to a secular term in the pressure equations which corresponds to that derived by Longuet-Higgens (1953), who calculated the effect of molecular viscosity on waves.

5. When the resonant coupling term outlined above is neglected, the equation for the homogeneous pressure field becomes

$$
\begin{aligned}
\text { (A-37) } & \int_{0}^{\infty} \frac{\partial^{2} \mathrm{p}_{3}^{\mathrm{oH}}}{\partial \mathrm{t}^{\prime 2}}(\mathrm{f}, \mathrm{t})+\mathrm{f}^{2} \frac{\partial \mathrm{p}_{3}^{\mathrm{oH}}}{\partial \mathrm{z}}(\mathrm{f}, \mathrm{t}) \mathrm{df}=\int_{0}^{\infty}\left(\frac{\partial \mathrm{a}(\mathrm{f}, \mathrm{t})}{\partial \mathrm{t}}+2 \mathrm{Laf}{ }^{4} \mathrm{a}(\mathrm{f}, \mathrm{t})\right) \sin \left(\mathrm{f}^{2} \mathrm{y}-\mathrm{ft} \mathrm{f}^{\prime}+\phi(\mathrm{f}, \mathrm{t})\right) \mathrm{df} \\
& \int_{0}^{\infty}\left(\frac{\partial \phi(f, \mathrm{t})}{\partial \mathrm{t}}+\frac{\mathrm{a}(\mathrm{f}, \mathrm{t})^{2}}{2}+\frac{\mathrm{F}_{\mathrm{x}}}{2}+\int_{-\infty}^{0}\left\langle\mathrm{v}_{2}^{\mathrm{o}}\left(\mathrm{z}^{\prime}\right)>\mathrm{e}^{2 \mathrm{z}^{\prime}} \mathrm{dz} \mathrm{z}^{\prime}\right) \cos \left(\mathrm{f}^{2} \mathrm{y}-\mathrm{ft}^{\prime}+\phi(\mathrm{f}, \mathrm{t})\right) \mathrm{df}\right.
\end{aligned}
$$

where $<>$ is used to denote averaging along the wave direction of propagation. In order to avoid secularity either there must be an atmospheric component which balances the terms on the right-hand side, or $\partial \mathrm{a} / \partial \mathrm{t}$ and $\partial \phi / \partial \mathrm{t}$ are nonzero. The first of these corresponds to a damping term due to viscosity, the second to a correction to the wave phase speed due to nonlinear dispersion, advection, and any cross-wave horizontal component of the Coriolis force.

6 . The third-order velocities associated with the irrotational portion of the flow and third-order wave profile may be solved for here. However, as we are only interested in the second-order flow (and these components do not affect it directly) they are not presented here. 
7. Solving the horizontal internal momentum equation yields the boundary conditions for $\mathbf{u}_{2}^{o}$.

$$
\begin{gathered}
\left.\frac{\partial u_{2}^{o}}{\partial z}\right|_{z=0}=\tau_{x} \\
\left.\frac{\partial v_{2}^{o}}{\partial z}\right|_{z=0} ^{\infty}=\tau_{y}+2 \int_{0}^{\infty} f^{4} L a a(f, t)^{2} d f=\tau_{y}+\left.\frac{\partial v_{s}}{\partial z}\right|_{z=0}
\end{gathered}
$$

So that there is one term due to frictional stresses and a second term due to wave decay.

8. The wave velocities result in an order $\mathrm{e}^{3}$ fluctuating density which is given by

$$
\rho_{3}^{o}=\int_{f=0}^{\infty} a(f, t) f^{2} e^{2} z\left(\sin \left(f^{2} y-f t^{\prime}+\phi(f, t)\right) \frac{\partial \rho_{2}^{o}}{\partial y}-\cos \left(f^{2} y-f t^{\prime}+\phi(f, t)\right) \frac{\partial \rho_{2}^{o}}{\partial z}\right) d f
$$

\section{Solution at order $\varepsilon^{4}$}

At this order, only the velocity and density equations are of interest. As a result, only these equations, and their solutions are considered below. The relevant equations are

$$
\text { (A-40a) } \begin{aligned}
\frac{\partial \mathbf{u}_{2}^{0}}{\partial \mathrm{t}}+\frac{\partial \mathbf{u}_{4}^{\mathrm{o}}}{\partial \mathrm{t}^{\prime}}+\left(\mathbf{u}_{3}^{\mathrm{o}} * \nabla\right) \mathbf{u}_{1}^{\mathrm{o}}+\left(\mathbf{u}_{2}^{\mathrm{o}} * \nabla\right) \mathbf{u}_{2}^{\mathrm{o}}+\left(\mathbf{u}_{1}^{\mathrm{o}} * \nabla\right) \mathbf{u}_{3}^{\mathrm{o}}+\mathbf{F} \times \mathbf{u}_{2}^{\mathrm{o}}= \\
-\nabla \mathrm{p}_{4}^{\mathrm{o}}+\mathrm{Ri} \rho_{2}^{\mathrm{o}} \mathbf{k}+\mathrm{La} \nabla^{2} \mathbf{u}_{2}^{\mathrm{o}} \\
\text { (A-40b) } \\
\frac{\partial \rho_{2}}{\partial \mathrm{t}}+\frac{\partial \rho_{4}}{\partial \mathrm{t}^{\prime}}+\left(\mathbf{u}_{1}^{\mathrm{o}} * \nabla\right) \rho_{3}+\left(\mathbf{u}_{2}^{\mathrm{o}} * \nabla\right) \rho_{2}=\mathrm{La} \nabla^{2} \rho_{2}
\end{aligned}
$$

Taking the curl of the the momentum equation yields the following equation for vorticity:

$$
\begin{gathered}
(\mathrm{A}-41) \frac{\partial \omega_{2}^{\mathrm{o}}}{\partial \mathrm{t}}+\frac{\partial \omega_{4}^{\mathrm{o}}}{\partial \mathrm{t}^{\mathrm{\prime}}}+\left(\mathbf{u}_{1}^{\mathrm{o}} * \nabla\right) \omega_{3}^{\mathrm{o}}-\left(\omega_{3}^{\mathrm{o}} * \nabla\right) \mathbf{u}_{1}^{\mathrm{o}}+\left(\mathbf{u}_{2}^{\mathrm{o}} * \nabla\right) \omega_{2}^{\mathrm{o}}-\left(\omega_{2}^{\mathrm{o}} * \nabla\right) \mathbf{u}_{2}^{\mathrm{o}}= \\
(\mathbf{F} * \nabla) \mathbf{u}_{2}^{\mathrm{o}}+\operatorname{Ri} \nabla \times \mathbf{k} \rho_{2}+\mathrm{La} \nabla^{2} \omega_{2}^{\mathrm{o}}
\end{gathered}
$$

The terms of interest involve the interaction of the order $\varepsilon$ wave velocity with the fluctuating order $\varepsilon^{3}$ vorticities. These interactions give rise to terms which have no $t^{\prime}$ dependence and so must be balanced by the long-time variability in the order $\varepsilon^{2}$ vorticity. Substituting the formulations in (A-33), and applying the condition that only terms with no $t$ ' dependence be balanced by the long-time variation in $\omega_{2}$ yields the following equations: 
$(\mathrm{A}-42 \mathrm{a})$

$$
\begin{gathered}
\frac{\partial \xi_{2}^{0}}{\partial \mathrm{t}}+\left(\mathbf{u}_{2}^{0} * \nabla\right) \xi_{2}^{0}-\left(\omega_{2}^{0} * \nabla\right) \mathrm{u}_{2}^{\mathrm{o}}+\int_{0}^{\infty} \mathrm{a}(\mathrm{f}, \mathrm{t}) \mathrm{f}^{2} \mathrm{e}^{2 \mathrm{f}_{\mathrm{z}} \mathrm{z}} \frac{\partial \xi_{2}^{\mathrm{o}}}{\partial \mathrm{y}} \mathrm{df}= \\
(\mathbf{F} * \nabla) \mathrm{u}_{2}^{\mathrm{o}}+\mathrm{Ri} \frac{\partial \rho_{2}}{\partial \mathrm{y}}+\mathrm{La} \nabla 2 \xi_{2}^{\mathrm{o}}
\end{gathered}
$$

$(\mathrm{A}-42 \mathrm{~b})$

$$
\frac{\partial \Omega_{2}^{0}}{\partial \mathrm{t}}+\left(\mathbf{u}_{2}^{\mathrm{o}} * \nabla\right) \Omega_{2}^{\mathrm{o}}-\left(\omega_{2}^{\mathrm{o}} * \nabla\right) \mathrm{v}_{2}^{\mathrm{o}}+\int_{0}^{\infty} \mathrm{f} \mathrm{e} \mathrm{e}^{2 \mathrm{f} z} \frac{\partial \Omega_{2}^{0}}{\partial y} \mathrm{df}=
$$

$$
\int_{0}^{\infty} \mathrm{a}(\mathrm{f}, \mathrm{t})^{2} \mathrm{f} \mathrm{e}^{2 \mathrm{f}^{2} \mathrm{z}}\left(\varsigma_{2}^{\mathrm{o}}+\mathrm{F}_{\mathrm{z}}\right) \mathrm{df}+(\mathrm{F} * \nabla) \mathrm{v}_{2}^{\mathrm{o}}+\mathrm{Ri} \frac{\partial \rho_{2}}{\partial \mathrm{x}}+\mathrm{La} \nabla^{2} \Omega_{2}^{\mathrm{o}}
$$

$(\mathrm{A}-42 \mathrm{c})$

$$
\frac{\partial \zeta_{2}^{0}}{\partial \mathrm{t}}+\left(\mathbf{u}_{2}^{0} * \nabla\right) \zeta_{2}^{0}-\left(\omega_{2}^{0} * \nabla\right) \mathrm{w}_{2}^{0}+\int_{0}^{\infty} \mathrm{a}(\mathrm{f}, \mathrm{t})^{2} \mathrm{f} 3 \mathrm{e}^{2 \mathrm{f} 2} \frac{\partial \zeta_{2}^{\mathrm{o}}}{\partial \mathrm{y}} \mathrm{df}=
$$

$$
(\mathrm{F} * \nabla) \mathrm{w}_{2}^{0}+\mathrm{La} \nabla 2 \xi_{2}^{0}
$$

But since the Stokes drift and Stokes drift shear are

$$
\begin{aligned}
& v_{s}(z)=\int_{0}^{\infty} a(f, t)^{2 f} e^{2 f f^{2}} d f \\
& \frac{\partial v_{s}}{\partial z}(z)=\int_{0}^{\infty} a(f, t)^{2} f^{5} e^{2 f 2} d f
\end{aligned}
$$

In vector form (A-42) may be rewritten

$$
\begin{gathered}
\text { (A-44) } \frac{\partial \omega_{2}^{0}}{\partial \mathrm{t}}+\left(\mathbf{u} \mathbf{u}^{*} \nabla\right) \omega_{2}^{\mathrm{o}}+\left(\mathbf{u}_{2}^{\mathrm{o}} * \nabla\right) \omega_{2}^{\mathrm{o}}-\left(\omega_{2}^{\mathrm{o}} * \nabla\right) \mathbf{u}_{2}^{0}=\left(\omega_{2}^{\mathrm{o}} * \nabla\right) \mathbf{u}_{\mathrm{s}} \\
(\mathbf{F} * \nabla)\left(\mathbf{u}_{2}^{\mathrm{o}}+\mathbf{u}_{\mathrm{s}}\right)+\operatorname{Ri} \nabla \times \mathbf{k} \rho_{2}+\mathrm{La} \nabla^{2} \omega_{2}^{\mathrm{o}}
\end{gathered}
$$

If the coordinate system is now changed into one in which the $y$ axis is parallel to the cell axis and the variation of $\mathrm{u}_{2}^{o}$ in the alongcell direction is taken to be order $\varepsilon$ in comparison with its variation in the crosscell direction then the vorticity may be written

$$
\omega_{2}^{0}=\left(-\frac{\partial v}{\partial z}, \frac{\partial u}{\partial z}-\frac{\partial w}{\partial x}, \frac{\partial v}{\partial x}\right)
$$

where the subscripts and superscripts have now been dropped. Letting $\Omega=\frac{\partial u}{\partial z}-$ $\frac{\partial \mathrm{w}}{\partial \mathrm{x}}$, equation (A-43) gives: 


$$
\begin{aligned}
& (\mathrm{A}-46 \mathrm{a})-\frac{\partial \partial \mathrm{v}}{\partial \mathrm{t} \partial \mathrm{z}}-\left(\left(\mathbf{u}+\mathrm{u}_{\mathrm{S}}\right) * \nabla\right) \frac{\partial \mathrm{v}}{\partial \mathrm{z}}-(\mathbf{u} * \nabla) \mathrm{F}(\partial \mathrm{v}, \partial \mathrm{z})+\frac{\partial \mathrm{v}}{\partial \mathrm{z}} \frac{\partial \mathrm{u}}{\partial \mathrm{x}}-\frac{\partial \mathrm{v}}{\partial \mathrm{x}} \frac{\partial \mathrm{u}}{\partial \mathrm{z}}=\frac{\partial \mathrm{v} \partial \mathrm{u}_{\mathrm{s}}}{\partial \mathrm{x} \partial \mathrm{z}} \\
& \mathrm{F}_{\mathrm{x}} \frac{\partial \mathrm{u}}{\partial \mathrm{x}}+\mathrm{F}_{\mathrm{z}} \frac{\partial \mathrm{u}}{\partial \mathrm{z}}+\mathrm{La} \nabla 2 \frac{\partial \mathrm{v}}{\partial \mathrm{z}} \\
& (\mathrm{A}-46 \mathrm{~b}) \frac{\partial \Omega}{\partial \mathrm{t}}+\left(\left(\mathrm{u}+\mathrm{u}_{\mathrm{S}}\right) * \nabla\right) \Omega=\frac{\partial \mathrm{v} \partial \mathrm{v}_{\mathrm{s}}}{\partial \mathrm{x} \partial \mathrm{z}}+\mathrm{F}_{\mathrm{z}} \frac{\partial}{\partial \mathrm{z}}\left(\mathrm{v}+\mathrm{v}_{\mathrm{s}}\right)+\mathrm{F}_{\mathrm{x}} \frac{\partial \mathrm{v}}{\partial \mathrm{x}} \mathrm{Ri} \frac{\partial \rho_{2}}{\partial \mathrm{x}}+\mathrm{La} \nabla^{2} \Omega \\
& \text { (A-46c) } \quad \frac{\partial \partial \mathrm{v}}{\partial \mathrm{t} \partial \mathrm{x}}+\left(\left(\mathbf{u}+\mathbf{u}_{\mathrm{S}}\right) * \nabla\right) \frac{\partial \mathrm{v}}{\partial \mathrm{x}}-(\mathbf{u} * \nabla) \frac{\partial \mathrm{w}}{\partial \mathrm{x}}+\frac{\partial \mathrm{v}}{\partial \mathrm{z}} \frac{\partial \mathrm{w}}{\partial \mathrm{x}}-\frac{\partial \mathrm{v}}{\partial \mathrm{x}} \frac{\partial \mathrm{w}}{\partial \mathrm{z}}= \\
& \mathrm{F}_{\mathrm{x}} \frac{\partial \mathrm{w}}{\partial \mathrm{x}}+\mathrm{F}_{\mathrm{z}} \frac{\partial \mathrm{w}}{\partial \mathrm{z}}+\mathrm{La} \nabla 2 \frac{\partial \mathrm{v}}{\partial \mathrm{x}}
\end{aligned}
$$

When $F_{x}=0$ the second equation is just that for vorticity used throughout this thesis. When the substitution $\frac{\partial u}{\partial x}=-\frac{\partial w}{\partial z}$ is made in (A-46a) and (A-46c) they turn out to be the $\mathrm{z}$ and $\mathrm{x}$ derivatives, respectively, of

$$
\frac{\partial \mathrm{v}}{\partial \mathrm{t}}+\left(\mathrm{u}+\mathrm{u}_{\mathrm{s}}\right) \frac{\partial \mathrm{v}}{\partial \mathrm{x}}+\mathrm{w} \frac{\partial \mathrm{v}}{\partial \mathrm{z}}=\mathrm{F}_{\mathrm{x}} \mathrm{w}-\mathrm{F}_{\mathrm{z}} \mathrm{u}+\mathrm{La} \nabla^{2} \mathrm{v}
$$

which (when $\mathrm{F}_{\mathrm{x}}=0$ ) is just the equation for alongcell velocity used throughout this work.

The density equation may also be solved for the long-term variation in density. It turns out that if $\rho=\rho_{2}$ the fluctuating order $\varepsilon^{3}$ density interacts with the wave velocities to give a term of the form $v_{s} \frac{\partial \rho}{\partial y}$ so that the consistency condition on density is:

$$
\frac{\partial \rho}{\partial t}+\left(u+u_{s}\right) \frac{\partial \rho}{\partial x}+w \frac{\partial \rho}{\partial z}=L a \nabla^{2} \rho
$$

This gives the full set of equations used in this work, with two heuristic modifications. These are

1. The scattering of waves off of cells is not calculated.

2. The surface stress does not include a term which depends on the Stokes drift. Additionally, the potential effects of the horizontal component of the Coriolis force are neglected. This means that any dependence of cell dynamics on whether the cells are oriented in the zonal or meridional direction is not included.

This work along with that of previous authors neglects these effects, in large part because they impede comparison with data (where the Stokes drift and surface stress are taken as given, and the surface stress includes topographic form drag). It should be noted that the data presented in this work does not support the idea that the surface Eulerian shear goes as $\mathrm{La} \frac{\partial \mathrm{v}_{\mathrm{S}}}{\partial \mathrm{z}}$ (it is much smaller, and not well correlated with the Eulerian shear). This highlights the importance of a better parameterization of eddy viscosity near the surface. 


\section{Appendix B: The Spectral Instability Code}

The equations for linear instability are derived below following the procedure outlined in Chapter 2. Let

$$
\psi=\Psi(z)+\psi(x, z, t)=\sum_{n=1}^{\infty} \Psi_{n} \sin \left(\frac{n \pi z}{D}\right)+\sum_{n=1}^{\infty} \Psi_{n}(t) \sin \left(\frac{n \pi z}{D}\right) e^{i k x}
$$

$$
v=V(z)+v(x, z, t)=\sum_{n=1}^{\infty} v_{n} \cos \left(\frac{n \pi z}{D}\right)+\sum_{n=1}^{\infty} v_{n}(t) \sin \left(\frac{n \pi z}{D}\right) e^{i k x}
$$

$$
\mathrm{u}_{\mathrm{S}}=\mathrm{u}_{\mathrm{S}}(\mathrm{z}) \sum_{\mathrm{n}=1}^{\infty} \mathrm{u}_{\mathrm{s}_{\mathrm{n}}} \cos \left(\frac{\mathrm{n} \pi \mathrm{z}}{\mathrm{D}}\right)
$$

$$
\mathrm{v}_{\mathrm{S}}=\mathrm{v}_{\mathrm{S}}(\mathrm{z})=\sum_{\mathrm{n}=1}^{\infty} \mathrm{v}_{\mathrm{S}_{\mathrm{n}}} \cos \left(\frac{\mathrm{n} \pi \mathrm{z}}{\mathrm{D}}\right)
$$

Two different representations of the density field are used, one in which the density is fixed on the upper and lower boundaries

$$
\rho=P(z)+\rho(x, z, t)=\sum_{n=0}^{\infty} P_{n} \cos \left(\frac{n \pi z}{D}\right)+\sum_{n=1}^{\infty} \rho_{n}(t) \sin \left(\frac{n \pi z}{D}\right) e^{i k x}
$$

and another for which the density is only fixed on the lower boundary while the density flux is fixed on the upper boundary.

$$
\rho=P(z)+\rho(x, z, t)=\sum_{n=1}^{\infty} P_{n} \cos \left(\frac{(2 n-1) \pi z}{2 D}\right)+\sum_{n=1}^{\infty} \rho_{n}(t) \sin \left(\frac{(2 n-1) \pi z}{D}\right) e^{i k x}
$$

Care needs to be taken to avoid singularites in the representation of the fields in which vertical gradients are important (in particular, density, Stokes' drift and alongcell velocity). In everything that follows, the horizontally varying series will be considered the perturbation. These series are truncated at some number $\mathrm{N}$ for the horizontally varying part (the second summation) and at $2 \mathrm{~N}+1$ for the horizontal mean part. A matrix equation for the $\psi_{n}, v_{n}, \rho_{n}$ can then be defined. 
(B-4a) $\frac{\partial \psi_{n}}{\partial t}=\sum_{m=1}^{N}\left(U s p_{n m}+P p_{n m}+D p_{n m}\right) \psi_{m}+\sum_{m=0}^{N}\left(E v_{n m}+V s_{n m}\right) v_{m}+\sum_{m=1}^{N} R_{n m} \rho_{m}$

$$
\begin{gathered}
\frac{\partial v_{n}}{\partial t}=\sum_{m=0}^{N}\left(U s v_{n m}+P v_{n m}+D v_{n m}\right) v_{m}+\sum_{m=1}^{N}\left(E p_{n m}+V p_{n m}\right) \psi_{m} \\
\frac{\partial \rho_{n}}{\partial t}=\sum_{m=1}^{N}\left(U s r_{n m}+\operatorname{Pr}_{n m}+D r_{n m}\right) \rho_{m}+\sum_{m=1}^{N} R p_{n m} \psi_{m}
\end{gathered}
$$

Pp represents the interaction of the streamfunction perturbation with the mean crosstream shear corresponding to the Jacobian terms in the linearized vorticity equation $-\frac{\partial \psi}{\partial \mathrm{x}} \frac{\partial^{3}}{\partial \mathrm{z}^{3}} \Psi-\frac{\partial \Psi}{\partial \mathrm{z}} \frac{\partial}{\partial \mathrm{x}} \nabla^{2} \Psi$. Pv and Pr represent the advection of the alongcell velocity and density perturbations respectively by the crosstream flow, corresponding to $-\frac{\partial \Psi \partial v}{\partial z \partial x}$ and $-\frac{\partial \Psi}{\partial z} \frac{\partial \rho}{\partial x}$. Vp and Rp represent the advection of the mean alongcell velocity and density fields respectively by the perturbation flow corresponding to the terms $\frac{\partial \mathrm{V}}{\partial \mathrm{z}} \frac{\partial \psi}{\partial \mathrm{x}}$ and $\frac{\partial \mathrm{P}}{\partial \mathrm{z}} \frac{\partial \psi}{\partial \mathrm{x}}$. Usp,Usv, and Usr represent the advection of the streamfunction, alongcell velocity, and density perturbations by the crosstream Stokes' drift, corresponding to the terms $\mathrm{u}_{\mathrm{s}} \frac{\partial}{\partial \mathrm{x}} \nabla^{2} \psi, \mathrm{u}_{\mathrm{s}} \frac{\partial \mathrm{v}}{\partial \mathrm{x}}$, and $\mathrm{u}_{\mathrm{s}} \frac{\partial \rho}{\partial \mathrm{x}}$. $\mathrm{Dp}, \mathrm{Dv}$, and Dr represent the diffusive damping terms $-\mathrm{La}\left(\frac{\partial^{2}}{\partial \mathrm{x}^{2}}+\frac{\partial^{2}}{\partial \mathrm{z}^{2}}\right)(\Omega, \mathrm{v}, \rho)$. Ev and Ep represent the effects of Coriolis force turning the perturbation velocities, $\mathrm{Ek} \frac{\partial \mathrm{v}}{\partial \mathrm{z}}$ and $-\mathrm{Ek} \frac{\partial \psi}{\partial \mathrm{z}}$. Vsp is the CL2 vortex force, corresponding to the term $\frac{\partial v_{\mathrm{s}} \partial \mathrm{v}}{\partial \mathrm{z} \partial \mathrm{x}}$ and $\mathrm{R}$ is the density vortex force $\mathrm{Ri} \frac{\partial \rho}{\partial \mathrm{x}}$. Each matrix is derived below.

The damping matrices are the easy to find. Substituting the modal expansions in (B-1) and (B-2) yields

$$
D v_{n m}=D p_{n m}=D r_{n m}=-L a\left(k^{2}+\frac{n^{2} \pi^{2}}{D^{2}}\right) \delta_{n m}
$$

When the density flux is fixed on the upper boundary, the damping matrix for density is

$$
\operatorname{Dr}_{\mathrm{nm}}=-\mathrm{La}\left(\mathrm{k}^{2}+\frac{(2 \mathrm{n}-1)^{2} \pi^{2}}{\mathrm{D}^{2}}\right) \delta_{\mathrm{nm}}
$$

The Ekman velocity forcing matrices are equally easy to find. Substituting the summations for $\mathrm{v}$ and $\psi$ yields

$$
\mathrm{Ev}_{\mathrm{nm}}=\frac{\mathrm{Ekn} \pi / \mathrm{D}}{\mathrm{k}^{2}+\mathrm{n}^{2} \pi^{2} / \mathrm{D}^{2}} \delta_{\mathrm{nm}}
$$




$$
\mathrm{Epnm}=-\mathrm{Ek} \frac{\mathrm{n} \pi}{\mathrm{D}} \delta_{\mathrm{nm}}
$$

Note that these matrices will, left to themselves, produce an inertial oscillation whose frequency asymptotes to a minimum of Ek at $\mathrm{k}=0$.

The density forcing matrix is simple for the basis chosen in (B-2). The equation

$$
\frac{\partial \Omega}{\partial t}=\operatorname{Ri} \frac{\partial \rho}{\partial x}
$$

yields

$$
\mathrm{R}_{\mathrm{nm}}=-\frac{\mathrm{ikRi}}{\mathrm{k}^{2}+\mathrm{n}^{2} \pi^{2} / \mathrm{D}^{2}} \delta_{\mathrm{nm}}
$$

When however, the density flux is fixed on the upper surface, (B-8) becomes in summation form

$$
\sum_{n=1}^{N}-\left(k^{2}+\frac{n^{2} \pi^{2}}{D^{2}}\right) \frac{\partial \psi_{n}}{\partial t} \sin \left(\frac{n \pi z}{D}\right)=\sum_{n=1}^{N} \text { ik Ri } \rho_{m} \cos \left(\frac{(m-1 / 2) \pi z}{D}\right)
$$

Multiplying both sides by $\sin \left(\frac{\mathrm{n} \pi \mathrm{z}}{\mathrm{D}}\right)$ and integrating yields.

$$
\begin{aligned}
R_{n m}=- & \frac{i k R i}{k^{2}+n^{2} \pi^{2} / D^{2}} \frac{2}{D} \int_{-D} \sin \left(\frac{n \pi z}{D}\right) \cos \left(\frac{(n-1 / 2) \pi z}{D}\right) d z \\
= & \frac{i k R i / \pi}{k^{2}+n^{2} \pi^{2} / D^{2}}\left(\frac{1}{n+m-1 / 2}+\frac{1}{n-m+1 / 2}\right)
\end{aligned}
$$

The vorticity advection matrix Pp is derived in a similar fashion. Substituting (B-

1) into the vorticity transport equation gives

(B-12) $\mathrm{Pp}_{\mathrm{nm}}=\frac{-\mathrm{ik \pi /2 \textrm {D }}}{\mathrm{k}^{2}+\mathrm{n}^{2} \pi^{2} / \mathrm{D}^{2}}$

$$
\left(\operatorname{lm}-\mathrm{nl}\left(\mathrm{k}^{2}+\frac{\mathrm{n}^{2} \pi^{2}}{\mathrm{D}^{2}}-\frac{|\mathrm{m}-\mathrm{n}|^{2} \pi^{2}}{\mathrm{D}^{2}}\right) \Psi_{|\mathrm{m}-\mathrm{n}|}-(\mathrm{m}+\mathrm{n})\left(\frac{\mathrm{k}^{2}+\mathrm{n}^{2} \pi^{2}}{\mathrm{D}^{2}}-\frac{(\mathrm{m}+\mathrm{n})^{2} \pi^{2}}{\mathrm{D}^{2}}\right) \Psi_{\mathrm{m}+\mathrm{n}}\right)
$$

Similarly, the matrix Usp, respresenting vorticity advection due to crosstream Stokes drift is given by:

$$
U \mathrm{sp}_{n \mathrm{~m}}=-\frac{i \mathrm{k}}{2} \frac{\mathrm{k}^{2}+\frac{\mathrm{m}^{2} \pi^{2}}{\mathrm{D}^{2}}}{\mathrm{k}^{2}+\frac{\mathrm{n}^{2} \pi^{2}}{\mathrm{D}^{2}}}\left(\mathrm{u}_{\mathrm{S}_{\mathrm{n}-\mathrm{ml}}}-\mathrm{u}_{\mathrm{S}_{\mathrm{n}+\mathrm{m}}}\right)
$$

The remaining term in the vorticity equation is the Craik-Leibovich vortex force. The truncated series representation yields the following expression for $\mathrm{Vs}_{\mathrm{nm}}$.

$$
\mathrm{Vs}_{\mathrm{nm}}=\frac{\mathrm{ik \pi /2D}}{\mathrm{k}^{2}+\mathrm{n}^{2} \pi^{2} / \mathrm{D}^{2}}\left((\mathrm{n}-\mathrm{m}) \mathrm{v}_{\mathrm{S}_{|\mathrm{n}-\mathrm{m}|}}+(\mathrm{n}+\mathrm{m}) \mathrm{v}_{\mathrm{S}_{\mathrm{n}+\mathrm{m}}}\right)
$$


Turning to the alongcell velocity advection terms, we first consider the cross tream advection of perturbation velocity.

$$
\sum_{n=0}^{N} \frac{\partial v_{n}}{\partial t} \cos \left(\frac{n \pi z}{D}\right)=-\sum_{1=1}^{2 N+1} \frac{1 \pi}{D} \Psi_{1} \sum_{m=0}^{N} i k \cos \left(\frac{1 \pi z}{D}\right) \cos \left(\frac{m \pi z}{D}\right) v_{m}
$$

To isolate single modes in this case it is necessary to multiply by $\frac{2}{D} \cos \left(\frac{n \pi z}{D}\right)$ for $\mathrm{n}>0$ and $\frac{1}{\mathrm{D}}$ for $\mathrm{n}=0$. Integrating yields the following expression for $\mathrm{Pv}$.

$$
\begin{aligned}
& \mathrm{Pv}_{\mathrm{nm}}=-\frac{\mathrm{ik} \pi}{2 \mathrm{D}}\left(\ln -\mathrm{m} \mid \Psi_{|\mathrm{n}-\mathrm{m}|}+(\mathrm{n}+\mathrm{m}) \Psi_{\mathrm{n}+\mathrm{m}}\right) \quad \mathrm{n}>0 \\
& \mathrm{Pv}_{0 \mathrm{~m}}=-\frac{\mathrm{ik} \pi}{2 \mathrm{D}} \mathrm{m} \Psi_{\mathrm{m}}
\end{aligned}
$$

Similarly, the advection of mean alongcell velocity by the perturbation flow is given by:

$$
\begin{gathered}
V_{p_{n m}}=-\frac{i k \pi}{2 D}\left(-(n-m) V_{\mid n-m l}+(n+m) V_{n+m}\right) \quad n>0 \\
V p_{0 m}=-\frac{i k \pi}{2 D}\left(-m V_{m}\right)
\end{gathered}
$$

and the matrix representation of the advection of the alongcell velocity by crossstream Stokes drift is:

$$
\begin{gathered}
\mathrm{Usv}_{\mathrm{nm}}=-\frac{\mathrm{ik}}{2}\left(\mathrm{u}_{\mathrm{S} \mathrm{ln}_{\mathrm{m} l} \mathrm{l}}+\mathrm{u}_{\mathrm{S}_{\mathrm{n}+\mathrm{m}}}\right) \quad \mathrm{n}>0 \\
\mathrm{Usv}_{0 \mathrm{~m}}=-\frac{\mathrm{ik}}{2}\left(\mathrm{~m} \mathrm{u}_{\mathrm{S}_{\mathrm{m}}}\right)
\end{gathered}
$$

Lastly, we turn to the density equation. The crosstream advection of the density perturbation by the mean flow is (for the two different sets of boundary conditions)

$$
\sum_{n=1}^{N} \frac{\partial \rho_{n}}{\partial t} \sin \left(\frac{n \pi z}{D}\right)=-\sum_{1=1}^{2 N+1} \frac{1 \pi}{D} \Psi_{1} \sum_{m=1}^{N} i k \cos \left(\frac{1 \pi z}{D}\right) \sin \left(\frac{m \pi z}{D} \rho_{m}\right)
$$

$$
\sum_{n=1}^{N} \frac{\partial \rho_{n}}{\partial t} \cos \left(\frac{(n-1 / 2) \pi \mathrm{z}}{D}\right)=-\sum_{1=1}^{2 N+1} \frac{1 \pi}{D} \Psi_{1} \sum_{m=1}^{N} i k \cos \left(\frac{1 \pi \mathrm{z}}{D}\right) \cos \left(\frac{(m-1 / 2) \pi \mathrm{z}}{\mathrm{D}} \rho_{m}\right)
$$

which yields the following expressions for Pr.

$$
\begin{aligned}
\operatorname{Pr}_{n m}=-\frac{i k \pi}{2 D}\left(\ln -m \mid \Psi_{|n-m|}-(n+m) \Psi_{n+m}\right) & \rho \text { fixed @ } \mathrm{z}=0 \\
\operatorname{Pr}_{n m}=-\frac{i k \pi}{2 D}\left(\ln -m \mid \Psi_{|n-m|+}(n+m-1) \Psi_{n+m}\right) & \frac{\partial \rho}{\partial z} \text { fixed @ } \mathrm{z}=0
\end{aligned}
$$


The advection of the mean density field by the perturbation flow is the most complicated of the expressions since it does not permit the neat separation into kronecker delta functions

$$
\sum_{n=1}^{N} \frac{\partial \rho_{n}}{\partial t} \sin \left(\frac{n \pi z}{D}\right)=-\sum_{1=1}^{2 N+1} \frac{1 \pi}{D} P_{1} \sum_{m=1}^{N} i k \sin \left(\frac{1 \pi z}{D}\right) \sin \left(\frac{m \pi z}{D}\right) \psi m
$$

$$
\sum_{n=1}^{N} \frac{\partial \rho_{n}}{\partial t} \cos \left(\frac{(n-1 / 2) \pi \mathrm{z}}{D}\right)=-\sum_{1=1}^{2 N+1} \frac{1 \pi}{D^{P_{1}}} \sum_{m=1}^{N} i k \sin \left(\frac{(1-1 / 2) \pi \mathrm{z}}{D}\right) \cos \left(\frac{m \pi z}{D}\right) \psi_{m}
$$

Multiplying by the appropriate basis function and integrating yields (B-22a) $\mathrm{Rp}_{\mathrm{nm}}=$

\section{$2 \mathrm{~N}+1$}

$\sum_{1=1}^{i \frac{k}{D}} 1 P_{1}\left(1+(-1)^{n+l+m}\right)\left(\frac{1}{n+l-m}+\frac{1}{n-1+m}-\frac{1}{n+l+m}-\frac{1}{n-1-m}\right) \rho$ fixed @z=0

(B-22b) $\quad \mathrm{Rp}_{\mathrm{nm}}=$

$$
\sum_{1=1}^{2 N+1} \frac{i k}{D} 1 P_{1}\left(\frac{1}{1+m+n-1}+\frac{1}{1+m-n}-\frac{1}{1-m+n}-\frac{1}{1-m-n+1}\right) \frac{\partial \rho}{\partial z} \text { fixed @ } z=0
$$

Finally, we turn to the advection of the density by cross-stream Stokes drift. Proceeding as done above gives the following expressions for Usr.

$$
\begin{array}{ll}
\mathrm{Usr}_{\mathrm{nm}}=-\frac{\mathrm{ik}}{2}\left(\mathrm{u}_{\mathrm{S}_{|\mathrm{n}-\mathrm{m}|}}-\mathrm{u}_{\mathrm{S}_{\mathrm{n}+\mathrm{m}}}\right) & \rho \text { fixed @ } \mathrm{z}=0 \\
\mathrm{Usr} r_{\mathrm{nm}}=-\frac{\mathrm{ik}}{2}\left(\mathrm{u}_{\mathrm{S}_{|\mathrm{n}-\mathrm{m}|}}+\mathrm{u}_{\mathrm{S}_{\mathrm{n}+\mathrm{m}-1}}\right) & \frac{\partial \rho}{\partial \mathrm{z}} \text { fixed @ } \mathrm{z}=0
\end{array}
$$

If the density flux is fixed on both top and bottom boundaries, the density basis functions will have the representation $\cos \left(\frac{n \pi \mathrm{z}}{\mathrm{D}}\right)$. In this case the advection matrices for density Pr, Usr, and Rpassume the same form for as those for downstream velocity. The density forcing matrix is now given by:

$$
R_{n m}=\frac{i k R i}{k^{2}+n^{2} \pi^{2} D^{2}}(1-(-1) n+m)\left(\frac{1}{n+m}+\frac{1}{n-m}\right)
$$

The code was tested for a number of cases where analytic solutions were available.

1. Viscous Rayleigh-Taylor instability 
2. Inviscid Craik-Leibovich instability with and without stratification.

For viscous Rayleigh-Taylor instability, the normal modes are

$$
(\psi, \rho)=\left(\psi_{n} \sin \left(\frac{n \pi z}{D}\right) \rho_{n} \sin \left(\frac{n \pi z}{D}\right)\right)
$$

The growth rates for such modes are

$$
\gamma=-\mathrm{La}\left(\mathrm{k}^{2}+\left(\frac{\mathrm{n} \pi}{\mathrm{D}}\right)^{2}\right)+\sqrt{\frac{\mathrm{Rik}^{2} \partial \rho_{0} / \partial \mathrm{z}}{\mathrm{k}^{2}+(\mathrm{n} \pi / \mathrm{D})^{2}}}
$$

For stratified Craik-Leibovich instability if the Eulerian and Stokes drift shears are linear and $\mathrm{La}=0$, the normal modes have the form

$$
(\psi, \rho, v)=\left(\psi_{n} \sin \left(\frac{n \pi z}{D}\right) \rho_{n} \sin \left(\frac{n \pi z}{D}\right) v_{n} \sin \left(\frac{n \pi z}{D}\right)\right)
$$

and the growth rates are given by

$$
\gamma=\sqrt{\left(\frac{k^{2}}{k^{2}+(n \pi / D)^{2}}\right)\left(\frac{\partial V \partial v_{s}}{\partial z \partial z}+R i \frac{\partial \rho_{0}}{\partial z}\right)}
$$

Table B. 1 shows the growth rates given $\mathrm{k}=1, \mathrm{D}=\pi$ for viscous Rayleigh-Taylor and inviscid Craik Leibovich instability for different values of $\mathrm{N}$. For relatively small values of $\mathrm{N}(\mathrm{N}=10)$, the code yields results within $0.01 \%$ of the correct theoretical answer.

\begin{tabular}{|l|l|l|l|l|l|l|}
\hline $\mathrm{La}$ & $\frac{\partial \mathrm{V}}{\partial \mathrm{z}}$ & $\mathrm{Ri}$ & $\mathrm{N}$ & $\begin{array}{l}\text { Theoretical } \\
\text { Growth Rate }\end{array}$ & $\begin{array}{l}\text { Error in } \\
\text { Growth Rate } \\
(\rho \text { fixed })\end{array}$ & $\begin{array}{l}\text { Error in } \\
\text { Growth Rate } \\
(\partial \rho / \partial \mathrm{z} \text { fixed })\end{array}$ \\
\hline 0 & 0 & -1 & 10 & 0.7071 & $-2.4 \times 10^{-16}$ & $-5.2 \times 10^{-5}$ \\
\hline 0 & 0 & -1 & 20 & 0.7071 & $-2.3 \times 10^{-16}$ & $-1.5 \times 10^{-5}$ \\
\hline 0 & 0 & -1 & 40 & 0.7071 & $-2.2 \times 10^{-16}$ & $-1.0 \times 10^{-5}$ \\
\hline 0.05 & 0 & -1 & 10 & 0.6071 & $-1.9 \times 10^{-16}$ & $* * * * * *$ \\
\hline 0.1 & 0 & -1 & 10 & 0.5071 & $-5.5 \times 10^{-16}$ & $* * * * * *$ \\
\hline 0 & 1 & 0 & 10 & 0.7071 & $-7.1 \times 10^{-5}$ & $-7.1 \times 10^{-5}$ \\
\hline 0 & 1 & 0 & 20 & 0.7071 & $-1.0 \times 10^{-5}$ & $-1.0 \times 10^{-5}$ \\
\hline 0 & 1 & 0 & 40 & 0.7071 & $-1.4 \times 10^{-6}$ & $-1.4 \times 10^{-6}$ \\
\hline
\end{tabular}

Table B.1: Error introduced by the truncation in the growth rates of the most unstable modes for some cases where analytic growth rates are available. Density stratification and Eulerian velocity shear $\frac{\partial \mathrm{V}}{\partial \mathrm{z}}$ are taken to be constant with depth. *** mark two cases where an analytic solution is not available given the upper boundary condition. 


\section{Appendix C: The Finite-Difference Code}

The finite-difference code was developed using techniques outlined in Roache (1977). The various terms were handled as follows:

1. Partial time derivatives were handled using simple forward differencing.

2. The Jacobian terms were coded using the parameterization of Arakawa (1966) which preserves the integrals of momentum, angular momentum, and density, as well as the energy and enstrophy.

3. The source terms for vorticity $\frac{\partial v}{d x} \frac{\partial v_{s}}{\partial z}$ (Craik-Leibovich) and Ri $\frac{\partial \rho}{\partial x}$ were parameterized using simple centered differencing. (Note: this is more accurate than using the calculated value of the gradient at the center of the box, since the integrated force over the box is better represented, particular when the Stokes drift profile has a high degree of curvature).

4. The Coriolis force term was handled by rotating the velocity and shear, thus avoiding growth of inertial oscillations due to simple time-stepping.

5. The streamfunction was solved using a Poisson equation solver developed at NCAR the results of which were compared to some results obtained using simple Richardson relaxation. The two agreed extremely well.

A number of tests of the code were carried out using results from simple linear theory. These were

1. Simple decay of an unforced vortex with different values of La. This test was done to test whether or not the time-stepping scheme introduced significant numerical viscosity. Even with decay rates of order 1, the finite-difference code reproduced the predicted decay rates to within $1.5 \%$.

2. Craik-Leibovich instability given linear Stokes drift and Eulerian shears.

Growth rates ranging from 1 to 4 were reproduced to within $3.6 \%$ (at least some of the error is due to the fact that as the cells grow they reduce the velocity shear, reducing the growth rate as shown in Chapter 5).

3. Rayleigh-Taylor instability given a linear density profile which was unstably stratified. Growth rates ranging from $0.2-0.9$ were reproduced to within $3.5 \%$.

To summarize, the code reproduced both decay rates as well as unstable growth rates which are within the range of interest for this work (order 1 nondimensional) to within a few percent. This is evidence that the fundamental dynamics forcing the system are accurately represented (the numerical viscosity is not too large and the forcing functions are correctly parameterized). 


\section{Appendix D: Measurement Errors from Current Meters on FLIP}

Since the observational chapters of this thesis focus on current meter measurements it is important at the outset to mention and quantify the error introduced by surface gravity waves. As noted in the last section, the VMCMs during MILDEX were set to average over periods of one minute. If the wave field is given by a sinusoidal gravity wave with velocity amplitude $V_{0}$ and period $T_{w}$, the error from averaging over a period $\mathrm{T}_{\mathrm{av}}$ goes as

$$
\mathrm{V}_{\mathrm{err}} \sim \frac{\mathrm{V}_{0} \mathrm{~T}_{\mathrm{w}}}{2 \pi \mathrm{T}_{\mathrm{av}}}
$$

so that for $V_{0}$ of order $1 \mathrm{~m} / \mathrm{s}$, aliased signals of order $2-4 \mathrm{~cm} / \mathrm{s}$ could be seen given $\mathrm{T}_{\mathrm{av}}$ of 1 minute and $\mathrm{T}_{\mathrm{w}}$ of 7-15 seconds. During SWAPP, the current meters sampled at $0.5 \mathrm{~Hz}$, so that wave aliasing over long averaging periods is less of a problem.

The problem gets more complicated when the fact that current meters hanging from a string are not fixed in space but may move and tilt in phase with the waves and their associated currents is taken into account. Suppose a current meter has a periodic trajectory $\left(\mathrm{x}_{\mathrm{c}}(\mathrm{t}), \mathrm{z}_{\mathrm{c}}(\mathrm{t})\right)$ and tilts with an angle $\mathrm{q}(\mathrm{t})$. Defining $<>$ as a time-averaging operator and letting

$$
<\left(\mathrm{x}_{\mathrm{c}}(\mathrm{t}), \mathrm{z}_{\mathrm{c}}(\mathrm{t})\right)>=\left(\mathrm{x}_{0}, \mathrm{z}_{0}\right)
$$

then if $\mathrm{u}_{\mathrm{m}}$ is the velocity measured by the current meter, if the current meter tilt is small, the measured current is related to the actual current through the following relationship.

$$
\begin{gathered}
(\mathrm{D}-3)<\mathrm{u}_{\mathrm{m}}\left(\mathrm{x}_{\mathrm{c}}, \mathrm{z}_{\mathrm{c}}\right)>=<\mathrm{u}\left(\mathrm{x}_{0}, \mathrm{z}_{0}\right)>\cos (\theta(\mathrm{t}))+<\left(\left(\mathrm{x}-\mathrm{x}_{0}\right) \frac{\partial \mathrm{u}}{\partial \mathrm{x}}+\left(\mathrm{z}-\mathrm{z}_{0}\right) \frac{\partial \mathrm{u}}{\partial \mathrm{z}}\right) \cos (\theta(\mathrm{t}))+ \\
<\mathrm{w}\left(\mathrm{x}_{0}, \mathrm{z}_{0}\right) \sin (\theta(\mathrm{t}))>+\ldots
\end{gathered}
$$

If the motion of the current meter is in phase with the shears induced by the waves a wave-induced bias appears. The size of this term is the same order of magnitude as the Stokes drift, $5 \mathrm{~cm} / \mathrm{s}$. Likewise if the current meter tilts in phase with the waves, there is a potential velocity bias.

In order to evaluate the size of these errors, a model of a current meter string as a multiple pendulum was constructed. The model was forced with pressure and velocity fields derived from the wave height time series. The 
resulting velocity fields had no mean velocity and no time-varying velocities with period more than about 20 seconds. The model is derived below.

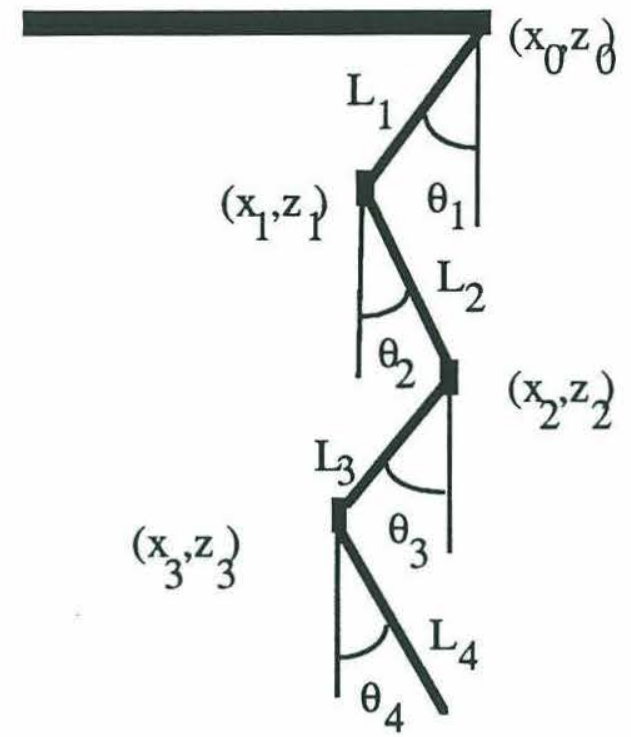

Figure D.1: Schematic of the multiple pendulum problem
Suppose that the current meter string is represented as a multiple pendulum as at left, with the $\mathrm{N}$ current meters being point masses with masses $\mathrm{M}_{1}, \mathrm{M}_{2}, \mathrm{M}_{3}, \ldots, \mathrm{M}_{\mathrm{N}}$ and connected by rigid elements of length $\mathrm{L}_{1}, \mathrm{~L}_{2}$, $\mathrm{L}_{3}, \ldots \mathrm{L}_{\mathrm{N}}$. The fulcrum of the pendulum has coordinates $\mathrm{x}_{0}, \mathrm{z}_{0}$ and may be allowed to move. The equation of motion may be derived using Lagrangian dynamics (Marion, 1965).

The total potential energy of the system is

(D-4) $P E=\sum_{i=1}^{N} M_{i} g z_{i}=\sum_{i=1}^{N} M_{i} g \sum_{j=1}^{i} L_{j} \cos \theta_{j}$

While the total kinetic energy is

$$
\begin{gathered}
\mathrm{KE}=\sum_{\mathrm{i}=1}^{N} \frac{\mathrm{M}_{\mathrm{i}}}{2}\left(\frac{\partial \mathrm{x}_{\mathrm{i}}^{2}}{\partial \mathrm{t}}+\frac{\partial \mathrm{z}_{\mathrm{i}}^{2}}{\partial \mathrm{t}}\right)= \\
\sum_{\mathrm{i}=1}^{\mathrm{N}} \frac{\mathrm{M}_{\mathrm{i}}}{2}\left(\frac{\partial \mathrm{x}_{0}}{\partial \mathrm{t}}+\sum_{\mathrm{j}=1}^{\mathrm{i}} \frac{\partial \theta_{\mathrm{i}}}{\partial \mathrm{t}} \mathrm{L}_{\mathrm{j}} \cos \theta_{\mathrm{j}}\right)^{2}+\left(\frac{\partial \mathrm{z}_{0}}{\partial \mathrm{t}}+\sum_{\mathrm{j}=1}^{\mathrm{i}} \frac{\partial \theta_{\mathrm{i}}}{\partial \mathrm{t}} \mathrm{L}_{\mathrm{j}} \sin \theta_{\mathrm{j}}\right)^{2}
\end{gathered}
$$

The unforced equations of motion are derived by letting $L=\mathrm{KE}-\mathrm{PE}$, and $\mathrm{p}_{\mathrm{j}}=\frac{\partial \theta_{\mathrm{j}}}{\partial \mathrm{t}}$ so that:

$$
\frac{\mathrm{d}}{\mathrm{dt}} \frac{\partial L}{\partial \mathrm{p}_{\mathrm{j}}}-\frac{\partial L}{\partial \theta_{\mathrm{j}}}=0
$$

The resulting equation is shown below

$$
\begin{gathered}
\text { (D-7) } \frac{d}{d t} \frac{\partial L}{\partial p_{j}}-\frac{\partial L}{\partial \theta_{j}}=\sum_{k=j}^{N} M_{k}\left\{g L_{j} \sin \left(\theta_{j}\right)+\frac{\partial^{2} x_{0}}{\partial t^{2}} L_{j} \cos \left(\theta_{j}\right)+\frac{\partial^{2} z_{0}}{\partial t^{2}} L_{j} \sin \left(\theta_{j}\right)+\right. \\
\left.\sum_{i=1}^{N} L_{i} L_{j}\left(\cos \left(\theta_{i}-\theta_{j}\right) \frac{\partial^{2} \theta_{i}}{\partial t^{2}}-\sin \left(\theta_{i}-\theta_{j}\right) \frac{\partial \theta_{i}{ }^{2}}{\partial t}\right)\right\}=0
\end{gathered}
$$


This equation can be simplified by letting $M_{\mathrm{j}}=\sum_{\mathrm{k}=\mathrm{j}}^{\mathrm{N}} \mathrm{M}_{\mathrm{k}}$ and making the small angle approximation so that all $\theta_{\mathrm{k}}$ are small. Then the equations can be linearized so that

$$
M_{j}\left(\frac{\partial^{2} x_{0}}{\partial t^{2}} L_{j}+g L_{j} \theta_{j}\right)+\sum_{k=j}^{N} L_{j} \sum_{i=1}^{N} L_{i} \frac{\partial^{2} \theta_{i}}{\partial t^{2}}=0
$$

or in matrix form

$$
\sum_{i=1}^{N} A_{k i} \frac{\partial^{2} \theta_{i}}{\partial t^{2}}+M_{j}\left(\frac{\partial^{2} x_{0}}{\partial t^{2}} L_{j}+g L_{j} \theta_{j}\right)=0
$$

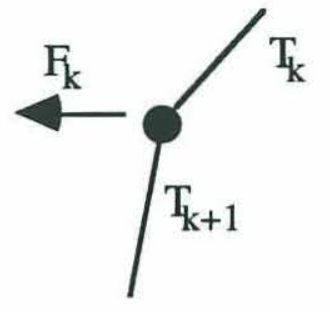

The inclusion of forcing into the problem can be understood by considering the diagram at left. Assuming that the system is at rest,

$$
\text { (D-10a) } \quad \mathrm{T}_{\mathrm{j}} \sin \left(\theta_{\mathrm{k}}\right)-\mathrm{T}_{\mathrm{j}+1} \sin \left(\theta_{\mathrm{k}+1}\right)=\mathrm{F}_{\mathrm{j}}
$$

$$
\mathrm{T}_{\mathrm{j}} \cos \left(\theta_{\mathrm{k}}\right)-\mathrm{T}_{\mathrm{j}+1} \cos \left(\theta_{\mathrm{k}+1}\right)=\mathrm{M}_{\mathrm{j}} \mathrm{g}
$$

Applying the small angle approximation, this means that $\mathrm{T}_{\mathrm{j}}=M_{\mathrm{j}} \mathrm{g}$. Then at equilibrium summing the first equation to the end of the string yields

$$
\theta_{\mathrm{j}}=\sum_{\mathrm{k}=\mathrm{j}}^{\mathrm{N}} \mathrm{F}_{\mathrm{j}} / M_{\mathrm{j}} \mathrm{g}=\frac{F_{\mathrm{j}}}{M_{\mathrm{j}} \mathrm{g}}
$$

This means that the equation of motion is

$$
\sum_{\mathrm{i}=1}^{N} \mathrm{~A}_{\mathrm{ki}} \frac{\partial^{2} \theta_{\mathrm{i}}}{\partial \mathrm{t}^{2}}+M_{\mathrm{j}}\left(\frac{\partial^{2} \mathrm{x}_{0}}{\partial \mathrm{t}^{2}} \mathrm{~L}_{\mathrm{j}}+\mathrm{g} \mathrm{L}_{\mathrm{j}} \theta_{\mathrm{j}}\right)=\sum_{\mathrm{k}=\mathrm{j}}^{\mathrm{N}} \mathrm{F}_{\mathrm{j}}
$$

The external force on the current meters was defined as the sum of the frictional and pressure forces

$$
F_{j}=A\left(\rho C_{d} l U-u_{w} l\left(U-u_{w}\right)+\frac{\partial p}{\partial x}\right)
$$

where $\mathrm{A}$ is the cross-sectional area of the current meter, $\mathrm{u}_{\mathrm{c}}$ is the current meter velocity, $u_{w}$ is the wave velocity, and $C_{d}$ is a drag coefficient set to 0.75 (Tupper, pers, comm.). The mass of the current meters is taken to be $78 \mathrm{~kg}$. The model was forced with a velocity field and fulcrum time series calculated from the wave 
records measured from FLIP. The resulting trajectories of the current meters at various depths could then be calculated.

During MILDEX, FLIP was taken to move very little with respect to the waves so that the only errors were calculated as the result of the current meters moving in response to wave forcing. The velocities "measured" by the model current meters given the observed wave field were averaged over 1 minute and mean and standard deviations computed for 15 minute periods. The results provide an estimate of the biases in current and shear introduced by the waves, as well as the contribution of wave aliasing to the velocity and shear variability. Because the wave field used is nondirectional, the multiple pendulum model will tend to overestimate the size of the error. The degree to which this is the case is a function of the spread of the waves. If the waves have $a \cos ^{2}(\theta)$ spread in direction for example, the method here will overestimate the wave velocities by a factor of 2 .

The output of the multiple pendulum model is considered for a period which included the roughest wind and wave conditions seen over the course of the experiment, the passage of the low pressure system on November 9th and 10th. Forty-one fifteen minute time series were taken and used to run the multiple pendulum model. The results are shown in Figure D.2. The average velocities produced by a wavefield in the absence of any mean Eulerian current are shown for depths of 2, 6.5, and 12 meters. The velocity bias was quite small (less than 1 $\mathrm{cm} / \mathrm{s}$ most of the time). Interestingly the velocity bias near the surface ran counter to the wave propagation direction. The averaged shear produced between "current meters" fixed at 2 and 6.5 meters (solid lines) and 6.5 and 12 meters (dashed lines) are shown in Figures D.2b. The shear is "upwave" with a magnitude of $0.0015 / \mathrm{s}$. This is an order of magnitude smaller than the observed shear.

We now turn to the time-varying currents produced by the waves as a result of aliasing. The standard deviation of the velocity "measured" by current meters at 2, 6.5, and 12 meters in the presence of waves alone is shown in Figure the fourth panel in Figure D.2. The standard deviations are quite a bit larger than the mean, reaching $3.2 \mathrm{~cm} / \mathrm{s}$. The standard deviation of the shear is shown in Figure D.2d. The time-varying shears are in general quite small, nowhere exceeding $0.004 \mathrm{~s}^{-1}$. They do not decrease with depth in the same way that the mean shear does. 

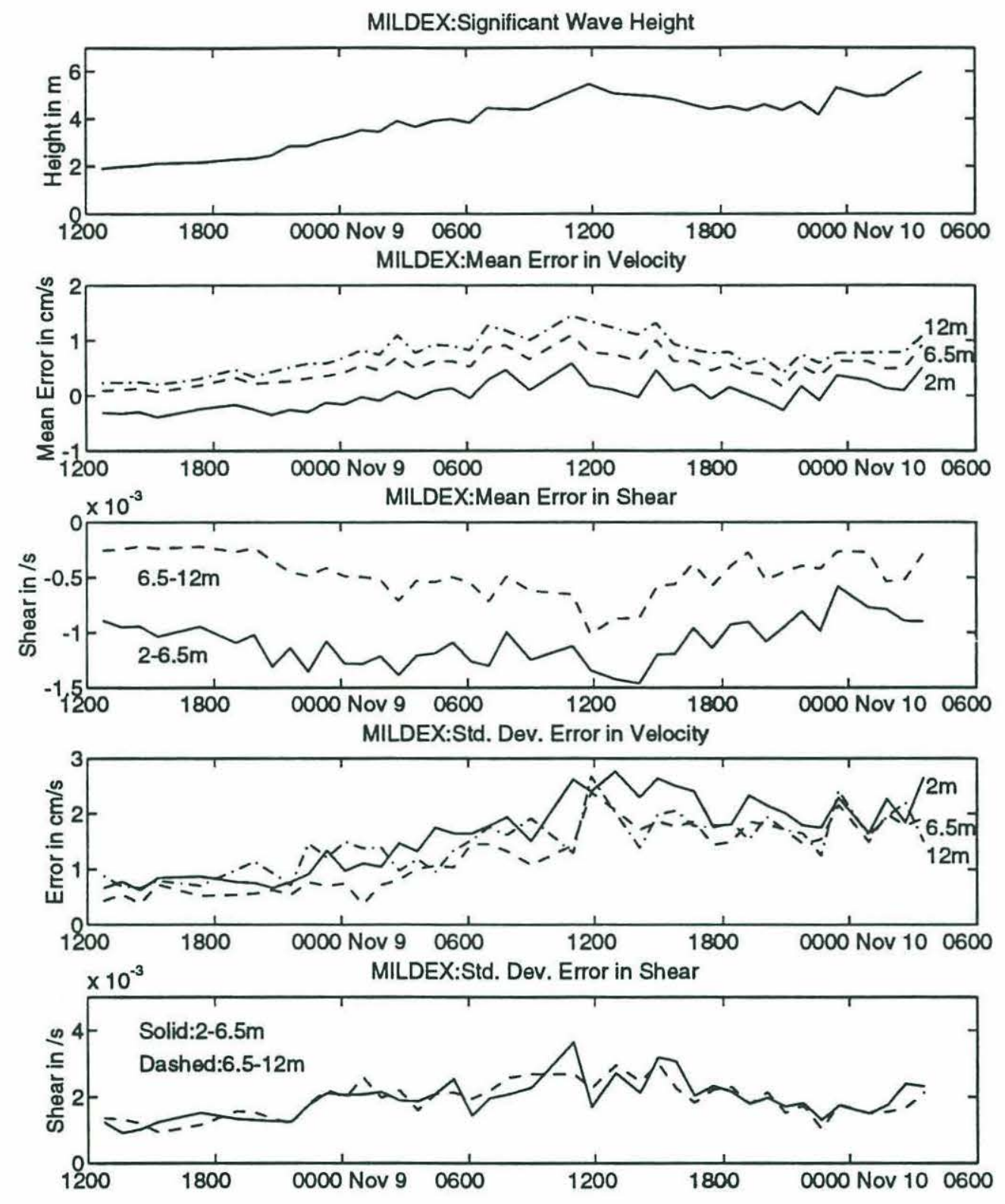

Figure D.2: Errors introduced during MILDEX by current meter motion in phase with the wave field. Period shown is one in which the Langmuir cells and waves reach their maximum strength. From top to bottom: Significant wave height in m, Error in mean velocity at 2, 6.5 and 12 meters depths, Error in mean shear between 2 and 6.5, 6.5 and 12 meters, Error in the standard deviation of timevarying $(1-30 \mathrm{cph})$ velocity, 2, 6.5 and 12 meters, Error in the standard devation of the high-frequency (1-30cph) shear between 2 and 6.5 and 6.5 and 12 meters. 


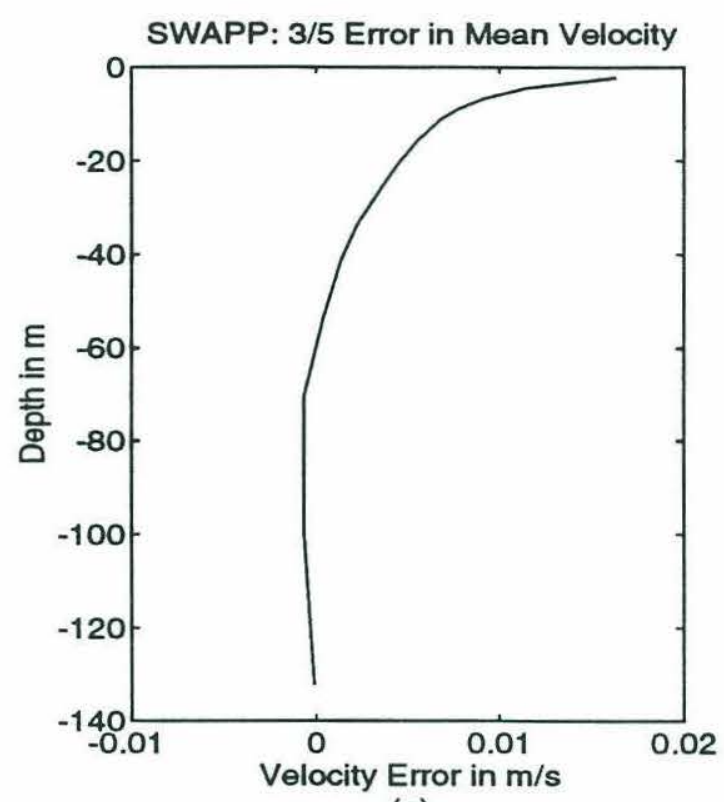

(a)

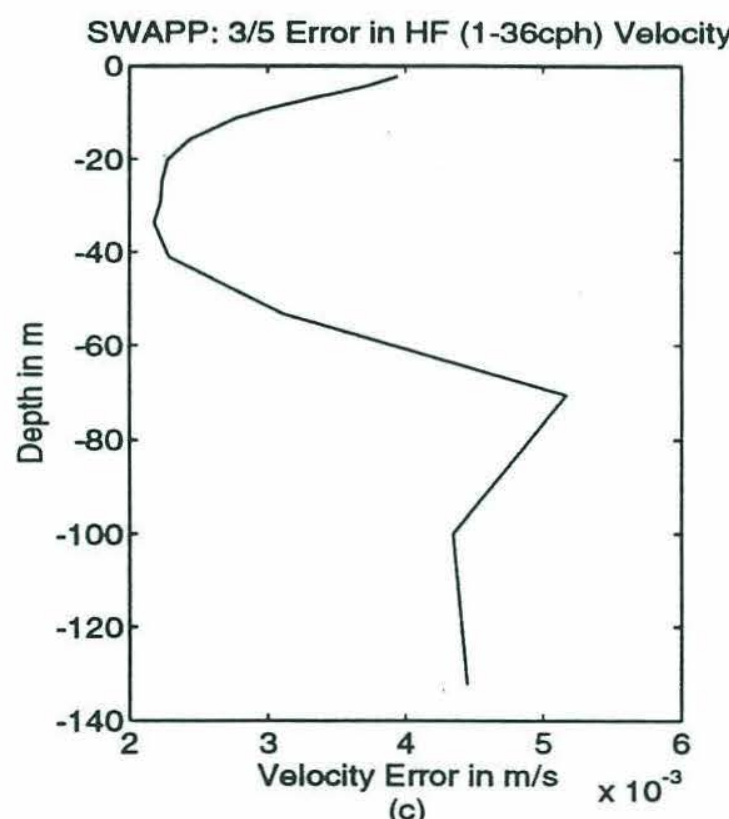

(c)

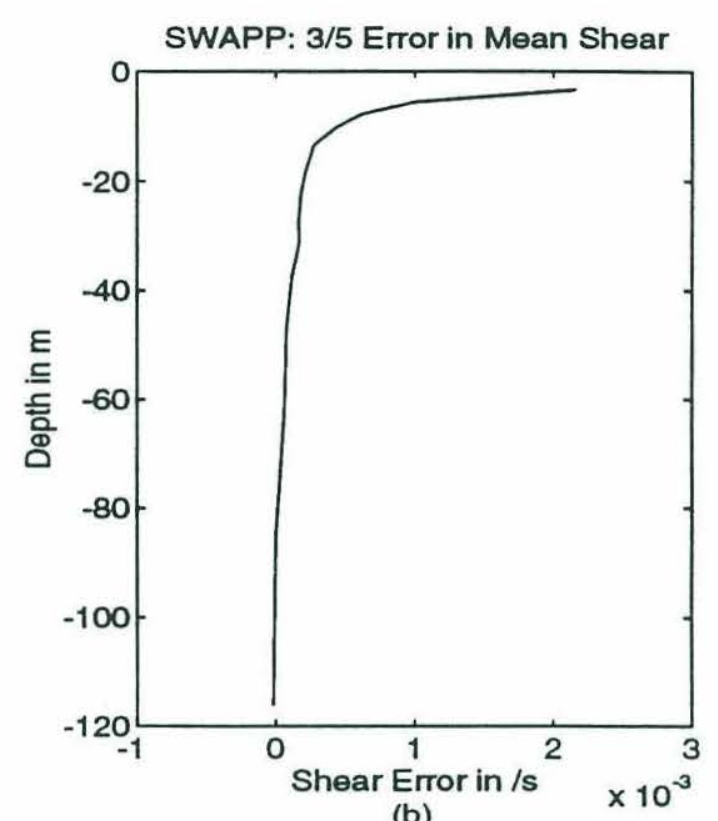

(b)

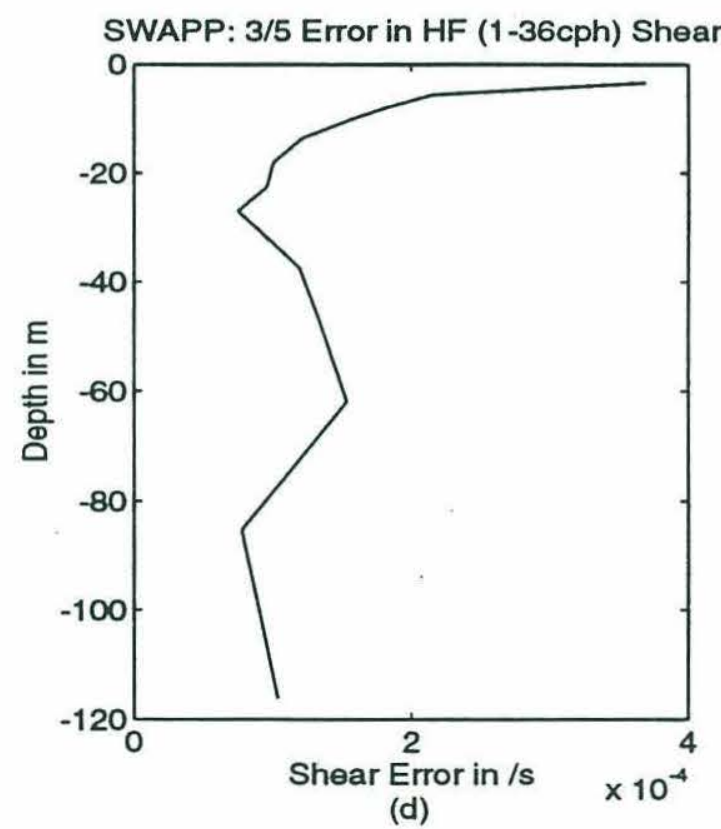

Figure D.3: Profiles of the velocity and shear error during SWAPP after the first wind event near time of highest waves. (a) Time-mean velocity error in $\mathrm{m} / \mathrm{s}$. (b) Time varying (1-36cph) velocity error in $\mathrm{m} / \mathrm{s}$. (c) Time-mean shear error in $/ \mathrm{s}$. Time varying (1-36) $\mathrm{cph}$ shear error in /s. 
The mean shear and time-varying shear associated with wave aliasing are much smaller than the shears observed during MILDEX which were linked to the presence of Langmuir cells. The mean upwind shears seen during MILDEX was an order of magnitude larger than the error predicted here, while the highfrequency shear had amplitudes at least 4 times as large as the predicted errors.

The story during SWAPP is similar, although the potential errors are somewhat larger. During SWAPP the presence of a three-point mooring resulted in FLIP's being closely coupled to the surface gravity wave field, tilting and changing its heading at frequencies near those of the gravity waves. Although the much heavier current meter strings deployed during SWAPP reduced this effect somewhat the mean shears were affected by the presence of motions in the vertical which were phase-locked to the surface waves. Figure D.3 shows profiles of the error introduced by current meter motion into the mean current and shear, and the time-varying current and shear in a frequency band from 1-36 cph. The time period shown is early on March 6th, when the waves were still quite high, but the winds were low. The errors are generally small in comparison to the observed signals. The only exception is the mean shear error which is about half the size of the observed mean shear $\left(0.004 \mathrm{~s}^{-1}\right)$. Using a directional spread to reduce the wave velocities results in the mean shear error being about one quarter of the observed near-surface shear. The most important term in producing the mean shear error is tilting of the platform in phase with the waves, which moves the current meters up and down in phase with the wave velocity signal. The timevarying shear error, however, is about one tenth of the observed high-frequency shear amplitude of $0.004 \mathrm{~s}^{-1}$. 


\section{Appendix E: Thruster Contamination during MILDEX}

During MILDEX, a thruster at a nominal depth of 15 meters on FLIP's hull was used to orient FLIP relative to the wind. A schematic of the orientation of the thruster and the current meters used during the experiment is shown in Figure E.1.

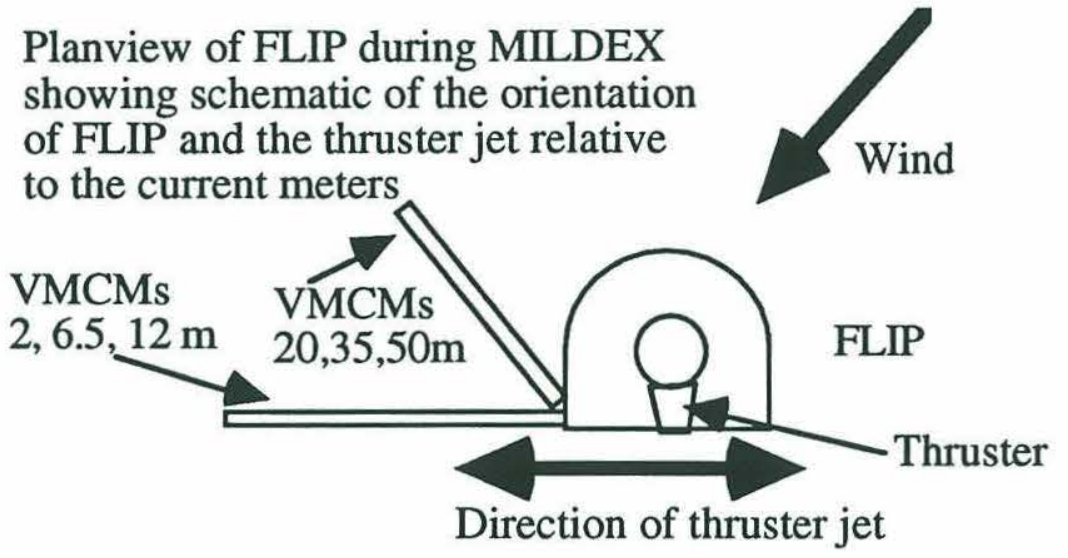

Figure E.1: Planview of FLIP during MILDEX showing orientation of the thruster relative to the wind and current meters.

When the thruster was on, it generated an intense jet, sometimes directed off the port side of FLIP and sometimes off the starboard side. Unfortunately the current meter string was placed in the path of this jet. As a result the current meter at 12 meters was strongly affected by the thruster. Figure E.2a and b show the hourly averaged velocity from this current meter (solid lines) in the east and north directions. These velocities were found to differ sharply from velocities measured at $6.5 \mathrm{~m}$ and $20 \mathrm{~m}$, which agreed relatively well with each other. The interpolated velocity from these current meters is shown by the dashed lines in E.2a and b. There were a number of bursts of thruster activity during the experiment, with particularly strong velocities recorded during the passage of the low on November 8-10.

The velocity signal due to the thruster needed to be removed in order for the binned data to be useful in calculating the mixed layer response to surface forcing. This was done by hand, taking times when the velocity at 12 meters was grossly different from the velocities around it and interpolating across the contaminated depth.

The mean velocity of the current meters at 6.5 and 20 meters does not seem to have been greatly affected by the presence of the thruster. The current 


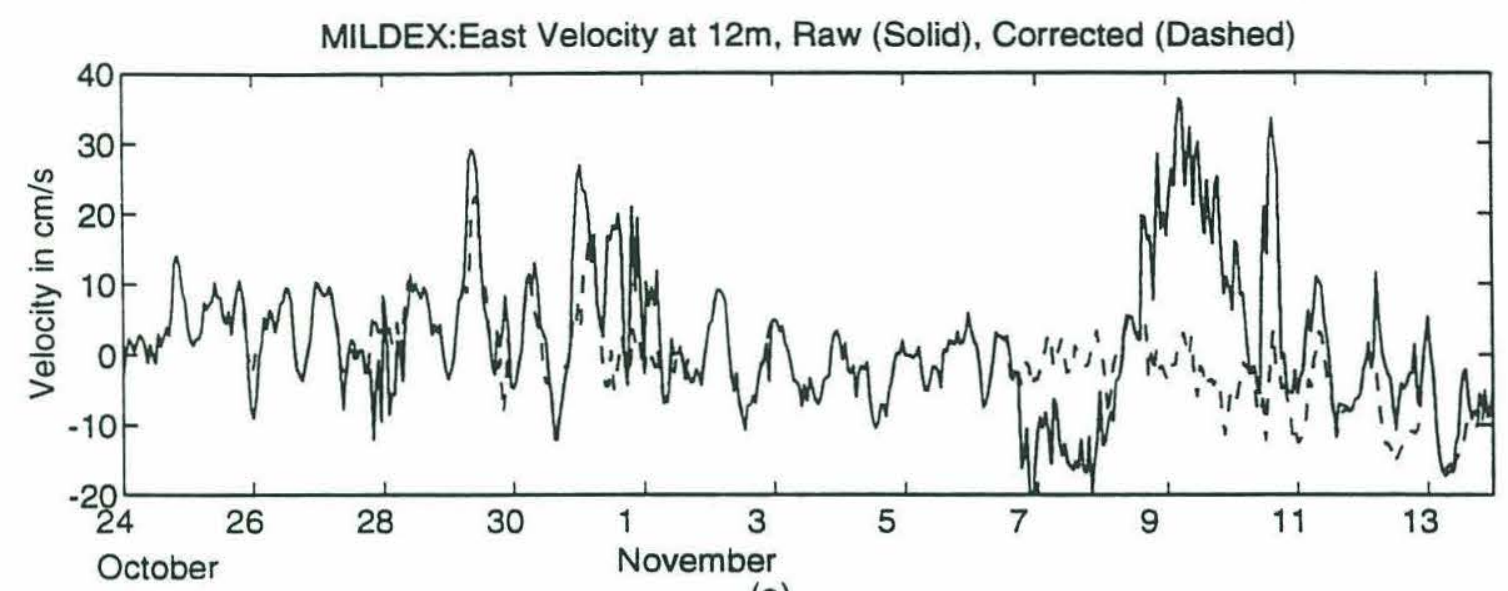

(a)

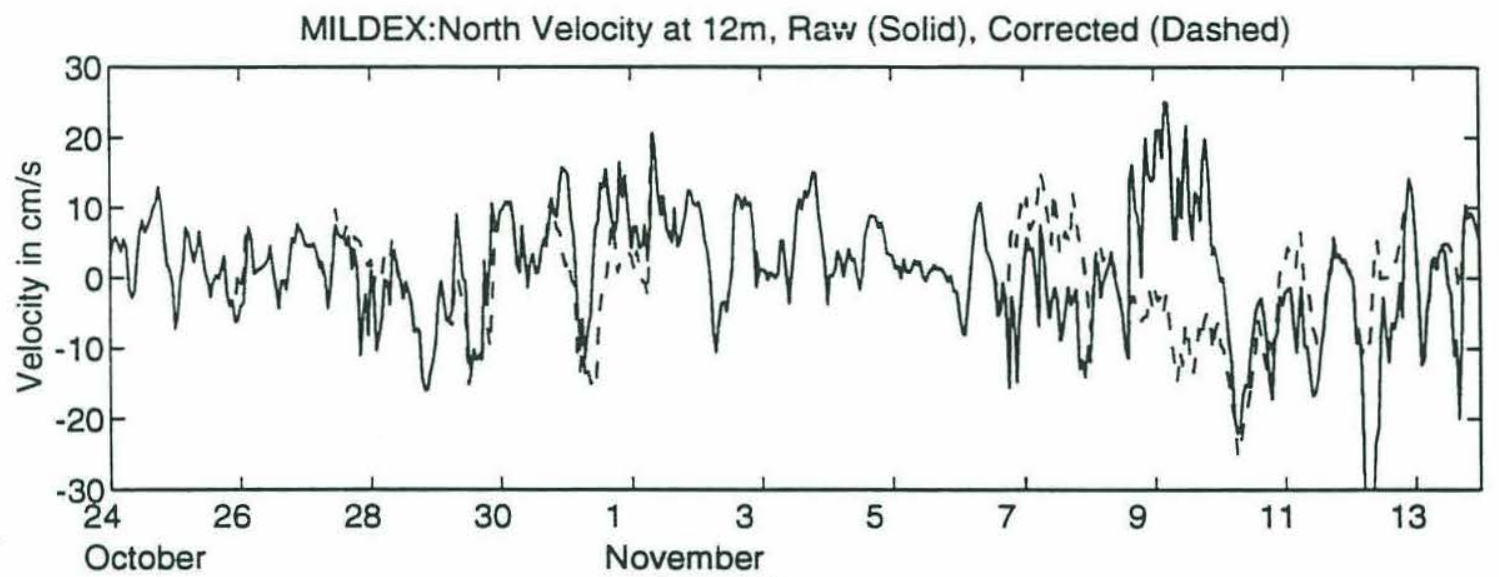

(b)

Figure E.2: Errors in velocity due to thruster contamination. Effect of the thruster on the binned,averaged data is shown. Raw velocities are shown by the solid lines, corrected velocities by the dashed lines. (a) East velocity at 12 meters. (b) North velocity at $12 \mathrm{~m}$. 
meter at 20 meters was not in a position where it could be contaminated as easily by the thruster, since it was placed forward of the axis along which the thruster acted rather than being in line. The velocities at 6.5 and 20 meters were in close agreement, providing evidence that the current meter at $6.5 \mathrm{~m}$ was not affected by the thruster in the mean. Thruster contamination would have biased Ekman response in the crosswind direction, increasing the overall transport significantly. However, the total Ekman transport during MILDEX was close to the theoretical value, so that it is unlikely that the thruster jet was picked up in the mean by either of these two current meters.

In order to evaluate whether the thruster affected the high-frequency (up to $30 \mathrm{cph}$, the Nyquist frequency for the VMCMs) variability in the current meters above and below it Figure E.3 examines the high frequency velocity field on November 9. During this day there are large differences between the velocity measured at 12 meters and those above and below it. The wind on November 9 was largely to the north. Figure E.3a and E.3b show the coherence between the east and north velocities, respectively, at 12 meters and those at 6.5 (solid) and 20 meters (dashed) for frequencies between 0.5 and $30 \mathrm{cph}$. The $95 \%$ confidence level is 0.125 and is shown by a horizontal chain-dotted line. The coherence is in general very low. Significant values are seen at around $2-5 \mathrm{cph}$, but even here the coherence is only 0.3. Except for this frequency band, there is little evidence that the thruster contaminates the high-frequency velocity at levels above and below it. Within this frequency band, there is still a question of whether the thruster is producing velocity perturbations in the VMCMs at 6.5 and 20 meters, or whether the physical mechanisms producing variability in the current meters at 6.5 and 20 meters also affect the 12 meter VMCM.

Figure E.3c shows spectra of the north velocity on November 9 at 6.5 and 20 meters. There are strong signals at about $4 \mathrm{cph}$ in both current meters. Figure E.3d shows the coherence between the meridional velocities in these current meters. The coherence is quite high, reaching 0.6 near the peak frequencies. The coherence between the current meters at 6.5 and 20 meters is twice as large as the coherence between these current meters and the one at 12 meters, presented in E.3a.

There are two possibilities for explaining the significant coherence found in the north velocity on November 9 . The first is that the thruster contaminated the current meters at 6.5 and 20 meters. The second is that Langmuir cells, 
Coherence Between 6.5 and $12 \mathrm{~m}$ Nov. 9

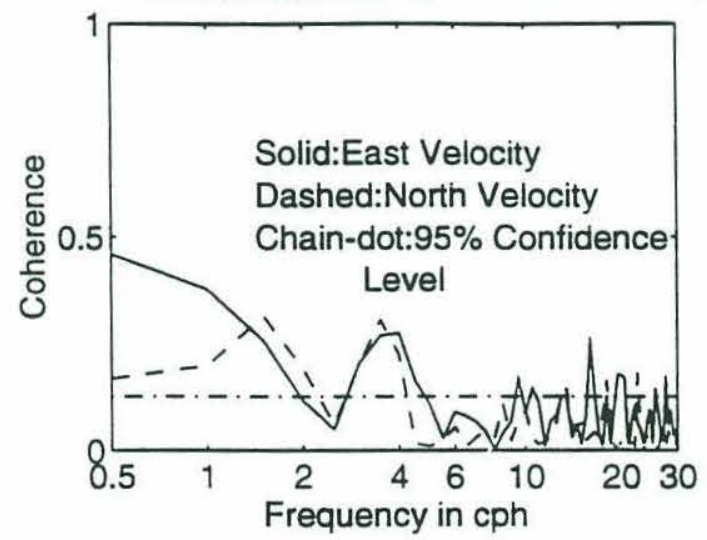

a

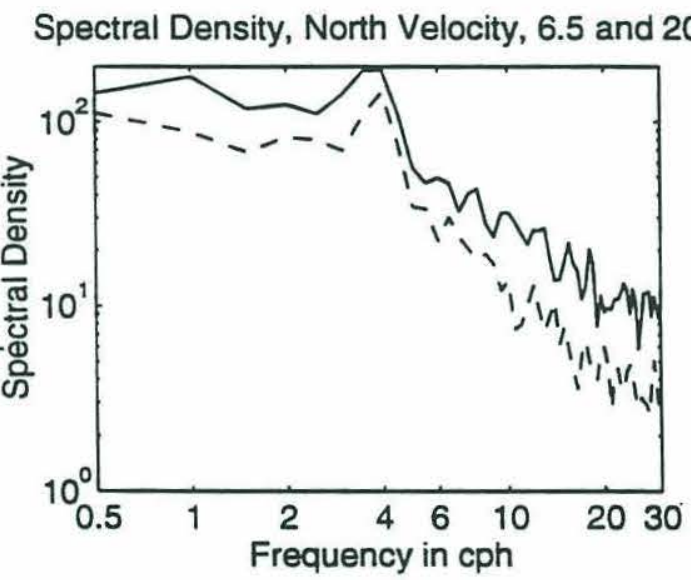

c

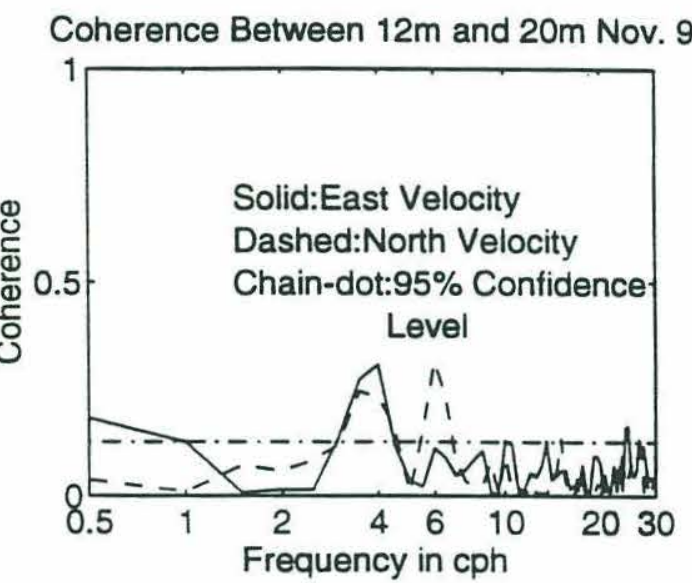

b

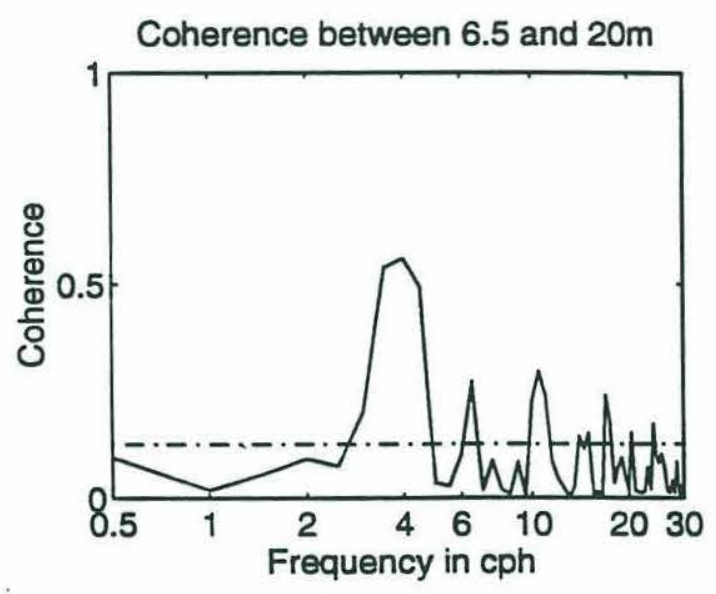

d

Figure E.3: Spectra and coherence of velocities at 6.5 and 20 meters during MILDEX. November 9. (a) Coherence between $6.5 \mathrm{~m}$ and $12 \mathrm{~m}$ (solid is east-west velocity, dashed is north-south velocity. (b) Same as (a) but between $12 \mathrm{~m}$ and $20 \mathrm{~m}$. (c) Spectrum of north-south velocity, Nov. $9,6.5 \mathrm{~m}$ (solid) and $20 \mathrm{~m}$ (dashed). (d) Coherence between north-south velocity at $6.5 \mathrm{~m}$ and that at $20 \mathrm{~m}$. 
perhaps associated with strong downwelling, interacted with the thruster jet and produced the variability at all three depths. If the first hypothesis were true, the coherence between the VMCMs flanking the thruster should have been lower than the coherence between the either of the VMCMs flanking the thruster and the VMCM within the thruster jet. Since this is not the case, it is argued that the second hypothesis is in fact the correct one. 


\section{References}

Agarwal, Y.,G., E.A. Terray, M.A. Donelan, P.A. Hwang, A. J. Williams, W.M. Drennan, K.K. Kahma, and S.A. Kitaigorodskii, (1992), Enhanced dissipation of kinetic energy beneath surface waves. Nature, 359: 219-221.

Arakawa, A., (1966), Computational design for long-term numerical integration of fluid motion, Two-dimensional incompressible flow: Part I., J. Comput. Phys., 1: 119-143.

Batchelor, G.K., (1969), The computation of the energy spectrum of twodimensional turbulence, Phys. Fluids, 12: 233-239.

Chapman, C.J., and M. R. E. Proctor, (1980), Nonlinear Rayleigh-Benard convection between poorly conducting boundaries, J. Fluid Mech., 101: 759782.

Chapman, R.P., and J. H. Harris, (1962), Surface backscattering strengths measured with explosive sound sources, J. Acous. Soc. Am., 52: 944-960.

Crawford W.R., (1992), Turbulence observations in the upper ocean during the Surface Waves Processes Program in the northeast Pacific, February to March, 1990. Canadian Data Report of Hydrography and Ocean Sciences No. 106., Sidney, B.C. Canada, Institute for Ocean Sciences.

Crawford W.R. and A. E. Gargett, (1988), Multiple thermoclines are barriers to vertical exchange in the subarctic Pacific during SUPER, May 1984, J. Mar. Res., 46: 77-103.

Craik, A. D. D., (1970), A wave-interaction model for the generation of windrows, J. Fluid Mech., 41: 802-822.

Craik, A.D.D., and S. Leibovich, (1976), A rational model for Langmuir Circulation, J. Fluid Mech., 73: 401-426.

Cox, S. M. and S. Leibovich, (1993), Langmuir circulations in a surface layer bounded by a strong thermocline, J. Phys. Oceanogr., 23: 1330-1345.

Davis. R.E, R. DeSzoeke, and P. Niiler, (1981), Variability in the upper ocean during MILE: Part II, Modelling the mixed layer response. Deep Sea Res., 28: 1427-1452.

Denman, K.L., (1973), A time-dependent model of the upper ocean, J. Phys. Oceanogr., 3: 173-185.

Denman, K.L., and M. Miyake, (1973), Upper layer modification at Ocean Station Papa: Observation and simulation, J. Phys. Oceanogr., 3: 186-196. 
Ekman, V.W., (1905), On the influence of the earth's rotation on ocean currents, Arkiv. Math. Astron. Phys., vol. 2, no. 11.

Faller, A. J., (1964), The angle of windrows in the ocean, Tellus, 16: 363-370.

Faller A.J. and A. H. Woodcock, (1964), The spacing of windrows of Sargassum in the ocean, J. Mar. Res., 22:22-29.

Fiedler, B.H., (1989), Scale selection in nonlinear thermal convection between poorly conducting boundaries, Geophys. and Astrophys. Fluid Dyn., 46:191201.

Foster, T.D., (1969), The effect of initial conditions and lateral boundaries on convection, J. Fluid Mech., 37:81-94.

Gammelsrød, T., (1975), Instability of Couette flow in a rotating fluid and the origin of Langmuir circulations. J. Geophys. Res., 80:5069-5075.

Gordon, A. L., (1970), Vertical momentum fluxes accomplished by Langmuir circulation, J. Geophys. Res., 75:4177-4179.

Gnanadesikan, A., (1990), XBT Measurements taken during the Surface Waves Processes Program Main Cruise. unpublished ms. Woods Hole Oceanographic Institution.

Gnanadesikan, A., (1994, subm.) Effects of mixed layer dynamics on the diurnal cycle of photochemically produced trace species: Special focus on carbon monoxide, submitted, J. Geophys. Res.

Harris, G.P., and J. N. A. Lott, (1973), Observations of Langmuir circulations in Lake Ontario, Limnol. Oceanogr., 18:584-589.

Hasselmann, K., (1961), On the nonlinear energy transfer in a gravity-wave spectrum, J. Fluid Mech., 12: 481-500.

Hasselmann, K., (1970), Wave-driven inertial oscillations, Geophys. Fluid Dyn., 1: 463-502.

Howard, L.N., and R. Krishnamurti, (1986), Large-scale flow in turbulent convection: A mathematical model, J. Fluid Mech., 170: 385-410.

Huang, N.E., (1979), On the surface drift currents in the ocean. J. Fluid Mech., 91:191-208.

Kenney, B.C., (1977), An experimental investigation of the fluctuating currents responsible for the generation of windrows, Ph.D. Thesis, Univ. Waterloo, Ont., 163 pp. 
Klein, P. and B. Coste, (1984), Effects of wind stress variability on nutrient transport into the mixed layer, Deep Sea Res., 31: 21-37.

Krishnamurti, R. and L.N. Howard, (1981), Large-scale flow generation in turbulent convection, Proc. Nat. Acad. Sci., 78: 1981-1985.

Langmuir, I., (1938), Surface motion of water induced by wind, Science, 87: 119123.

Large, W.G., and S. Pond, (1981), Open ocean momentum flux measurements in moderate to strong winds, J. Phys. Oceanogr., 11: 324-336.

Large, W.G., and S. Pond, (1982), Sensible and latent heat flux measurements over the ocean, J. Phys. Oceanogr., 12: 464-482.

Leibovich, S., (1977a), On the evolution of the system of surface wind drift currents and Langmuir circulation in the ocean. Part I: Theory and averaged current. J. Fluid Mech., 79:715-743.

Leibovich, S., (1977b), Convective instability of stably stratified water in the ocean, J. Fluid Mech., 82:561-583.

Leibovich, S., (1983), The form and dynamics of Langmuir Circulation, Ann. Rev. Fluid Mech., 15:391-427.

Leibovich, S., S.K. Lele, and I.M. Moroz, (1989), Nonlinear dynamics in Langmuir circulations and in thermosolutal convection, J. Fluid Mech., 198: 471-511.

Leibovich, S. and S. Paolucci, (1981), The instability of the ocean to Langmuir circulations, J. Fluid Mech., 102: 141-167.

Lele, S., (1985), Some problems of hydrodynamic stability arising in geophysical fluid dynamics, Ph.D. Thesis, Cornell Univ., 302pp.

Li, M., and C. Garrett, (1993a), Cell merging and the jet/downwelling ratio in Langmuir circulation, J. Mar. Res., 51:737-769.

Li, M., and C. Garrett (1993b), Is Langmuir circulation driven by surface waves or surface cooling? preprint, submitted to J. Phys. Oceanogr.

Linden, P.F., (1980), Mixing across a density interface produced by grid turbulence, J. Fluid Mech., 100: 691-703.

List, R.J., (1984) Smithsonian Meteorological Tables, Smithsonian Institution Press, Washington, D.C., 527 pp.

Longuet-Higgens, M.S., (1953), Mass transport in water waves, Phil. Trans. Roy. Soc. Lon. A, 145: 535-581. 
Lueck, R.E., (1988), Near-surface turbulence during the passage of a storm, unpublished ms. Johns Hopkins University.

McIntyre, M.E., (1981), On the "wave-momentum" myth., J. Fluid Mech.,.106: 331-347.

Malkus, W.V. R., and G. Veronis, (1958), Finite amplitude cellular convection, J. Fluid Mech., 4: 225-260.

Marion, J.B., (1965), Classical Dynamics of Particles and Systems, New York, Academic Press, 576 pp.

Mellor, G., and T. Yamada, (1974), A hierarchy of turbulence closure models for planetary boundary layers. J. Atmos. Sci., 31: 1791-1806.

Olson, R.J, S.W. Chisholm, E.R. Zettler, and E.V. Armbrust, (1990), Pigments, size, and distribution of Synechococcus in the North Atlantic and Pacific Oceans, Limnol. Oceanogr., 35: 45-58.

Owen, R.W., (1966), Small-scale, horizontal vortices in the surface layer of the sea, J. Mar. Res., 24:56-65.

Paduan, J,A. R.D. DeSzoeke, and R.A. Weller, (1989), Inertial oscillations in the mixed layer during the Mixed Layer Dynamics Experiment (MILDEX), $J$. Geophys. Res., 94:(C4) 4835-4842.

Park, Y.G., J.A. Whitehead and A. Gnanadesikan, (1994), Stirring a stratified fluid: Layer formation and energetics, in press J. Fluid Mech,

Payne, R.E., (1972), Albedo of the sea surface, J. Atmos. Sci., 29: 959-970.

Pedlosky, J., (1981), The nonlinear dynamics of baroclinic wave ensembles, $J$. Fluid Mech., 102: 169-209.

Phillips, O.M., (1960), The mean horizontal momentum and surface velocity of finite-amplitude random gravity waves, J. Geophys. Res., 65: 3473-3476.

Pierson, W.J., and L. Moskowitz, (1964), A proposed spectral form for fully developed wind seas based on the similarity theory of S.A. Kitaigorodskii, $J$. Geophys. Res., 69: 5191-5203.

Pinkel, R. and J. Smith, (1987), Open ocean surface wave measurement using Doppler sonar, J. Geophys. Res., 92: 12,967-12,973.

Pollard., R.T., (1970), Surface waves with rotation: An exact solution. J. Geophys. Res., 75: 5895-5898.

Pollard, R.T., P.B. Rhines, and R.O.R.Y. Thompson, (1973), The deepening of the wind-mixed layer, Geophys. Fluid Dyn., 3: 381-404. 
Plueddemann, A.J., J.A. Smith, D.M. Farmer, R.A. Weller, W.R. Crawford, R. Pinkel, S. Vagle, and A. Gnanadesikan, (1994), From drifters to Dopplers, Measurements of Langmuir circulation during the Surface Waves Processes Program, submitted J. Geophys. Res.

Price, J.F., R.A. Weller, and R. Pinkel, (1986), Diurnal cycling: Observations and models of the upper ocean response to diurnal heating, cooling and wind mixing, J. Geophys. Res., 91: 8411-8427.

Price, J.F., R.A. Weller, and R.R. Schudlich, (1987), Wind-driven ocean currents and Ekman transport, Science, 288: 1534-1538.

Price, J.F., R.A. Weller, C.M. Bowers, and M.G. Briscoe, (1987), Diurnal response of the sea-surface temperature observed at the Long-term Upper Ocean Study $\left(34^{\circ} \mathrm{N} 70^{\circ} \mathrm{W}\right)$ in the Sargasso Sea, J. Geophys. Res., 92: 14,480-14,490.

Roache, P.J. (1977), Computational Fluid Dynamics, Hermosa Publishers, Albequerque, NM., 434 pp.

Robinson, S.K., (1991), Coherent motions in the turbulent boundary layer, Ann. Rev. Fluid Mech., 23: 601-639.

Rosenhead, L., (1929), The Karman street of vortices in a channel of finite breadth, Phil. Trans. Roy. Soc. Lon., 228: 275-330.

Ryanzhin,S. V., (1983), The kinematics of horizontal flows in circulating Langmuir cells, Isvestiya, Atmos. and Oceanic Phys., 18: 41-46.

Salmon, R., (1980), Baroclinic instability and geostrophic turbulence, Geophys. Astrophys. Fluid Dyn., 15: 167-211.

Scott, J. T., G. E. Myer, R. Stewart, and E. G. Walther, (1969), On the mechanism of Langmuir circulations and their role in epilimnion mixing, Limnol. Oceanogr., 14:493-503.

Smith, J., (1993), Observations of Langmuir Circulations, waves, and the mixed layer, in Proc. Air-Sea Interaction Symposium, Marseilles, France.

Smith, J., R.A. Weller, and R. Pinkel, (1987) Velocity structure in the mixed layer during MILDEX, J. Phys. Oceanogr., 17: 425-439.

Stramma, L., P.Cornillon, R.A. Weller, J.F. Price, and M.G. Briscoe, (1986), Large diurnal sea-surface termperature variability: Satellite and in situ measurements. J. Phys. Oceanogr., 16: 827-837.

Sutcliffe, W.H., E. R. Baylor, and D. W. Menzel, (1963), Sea surface chemistry and Langmuir circulations, Deep Sea Res., 10:233-243. 
Thorpe, S.A., (1984), The effect of Langmuir circulation on the distribution of submerged bubbles caused by breaking wind waves, J. Fluid Mech., 142: 151170.

Thorpe, S. A., (1992), The breakup of Langmuir circulation and the instability of an array of vortices, J. Phys. Oceanogr., 16: 1462-1478.

Ursell, F., (1950), On the theoretical form of ocean swell on a rotating earth, Mon. Not. Roy. Astron. Soc. Geophys. Suppl. 6: 1-8.

Van Straaten, L. M. J. J., (1950), Periodic patterns of rippled and smooth areas on water surfaces, induced by wind action, K. Ned. Akad. Wet. Ser. B, 53:2-12.

Weber, J.E., (1983), Steady wind and wave-induced currents in the open ocean, $J$. Phys. Oceanogr., 13: 524-530.

Weller, R.A. (1978), Observations of horizontal velocity in the upper ocean made with a new vector-measuring current meter, Ph.D. Thesis, University of California at San Diego.

Weller, R.A., (1981), Observations of the velocity response to wind forcing in the upper ocean, J. Geophys. Res., 86:1969-1977.

Weller, RA., J.P. Dean, J. Marra, J.F. Price, E.A. Francis, and D.C. Boardman, (1985), Three-dimensional flow in the upper ocean, Science, 227: 1552-1556.

Weller, R.A., M.A. Donelan, M.G. Briscoe, and N.E. Huang, (1990), Riding the crest: A tale of two wave experiments, Bull. Amer. Met. Soc., 72: 163-183.

Weller, R.A. and A.J. Plueddemann, (1994) Observations of the mean vertical structure of the oceanic boundary layer, submitted, J. Geophys. Res.

Weller, R.A., and J. F. Price, (1988), Langmuir circulations in the oceanic surface layer, Deep Sea Res., 35: 711-747.

Woodcock, A.H., (1944), A theory of surface water motion deduced from the wind-induced motion of the Physalia, J. Mar. Res., 9:77-92..

Wu, J., (1975), Wind-induced drift currents, J. Fluid Mech., 68: 49-70.

Yamazaki T. and R.G., Lueck, (1987), Turbulence in the California Undercurrent, J. Phys. Oceanogr., 17: 1378-1396.

Zedel, L., and D. Farmer, (1991), Organized structures in subsurface bubble clouds: Langmuir circulation in the open ocean, J. Geophys. Res., 91:(C5) 8889-8900. 


\section{Vita}

\section{Anand Gnanadesikan}

Born January 13, 1967 in Summit, N.J. Graduated the Pingry School, June, 1984. Attended Princeton University, majoring in Physics. Summer Student Fellow at Woods Hole Oceanographic Institution, Summer, 1986. Graduated with high honors, Sigma Xi, Phi Beta Kappa, in June, 1988. Married Amalia Elisabeth Bachman in June, 1990. Daughter Gitanjali Elisabeth, born June, 1992.

Publications:

Cook, K.H. and A. Gnanadesikan, 1991, Effect of saturated and dry land surfaces on the tropical circulation and precipitation in a general circulation model, J.Climate, 4: 873-889.

Galbraith, N.R., A. Gnanadesikan, G.H. Tupper, B.S. Way, and E. A. Terray, 1994, Meteorological and Oceanographic Measurements Taken During the 1991 Acoustic Surface Reverberation Experiment, Technical Report, Woods Hole Oceanographic Inst., Woods Hole, MA in press.

Gnanadesikan, A.,1994, Comment, Statistical Science, 9: 208-212.

Effects of mixing on the diurnal cycle of photochemically produced trace species: Special focus on carbon monoxide, 1994, submitted, J. Geophys. Res.

, and E.A. Terray, 1994, A comparison of three wave-measuring buoys, in O.T Magoon and J. M. Hemsley, eds., Ocean Wave Measurement and Analysis, New York, Amer. Soc. Civ. Eng., pp 287-301.

Park, Y.-G., J.A. Whitehead, and A. Gnanadesikan, 1994, Mixing in a Stratified Fluid: Energetics and Layer Formation, in press, J. Fluid Mech.

Plueddemann, A.J., J.A. Smith, D.M. Farmer, R.A. Weller, W.R. Crawford, R. Pinkel, S. Vagle, and A. Gnanadesikan, 1994, From drifters to Dopplers, Measurements of Langmuir circulation during the Surface Waves Processes Program, submitted J. Geophys. Res.

Weller, R.A., A. Gnanadesikan, A.J. Plueddemann, and M. Park-Samelson, 1993, Langmuir Circulations and their effect on the oceanic surface layer, Preprint Volume, Ninth Conference on Waves and Stability, American Meteorological Society. 